\title{
Domain Decomposition Methods for Uncertainty Quantification
}

\author{
A thesis submitted to \\ the Faculty of Graduate and Postdoctoral Affairs \\ in partial fulfillment of the requirements for the degree \\ Doctor of Philosophy \\ by \\ Waad Subber \\ Department of Civil and Environmental Engineering \\ Carleton University \\ Ottawa-Carleton Institute of Civil and Environmental Engineering \\ November 2012 \\ (C)2012 - Waad Subber
}


Library and Archives

Canada

Published Heritage

Branch

395 Wellington Street

Ottawa ON K1A ON4

Canada
Bibliothèque et

Archives Canada

Direction du

Patrimoine de l'édition

395 , rue Wellington

Ottawa ON K1A ON4

Canada
Your file Votre référence

ISBN: 978-0-494-94227-7

Our file Notre référence

ISBN: $978-0-494-94227-7$
NOTICE:

The author has granted a nonexclusive license allowing Library and Archives Canada to reproduce, publish, archive, preserve, conserve, communicate to the public by telecommunication or on the Internet, loan, distrbute and sell theses worldwide, for commercial or noncommercial purposes, in microform, paper, electronic and/or any other formats.

The author retains copyright ownership and moral rights in this thesis. Neither the thesis nor substantial extracts from it may be printed or otherwise reproduced without the author's permission.
AVIS:

L'auteur a accordé une licence non exclusive permettant à la Bibliothèque et Archives Canada de reproduire, publier, archiver, sauvegarder, conserver, transmettre au public par télécommunication ou par l'Internet, prêter, distribuer et vendre des thèses partout dans le monde, à des fins commerciales ou autres, sur support microforme, papier, électronique et/ou autres formats.

L'auteur conserve la propriété du droit d'auteur et des droits moraux qui protege cette thèse. $\mathrm{Ni}$ la thèse ni des extraits substantiels de celle-ci ne doivent être imprimés ou autrement reproduits sans son autorisation.
In compliance with the Canadian Privacy Act some supporting forms may have been removed from this thesis.

While these forms may be included in the document page count, their removal does not represent any loss of content from the thesis.
Conformément à la loi canadienne sur la protection de la vie privée, quelques formulaires secondaires ont été enlevés de cette thèse.

Bien que ces formulaires aient inclus dans la pagination, il n'y aura aucun contenu manquant. 


\begin{abstract}
In conjunction with modern high performance computing platforms, domain decomposition methods permit computational simulations of physical and engineering systems using extremely high resolution numerical models. Such numerical models substantially reduce discretization error. In realistic simulations of numerous physical and engineering systems, it is however necessary to quantify confidence in numerical simulations for their acceptability as reliable alternatives to field experiments. The spectral stochastic finite element method is a popular computational tool for uncertainty quantification in many engineering systems of practical interest. For large-scale engineering systems, the spectral stochastic finite element method becomes computationally challenging as the size of the resulting algebraic linear system grows rapidly with the spatial mesh resolution and the order of the stochastic dimension. To address this issue, domain decomposition methods are described for uncertainty quantification of large- scale engineering systems. The mathematical framework is based on non-overlapping domain decomposition in the geometric space and functional expansion along the stochastic dimension. For numerical illustrations, two-dimensional and three-dimensional flow through porous media and elasticity problems with spatially varying non-Gaussian material properties are considered. The parallel performance of the algorithms are investigated with respect to the mesh size, subdomain size, fixed problem size per subdomain, level of uncertainty and the order of stochastic dimen-
\end{abstract}


sion. For a fixed mesh resolution, the probabilistic domain decomposition techniques cannot reduce the total solution time beyond a certain number of processors due to hardware related issues such as latency and bandwidth of the communication network. To fully exploit the massively parallel computers, parallelization is exploited in temporal dimension for uncertain dynamical systems. In particular, a probabilistic version of a parallel time integration scheme is developed to simulate the trajectories of a noisy non-linear dynamical system described by stochastic differential equations. The numerical experiments are performed on a Linux cluster using MPI and PETSc parallel libraries. 
Dedicated to the living memory of my beloved Mother. 
"Finding a good preconditioner to solve a given sparse linear system is often viewed as a combination of art and science."

Yousef Saad, Iterative methods for sparse linear systems, 2003. 


\section{Acknowledgments}

I would like to express my sincere gratitude and appreciation to my thesis advisor Professor Abhijit Sarkar for his continued guidance and support throughout this research. Without his assistance and inspiration, I could not reach this far. I am very grateful to him for introducing me to the world of Uncertainty Quantification and High Performance Computing. I would like to deeply thank my friend and colleague Mohammad Khalil for the invaluable discussions, support and encouragement during the course of this study. I sincerely thank Professor John Goldak who has profoundly contributed to my knowledge and understanding of the finite element method. I am grateful to Professor Dominique Poirel for given me the opportunity to learn the fundamentals of computational fluid dynamics. Furthermore, I would like to thank Professor Heng Khoo and Professor Edward Sherwood for their helpful discussions and support throughout my graduate studies. Thanks and gratitude are also due to my fellow graduate students and friends especially Anton Matachniouk and Amin Fereidooni with whom I had wonderful times. Very special thanks to our Graduate Administrator Mrs. Payal Chadha for continuous and kind support during my graduate career. Finally, I would like to extend my gratitude and appreciation to my family who I am indebted forever for their support and encouragement in all of my accomplishments. 


\section{Citations}

Chapter 3 closely follows the following references:

1. A. Toselli and O. Widlund. Domain Decomposition Methods - Algorithms and Theory, volume 34 of Springer Series in Computational Mathematics. Springer, Berlin, 2005 .

2. B. Smith, P. Bjorstad, and W. Gropp. Domain Decomposition: Parallel Multilevel Methods for Elliptic Partial Differential Equations. Cambridge University Press, New York, 1996.

3. T. Mathew. Domain Decomposition Methods for the Numerical Solution of Partial Differential Equations. Number 61 in Lecture Notes in Computational Science and Engineering. Springer, Berlin, 2008.

4. A. Quarteroni and A. Valli. Domain Decomposition Methods for Partial Differential Equations. Numerical Mathematics and Scientific Computation. Oxford University Press, USA, 1999.

Chapters 4 and 5 are closely based on the following references:

1. W. Subber and A. Sarkar. Domain decomposition method of stochastic PDEs: An iterative solution technique using one-level parallel preconditioner. 2012. Submitted to Journal of Probabilistic Engineering Mechanics.

2. W. Subber and A. Sarkar. Domain decomposition of stochastic PDEs: A novel preconditioner and its parallel performance. In High Performance Computing Symposium, volume 5976 of Lecture Notes in Computer Science, pages 251268. Springer, 2010 .

3. W. Subber, H. Monajemi, M. Khalil, and A. Sarkar. A scalable parallel uncertainty analysis and data assimilation framework applied to some hydraulic problems. In International Symposium on Uncertainties in Hydrologic and Hydraulic. Montreal, Canada, 2008.

Chapter 6 is closely based on the following references:

1. W. Subber and A. Sarkar. A domain decomposition method of stochastic PDEs: An iterative solution techniques using a two-level scalable preconditioner. 2012. Submitted to Journal of Computational Physics.

2. W. Subber and A. Sarkar. Domain decomposition methods of stochastic PDEs: A two-level scalable preconditioner. Journal of Physics: Conference Series, 341(1), 2012. 
Chapter 7 is closely based on the following references:

1. W. Subber and A. Sarkar. Primal and dual-primal iterative substructuring methods of stochastic PDEs. Journal of Physics: Conference Series, 256(1), 2010.

2. W. Subber and A. Sarkar. Domain decomposition methods of stochastic PDEs. In The Twentieth International Conference on Domain Decomposition Methods (DD20). California, USA, 2011. Accepted.

Chapter 8 is closely based on the following references:

1. W. Subber and A. Sarkar. Dual-primal domain decomposition method for uncertainty quantification. 2012. To be Submitted to Journal of Computer Methods in Applied Mechanics and Engineering.

2. W. Subber and A. Sarkar. Primal and dual-primal iterative substructuring methods of stochastic PDEs. Journal of Physics: Conference Series, 256(1), 2010.

3. W. Subber and A. Sarkar. Domain decomposition methods of stochastic PDEs. In The Twentieth International Conference on Domain Decomposition Methods (DD20). California, USA, 2011. Accepted.

Chapter 9 is closely based on the following references:

1. W. Subber and A. Sarkar. Domain decomposition method of stochastic PDEs: An iterative solution technique using one-level parallel preconditioner. 2012. Submitted to Journal of Probabilistic Engineering Mechanics.

2. W. Subber and A. Sarkar. A domain decomposition method of stochastic PDEs: An iterative solution techniques using a two-level scalable preconditioner. 2012. Submitted to Journal of Computational Physics.

3. W. Subber and A. Sarkar. Dual-primal domain decomposition method for uncertainty quantification. 2012. To be Submitted to Journal of Computer Methods in Applied Mechanics and Engineering.

Chapter 10 is closely based on the following references:

1. W. Subber and A. Sarkar. Two-level domain decomposition methods for uncertainty quantification. 2013. Submitted to 54th AIAA/ASME/ASCE/AHS/ASC Structures, Structural Dynamics, and Materials Conference.

2. W. Subber and A. Sarkar. Scalable Two-Level Domain Decomposition Algorithms for Stochastic Systems. 2013. Submitted to 11th International Conference on Structural Safety and Reliability. 
Chapter 11 is closely based on the following references:

1. W. Subber and A. Sarkar. Performance of a parallel time integrator for noisy nonlinear system. In Non-Deterministic Approaches Forum, 49th AIAA/ASME/ASCE/AHS/ASC Structures, Structural Dynamics, and Materials Conference. Paper no: AIAA-20081924, 2008.

2. W. Subber and A. Sarkar. A parallel time integrator for noisy nonlinear system. Journal of Probabilistic Engineering Mechanics, 2008. Accepted pending revision. 


\section{Contents}

1 Introduction 1

1.1 Background ........................... 1

1.2 Statement of the Problem . . . . . . . . . . . . . . 6

1.3 Research Objective and Scope . . . . . . . . . . . . . . . . . 9

1.4 Organization of the Thesis . . . . . . . . . . . . . 14

2 Finite Element Method for Stochastic PDEs 15

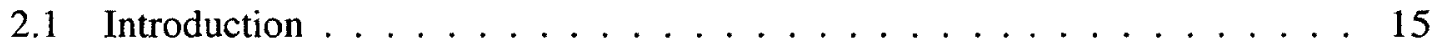

2.2 Uncertainty Representation by Stochastic Processes . . . . . . . . . . . 17

2.2.1 Karhunen-Loeve Expansion . . . . . . . . . . . . . . 17

2.2.2 Polynomial Chaos Expansion _............... 18

2.3 Spectral Stochastic Finite Element Method . . . . . . . . . . . . . 19

2.3.1 Galerkin (Intrusive) Approach . . . . . . . . . . . 20

2.3.2 Pseudo-Spectral (Non-Intrusive) Approach $\ldots \ldots \ldots . . \ldots 21$

2.3.3 Intrusive Versus Non-Intrusive . . . . . . . . . . . 21

2.4 Solution Methods for the SSFEM . . . . . . . . . . . . . . . 22 
2.4.1 Literature Review . . . . . . . . . . . . . . . . 22

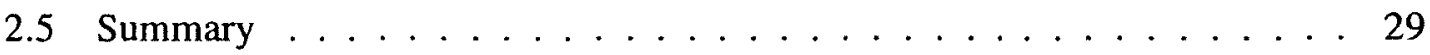

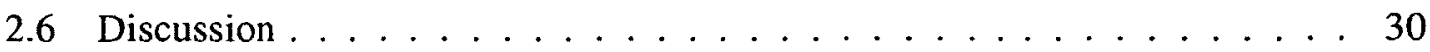

3 Domain Decomposition Methods for Deterministic PDEs 31

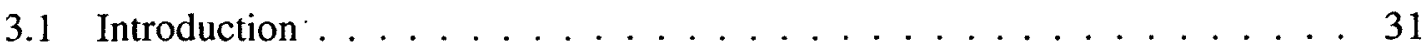

3.2 Overlapping Domain Decomposition Methods . . . . . . . . . . . . . . . 34

3.2.1 The Alternating Schwarz Method . . . . . . . . . . . 35

3.2.2 Schwarz Method as a Two-Steps Algorithm . . . . . . . . . . . 40

3.2.3 Schwarz Method as a Richardson's Iteration . . . . . . . . . . . 42

3.2.4 Schwarz Method as a Preconditioner . . . . . . . . . . . . . 45

3.2.5 Schwarz Method for Many Subdomains . . . . . . . . . . . . . . 47

3.3 Non-Overlapping Domain Decomposition Methods . . . . . . . . . . . . . 48

3.3.1 Primal Iterative Substructuring Method $\ldots \ldots . \ldots 53$

3.3.2 Primal-Primal Iterative Substructuring Method . . . . . . . . 82

3.3.3 Dual Iterative Substructuring Method . . . . . . . . . . . . . 89

3.3.4 Dual-Primal Iterative Substructuring Method . . . . . . . . . . 96

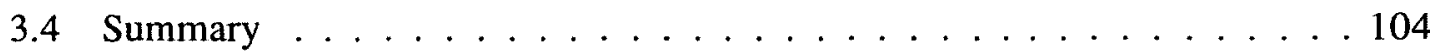

4 Domain Decomposition Methods for Stochastic PDEs 107

4.1 Introduction . . . . . . . . . . . . . . . 107

4.2 Mathematical Formulation . . . . . . . . . . . . . . . . 108

4.3 The Extended Schur Complement Matrix _. . . . . . . . . . . . . 112

4.4 Iterative Substructuring Methods for Stochastic PDEs . . . . . . . . . . . 113 
4.4.1 Iterative Solution of the Interface Problem .......... 115

4.4.2 Parallel Matrix-Vector Product . . . . . . . . . . . . . 118

4.4.3 Parallel Preconditioner Effect . . . . . . . . . . . . . 120

4.4.4 Parallel Preconditioned Conjugate Gradient Method . . . . . . . . 121

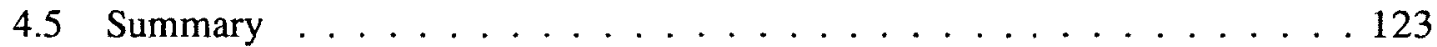

5 One-Level Preconditioners for Stochastic PDEs 124

5.1 Introduction . . . . . . . . . . . . . . . . 124

5.2 Extended Lumped Preconditioner . . . . . . . . . . . . . 125

5.2.1 Parallel Implementation $\ldots \ldots . \ldots . \ldots . \ldots . \ldots$

5.3 Extended Weighted Lumped Preconditioner . . . . . . . . . . . . 126

5.3.1 Parallel Implementation . . . . . . . . . . . . . . 127

5.4 Extended Neumann-Neumann Preconditioner . . . . . . . . . . 128

5.4.1 Parallel Implementation . . . . . . . . . . . . . 132

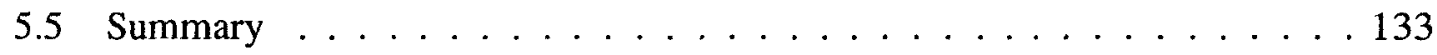

6 Two-Level Preconditioner for Stochastic PDEs 134

6.1 Introduction . . . . . . . . . . . . . . . . 134

6.2 Solution of the Interface Problem using Two-Level Preconditioner . . . . 135

6.3 Extended Two-Level Preconditioner . . . . . . . . . . . . . . 140

6.4 Parallel Implementation . . . . . . . . . . . . . . . . . . 145

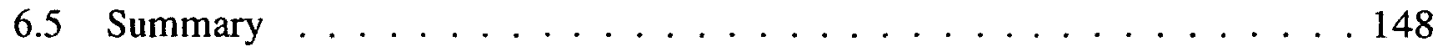

7 Primal-Primal Domain Decomposition Method for Stochastic PDEs 150

7.1 Introduction . . . . . . . . . . . . . 150 
7.2 Primal-Primal Method for Stochastic PDEs . . . . . . . . . . . . 151

7.3 Primal-Primal Preconditioner . . . . . . . . . . . . . 155

7.4 Parallel Implementation . . . . . . . . . . . . . . . . . 158

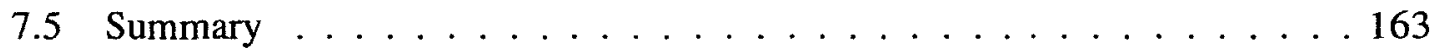

8 Dual-Primal Domain Decomposition Method for Stochastic PDEs 164

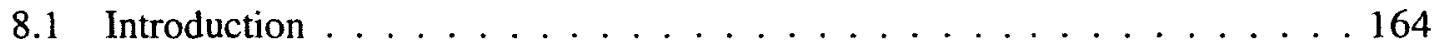

8.2 Dual-Primal Domain Decomposition Method for SPDEs . . . . . . . . 165

8.3 Preconditioners for the Probabilistic FETI-DP . . . . . . . . . . . 172

8.4 Parallel Implementation . . . . . . . . . . . . . . . . 173

8.5 Summary . . . . . . . . . . . . . . . 177

9 Numerical Results: Two-Dimensional Problems 179

9.1 Introduction . . . . . . . . . . . . . . . . 179

9.2 One-Level Preconditioner . . . . . . . . . . . . . . . . 182

9.2.1 Flow Through Random Media . . . . . . . . . . . . . . 182

9.3 Two-Level Preconditioner . . . . . . . . . . . . . . 200

9.3.1 Flow Through Random Media . . . . . . . . . . . . 201

9.3.2 Two-Dimensional Elasticity . . . . . . . . . . . . 206

9.4 Dual-Primal Domain Decomposition Method . . . . . . . . . . . . . 214

9.4.1 Flow Through Random Media and Elasticity Problems . . . . . . 215

9.5 Summary . . . . . . . . . . . . . . . . 221

10 Numerical Results: Three-Dimensional Problems 223

10.1 Introduction . . . . . . . . . . . . . . . 223

xiii 
10.2 Three-Dimensional Flow Through Random Media . . . . . . . . . . . 225

10.2.1 Stochastic Features of the Solution Field . . . . . . . . . 235

10.2.2 Numerical Scalability . . . . . . . . . . . . . . . . 241

10.2.3 Parallel Scalability . . . . . . . . . . . . . 245

10.3 Application to Large-Scale Heat Transfer Problem . . . . . . . . . . . . 247

10.3.1 Finite Element Discretization and Mesh Decomposition . . . . 248

10.3.2 Stochastic Field Representation _ . . . . . . . . . . . . 248

10.3.3 Stochastic Features of the Solution Field . . . . . . . . . 252

10.3.4 Numerical Scalability . . . . . . . . . . . . . 256

10.3.5 Parallel Scalability . . . . . . . . . . . . . 259

10.3.6 Performance Study for Large-Scale Systems _. . . . . . . . . 261

10.4 Three-Dimensional Elasticity Problem . . . . . . . . . . . . 261

10.5 Summary . . . . . . . . . . . . . . . . . . . 269

11 Time-Domain Decomposition for Stochastic ODEs 270

11.1 Introduction . . . . . . . . . . . . . . 270

11.2 Numerical Solution of Ito SDEs . . . . . . . . . . . . . . . . 272

11.3 Parareal Algorithm for SDEs . . . . . . . . . . . . . . . . . 274

11.4 Parallel Implementation . . . . . . . . . . . . . . . . 278

11.5 Numerical Results . . . . . . . . . . . . . . . . . . . . . . . 281

11.6 Parallel Performance . . . . . . . . . . . . . . . 301

11.7 Summary . . . . . . . . . . . . . . . . . . 307

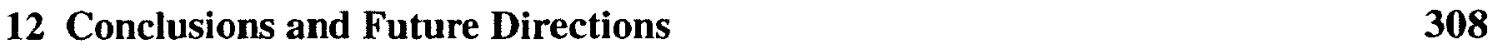


12.1 Summary of Findings . . . . . . . . . . . . . 308

12.2 Directions for Future Research . . . . . . . . . . . . . . . 313

Appendices

A Uncertainty Representation by Stochastic Processes $\quad 317$

B Polynomial Chaos Expansion of Lognormal Stochastic Process 325

C Spectral Stochastic Finite Element Method 330

D Domain Decomposition Methods for SPDEs 334

$\begin{array}{lll}\text { E Numerical Validation } & 340\end{array}$

F Comparison among Jacobi, Block-Jacobi and Lumped Preconditioners 348

$\begin{array}{ll}\text { References } & 352\end{array}$ 


\section{List of Figures}

$3.1 \quad$ A typical domain $\ldots \ldots \ldots \ldots \ldots \ldots \ldots \ldots \ldots$

3.2 A typical domain partitioned into two overlapping subdomains . . . . . 36

3.3 A typical domain partitioned into $n_{s}$ non-overlapping subdomains $\ldots . . .49$

3.4 Partitioning the subdomain nodes into: interior ( ), remaining ( $\boldsymbol{\square})$ and

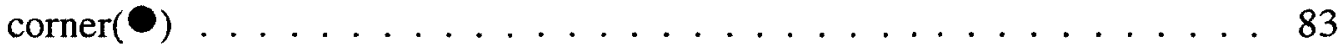

3.5 The interface boundary nodes split into: remaining $(\mathbf{\theta})$ and corner $(\bullet)$ nodes 85

3.6 Partial assembly of corner nodes $(\bullet) \ldots \ldots \ldots 7$

3.7 Lagrange multiples are the force required to enforce continuity constraint . 94

3.8 Lagrange multipliers are the forces required to connect the tore interface

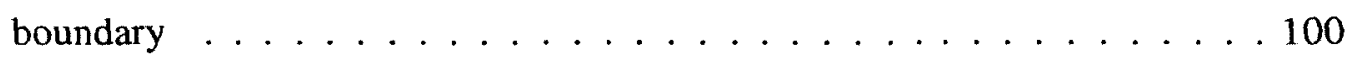

4.1 Partitioning the subdomain nodes into: interior ( ) and interface ( $\mathbf{0})$. 109

4.2 The sparsity structure of the stochastic block matrices . . . . . . . . 112

4.3 Flow chart of iterative substructuring technique . . . . . . . . 123

6.1 Partitioned interface nodes into remaining $(\boldsymbol{G})$ and corner $(\bullet)$ nodes. . . . 141

6.2 Flow chart of parallel implementation of the two-level extended NeumannNeumann algorithm . . . . . . . . . . . . . . . 148 
7.1 Partitioning the subdomain nodes into: interior $(\vartheta)$, remaining ( $\square$ ) and

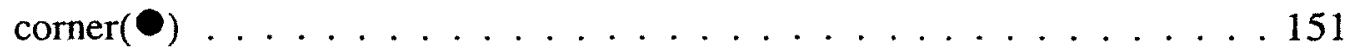

7.2 Fully assembled system: remaining $(\mathbb{})$ and corner $(\bullet)$ nodes . . . . . . . 154

7.3 Partial assembly of the corner nodes $(0) \ldots \ldots \ldots 5$

7.4 Flow chart of parallel implementation of the primal-primal method . . . . 163

8.1 Partitioning the boundary nodes into: remaining $(\boldsymbol{\square})$ and corner $(\bullet)$ nodes $\quad 167$

8.2 Lagrange multipliers: enforcing continuity of the polynomial chaos coefficients of the solution process ................. 171

8.3 Flow chart of parallel implementation of FETI-DP algorithm . . . . . . 177

9.1 Finite element discretization and mesh decomposition . . . . . . . . . . 183

9.2 The decay of the eigenvalues of the exponential covariance function . . . 184

9.3 The first fourth eigenfunctions of the exponential covariance function . . . 185

9.4 Selected PC coefficients of the hydraulic conductivity . . . . . . . . 186

9.5 The mean and the standard deviation of the hydraulic head . . . . . . 187

9.6 Selected PC coefficients of the hydraulic head . . . . . . . . . . . 188

9.7 The history of the relative PCGM residual . . . . . . . . . . . . . 189

9.8 Iteration counts with respect to the $\mathrm{CoV} \ldots \ldots \ldots$

9.9 Iteration counts with respect to mesh size for fixed number of subdomains: Different order of PCE . . . . . . . . . . . . . . . . 192

9.10 Iteration counts with respect to mesh size for fixed number of subdomains: Different preconditioners . . . . . . . . . . . . . . 193 
9.11 Iteration counts with respect to the number of subdomains for fixed problem size: Different order of PCE . . . . . . . . . . . . . . . 194

9.12 Iteration counts with respect to the number of subdomains for fixed problem size: Different preconditioners . . . . . . . . . . . . 195

9.13 Iteration counts with respect to the number of subdomains for fixed problem size per subdomain: Different order of PCE . . . . . . . . . . 196

9.14 Iteration counts with respect to the number of subdomains for fixed problem size per subdomain: Different preconditioners . . . . . . . . . . 197

9.15 Strong scalability . . . . . . . . . . . . . . . . . . . . 199

9.16 Weak scalability . . . . . . . . . . . . . . . . 200

9.17 The history of the relative PCGM residual . . . . . . . . . . . 201

9.18 Iteration counts versus mesh size for fixed number of subdomains . . . . 202

9.19 Iteration counts versus the number of subdomains for fixed problem size . 203

9.20 Iteration counts versus the number of subdomains for fixed problem size per subdomain . . . . . . . . . . . . . . . . . . 204

9.21 Strong scalability . . . . . . . . . . . . . . . . . . . 204

9.22 Weak scalability . . . . . . . . . . . . . . . . 205

9.23 The geometry of the two-dimensional plane-stress problem (all dimensions

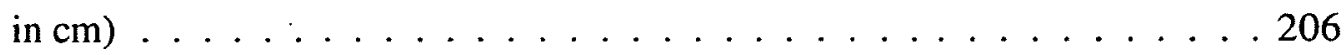

9.24 Selected PC coefficients of the Young's modules . . . . . . . . . . . . . 208

9.25 Finite element discretization and domain decomposition . . . . . . 209

9.26 The mean and standard deviation of the magnitude of the displacement field 209

9.27 Selected PC coefficients of the magnitude of the displacement field . . . . 210 
9.28 Iteration counts versus the order of PCE . . . . . . . . . 211

9.29 Iteration counts versus mesh size for fixed number of subdomains . . . . . 212

9.30 Iteration counts versus the number of subdomains for fixed problem size . . 212

9.31 Iteration counts versus the number of subdomains for fixed problem size per subdomain . . . . . . . . . . . . . . . . . . 213

9.32 Strong scalability . . . . . . . . . . . . . . . . . . 214

9.33 Weak scalability . . . . . . . . . . . . . . . . . 214

9.34 The history of the relative PCGM residual . . . . . . . . . . 215

9.35 Iteration counts with respect to the order of PCE . . . . . . . 216

9.36 Iteration counts with respect to mesh size for fixed number of subdomains .217

9.37 Iteration counts with respect to number of subdomains for fixed mesh size . 218

9.38 Iteration counts with respect to the number of subdomains for fixed problem size per subdomain . . . . . . . . . . . . . . . . . . 219

9.39 Strong scalability . . . . . . . . . . . . . . . . . 220

9.40 Weak scalability . . . . . . . . . . . . . . . 221

10.1 Finite element discretization and mesh decomposition . . . . . . . . . 226

10.2 Eigenvalues $\lambda_{i}$ of the covariance function $\ldots \ldots \ldots . \ldots 227$

10.3 Selected eigenfunctions: $b_{x}=b_{y}=b_{z}=1 \ldots \ldots 228$

10.4 Selected eigenfunctions: $b_{x}=b_{y}=b_{z}=10 \ldots . \ldots . \ldots 229$

10.5 Selected PC coefficients of the hydraulic conductivity: $b_{x}=b_{y}=b_{z}=1.232$

10.6 Selected PC coefficients of the hydraulic conductivity: $b_{x}=b_{y}=b_{z}=10.233$

10.7 Samples of the random hydraulic conductivity field: $b_{x}=b_{y}=b_{z}=1 \ldots 234$

10.8 Samples of the random hydraulic conductivity field: $b_{x}=b_{y}=b_{z}=10 \ldots 235$ 
10.9 The mean and standard deviation of the hydraulic head for different order

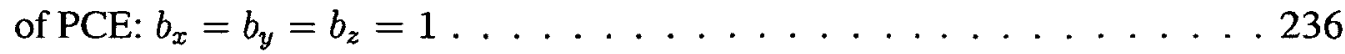

10.10The mean and standard deviation of the hydraulic head for different order of PCE: $b_{x}=b_{y}=b_{z}=10 \ldots 237$

10.11 Selected PC coefficients of the hydraulic head: $b_{x}=b_{y}=b_{z}=1 \ldots 239$

10.12 Selected PC coefficients of the hydraulic head: $b_{x}=b_{y}=b_{z}=10 \ldots 240$

10.13 Iteration counts versus the order of PCE . . . . . . . . . . 241

10.14Running time versus the order of PCE . . . . . . . . . 242

10.15Iteration counts with respect to mesh size for fixed number of subdomains . 243

10.16Iteration counts with respect to number of subdomains for fixed problem size 244

10.17Iteration counts with respect to number of subdomains for fixed problem

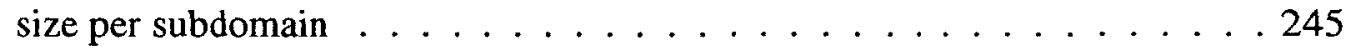

10.18Strong scalability . . . . . . . . . . . . . 246

10.19 Weak scalability . . . . . . . . . . . . . . . . 246

10.20The geometry of the crankshaft (all dimensions in $\mathrm{cm}$ ) . . . . . . 247

10.21 Finite element discretization and mesh decomposition . . . . . . . . . . 248

10.22The first fourth eigenfunctions of the covariance function $\ldots \ldots 250$

10.23 Selected PC coefficients of the diffusivity field on the extended domain . . 251

10.24 Selected PC coefficients of the diffusivity field on the original domain . . 252

10.25The mean and the standard deviation of the temperature field . . . . . 253

10.26Selected PC coefficients of the temperature field . . . . . . . . 254

10.27Lagrange multipliers corresponding to the first four PC coefficient of the

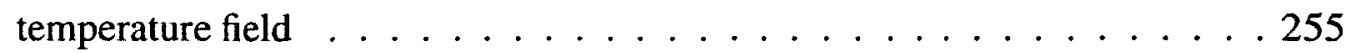


10.28Iteration counts versus the order of PCE . . . . . . . . . 256

10.29Iteration counts with respect to mesh size for fixed number of subdomains $\quad 257$

10.30Iteration counts with respect to the number of subdomains for fixed prob-

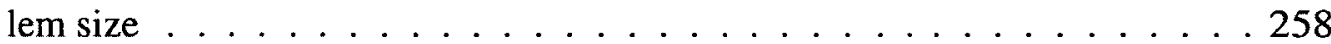

10.31 Iteration counts with respect to the number of subdomains for fixed prob-

lem size per subdomain . . . . . . . . . . . . . . . 259

10.32Strong scalability . . . . . . . . . . . . . 260

10.33 Weak scalability . . . . . . . . . . . . . . . 260

10.34The geometry of three-dimensional cantilever beam (all dimensions in $\mathrm{m}$ ) .262

10.35The mean and standard deviation of the displacement field $\ldots \ldots 265$

10.36 Selected chaos coefficients of the displacement field . . . . . . . . 266

10.37Selected Lagrange multipliers for the chaos coefficients of the displace-

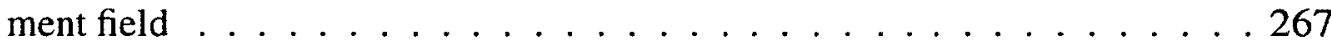

10.38The interface boundary $\Gamma$ in three dimensions: Vertices $(\bullet)$, Edges $(-)$ and Faces $(\equiv) \ldots \ldots \ldots \ldots$. . . . . . . . . . . . . . . . . . . . .

11.1 Schematic Diagram: $S_{i}$ is the time slice $i$ and $X\left(\omega_{j}, t\right)$ denotes $j$-th realization 275

11.2 Performance of The Stochastic Parareal Integrator: $\Gamma=0.3, \sigma=0 \ldots . .284$

11.3 Convergence of the parareal algorithm $(k=1, k=3, k=5, k=23$, $k=35$ and $k=59$ ): $\Gamma=0.3, \sigma=0 \ldots \ldots \ldots . \ldots . \ldots . \ldots . \ldots . \ldots$

11.4 Samples of the response of the Duffing oscillator: $\Gamma=0.3, \sigma=0.1 \Gamma \ldots 287$

11.5 Samples of the response of the Duffing oscillator: $\Gamma=0.3, \sigma=0.5 \Gamma \ldots 289$

11.6 Samples of the response of the Duffing oscillator: $\Gamma=0.3, \sigma=0.9 \Gamma \ldots 291$

11.7 Performance of The Stochastic Parareal Integrator: $\Gamma=0.37, \sigma=0 \ldots 292$ 
11.8 Convergence of the parareal algorithm $(k=1, k=3, k=5, k=6$, $k=35$ and $k=59): \Gamma=0.37, \sigma=0 \ldots \ldots . \ldots . \ldots . \ldots 294$

11.9 Samples of the response of the Duffing oscillator: $\Gamma=0.37, \sigma=0.1 \Gamma \ldots 296$

11.10Samples of the response of the Duffing oscillator: $\Gamma=0.37, \sigma=0.5 \Gamma \ldots 298$

11.11 Samples of the response of the Duffing oscillator: $\Gamma=0.37, \sigma=0.9 \Gamma \ldots 300$

11.12Parallel scalability and speedup: $\Gamma=0.3 \ldots \ldots . \ldots$. . . . . . 304

11.13Parallel scalability and speedup: $\Gamma=0.37 \ldots \ldots \ldots 5$

A.1 The finite element mesh . . . . . . . . . . . . . . . . 319

A.2 Samples of the permeability measurements . . . . . . . . . . 320

A.3 Selected PC coefficients of the permeability random field . . . . . . . . 322

A.4 The deterministic solution field . . . . . . . . . . . . . 323

A.5 The mean and standard deviation of the solution process using PCE and MCS324

E.1 The decay of the eigenvalues of the covariance function $\ldots \ldots . \ldots 341$

E.2 Selected eigenfunctions of the covariance function . . . . . . . . 342

E.3 The mean and standard deviation of the solution process . . . . . . . 342

E.4 The decomposed domain $\ldots \ldots \ldots \ldots$. . . . . . . . . . . . . . . . . . . . .

E.5 The mean and standard deviation of the solution process . . . . . . . 344

E.6 The convergence of MCS and PCE . . . . . . . . . . . . . . 345

E.7 The total execution time of MCS and PCE . . . . . . . . . . . 345

E.8 The mean and standard deviation of the solution process $\ldots \ldots \ldots 346$

E.9 The convergence of MCS and PCE . . . . . . . . . . . . . . 347

E.10 The total execution time of MCS and PCE . . . . . . . . . . 347 
F.1 The mean and standard deviation of the solution process . . . . . . 350

F.2 Iteration counts for fixed $\mathrm{CoV}$ while increasing the order of PCE . . . 350

F.3 Iteration counts for fixed order of PCE while increasing the value of the

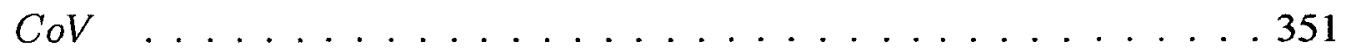




\section{List of Tables}

10.1 Iteration counts and timing for the probabilistic DP-DDM . . . . . . 261

10.2 Iteration counts and timing for $3 \mathrm{D}$ elasticity problem $\ldots \ldots 263$

11.1 Execution time in second for the parareal algorithm . . . . . . . . . 302

12.1 Connection among the two-level probabilistic Neumann-Neumann preconditioner, primal-primal preconditioner and dual-primal operator . . . . . . 312

B.1 The expectation and variance of the $\mathrm{PC}$ basis $\ldots \ldots . \ldots 328$ 


\section{List of Algorithms}

1 Alternating Schwarz Procedure $\ldots \ldots \ldots$

2 Iterative Substructuring Procedure . . . . . . . . . . . . 114

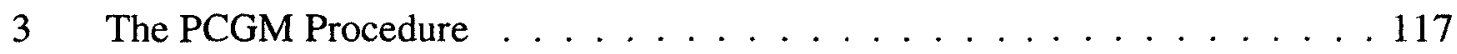

4 Parallel Matrix-Vector Product Procedure . . . . . . . . . . . . . . 119

5 Parallel Preconditioner Effect Procedure . . . . . . . . . . . . . . 120

6 The Parallel PCGM Procedure . . . . . . . . . . . . . . . . . . 122

7 Extended Lumped Preconditioner Effect Procedure . . . . . . . . . . . . 126

8 Extended Weighted Lumped Preconditioner Effect Procedure . . . . . . . 128

9 Extended Neumann-Neumann Preconditioner Effect Procedure . . . . . . 132

10 The Parallel PCGM Procedure . . . . . . . . . . . . . . . . . . . 137

11 Dirichlet-Solver Procedure . . . . . . . . . . . . . . . 138

12 Two-Level Neumann-Neumann Preconditioner Procedure . . . . . . . . 146

13 Neumann-Solver Procedure . . . . . . . . . . . . . . . . 147

14 Parallel Matrix-Vector Product for Primal-Primal Method . . . . . . . . 159

15 Dirichlet-Solver Procedure . . . . . . . . . . . . . . 160 
16 Primal-Primal Preconditioner Effect Procedure . . . . . . . . . . 161

17 Neumann-Solver Procedure ... . . . . . . . . . . . . . 162

18 Parallel Matrix-Vector Product for Dual-Primal Method . . . . . . . . . . 174

19 Neumann-Solver Procedure . . . . . . . . . . . . . . . 175

20 Dirichlet-Solver Procedure . . . . . . . . . . . . . . . 175

21 The Parareal Procedure . . . . . . . . . . . . . . . . 280 


\section{List of Acronyms}

PDEs Partial Differential Equations

SPDEs Stochastic Partial Differential Equations

SSFEM Spectral Stochastic Finite Element Method

MC Monte Carlo

PCGM Preconditioned Conjugate Gradient Method

MINRES Minimal Residual Method

KLE Karhunen-Loeve Expansion

PCE Polynomial Chaos Expansion

gPCE generalized Polynomial Chaos Expansion

DDM Domain Decomposition Method

L Lumped preconditioner

WL Wighted Lumped preconditioner

NN Neumann-Neumann preconditioner

NNC Neumann-Neumann with a Coarse problem

PP-DDM Primal-Primal Domain Decomposition Method

DP-DDM Dual-Primal Domain Decomposition Method 
BDDM Balancing Domain Decomposition Method

BDDC Balancing Domain Decomposition by Constraints

FETI Finite Element Tearing and Interconnecting

FETI-DP Dual-Primal Finite Element Tearing and Interconnecting

PETSc Portable, Extensible Toolkit for Scientific Computation

MPI Message Passing Interface

SRBM Stochastic Reduced Basis Method 


\section{Chapter 1}

\section{Introduction}

\subsection{Background}

High resolution numerical models can offer a cost-effective alternative to expensive physical experiments [1-3]. Aided by high performance computing platforms, parallel numerical algorithms such as domain decomposition methods permit numerical simulations with substantial model resolution and thereby significantly reduce the discretization error [4-7]. In modeling numerous practical engineering systems, the random heterogeneity of the model parameters can adversely affect the numerical prediction $[1,3,8-10]$. For realistic simulation, it is therefore necessary to quantify uncertainty in the numerical simulations to assess the confidence in the computer predictions $[3,9-11]$.

Uncertainty quantification involves investigating the discrepancy between a physical phenomenon and its computational simulations [12]. Uncertainty quantification methods aim to characterize such discrepancies in order to provide a confidence bound on the numer- 
ical predictions $[3,13-17]$. To this end, the probability theory offers a rich mathematical framework to represent uncertainty in terms of random variables and stochastic processes, provided sufficient information is available to statistically characterize the physical properties of the random medium $[1,9,11]$. Within the probabilistic framework, uncertainty propagation may be construed to be identifying the dependency of the solution process on the random variables and stochastic processes used to characterize uncertainty in the system parameters [3].

The probabilistic methods for uncertainty quantification in engineering can be broadly classified into sampling and non-sampling based approaches $[2,3,9,17,18]$. The choice of a specific methodology for the analysis and the selection of a particular numerical solution strategy depends on the nature of uncertainty and complexity of the problem $[1,2,9]$.

Monte Carlo (MC) simulation is a robust and straightforward method for uncertainty propagation through the system $[3,17,19]$. MC method involves repeatedly generating independent realizations of the uncertain system parameters based on their prescribed probability distributions $[3,8]$. For each of these realizations, the deterministic model is used in a black box fashion to generate the corresponding output. Statistical analysis on the ensemble of solution process (constructed from the realizations) is conducted to extract the desired statistical properties of the solution process. The rate of convergence of the MC method is independent of the number of the uncertain system parameters $[3,17]$. The slow convergence rate of $\mathrm{MC}$ method however, is the main limitation of this sampling technique $[3,17]$. MC method is an embarrassingly parallel algorithm. In practice however, 
MC simulation may become impractical for high resolution models where a single deterministic computation requires high computational effort $[20,21]$.

Alternative to $\mathrm{MC}$ sampling technique is the perturbation method. In the perturbation method, the solution process and the stochastic operator are expanded about their respective mean values using Taylor series $[9,11,17,22]$. Taylor series expansion in the perturbation method is limited to small coefficient of variation $[9,23]$. The perturbation method is often used to evaluate the first two moments of the solution process as for higher order moments the resulting expansion becomes difficult to handle in general $[9,23]$. For high level of uncertainty, more terms in Taylor series are required. While increasing the order of the expansion, secular terms (terms growing without bound) appear in the higher-order expansion [11]. These terms lead to divergence of the series expansion [11].

Another non-sampling method is the Neumann series expansion. In this approach, the inversion of the stochastic stiffness matrix is approximated using Neumann series $[8,11$, 22]. The advantage of the Neumann series expansion approach is that only a deterministic matrix needs to be inverted once $[9,20]$. Similar to the perturbation method, the Neumann expansion is often limited to problems with a small magnitude of uncertainty $[8,17,20]$.

Due to the high computational cost of MC simulation and the limitations of the perturbation and Neumann series expansion approaches, significant attention has been focused on the Spectral Stochastic Finite Element Method (SSFEM) $[2,11,24]$. The SSFEM has been applied successfully to a wide variety of problems including structural mechanics [25-28], 
computational fluid dynamics $[3,16,29,30]$, transport in porous medium $[25,31]$, fluidstructure interactions [32], chemistry [33, 34] biomedical engineering [35] and acoustic scattering [36].

In the context of SSFEM, randomness in the system is treated as an additional dimension to the problem [37] with an associated Hilbert space of real-valued second order random variables $[3,19,38]$. The solution process is represented by a functional expansion along the new dimension and the coefficients of the expansion are determined either through Galerkin (intrusive) or pseudo-spectral (non-intrusive) projection [30].

In the intrusive approach $[1,2,11,24,39,40]$, Galerkin projection onto the space spanned by orthogonal polynomial basis transforms the original stochastic problem into a coupled set of deterministic problems [41]. The solution of this coupled deterministic system provides the expansion coefficients of the solution process. In the non-intrusive approach $[15,42]$, the expansion coefficients of the solution process are obtained through orthogonal projection on a selected set of prescribed basis functions. The inner product in the orthogonal projection (defined as a statistical average) is evaluated numerically using random sampling, quadrature rule or sparse grid techniques $[3,13,30,42]$.

Stochastic collocation method is another non-intrusive technique $[8,43]$. In the context of the stochastic collocation method, the solution process is approximated by Lagrange interpolation polynomials. The basis of the polynomials is constructed using a set of prescribed structured collocation points (defined by a tensor product or sparse grid) and the coefficients of the polynomial are the solution at the collocation points $[13,43,44]$. 
The implementations of the non-intrusive (collocation and pseudo-spectral) methods involve repeatedly solving deterministic problems corresponding to the collocation points or realizations of the random variables used to represent the system parameters $[3,44,45]$. The advantage of the non-intrusive approach lies in its ability to reuse existing deterministic legacy codes. To achieve the same level of accuracy, the non-intrusive methods may require solutions of considerably larger number of equations than the size of the linear systems arising from Galerkin projection in the intrusive approach $[44,45]$. In the Galerkin projection approach, the error due to the finite representation is minimum leading to optimal accuracy $[13,17]$. On the contrary, there is no explicit control of the error in the collocation methods except at the collocation points where the error is zero. Therefore the accuracy of the solution depends on the selection of the collocation points $[3,17,30,46]$.

The stochastic reduced basis methods (SRBMs) [47-49] is an efficient tool to reduce the computational complexity of the intrusive SSFEM approach. In the SRBMs, the solution process is represented as a linear combination of a set of basis vectors from the preconditioned stochastic Krylov subspace [47-49]. Galerkin projection scheme is employed to obtain a reduced order deterministic set of equations for the expansion coefficients [47-49]. The order of the resulting set of equations in the SRBMs is much less than that in the standard intrusive SSFEM. In contrast to the standard intrusive SSFEM approach in which the basis functions of the solution process are Hermite polynomials, the stochastic basis vectors are problem dependent in the SRBMs [28]. At much lower computational complexity, the accuracy of the first two statistical moments of the solution processes obtained from the 
SRBMs is comparable to those from the standard intrusive SSFEM [47-49]. The SRBMs rely on the preconditioned stochastic Krylov subspace to construct a reduced order expansion for the solution process. Efficient and scalable preconditioners therefore, could be beneficial in further reducing the computational complexity of the SRBMs for large-scale problems with many random variables.

\subsection{Statement of the Problem}

The computational cost of the SSFEM primarily depends on the size of the finite element mesh, the dimension and order of the orthogonal polynomial used in the expansion of the random system parameters and the solution process $[37,38]$. The Galerkin projection in the SSFEM leads to a set of deterministic linear systems with a block matrix structure, coupling all the coefficients of the solution process $[3,37,50]$. The size of the resulting linear systems grows rapidly with the spatial mesh resolution and the order of the stochastic dimension $[37,38]$. The solution of these systems of linear equations poses significant computational challenge and therefore efficient solvers are required $[3,14,51]$. To this end, the development of robust and efficient numerical solution strategies is of paramount importance for the applicability of the SSFEM for large-scale practical (industrially relevant) problems [28]. These numerical algorithms must be parallel and scalable in order to fully take advantage of the advances of high performance computing platforms. 
Iterative numerical methods based on Krylov subspace, carefully designed for the SSFEM problem, may provide a suitable solution technique $[3,50]$. The reuse of the existing iterative solvers developed for deterministic problem can be a feasible strategy [3]. However, the reliability and robustness of the iterative methods mainly depend on the preconditioning techniques [52]. Thus preconditioners, suitably customized for the SSFEM problem are needed to improve the performance of the ensuing linear solver [3, 51].

Previous work in the literature has been devoted to formulating robust and efficient iterative solution techniques to tackle the resulting linear systems arising in the context of the stochastic Galerkin projection based SSFEM, namely the intrusive SSFEM. However, the solution of the coupled set of deterministic systems in the SSFEM remains to be a major computational challenge for uncertainty quantification of large-scale systems. In general, the previous attempts to solve the linear systems for the SSFEM can be categorized into preconditioned iterative solvers $[27,37,50,53,54]$ and multigrid methods $[51,55,56]$.

For a symmetric positive definite system, the iterative solvers reported in the literature are Preconditioned Conjugate Gradient Method (PCGM) $[27,37,50,54]$ and block Gauss-Jacobi method [53]. The global stochastic stiffness matrix, arising from stochastic Galerkin projection is block-diagonally dominant (for small magnitude of fluctuations around the mean material properties), this fact motivates the use of block-Jacobi preconditioner to accelerate the rate of convergence of the iterative solvers $[3,37,50]$. Each diagonal block of the preconditioning matrix is constructed based on a factorization of the mean matrix. This type of preconditioner is termed as a mean-based preconditioner. Different 
deterministic techniques are proposed in the literature to factorize each building blocks of the preconditioning matrix in a black-box fashion. Indeed, Incomplete LU factorization [37, 53, 57], sparse inverse [50], algebraic multigrid [21, 57], domain decomposition method [27] and the existing deterministic parallel solver $[54,58]$ are used to solve each diagonal blocks of the block-Jacobi preconditioning matrix. Although the block-Jacobi preconditioner demonstrates an improvement of the convergence rate of the iterative solver compared to the traditional Jacobi preconditioner, the performance of the block-Jacobi preconditioner is sensitive to the level of uncertainty in the system parameters $[50,53,57]$. In addition, the convergence rate of the preconditioner may deteriorate when the system parameters are non-Gaussian in nature that leads to large contribution from the off-diagonal blocks to the SSFEM stiffness matrix $[37,57]$.

Multigrid methods are the second type of iterative solvers used in the literature to tackle the linear system of stochastic Galerkin projection $[51,55,56]$. The multigrid solver demonstrates a convergence rate that is independent of the size of the spatial discretization and the order of the stochastic dimension. However, the performance of the multigrid solver is substantially dependent on the level of the coefficient of variation of the system parameters [51]. Furthermore, some of the multigrid parameters may need to be carefully selected to gain the optimal convergence rate [51].

Domain decomposition methods have become major computational tools for parallel computing of large-scale numerical models of partial differential equations (PDEs) [4, 6, $7,52,59]$. Domain decomposition algorithms can achieve the highest level of paralleliza- 
tion in the context of the numerical solution of PDEs [52, 60-62]. Domain decomposition algorithms are particularly useful in developing efficient preconditioners to accelerate the convergence rate of the iterative solvers $[52,63-67]$.

To this end, a theoretical framework for domain decomposition method of uncertain systems defined by SPDEs is presented in [68]. The methodology is based on Schur complement based domain decomposition in the physical space and functional decomposition in the stochastic dimension [68]. This approach can take advantage of the well-developed parallel algorithms devised for deterministic systems [68]. A direct substructuring technique is implemented. The serial implementation of the algorithm demonstrates the effectiveness of the methodology in reducing the total execution time [69].

\subsection{Research Objective and Scope}

The main objective of this study is to develop the next generation uncertainty quantification algorithms which efficiently exploit the modern supercomputers in order to enable high fidelity simulations with new capability to quantify uncertainty in the prediction of computational mechanistic models. The investigation is directed towards devising efficient parallel and scalable computational algorithms to quantify and propagate uncertainty in large-scale engineering systems using high performance computing platforms.

The SSFEM is a popular computational tool for uncertainty quantification in numerous engineering problems of practical interest. For large-scale engineering systems, the 
application of SSFEM becomes computationally challenging as the size of the resulting algebraic linear system grows rapidly with the spatial mesh resolution and the order of the stochastic dimension. To address this issue, a non-overlapping domain decomposition approach (namely a substructuring method) of SPDEs is introduced in [68] in the framework of SSFEM. A dense direct solver is used to tackle the extended Schur complement system (the interface problem).

For large-scale problems, the solution of the extended Schur complement system using direct solvers becomes impractical due to overwhelming memory requirements and poor scalability to large number of processors [52]. In order to tackle the interface problem of the stochastic system using iterative substructuring technique, a parallel PCGM equipped with a parallel extended lumped preconditioner is adopted [70]. The iterative algorithm circumvents the need to construct both the local and global extended Schur complement matrices explicitly. Despite being superior to the direct solver (in terms of memory requirement and floating point operations), the PCGM based iterative solver equipped with the lumped preconditioner exhibits performance degradation as the number of subdomains increases and therefore scalable preconditioner is needed to enhance the performance of the algorithm [70].

As already demonstrated for the deterministic PDEs, the Neumann-Neumann preconditioner achieves a convergence rate that is independent of the spatial mesh size and exhibits a good scalability for a moderate number of subdomains $[4,5,7,71,72]$. The NeumannNeumann preconditioner approximates the inverse of the global Schur complement matrix 
by a weighted sum of the inverse of the local Schur complement matrices. This approximation is rational since the global Schur complement matrix is the sum of the local Schur complement matrices [73]. The Neumann-Neumann method paves the way for constructing two-level preconditioners. These facts motivate the development of Neumann-Neumann preconditioner in the context of stochastic PDEs [74, 7.5].

The PCGM equipped with the one-level Neumann-Neumann preconditioner exhibits a convergence rate that is almost insensitive to the level of uncertainty in the system parameters $[74,75]$. As no mechanism for global information exchange exists in the one-level Neumann-Neumann preconditioner, its performance deteriorates for relatively large number of subdomains. For scalability, the global communication is required to propagate information across the subdomains [4-7].

To this end, a two-level domain decomposition method is reported to tackle the largescale linear system within the framework of the intrusive SSFEM [76, 77]. The implementation of the algorithm involves solving a local problem on each subdomain that constructs the local part of the preconditioner [7] and a coarse problem that propagates information globally among the subdomains. This information exchange achieved by the coarse problem makes the preconditioner scalable. This method bears similarity with a version of the Balancing Domain Decomposition by Constraints (BDDC) for deterministic PDEs [66, 78].

A primal-primal iterative substructuring technique is introduced to solve the large-scale linear system arising from the finite element discretization of SPDEs $[79,80]$. The mathe- 
matical framework naturally leads to a coarse problem connecting the subdomains globally and provides a mechanism to propagate information across the subdomains. The global spread of information in the primal-primal preconditioner leads to a scalable performance. In an abstract sense, the primal-primal preconditioner may be viewed as an another extension of BDDC for SPDEs.

For deterministic PDEs, BDDC and dual-primal finite element tearing and interconnect FETI-DP $[64,81]$ are perhaps the most popular non-overlapping domain decomposition techniques for the iterative solution of large-scale deterministic linear systems [82]. It has already been demonstrated that the condition number and thus the parallel performance of BDDC and FETI-DP are quite similar $[65,67,78,83-85]$. It is therefore natural to ask whether the similarity of BDDC and FETI-DP extends to stochastic systems

To address this question, we formulate a probabilistic version of the FETI-DP iterative substructuring technique for the intrusive SSFEM $[79,80,86]$. In the probabilistic setting, the operator of the dual interface system in the dual-primal approach contains a coarse problem. The introduction of the coarse problem [65] leads to a scalable performance of the dual-primal iterative substructuring method for uncertainty quantification of large-scale computational models.

The parallel performances of the aforementioned algorithms are studied for two- and and three-dimensional stochastic PDEs with spatially varying non-Gaussian random coefficients. For efficient memory usage and minimum floating point operations, the numerical 
implementation of the algorithms exploit the multi-level sparsity structure of the coefficient matrix of the stochastic system, namely (a) the sparsity structure due to the finite element discretization and (b) the block sparsity structure arising from the orthogonal representation and projection of the stochastic processes. PETSc [87] and MPI [88] parallel libraries are used for efficient parallel implementation of the algorithms. The graph partitioning tool METIS [89] is used for optimal decomposition of the finite element mesh.

For a fixed mesh resolution, domain decomposition algorithms cannot reduce the total execution time beyond a critical number of subdomains/processors as the interprocessor communication overhead and memory bandwidth requirement predominate over floating point operations. In order to exploit the remaining idle processors in multi-processor computers, the recent research initiatives have been directed towards achieving time domain parallelism (e.g., $[90,91])$. To this end, we adapt the so-called parareal algorithm for ordinary differential equations (ODEs) to simulate the trajectories (sample paths) of a noisy non-linear dynamical system described by stochastic differential equations (SDEs). To assess the performance of the parallel algorithm, a numerical investigation is conducted to simulate the sample path of a Duffing oscillator driven by combined deterministic and random inputs. The parareal scheme may also be applied for efficient simulation of SPDEs in conjunction with the probabilistic domain decomposition methods developed in this thesis. 


\subsection{Organization of the Thesis}

An introduction to the probabilistic methods for uncertainty quantification, the motivation for the research and the thesis statement are presented in Chapter 1. The SSFEM formulation and a brief literature review on the solution strategies to tackle the associated linear systems of equations are provided in Chapter 2. In Chapter 3, a review of domain decomposition methods for the deterministic systems is provided. In addition, a novel primalprimal iterative substructuring algorithm equipped with a two-level scalable preconditioner is introduced. Iterative substructuring technique for the numerical solution of stochastic systems is described in Chapter 4. The mathematical framework and parallel implementations of one-level domain decomposition preconditioners for the stochastic systems are provided in Chapter 5. In Chapter 6, a two-level domain decomposition method is reported to tackle the large-scale linear system within the framework of the intrusive SSFEM. Chapter 7 provides the mathematical framework of the primal-primal iterative substructuring technique for the solution of the large-scale linear system in the SSFEM. In Chapter 8, we report a probabilistic version of the dual-primal domain decomposition method for the intrusive SSFEM. Numerical illustrations to the mathematical formulation is presented in Chapter 9 for two-dimensional problems. Chapter 10 provides numerical experiments for three-dimensional problems. In Chapter 11 , the development of the probabilistic parallel time integration scheme is provided. In Chapter 12, the main conclusions of the thesis and some suggestions for future research direction are summarized. Some relevant mathematical details are reviewed in the Appendices. 


\section{Chapter 2}

\section{Finite Element Method for Stochastic}

\section{PDEs}

\subsection{Introduction}

In modeling numerous engineering and physical systems, it is necessary to consider the random heterogeneity of the model parameters for more realistic computer simulation [3, $8,11,92$ ]. When sufficient statistical information is available, probability theory offers a rich mathematical framework to represent uncertainty in terms of random variables and stochastic processes(e.g. $[8,11,68,92])$.

We assume that the input data (containing sufficient statistical information) permit a representation of the model parameters as stochastic processes that span a function space [3, $11,68-70,74,76,79,80]$. Using Karhunen-Loeve expansion (KLE), a set of basis func- 
tions can be identified to characterize this space $[3,11,68,69]$. The KLE of a stochastic process is based on the spectral decomposition of its covariance function $[3,11,68,69]$. When the covariance function of a stochastic process, for example the solution process, is not known a priori, KLE cannot be used to represent the process $[3,11,68,69]$. For stochastic processes with a priori unknown covariance function, a set of basis functions in the space of second-order random variables can be used to approximate the processes $[3,11,68,69]$. The solution process is a function of the system parameters and nodal solution variables, therefore it can be represented as some nonlinear functional of the basis of the KLE used to expressed uncertainty in the system parameters $[3,11,68,69]$. It has been shown that this functional dependence can be expanded in terms of polynomials in Gaussian random variables, namely the Polynomial Chaos Expansion (PCE) $[11,93]$. The expansion coefficients of the solution process can be evaluated either through Galerkin (intrusive) or pseudo-spectral (non-intrusive) projection [30].

In the intrusive approach $[1,2,11,24,39,40]$, Galerkin projection on the space spanned by the PC basis is performed to minimize the error of the truncated PCE. The resulting coupled set of equations is solved to obtain the expansion coefficients of the solution process. In the non-intrusive approach $[15,42]$, the orthogonality of the PC basis is employed to evaluate the expansion coefficients of the solution process $[3,13,30,42]$. The inner product in the orthogonal projection (defined as a statistical average) involves a multidimensional integral. This integral is evaluated numerically using random sampling, quadrature rule or sparse grid approaches $[3,13,30]$. 
In this chapter, uncertainty representation using random variables and processes is described. In addition, the intrusive and non-intrusive SSFEM formulation is outlined. A brief literature review is provided on the solution strategies to tackle the linear systems of equations arising in the context of the intrusive SSFEM.

\subsection{Uncertainty Representation by Stochastic Processes}

\subsubsection{Karhunen-Loeve Expansion}

The KLE of a stochastic process $\alpha(\mathbf{x}, \theta)$ is based on the spectral expansion of its covariance function $C_{\alpha \alpha}\left(\mathbf{x}_{1}, \mathbf{x}_{2}\right)$ as $[3,11]$

$$
\alpha(\mathbf{x}, \theta)=\alpha(\mathbf{x})+\sum_{i=1}^{\infty} \sqrt{\lambda_{i}} \phi_{i}(\mathbf{x}) \xi_{i}(\theta),
$$

where $\alpha(\mathbf{x})$ is the mean function of the stochastic process, $\left\{\xi_{i}(\theta)\right\}$ is a set of uncorrelated random variables, and $\lambda_{i}$ are the eigenvalues and $\phi_{i}(\mathbf{x})$ are the eigenfunctions of the covariance kernel $C_{\alpha \alpha}\left(\mathbf{x}_{1}, \mathbf{x}_{2}\right)$ [94]. The eigenvalues and eigenfunctions can be obtained as the solution to the following interral amation $12 \quad 11 \quad 171$

$$
\begin{aligned}
& \int_{D} C_{\alpha \alpha}\left(\mathbf{x}_{1}, \mathbf{x}_{2}\right) \phi_{i}\left(\mathbf{x}_{1}\right) \mathrm{d} \mathbf{x}_{1}=\lambda_{i} \phi_{i}\left(\mathbf{x}_{2}\right) . \\
& \int_{D} C_{\alpha \alpha}\left(\mathbf{x}_{1}, \mathbf{x}_{2}\right) \phi_{i}\left(\mathbf{x}_{1}\right) \mathrm{d} \mathbf{x}_{1}=\lambda_{i} \phi_{i}\left(\mathbf{x}_{2}\right) .
\end{aligned}
$$

When the marginal probability density function (pdf) of the process is Gaussian, the uncorrelated random variables in KLE are Gaussian and hence they are independent random variables $[11,37]$. The KLE is an optimal representation of a stochastic process in the 
mean-square sense $[3,11]$. For a smooth stochastic process, only a finite number of KLE terms is sufficient to represent the process $[3,11,17]$.

\subsubsection{Polynomial Chaos Expansion}

As mentioned previously, the KLE is based on the spectral expansion of the covariance function and therefore, KLE cannot be used to represent a stochastic process with $a$ priori unknown covariance function $[11,37,95]$. In this case, the PCE is used to expand a stochastic process as a sum of its projections on an appropriate basis defined on the space of second-order random variables as $[11,24,95]$

$$
\alpha(\mathbf{x}, \theta)=\sum_{i=0}^{\infty} \alpha_{i}(\mathbf{x}) \Psi_{i}(\boldsymbol{\xi}),
$$

where $\alpha_{i}(\mathbf{x})$ are the PC coefficients and $\Psi_{i}(\boldsymbol{\xi})$ is a set of multi-dimensional orthogonal Hermite polynomials (with zero mean) in the sense that their inner product $\left\langle\Psi_{i}(\boldsymbol{\xi}) \Psi_{j}(\boldsymbol{\xi})\right\rangle$ defined as the statistical average of their product, is equal to zero for $i \neq j$, as

$$
\begin{aligned}
\left\langle\Psi_{i}(\boldsymbol{\xi}) \Psi_{j}(\boldsymbol{\xi})\right\rangle & =\int \Psi_{i}(\boldsymbol{\xi}) \Psi_{j}(\boldsymbol{\xi}) \mathrm{p}(\boldsymbol{\xi}) d \boldsymbol{\xi} \\
& =\delta_{i j}\left\langle\Psi_{i}^{2}(\boldsymbol{\xi})\right\rangle
\end{aligned}
$$

where $\mathrm{p}(\cdot)$ is the probability density function of $\boldsymbol{\xi}$ and $\delta_{i j}$ is the Kronecker delta.

For numerical implementation, the infinite PCE series in Eq.(2.3) is truncated in both the order $p$ of the polynomial chaoses and the dimension of the stochastic space $n$, resulting a finite $\mathrm{PC}$ representation as 


$$
\omega(\mathbf{x}, \theta)=\sum_{i=0}^{N} \omega_{i}(\mathbf{x}) \Psi_{i}\left(\xi_{1}, \xi_{2}, \cdots, \xi_{n}\right)
$$

where $N$ is the number of the terms used in PCE given as

$$
N+1=\frac{(n+p) !}{n ! p !}
$$

The truncated PCE series in Eq.(2.6) converges in the mean square sense $[11,96]$. The PCE series can be refined by increasing the size of the stochastic dimension (adding more random variables to the set $\{\xi\}$ ) to capture higher frequency random fluctuation of the underlying stochastic process $[96,97]$, or by increasing the order of the polynomials in PC series (adding more random function $\Psi_{i}(\xi)$ ) to capture strong nonlinear dependence of the solution process on the underlying random variables $[96,97]$.

\subsection{Spectral Stochastic Finite Element Method}

Consider a linear elliptic stochastic PDE defined on a domain $\Omega$ with a given data on the boundary $\partial \Omega$. The finite element discretization in the spatial domain leads to the following stochastic linear system $[3,11,17]$

$$
\mathbf{A}(\theta) \mathbf{u}(\theta)=\mathbf{f}
$$

where $\mathbf{A}(\theta)$ is the stiffness matrix with random coefficients, $\mathbf{u}(\theta)$ is the stochastic process representing the response vector and $\mathrm{f}$ is the applied force. 
For general non-Gaussian material properties, the PCE can be used to represent uncertainty in the model parameters as [98]

$$
\mathbf{A}(\theta)=\sum_{i=0}^{L} \Psi_{i} \dot{\mathbf{A}_{i}}
$$

The solution process can be expanded using the same PC basis as

$$
\mathbf{u}(\theta)=\sum_{j=0}^{N} \Psi_{j} \mathbf{u}_{j}
$$

Galerkin projection or pseudo-spectral (discrete projection) approach can be used to evaluate the PC coefficients $u_{j}$ in Eq.(2.10) as delineated next.

\subsubsection{Galerkin (Intrusive) Approach}

In the intrusive approach, Galerkin projection onto a PC basis $\Psi_{i}$ is performed to ensure that the error resulting from a finite representation is orthogonal to the space spanned by $\left\{\Psi_{i}\right\}$ [37]. Substituting Eq.(2.9) and Eq.(2.10) into Eq.(2.8) and performing Galerkin projection, the following coupled deterministic systems of equations is obtained

$$
\sum_{j=0}^{N} \sum_{i=0}^{L}\left\langle\Psi_{i} \Psi_{j} \Psi_{k}\right\rangle \mathbf{A}_{i} \mathbf{u}_{j}=\left\langle\Psi_{k} \mathbf{f}\right\rangle, \quad k=0,1, \ldots, N
$$

Equivalently, Eq.(2.11) can be rewritten as

$$
\mathcal{A U}=\mathcal{F}
$$

where

$$
\mathcal{A}_{j, k}=\sum_{i=\mathbf{0}}^{L}\left\langle\Psi_{i} \Psi_{j} \Psi_{k}\right\rangle \mathbf{A}_{i}, \quad \mathcal{U}=\left(\mathbf{u}_{0}, \ldots, \mathbf{u}_{N}\right)^{T} \quad \text { and } \quad \mathcal{F}_{k}=\left\langle\Psi_{k} \mathbf{f}\right\rangle
$$


Eq.(2.12) can be expressed as

$$
\left[\begin{array}{cccc}
\mathcal{A}_{0,0} & \mathcal{A}_{0,1} & \ldots & \mathcal{A}_{0, N} \\
\mathcal{A}_{1,0} & \mathcal{A}_{1,1} & \ldots & \mathcal{A}_{1, N} \\
\vdots & \vdots & \ddots & \vdots \\
\mathcal{A}_{N, 0} & \mathcal{A}_{N, 1} & \ldots & \mathcal{A}_{N, N}
\end{array}\right]\left\{\begin{array}{c}
\mathrm{u}_{0} \\
\mathrm{u}_{1} \\
\vdots \\
\mathbf{u}_{N}
\end{array}\right\}=\left\{\begin{array}{c}
\mathcal{F}_{0} \\
\mathcal{F}_{1} \\
\vdots \\
\mathcal{F}_{N}
\end{array}\right\}
$$

\subsubsection{Pseudo-Spectral (Non-Intrusive) Approach}

In the non-intrusive approach, the orthogonality property of the PC basis defined in Eq.(2.4) is employed to obtain the $\mathrm{PC}$ coefficients of the solution process. Consequently, projection the solution process $\mathbf{u}(\theta)$ given by Eq.(2.10) onto PC basis $\Psi_{k}$, leads to

$$
\left\langle\mathbf{u}(\theta) \Psi_{k}\right\rangle=\sum_{j=0}^{N}\left\langle\Psi_{j} \Psi_{k}\right\rangle \mathbf{u}_{j}, \quad k=0,1, \ldots, N
$$

Using Eq.(2.4), PC coefficients of the solution process can be evaluated from Eq.(2.15) as

$$
\mathbf{u}_{j}=\frac{\left\langle\mathbf{u}(\theta) \Psi_{j}\right\rangle}{\left\langle\Psi_{j}^{2}\right\rangle}=\frac{1}{\left\langle\Psi_{j}^{2}\right\rangle} \int \mathbf{u}(\boldsymbol{\xi}) \Psi_{j}(\boldsymbol{\xi}) \mathrm{p}(\boldsymbol{\xi}) d \boldsymbol{\xi} .
$$

The integral in Eq.(2.16) is evaluated numerically using random sampling, quadrature rule or sparse grid technique $[3,13,30]$. This integral can be viewed as the correlation between the response process $\mathbf{u}(\boldsymbol{\xi})$ and the PC basis $\Psi(\xi)[3]$.

\subsubsection{Intrusive Versus Non-Intrusive}

The implementation of the non-intrusive method involves repeatedly solving deterministic problems that correspond to prescribed values of the system parameters. This approach 
can readily exploit existing deterministic solvers $[3,44,45]$. To achieve the same level of accuracy, however, the non-intrusive method requires solution of much larger number of equations than the size of the linear system arising from Galerkin projection in the intrusive SSFEM $[44,45]$. In the non-intrusive approach, the error due to the integration rules can cause significant aliasing [17]. In the Galerkin (intrusive) approach, the error due to finite representation is minimum (orthogonal to the space spanned by PC basis) leading to an optimal accuracy of the solution $[17,45]$. For large-scale problems (where a single deterministic run is computationally demanding), Galerkin projection approach may be preferred provided efficient solvers can be developed $[17,45]$.

\subsection{Solution Methods for the SSFEM}

The Galerkin projection in the SSFEM leads to a coupled systems of linear equations with a block sparsity structure $[3,37,50]$. The solution of this systems of linear equations constitutes a computationally challenging task and therefore efficient solvers are required $[3,51]$. A brief literature review on the solution strategies to tackle the linear systems of the SSFEM is provided in the next section.

\subsubsection{Literature Review}

Ghanem and Kruger (1996) [37] proposed Preconditioned Conjugate Gradient Method (PCGM) equipped with a block-Jacobi preconditioner to solve the linear system arising in the context of SSFEM. Each building block of the preconditioning matrix is based on an incomplete LU factorization of the stiffness matrix [99] corresponding to the mean material 
properties as

$$
\mathcal{M}^{-1} \mathcal{A U}=\mathcal{M}^{-1} \mathcal{F}
$$

where

$$
\mathcal{M}^{-1}=\left[\begin{array}{cccc}
\mathcal{M}_{1,1}^{-1} & 0 & \ldots & 0 \\
0 & \mathcal{M}_{2,2}^{-1} & \ldots & 0 \\
\vdots & \vdots & \ddots & \vdots \\
0 & 0 & \ldots & \mathcal{M}_{N, N}^{-1}
\end{array}\right]
$$

and

$$
\mathcal{M}_{j, j}^{-1}=\left\langle\xi_{0} \Psi_{j} \Psi_{j}\right\rangle I L U\left(\mathbf{A}_{0}\right)
$$

Due to the block structure of the preconditioning matrix, the operation on each diagonal block can be performed independently. The block-Jacobi preconditioner demonstrated an improvement in the performance of the PCGM compared to the traditional Jacobi preconditioner. For moderate uncertainty and Gaussian material properties, the performance of the block-Jacobi preconditioner is shown to be nearly independent of the level of randomness in the system parameters. Nevertheless, the number of iterations required by the iterative solver to converge grows as the order of the PCE increases.

Pellissetti and Ghanem (2000) [50] applied PCGM equipped with a block-Jacobi preconditioner to solve the linear system arising from the SSFEM. The block-Jacobi preconditioner has the same structure as in Eq.(2.18). However, each individual block is an approximate inverse of the mean stiffness matrix as 


$$
\mathcal{M}_{j, j}^{-1}=\left\langle\xi_{0} \Psi_{j} \Psi_{j}\right\rangle \mathbf{A}_{0}^{-1}
$$

The inversion of the mean stiffness matrix is evaluated approximately to preserve a prescribed sparsity structure. For a small problem size and Gaussian material properties, the PCGM solver equipped with the block-Jacobi preconditioner showed good performance compared to the classical CGM solver. However, even for a small value of the coefficient of variation of the system parameters, the convergence rate of the block-Jacobi preconditioner deteriorates as the order of PCE increases. One the other hand, finding a good approximate inverse for the mean stiffness matrix is computational expensive and the cost increases rapidly as the size of the matrix grows.

Maitre et al. (2003) [51] adapted a geometric multigrid method to solve the linear system arising from the stochastic Galerkin projection. A simple V-cycle algorithm is implemented. The multigrid solver showed a convergence rate that is nearly independent of the mesh size. Furthermore, the convergence rate of the multigrid solver does not degenerate as the number of random variables in KLE increases. On the other hand, the convergence rate of the multigrid method decays slightly as the order of the PCE increases. The performance of the multigrid solver is substantially dependent on the level of the coefficient of variation of the system parameters. The structure of the multilevel iteration cycle and some of the multigrid parameters (such as the number of iterations on each grid) need to be carefully selected to gain the optimal convergence rate.

Keese and Matthies (2005) $[54,58]$ used a hierarchical parallelization approach on a 
distributed memory machine to solve the linear system of SSFEM. A coarse-grained parallelization is implemented by constructing each block of the stochastic stiffness matrix. A fine level parallelization is implemented within each individual block. The fine level of parallelization exploits the available deterministic parallel libraries to compute the matrixvector multiplication and the preconditioner effect. Instead of computing the inverse of each block in the block-Jacobi preconditioner in Eq.(2.18), a system of the following form is solved

$$
\mathbf{A}_{0} \mathbf{z}=\mathbf{r}
$$

The mean stiffness matrix $A_{0}$ is a deterministic and thus any existing deterministic parallel solver can be used to tackle it in a black-box fashion. In this hierarchical parallelization approach, the time required to compute the preconditioner effect is inversely proportional to the number of processors.

Chung et. al. (2005) [53] used a block Gauss-Jacobi iterative method equipped with an incomplete block-Jacobi preconditioner defined in Eq.(2.18) to solve the linear system of SSFEM. An element-by-element strategy is proposed to evaluate the matrix-vector product required by the iterative solver. The element-by-element approach only requires the storage of the element stochastic stiffness matrix which is less expensive in terms of memory requirements than that of the full stochastic stiffness matrix. After each multiplication step, the resulting vector however needs to be assembled which consumes a large computation time. Increasing the number of random variables in KLE and the order of PCE do not significantly affect the performance of the block Gauss-Jacobi solver. As the coefficient of 
variation of the system parameters increases, the iteration counts grow.

Chao et. al. (2007) [100] used a recycling Krylov subspace method equipped with a black-box additive Schwarz domain decomposition preconditioner to solve the SSFEM linear systems. Double orthogonal basis are used to decouple the probabilistic space resulting a sequence of uncoupled systems of linear equations. The recycling technique is used to reduce the computational cost of solving a sequence of related linear systems with multiple right hand sides. Some difficulties arose in using the recycling Krylov subspace method as the linear stochastic systems are not exactly the same. Therefore, a grouping algorithm is proposed to collect the closely related linear systems in subsets and then tackle them using the recycling Krylov subspace method. The recycling technique efficiently reduces the total execution time. The additive Schwarz preconditioner achieves a scalable performance with respect to mesh size and number of subdomains. The double orthogonal basis is a special case and cannot be applied to the general type of uncertainty.

Elman and Furnival (2007) [55] applied geometric multigrid method to solve the linear system in SSFEM. A two-grid correlation scheme based on the spatial discretization is implemented. The convergence rate of the multigrid solver is shown to be independent of the spatial mesh size and the order of the stochastic dimension.

Rosseel et al. (2008) [56] used algebraic multigrid method to solve the linear system arising in the context of SSFEM. The mean stiffness matrix is used to build the hierarchy of meshes for multigrid method. For a Gaussian and non-Gaussian material properties, the 
number of multigrid iterations is shown to be independent of the geometric mesh size. The convergence rate of the multigrid solver is shown to be asymptotically independent of the order of PCE in case of material properties with uniform probability density (pdf) but not in case of Gaussian pdf.

Powell and Elman (2009) [21, 57] provided a theoretical basis for the block-Jacobi preconditioner in Eq.(2.18) and established a bound for the condition number. The theoretical results show that, the condition number is independent of the spatial mesh size, but depends on the COV of the system parameters, and additionally, on the order of PCE if unbounded random variables are used. An incomplete Cholesky factorization of the mean stiffness matrix and a black-box algebraic multigrid method are used to implement the block-Jacobi preconditioner. The performance of the algebraic multigrid scheme is independent of the size of the spatial discretization.

Ghosh et al. (2009) [27] used PCGM equipped with the block-Jacobi preconditioner in Eq.(2.18) to solve the linear system in the context of SSFEM. The FETI-DP domain decomposition method is used to compute the preconditioned residual $\mathrm{z}$ in Eq.(2.21). In particular, the FETI-DP solver is used to tackle the linear systems of the following form

$$
\frac{1}{\left\langle\Psi_{j}^{2}\right\rangle} \mathbf{A}_{0} \mathrm{z}=\mathbf{r}
$$

The overall iterative scheme consists of two nested PCGM solvers. An outer solver is used to tackle the full stochastic linear system, while an inner solver is used to tackle each individual block of the preconditioner. A recycling Krylov subspace method is utilized to 
speed-up the convergence rate of the inner solver. The multiple right-hand sides version of FETI-DP solver helped in reducing the computational cost of the block-Jacobi preconditioner. The iterative scheme demonstrated good scalability with respect to the size of the problem and number of subdomains. It is again worth emphasizing that the FETI-DP method is only used to solve the blocks of the block-Jacobi preconditioner for the global stochastic system.

Sarkar et al. (2009) [68] presented a theoretical framework for the domain decomposition method of uncertain systems defined by SPDEs. The methodology based on Schur complement based domain decomposition in the physical space and functional decomposition in the stochastic dimension. This approach gives the ability to exploit the deterministic domain decomposition algorithms in the context of stochastic PDEs. For numerical validation of the methodology, a direct substructuring technique is implemented to solve a steady state wave propagation problem in one-dimensional random media. A dense direct solver is used to tackle the stochastic Schur complement system.

Ullmann (2010) [101] introduced a Kronecker product preconditioner to improve the performance of the iterative solver for the linear system in SSFEM. The preconditioner exploits the block sparsity structure of the stochastic stiffness matrix. The Kronecker product preconditioner enhances the performance of the PCGM compared to the mean-based preconditioner [101]. Increasing the level of uncertainty in the system parameters and the order of the stochastic expansion leads to a moderate increase in the iteration counts of the PCGM [101]. Unlike the block-Jacobi preconditioner, the Kronecker product precon- 
ditioner is not a block-diagonal matrix and therefore it is not an embarrassingly parallel preconditioner.

\subsection{Summary}

In the SSFEM, uncertainty in the system is treated as an additional dimension to the problem whereby a suitable basis functions is used to discretize the stochastic dimension [37]. The PCE is used to represent the solution process. Using the PC basis, a Galerkin projection is performed to minimize the error of the truncated PCE that leads to a set of coupled deterministic linear system. The solution of this coupled linear system provides the PC coefficients of the solution process. The solution of this large-scale linear system associated with SSFEM constitutes a computationally challenging task and therefore efficient solvers are required.

In general, an iterative solver is used to tackle the linear system in SSFEM. The fact that the full stochastic linear system consists of block submatrices and is diagonally dominant motivates the use of block-Jacobi preconditioner to accelerate the convergence rate of the iterative method. Each building block of the preconditioner is constructed based on a factorization of the mean stiffness matrix. Different deterministic techniques are proposed in the literature to solve each diagonal block of the preconditioner in a black-box fashion such as incomplete LU factorization [37, 53, 57], sparse inverse [50], algebraic multigrid [56, 57], domain decomposition method [27], or use of existing deterministic parallel solver $[54,58]$. Due to the block diagonal structure of the block-Jacobi precondi- 
tioner, its application requires repeated solution of a linear system with different right hand side which can be performed concurrently. However, the performance of the block-Jacobi preconditioner appears to be sensitive to the level of uncertainty of the system parameters $[50,53,57]$. Furthermore, the convergence rate of the preconditioner may deteriorate when the system parameters are non-Gaussian in nature [37, 57].

\subsection{Discussion}

The preconditioning technique is the essential ingredient in enhancing the reliability, robustness and performance of the iterative methods used to tackle the linear system arising in SSFEM. The combination of a scalable parallel preconditioner and the CGM solver makes the iterative method well-suited to tackle the SSFEM linear system using high performance computing platforms. The block-Jacobi preconditioner and its generalization (additive Schwarz domain decomposition method) are one-level preconditioning techniques and thus their performance deteriorate as the number of subdomains increases leading to poor scalability $[6,52,102]$. This performance degradation can be attributed to the fact that the information is exchanged only among the neighboring subdomains in one-level preconditioners. There is no mechanism for global communication in the one-level preconditioners [103]. For large-scale stochastic systems, a two-level preconditioner consisting of local (fine) and global (coarse) components is required to make the convergence rate of the iterative solver (nearly) independent of the number of the subdomains. 


\section{Chapter 3}

\section{Domain Decomposition Methods for}

\section{Deterministic PDEs}

\subsection{Introduction}

This chapter closely follows the references [4-7]. Domain decomposition methods are generally used to partition a large computational domain into a number of subdomains whereby a smaller problem on each subdomain can be solved independently and concurrently [4$7,104,105]$. In multi-physics problems, domain decomposition may arise naturally from partitioning the physical system into subregions based on phenomenological considerations, and the physics on each subregion is modeled using a suitable partial differential equation [6]. In parallel computing, domain decomposition method is defined as the process of dividing a data set into a smaller groups that can be distributed among the processors in a distributed memory architecture computers [6]. From algebraic point of view, domain 
decomposition method can be described as a parallel algorithm for solving the large-scale linear system arising from the discretization of partial differential equations [6]. In this context, the large-scale linear system is subdivided into a smaller subsystems whose solutions can be used to produce a scalable preconditioner for the original system [106]. Domain decomposition preconditioners whose computational work grows linearly or log-linearly with the number of subdomains are said to be scalable preconditioners $[4,5,7]$. Most of the current interest in domain decomposition algorithms lies in developing scalable parallel preconditioners $[4,64,107,108]$. Domain decomposition methods have been applied successfully to a wide variety of problems [109] including structural mechanics [64, 65, 81, 110], computational fluid dynamics $[60,111,112]$, acoustic scattering [113] and biomechanics [61].

Domain decomposition methods are broadly categorized into overlapping methods ( Schwarz methods) and non-overlapping or substructuring methods (Schur complement methods ) [4-7, 72]. In Schwarz methods, the solution domain is decomposed into a number of overlapping subdomains and a smaller problem is solved alternately on each subdomain with an appropriate approximate solution on the artificial boundary [4-7]. The solution on the artificial boundary is updated by propagating the solution of the subdomain problem to the boundary [4-7]. The solution converges when the change in the successive solutions on the artificial boundary is less than a prescribed tolerance value [4-7]. Schwarz methods can be viewed as a generalizations of block Jacobi and block GaussSeidel iterative methods $[4,5,114]$. The alternating Schwarz method can be used as a preconditioner for Krylov subspace iterative methods, whereby each application of the pre- 
conditioner is one iteration of the alternating Schwarz method with zero initial guess $[5,6]$. In Schur complement methods, the solution domain is partitioned into a number of nonoverlapping subdomains $[4-7,72,115]$. On each subdomain, for example, the finite element stiffness matrix and the load vector are assembled independently, and the degrees of freedom on each subdomains are split into interior and interface variables. Block Gaussian elimination is performed to reduce the global system to the Schur complement system [47, 72]. The solution of the Schur complement system gives the interface variables. The interior variables are obtained by solving the interior problem on each subdomain concurrently $[63,78]$. Depending on the solution method used to solve the Schur complement system, non-overlapping domain decomposition techniques are referred to as a direct substructuring or iterative substructuring method $[6,7]$.

The most popular and widely used Schur complement based iterative substructuring techniques can be divided into the primal and the dual methods $[65,110]$. In the primal iterative substructuring methods, the continuity condition along the interfaces boundaries are enforced strongly by global assembly $[65,78,83]$. The primal methods contain NeumannNeumann [71], Balancing Domain Decomposition (BDD) [116, 117], and Balancing Domain Decomposition by Constraints (BDDC) $[107,108]$. In the dual iterative substructuring methods the continuity condition along the interfaces boundaries are enforced weakly by Lagrange multipliers $[61,65,78,118]$. The Finite Element Tearing and Interconnecting (FETI) $[119,120]$ and the Dual-Primal Finite Element Tearing and Interconnecting (FETIDP) $[64,81]$ belong to this class of domain decomposition method. 
In this chapter, we review the overlapping and non-overlapping domain decomposition methods. Specifically, we first describe the alternating Schwarz method and represent it as a preconditioner. Next, we describe the well-known primal, dual and dual-primal iterative substructuring techniques. In addition, we present a novel primal-primal iterative substructuring algorithm equipped with a two-level scalable preconditioner.

\subsection{Overlapping Domain Decomposition Methods}

The Schwarz method is the earliest domain decomposition method $[4,5,121]$ which was published in 1890. In the classical Schwarz method, a Laplace equation defined on an irregular domain is solved iteratively on a union of regular subdomains that forms the irregular original domain such as rectangular and circular subdomains [7] (see Fig.(3.1) and Fig.(3.2)).

The Schwarz alternating method is based on splitting the computational domain into a number of overlapping subdomains. The algorithm starts with a suitable initial guess for the solution and precedes by constructing a sequence of improved approximations [72]. The approximate solutions are obtained by alternately solving a local Dirichlet problem with an inhomogeneous boundary condition on the artificial boundary. When the most recent available solution from the adjacent subdomain is used to approximate the artificial boundary condition, the procedure is known as multiplicative Schwarz method which can be viewed as a generalization of the block Gauss-Seidel iteration method [4-6]. The multiplicative Schwarz method is serial algorithm in nature because it requires the solution of one sub- 
domain problem prior to another $[5,7]$. On the other hand, when the previous solution is used to approximate the artificial boundary condition, the procedure is known as additive Schwarz method which corresponds to a generalized block Jacobi method $[5,6,72]$. The additive Schwarz method is embarrassingly parallel algorithm because there is no dependency between the concurrent approximate solution of the subdomains problems. However, the convergence rate of the additive Schwarz method is slower than that of the multiplicative Schwarz method [4-7]. In practice, the additive Schwarz method is typically employed as a parallel preconditioner to accelerate the the convergence rate of Krylov subspace methods [7].

\subsubsection{The Alternating Schwarz Method}

Consider a linear second-order elliptic partial differential equation (PDE) defined on a domain $\Omega$ with a given Dirichlet boundary condition on $\partial \Omega$ as shown in Fig.(3.1). The finite element discretization of the PDE leads to the following sparse symmetric positive definite linear system

$$
\mathrm{Au}=\mathrm{f}
$$

where $\mathbf{A} \in \Re^{n \times n}$ is the stiffness matrix, $\mathbf{u} \in \Re^{n}$ is the response, and $\mathrm{f} \in \Re^{n}$ is the force vector. 


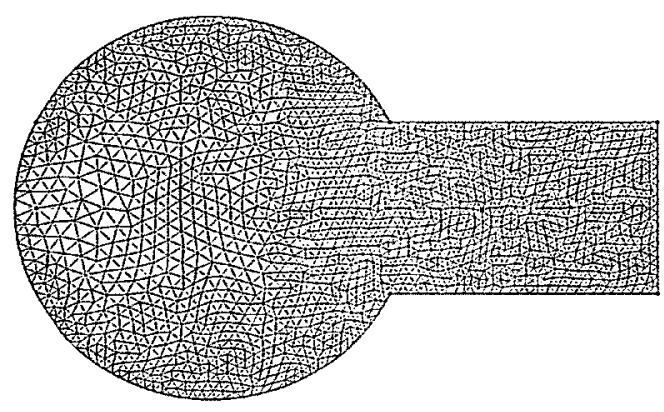

Figure 3.1: A typical domain

Let the spatial domain $\Omega$ be partitioned into two overlapping subdomains as shown in Fig.(3.2) and such that

$$
\Omega=\Omega_{1} \cup \Omega_{2}, \quad \Omega_{1} \cap \Omega_{2} \neq 0, \quad \text { and } \quad \Gamma=\Gamma_{1} \cup \Gamma_{2}
$$

where $\Gamma$ represents the artificial boundary between the two overlapped subdomains.

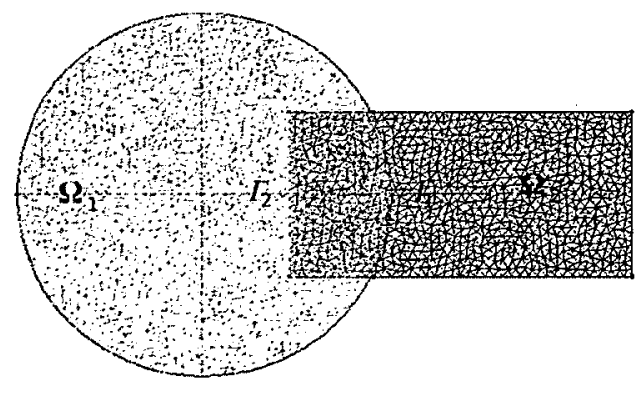

$\underline{Z} x$

Figure 3.2: A typical domain partitioned into two overlapping subdomains 
For a typical subdomain $\Omega_{s}$, the solution vector $\mathbf{u}^{s}$ can be split as

$$
\mathbf{u}^{s}=\left\{\begin{array}{c}
\mathbf{u}_{I}^{s} \\
\mathbf{u}_{\Gamma}^{s}
\end{array}\right\}
$$

where $u_{I}^{s}$ is the interior solution vector corresponding to the nodes in the interior of the subdomain $\Omega_{s}$ and $\mathbf{u}_{\Gamma}^{s}$ is the interface solution vector corresponding to the nodes on the artificial boundary $\Gamma_{s}$. Consequently, the equilibrium equation for a typical subdomain $\Omega_{s}$ can be expressed in the following block form

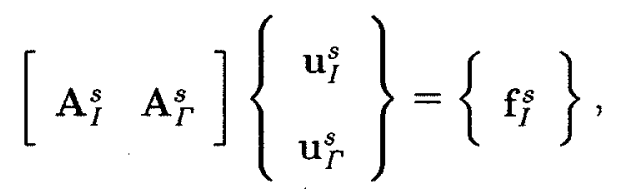

where $\mathbf{A}_{I}^{s} \in \Re^{n_{I}^{s} \times n_{I}^{s}}$ represents the stiffness submatrix associated with the nodes in the interior of the subdomain and $\mathrm{A}_{\Gamma}^{s} \in \Re^{n_{I}^{s} \times n_{\Gamma}^{s}}$ denotes the stiffness submatrix corresponding to the interaction among the interior and the artificial boundary nodes.

Let $\mathcal{I}_{I_{r} \rightarrow \Gamma_{s}}$ be a discrete operator which interpolates the solution of the interior nodes of subdomain $\Omega_{r}$ to the nodes on the artificial boundary $\Gamma_{s}$ of the subdomain $\Omega_{s}$ as

$$
\mathbf{u}_{\Gamma}^{s}=\mathcal{I}_{I_{r} \rightarrow \Gamma_{s}} \mathbf{u}_{I}^{r}
$$

The alternating Schwarz method starts with an initial guess for the solution in one of the subdomains, let us say the second subdomain $\Omega_{2}$, as

$$
\left(\mathbf{u}_{I}^{2}\right)^{k-1}=\left(\mathbf{u}_{I}^{2}\right)^{0}
$$

where $k$ denotes the iteration count. 
Next, the solution from the second subdomain $\Omega_{2}$ is interpolated to the artificial interface boundary of the first subdomain $\Gamma_{1}$ as

$$
\left(\mathrm{u}_{\Gamma}^{1}\right)^{k}=\mathcal{I}_{I_{2} \rightarrow \Gamma_{1}}\left(\mathrm{u}_{I}^{2}\right)^{k-1} .
$$

A local Dirichlet problem with non-homogeneous boundary condition is solved next on the first subdomain $\Omega_{1}$ as

$$
\mathbf{A}_{I}^{1}\left(\mathbf{u}_{I}^{1}\right)^{k}=\mathbf{f}_{I}^{1}-\mathbf{A}_{\Gamma}^{1}\left(\mathbf{u}_{\Gamma}^{1}\right)^{k} .
$$

The current available solution from the first subdomain $\Omega_{1}$ is propagated to the artificial interface boundary of the second subdomain $\Gamma_{2}$ as

$$
\left(\mathbf{u}_{\Gamma}^{2}\right)^{k}=\mathcal{I}_{I_{1} \rightarrow \Gamma_{2}}\left(\mathbf{u}_{I}^{1}\right)^{k} .
$$

A second subdomain level Dirichlet problem with non-homogeneous boundary condition is solved next on the second subdomain $\Omega_{2}$ as

$$
\mathbf{A}_{I}^{2}\left(\mathbf{u}_{I}^{2}\right)^{k}=\mathbf{f}_{I}^{2}-\mathbf{A}_{\Gamma}^{2}\left(\mathbf{u}_{\Gamma}^{2}\right)^{k}
$$

The iteration continues until convergence. Different stopping criteria can be used to determine the convergence of the alternating Schwarz algorithm, such as the difference in the current and the previous interior solution of both the subdomains is less than a tolerance prescribed value $\epsilon_{I_{s}}$ as

$$
\frac{\left\|\left(\mathbf{u}_{I}^{1}\right)^{k}-\left(\mathbf{u}_{I}^{1}\right)^{k-1}\right\|}{\left\|\left(\mathbf{u}_{I}^{1}\right)^{k-1}\right\|} \leqslant \epsilon_{I_{1}} \quad \text { and } \quad \frac{\left\|\left(\mathbf{u}_{I}^{2}\right)^{\mathrm{k}}-\left(\mathbf{u}_{\mathrm{I}}^{2}\right)^{\mathrm{k}-1}\right\|}{\|\left(\left(\mathbf{u}_{\mathrm{I}}^{2}\right)^{\mathrm{k}-1} \|\right.} \leqslant \epsilon_{I_{2}} \text {. }
$$


Clearly, the basic Schwarz alternating algorithm to solve Eq.(3.1) starts with an initial guess for the solution on one subdomain and then iteratively constructs a sequence of improved approximate solutions by alternately solving the subdomains problems $[4,5]$.

The alternating Schwarz method can be outlined in the following algorithm [4-7]:

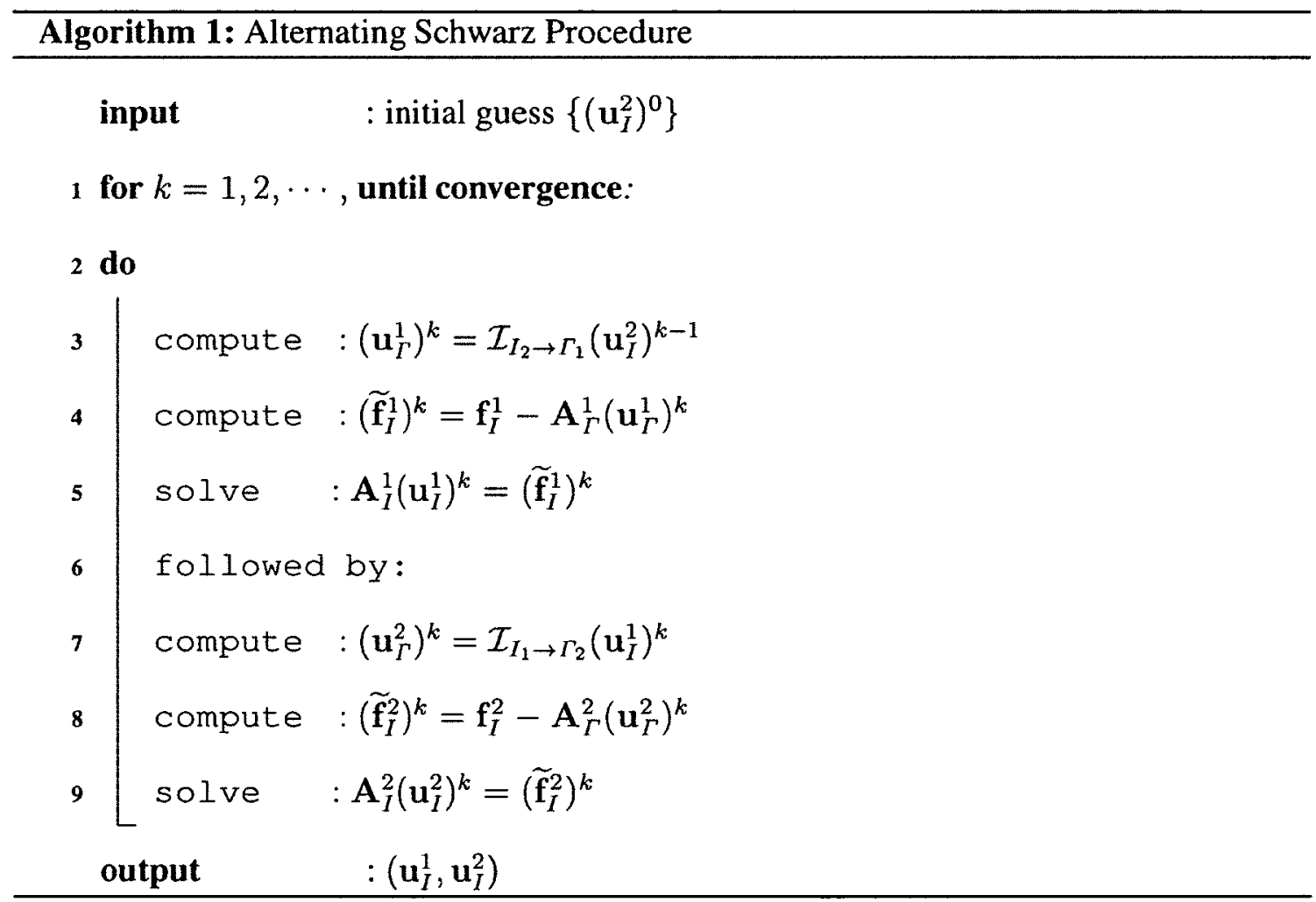

Note that the most current available value of the solution in $\Omega_{1}$ is used to generate the values for the inhomogeneous boundary condition on the artificial interface for the solution in $\Omega_{2}$. This dependency of the second problem on the first problem makes the basic Schwarz algorithm serial in natural when applied to the case of two subdomains $[6,72]$. 
However, this coupling is useful to exchange information between the adjacent subdomains, and as a result improves the convergence rate of the Schwarz method.

\subsubsection{Schwarz Method as a Two-Steps Algorithm}

The alternating Schwarz algorithm can be summarized in the following form [6]

$$
\begin{aligned}
& \mathbf{A}_{I}^{1}\left(\mathbf{u}_{I}^{1}\right)^{k}=\mathbf{f}_{I}^{1}-\mathbf{A}_{\Gamma}^{1} \mathcal{I}_{I_{2} \rightarrow \Gamma_{1}}\left(\mathbf{u}_{I}^{2}\right)^{k-1} \\
& \mathbf{A}_{I}^{2}\left(\mathbf{u}_{I}^{2}\right)^{k}=\mathbf{f}_{I}^{2}-\mathbf{A}_{\Gamma}^{2} \mathcal{I}_{I_{1} \rightarrow \Gamma_{2}}\left(\mathbf{u}_{I}^{1}\right)^{k}
\end{aligned}
$$

Adding and subtracting $\mathbf{A}_{I}^{s}\left(\mathbf{u}_{I}^{s}\right)^{k-1}, s=1,2$ from Eq.(3.12) and Eq.(3.13), the alternating Schwarz algorithm can be expressed as a two-step algorithm as

$$
\begin{aligned}
& \left(\mathbf{u}_{I}^{1}\right)^{k}=\left(\mathbf{u}_{I}^{1}\right)^{k-1}+\left[\mathbf{A}_{I}^{1}\right]^{-1}\left(\mathbf{f}_{I}^{1}-\mathbf{A}_{I}^{1}\left(\mathbf{u}_{I}^{1}\right)^{k-1}-\mathbf{A}_{\Gamma}^{1} \mathcal{I}_{I_{2} \rightarrow \Gamma_{1}}\left(\mathbf{u}_{I}^{2}\right)^{k-1}\right), \\
& \left(\mathbf{u}_{I}^{2}\right)^{k}=\left(\mathbf{u}_{I}^{2}\right)^{k-1}+\left[\mathbf{A}_{I}^{2}\right]^{-1}\left(\mathbf{f}_{I}^{2}-\mathbf{A}_{I}^{2}\left(\mathbf{u}_{I}^{2}\right)^{k-1}-\mathbf{A}_{\Gamma}^{2} \mathcal{I}_{I_{1} \rightarrow \Gamma_{2}}\left(\mathbf{u}_{I}^{1}\right)^{k}\right) .
\end{aligned}
$$

In case of matching grids, the interpolation operator becomes an identity matrix and two-steps Schwarz scheme reduces into the following form

$$
\begin{aligned}
& \left(\mathbf{u}_{I}^{1}\right)^{k}=\left(\mathbf{u}_{I}^{1}\right)^{k-1}+\left[\mathbf{A}_{I}^{1}\right]^{-1}\left(\mathbf{f}_{I}^{1}-\mathbf{A}_{I}^{1}\left(\mathbf{u}_{I}^{1}\right)^{k-1}-\mathbf{A}_{\Gamma}^{1}\left(\mathbf{u}_{I}^{2}\right)^{k-1}\right), \\
& \left(\mathbf{u}_{I}^{2}\right)^{k}=\left(\mathbf{u}_{I}^{2}\right)^{k-1}+\left[\mathbf{A}_{I}^{2}\right]^{-1}\left(\mathbf{f}_{I}^{2}-\mathbf{A}_{I}^{2}\left(\mathbf{u}_{I}^{2}\right)^{k-1}-\mathbf{A}_{\Gamma}^{2}\left(\mathbf{u}_{I}^{1}\right)^{k}\right) .
\end{aligned}
$$

The scheme in Eq.(3.16) and Eq.(3.17) can be viewed as a generalized block GaussSeidel scheme to solve the following system $[6,72]$ 


$$
\left[\begin{array}{cc}
\mathrm{A}_{I}^{1} & \mathbf{A}_{\Gamma}^{1} \\
\mathrm{~A}_{\Gamma}^{2} & \mathbf{A}_{I}^{2}
\end{array}\right]\left\{\begin{array}{c}
\mathbf{u}_{I}^{1} \\
\mathbf{u}_{I}^{2}
\end{array}\right\}=\left\{\begin{array}{c}
\mathbf{f}_{I}^{1} \\
\mathbf{f}_{I}^{2}
\end{array}\right\} .
$$

The residual vectors in the interior of the subdomains $\Omega_{1}$ and $\Omega_{2}$ can be obtained from Eq.(3.16) and Eq.(3.17) as

$$
\begin{aligned}
& \left(\mathbf{r}^{1}\right)^{k}=\mathbf{f}_{I}^{1}-\mathbf{A}_{I}^{1}\left(\mathbf{u}_{I}^{1}\right)^{k-1}-\mathbf{A}_{\Gamma}^{1}\left(\mathbf{u}_{I}^{2}\right)^{k-1} \\
& \left(\mathbf{r}^{2}\right)^{k}=\mathbf{f}_{I}^{2}-\mathbf{A}_{I}^{2}\left(\mathbf{u}_{I}^{2}\right)^{k-1}-\mathbf{A}_{\Gamma}^{2}\left(\mathbf{u}_{I}^{1}\right)^{k}
\end{aligned}
$$

In a vector form, the residual vector can be expressed as

$$
\mathbf{r}=\left\{\begin{array}{c}
\mathbf{r}^{1} \\
\mathrm{r}^{2}
\end{array}\right\}=\mathbf{f}-\mathbf{A u}
$$

Thus the alternating Schwarz method can be rewritten in a two-step algorithm as [6]

$$
\begin{aligned}
& \mathbf{u}^{k+\frac{1}{2}}=\mathbf{u}^{k}+\left[\begin{array}{rr}
{\left[\mathbf{A}_{I}^{1}\right]^{-1}} & 0 \\
0 & 0
\end{array}\right]\left(\mathbf{f}-\mathbf{A} \mathbf{u}^{k}\right) \\
& \mathbf{u}^{k+1}=\mathbf{u}^{k+\frac{1}{2}}+\left[\begin{array}{cc}
0 & 0 \\
0 & {\left[\mathbf{A}_{I}^{2}\right]^{-1}}
\end{array}\right]\left(\mathbf{f}-\mathbf{A} \mathbf{u}^{k+\frac{1}{2}}\right)
\end{aligned}
$$

Note that, this form of the iteration scheme does not explicitly depend on the artificial boundaries.

In case of the additive Schwarz method, the two-step scheme is expressed as 


$$
\begin{aligned}
& \mathbf{u}^{k+\frac{1}{2}}=\mathbf{u}^{k}+\left[\begin{array}{rr}
{\left[\mathbf{A}_{I}^{1}\right]^{-1}} & 0 \\
0 & 0
\end{array}\right]\left(\mathbf{f}-\mathbf{A} \mathbf{u}^{k}\right) \\
& \mathbf{u}^{k+1}=\mathbf{u}^{k+\frac{1}{2}}+\left[\begin{array}{cc}
0 & 0 \\
0 & {\left[\mathbf{A}_{I}^{2}\right]^{-1}}
\end{array}\right]\left(\mathbf{f}-\mathbf{A} \mathbf{u}^{k}\right)
\end{aligned}
$$

Note that the current approximate solution $\mathrm{u}^{k}$ is used to calculate the residual vector in the second step.

Equivalently, Eq.(3.24) and Eq.(3.25) can be combined as

$$
\mathbf{u}^{k+1}=\mathbf{u}^{k}+\left[\begin{array}{cc}
{\left[\mathbf{A}_{I}^{1}\right]^{-1}} & 0 \\
0 & {\left[\mathbf{A}_{I}^{2}\right]^{-1}}
\end{array}\right]\left(\mathbf{f}-\mathbf{A u}^{k}\right)
$$

which can be viewed as a generalization of block-Jacobi method $[4,6]$.

\subsubsection{Schwarz Method as a Richardson's Iteration}

The Schwarz alternating method can be viewed as a Richardson's iterative method as explained next $[4,6]$.

Let $\mathbf{R}_{s}$ be a restriction operator such that 


$$
\begin{aligned}
& \mathbf{u}_{I}^{1}=\mathbf{R}_{1} \mathbf{u}=\left[\begin{array}{ll}
\mathbf{I} & 0
\end{array}\right]\left\{\begin{array}{l}
\mathbf{u}_{I}^{1} \\
\mathbf{u}_{I}^{2}
\end{array}\right\}, \\
& \mathbf{u}_{I}^{2}=\mathbf{R}_{2} \mathbf{u}=\left[\begin{array}{ll}
0 & \mathbf{I}
\end{array}\right]\left\{\begin{array}{l}
\mathbf{u}_{I}^{1} \\
\mathbf{u}_{I}^{2}
\end{array}\right\} .
\end{aligned}
$$

Using the definition of the restriction operators, the residual vectors in subdomains $\Omega_{1}$ and $\Omega_{2}$ can be expressed respectively as [6]

$$
\begin{aligned}
& \mathbf{r}_{1}=\mathbf{R}_{1}\left(\mathrm{f}-\mathbf{A} \mathbf{u}^{k}\right) \\
& \mathbf{r}_{2}=\mathbf{R}_{2}\left(\mathrm{f}-\mathbf{A} \mathbf{u}^{k+\frac{1}{2}}\right) .
\end{aligned}
$$

The stiffness matrix of the subdomains $\Omega_{1}$ and $\Omega_{2}$, can be obtained as

$$
\begin{aligned}
& \mathbf{A}_{I}^{1}=\mathbf{R}_{1} \mathbf{A R}_{1}^{T} \\
& \mathbf{A}_{I}^{2}=\mathbf{R}_{2} \mathbf{A R}_{2}^{T} .
\end{aligned}
$$

Consequently, the multiplicative Schwarz method (see Eq.(3.22) and Eq.(3.23)) can be rewritten as $[5,6]$

$$
\begin{aligned}
& \mathbf{u}^{k+\frac{1}{2}}=\mathbf{u}^{k}+\mathbf{R}_{1}^{T}\left(\mathbf{R}_{1} \mathbf{A} \mathbf{R}_{1}^{T}\right)^{-1} \mathbf{R}_{1}\left(\mathbf{f}-\mathbf{A} \mathbf{u}^{k}\right) \\
& \mathbf{u}^{k+1}=\mathbf{u}^{k+\frac{1}{2}}+\mathbf{R}_{2}^{T}\left(\mathbf{R}_{2} \mathbf{A} \mathbf{R}_{2}^{T}\right)^{-1} \mathbf{R}_{2}\left(\mathbf{f}-\mathbf{A} \mathbf{u}^{k+\frac{1}{2}}\right)
\end{aligned}
$$


Let $\mathbf{B}_{s}$ be a matrix that restricts the residual to subdomain $\Omega_{s}$, solves the problem on that subdomain to generate a correction and then extends the correction back to the entire domain as [6]

$$
\mathbf{B}_{s}=\mathbf{R}_{s}^{T}\left(\mathbf{R}_{s} \mathbf{A} \mathbf{R}_{s}^{T}\right)^{-1} \mathbf{R}_{s}
$$

In terms of $\mathrm{B}_{s}$ matrix, the two-step multiplicative Schwarz algorithm can be expressed as

$$
\begin{aligned}
& \mathbf{u}^{k+\frac{1}{2}}=\mathbf{u}^{k}+\mathbf{B}_{1}\left(\mathbf{f}-\mathbf{A} \mathbf{u}^{k}\right) \\
& \mathbf{u}^{k+1}=\mathbf{u}^{k+\frac{1}{2}}+\mathbf{B}_{2}\left(\mathbf{f}-\mathbf{A} \mathbf{u}^{k+\frac{1}{2}}\right)
\end{aligned}
$$

Substituting Eq.(3.36) into Eq.(3.37), leads to

$$
\mathbf{u}^{k+1}=\mathbf{u}^{k}+\left(\mathbf{B}_{1}+\mathbf{B}_{2}-\mathbf{B}_{2} \mathbf{A} \mathbf{B}_{1}\right)\left(\mathbf{f}-\mathbf{A u}^{k}\right)
$$

which is a one-step Richardson iterative procedure [6].

Note that the coupling term $\mathrm{B}_{2} \mathrm{AB}_{1}$ in Eq.(3.38) prevents the parallel implementation of the multiplicative Schwarz scheme. When this term is dropped, the scheme becomes the embarrassingly parallel additive Schwarz method as $[5,6]$

$$
\mathbf{u}^{k+1}=\mathbf{u}^{k}+\left(\mathbf{B}_{1}+\mathbf{B}_{2}\right)\left(\mathbf{f}-\mathbf{A} \mathbf{u}^{k}\right)
$$


Although the additive Schwarz method is embarrassingly parallel procedure, it has a convergence rate that is slower than that of the multiplicative Schwarz. The difference of the convergence properties of the additive and multiplicative Schwarz methods is analogues to that of Jacobi and Gauss-Seidel methods $[5,6]$.

\subsubsection{Schwarz Method as a Preconditioner}

The Schwarz method can be used as a preconditioner to accelerate the convergence rate of Krylov subspace iterative methods as explained next [5, 6].

Eq.(3.38) can be rearranged as

$$
\mathbf{u}^{k+1}-\mathbf{u}^{k}=\left(\mathbf{B}_{1}+\mathbf{B}_{2}-\mathbf{B}_{2} \mathbf{A B}_{1}\right)\left(\mathbf{f}-\mathbf{A u}^{k}\right)
$$

Equivalently, Eq.(3.40) can be rewritten as

$$
\mathbf{e}^{k}=\mathbf{M}^{-1} \mathbf{r}^{k}
$$

where the multiplicative Schwarz preconditioner (Richardson Preconditioner) is defined as

$$
\mathbf{M}^{-1}=\mathbf{B}_{1}+\mathbf{B}_{2}-\mathbf{B}_{2} \mathbf{A B}_{1}
$$

Note that the multiplicative Schwarz preconditioner in Eq.(3.42) is not symmetric. To symmetrize the preconditioner, a third sub-step is needed as $[5,6]$ 


$$
\begin{aligned}
& \mathbf{u}^{k+\frac{1}{3}}=\mathbf{u}^{k}+\mathrm{B}_{1}\left(\mathbf{f}-\mathbf{A} \mathbf{u}^{k}\right) \\
& \mathbf{u}^{k+\frac{2}{3}}=\mathbf{u}^{k+\frac{1}{3}}+\mathbf{B}_{2}\left(\mathbf{f}-\mathbf{A} \mathbf{u}^{k+\frac{1}{3}}\right) \\
& \mathbf{u}^{k+1}=\mathbf{u}^{k+\frac{2}{3}}+\mathbf{B}_{1}\left(\mathbf{f}-\mathbf{A} \mathbf{u}^{k+\frac{2}{3}}\right)
\end{aligned}
$$

As a one-step scheme the symmetrized multiplicative Schwarz can be rewritten as [6]

$$
\mathbf{u}^{k+1}=\mathbf{u}^{k}+\left(\mathbf{B}_{1}+\mathbf{B}_{2}-\mathbf{B}_{2} \mathbf{A B}_{1}-\mathbf{B}_{1} \mathbf{A B}_{2}+\mathbf{B}_{1} \mathbf{A B}_{2} \mathbf{A B}_{1}\right)\left(\mathbf{f}-\mathbf{A u}^{k}\right)
$$

Eq.(3.46) can be expressed in a factored form as

$$
\mathbf{u}^{k+1}=\mathbf{u}^{k}+\left[\mathbf{B}_{1}+\left(\mathbf{I}-\mathbf{B}_{1} \mathbf{A}\right) \mathbf{B}_{2}\left(\mathbf{I}-\mathbf{A B}_{1}\right)\right]\left(\mathbf{f}-\mathbf{A} \mathbf{u}^{k}\right)
$$

and the symmetrized multiplicative Schwarz preconditioner can be expressed as

$$
\mathbf{M}^{-1}=\mathbf{B}_{1}+\left(\mathbf{I}-\mathbf{B}_{1} \mathbf{A}\right) \mathbf{B}_{2}\left(\mathbf{I}-\mathbf{A B}_{1}\right)
$$

which can be viewed as a generalization of block Gauss-Seidel method $[5,6,102]$.

On the other hand, the additive Schwarz preconditioner can be expressed as

$$
\mathbf{M}^{-1}=\left[\mathbf{B}_{1}+\mathbf{B}_{2}\right]
$$

which is a generalization of a block Jacobi method $[5,6]$. 


\subsubsection{Schwarz Method for Many Subdomains}

The multiplicative Schwarz scheme Eq.(3.36) and Eq.(3.37) can be generalized to the case of many overlapping subdomains $\left(\Omega_{s}, s=1,2, \cdots, n_{s}\right)$ as [6]

$$
\begin{aligned}
\mathbf{u}^{k+\frac{1}{n_{s}}} & =\mathbf{u}^{k}+\mathbf{B}_{1}\left(\mathbf{f}-\mathbf{A} \mathbf{u}^{k}\right) \\
\mathbf{u}^{k+\frac{2}{n_{s}}} & =\mathbf{u}^{k+\frac{1}{n_{s}}}+\mathbf{B}_{2}\left(\mathbf{f}-\mathbf{A} \mathbf{u}^{k+\frac{1}{n_{s}}}\right), \\
\vdots & \\
\mathbf{u}^{k+1} & =\mathbf{u}^{k+\frac{\left(n_{s}-1\right)}{n_{s}}}+\mathbf{B}_{n_{s}}\left(\mathbf{f}-\mathbf{A} \mathbf{u}^{k+\frac{\left(n_{s}-1\right)}{n_{s}}}\right) .
\end{aligned}
$$

This form of the multiplicative Schwarz scheme has a limited potential for parallelism [6, 72]. In practice however, there are often many subdomains that do not share a common interface boundary. Thus the solution on these subdomains can be updated concurrently and in parallel. This strategy can be implemented using a multi-coloring technique whereby each subdomain is identified with a color such that subdomains of the same color do not share a common interface boundary $[6,72]$.

If $q$ be the number of colors used, the $q$-step multiplicative Schwarz scheme can be defined as [6] 


$$
\begin{aligned}
\mathbf{u}^{k+\frac{1}{q}} & =\mathbf{u}^{k}+\sum_{i \in \text { Color }_{1}} \mathbf{B}_{i}\left(\mathbf{f}-\mathbf{A} \mathbf{u}^{k}\right), \\
\mathbf{u}^{k+\frac{2}{q}} & =\mathbf{u}^{k+\frac{1}{q}}+\sum_{i \in \text { Color }_{2}} \mathbf{B}_{i}\left(\mathbf{f}-\mathbf{A} \mathbf{u}^{k+\frac{1}{q}}\right), \\
\vdots & \\
\mathbf{u}^{k+1} & =\mathbf{u}^{k+\frac{(q-1)}{q}}+\sum_{i \in \text { Color }_{q}} \mathbf{B}_{i}\left(\mathbf{f}-\mathbf{A} \mathbf{u}^{\left.k+\frac{(q-1)}{q}\right)} .\right.
\end{aligned}
$$

Generally, the convergence rate of the multi-coloring scheme depends on the number of colors. Increasing the number of colors leads to slow convergence rate of the scheme [6].

\subsection{Non-Overlapping Domain Decomposition Methods}

In non-overlapping domain decomposition methods or substructuring techniques, the solution domain is partitioned into a number of non-overlapping subdomains. On each subdomain, the finite element stiffness matrix and load vector are assembled independently. The subdomain local variables are split into interior and interface variables. The interior variables of each subdomain are concurrently eliminated by static condensation leading to a smaller but denser interface problem, namely the Schur complement system. The solution of the Schur complement system gives the interface variables. Once the interface variables are available, the interior variable can be obtained by solving the interior problem concurrently on each subdomain. The methodology can be described as follow [4-7].

Consider a linear second-order elliptic partial differential equation (PDE) defined on a domain $\Omega$ with given boundary conditions on $\partial \Omega$. The finite element discretization of the 
PDE leads to the following sparse symmetric positive definite linear system

$$
\mathbf{A u}=\mathbf{f}
$$

where $\mathbf{A} \in \Re^{n \times n}$ is the stiffness matrix, $\mathbf{u} \in \Re^{n}$ is the response, and $\mathbf{f} \in \Re^{n}$ is the forcing term.

Let the spatial domain $\Omega$ be partitioned into $n_{s}$ non-overlapping subdomains $\left\{\Omega_{s}, 1 \leq\right.$ $\left.s \leq n_{s}\right\}$ as shown schematically in Fig.(3.3). Each of the subdomains is a union of elements and such that

$$
\Omega=\bigcup_{s=1}^{n_{s}} \Omega_{s}, \quad \Omega_{s} \bigcap \Omega_{r}=0 \quad s \neq r
$$

and

$$
\Gamma=\bigcup_{s=1} \Gamma_{s} \text { where } \Gamma_{s}=\partial \Omega_{s} \backslash \partial \Omega
$$

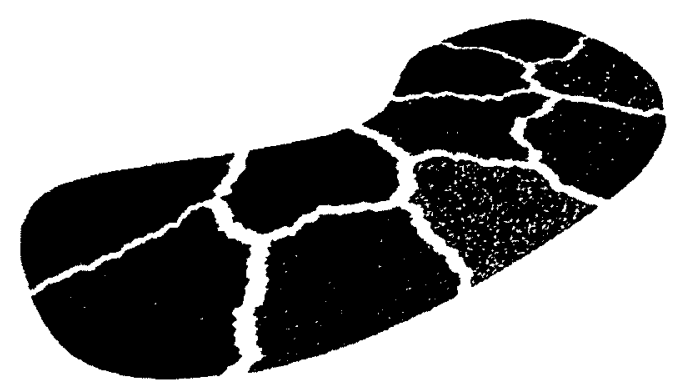

Figure 3.3: A typical domain partitioned into $n_{s}$ non-overlapping subdomains

For a typical subdomain $\Omega_{s}$, the solution vector $\mathbf{u}^{s}$ can be split into two subvectors as 


$$
\mathbf{u}^{s}=\left\{\begin{array}{c}
\mathbf{u}_{I}^{s} \\
\mathbf{u}_{\Gamma}^{s}
\end{array}\right\}
$$

where $u_{I}^{s}$ is the interior solution vector which corresponds to the nodes in the interior of the subdomain and $u_{\Gamma}^{s}$ is the interface solution vector that relates to the nodes on the interface boundary between two or more neighboring subdomains.

Consequently, the equilibrium equation for a typical subdomain $\Omega_{s}$ can be written in a block form as

$$
\left[\begin{array}{cc}
\mathbf{A}_{I I}^{s} & \mathbf{A}_{I \Gamma}^{s} \\
\mathbf{A}_{\Gamma I}^{s} & \mathbf{A}_{\Gamma \Gamma}^{s}
\end{array}\right]\left\{\begin{array}{c}
\mathbf{u}_{I}^{s} \\
\mathbf{u}_{\Gamma}^{s}
\end{array}\right\}=\left\{\begin{array}{c}
\mathbf{f}_{I}^{s} \\
\mathbf{f}_{\Gamma}^{s}
\end{array}\right\} .
$$

The submatrix $\mathbf{A}_{I I}^{s}$ relates to the stiffness matrix of the interior nodes, and the stiffness submatrices $\mathrm{A}_{I \Gamma}^{s}$ and $\mathrm{A}_{\Gamma I}^{s}$ relate to the coupling between the interior and interface boundary nodes, and the submatrix $\mathbf{A}_{\Gamma \Gamma}^{s}$ represents the stiffness matrix of the interface boundary nodes.

For a typical subdomain $\Omega_{s}$, let $\mathbf{R}_{s}$ be a restriction matrix (consisting of zeros and ones) that maps the entire set of interface degrees of freedom $u_{\Gamma}$ to the local subdomain interface degree of freedom $\mathbf{u}_{\Gamma}^{s}$ on $\Gamma_{s}$ as

$$
\mathbf{u}_{\Gamma}^{s}=\mathbf{R}_{s} \mathbf{u}_{\Gamma}
$$

In parallel implementation, $\mathbf{R}_{s}$ acts as a scatter operator while $\mathbf{R}_{s}^{T}$ acts as a gather operator and are not constructed explicitly. 
Enforcing the transmission conditions (compatibility and equilibrium) along the interfaces boundaries by global assembly, yields the following global equilibrium equation [66]

$$
\left[\begin{array}{cccc}
\mathbf{A}_{I I}^{1} & \cdots & 0 & \mathbf{A}_{I \Gamma}^{1} \mathbf{R}_{1} \\
\vdots & \ddots & \vdots & \vdots \\
0 & \cdots & \mathbf{A}_{I I}^{n_{s}} & \mathbf{A}_{I \Gamma}^{n_{s}} \mathbf{R}_{n_{s}} \\
\mathbf{R}_{1}^{T} \mathbf{A}_{\Gamma I}^{1} & \cdots & \mathbf{R}_{n_{s}}^{T} \mathbf{A}_{\Gamma I}^{n_{s}} & \sum_{s=1}^{n_{s}} \mathbf{R}_{s}^{T} \mathbf{A}_{\Gamma \Gamma}^{s} \mathbf{R}_{s}
\end{array}\right]\left\{\begin{array}{c}
\mathbf{u}_{I}^{1} \\
\vdots \\
\mathbf{u}_{I}^{n_{s}} \\
\mathbf{u}_{\Gamma}
\end{array}\right\}=\left\{\begin{array}{c}
\mathbf{f}_{I}^{1} \\
\vdots \\
\mathbf{f}_{I}^{n_{s}} \\
\sum_{s=1}^{n_{s}} \mathbf{R}_{s}^{T} \mathbf{f}_{\Gamma}^{s}
\end{array}\right\} .
$$

Performing the Gaussian elimination, Eq.(3.62) can be recast as

$$
\left[\begin{array}{cccc}
\mathbf{A}_{I I}^{1} & \cdots & 0 & \mathbf{A}_{I T}^{1} \mathbf{R}_{1} \\
\vdots & \ddots & \vdots & \vdots \\
0 & \cdots & \mathbf{A}_{I I}^{n_{s}} & \mathbf{A}_{I \Gamma}^{n_{s}} \mathbf{R}_{n_{s}} \\
0 & \ldots & 0 & \sum_{s=1}^{n_{s}} \mathbf{R}_{s}^{T} \mathbf{S}_{s} \mathbf{R}_{s}
\end{array}\right]\left\{\begin{array}{c}
\mathbf{u}_{I}^{1} \\
\vdots \\
\mathbf{u}_{I}^{n_{s}} \\
\mathbf{u}_{\Gamma}
\end{array}\right\}=\left\{\begin{array}{c}
\mathrm{f}_{I}^{1} \\
\vdots \\
\mathbf{f}_{I}^{n_{s}} \\
\sum_{s=1}^{n_{s}} \mathbf{R}_{s}^{T} \mathbf{g}_{\Gamma}^{s}
\end{array}\right\}
$$

where the local Schur complement matrix $\mathbf{S}_{s}$ and the corresponding right-hand side vector $\mathrm{g}_{\Gamma}^{s}$ are respectively defined as

$$
\begin{aligned}
\mathbf{S}_{s} & =\mathbf{A}_{\Gamma \Gamma}^{s}-\mathbf{A}_{\Gamma I}^{s}\left[\mathbf{A}_{I I}^{s}\right]^{-1} \mathbf{A}_{I \Gamma}^{s}, \\
\mathbf{g}_{\Gamma}^{s} & =\mathbf{f}_{\Gamma}^{s}-\mathbf{A}_{\Gamma I}^{s}\left[\mathbf{A}_{I I}^{s}\right]^{-1} \mathbf{f}_{I}^{s} .
\end{aligned}
$$

The global interface problem or the Schur complement system is defined as

$$
\mathbf{S} \mathbf{u}_{\Gamma}=\mathbf{g}_{\Gamma}
$$


where the global Schur complement matrix $\mathbf{S}$ and the corresponding right-hand side vector $\mathbf{g}_{\Gamma}$ are respectively given by

$$
\begin{aligned}
\mathbf{S} & =\sum_{s=1}^{n_{s}} \mathbf{R}_{s}^{T} \mathbf{S}_{s} \mathbf{R}_{s} \\
\mathbf{g}_{\Gamma} & =\sum_{s=1}^{n_{s}} \mathbf{R}_{s}^{T} \mathbf{g}_{\Gamma} .
\end{aligned}
$$

The solution of the Schur complement system gives the unknowns $\mathbf{u}_{\Gamma}$ along the interfaces boundaries. Once these unknowns are available, the interior unknowns $\mathbf{u}_{I}^{s}$ can be obtained by solving the following interior problem on each subdomain in parallel as

$$
\mathbf{A}_{I I}^{s} \mathbf{u}_{I}^{s}=\mathbf{f}_{I}^{s}-\mathbf{A}_{\Gamma I}^{s} \mathbf{R}_{s} \mathbf{u}_{\Gamma}
$$

The global Schur complement of the stiffness matrix of a second-order elliptic PDE is symmetric and positive definite [4-7]. However, the local Schur complement matrix is singular for the case of floating subdomains (subdomains without enough Dirichlet boundary conditions to constrain rigid body motion at the null space), and hence care must be taken while tackling the subdomain-level (local) Schur complement system [4-7]. The Schur complement matrix is generally dense and expensive to construct explicitly [4-7].

The condition number of the Schur complement matrix is generally better than the original stiffness matrix $[6,72,78]$. For instance, if $h$ is the finite element mesh size, the condition number of the stiffness matrix for many second order PDEs is [4-7]

$$
\kappa(\mathbf{A})=\mathcal{O}\left(\frac{1}{h^{2}}\right)
$$


On the other hand, the condition number of the Schur complement matrix grows as [4$7,122]$

$$
\kappa(\mathbf{S})=\mathcal{O}\left(\frac{1}{h H}\right)
$$

where $H$ denotes the subdomain size. As $H$ is much greater than $h$, the Schur complement matrix is better conditioned than the original stiffness matrix.

Depending on the solution method used to solve the Schur complement system Eq.(3.66), substructuring techniques are divided into direct and iterative methods $[6,7]$. In the direct substructuring method, the Schur complement system is explicitly constructed and solved using a dense direct solver (usually a multi-frontal solver). The computational cost and the memory requirements for constructing the Schur complement system limit the capability of the direct substructuring method to tackle large-scale practical problems using parallel computing. On the other hand, in the iterative substructuring techniques, the interface problem is solved iteratively without explicitly constructing the Schur complement system.

\subsubsection{Primal Iterative Substructuring Method}

In practice, it is expensive to construct the Schur complement system explicitly. Instead an iterative method, which only requires the action of the Schur complement matrix on a vector [115], is used to solve the Schur complement system. In general the performance of the iterative method can be dramatically improved by using an efficient preconditioner [52].

Iterative substructuring can be viewed as a preconditioned iterative method for solving the Schur complement system of the form [4] 


$$
\mathbf{S} \mathbf{u}_{\Gamma}=\mathbf{g}_{\Gamma}
$$

For symmetric positive-definite system such as Schur complement system, the Conjugate Gradient Method (CGM) is generally used. The performance of CGM mainly depends on the spectrum of the coefficient matrix. However, the rate of convergence of the iterative method can generally be improved by transforming the original system into an equivalent system that has better spectral properties (i.e. lower condition number) [52]. This transformation is called preconditioning and the matrix used in the transformation is called the preconditioner. In other words, the transformed linear system becomes

$$
\mathbf{M}^{-1} \mathbf{S} \mathbf{u}_{\Gamma}=\mathbf{M}^{-1} \mathbf{g}_{\Gamma}
$$

In general, $\kappa\left(\mathbf{M}^{-1} \mathbf{S}\right)$ is much smaller than $\kappa(\mathbf{S})$ and the eigenvalues of $\mathbf{M}^{-1} \mathbf{S}$ are clustered near one [52]. This procedure is known as the Preconditioned Conjugate Gradient Method (PCGM). In practice, the explicit construction of $\mathrm{M}^{-1}$ is not needed. Instead, for a given vector $\mathbf{r}_{\Gamma}$, a system of the the following form is solved

$$
\mathbf{M z}=\mathbf{r}_{\Gamma}
$$

In the following sections, we review various types of popular iterative substructuring methods which are used to develop an efficient preconditioner $\mathrm{M}^{-1}$ for the Schur complement system. 


\section{Neumann-Neumann Method}

Neumann-Neumann preconditioner approximates the inverse of the global Schur complement matrix by a weighted sum of the inverse of the local Schur complement matrices [71]. The implementation of the preconditioner requires a local solve of a Neumann problem in each iteration of the iterative solver. The algorithm can be described as follow $[4,5,7]$.

For a typical subdomain $\Omega_{s}$, the equilibrium equation can be written as

$$
\left[\begin{array}{cc}
\mathbf{A}_{I I}^{s} & \mathbf{A}_{I \Gamma}^{s} \\
\mathbf{A}_{\Gamma I}^{s} & \mathbf{A}_{\Gamma \Gamma}^{s}
\end{array}\right]\left\{\begin{array}{l}
\mathbf{u}_{I}^{s} \\
\mathbf{u}_{\Gamma}^{s}
\end{array}\right\}=\left\{\begin{array}{l}
\mathbf{f}_{I}^{s} \\
\mathbf{f}_{\Gamma}^{s}
\end{array}\right\} .
$$

Starting with an initial guess for the interface unknown $\mathbf{u}_{\Gamma_{j}}$ (where $j$ represents the iteration count), a local Dirichlet problem with non-homogeneous boundary condition is solved to obtain the interior unknown. Hence from the first row of the Eq.(3.75), the local interior unknown $\mathbf{u}_{I}^{s}$ can be obtained as

$$
\mathbf{u}_{I}^{s}=\left[\mathbf{A}_{I I}^{s}\right]^{-1}\left(\mathbf{f}_{I}^{s}-\mathbf{A}_{I \Gamma}^{s} \mathbf{u}_{\Gamma}^{s}\right)
$$

where

$$
\mathbf{u}_{\Gamma}^{s}=\mathbf{R}_{s} \mathbf{u}_{\Gamma_{j}}
$$

The corresponding subdomain level residual vector $\mathbf{r}_{\Gamma}^{s}$ can be computed from the second row of Eq.(3.75) as

$$
\mathbf{r}_{\Gamma}^{s}=\mathbf{A}_{\Gamma I}^{s} \mathbf{u}_{I}^{s}+\mathbf{A}_{\Gamma \Gamma}^{s} \mathbf{u}_{\Gamma}^{s}-\mathbf{f}_{\Gamma}^{s}
$$

Substituting Eq.(3.76) into Eq.(3.78) leads to 


$$
\mathbf{r}_{\Gamma}^{s}=\left(\mathbf{A}_{\Gamma \Gamma}^{s}-\mathbf{A}_{\Gamma I}^{s}\left[\mathbf{A}_{I I}^{s}\right]^{-1} \mathbf{A}_{I \Gamma}^{s}\right) \mathbf{u}_{\Gamma}^{s}-\left(\mathbf{f}_{\Gamma}^{s}-\mathbf{A}_{\Gamma I}^{s}\left[\mathbf{A}_{I I}^{s}\right]^{-1} \mathbf{f}_{I}^{s}\right)
$$

Using the definition of the local Schur complement matrix and the corresponding righthand side, the local residual in Eq.(3.79) can be rewritten as

$$
\mathbf{r}_{\Gamma}^{s}=\mathbf{S}_{s} \mathbf{u}_{\Gamma}^{s}-\mathbf{g}_{\Gamma}^{s}
$$

where

$$
\begin{aligned}
& \mathbf{S}_{s}=\mathbf{A}_{\Gamma \Gamma}^{s}-\mathbf{A}_{\Gamma I}^{s}\left[\mathbf{A}_{I I}^{s}\right]^{-1} \mathbf{A}_{I \Gamma}^{s} \\
& \mathbf{g}_{\Gamma}^{s}=\mathbf{f}_{\Gamma}^{s}-\mathbf{A}_{\Gamma I}^{s}\left[\mathbf{A}_{I I}^{s}\right]^{-1} \mathbf{f}_{I}^{s}
\end{aligned}
$$

The global equilibrium is enforced next by gathering the local residuals from all the subdomains as

$$
\mathbf{r}_{\Gamma_{j}}=\sum_{s=1}^{n_{s}} \mathbf{R}_{s}^{T} \mathbf{r}_{\Gamma}^{s},
$$

where $\mathrm{r}_{\Gamma_{j}}$ is the global residual vector at the $j^{\text {th }}$ iteration which represents the unbalanced force over the boundary interfaces due to the erroneous initial guess for $\mathbf{u}_{\Gamma_{j}}$. Note that when $\mathbf{r}_{\Gamma_{j}}=0$, the system is in equilibrium.

Let $\mathbf{D}_{s}$ represent a diagonal scaling matrix that constitutes a decomposition of unity as

$$
\sum_{s=1}^{n_{s}} \mathbf{R}_{s}^{T} \mathbf{D}_{s} \mathbf{R}_{s}=\mathbf{I}
$$


where the diagonal entries of $\mathrm{D}_{s}$ are the reciprocal of the number of subdomains that share the interface boundary nodes $[4,6,7]$.

The global residual (the unbalanced force) given by Eq.(3.83) can be distributed over the subdomains as

$$
\mathbf{r}_{\Gamma}^{s}=\mathbf{D}_{s} \mathbf{R}_{s} \mathbf{r}_{\Gamma_{j}}
$$

The corresponding unknowns, $\mathbf{u}_{\Gamma}^{s}$ due to the local unbalanced force given by Eq.(3.85) can be computed by solving a local Schur complement system as

$$
\mathbf{S}_{s} \mathbf{u}_{\Gamma}^{s}=\mathbf{r}_{\Gamma}^{s}
$$

In practice, the local Schur complement matrix $\mathbf{S}_{s}$ is not formed explicitly. Instead the effect of its inverse on the residual vector is computed by solving the following subdomain level Neumann problem

$$
\left[\begin{array}{cc}
\mathbf{A}_{I I}^{s} & \mathbf{A}_{I \Gamma}^{s} \\
\mathbf{A}_{\Gamma I}^{s} & \mathbf{A}_{\Gamma \Gamma}^{s}
\end{array}\right]\left\{\begin{array}{c}
\mathbf{x}^{s} \\
\mathbf{u}_{\Gamma}^{s}
\end{array}\right\}=\left\{\begin{array}{c}
\mathbf{0} \\
\mathbf{r}_{\Gamma}^{s}
\end{array}\right\} .
$$

For the case of floating subdomain (subdomains without sufficient Dirichlet boundary conditions), the solution to the local Neumann problem defined in Eq.(3.87) is only unique up to an additive constant (see Eq.(3.98)) and only if the right hand side vector satisfies the compatibility condition (the residual vector is orthogonal to the null space of the subdomain stiffness matrix) $[4,7]$. Therefore, a small value can be added to the diagonal entries of the subdomain stiffness matrices to ensure the solvability of the subdomain level Neu- 
mann problem in Eq. $(3.87)[4,6]$. Note that this regularization does not change the original problem since it only affects the preconditioner matrix. Alternatively, the computationally expensive Moore-Penrose pseudo-inverse can be used. However, the accuracy of the approximate inversion highly influences the performance of the preconditioner [6].

The new approximate solution $\mathbf{u}_{\Gamma_{j+1}}$ can be obtained by updating the initial guess $\mathbf{u}_{\Gamma_{j}}$ with the average local solutions $\mathbf{u}_{\Gamma}^{s}$ as

$$
\mathbf{u}_{\Gamma_{j+1}}=\mathbf{u}_{\Gamma_{j}}+\sum_{s=1}^{n_{s}} \mathbf{R}_{s}^{T} \mathbf{D}_{s}^{T} \mathbf{u}_{\Gamma}^{s} .
$$

Consequently, the corresponding error vector at the $j^{\text {th }}$ iteration can be obtained as

$$
\mathbf{e}_{\Gamma j}=\mathbf{u}_{\Gamma_{j+1}}-\mathbf{u}_{\Gamma_{j}}
$$

Substituting Eq.(3.88) into Eq.(3.89) and using Eq.(3.86) gives

$$
\mathbf{e}_{\Gamma j}=\sum_{s=1}^{n_{s}} \mathbf{R}_{s}^{T} \mathbf{D}_{s}^{T} \mathbf{S}_{s}^{-1} \mathbf{D}_{s} \mathbf{R}_{s} \mathbf{r}_{\Gamma j}
$$

From preconditioning perspective, Eq.(3.90) can be rewritten as

$$
\mathrm{e}_{\Gamma j}=\mathbf{M}_{N N}^{-1} \mathbf{r}_{\Gamma_{j}}
$$

where the Neumann-Neumann preconditioner formally defined as

$$
\mathbf{M}_{N N}^{-1}=\sum_{s=1}^{n_{s}} \mathbf{R}_{s}^{T} \mathbf{D}_{s}^{T} \mathbf{S}_{s}^{-1} \mathbf{D}_{s} \mathbf{R}_{s}
$$




\section{Condition Number Bound}

If $h$ is the finite element mesh size and $H$ is a typical subdomain size, the condition condition number of the Neumann-Neumann method for both two and three dimensional problems grows as $[4,5,7,72]$

$$
\kappa\left(\mathbf{M}_{N N}^{-1} \mathbf{S}\right) \leq \frac{C}{H^{2}}\left(1+\log \frac{H}{h}\right)^{2}
$$

where $C$ is a positive coefficient independent of the geometric parameters $h$ and $H$.

According to the condition number estimate in Eq.(3.93) for Neumann-Neumann method, the convergence rate of the iterative method used to solve the preconditioned system $\left(\mathbf{M}^{-1} \mathbf{S}\right)$ deteriorates as the size of the subdomain becomes small (i.e., the number of subdomains grows). This fact is due to the dependency of the conditioned number on $\mathrm{H}^{-2}$ which is typical for one-level domain decomposition preconditioners $[4,5,7,78]$.

\section{Balancing Domain Decomposition Method}

For a small number of subdomains, the Neumann-Neumann preconditioner exhibits a good performance [6]. However, its performance deteriorates as the number of subdomains increases $[6,72]$. The Balancing Domain Decomposition (BDD) is introduced to address this problem $[116,117]$.

The Balancing Domain Decomposition (BDD) $[116,117]$ corrects (balances) the residual vector forcing it to be in the range of the local Schur complement matrix (i.e., makes 
the local Schur complement system consistent to ensure the solvability of the singular system). Additionally, the BDD provides a coarse grid correction to the one-level NeumannNeumann method. In the preconditioning step of the iterative solver, the coarse grid serves as a mechanism to propagate information globally over the subdomains $[116,117]$. This spread of information prevents the growth of the condition number as increasing the number of subdomains $[116,117]$. The coarse problem consists of a few unknowns per each subdomain. This coarse grid is purely algebraic and hence no geometric information about the mesh is needed to implement the BDD method $[116,117]$.

The effect of the Neumann-Neumann preconditioner Eq.( 3.92) on a residual vector $\mathbf{r}$ is computed as

$$
\mathbf{M}_{N N}^{-1} \mathbf{r}=\sum_{s=1}^{n_{s}} \mathbf{R}_{s}^{T} \mathbf{D}_{s}^{T} \mathbf{w}_{s}
$$

where $\mathbf{w}_{s}$ is the solution vector of the following system

$$
\mathrm{S}_{s} \mathbf{w}_{s}=\mathbf{D}_{s} \mathbf{R}_{s} \mathbf{r}
$$

As previously alluded, the local Schur complement matrix in Eq.(3.95) is singular for floating subdomains. The system has a solution (not necessarily unique) only if the system is consistent (i.e., the right-hand side vector is orthogonal to the null space of the singular matrix $\mathbf{S}_{s}$ ).

Let $\mathbf{N}_{s}$ be a matrix of size $n_{\Gamma_{s}} \times d_{s}$, where $n_{\Gamma_{s}}$ is the size of the interface boundary 
nodes for subdomain $\Omega_{s}$, and $d_{s}$ is the dimension of the null space of $\mathbf{S}_{s}$. Let the column vector of the matrix $N_{s}$ span the null space of the singular matrix $S_{s}$, as

$$
\operatorname{Range}\left(\mathbf{N}_{s}\right)=\operatorname{Kernel}\left(\mathbf{S}_{s}\right)
$$

when the matrix $S_{s}$ is singular, the subdomain matrix is solvable only if the compatibility condition holds as [7]

$$
\mathrm{N}_{s}^{T}\left(\mathrm{D}_{s} \mathbf{R}_{s} \mathbf{r}\right)=0
$$

The solution to the Schur complement Eq.(3.95) is not unique and has the following general form

$$
\mathbf{w}_{s}=\mathbf{v}_{s}+\mathrm{N}_{s} \boldsymbol{\alpha}_{s}
$$

where $\mathbf{v}_{s} \in \Re^{n_{r_{s}}}$ is a particular solution and $\mathbf{N}_{s} \boldsymbol{\alpha}_{s}$ is a general term in the null space of $\mathbf{S}_{s}$ for $\alpha_{s} \in \Re^{d_{s}}$.

The global solution can be obtained by averaging the subdomain level solutions as

$$
\mathbf{w}=\sum_{s=1}^{n_{s}} \mathbf{R}_{s}^{T} \mathbf{D}_{s}^{T} \mathbf{w}_{s} .
$$

Substituting Eq.(3.98) into Eq.(3.99) gives

$$
\mathbf{w}=\sum_{s=1}^{n_{s}} \mathbf{R}_{s}^{T} \mathbf{D}_{s}^{T} \mathbf{v}_{s}+\sum_{s=1}^{n_{s}} \mathbf{R}_{s}^{T} \mathbf{D}_{s}^{T} \mathbf{N}_{s} \boldsymbol{\alpha}_{s}
$$


Eq.(3.100) can be rewritten as

$$
\mathbf{w}=\mathbf{v}+\sum_{s=1}^{n_{s}} \mathbf{R}_{s}^{T} \mathbf{D}_{s}^{T} \mathbf{N}_{s} \alpha_{s}
$$

where

$$
\mathbf{v}=\sum_{s=1}^{n_{s}} \mathbf{R}_{s}^{T} \mathbf{D}_{s}^{T} \mathbf{v}_{s}
$$

Premultiplying Eq.(3.101) by the global Schur complement matrix $\mathbf{S}$ to calculate the global residual, one obtains

$$
\mathbf{S w}=\mathbf{S v}+\mathbf{S} \sum_{s=1}^{n_{s}} \mathbf{R}_{s}^{T} \mathbf{D}_{s}^{T} \mathbf{N}_{s} \boldsymbol{\alpha}_{s}
$$

Equivalently, Eq.(3.103) can be expressed as

$$
\mathbf{S w}=\mathbf{S v}+\mathbf{S}\left[\begin{array}{llll}
\mathbf{R}_{1}^{T} \mathbf{D}_{1}^{T} \mathbf{N}_{1} & \mathbf{R}_{2}^{T} \mathbf{D}_{2}^{T} \mathbf{N}_{2} & \cdots & \mathbf{R}_{n_{s}}^{T} \mathbf{D}_{n_{s}}^{T} \mathbf{N}_{n_{s}}
\end{array}\right]\left\{\begin{array}{c}
\boldsymbol{\alpha}_{1} \\
\boldsymbol{\alpha}_{2} \\
\vdots \\
\boldsymbol{\alpha}_{n_{s}}
\end{array}\right\}
$$

Let $\mathbf{R}_{0}$ represent a matrix of size $n_{\Gamma_{s}} \times d$ where $d=\left(d_{1}+\cdots+d_{n_{s}}\right)$ and $n_{s}$ is the number of subdomains. The columns of $\mathbf{R}_{\mathbf{0}}$ are defined as

$$
\mathbf{R}_{\mathbf{0}}=\left[\begin{array}{llll}
\mathbf{R}_{1}^{T} \mathbf{D}_{1}^{T} \mathbf{N}_{1} & \mathbf{R}_{2}^{T} \mathbf{D}_{2}^{T} \mathbf{N}_{2} & \cdots & \mathbf{R}_{n_{s}}^{T} \mathbf{D}_{n_{s}}^{T} \mathbf{N}_{n_{s}}
\end{array}\right]
$$

In the case of non-floating subdomain, $\alpha_{s}=0$ and hence the corresponding column in the matrix $\mathbf{R}_{0}$ is void. However, it may be advantageous to set the corresponding column 
in the matrix $\mathbf{R}_{\mathbf{0}}$ to a non-zero value for computer implementation [7].

Hence, Eq.(3.104) can be rewritten as

$$
\mathbf{r}=\widetilde{\mathbf{r}}+\mathrm{SR}_{\mathbf{0}} \boldsymbol{\alpha}
$$

where

$$
\mathbf{r}=\mathbf{S w}, \quad \widetilde{\mathbf{r}}=\mathbf{S v}, \quad \alpha=\left\{\begin{array}{c}
\alpha_{1} \\
\alpha_{2} \\
\vdots \\
\alpha_{n_{s}}
\end{array}\right\}
$$

Consequently, the compatibility condition defined in Eq.(3.97) can now be expressed as

$$
\mathbf{R}_{\mathbf{0}}^{T} \mathbf{r}=\mathbf{0}
$$

When the residual vector is not balanced (i.e., $\mathbf{R}_{0}^{T} \mathbf{r} \neq 0$ ), a correction term $\left(\mathbf{S R}_{0} \boldsymbol{\alpha}\right)$ can be sought such that the new residual $\widetilde{\mathbf{r}}$ is balanced as

$$
\mathbf{R}_{0}^{T}(\widetilde{\mathbf{r}})=0
$$

Substituting Eq.(3.106) into Eq.(3.109) leads to

$$
\mathbf{R}_{0}^{T}\left(\mathbf{r}-\mathbf{S R}_{0} \alpha\right)=0
$$


Therefore the following linear system is solved to obtained the vector $\alpha$

$$
\left[\mathbf{R}_{0}^{T} \mathbf{S} \mathbf{R}_{0}\right] \alpha=\mathbf{R}_{0}^{T} \mathbf{r}
$$

This system is uniquely solvable due to the positive definiteness of the global Schur complement matrix $\mathbf{S}$. The system can be rewritten in a block structure as [7]

$$
\left[\begin{array}{ccc}
\mathbf{R}_{0}^{1^{T}} \mathbf{S R}_{0}^{1} & \cdots & \mathbf{R}_{0}^{1^{T}} \mathbf{S R}_{\mathbf{0}}^{n_{s}} \\
\vdots & \cdots & \vdots \\
\mathbf{R}_{0}^{1^{T}} \mathbf{S R}_{0}^{n_{s}} & \cdots & \mathbf{R}_{0}^{n_{s} T} \mathbf{S R}_{0}^{n_{s}}
\end{array}\right]\left\{\begin{array}{c}
\boldsymbol{\alpha}_{1} \\
\vdots \\
\boldsymbol{\alpha}_{n_{s}}
\end{array}\right\}=\left\{\begin{array}{c}
\mathbf{R}_{0}^{1^{T}} \mathbf{r} \\
\vdots \\
\mathbf{R}_{0}^{n_{s}} \mathbf{r}
\end{array}\right\},
$$

where $\mathbf{R}_{0}^{s}$ defines the columns of the matrix $\mathbf{R}_{0}$, see Eq.(3.105).

Thus, the vector $\alpha$ can be obtained as

$$
\alpha=\mathbf{S}_{0}^{-1} \mathbf{R}_{0}^{T} \mathbf{r}
$$

where the coarse grid operator is defined as

$$
\mathbf{S}_{0}=\mathbf{R}_{0}^{T} \mathbf{S R}_{0}
$$

The Neumann-Neumann preconditioner now can be applied to find any solution, since the system now is consistent (the residual vector is balanced $\widetilde{\mathbf{r}}$ ). Thus, applying the NeumannNeumann preconditioner, one obtains 


$$
\mathbf{v}=\mathbf{M}_{N N}^{-1} \widetilde{\mathbf{r}}
$$

Substituting Eq.(3.106) into Eq.(3.115) gives

$$
\mathbf{v}=\mathbf{M}_{N N}^{-1}\left(\mathbf{r}-\mathbf{S R}_{0} \alpha\right)
$$

The residual needs to be balanced again in order to have a symmetric preconditioner. In other words, a correction term $\mathrm{SR}_{0} \beta$ is sought such that the new residual is balanced again. The residual vector can be expressed as

$$
\mathbf{r}=\widetilde{\mathbf{r}}+\widehat{\mathbf{r}}+\mathbf{S R}_{0} \boldsymbol{\beta}
$$

Next the correction term can be calculated such that $\widehat{\mathbf{r}}$ is balanced as

$$
\mathbf{R}_{0}^{T}(\widehat{\mathbf{r}})=0
$$

Substituting Eq.(3.117) into Eq.(3.118), gives

$$
\mathbf{R}_{0}^{T}\left(\mathbf{r}-\widetilde{\mathbf{r}}-\mathbf{S R}_{0} \boldsymbol{\beta}\right)=0
$$

Solving for the vector $\beta$, one obtains

$$
\beta=\mathbf{S}_{0}^{-1} \mathbf{R}_{0}^{T}(\mathbf{r}-\hat{\mathbf{r}})
$$


Finally the solution can be written as

$$
\begin{aligned}
\mathbf{u} & =\mathbf{v}+\mathbf{R}_{0} \boldsymbol{\beta} \\
& =\mathbf{v}+\mathbf{R}_{0} \mathbf{S}_{0}^{-1} \mathbf{R}_{0}^{T}(\mathbf{r}-\tilde{\mathbf{r}}) \\
& =\mathbf{v}+\mathbf{R}_{0} \mathbf{S}_{0}^{-1} \mathbf{R}_{0}^{T}(\mathbf{r}-\mathbf{S} \mathbf{v}) \\
& =\mathbf{R}_{0} \mathbf{S}_{0}^{-1} \mathbf{R}_{0}^{T} \mathbf{r}+\left(\mathbf{I}-\mathbf{R}_{0} \mathbf{S}_{0}^{-1} \mathbf{R}_{0}^{T} \mathbf{S}\right) \mathbf{v} \\
& =\mathbf{R}_{0} \mathbf{S}_{0}^{-1} \mathbf{R}_{0}^{T} \mathbf{r}+\left(\mathbf{I}-\mathbf{R}_{0} \mathbf{S}_{0}^{-1} \mathbf{R}_{0}^{T} \mathbf{S}\right) \mathbf{M}_{N N}^{-1}\left(\mathbf{r}-\mathbf{S R}_{0} \alpha\right) \\
& =\mathbf{R}_{0} \mathbf{S}_{0}^{-1} \mathbf{R}_{0}^{T} \mathbf{r}+\left(\mathbf{I}-\mathbf{R}_{0} \mathbf{S}_{0}^{-1} \mathbf{R}_{0}^{T} \mathbf{S}\right) \mathbf{M}_{N N}^{-1}\left(\mathbf{I}-\mathbf{S R}_{0} \mathbf{S}_{0}^{-1} \mathbf{R}_{0}^{T}\right) \mathbf{r} \\
& =\left[\mathbf{P}_{0}+\left(\mathbf{I}-\mathbf{P}_{0} \mathbf{S}\right) \mathbf{M}_{N N}^{-1}\left(\mathbf{I}-\mathbf{S} \mathbf{P}_{0}\right)\right] \mathbf{r}
\end{aligned}
$$

where

$$
\mathbf{P}_{0}=\mathbf{R}_{0} \mathbf{S}_{0}^{-1} \mathbf{R}_{0}^{T}
$$

Note that $\mathrm{P}_{0}$ denotes the $\mathrm{S}$-orthogonal projection onto the coarse space spanned by $\mathbf{R}_{0}$.

The balancing domain decomposition preconditioner can be formally written as

$$
\mathbf{M}_{B D D}^{-1}=\mathbf{P}_{0}+\left(\mathbf{I}-\mathbf{P}_{0} \mathbf{S}\right) \mathbf{M}_{N N}^{-1}\left(\mathbf{I}-\mathbf{S P}_{0}\right)
$$

where

$$
\mathbf{M}_{N N}^{-1}=\sum_{s=1}^{n_{s}} \mathbf{R}_{s}^{T} \mathbf{D}_{s}^{T} \mathbf{S}_{s}^{-1} \mathbf{D}_{s} \mathbf{R}_{s}, \quad \text { and } \quad \mathbf{S}=\sum_{s=1}^{n_{s}} \mathbf{R}_{s}^{T} \mathbf{S}_{s} \mathbf{R}_{s}
$$




\section{Condition Number Bound}

The theoretical bound for the condition number of the BDD preconditioner for both two and three dimensional problems satisfies $[5,7,72,116]$

$$
\kappa\left(\mathbf{M}_{B D D}^{-1} \mathbf{S}\right) \leq C\left(1+\log \frac{H}{h}\right)^{2}
$$

where $C$ is a positive constant (independent of the geometric parameters) and $h$ and $H$ are the finite element mesh size and subdomain size respectively.

\section{Neumann-Neumann with a Coarse Grid Preconditioner}

The BDD method is an efficient domain decomposition technique. However, the coarse problem is costly to build as it involves a product with the global Schur complement matrix, generally not available explicitly [66]. In addition, characterization of the null space of the singular Schur complement matrix may not be a trivial task for some problems [4].

Therefore, a geometric coarse problem based on a collection of corner nodes in the physical domain can be added to the Neumann-Neumann method [66]. The coarse grid is constructed to impose sufficient artificial Dirichlet boundary conditions to remove the singularity in the stiffness matrix of the floating subdomain. This methodology is described below [66].

For a typical subdomain $\Omega_{s}$, the equilibrium equation can be written as 


$$
\left[\begin{array}{ll}
\mathbf{A}_{i i}^{s} & \mathbf{A}_{i \Gamma}^{s} \\
\mathbf{A}_{\Gamma i}^{s} & \mathbf{A}_{\Gamma \Gamma}^{s}
\end{array}\right]\left\{\begin{array}{c}
\mathbf{u}_{i}^{s} \\
\mathbf{u}_{\Gamma}^{s}
\end{array}\right\}=\left\{\begin{array}{c}
\mathbf{f}_{i}^{s} \\
\mathbf{f}_{\Gamma}^{s}
\end{array}\right\} .
$$

Starting with an initial guess for the global interface unknowns $u_{\Gamma_{j}}$ ( where $j$ represents the iteration count), the local interface unknowns $\mathrm{u}_{\Gamma}^{s}$ can be obtained as

$$
\mathbf{u}_{\Gamma}^{s}=\mathbf{R}_{s} \mathbf{u}_{\Gamma_{j}}
$$

Next a local Dirichlet problem with non-homogeneous boundary condition can be solved on each subdomain to obtain the interior unknowns. Thus from the first row of Eq.(3.126), the local interior unknown $u_{i}^{s}$ can be obtained as

$$
\mathbf{u}_{i}^{s}=\left[\mathbf{A}_{i i}^{s}\right]^{-1}\left(\mathbf{f}_{i}^{s}-\mathbf{A}_{i \Gamma}^{s} \mathbf{u}_{\Gamma}^{s}\right)
$$

The corresponding subdomain level residual vector $\mathrm{r}_{\Gamma}^{s}$ can be computed from the second row of Eq.(3.126) as

$$
\mathbf{r}_{\Gamma}^{s}=\mathbf{A}_{\Gamma i}^{s} \mathbf{u}_{i}^{s}+\mathbf{A}_{\Gamma \Gamma}^{s} \mathbf{u}_{\Gamma}^{s}-\mathbf{f}_{\Gamma}^{s} .
$$

Substituting Eq.(3.128) into Eq.(3.129) leads to

$$
\mathbf{r}_{\Gamma}^{s}=\left(\mathbf{A}_{\Gamma \Gamma}^{s}-\mathbf{A}_{\Gamma i}^{s}\left[\mathbf{A}_{i i}^{s}\right]^{-1} \mathbf{A}_{i \Gamma}^{s}\right) \mathbf{u}_{\Gamma}^{s}-\left(\mathbf{f}_{\Gamma}^{s}-\mathbf{A}_{\Gamma i}^{s}\left[\mathbf{A}_{i i}^{s}\right]^{-1} \mathbf{f}_{i}^{s}\right)
$$

In terms of Schur complement matrix $\mathrm{S}_{s}$, the local residual in Eq.(3.130) can be rewritten as

$$
\mathbf{r}_{\Gamma}^{s}=\mathbf{S}_{s} \mathbf{u}_{\Gamma}^{s}-\mathbf{g}_{\Gamma}^{s}
$$


where

$$
\begin{aligned}
& \mathbf{S}_{s}=\mathbf{A}_{\Gamma \Gamma}^{s}-\mathbf{A}_{\Gamma i}^{s}\left[\mathbf{A}_{i i}^{s}\right]^{-1} \mathbf{A}_{i \Gamma}^{s}, \\
& \mathbf{g}_{\Gamma}^{s}=\mathbf{f}_{\Gamma}^{s}-\mathbf{A}_{\Gamma i}^{s}\left[\mathbf{A}_{i i}^{s}\right]^{-1} \mathbf{f}_{i}^{s} .
\end{aligned}
$$

The global residual vector $\mathbf{r}_{\Gamma_{j}}$ is obtained next by gathering the local residuals from all the subdomains as

$$
\mathbf{r}_{\Gamma_{j}}=\sum_{s=1}^{n_{s}} \mathbf{R}_{s}^{T} \mathbf{r}_{\Gamma}^{s} .
$$

Let $\mathbf{D}_{s}$ represent a diagonal scaling matrix that forms a decomposition of unity $[4,6,7]$ as

$$
\sum_{s=1}^{n_{s}} \mathbf{R}_{s}^{T} \mathbf{D}_{s} \mathbf{R}_{s}=\mathbf{I}
$$

where the diagonal entries of $\mathbf{D}_{s}$ are the reciprocal of the number of subdomains that share the interface nodes $[4,6,7]$.

The global residual given by Eq.(3.134) can be distributed over the subdomains as

$$
\mathbf{r}_{\Gamma}^{s}=\mathbf{D}_{s} \mathbf{R}_{s} \mathbf{r}_{\Gamma_{j}}
$$

The corresponding unknowns $\mathbf{u}_{\Gamma}^{s}$ due to $\mathbf{r}_{\Gamma}^{s}$ in Eq.(3.136) can be computed by solving a local Schur complement system as

$$
\mathbf{S}_{s} \mathbf{u}_{\Gamma}^{s}=\mathbf{r}_{\Gamma}^{s}
$$


In practice, the local Schur complement matrix $\mathbf{S}_{s}$ is never formed explicitly. Instead the following subdomain level Neumann problem is solved to obtain $\mathbf{u}_{\Gamma}^{s}$

$$
\left[\begin{array}{cc}
\mathbf{A}_{i i}^{s} & \mathbf{A}_{i \Gamma}^{s} \\
\mathbf{A}_{\Gamma i}^{s} & \mathbf{A}_{\Gamma \Gamma}^{s}
\end{array}\right]\left\{\begin{array}{l}
\mathbf{x}^{s} \\
\mathbf{u}_{\Gamma}^{s}
\end{array}\right\}=\left\{\begin{array}{c}
\mathbf{0} \\
\mathbf{r}_{\Gamma}^{s}
\end{array}\right\} .
$$

To ensure the solvability of the subdomain level Neumann problem defined in Eq.(3.138), sufficient artificial Dirichlet boundary conditions are necessary to be provided in order to remove the singularity from the stiffness matrix of the floating subdomains. Therefore, for a typical subdomain $\Omega_{s}$, the interface unknown vector $u_{\Gamma}^{s}$ can be split as follows

$$
\mathbf{u}_{\Gamma}^{s}=\left\{\begin{array}{l}
\mathbf{u}_{r}^{s} \\
\mathbf{u}_{c}^{s}
\end{array}\right\}
$$

where $u_{r}^{s}$ is the remaining nodes (boundary nodes shared between two adjacent subdomains only) and $\mathbf{u}_{c}^{s}$ is the corner nodes (boundary nodes shared between more than two subdomains plus the nodes on the ends of interface edges) $[64,66]$. The corner nodes provide a mechanism to impose the artificial Dirichlet boundary conditions to remove singularity from the local Schur complement matrix of the floating subdomains. On the other hand, the corner nodes introduces a coarse grid that connects all the subdomains to provide information transfer mechanism globally.

For a typical subdomain $\Omega_{s}$, the Boolean splitting operators $\mathbf{R}_{s}^{r}$ and $\mathbf{R}_{s}^{c}$ extract the remaining interface variable $\mathbf{u}_{r}^{s}$ and corner interface unknown $\mathbf{u}_{c}^{s}$ from the subdomain interface vector $\mathbf{u}_{\Gamma}^{s}$ as 


$$
\mathbf{u}_{r}^{s}=\mathbf{R}_{s}^{r} \mathbf{u}_{\Gamma}^{s}
$$

and

$$
\mathbf{u}_{c}^{s}=\mathbf{R}_{s}^{c} \mathbf{u}_{\Gamma}^{s}
$$

Furthermore, the Boolean restriction operator $\mathrm{B}_{c}^{s}$ that maps the global corner interface vector $\mathbf{u}_{c}$ into the local corner interface vector $\mathbf{u}_{c}^{s}$ is defined as

$$
\mathbf{u}_{c}^{s}=\mathbf{B}_{c}^{s} \mathbf{u}_{c}
$$

Consequently, the following partially assembled equilibrium system can be obtained

$$
\left[\begin{array}{ccc}
\mathbf{A}_{i i}^{s} & \mathbf{A}_{i r}^{s} & \mathbf{A}_{i c}^{s} \mathbf{B}_{c}^{s} \\
\mathbf{A}_{r i}^{s} & \mathbf{A}_{r r}^{s} & \mathbf{A}_{r c}^{s} \mathbf{B}_{c}^{s} \\
\sum_{s=1}^{n_{s}} \mathbf{B}_{c}^{s T} \mathbf{A}_{c i}^{s} & \sum_{s=1}^{n_{s}} \mathbf{B}_{c}^{s T} \mathbf{A}_{c r}^{s} & \sum_{s=1}^{n_{s}} \mathbf{B}_{c}^{s T} \mathbf{A}_{c c}^{s} \mathbf{B}_{c}^{s}
\end{array}\right]\left\{\begin{array}{c}
\mathbf{x}_{i}^{s} \\
\mathbf{u}_{r}^{s} \\
\mathbf{u}_{c}
\end{array}\right\}=\left\{\begin{array}{c}
0 \\
\mathbf{f}_{r}^{s} \\
\sum_{s=1}^{n_{s}} \mathbf{B}_{c}^{s T} \mathbf{f}_{c}^{s}
\end{array}\right\},
$$

where

$$
\begin{aligned}
& \mathbf{f}_{r}^{s}=\mathbf{R}_{s}^{r} \mathbf{r}_{\Gamma}^{s}, \\
& \mathbf{f}_{c}^{s}=\mathbf{R}_{s}^{c} \mathbf{r}_{\Gamma}^{s},
\end{aligned}
$$

The dummy variable $x_{i}^{s}$ can be concurrently eliminated from Eq.(3.143) by solving a subdomain level Dirichlet problem as

$$
\mathbf{x}_{i}^{s}=-\left[\mathbf{A}_{i i}^{s}\right]^{-1}\left(\mathbf{A}_{i r}^{s} \mathbf{u}_{r}^{s}+\mathbf{A}_{i c}^{s} \mathbf{B}_{c}^{s} \mathbf{u}_{c}\right)
$$


Substituting Eq.(3.146) into the Eq.(3.143) leads to the following partially assembled Schur complement system

$$
\left[\begin{array}{cc}
\mathbf{S}_{r r}^{s} & \mathbf{S}_{r c}^{s} \mathbf{B}_{c}^{s} \\
\sum_{s=1}^{n_{s}} \mathbf{B}_{c}^{s T} \mathbf{S}_{c r}^{s} & \sum_{s=1}^{n_{s}} \mathbf{B}_{c}^{s T} \mathbf{S}_{c c}^{s} \mathbf{B}_{c}^{s}
\end{array}\right]\left\{\begin{array}{c}
\mathbf{u}_{r}^{s} \\
\mathbf{u}_{c}
\end{array}\right\}=\left\{\begin{array}{c}
\mathbf{f}_{r}^{s} \\
\sum_{s=1}^{n_{s}} \mathbf{B}_{c}^{s T} \mathbf{f}_{c}^{s}
\end{array}\right\}
$$

where

$$
\mathbf{S}_{\alpha \beta}^{s}=\mathbf{A}_{\alpha \beta}^{s}-\mathbf{A}_{\alpha i}^{s}\left[\mathbf{A}_{i i}^{s}\right]^{-1} \mathbf{A}_{i \beta}^{s}
$$

where the subscripts $\alpha$ and $\beta$ relate to $r$ and $c$.

Due to the artificial Dirichlet boundary conditions imposed by the corner nodes, the local Schur complement matrix $\mathbf{S}_{r r}^{s}$ in Eq.(3.147) is positive-definite and hence invertible for the floating subdomains. Thus, the remaining interface unknown $\mathrm{u}_{r}^{s}$ can be obtained by solving the fine Schur complement problem as

$$
\mathbf{S}_{r r}^{s} \mathbf{u}_{r}^{s}=\mathbf{f}_{r}^{s}-\mathbf{S}_{r c}^{s} \mathbf{B}_{c}^{s} \mathbf{u}_{c}
$$

Substituting the subdomain level solution $\mathbf{u}_{r}^{s}$ from Eq.(3.149) into Eq.(3.147) leads to the following global coarse Schur complement problem

$$
\mathbf{F}_{c c} \mathbf{u}_{c}=\mathbf{d}_{c}
$$


where

$$
\begin{aligned}
\mathbf{F}_{c c} & =\sum_{s=1}^{n_{s}} \mathbf{B}_{c}^{s T}\left(\mathbf{S}_{c c}^{s}-\mathbf{S}_{c r}^{s}\left[\mathbf{S}_{r r}^{s}\right]^{-1} \mathbf{S}_{r c}^{s}\right) \mathbf{B}_{c}^{s} \\
\mathrm{~d}_{c} & =\sum_{s=1}^{n_{s}} \mathbf{B}_{c}^{s T}\left(\mathbf{f}_{c}^{s}-\mathbf{S}_{c r}^{s}\left[\mathbf{S}_{r r}^{s}\right]^{-1} \mathbf{f}_{r}^{s}\right) .
\end{aligned}
$$

The coarse Schur complement problem Eq.(3.150) couples the subdomains by the corner nodes constituting a global grid to transfer information across the subdomains.

Next, the local interface unknowns can be obtained as

$$
\mathbf{u}_{\Gamma}^{s}=\mathbf{R}_{s}^{r T} \mathbf{u}_{r}^{s}+\mathbf{R}_{s}^{c T} \mathbf{u}_{c}^{s}
$$

The new approximate solution $\mathbf{u}_{\Gamma_{j+1}}$ can be expressed by updating the initial guess $\mathbf{u}_{\Gamma_{j}}$ with the average local solutions $\mathbf{u}_{\Gamma}^{s}$ as

$$
\mathbf{u}_{\Gamma_{j+1}}=\mathbf{u}_{\Gamma_{j}}+\sum_{s=1}^{n_{s}} \mathbf{R}_{s}^{T} \mathbf{D}_{s}^{T} \mathbf{u}_{\Gamma}^{s}
$$

After some algebraic manipulations, the Neumann-Neumann with a coarse grid preconditioner can be expressed as

$$
\mathbf{M}_{N N C}^{-1}=\sum_{s=1}^{n_{s}} \mathbf{R}_{s}^{T} \mathbf{D}_{s}^{T}\left(\mathbf{R}_{s}^{r T}\left[\mathbf{S}_{r r}^{s}\right]^{-1} \mathbf{R}_{s}^{r}\right) \mathbf{D}_{s} \mathbf{R}_{s}+\mathbf{R}_{0}^{T}\left[\mathbf{F}_{c c}\right]^{-1} \mathbf{R}_{0}
$$

where

$$
\mathbf{R}_{0}=\sum_{s=1}^{n_{s}} \mathbf{B}_{s}^{T}\left(\mathbf{R}_{s}^{c}-\mathbf{S}_{c r}^{s}\left[\mathbf{S}_{r r}^{s}\right]^{-1} \mathbf{R}_{s}^{r}\right) \mathbf{D}_{s} \mathbf{R}_{s}
$$




\section{Balancing Domain Decomposition by Constraints}

The Balancing Domain Decomposition by Constraint (BDDC) is a non-overlapping domain decomposition preconditioner based on constrained energy minimization $[67,107$, $108,123]$. The BDDC can be viewed as an evolution of the balancing domain decomposition (BDD) method. While BDD uses a multiplicative coarse problem, the BDDC method applies an additive coarse problem [108]. The use of an additive rather than multiplicative coarse problem leads to a favorable sparser coarse matrix [107].

The basic idea of BDDC is to find a set of coarse basis functions using the interface nodes and minimize the energy norm, subjected to some constraints. If primal constraints are used, the coarse basis functions have a value of unity at the corresponding coarse nodes and zero value on the remaining coarse nodes $[4,78]$. The basis functions span a coarse space. Projection of the Schur complement operator onto this space leads to a coarse problem. The method can be explained as follows $[7,67,78,83,107]$.

Let the physical domain $\Omega$ be decomposed into $n_{s}$ non-overlapping subdomains. The equilibrium system of a typical subdomain $\Omega_{s}$ can be written as

$$
\mathbf{A}^{s} \mathbf{u}^{s}=\mathbf{f}^{s}
$$

The discrete internal energy of subdomain $\Omega_{s}$ can be represented as [107]

$$
E_{s}=\frac{1}{2} \mathbf{u}^{s T} \mathbf{A}^{s} \mathbf{u}^{s}
$$


Let $\psi_{s}^{j}$ be the solution to the minimization problem of the subdomain internal energy $E_{s}$ in Eq.(3.158) subject to the following constraints [107]

$$
\mathrm{C}_{s} \mathbf{u}^{s}=\mathbf{e}_{j}
$$

where $\mathbf{C}_{s}$ represent a constraint matrix and $\mathbf{e}_{j}$ denotes the $j^{\text {th }}$ column of the identity matrix. The matrix $\mathrm{C}_{s}$ contains constraints to couple the subdomains globally which naturally leads to a coarse problem [107, 123].

A constrained minimization problem can be solved to minimize the energy norm as

$$
\begin{aligned}
& \text { minimize } E_{s}\left(\boldsymbol{\Psi}_{s}\right)=\frac{1}{2} \boldsymbol{\Psi}_{s}^{T} \mathbf{A}^{s} \Psi_{s} \\
& \text { subject to } \mathrm{C}_{s} \boldsymbol{\Psi}_{s}=\mathbf{I},
\end{aligned}
$$

where $\mathbf{I}$ is the identity matrix and $\Psi_{s}=\left[\psi_{s}^{1}, \cdots, \psi_{s}^{n c s}\right]$ and $n c s$ is the size of the local (subdomain) constraints. These constraints make the subdomain problem well-posed.

Introducing a vector of Lagrange multipliers, the following Lagrangian function can be obtained for the above constrained minimization problem

$$
\mathcal{L}\left(\Psi_{s}, \Lambda_{s}\right)=\frac{1}{2} \boldsymbol{\Psi}_{s}^{T} \mathbf{A}^{s} \boldsymbol{\Psi}_{s}+\boldsymbol{\Lambda}_{s}^{T}\left(\mathbf{C}_{s} \boldsymbol{\Psi}_{s}-\mathbf{I}\right)
$$

Minimizing the Lagrangian function in Eq.(3.162) with respect to $\boldsymbol{\Psi}_{s}$ and $\boldsymbol{\Lambda}_{s}$, the following linear system is obtained 


$$
\left[\begin{array}{cc}
\mathrm{A}^{s} & \mathrm{C}_{s}^{T} \\
\mathrm{C}_{s} & \mathbf{0}
\end{array}\right]\left\{\begin{array}{l}
\boldsymbol{\Psi}_{s} \\
\boldsymbol{\Lambda}_{s}
\end{array}\right\}=\left\{\begin{array}{l}
\mathbf{0} \\
\mathrm{I}
\end{array}\right\} .
$$

If primal constraints are used (e.g. the constrains represent continuity at the subdomain corner nodes), Lagrange multipliers are not required in order to enforce the constraints. In this case, the functions $\Psi_{s}$ are discrete harmonic functions (i.e. the functions $\Psi_{s}$ have a zero value at all the corner nodes except one where they have a value of unity, and they have minimal energy on the remaining interface nodes) $[4,78,83,124,125]$. Consequently, the coarse basis functions $\Psi_{s}$ can be obtained via an analogy to block Cholesky factorization as explained next $[78,83]$.

Let the subdomain unknown $\mathbf{u}^{s}$ be partitioned into the interior unknown $\mathbf{u}_{i}^{s}$, remaining unknown $\mathbf{u}_{r}^{s}$ and corner unknown $\mathbf{u}_{c}^{s}$. The equilibrium of a typical subdomain $\Omega_{s}$ can be expressed as

$$
\left[\begin{array}{lll}
\mathbf{A}_{i i}^{s} & \mathbf{A}_{i r}^{s} & \mathbf{A}_{i c}^{s} \\
\mathbf{A}_{r i}^{s} & \mathbf{A}_{r r}^{s} & \mathbf{A}_{r c}^{s} \\
\mathbf{A}_{c i}^{s} & \mathbf{A}_{c r}^{s} & \mathbf{A}_{c c}^{s}
\end{array}\right]\left\{\begin{array}{l}
\mathbf{u}_{i}^{s} \\
\mathbf{u}_{r}^{s} \\
\mathbf{u}_{c}^{s}
\end{array}\right\}=\left\{\begin{array}{l}
\mathbf{f}_{i}^{s} \\
\mathbf{f}_{r}^{s} \\
\mathbf{f}_{c}^{s}
\end{array}\right\} .
$$

Partial assembly of the corner node unknown $u_{c}^{s}$ in Eq.(3.164) leads to the following equilibrium equation 


$$
\left[\begin{array}{ccc}
\mathbf{A}_{i i}^{s} & \mathbf{A}_{i r}^{s} & \mathbf{A}_{i c}^{s} \mathbf{B}_{c}^{s} \\
\mathbf{A}_{r i}^{s} & \mathbf{A}_{r r}^{s} & \mathbf{A}_{r c}^{s} \mathbf{B}_{c}^{s} \\
\sum_{s=1}^{n_{s}} \mathbf{B}_{c}^{s T} \mathbf{A}_{c i}^{s} & \sum_{s=1}^{n_{s}} \mathbf{B}_{c}^{s T} \mathbf{A}_{c r}^{s} & \sum_{s=1}^{n_{s}} \mathbf{B}_{c}^{s T} \mathbf{A}_{c c}^{s} \mathbf{B}_{c}^{s}
\end{array}\right]\left\{\begin{array}{c}
\mathbf{u}_{i}^{s} \\
\mathbf{u}_{r}^{s} \\
\mathbf{u}_{c}
\end{array}\right\}=\left\{\begin{array}{c}
\mathbf{f}_{i}^{s} \\
\mathbf{f}_{r}^{s} \\
\sum_{s=1}^{n_{s}} \mathbf{B}_{c}^{s T} \mathbf{f}_{c}^{s}
\end{array}\right\},
$$

where $\mathbf{B}_{c}^{s}$ is a Boolean restriction operator that maps the global corner vector $\mathbf{u}_{c}$ to the local corner vector $\mathbf{u}_{c}^{s}$ as

$$
\mathbf{u}_{c}^{s}=\mathbf{B}_{c}^{s} \mathbf{u}_{c}
$$

The interior variable $\mathbf{u}_{i}^{s}$ can be obtained from the first row of Eq.(3.165) as

$$
\mathbf{u}_{i}^{s}=\left[\mathbf{A}_{i i}^{s}\right]^{-1}\left(\mathbf{f}_{i}^{s}-\mathbf{A}_{i r}^{s} \mathbf{u}_{r}^{s}-\mathbf{A}_{i c}^{s} \mathbf{B}_{c}^{s} \mathbf{u}_{c}^{s}\right)
$$

Substituting $\mathbf{u}_{i}^{s}$ in the second and third rows of Eq.(3.165), the following Schur complement system is obtained

$$
\left[\begin{array}{cc}
\mathbf{S}_{r r}^{s} & \mathbf{S}_{r c}^{s} \mathbf{B}_{c}^{s} \\
\sum_{s=1}^{n_{s}} \mathbf{B}_{c}^{s T} \mathbf{S}_{c r}^{s} & \sum_{s=1}^{n_{s}} \mathbf{B}_{c}^{s T} \mathbf{S}_{c c}^{s} \mathbf{B}_{c}^{s}
\end{array}\right]\left\{\begin{array}{c}
\mathbf{u}_{r}^{s} \\
\mathbf{u}_{c}
\end{array}\right\}=\left\{\begin{array}{c}
\mathbf{g}_{r}^{s} \\
\sum_{s=1}^{n_{s}} \mathbf{B}_{c}^{s T} \mathbf{g}_{c}^{s}
\end{array}\right\}
$$

where

$$
\begin{aligned}
\mathbf{S}_{\alpha \beta}^{s} & =\mathbf{A}_{\alpha \beta}^{s}-\mathbf{A}_{\alpha i}^{s}\left[\mathbf{A}_{i i}^{s}\right]^{-1} \mathbf{A}_{i \beta}^{s} \\
\mathbf{g}_{\alpha}^{s} & =\mathbf{f}_{\alpha}^{s}-\mathbf{A}_{\alpha i}^{s}\left[\mathbf{A}_{i i}^{s}\right]^{-1} \mathbf{f}_{i}^{s}
\end{aligned}
$$

and $\alpha$ and $\beta$ denote the subscripts $r$ and $c$. 
Equivalently, the Schur complement system in Eq.(3.168) can be expressed as

$$
\widetilde{\mathbf{S}}^{s} \widetilde{\mathbf{u}}_{\Gamma}^{s}=\widetilde{\mathrm{g}}^{s}
$$

Performing Cholesky factorization to the partially assembled Schur complement matrix $\widetilde{\mathbf{S}}^{s}$ in Eq.(3.171), one obtains

$$
\operatorname{Fact}\left(\widetilde{\mathbf{S}}^{s}\right)=\left[\begin{array}{cc}
\mathbf{I} & \mathbf{0} \\
\mathbf{S}_{c r}^{s}\left[\mathbf{S}_{r r}^{s}\right]^{-1} & \mathbf{I}
\end{array}\right]\left[\begin{array}{cc}
\mathbf{S}_{r r}^{s} & \mathbf{0} \\
\mathbf{0} & \mathbf{F}_{c c}
\end{array}\right]\left[\begin{array}{cc}
\mathbf{I} & {\left[\mathbf{S}_{r r}^{s}\right]^{-1} \mathbf{S}_{r c}^{s}} \\
\mathbf{0} & \mathbf{I}
\end{array}\right],
$$

where

$$
\mathbf{F}_{c c}=\sum_{s=1}^{n_{s}} \mathbf{B}_{c}^{s T}\left(\mathbf{S}_{c c}^{s}-\mathbf{S}_{c r}^{s}\left[\mathbf{S}_{r r}^{s}\right]^{-1} \mathbf{S}_{r c}^{s}\right) \mathbf{B}_{c}^{s}
$$

Alternatively, $\mathbf{F}_{c c}$ can be expressed as

$$
\mathbf{F}_{c c}=\sum_{s=1}^{n_{s}} \mathbf{B}_{c}^{s T} \mathbf{F}_{c c}^{s} \mathbf{B}_{c}^{s}
$$

where the local coarse grid operator is represented as

$$
\mathbf{F}_{c c}^{s}=\mathbf{S}_{c c}^{s}-\mathbf{S}_{c r}^{s}\left[\mathbf{S}_{r r}^{s}\right]^{-1} \mathbf{S}_{r c}^{s}
$$

Inverting the factored partially assembled Schur complement matrix in Eq.(3.172) as [83]

$$
\left[\widetilde{\mathbf{S}}^{s}\right]^{-1}=\left[\begin{array}{cc}
\mathbf{I} & -\left[\mathbf{S}_{r r}^{s}\right]^{-1} \mathbf{S}_{r c}^{s} \\
\mathbf{0} & \mathbf{I}
\end{array}\right]\left[\begin{array}{cc}
{\left[\mathbf{S}_{r r}^{s}\right]^{-1}} & \mathbf{0} \\
\mathbf{0} & \mathbf{F}_{c c}^{-1}
\end{array}\right]\left[\begin{array}{cc}
\mathbf{I} & \mathbf{0} \\
-\mathbf{S}_{c r}^{s}\left[\mathbf{S}_{r r}^{s}\right]^{-1} & \mathbf{I}
\end{array}\right]
$$


Let the function $\Psi_{s}$ be defined as

$$
\boldsymbol{\Psi}_{s}=\left[\begin{array}{c}
-\left[\mathbf{S}_{r r}^{s}\right]^{-1} \mathbf{S}_{r c}^{s} \\
\mathbf{I}
\end{array}\right]
$$

Then the inverse of the the partially assembled Schur complement matrix in Eq.(3.176) can be expressed as

$$
\left[\widetilde{\mathbf{S}}^{s}\right]^{-1}=\left[\begin{array}{cc}
{\left[\mathbf{S}_{r r}^{s}\right]^{-1}} & 0 \\
0 & 0
\end{array}\right]+\Psi_{s} \mathbf{F}_{c c}^{-1} \Psi_{s}^{T}
$$

Clearly, the partially assembled Schur complement problem in Eq.(3.178) consists of a local (fine) and global (coarse) components respectively defined as

$$
\begin{aligned}
\mathbf{T}_{s u b}^{s} & =\left[\mathbf{S}_{r r}^{s}\right]^{-1} \\
\mathbf{T}_{0}^{s} & =\Psi_{s} \mathbf{F}_{c c}^{-1} \Psi_{s}^{T}
\end{aligned}
$$

Note that the local coarse grid operator $\mathbf{F}_{c c}^{s}$ in Eq.(3.175) can be expressed in terms of the coarse basis functions $\Psi_{s}$ as

$$
\begin{aligned}
\mathbf{F}_{c c}^{s} & =\mathbf{S}_{c c}^{s}-\mathbf{S}_{c r}^{s}\left[\mathbf{S}_{r r}^{s}\right]^{-1} \mathbf{S}_{r c}^{s} \\
& =\left[\begin{array}{ll}
-\mathbf{S}_{c r}^{s}\left[\mathbf{S}_{r r}^{s}\right]^{-1} & \mathbf{I}
\end{array}\right]\left[\begin{array}{cc}
\mathbf{S}_{r r}^{s} & \mathbf{S}_{r c}^{s} \\
\mathbf{S}_{c r}^{s} & \mathbf{S}_{c c}^{s}
\end{array}\right]\left[\begin{array}{c}
-\left[\mathbf{S}_{r r}^{s}\right]^{-1} \mathbf{S}_{r c}^{s} \\
\mathbf{I}
\end{array}\right] \\
& =\Psi_{s}^{T} \mathbf{S}_{s} \Psi_{s} .
\end{aligned}
$$

Accordingly, the global coarse operator $\mathbf{F}_{c c}$ can be expressed as 


$$
\mathbf{F}_{c c}=\sum_{s=1}^{n_{s}} \mathbf{B}_{c}^{s T} \Psi_{s}^{T} \mathbf{S}_{s} \Psi_{s} \mathbf{B}_{c}^{s}
$$

where

$$
\mathbf{S}_{s}=\left[\begin{array}{ll}
\mathbf{S}_{r r}^{s} & \mathbf{S}_{r c}^{s} \\
\mathbf{S}_{c r}^{s} & \mathbf{S}_{c c}^{s}
\end{array}\right] .
$$

Given a global residual vector $\mathbf{r}_{\Gamma}$, its local component $\mathbf{r}_{r}^{s}$ can be obtained as

$$
\mathbf{r}_{r}^{s}=\mathbf{R}_{s}^{r} \mathbf{r}_{\Gamma}^{s}
$$

where

$$
\mathbf{r}_{\Gamma}^{s}=\mathbf{D}_{s} \mathbf{R}_{s} \mathbf{r}_{\Gamma}
$$

Consequently, the local (fine) solution $\mathbf{u}_{r}^{s}$ is obtained as

$$
\begin{aligned}
\mathbf{u}_{r}^{s} & =\left[\mathbf{S}_{r r}^{s}\right]^{-1} \mathbf{r}_{r}^{s}, \\
& =\left[\mathbf{S}_{r r}^{s}\right]^{-1} \mathbf{R}_{s}^{r} \mathbf{r}_{\Gamma}^{s} .
\end{aligned}
$$

The coarse component $\mathbf{u}_{c}^{s}$ is

$$
\mathbf{u}_{c}^{s}=\Psi_{s} \mathbf{F}_{c c}^{-1} \Psi_{s}^{T} \mathbf{r}_{\Gamma}^{s} .
$$

For the subdomain $\Omega_{s}$, the interface unknown $\mathbf{u}_{\Gamma}^{s}$ can be expressed as

$$
\begin{aligned}
\mathbf{u}_{\Gamma}^{s} & =\mathbf{R}_{s}^{r T} \mathbf{u}_{r}^{s}+\mathbf{u}_{c}^{s}, \\
& =\mathbf{R}_{s}^{r T}\left[\mathbf{S}_{r r}^{s}\right]^{-1} \mathbf{R}_{s}^{r} \mathbf{r}_{\Gamma}^{s}+\Psi_{s} \mathbf{F}_{c c}^{-1} \Psi_{s}^{T} \mathbf{r}_{\Gamma}^{s}
\end{aligned}
$$


The global interface unknown $\mathbf{u}_{\Gamma}$ can be constructed from the local interface unknown $\mathbf{u}_{\Gamma}^{s}$ gathered from all the subdomains as

$$
\mathbf{u}_{\Gamma}=\sum_{s=1}^{n_{s}} \mathbf{R}_{s}^{T} \mathbf{D}_{s}^{T} \mathbf{u}_{\Gamma}^{s}
$$

Now $\mathbf{u}_{\Gamma}$ can be expressed in terms of the global interface residual $\mathbf{r}_{\Gamma}$ as

$$
\mathbf{u}_{\Gamma}=\sum_{s=1}^{n_{s}} \mathbf{R}_{s}^{T} \mathbf{D}_{s}^{T}\left(\mathbf{R}_{s}^{r T}\left[\mathbf{S}_{r r}^{s}\right]^{-1} \mathbf{R}_{s}^{r}+\Psi_{s} \mathbf{F}_{c c}^{-1} \Psi_{s}^{T}\right) \mathbf{D}_{s} \mathbf{R}_{s} \mathbf{r}_{\Gamma}
$$

Therefore the BDDC preconditioner takes the following form

$$
\mathbf{M}_{B D D C}^{-1}=\sum_{s=1}^{n_{s}} \mathbf{R}_{s}^{T} \mathbf{D}_{s}^{T}\left(\mathbf{T}_{s u b}+\mathbf{T}_{0}\right) \mathbf{D}_{s} \mathbf{R}_{s}
$$

where

$$
\mathbf{T}_{s u b}=\mathbf{R}_{s}^{r T}\left[\mathbf{S}_{r r}^{s}\right]^{-1} \mathbf{R}_{s}^{r}, \quad \text { and } \quad \mathbf{T}_{0}=\Psi_{s} \mathbf{F}_{c c}^{-1} \Psi_{s}^{T}
$$

\section{Condition Number Bound}

If $h$ is the finite element mesh size and $H$ is a typical subdomain size, the condition number of the BDDC method is bounded by $[67,108]$

$$
\kappa\left(\mathrm{M}_{B D D C}^{-1} \mathrm{~S}\right) \leq C\left(1+\log \left(\frac{H}{h}\right)\right)^{2},
$$

where $C$ is a positive coefficient independent of the the number of subdomains and the size of the elements. 


\subsubsection{Primal-Primal Iterative Substructuring Method}

In this section, we describe a novel primal version of iterative substructuring methods for the solution of the large-scale linear system arising from the finite element method. The method offers a straightforward approach to formulate a two-level scalable preconditioner. In this method, the interior and corner degrees of freedom are eliminated first to obtain a reduced interface problem for the remaining degrees of freedom. Next, the two-level preconditioner for the reduced interface problem is formulated by strictly enforcing the continuity condition on the corner nodes of the interface by global assembly. The continuity condition on the remaining interface nodes is satisfied weakly (in an average sense). The continuity of the solution field across the entire interface is eventually satisfied at the convergence of the iterations. This approach naturally leads to a coarse grid which connects the subdomains globally via the corner nodes. The coarse grid provides a mechanism to propagate information globally which makes the algorithm scalable with respect to subdomain size.

For a typical subdomain $\Omega_{s}$, we partition the subdomain nodal vector $\mathbf{u}^{s}$ into a set of interior unknowns $u_{i}^{s}$, remaining unknowns $\mathbf{u}_{r}^{s}$ and corner unknowns $\mathbf{u}_{c}^{s}$ as schematically shown in Fig.(3.4). 

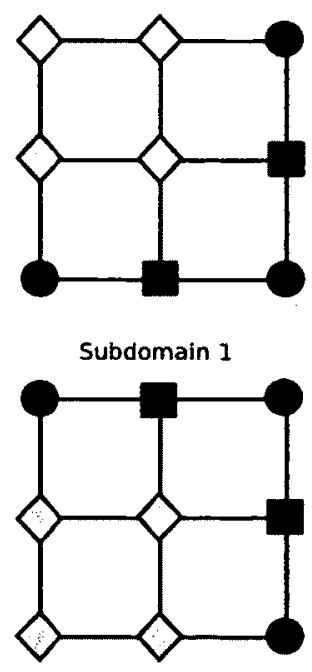

Subdomain 3

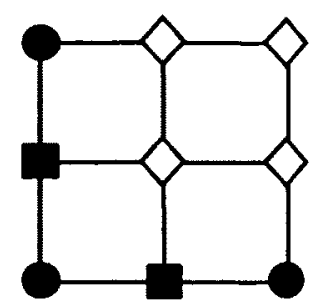

Subdomain 2

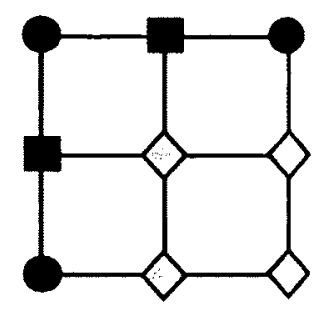

Subdomain 4

Figure 3.4: Partitioning the subdomain nodes into: interior $(\diamond)$, remaining

(ש) and corner( $)$

According to this partitioning scheme, the subdomain equilibrium equation can be represented as

$$
\left[\begin{array}{lll}
\mathbf{A}_{i i}^{s} & \mathbf{A}_{i r}^{s} & \mathbf{A}_{i c}^{s} \\
\mathbf{A}_{r i}^{s} & \mathbf{A}_{r r}^{s} & \mathbf{A}_{r c}^{s} \\
\mathbf{A}_{c i}^{s} & \mathbf{A}_{c r}^{s} & \mathbf{A}_{c c}^{s}
\end{array}\right]\left\{\begin{array}{c}
\mathbf{u}_{i}^{s} \\
\mathbf{u}_{r}^{s} \\
\mathbf{u}_{c}^{s}
\end{array}\right\}=\left\{\begin{array}{c}
\mathbf{f}_{i}^{s} \\
\mathbf{f}_{r}^{s} \\
\mathbf{f}_{c}^{s}
\end{array}\right\} .
$$

Enforcing the transmission conditions along the boundary interfaces, the subdomain equilibrium equation can be expressed as 


$$
\left[\begin{array}{ccc}
\mathbf{A}_{i i}^{s} & \mathbf{A}_{i r}^{s} \mathbf{B}_{r}^{s} & \mathbf{A}_{i c}^{s} \mathbf{B}_{c}^{s} \\
\sum_{s=1}^{n_{s}} \mathbf{B}_{r}^{s T} \mathbf{A}_{r i}^{s} & \sum_{s=1}^{n_{s}} \mathbf{B}_{r}^{s T} \mathbf{A}_{r r}^{s} \mathbf{B}_{r}^{s} & \sum_{s=1}^{n_{s}} \mathbf{B}_{r}^{s T} \mathbf{A}_{r c}^{s} \mathbf{B}_{c}^{s} \\
\sum_{s=1}^{n_{s}} \mathbf{B}_{c}^{s T} \mathbf{A}_{c i}^{s} & \sum_{s=1}^{n_{s}} \mathbf{B}_{c}^{s T} \mathbf{A}_{c r}^{s} \mathbf{B}_{r}^{s} & \sum_{s=1}^{n_{s}} \mathbf{B}_{c}^{s T} \mathbf{A}_{c c}^{s} \mathbf{B}_{c}^{s}
\end{array}\right]\left\{\begin{array}{c}
\mathbf{u}_{i}^{s} \\
\mathbf{u}_{r} \\
\mathbf{u}_{c}
\end{array}\right\}=\left\{\begin{array}{c}
\mathbf{f}_{i}^{s} \\
\sum_{s=1}^{n_{s}} \mathbf{B}_{r}^{s T} \mathbf{f}_{r}^{s} \\
\sum_{s=1}^{n_{s}} \mathbf{B}_{c}^{s T} \mathbf{f}_{c}^{s}
\end{array}\right\}
$$

where $\mathrm{B}_{r}^{s}$ is a Boolean rectangular matrix that maps the global remaining vector $\mathbf{u}_{r}$ to the local remaining vector $\mathbf{u}_{r}^{s}$ as

$$
\mathbf{u}_{r}^{s}=\mathbf{B}_{r}^{s} \mathbf{u}_{r}
$$

Similarly, the restriction operator $\mathbf{B}_{c}^{s}$ is a Boolean rectangular matrix that maps the global corner vector $\mathbf{u}_{c}$ to the local corner vector $\mathbf{u}_{c}^{s}$ as

$$
\mathbf{u}_{c}^{s}=\mathbf{B}_{c}^{s} \mathbf{u}_{c}
$$

In parallel implementation both $\mathbf{B}_{r}^{s}$ and $\mathbf{B}_{c}^{s}$ act as scatter operators, while $\mathbf{B}_{r}^{s T}$ and $\mathbf{B}_{c}^{s^{T}}$ act as gather operators.

The first block equation in Eq.(3.197) can be solved concurrently for $\mathbf{u}_{i}^{s}$ as

$$
\mathbf{u}_{i}^{s}=\left[\mathbf{A}_{i i}^{s}\right]^{-1}\left(\mathbf{F}_{i}^{s}-\mathbf{A}_{i r}^{s} \mathbf{B}_{r}^{s} \mathbf{u}_{r}-\mathbf{A}_{i c}^{s} \mathbf{B}_{c}^{s} \mathbf{u}_{c}\right)
$$

Substituting Eq.(3.200) into Eq.(3.197) leads to the following condensed system 


$$
\left[\begin{array}{ll}
\sum_{s=1}^{n_{s}} \mathbf{B}_{r}^{s T} \mathbf{S}_{r r}^{s} \mathbf{B}_{r}^{s} & \sum_{s=1}^{n_{s}} \mathbf{B}_{r}^{s T} \mathbf{S}_{r c}^{s} \mathbf{B}_{c}^{s} \\
\sum_{s=1}^{n_{s}} \mathbf{B}_{c}^{s T} \mathbf{S}_{c r}^{s} \mathbf{B}_{r}^{s} & \sum_{s=1}^{n_{s}} \mathbf{B}_{c}^{s T} \mathbf{S}_{c c}^{s} \mathbf{B}_{c}^{s}
\end{array}\right]\left\{\begin{array}{c}
\mathbf{u}_{r} \\
\mathbf{u}_{c}
\end{array}\right\}=\left\{\begin{array}{l}
\sum_{s=1}^{n_{s}} \mathbf{B}_{r}^{s T} \mathbf{g}_{r}^{s} \\
\sum_{s=1}^{n_{s}} \mathbf{B}_{c}^{s T} \mathbf{g}_{c}^{s}
\end{array}\right\}
$$

where

$$
\begin{aligned}
\mathbf{S}_{\alpha \beta}^{s} & =\mathbf{A}_{\alpha \beta}^{s}-\mathbf{A}_{\alpha i}^{s}\left[\mathbf{A}_{i i}^{s}\right]^{-1} \mathbf{A}_{i \beta}^{s} \\
\mathbf{g}_{\alpha}^{s} & =\mathbf{f}_{\alpha}^{s}-\mathbf{A}_{\alpha i}^{s}\left[\mathbf{A}_{i i}^{s}\right]^{-1} \mathbf{f}_{i}^{s}
\end{aligned}
$$

The interface system in Eq.(3.201) involves the remaining nodal vector $\mathbf{u}_{r}$ and corner node unknown $\mathbf{u}_{c}$ as shown in Fig.(3.5).

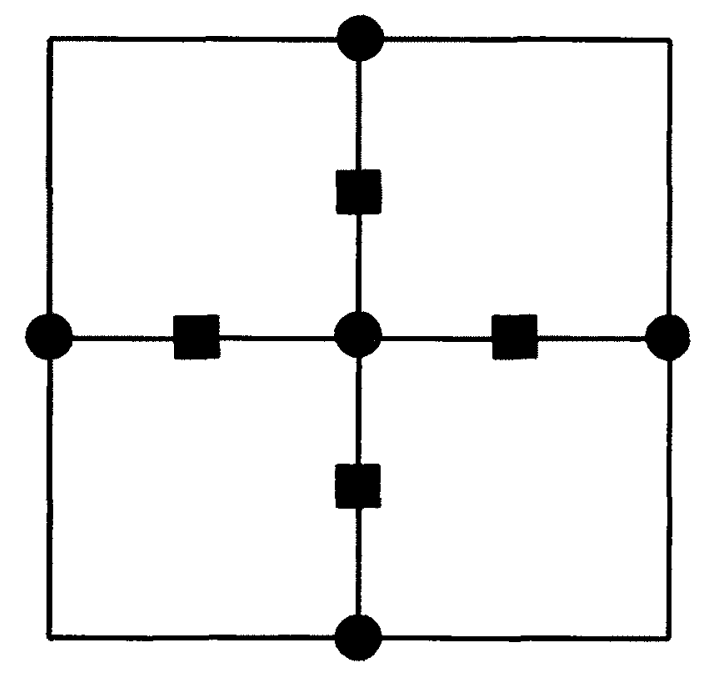

Figure 3.5: The interface boundary nodes split into: remaining (a) and corner( $)$ nodes

The corner nodal vector $\mathbf{u}_{c}$ in Eq.(3.201) is eliminated next to obtain the following symmetric positive definite reduced interface problem 


$$
\left(\mathbf{F}_{r r}-\mathbf{F}_{r c}\left[\mathbf{F}_{c c}\right]^{-1} \mathbf{F}_{c r}\right) \mathbf{u}_{r}=\mathbf{d}_{r}-\mathbf{F}_{r c}\left[\mathbf{F}_{c c}\right]^{-1} \mathrm{~d}_{c}
$$

where

$$
\begin{aligned}
\mathbf{F}_{\alpha \beta} & =\sum_{s=1}^{n_{s}} \mathbf{B}_{\alpha}^{s T} \mathbf{S}_{\alpha \beta}^{s} \mathbf{B}_{\beta}^{s} \\
\mathbf{d}_{\alpha} & =\sum_{s=1}^{n_{s}} \mathbf{B}_{\alpha}^{s T} \mathbf{g}_{\alpha}^{s}
\end{aligned}
$$

and $\alpha$ and $\beta$ denotes subscripts $r$ and $c$. The reduced interface problem can be solved using PCGM with an appropriate preconditioner defined in the next section.

\section{Primal-Primal Preconditioner}

As alluded previously, the continuity condition on the remaining interface nodes is enforced weakly. At the corner nodes, the continuity is enforced strictly. This fact is schematically illustrated in Fig.(3.6). As the iterations converges the continuity condition is enforced strictly for the entire interface nodal vector. Consequently, the following equilibrium equation is obtained

$$
\left[\begin{array}{ccc}
\mathbf{A}_{i i}^{s} & \mathbf{A}_{i r}^{s} & \mathbf{A}_{i c}^{s} \mathbf{B}_{c}^{s} \\
\mathbf{A}_{r i}^{s} & \mathbf{A}_{r r}^{s} & \mathbf{A}_{r c}^{s} \mathbf{B}_{c}^{s} \\
\sum_{s=1}^{n_{s}} \mathbf{B}_{c}^{s T} \mathbf{A}_{c i}^{s} & \sum_{s=1}^{n_{s}} \mathbf{B}_{c}^{s T} \mathbf{A}_{c r}^{s} & \sum_{s=1}^{n_{s}} \mathbf{B}_{c}^{s T} \mathbf{A}_{c c}^{s} \mathbf{B}_{c}^{s}
\end{array}\right]\left\{\begin{array}{c}
\mathbf{x}_{i}^{s} \\
\mathbf{u}_{r}^{s} \\
\mathbf{u}_{c}
\end{array}\right\}=\left\{\begin{array}{c}
0 \\
\mathbf{f}_{r}^{s} \\
0
\end{array}\right\}
$$

where $\mathbf{f}_{r}^{s}=\mathbf{D}_{r}^{s} \mathbf{B}_{r}^{s} \mathbf{r}_{j}$ and $\mathbf{r}_{j}$ is the residual at the $j^{t h}$ iteration of the iterative solver and $\mathbf{D}_{r}^{s}$ represents a diagonal weighting matrix which satisfies the following property 


$$
\sum_{s=1}^{n_{s}} \mathbf{B}_{r}^{s T} \mathbf{D}_{r}^{s} \mathbf{B}_{r}^{s}=\mathbf{I}
$$

The diagonal entries of each block of $\mathrm{D}_{r}^{s}$ are the reciprocal of the number of subdomains that share the remaining interface boundary nodes.

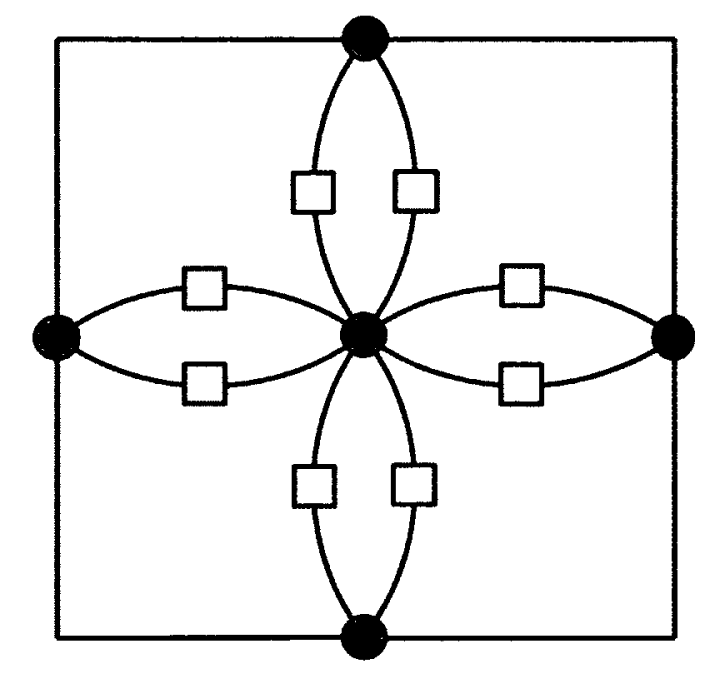

Figure 3.6: Partial assembly of corner nodes $(\bullet)$

The dummy variable $\mathbf{x}_{i}^{s}$ can be eliminated from Eq.(3.207) in parallel by solving a subdomain level Dirichlet problem as

$$
\mathbf{x}_{i}^{s}=-\left[\mathbf{A}_{i i}^{s}\right]^{-1}\left(\mathbf{A}_{i r}^{s} \mathbf{u}_{r}^{s}+\mathbf{A}_{i c}^{s} \mathbf{B}_{s} \mathbf{u}_{c}\right) .
$$

Substituting Eq.(3.209) into the Eq.(3.207) leads to

$$
\left[\begin{array}{cc}
\mathbf{S}_{r r}^{s} & \mathbf{S}_{r c}^{s} \mathbf{B}_{c}^{s} \\
\sum_{s=1}^{n_{s}} \mathbf{B}_{c}^{s T} \mathbf{S}_{c r}^{s} \mathbf{B}_{r}^{s} & \sum_{s=1}^{n_{s}} \mathbf{B}_{c}^{s T} \mathbf{S}_{c c}^{s} \mathbf{B}_{c}^{s}
\end{array}\right]\left\{\begin{array}{l}
\mathbf{u}_{r}^{s} \\
\mathbf{u}_{c}
\end{array}\right\}=\left\{\begin{array}{c}
\mathbf{f}_{r}^{s} \\
0
\end{array}\right\} .
$$

For each subdomain, $\mathbf{u}_{r}^{s}$ can be eliminated in parallel from the first row of Eq.(3.210) as 


$$
\mathbf{u}_{r}^{s}=\left[\mathbf{S}_{r r}^{s}\right]^{-1}\left(\mathbf{f}_{r}^{s}-\mathbf{S}_{r c}^{s} \mathbf{B}_{c}^{s} \mathbf{u}_{c}\right)
$$

Substituting $\mathbf{u}_{r}^{s}$ into the second row of Eq.(3.210) leads to the following coarse problem

$$
\mathbf{F}_{c c} \mathbf{u}_{c}=\mathrm{d}_{c}
$$

where

$$
\begin{aligned}
\mathbf{F}_{c c} & =\sum_{s=1}^{n_{s}} \mathbf{B}_{c}^{s T}\left(\mathbf{S}_{c c}^{s}-\mathbf{S}_{c r}^{s}\left[\mathbf{S}_{r r}^{s}\right]^{-1} \mathbf{S}_{r c}^{s}\right) \mathbf{B}_{c}^{s} \\
\mathbf{d}_{c} & =-\sum_{s=1}^{n_{s}} \mathbf{B}_{c}^{s T} \mathbf{S}_{c r}^{s}\left[\mathbf{S}_{r r}^{s}\right]^{-1} \mathbf{f}_{r}^{s}
\end{aligned}
$$

The continuity of the solution field on the remaining interface nodes $\mathbf{u}_{r}$ is satisfied next by averaging $\mathbf{u}_{r}^{s}$ for all the subdomains as

$$
\mathbf{u}_{r}=\sum_{s=1}^{n_{s}} \mathbf{B}_{r}^{s T} \mathbf{D}_{r}^{s} \mathbf{u}_{r}^{s}
$$

,After some algebraic manipulations, the primal-primal preconditioner can be expressed as

$$
\mathbf{M}_{P P}^{-1}=\sum_{s=1}^{n_{s}} \mathbf{B}_{r}^{s T} \mathbf{D}_{r}^{s}\left[\mathbf{S}_{r r}^{s}\right]^{-1} \mathbf{D}_{r}^{s} \mathbf{B}_{r}^{s}+\mathbf{R}_{0}^{T}\left[\mathbf{F}_{c c}\right]^{-1} \mathbf{R}_{0}
$$

where

$$
\mathbf{R}_{0}=\sum_{s=1}^{n_{s}} \mathbf{B}_{c}^{s T} \mathbf{S}_{c r}\left[\mathbf{S}_{r r}^{s}\right]^{-1} \mathbf{D}_{r}^{s} \mathbf{B}_{r}^{s}
$$




\subsubsection{Dual Iterative Substructuring Method}

The Finite Element Tearing and Interconnecting (FETI) is a Lagrange multiplier based technique for solving the linear system arising from the discretization of self-adjoint elliptic PDEs $[119,120,126-129]$. In the FETI method, the solution domain is decomposed into a number of non-overlapping subdomains [130]. On each subdomain, a local stiffness matrix and a local load vector are obtained from the finite element discretization of a subdomain level problem. The continuity of the solution field along the subdomain interfaces is enforced by Lagrange multipliers [131]. The interior variables are then eliminated by solving a subdomain level Neumann problems resulting a dual interface problem containing Lagrange multipliers. The dual interface problem is solved using a projected PCGM algorithm equipped with lumped or Dirichlet preconditioners. In each iteration of the iterative solver, the equilibrium in each subdomain is satisfied. However, the continuity is strictly enforced only when the iterative solver converges $[119,120,126]$.

In the FETI method, an algebraic coarse problem handles the issue of floating subdomains (having singular stiffness matrices). The coarse problem enables a global propagation of information across the subdomains $[7,110,132]$. In particular, the coarse problem provides a global mechanism to exchange information among non-neighboring subdomains without requiring the transmission of information through the intermediate subdomains $[7,110,132]$. The coarse problem forces the condition number of FETI method to be bounded and independent on the number of subdomains [120]. The FETI iterative substructuring method can be summarized as follows $[4,7,119]$. 
Let the spatial domain $\Omega$ be partitioned into $n_{s}$ non-overlapping subdomains $\left\{\Omega_{s}, 1 \leq\right.$ $\left.s \leq n_{s}\right\}$. For a typical subdomain $\Omega_{s}$, let $\mathbf{u}_{s}, \mathbf{A}_{s}$ and $\mathbf{f}_{s}$ be the local solution vector, the subdomain stiffness matrix and local load vector respectively. For the entire system, the extended stiffness matrix, solution and load vectors can be expressed as follows [7]

$$
\mathbf{A}=\left[\begin{array}{ccccc}
\mathbf{A}^{1} & & & & \\
& \ddots & & & \\
& & \mathbf{A}^{s} & & \\
& & \ddots & \\
& & & \mathbf{A}^{n_{s}}
\end{array}\right], \mathbf{u}=\left\{\begin{array}{c}
\mathbf{u}^{1} \\
\vdots \\
\mathbf{u}^{s} \\
\vdots \\
\\
\end{array}\right.
$$

The subdomain stiffness matrix is positive semidefinite for the case of floating subdomains. Let $\mathrm{Z}_{s}$ be a full rank matrix of size $n_{s} \times \min \left(1, d_{s}\right)$ whose columns span the null space of the subdomain stiffness matrix $\mathbf{A}_{s}$. When the subdomain stiffness matrix is positive definite, $\mathrm{Z}_{s}$ is a void matrix. In the block matrix form the null space matrix can be expressed as [7]

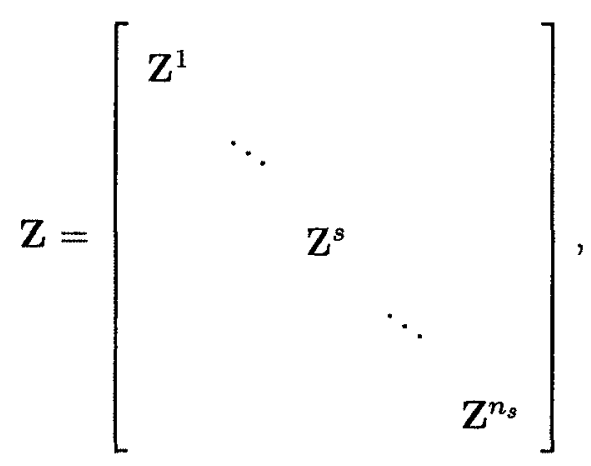

where Range $(\mathbf{Z})=\operatorname{Kernel}(\mathbf{A})$ and $\operatorname{Kernel}(\mathbf{Z})=\{0\}$. 
Let $\mathrm{B}^{s}$ be a signed Boolean matrix that enforces that the nodal interface variables have the same values for all the subdomains shearing the same boundaries. This constrain is represented by

$$
\mathbf{B}^{s} \mathbf{u}^{s}=0
$$

The global continuity matrix $\mathbf{B}$ takes the following form

$$
\mathbf{B}=\left[\begin{array}{lllll}
\mathbf{B}^{1} & & & & \\
& \ddots & & & \\
& & \mathbf{B}^{s} & & \\
& & & \ddots & \\
& & & & \mathbf{B}^{n_{s}}
\end{array}\right] .
$$

In FETI method, the original finite element problem is restated as follows $[4,7,78,133]$

Find $\mathbf{u}$ such that

$$
\begin{array}{r}
J(\mathbf{u})=\frac{1}{2} \mathbf{u}^{T} \mathbf{A} \mathbf{u}-\mathbf{u}^{T} \mathbf{f} \rightarrow \min \\
\text { subject to } \mathbf{B u}=0 .
\end{array}
$$

By introducing a vector of Lagrange multipliers $\lambda$, the constrained minimization problem in Eq.(3.222) can be reexpressed as a saddle point problem as

$$
\mathcal{L}(\mathbf{u}, \boldsymbol{\lambda})=\frac{1}{2} \mathbf{u}^{T} \mathbf{A} \mathbf{u}-\mathbf{u}^{T} \mathbf{f}+\mathbf{u}^{T} \mathbf{B}^{T} \boldsymbol{\lambda}
$$


The Lagrange multipliers $\boldsymbol{\lambda}$ weakly enforces the continuity condition of the nodal interface variables.

Minimizing the Lagrangian function in Eq.(3.223) with respect to $\mathbf{u}$ and $\boldsymbol{\lambda}$ leads to the following equilibrium system

$$
\left[\begin{array}{cc}
\mathrm{A} & \mathbf{B}^{T} \\
\mathrm{~B} & 0
\end{array}\right]\left\{\begin{array}{l}
\mathbf{u} \\
\boldsymbol{\lambda}
\end{array}\right\}=\left\{\begin{array}{l}
\mathbf{f} \\
\mathbf{0}
\end{array}\right\} .
$$

If $\mathbf{A}$ is singular, a solution vector $\mathbf{u}$ of the first equation in Eq.(3.224) exists if and only if $[4,7]$

$$
\left(\mathbf{f}-\mathbf{B}^{T} \boldsymbol{\lambda}\right) \in \operatorname{Range}(\mathbf{A})
$$

The general solution to the singular system can be written as $[4,7]$

$$
\mathbf{u}=\mathbf{A}^{+}\left(\mathbf{f}-\mathbf{B}^{T} \boldsymbol{\lambda}\right)+\mathbf{Z} \boldsymbol{\alpha} \quad \text { if } \quad\left(\mathbf{f}-\mathbf{B}^{\mathrm{T}} \boldsymbol{\lambda}\right) \perp \operatorname{Kernel}(\mathbf{A})
$$

where $\mathrm{A}^{+}$is a pseudo-inverse of $\mathbf{A}$ and $\alpha \in \Re^{d}$ is to be determined. Note that, the condition $\left(\mathbf{f}-\mathbf{B}^{T} \boldsymbol{\lambda}\right) \perp K \operatorname{ernel}(\mathbf{A})$ means that the action of applied forces does not excite the rigid body motions $[4,7]$.

Since the matrix $\mathrm{Z}$ has a full rank, and the column vectors of $\mathrm{Z}^{T}$ span the null space of the singular matrix $A$, the solution exists when

$$
\mathbf{Z}^{T}\left(\mathbf{f}-\mathbf{B}^{T} \boldsymbol{\lambda}\right)=\mathbf{0}
$$


Eq.(3.227) can be rewritten as

$$
\mathrm{G}^{T} \lambda=\mathrm{e}
$$

where $\mathrm{G}=\mathrm{BZ}$ and $\mathbf{e}=\mathbf{Z}^{T} \mathbf{f}$.

Using the constrain $\mathrm{Bu}=0$, Eq.(3.226) yields

$$
\mathbf{B A}^{+}\left(\mathbf{f}-\mathbf{B}^{T} \lambda\right)+\mathbf{B Z} \alpha=\mathbf{0}
$$

Eq.(3.229) can be expressed as

$$
\mathbf{F} \boldsymbol{\lambda}-\mathbf{G} \boldsymbol{\alpha}=\mathrm{d}
$$

where $\mathbf{F}=\mathbf{B A}^{+} \mathbf{B}^{T}, \mathbf{G}=\mathbf{B Z}$ and $\mathbf{d}=\mathbf{B A}^{+} \mathbf{f}$.

When Eq.(3.230) and Eq.(3.228) are combined, one obtains the following linear system

$$
\begin{aligned}
\mathbf{F} \boldsymbol{\lambda}-\mathbf{G} \boldsymbol{\alpha} & =\mathrm{d} . \\
\mathbf{G}^{T} \boldsymbol{\lambda} & =\mathbf{e} .
\end{aligned}
$$

Let $\mathbf{P}$ be an orthogonal projection operator onto $\operatorname{Kernel}\left(\mathbf{G}^{T}\right)$ defined as $[4,7]$

$$
\mathbf{P}=\mathbf{I}-\mathbf{G}\left(\mathbf{G}^{T} \mathbf{G}\right)^{-1} \mathbf{G}^{T}
$$


Multiplying the first equation of Eq.(3.231) by the orthogonal projector $\mathbf{P}$ yields

$$
\mathbf{P F} \boldsymbol{\lambda}=\mathbf{P d}
$$

The FETI method is a preconditioned conjugate gradient method applied to solve the system defined in Eq.(3.234) (as schematically shown in Fig.(3.7)) with an initial approximation $\lambda_{0}$ chosen such that

$$
\mathbf{G}^{T} \boldsymbol{\lambda}_{0}=\mathbf{e}
$$

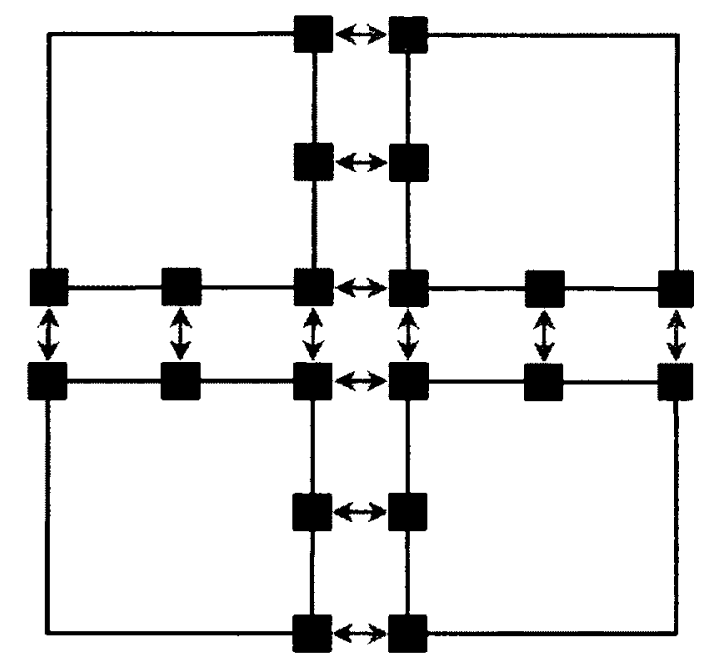

Figure 3.7: Lagrange multiples are the force required to enforce continuity constraint

Once $\lambda$ is determined, the unknown coefficient vector $\alpha$ can be obtained from Eq.(3.230) in a least square sense as 


$$
\alpha=\left(\mathbf{G}^{T} \mathbf{G}\right)^{-1} \mathbf{G}^{T}(\mathbf{F} \boldsymbol{\lambda}-\mathrm{d})
$$

Any solution $\lambda$ from Eq.(3.231) is unique up to an additive vector from $K \operatorname{ernel}\left(\mathbf{B}^{T}\right)$ [7]. However, any of these solutions of $\lambda$ are the solution to the minimization problem. Here $\mathbf{u}$ is given by Eq.(3.226) with $\alpha$ defined by Eq.(3.236). When the matrix $\mathbf{A}$ is not singular, the coefficient vector $\alpha$ is omitted. In this case $\mathbf{F}=\mathbf{B A}^{-1} \mathbf{B}^{T}, \mathbf{d}=\mathbf{B A}^{-1} \mathrm{f}, \mathbf{G}=\mathbf{0}, \mathbf{P}=\mathbf{I}$ and $\mathbf{e}=\mathbf{0}$

\section{Preconditioners for FETI}

The FETI method can be viewed as a projected preconditioned conjugate gradient iterative solver for the Lagrange multipliers as $[4,7,64]$

$$
\mathbf{P M}^{-1} \mathbf{P}^{T} \mathbf{F} \boldsymbol{\lambda}=\mathbf{P M}^{-1} \mathbf{P}^{T} \mathbf{d}
$$

Two types of preconditioner $\mathrm{M}^{-1}$ are available to FETI method, lumped and Dirichlet preconditioners. The lumped preconditioner approximates the local Schur complement matrix by its leading matrix as

$$
\mathbf{M}_{L}^{-1}=\sum_{s=1}^{n_{s}} \mathbf{B}^{s} \mathbf{D}^{s} \mathbf{A}_{\Gamma \Gamma}^{s} \mathbf{D}^{s} \mathbf{B}^{s T}
$$

This preconditioner is not expensive to apply, but not mathematically optimal. On the other hand, the Dirichlet preconditioner is defined as 


$$
\mathbf{M}_{D}^{-1}=\sum_{s=1}^{n_{s}} \mathbf{B}^{s} \mathbf{D}^{s} \mathbf{S}_{s} \mathbf{D}^{s} \mathbf{B}^{s T}
$$

The Dirichlet preconditioner is mathematically optimal [134] due to the fact that it leads to the boundness of the condition number estimate $[4,7,134]$. Application of the Dirichlet preconditioner requires the solution of a local subdomain problem with non-homogeneous Dirichlet boundary conditions.

\section{Condition Number Bound}

For the second order elliptic problems, the condition number of the preconditioned coefficient matrices in Eq.(3.237), equipped with the Dirichlet preconditioner $\mathrm{M}_{D}^{-1}$, grows asymptotically as $[4,126]$

$$
\kappa\left(\mathbf{P M}_{D}^{-1} \mathbf{P}^{T} \mathbf{F}\right) \leq C\left(1+\log \frac{H}{h}\right)^{2}
$$

where $C$ is a positive coefficient independent of the mesh size $h$ and subdomain size $H$. Clearly the condition number estimation of FETI method indicates that the method is numerically scalable with respect to problem size $h$ and subdomain size $H$ [129].

\subsubsection{Dual-Primal Iterative Substructuring Method}

The existence of non-trivial kernels of the local subdomain stiffness matrices is essential for the scalability of FETI method [4, 7, 135]. For complex problems, the characterization of these local kernels is not a trivial task $[4,7]$. 
In the Dual-Primal Finite Element Tearing and Interconnecting (FETI-DP) [64, 81, 136138], the continuity condition on the comer nodes (the primal variables) of the subdomains is enforced strictly in each iteration while Lagrange multipliers (the dual variables) are used to enforce the continuity constraint weakly on the remaining interface nodes. The continuity condition on the whole interface boundary is enforced only when the iterative solver converges. Strictly enforcing the continuity condition on the corner nodes at each iteration makes the local (subdomain) problems nonsingular. This fact also naturally provides a global coarse problem. The original FETI, on the other hand, is a projected preconditioned conjugate gradient which cannot start from an arbitrary guess $[4,7]$. In some cases, the initial guess can be faraway from the exact solution $[4,7]$. The FETI-DP, in contrast, employs the standard preconditioned conjugate gradient algorithm which can be start with any arbitrary initial guess $[4,7]$. The dual-primal FETI method can be described as follows $[4,7,64,81,136,137]$.

Let the physical domain $\Omega$ be decomposed into $n_{s}$ non-overlapping subdomains. The subdomain nodes are partitioned into interior $\mathbf{u}_{i}^{s}$, remaining $\mathbf{u}_{r}^{s}$ and corner $\mathbf{u}_{c}^{s}$ nodes as shown schematically in Fig.(3.4). The equilibrium system of a typical subdomain $\Omega_{s}$ can be expressed as $[4,7,64,81,136,137]$

$$
\left[\begin{array}{lll}
\mathbf{A}_{i i}^{s} & \mathbf{A}_{i r}^{s} & \mathbf{A}_{i c}^{s} \\
\mathbf{A}_{r i}^{s} & \mathbf{A}_{r r}^{s} & \mathbf{A}_{r c}^{s} \\
\mathbf{A}_{c i}^{s} & \mathbf{A}_{c r}^{s} & \mathbf{A}_{c c}^{s}
\end{array}\right]\left\{\begin{array}{l}
\mathbf{u}_{i}^{s} \\
\mathbf{u}_{r}^{s} \\
\mathbf{u}_{c}^{s}
\end{array}\right\}=\left\{\begin{array}{c}
\mathbf{f}_{i}^{s} \\
\mathbf{f}_{r}^{s} \\
\mathbf{f}_{c}^{s}
\end{array}\right\} .
$$

The global equilibrium equation is given by $[7,64,78,83]$ 


$$
\left[\begin{array}{ccc}
\mathbf{A}_{i i}^{s} & \mathbf{A}_{i r}^{s} & \mathbf{A}_{i c}^{s} \mathbf{B}_{c}^{s} \\
\mathbf{A}_{r i}^{s} & \mathbf{A}_{r r}^{s} & \mathbf{A}_{r c}^{s} \mathbf{B}_{c}^{s} \\
\sum_{s=1}^{n_{s}} \mathrm{~B}_{c}^{s T} \mathbf{A}_{c i}^{s} & \sum_{s=1}^{n_{s}} \mathbf{B}_{c}^{s T} \mathbf{A}_{c r}^{s} & \sum_{s=1}^{n_{s}} \mathbf{B}_{c}^{s T} \mathbf{A}_{c c}^{s} \mathbf{B}_{c}^{s}
\end{array}\right]\left\{\begin{array}{c}
\mathbf{u}_{i}^{s} \\
\mathbf{u}_{r}^{s} \\
\mathbf{u}_{c}
\end{array}\right\}=\left\{\begin{array}{c}
\mathrm{f}_{i}^{s} \\
\mathbf{f}_{r}^{s} \\
\sum_{s=1}^{n_{s}} \mathrm{~B}_{c}^{s T} \mathrm{f}_{c}^{s}
\end{array}\right\},
$$

where $\mathrm{B}_{c}^{s}$ is a Boolean restriction operator that maps the global corner vector $\mathrm{u}_{c}$ to the local corner vector $\mathbf{u}_{c}^{s}$ as

$$
\mathbf{u}_{c}^{s}=\mathbf{B}_{c}^{s} \mathbf{u}_{c}
$$

The partially assembled system in Eq.(3.242) can be rewritten in compact form as

$$
\tilde{\mathbf{A}}^{s} \tilde{\mathbf{u}}^{s}=\tilde{\mathbf{f}}^{s},
$$

where $\tilde{\mathbf{A}}^{s}$ denotes the partially assembled stiffness matrix, $\tilde{\mathbf{u}}^{s}$ is a partially assembled unknowns nodal vector, and $\tilde{\mathbf{f}}^{s}$ is the associated partially assembled load vector.

Let $\mathrm{B}_{r}^{s}$ be a signed Boolean continuity matrix defined as

$$
\sum_{s=1}^{n_{s}} \mathbf{B}_{r}^{s} \mathbf{u}_{r}^{s}=0
$$

Next, the original finite element problem can be reformulated as an equivalent constrained minimization problem as 


$$
\begin{array}{r}
\frac{1}{2} \tilde{\mathbf{u}}^{T} \tilde{\mathbf{A}} \tilde{\mathbf{u}}-\tilde{\mathbf{u}}^{T} \tilde{\mathbf{f}} \rightarrow \min \\
\text { subject to } \mathbf{B u}=\mathbf{0},
\end{array}
$$

where

$$
\begin{aligned}
& \tilde{\mathbf{A}}=\left[\begin{array}{ccccc}
\tilde{\mathbf{A}}^{1} & & & & \\
& \ddots & & & \\
& & \tilde{\mathbf{A}}^{s} & & \\
& & \ddots & \\
& & & \tilde{\mathbf{A}}^{n_{s}}
\end{array}\right], \tilde{\mathbf{u}}=\left\{\begin{array}{c}
\tilde{\mathbf{u}}^{1} \\
\vdots \\
\tilde{\mathbf{u}}^{s} \\
\vdots \\
\\
\end{array}\right. \\
& \mathbf{B}=\left[\left(\begin{array}{lll}
0 & \mathbf{B}_{r}^{1} & 0
\end{array}\right), \cdots,\left(\begin{array}{lll}
0 & \mathbf{B}_{r}^{s} & 0
\end{array}\right), \cdots,\left(\begin{array}{lll}
0 & \mathbf{B}_{r}^{n_{s}} & 0
\end{array}\right)\right]
\end{aligned}
$$

Introducing Lagrange multipliers to weakly enforce the compatibility constraint, the constrained minimization problem in Eq.(3.246) can be expressed as $[4,7]$

$$
\mathcal{L}(\tilde{\mathbf{u}}, \boldsymbol{\lambda})=\frac{1}{2} \tilde{\mathbf{u}}^{T} \tilde{\mathbf{A}} \tilde{\mathbf{u}}-\tilde{\mathbf{u}}^{T} \tilde{\mathbf{f}}+\tilde{\mathbf{u}}^{T} \mathbf{B}^{T} \boldsymbol{\lambda}
$$

Minimizing Eq.(3.249) with respect to $\tilde{\mathbf{u}}$ and $\boldsymbol{\lambda}$ leads to the following equilibrium system $[7,64,78,83]$ 


$$
\left[\begin{array}{cccc}
\mathbf{A}_{i i}^{s} & \mathbf{A}_{i r}^{s} & \mathbf{A}_{i c}^{s} \mathbf{B}_{c}^{s} & 0 \\
\mathbf{A}_{r i}^{s} & \mathbf{A}_{r r}^{s} & \mathbf{A}_{r c}^{s} \mathbf{B}_{c}^{s} & \mathbf{B}_{r}^{s T} \\
\sum_{s=1}^{n_{s}} \mathbf{B}_{c}^{s T} \mathbf{A}_{c i}^{s} & \sum_{s=1}^{n_{s}} \mathbf{B}_{c}^{s T} \mathbf{A}_{c r}^{s} & \sum_{s=1}^{n_{s}} \mathbf{B}_{c}^{s T} \mathbf{A}_{c c}^{s} \mathbf{B}_{c}^{s} & 0 \\
0 & \sum_{s=1}^{n_{s}} \mathbf{B}_{r}^{s} & 0 & 0
\end{array}\right]\left\{\begin{array}{c}
\mathbf{u}_{i}^{s} \\
\mathbf{u}_{r}^{s} \\
\mathbf{u}_{c} \\
\boldsymbol{\lambda}
\end{array}\right\}=\left\{\begin{array}{c}
\mathbf{f}_{i}^{s} \\
\mathbf{f}_{r}^{s} \\
\sum_{s=1}^{n_{s}} \mathbf{B}_{c}^{s T} \mathbf{f}_{c}^{s} \\
0
\end{array}\right\}
$$

where $\lambda$ are the nodal forces required to satisfy continuity at the remaining interface nodes as shown schematically in Fig.(3.8).

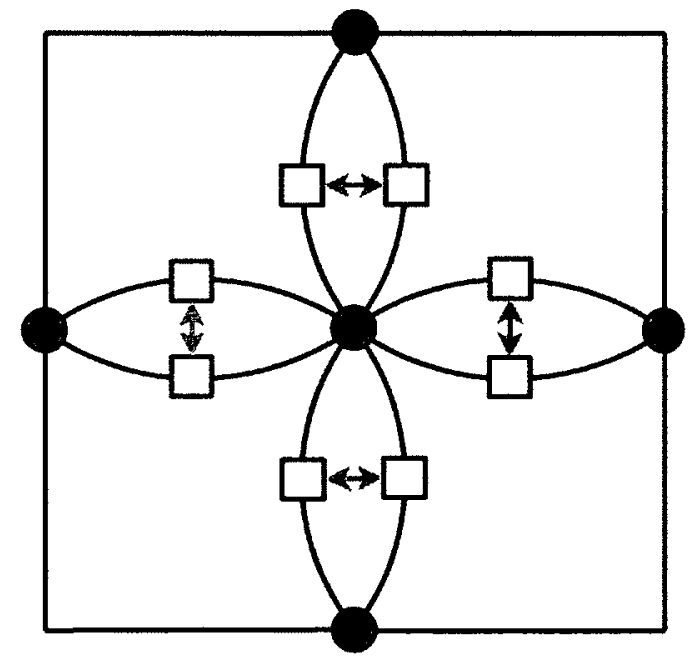

Figure 3.8: Lagrange multipliers are the forces required to connect the tore interface boundary

From the first row of Eq.(3.250), one obtains

$$
\mathbf{u}_{i}^{s}=\left[\mathbf{A}_{i i}^{s}\right]^{-1}\left(\mathbf{f}_{i}^{s}-\mathbf{A}_{i r}^{s} \mathbf{u}_{r}^{s}-\mathbf{A}_{i c}^{s} \mathbf{B}_{c}^{s} \mathbf{u}_{c}\right)
$$

Eliminating $u_{i}^{s}$ from Eq.(3.250), leads to the following compact system 


$$
\left[\begin{array}{ccc}
\mathbf{S}_{r r}^{s} & \mathbf{S}_{r c}^{s} \mathbf{B}_{c}^{s} & \mathbf{B}_{r}^{s T} \\
\sum_{s=1}^{n_{s}} \mathbf{B}_{c}^{n_{s} T} \mathbf{S}_{c r}^{s} & \sum_{s=1}^{n_{s}} \mathbf{B}_{c}^{s T} \mathbf{S}_{c c}^{s} \mathbf{B}_{c}^{s} & 0 \\
\sum_{s=1}^{n_{r}} \mathbf{B}_{r}^{s} & 0 & 0
\end{array}\right]\left\{\begin{array}{c}
\mathbf{u}_{r}^{s} \\
\mathbf{u}_{c} \\
\boldsymbol{\lambda}
\end{array}\right\}=\left\{\begin{array}{c}
\mathbf{g}_{r}^{s} \\
\sum_{s=1}^{n_{s}} \mathbf{B}_{c}^{s T} \mathbf{g}_{c}^{s} \\
0
\end{array}\right\}
$$

where

$$
\begin{aligned}
\mathbf{S}_{\alpha \beta}^{s} & =\mathbf{A}_{\alpha \beta}^{s}-\mathbf{A}_{\alpha i}^{s}\left[\mathbf{A}_{i i}^{s}\right]^{-1} \mathbf{A}_{i \beta}^{s} \\
\mathbf{g}_{\alpha}^{s} & =\mathbf{f}_{\alpha}^{s}-\mathbf{A}_{\alpha i}^{s}\left[\mathbf{A}_{i i}^{s}\right]^{-1} \mathbf{f}_{i}^{s} .
\end{aligned}
$$

The local Schur complement matrix $\mathbf{S}_{r r}^{s}$ associated with the remaining interface nodes in Eq.(3.252) is positive definite and hence invertible for the case of floating subdomains. Consequently, the subdomain level remaining unknown vector $u_{r}^{s}$ can be obtained from Eq.(3.252) as

$$
\mathbf{u}_{r}^{s}=\left[\mathbf{S}_{r r}^{s}\right]^{-1}\left(\mathbf{g}_{r}^{s}-\mathbf{S}_{r c}^{s} \mathbf{B}_{c}^{s} \mathbf{u}_{c}-\mathbf{B}_{r}^{s T} \boldsymbol{\lambda}\right)
$$

Substituting $\mathrm{u}_{r}^{s}$ from Eq.(3.255) into Eq.(3.252) leads to the following system

$$
\left[\begin{array}{cc}
\mathbf{F}_{c c} & -\mathbf{F}_{c r} \\
\mathbf{F}_{r c} & \mathbf{F}_{r r}
\end{array}\right]\left\{\begin{array}{c}
\mathbf{u}_{c} \\
\boldsymbol{\lambda}
\end{array}\right\}=\left\{\begin{array}{c}
\mathbf{d}_{c} \\
\mathrm{~d}_{r}
\end{array}\right\}
$$


where

$$
\begin{aligned}
\mathbf{F}_{c c} & =\sum_{s=1}^{n_{s}} \mathbf{B}_{c}^{s T}\left(\mathbf{S}_{c c}^{s}-\mathbf{S}_{c r}^{s}\left[\mathbf{S}_{r r}^{s}\right]^{-1} \mathbf{S}_{r c}^{s}\right) \mathbf{B}_{\mathbf{c}}^{\mathbf{s}} \\
\mathbf{F}_{c r} & =\sum_{s=1}^{n_{s}} \mathbf{B}_{c}^{s T} \mathbf{S}_{c r}^{s}\left[\mathbf{S}_{r r}^{s}\right]^{-1} \mathbf{B}_{\mathbf{r}}^{\mathbf{s}^{T}} \\
\mathbf{F}_{r c} & =\sum_{s=1}^{n_{s}} \mathbf{B}_{r}^{s}\left[\mathbf{S}_{r r}^{s}\right]^{-1} \mathbf{S}_{r c}^{s} \mathbf{B}_{\mathbf{c}}^{\mathbf{s}} \\
\mathbf{F}_{r r} & =\sum_{s=1}^{n_{s}} \mathbf{B}_{r}^{s}\left[\mathbf{S}_{r r}^{s}\right]^{-1} \mathbf{B}_{\mathbf{r}}^{\mathbf{s} T} \\
\mathbf{d}_{c} & =\sum_{s=1}^{n_{s}} \mathbf{B}_{c}^{s T}\left(\mathbf{G}_{c}^{s}-\mathbf{S}_{c r}^{s}\left[\mathbf{S}_{r r}^{s}\right]^{-1} \mathbf{g}_{r}^{s}\right) \\
\mathbf{d}_{r} & =\sum_{s=1}^{n_{s}} \mathbf{B}_{r}^{s}\left[\mathbf{S}_{r r}^{s}\right]^{-1} \mathbf{g}_{r}^{s} .
\end{aligned}
$$

Solving for $\mathrm{u}_{c}$ from Eq.(3.256) gives the following coarse problem

$$
\mathbf{F}_{c c} \mathbf{u}_{c}=\left(\mathbf{d}_{c}+\mathbf{F}_{c r} \lambda\right)
$$

Substituting $\mathrm{u}_{c}$ into Eq.(3.256) leads to the following symmetric positive-definite Lagrange multiplier system

$$
\left(\mathbf{F}_{r r}+\mathbf{F}_{r c}\left[\mathbf{F}_{c c}\right]^{-1} \mathbf{F}_{c r}\right) \boldsymbol{\lambda}=\mathrm{d}_{r}-\mathbf{F}_{r c}\left[\mathbf{F}_{c c}\right]^{-1} \mathrm{~d}_{c}
$$

Eq.(3.264) is the dual interface problem which can be tackled iteratively by the standard preconditioned conjugate gradient method equipped with a lumped or Dirichlet preconditioner. 


\section{Preconditioners for FETI-DP}

The dual interface problem in the FETI-DP method can be solved using the standard preconditioned conjugate gradient method. The preconditioned system takes the following form

$$
\mathbf{M}^{-1}\left(\mathbf{F}_{r r}+\mathbf{F}_{r c}\left[\mathbf{F}_{c c}\right]^{-1} \mathbf{F}_{c r}\right) \boldsymbol{\lambda}=\mathbf{M}^{-1}\left(\mathbf{d}_{r}-\mathbf{F}_{r c}\left[\mathbf{F}_{c c}\right]^{-1} \mathbf{d}_{c}\right)
$$

Since the coarse problem $\mathbf{F}_{c c}^{-1}$ is already embedded within the FETI-DP operator, a onelevel preconditioner such as a lumped or Dirichlet preconditioner is sufficient for numerical scalability. The lumped and Dirichlet preconditioners are respectively defined as

$$
\begin{gathered}
\mathbf{M}_{L}^{-1}=\sum_{s=1}^{n_{s}} \mathbf{B}_{r}^{s} \mathbf{D}_{r}^{s} \mathbf{A}_{r r}^{s} \mathbf{D}_{r}^{s} \mathbf{B}_{r}^{s T} \\
\mathbf{M}_{D}^{-1}=\sum_{s=1}^{n_{s}} \mathbf{B}_{r}^{s} \mathbf{D}_{r}^{s} \mathbf{S}_{r r}^{s} \mathbf{D}_{r}^{s} \mathbf{B}_{r}^{s T}
\end{gathered}
$$

The lumped preconditioner is not computationally expensive to apply, but not mathematically optimal. The Dirichlet preconditioner, on the other hand, is mathematically optimal as it provides a bound on the condition number estimate $[4,78,136]$.

\section{Condition Number Bound}

If $h$ and $H$ are the typical finite element mesh size and subdomain size repectively, the condition number of the preconditioned operator of the FETI-DP method is bounded by [4, $78,136]$ 


$$
\kappa\left(\mathbf{M}_{D}^{-1}\left(\mathbf{F}_{r r}+\mathbf{F}_{r c}\left[\mathbf{F}_{c c}\right]^{-1} \mathbf{F}_{c r}\right)\right) \leq C\left(1+\log \frac{H}{h}\right)^{2}
$$

where $C$ is a positive coefficient independent of the the number of subdomains and the size of the elements. Clearly, the condition number estimate is bounded as the number of subdomains increases [135].

\subsection{Summary}

An overview of various types of domain decomposition methods is presented in this chapter. In particular, we describe the overlapping and non-overlapping domain decomposition approaches and outline the primal, dual and dual-primal iterative substructuring methods. In addition, a novel primal-primal iterative substructuring method equipped with a twolevel scalable preconditioner is presented.

Domain decomposition methods can be viewed as a divide and conquer techniques for solving the linear system arising from the discretization of partial differential equations [4 7]. It involves iteratively or alternately solving subsystems defined on smaller subdomains while enforcing continuity requirements of the solution field across the subdomain interfaces $[4-7]$.

In the overlapping domain decomposition method (Schwarz methods), the computational domain is split into a number of overlapping subdomains whereby a smaller problem is solved alternately on each subdomain. The additive Schwarz method is an embar- 
rassingly parallel algorithm, but its convergence rate is slow compared to multiplicative Schwarz method. On the other hand, the classical multiplicative Schwarz method is sequential in nature and has a limited potential for parallelism as it requires the solution of one subdomain problem prior to another $[6,7]$. However, the multi-coloring technique can be used to improve the parallel performance of the multiplicative Schwarz method. In this approach, each subdomain is identified with a color such that the subdomains with the same color do not have a common interface boundary. Consequently the multiplicative Schwarz algorithm is implemented sequentially for the subdomains with different colors, but concurrently for subdomains with the same colors citechan 1994. In general the convergence rate depends on the number of colors as the fewer the colors the faster the convergence [6].

Non-overlapping domain decomposition methods are preconditioned iterative algorithms to solve the large-scale linear system arising from the discretization of partial differential equations. The computational domain is partitioned into a number of non-overlapping subdomains. At each iteration of the iterative solver loop, local problems are solved on each subdomain concurrently to construct a subdomain level preconditioner. This local preconditioner leads to an iterative method that is numerically scalable with respect to mesh size [4-7, 129]. Furthermore, a coarse problem with a few unknowns per each subdomain is solved in each iteration in order to make the iterative method numerically scalable with respect to subdomain size. The local part of the preconditioner is to eliminate the high frequency component of the error, while the coarse problem is to provide a global exchange of information about the error and thus quickly reducing the low frequency component of the error [4-7]. 
In general, the overlapping Schwarz domain decomposition methods are relatively easier to formulate and implement compared to the non-overlapping Schur complement methods. However, the computational complexity of one iteration of Schwarz algorithm can exceed the computational cost of the Schur complement algorithm by a factor, proportional to the overlap between the adjacent subdomain [7]. 


\section{Chapter 4}

\section{Domain Decomposition Methods for}

\section{Stochastic PDEs}

\subsection{Introduction}

This chapter is closely based on the references $[70,74,75]$. In the SSFEM, the finite element discretization of SPDEs involves tackling a large-scale deterministic linear system. The order of the linear system in SSFEM increases significantly with the mesh resolution and the size of the stochastic dimension. The solution of this system of linear equations poses a significant computational challenge. In this chapter, iterative substructuring technique for SPDEs is presented extending the basic formulation in [68]. In particular, a parallel PCGM is introduced to tackle the interface problem in the context of domain decomposition method of SPDEs. The iterative algorithm eliminates the need of explicit construction of the extended Schur complement system. 


\subsection{Mathematical Formulation}

Consider an elliptic SPDE, defined on a spatial domain $\Omega$ with given boundary conditions on $\partial \Omega$, whose differential operator (left hand side) contains spatially varying random coefficients. The finite element approximation of the SPDE yields the linear system with stochastically varying parameters as

$$
\mathbf{A}(\theta) \mathbf{u}(\theta)=\mathbf{f},
$$

where $A(\theta)$ denotes the stochastic stiffness matrix, $u(\theta)$ is the stochastic solution vector and $\mathbf{f}$ is the applied deterministic force. In the domain decomposition methods (for example, see [4-7]), the spatial domain $\Omega$ is divided into $n_{s}$ non-overlapping subdomains $\left\{\Omega_{s}\right\}_{s=1}^{n_{s}}$ and the subdomain degrees of freedom $\mathbf{u}^{s}(\theta)$ is split into two subsets: interior $\mathbf{u}_{I}^{s}(\theta)$ and interface $\mathbf{u}_{\Gamma}^{s}(\theta)$ degrees of freedom as shown schematically in Fig.(4.1). 

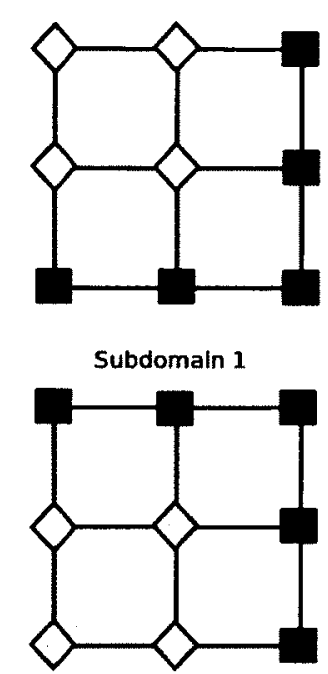

Subdomain 3
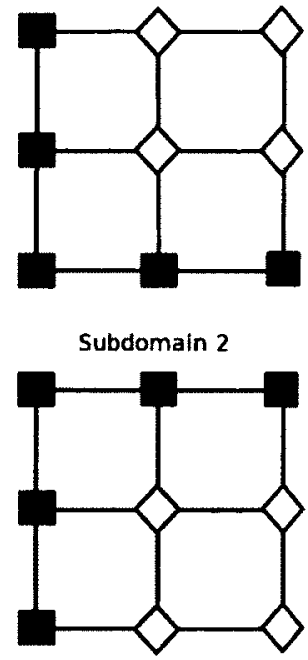

Subdomain 4

Figure 4.1: Partitioning the subdomain nodes into: interior $(\vartheta)$ and interface (ם)

The equilibrium equation for a typical subdomain $\Omega_{s}$ is expressed as

$$
\left[\begin{array}{ll}
\mathbf{A}_{I I}^{s}(\theta) & \mathbf{A}_{I \Gamma}^{s}(\theta) \\
\mathbf{A}_{\Gamma I}^{s}(\theta) & \mathbf{A}_{\Gamma \Gamma}^{s}(\theta)
\end{array}\right]\left\{\begin{array}{c}
\mathbf{u}_{I}^{s}(\theta) \\
\mathbf{u}_{\Gamma}^{s}(\theta)
\end{array}\right\}=\left\{\begin{array}{c}
\mathbf{f}_{I}^{s} \\
\mathbf{f}_{\Gamma}^{s}
\end{array}\right\} .
$$

When the PCE can be used to represent the non-Gaussian system parameters, the above equation becomes

$$
\sum_{i=0}^{L} \Psi_{i}(\theta)\left[\begin{array}{cc}
\mathbf{A}_{I I, i}^{s} & \mathbf{A}_{I \Gamma, i}^{s} \\
\mathbf{A}_{\Gamma I, i}^{s} & \mathbf{A}_{\Gamma \Gamma, i}^{s}
\end{array}\right]\left\{\begin{array}{c}
\mathbf{u}_{I}^{s}(\theta) \\
\mathbf{u}_{\Gamma}^{s}(\theta)
\end{array}\right\}=\left\{\begin{array}{c}
\mathbf{f}_{I}^{s} \\
\mathbf{f}_{\Gamma}^{s}
\end{array}\right\}
$$

The transmission conditions along the subdomain boundaries are enforced next by global assembly. Using the PCE of the solution vector and Galerkin projection, we obtain the following coupled linear systems $[68,70,74,76,79,80]$ : 


$$
\begin{aligned}
&\left\langle\sum_{i=0}^{L} \Psi_{i}(\theta)\left[\begin{array}{cccc}
\mathbf{A}_{I I, i}^{1} & \ldots & 0 & \mathbf{A}_{I \Gamma, i}^{1} \mathbf{R}_{1} \\
\vdots & \ddots & \vdots & \vdots \\
0 & \ldots & \mathbf{A}_{I I, i}^{n_{s}} & \mathbf{A}_{I \Gamma, i}^{n_{s}} \mathbf{R}_{n_{s}} \\
\mathbf{R}_{1}^{T} \mathbf{A}_{\Gamma I, i}^{1} & \ldots & \mathbf{R}_{n_{s}}^{T} \mathbf{A}_{\Gamma I, i}^{n_{s}} & \sum_{s=1}^{n_{s}} \mathbf{R}_{s}^{T} \mathbf{A}_{\Gamma \Gamma, i}^{s} \mathbf{R}_{s}
\end{array}\right] \sum_{j=0}^{N} \Psi_{j}(\theta)\left\{\begin{array}{c}
\mathbf{u}_{I, j}^{1} \\
\vdots \\
\mathbf{u}_{I, j}^{n_{s}} \\
\mathbf{u}_{\Gamma, j}
\end{array}\right\} \Psi_{k}(\theta)\right\rangle \\
&\left.=\left\{\begin{array}{c}
\mathbf{f}_{I}^{1} \\
\vdots \\
\mathbf{f}_{I}^{n_{s}} \\
\sum_{s=1}^{n_{s}} \mathbf{R}_{s}^{T} \mathbf{f}_{\Gamma}^{s}
\end{array}\right\} \Psi_{k}(\theta)\right\rangle, \quad k=0, \ldots, N .
\end{aligned}
$$

where $\mathbf{R}_{s}$ is a restriction matrix that relates the global interface unknown $\mathbf{u}_{\Gamma}(\theta)$ to the local interface unknown $\mathbf{u}_{\Gamma}^{s}(\theta)$ as

$$
\mathbf{u}_{\Gamma}^{s}(\theta)=\mathbf{R}_{s} \mathbf{u}_{\Gamma}(\theta) .
$$

In the concise form, Eq.(4.4) can be expressed as

$$
\left[\begin{array}{cccc}
\mathcal{A}_{I I}^{1} & \ldots & 0 & \mathcal{A}_{I \Gamma}^{1} \mathcal{R}_{1} \\
\vdots & \ddots & \vdots & \vdots \\
0 & \ldots & \mathcal{A}_{I I}^{n_{s}} & \mathcal{A}_{I \Gamma}^{n_{s}} \mathcal{R}_{n_{s}} \\
\mathcal{R}_{1}^{T} \mathcal{A}_{\Gamma I}^{1} & \ldots & \mathcal{R}_{n_{s}}^{T} \mathcal{A}_{\Gamma I}^{n_{s}} & \sum_{s=1}^{n_{s}} \mathcal{R}_{s}^{T} \mathcal{A}_{\Gamma \Gamma}^{s} \mathcal{R}_{s}
\end{array}\right]\left\{\begin{array}{c}
\mathcal{U}_{I}^{1} \\
\vdots \\
\mathcal{U}_{I}^{n_{s}} \\
\mathcal{U}_{\Gamma}
\end{array}\right\}=\left\{\begin{array}{c}
\mathcal{F}_{I}^{1} \\
\vdots \\
\mathcal{F}_{I}^{n_{s}} \\
\sum_{s=1}^{n_{s}} \mathcal{R}_{s}^{T} \mathcal{F}_{\Gamma}^{s}
\end{array}\right\},
$$

where

$$
\begin{gathered}
{\left[\mathcal{A}_{\alpha \beta}^{s}\right]_{j k}=\sum_{i=0}^{L}\left\langle\Psi_{i} \Psi_{j} \Psi_{k}\right\rangle \mathbf{A}_{\alpha \beta, i}^{s} ; \quad \mathcal{F}_{\alpha, k}^{s}=\left\langle\Psi_{k} \mathbf{f}_{\alpha}^{s}\right\rangle} \\
\mathcal{U}_{I}^{m}=\left(\mathbf{u}_{I, 0}^{m}, \ldots, \mathbf{u}_{I, N}^{m}\right)^{T} ; \quad \mathcal{U}_{\Gamma}=\left(\mathbf{u}_{\Gamma, 0}, \ldots, \mathbf{u}_{\Gamma, N}\right)^{T}
\end{gathered}
$$


The subscripts $\alpha$ and $\beta$ can assume the index $I$ and $\Gamma$. The new restriction matrix $\mathcal{R}_{s}$ is given by

$$
\mathcal{R}_{s}=\operatorname{blockdiag}\left(\mathbf{R}_{s}^{0}, \ldots, \mathbf{R}_{s}^{N}\right)
$$

After eliminating the interior degrees of freedom, Eq.(4.6) reduces to the following $e x$ tended Schur complement system

$$
\mathcal{S} \mathcal{U}_{\Gamma}=\mathcal{G}_{\Gamma}
$$

where the extended Schur complement matrix $\mathcal{S}$ is defined by the local Schur complement matrices as

$$
\mathcal{S}=\sum_{s=1}^{n_{s}} \mathcal{R}_{s}^{T}\left[\mathcal{A}_{\Gamma \Gamma}^{s}-\mathcal{A}_{\Gamma I}^{s}\left(\mathcal{A}_{I I}^{s}\right)^{-1} \mathcal{A}_{I \Gamma}^{s}\right] \mathcal{R}_{s}
$$

and the right hand side vector $\mathcal{G}_{\Gamma}$ is defined by

$$
\mathcal{G}_{\Gamma}=\sum_{s=1}^{n_{s}} \mathcal{R}_{s}^{T}\left[\mathcal{F}_{\Gamma}^{s}-\mathcal{A}_{\Gamma I}^{s}\left(\mathcal{A}_{I I}^{s}\right)^{-1} \mathcal{F}_{I}^{s}\right] .
$$

The solution of the extended Schur complement system in Eq.(4.9) gives the unknowns $\mathcal{U}_{\Gamma}$ along the interface. Once these unknowns are available, the interior unknowns $\mathcal{U}_{I}^{s}$ can be obtained by solving the following stochastic interior problem on each subdomain concurrently

$$
\mathcal{A}_{I I}^{s} \mathcal{U}_{I}^{s}=\mathcal{F}_{I}^{s}-\mathcal{A}_{\Gamma I}^{s} \mathcal{R}_{s} \mathcal{U}_{\Gamma}
$$




\subsection{The Extended Schur Complement Matrix}

The extended Schur complement matrix of stochastic PDEs is generally dense. However, the block matrices forming the extended Schur complement matrix have two distinct level of sparsity structure. The first level relates to the finite element discretization whereas the second level arises due to the stochastic features of the problem. In the computer implementation of the algorithm, this multilevel sparsity structure is exploited in order to minimize the storage requirements and to reduce the unnecessary floating point operations associated with zero-valued elements. Fig.(4.2) shows the non-zero block pattern of the block matrices forming the stochastic Schur complement matrix for the first, second and third order PC expansions. The size of Schur complement matrix is $\left[n_{\Gamma}(N+1)\right] \times\left[n_{\Gamma}(N+\right.$ 1)] where $n_{\Gamma}$ is the number of the global interface unknowns and $(N+1)$ is the order of PC expansion. The size of each individual block is $n_{\Gamma} \times n_{\Gamma}$ and the number of blocks is $(N+1)$.

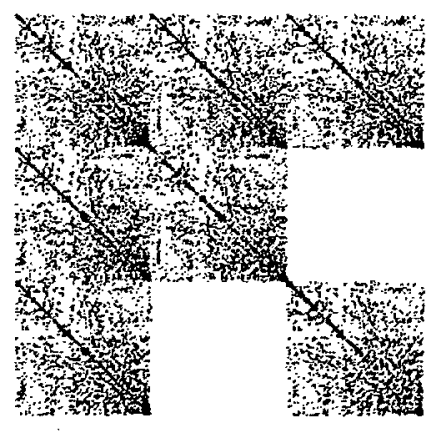

(a) Ist order PCE

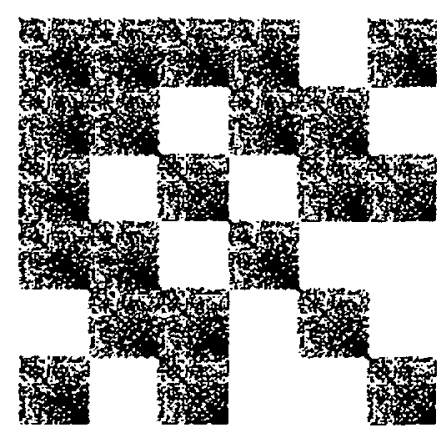

(b) 2nd order PCE

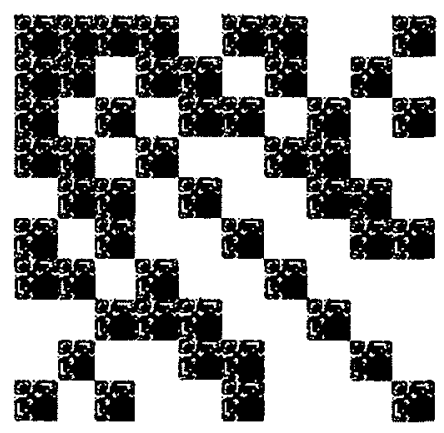

(c) 3rd order PCE

Figure 4.2: The sparsity structure of the stochastic block matrices 
Note that we do not construct or store the extended Schur complement matrix. Instead we only store the non-zero elements of the subdomain stiffness matrices $\mathcal{A}_{\alpha \beta}^{s}$ in Eq.(4.10). PETSc [87] sparse matrix (compressed row) format is used for efficient memory management. For better performance of PETSc matrix assembly process, the memory required for the sparse matrix storage need to be preallocated by setting the number of non-zeros entries per each row. This task is accomplished by defining an integer array of size $n_{\alpha}$ where $\alpha$ represents the index $I$ and $\Gamma$. The entries of this array is obtained by looping over the rows of the $\mathcal{A}_{\alpha \beta, i}^{s}$ matrix in Eq.(4.7) and counting the number of non-zeros for each row. This array represents the first sparsity level of the stochastic matrix. A second array of size $(N+1)$ containing the number of non-zeros block per row is constructed by counting the non-zeros coefficients of the $\left\langle\Psi_{i} \Psi_{j} \Psi_{k}\right\rangle$ (see Eq.(4.7)) which provides the second level of sparsity. Finally, an array of size $n_{\alpha}(N+1)$ is formed by multiplying the first array by the corresponding element of the second array. This array contains the sparsity pattern of the stochastic submatrices $\mathcal{A}_{\alpha \beta}^{s}$ in Eq.(4.10). Recall that $\mathcal{A}_{\alpha \beta}^{s}$ relates to $\mathcal{S}$ through Eq.(4.10).

\subsection{Iterative Substructuring Methods for Stochastic PDEs}

For a large-scale system, the solution of the interface problem in Eq.(4.9) using direct solvers becomes impractical due to overwhelming memory requirements and poor scalability to large number of processors. Therefor, iterative solution techniques are generally adopted [52]. When an iterative solver is used to tackle the global Schur complement system, domain decomposition method is referred to as iterative substructuring $[6,7]$. The iterative substructuring algorithm can be outlined as follows $[4,6,7,52]$ : 


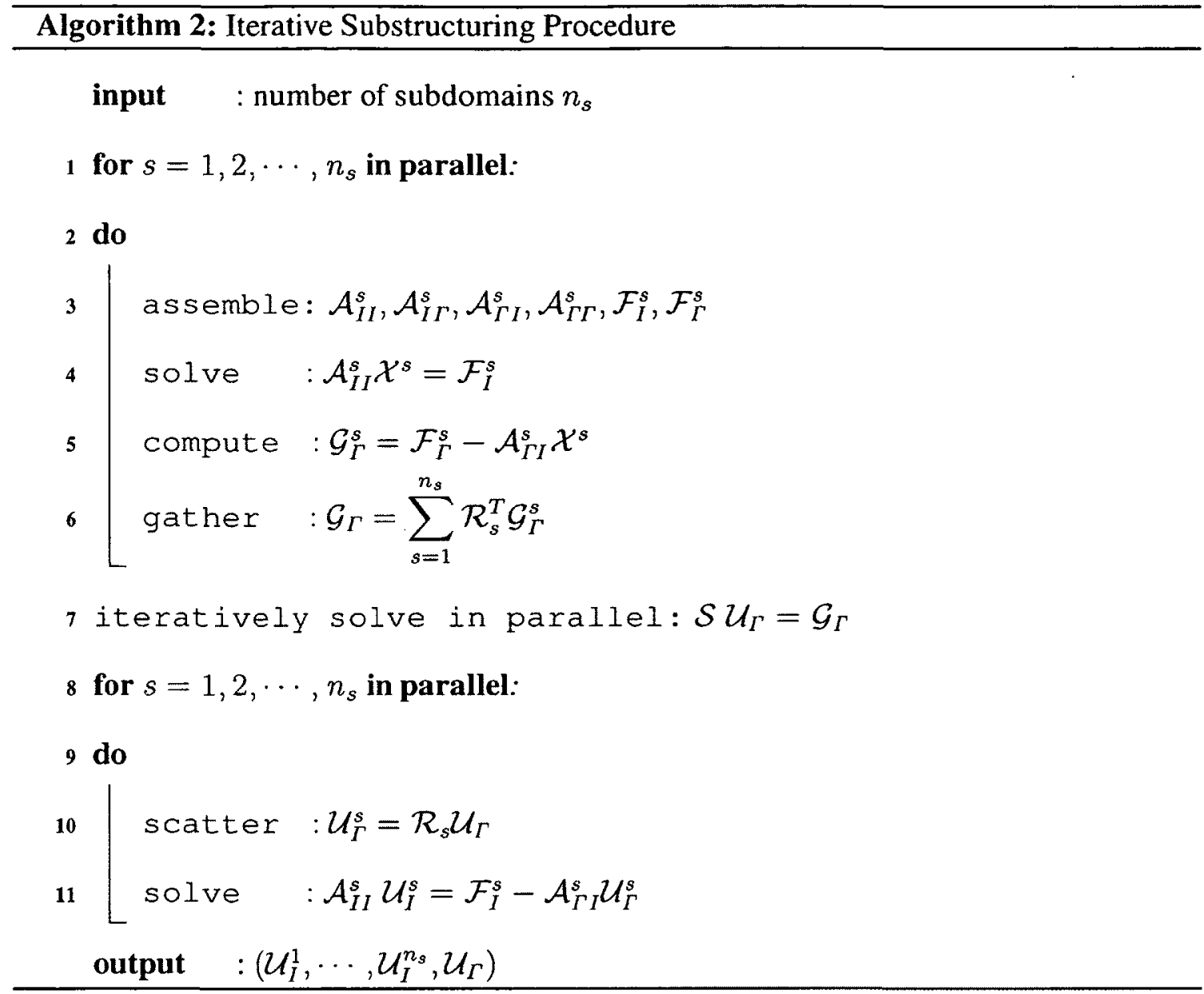




\subsubsection{Iterative Solution of the Interface Problem}

In domain decomposition approach, the Schur complement system is typically solved using preconditioned Krylov subspace method such as Precondition Conjugate Gradient Method (PCGM) (e.g. [52]). The preconditioned Krylov subspace method circumvents the need to construct the Schur complement matrix explicitly. Instead only the action of the Schur complement matrix on a vector is needed $[4,7]$. Non-overlapping domain decomposition method or iterative substructuring can be viewed as a preconditioned iterative method to solve the Schur complement system defined by Eq.(4.9) equipped with a parallel preconditioner $[4,7]$.

For symmetric positive-definite system such as the Schur complement system considered here, the Conjugate Gradient Method (CGM) is generally used. The performance of CGM mainly depends on the spectrum of the coefficient matrix [52]. However, the rate of convergence of the iterative method can generally be improved by transforming the original system into an equivalent system that has better spectral properties (i.e. lower condition number). This transformation is called preconditioning and the matrix used in the transformation is called the preconditioner. In other words, the transformed linear system becomes:

$$
\mathcal{M}^{-1} \mathcal{S} \mathcal{U}_{\Gamma}=\mathcal{M}^{-1} \mathcal{G}_{\Gamma}
$$

where $\mathcal{M}^{-1}$, the preconditioner, is a symmetric and invertible matrix that approximates $\mathcal{S}^{-1}$, the inverse of the coefficient matrix, in the sense that the condition number of the preconditioned system $\kappa\left(\mathcal{M}^{-1} \mathcal{S}\right)$ is much smaller than that of the original system $\kappa(\mathcal{S})$ 
and the eigenvalues of the preconditioned system $\mathcal{M}^{-1} \mathcal{S}$ are clustered near one $[52,139]$. This procedure known as Preconditioned Conjugate Gradient Method (PCGM). In practice, the explicit construction of $\mathcal{M}^{-1}$ is not needed. Instead, for a given vector $r_{\Gamma}$, a system of the the following form is solved

$$
\mathcal{M Z}=r_{\Gamma}
$$

The combination of a good parallel preconditioning technique and the CGM solver makes the iterative solver well-suited to tackle large-scale systems in parallel computers [52]. The preconditioned conjugate gradient algorithm applied to solve the extended Schur complement system proceeds as follows [52] 


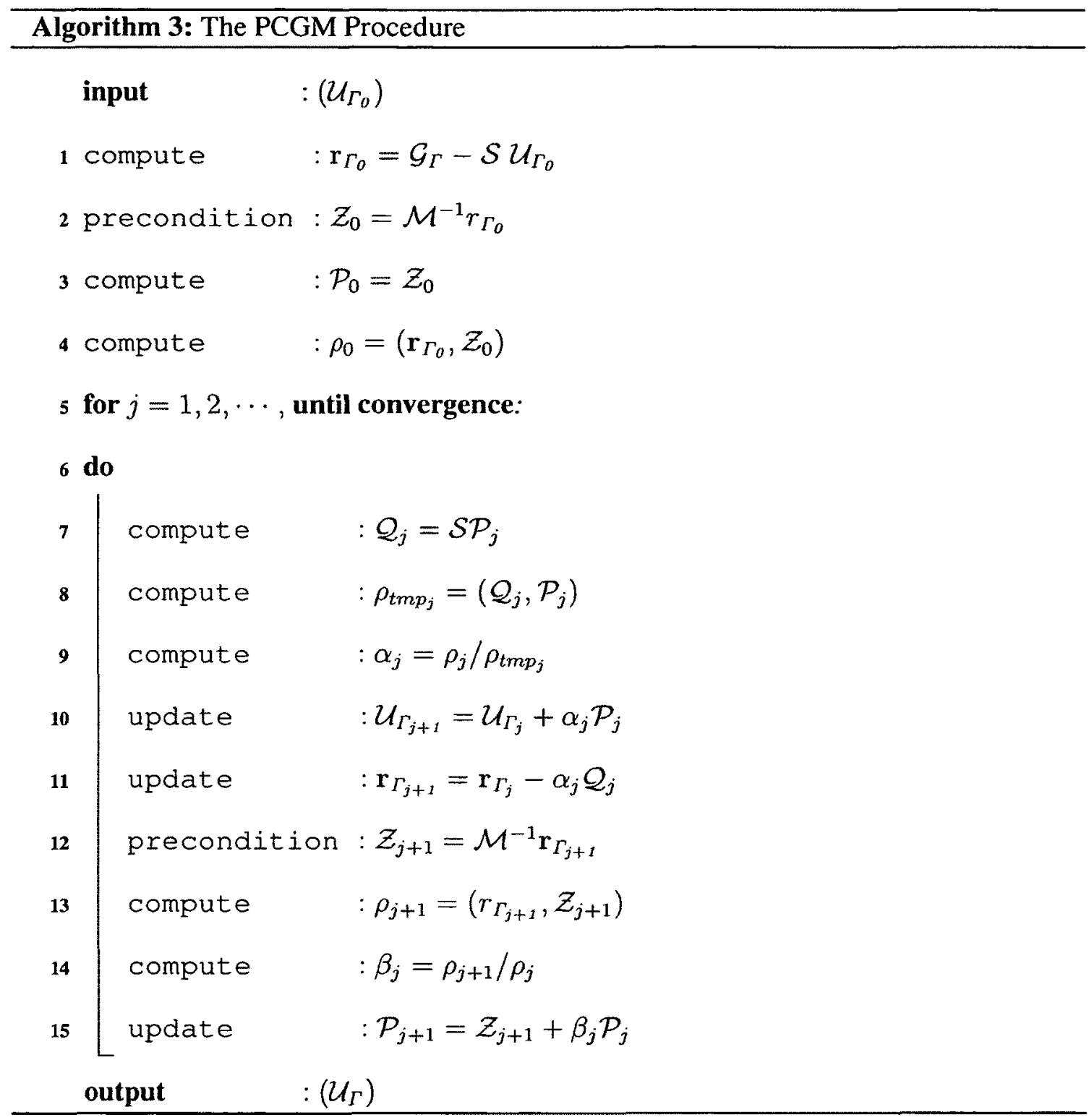

The PCGM algorithm indicates that the essential computational operations are calculating the matrix-vector product $(\mathcal{Q}=\mathcal{S P})$ in step 7 and computing the preconditioner effect on the residual vector $\left(\mathcal{Z}=\mathcal{M}^{-1} r_{\Gamma}\right)$ in steps 2 and 12 . Iterative substructuring tech- 
nique allows to perform these operations at the subdomain level (local). In other words, the global matrix-vector operations can be transformed into a series of subdomain level (local) computations. This fact paves the way for concurrency or parallelism as delineated in the next sections.

\subsubsection{Parallel Matrix-Vector Product}

While using PCGM, the Schur complement matrix need not be constructed explicitly, only its effect on a vector is required. This matrix-vector product can be obtained by solving an auxiliary Dirichlet problem on each subdomain concurrently and then gathering the resulting vectors. For the $j^{\text {th }}$ iteration of Algorithm (3), the matrix-vector product in step 7 is computed as

$$
\begin{aligned}
\mathcal{Q} & =\mathcal{S P} \\
& =\sum_{s=1}^{n_{s}} \mathcal{R}_{s}^{T} \mathcal{S}_{s} \mathcal{R}_{s} \mathcal{P} \\
& =\sum_{s=1}^{n_{s}} \mathcal{R}_{s}^{T}\left(\mathcal{A}_{\Gamma \Gamma}^{s}-\mathcal{A}_{\Gamma I}^{s}\left[\mathcal{A}_{I I}^{s}\right]^{-1} \mathcal{A}_{I \Gamma}^{s}\right) \mathcal{R}_{s} \mathcal{P}
\end{aligned}
$$

This procedure is summarized in the following Algorithm: 


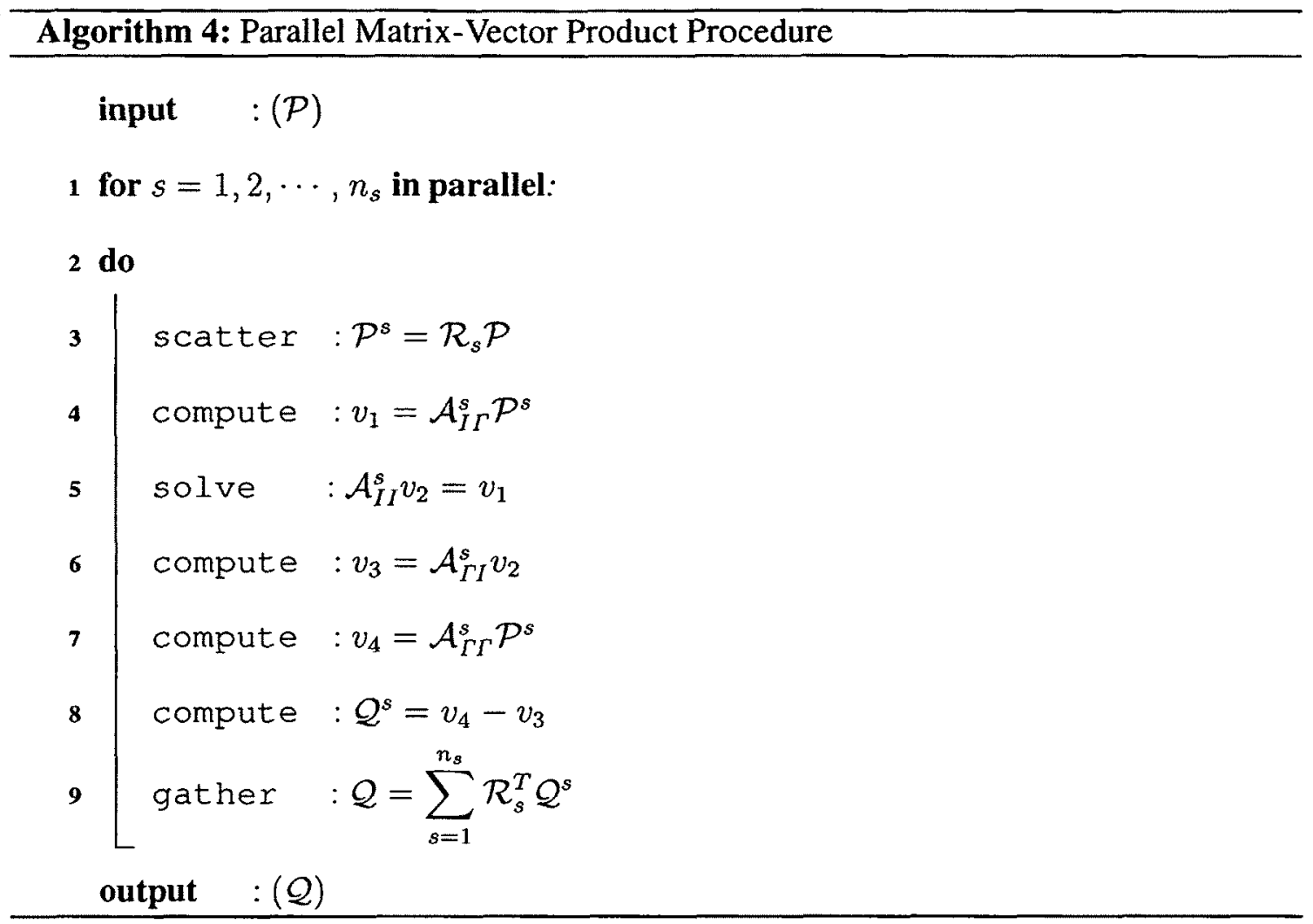

The action of the Schur complement $\mathcal{S}$ on a vector $\mathcal{P}$ is accomplished by three matrix vector products and one direct solve of a local Dirichlet problem with non-homogeneous boundary condition on each subdomain. This approach is computational less expensive than constructing the dense Schur complement matrix $\mathcal{S}$ explicitly. A drawback associated with the approach of not constructing the Schur complement matrix explicitly is that preconditioners for the Schur complement matrix such as Jacobi preconditioner (the inverse of the diagonal of $\mathcal{S}$ ) or probing technique (the inverse of the tridiagonal part of $\mathcal{S}$ ) cannot be used directly. 


\subsubsection{Parallel Preconditioner Effect}

The preconditioned residual vector $\mathcal{Z}$ at step 12 of Algorithm (3), is computed by solving a local system of the form $\mathcal{M}^{s} \mathcal{Z}^{s}=r_{\Gamma}^{s}$ on each subdomain in parallel followed by a global assembly to calculate the global effect as

$$
\begin{aligned}
\mathcal{Z} & =\mathcal{M}^{-1} r_{\Gamma} \\
& =\sum_{s=1}^{n_{s}} \mathcal{R}_{s}^{T} \mathcal{M}_{s}^{-1} \mathcal{R}_{s} r_{\Gamma},
\end{aligned}
$$

where $\mathcal{M}_{s}^{-1}$ is a subdomain level preconditioner. This procedure is outlined in the following algorithm

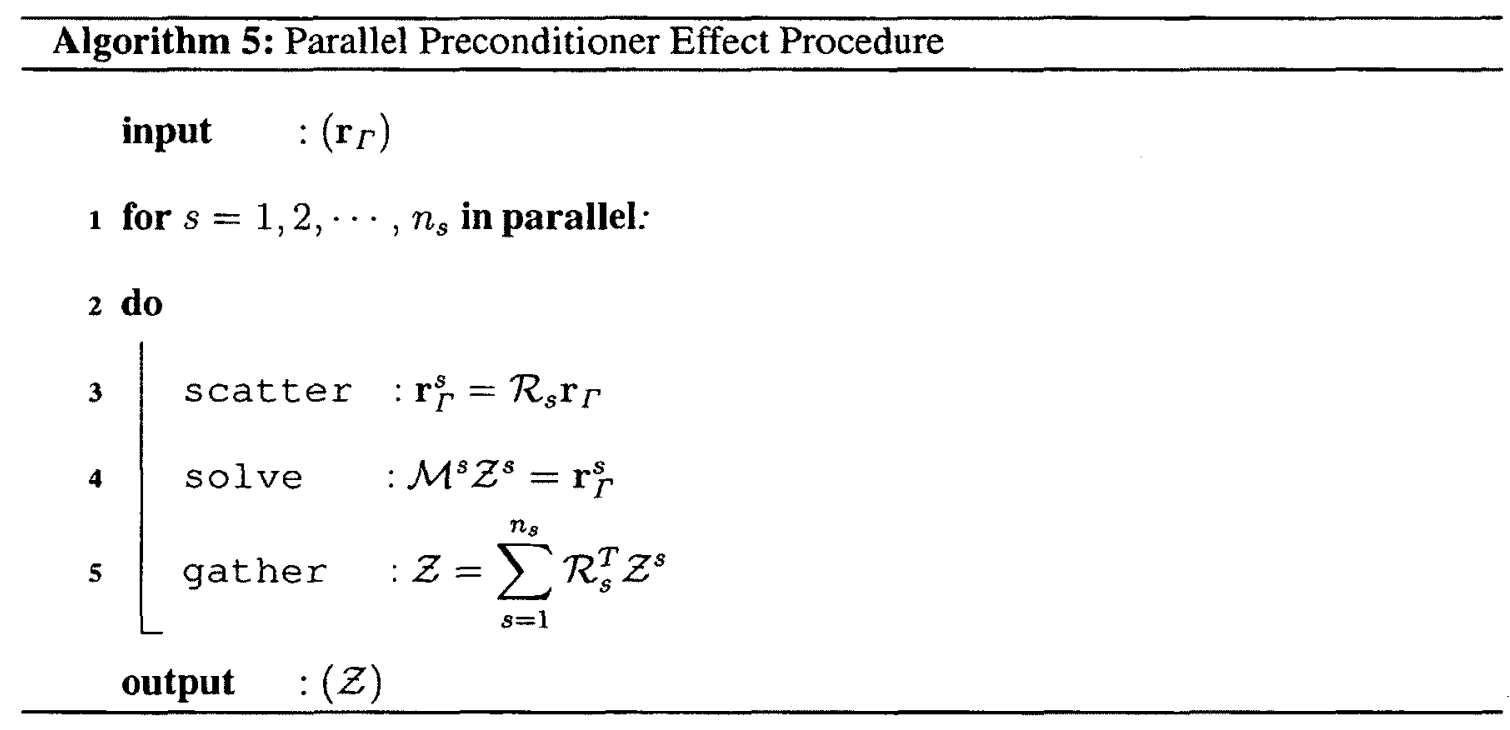

The working vectors $r_{\Gamma}^{s}$ and $\mathcal{Z}^{s}$ are defined on the subdomain level. 


\subsubsection{Parallel Preconditioned Conjugate Gradient Method}

Exploiting Algorithms(4 \& 5), a parallel version of the PCGM algorithm can be implemented as outlined in the following algorithm. The summation steps in the following algorithm can be performed in parallel. 


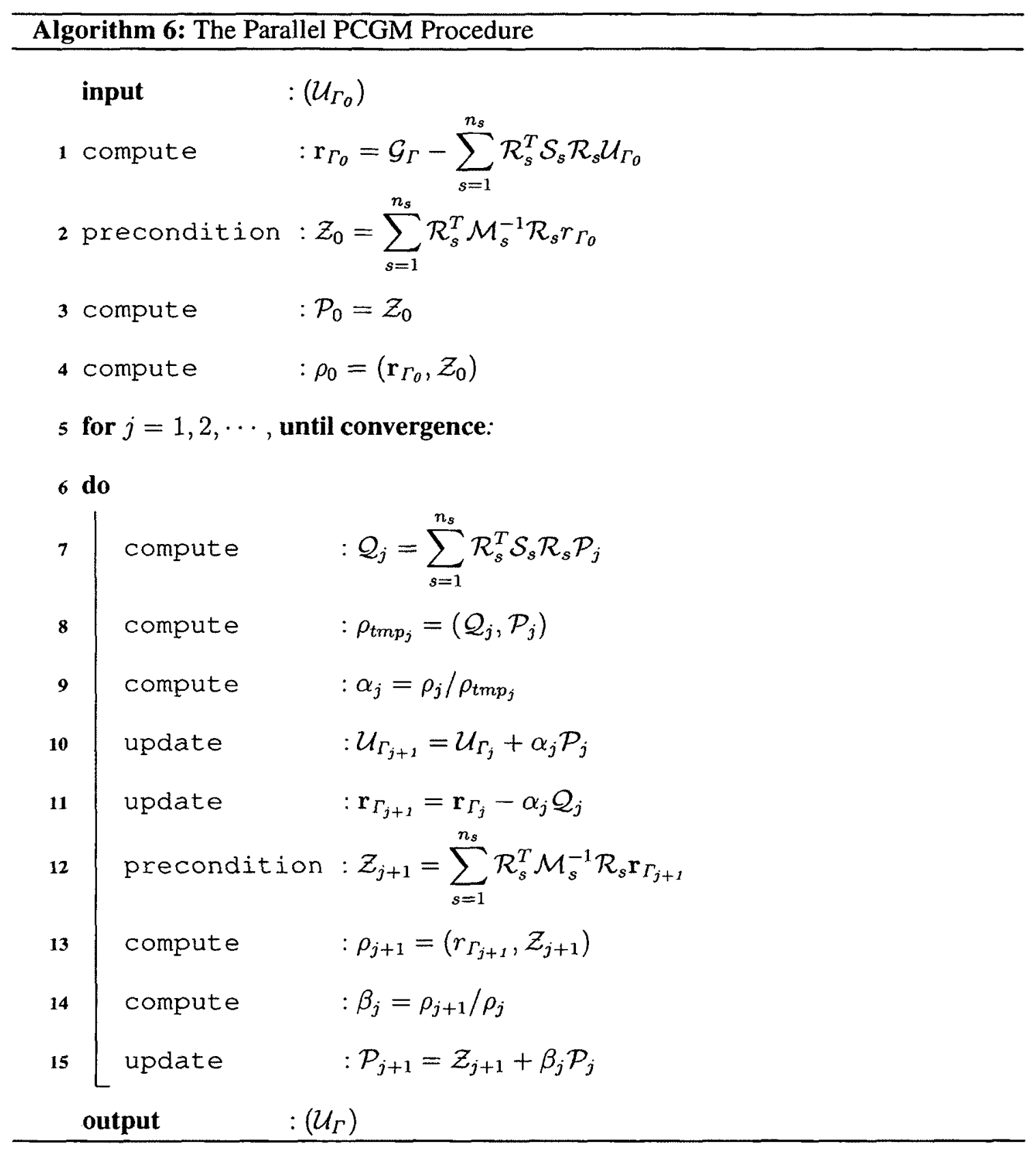

The vector $\mathcal{Q}_{j}$ in step 7 of Algorithm (6) is computed in parallel using Algorithm (4), and the preconditioned residual vector $\mathcal{Z}_{j}$ in step 12 of Algorithm (6) is obtained in parallel using Algorithm (5). All the inner products of the vectors are preformed in parallel on a 
distributed data set.

Finally, A flow chart of iterative substructuring technique for stochastic PDEs is shown in Fig. (4.3).

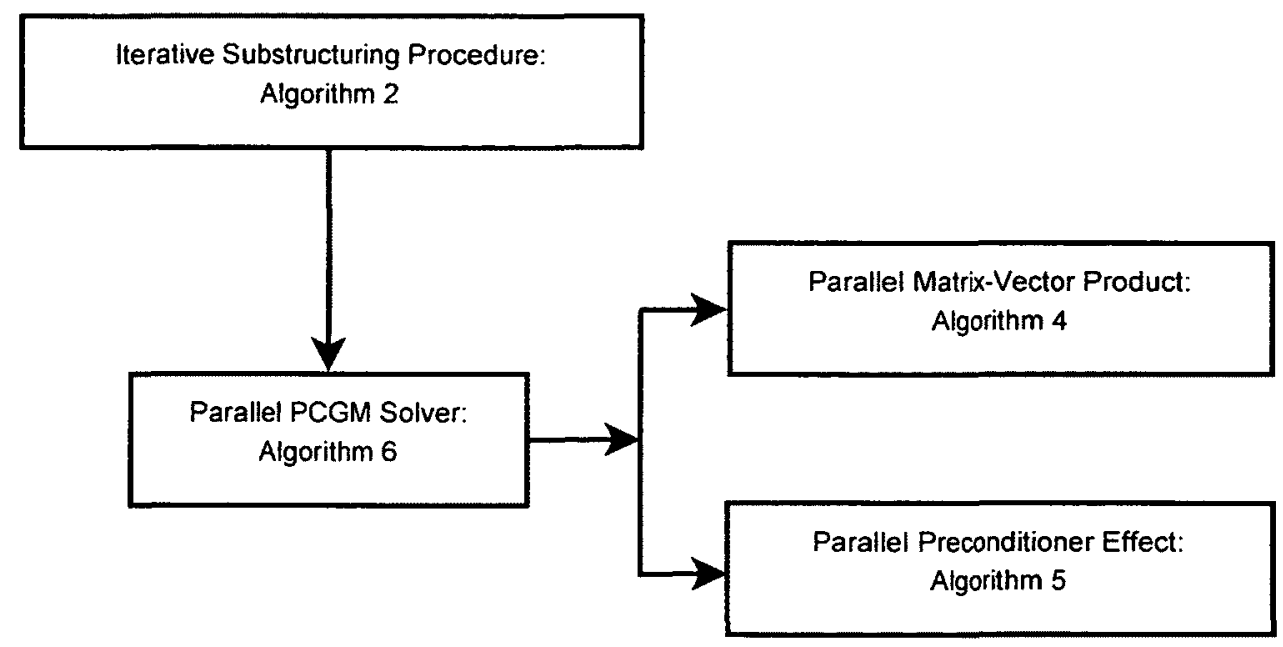

Figure 4.3: Flow chart of iterative substructuring technique

\subsection{Summary}

Iterative substructuring technique for SPDEs is described in this chapter. In particular, a parallel PCGM is introduced to tackle the interface problem in the context of domain decomposition method of SPDEs. The iterative algorithm eliminates the need of explicit construction of the extended Schur complement system. 


\section{Chapter 5}

\section{One-Level Preconditioners for}

\section{Stochastic PDEs}

\subsection{Introduction}

This chapter is closely based on the references $[70,74,75]$. The preconditioning technique is the essential ingredient in enhancing the reliability, robustness and performance of the iterative methods used to tackle the interface problem arising in the domain decomposition of SPDEs. The combination of a scalable parallel preconditioner suitably customized for the stochastic systems and the CGM solver makes the iterative method well-suited to tackle the interface problem using high performance computing platforms. In this chapter, we describe the mathematical frameworks and parallel implementations of one-level preconditioners namely Lumped (L), Weighted Lumped (WL) and Neumann-Neumann (NN) to tackle the extended Schur complement system of SPDEs. 


\subsection{Extended Lumped Preconditioner}

As previously alluded, iterative substructuring technique is a preconditioned Krylov subspace iteration method for solving the extended Schur complement system as

$$
\mathcal{M}^{-1} \mathcal{S} \mathcal{U}_{\Gamma}=\mathcal{M}^{-1} \mathcal{G}_{\Gamma}
$$

where $\mathcal{M}^{-1}$ being the preconditioning matrix.

A preliminary preconditioner for the extended Schur complement system is devised by approximating the inverse of the extended Schur compliment matrix by the sum of the inverse of the local interface coupling matrices as [70]

$$
\mathcal{M}_{L}^{-1}=\sum_{s=1}^{n_{s}} \mathcal{R}_{s}^{T}\left[\mathcal{A}_{\Gamma \Gamma}^{s}\right]^{-1} \mathcal{R}_{s} .
$$

The inverse of the subdomain interface coupling matrix $\left[\mathcal{A}_{\Gamma \Gamma}^{s}\right]^{-1}$ is not constructed explicitly. Instead the following system is solved on each subdomain in parallel followed by a global assembly in order to calculate the global effect of the preconditioner as

$$
\mathcal{A}_{\Gamma \Gamma}^{s} \mathcal{Z}^{s}=r_{\Gamma}^{s} .
$$

The interface coupling matrix is much more diagonally dominant than the Schur complement matrix. However, this elementary preconditioner reduces the arithmetic complexity in the preconditioning step of the PCGM iteration loop. 


\subsubsection{Parallel Implementation}

In the framework of the parallel PCGM, the extended Lumped preconditioner can be implemented in parallel as outlined in the following algorithm

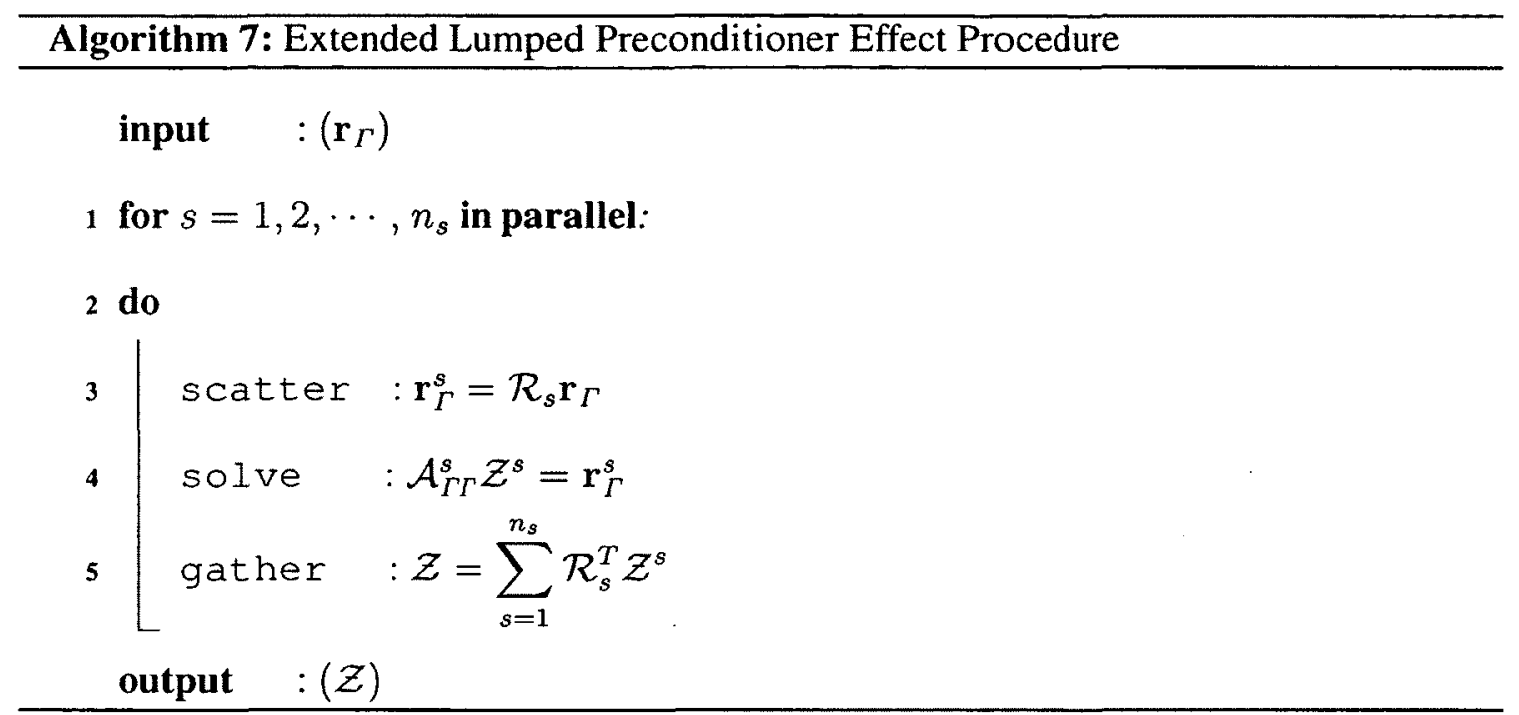

Cholesky factorization of the matrix $\mathcal{A}_{\Gamma \Gamma}^{s}$ is performed only once, and the solution of the local system proceeds by forward and backward substitutions. This fact highlights the efficiency of the Lumped preconditioner in reducing the arithmetic complexity in the preconditioning step.

\subsection{Extended Weighted Lumped Preconditioner}

The performance of the Lumped preconditioner can be improved by employing a scaling matrix $\mathcal{D}_{s}$, namely a partition of identity matrix (e.g $[4,6,7]$ obtained as 


$$
\sum_{s=1}^{n_{s}} \mathcal{R}_{s}^{T} \mathcal{D}_{s} \mathcal{R}_{s}=I,
$$

where $\mathcal{D}_{s}$ is a block diagonal matrix of the following form

$$
\mathcal{D}_{s}=\operatorname{blockdiag}\left(\mathbf{D}_{s}^{0}, \cdots, \mathbf{D}_{s}^{N}\right)
$$

The diagonal entries of the deterministic weighting matrix $\mathbf{D}_{s}^{i}$ are equal to the reciprocal of the number of subdomains which contain the same interface node $[4,6,7]$.

Consequently, the extended Weighted Lumped preconditioner which approximates the inverse of the global extended Schur compliment matrix by a weighted sum of the inverse of the local interface coupling matrices can be expressed as

$$
\mathcal{M}_{W L}^{-1}=\sum_{s=1}^{n_{s}} \mathcal{R}_{s}^{T} \mathcal{D}_{s}\left[\mathcal{A}_{\Gamma \Gamma}^{s}\right]^{-1} \mathcal{D}_{s} \mathcal{R}_{s}
$$

\subsubsection{Parallel Implementation}

The parallel implementation of the extended Weighted Lumped preconditioner resembles that of the extended Lumped preconditioner but with the additional two steps to calculate the multiplication of a vector by the scaling matrix $\mathcal{D}_{s}$. The parallel implementation is summarized below: 


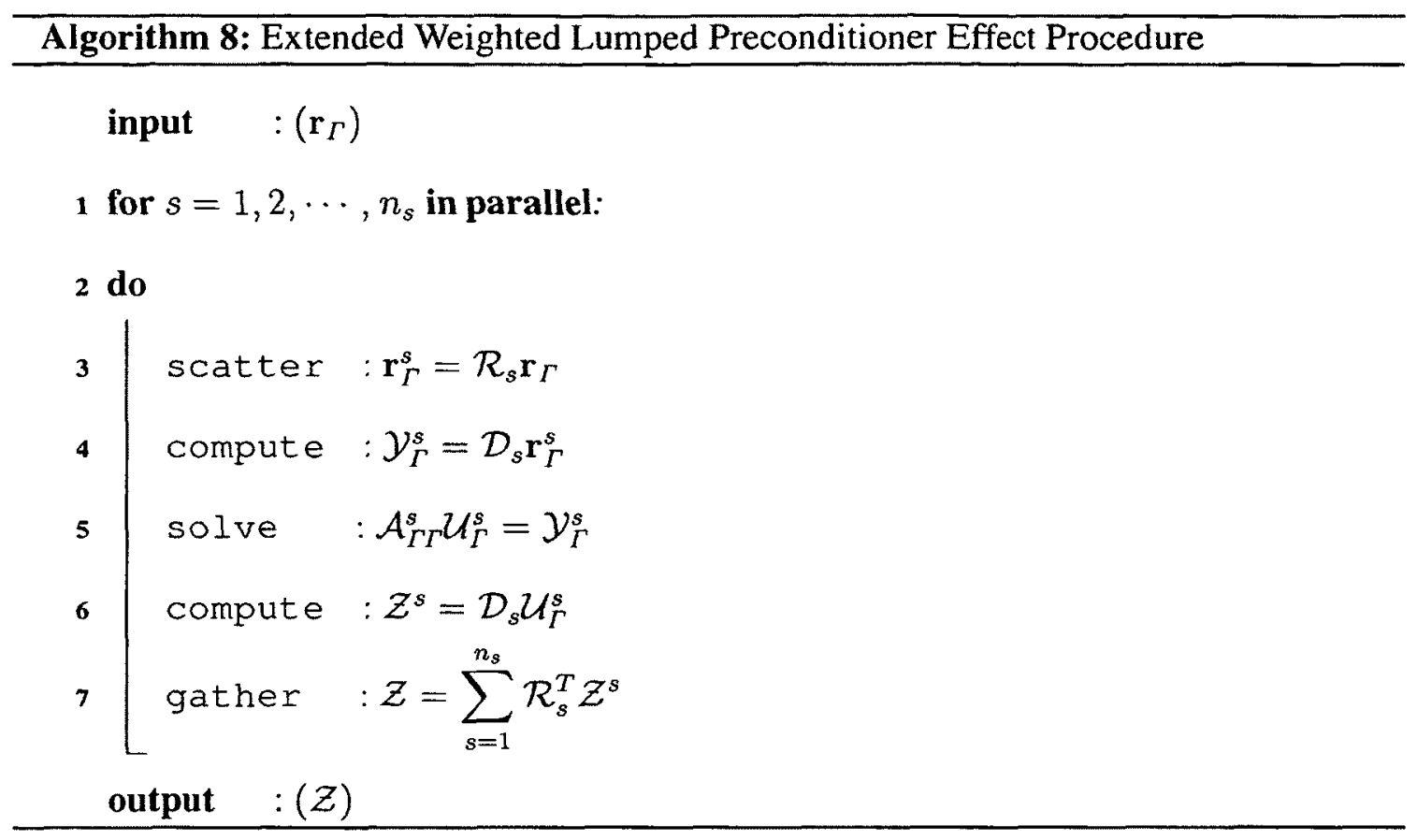

\subsection{Extended Neumann-Neumann Preconditioner}

In this section, a one-level Neumann-Neumann domain decomposition preconditioner [4$7,71,74,76]$ is introduced to enhance the performance of the PCGM to tackle the interface system. The global extended Schur complement matrix can be constructed by the aggregated local extended Schur complement matrices. It is therefore natural to design a preconditioner for SPDEs by the weighted average of the inverse extended subdomain Schur complement matrices. This preconditioner may be construed to be analogous to the Neumann-Neumann preconditioner devised for the deterministic PDEs [4-7], albeit with more complex structure and higher dimension due to the stochastic aspects. The construction of the Neumann-Neumann preconditioner for SPDEs is described next in details $[74,76]$. 
In particular, we describe the details of computing preconditioned residual $\mathcal{Z}=\mathcal{M}_{N N}^{-1} r_{\Gamma}$ where $\mathcal{M}_{N N}^{-1}$ and $r_{\Gamma}$ are the Neumann-Neumann preconditioner and the residual vector at a given PCGM iteration. Subsequently it is demonstrated that the Neumann-Neumann preconditioner is the weighted sum of the inverse local Schur complement matrices of the stochastic subsystems. This fact can be mathematically stated as:

$$
\mathcal{M}_{N N}^{-1}=\sum_{s=1}^{n_{s}} \mathcal{R}_{s}^{T} \mathcal{D}_{s} \mathcal{S}_{s}^{-1} \mathcal{D}_{s} \mathcal{R}_{s}
$$

At the $j^{\text {th }}$ iteration of PCGM, assume $\mathcal{U}_{\Gamma_{j}}$ to be an approximation of the global interface variable which in turn provides the local interface variable $\mathcal{U}_{\Gamma}^{s}$ as

$$
\mathcal{U}_{\Gamma}^{s}=\mathcal{R}_{s} \mathcal{U}_{\Gamma_{j}}
$$

Next we describe the procedure to compute the new residual $r_{\Gamma_{j}}$ using $\left\{\mathcal{U}_{\Gamma}^{s}\right\}_{s=1}^{n_{s}}$. For each subdomain, it involves first computing the local interior variable $\mathcal{U}_{I}^{s}$ which is then used to obtain the local residual $\mathbf{r}_{\Gamma}^{s}$. The global residual $\mathbf{r}_{\Gamma_{j}}$ is calculated by aggregating the local residuals $\left\{\mathbf{r}_{\Gamma}^{s}\right\}_{s=1}^{n_{s}}$.

The computation of the interior unknown $\mathcal{U}_{I}^{s}$ translates to tackling a stochastic local problem defined by

$$
\left[\begin{array}{cc}
\mathcal{A}_{I I}^{s} & \mathcal{A}_{I \Gamma}^{s} \\
\mathcal{A}_{\Gamma I}^{s} & \mathcal{A}_{\Gamma \Gamma}^{s}
\end{array}\right]\left\{\begin{array}{c}
\mathcal{U}_{I}^{s} \\
\mathcal{U}_{\Gamma}^{s}
\end{array}\right\}=\left\{\begin{array}{c}
\mathcal{F}_{I}^{s} \\
\mathcal{F}_{\Gamma}^{s}
\end{array}\right\} .
$$

From the first row of Eq.(5.9), the solution of a stochastic Dirichlet problem computes the unknown $\mathcal{U}_{I}^{s}$ as 


$$
\mathcal{U}_{I}^{s}=\left[\mathcal{A}_{I I}^{s}\right]^{-1}\left(\mathcal{F}_{I}^{s}-\mathcal{A}_{I \Gamma}^{s} \mathcal{U}_{\Gamma}^{s}\right)
$$

Consequently, the local residual $\mathbf{r}_{\Gamma}^{s}$ is computed from the second row of Eq.(5.9) as

$$
\mathbf{r}_{\Gamma}^{s}=\mathcal{S}_{s} \mathcal{U}_{\Gamma}^{s}-\mathcal{G}_{\Gamma}^{s}
$$

where the local extended Schur complement matrix $\mathcal{S}_{s}$ and the corresponding right hand side vector $\mathcal{G}_{\Gamma}^{s}$ are given by

$$
\begin{aligned}
\mathcal{S}_{s} & =\mathcal{A}_{\Gamma \Gamma}^{s}-\mathcal{A}_{\Gamma I}^{s}\left[\mathcal{A}_{I I}^{s}\right]^{-1} \mathcal{A}_{I \Gamma}^{s}, \\
\mathcal{G}_{\Gamma}^{s} & =\mathcal{F}_{\Gamma}^{s}-\mathcal{A}_{\Gamma I}^{s}\left[\mathcal{A}_{I I}^{s}\right]^{-1} \mathcal{F}_{I}^{s} .
\end{aligned}
$$

The local residual vectors $\left\{\mathbf{r}_{\Gamma}^{s}\right\}_{s=1}^{n_{s}}$ are then aggregated to obtain the global residual vector $\mathbf{r}_{\Gamma_{j}}$ as

$$
\mathbf{r}_{\Gamma_{j}}=\sum_{s=1}^{n_{s}} \mathcal{R}_{s}^{T} \mathbf{r}_{\Gamma}^{s}
$$

The global residual $\mathbf{r}_{\Gamma_{j}}$ is scattered on the subdomain interfaces to obtain the new local residuals as

$$
\mathbf{r}_{\Gamma}^{s}=\mathcal{D}_{s} \mathcal{R}_{s} \mathbf{r}_{\Gamma_{j}}, \quad s=1, \ldots, n_{s}
$$

Using the new local residual $\mathbf{r}_{\Gamma}^{s}$, the computation of the local interface variable $\mathcal{U}_{\Gamma}^{s}$ involves the solution of a local stochastic Neumann problem defined by 


$$
\left[\begin{array}{cc}
\mathcal{A}_{I I}^{s} & \mathcal{A}_{I \Gamma}^{s} \\
\mathcal{A}_{\Gamma I}^{s} & \mathcal{A}_{\Gamma \Gamma}^{s}
\end{array}\right]\left\{\begin{array}{c}
\mathcal{X}^{s} \\
\mathcal{U}_{\Gamma}^{s}
\end{array}\right\}=\left\{\begin{array}{c}
0 \\
\mathrm{r}_{\Gamma}^{s}
\end{array}\right\} .
$$

It is straightforward to prove that the solution of the above Neumann problem relates to tackling the local Schur complement system defined by

$$
\mathcal{S}_{s} \mathcal{U}_{\Gamma}^{s}=\mathbf{r}_{\Gamma}^{s}
$$

It is worthwhile to point out at this stage that the local Schur complement matrix is singular for the floating subdomains, namely the subdomains containing insufficient Dirichlet boundary conditions. This singularity issue is circumvented by adding a small number to the diagonal entries of the subdomain stiffness matrix. This numerical artifact only affects the computation of the preconditioned residual, but does not alter the original problem to be solved.

Next the PCGM update of the solution vector $\mathcal{U}_{\Gamma_{j+1}}$ is computed by gathering the local interface vectors $\left\{\mathcal{U}_{\Gamma}^{s}\right\}_{s=1}^{n_{s}}$ as

$$
\mathcal{U}_{\Gamma_{j+1}}=\mathcal{U}_{\Gamma_{j}}+\sum_{s=1}^{n_{s}} \mathcal{R}_{s}^{T} \mathcal{D}_{s} \mathcal{U}_{\Gamma}^{s}
$$

Using Eq.(5.17) and Eq.(5.15), the new solution vector $\mathcal{U}_{\Gamma_{j+1}}$ can be formally written as

$$
\mathcal{U}_{\Gamma_{j+1}}=\mathcal{U}_{\Gamma_{j}}+\sum_{s=1}^{n_{s}} \mathcal{R}_{s}^{T} \mathcal{D}_{s} \mathcal{S}_{s}^{-1} \mathcal{D}_{s} \mathcal{R}_{s} \mathbf{r}_{\Gamma_{j}}
$$

Therefore the error vector $\mathrm{e}_{\Gamma_{j}}$ can be expressed as 


$$
\mathbf{e}_{\Gamma_{j}}=\sum_{s=1}^{n_{s}} \mathcal{R}_{s}^{T} \mathcal{D}_{s} \mathcal{S}_{s}^{-1} \mathcal{D}_{s} \mathcal{R}_{s} \mathbf{r}_{\Gamma_{j}},
$$

leading to the following expression of the extended Neumann-Neumann preconditioner for the stochastic system

$$
\mathcal{M}_{N N}^{-1}=\sum_{s=1}^{n_{s}} \mathcal{R}_{s}^{T} \mathcal{D}_{s} \mathcal{S}_{s}^{-1} \mathcal{D}_{s} \mathcal{R}_{s}
$$

\subsubsection{Parallel Implementation}

The parallel implementation of the extended Neumann-Neumann preconditioner can be outline in Algorithm(9).

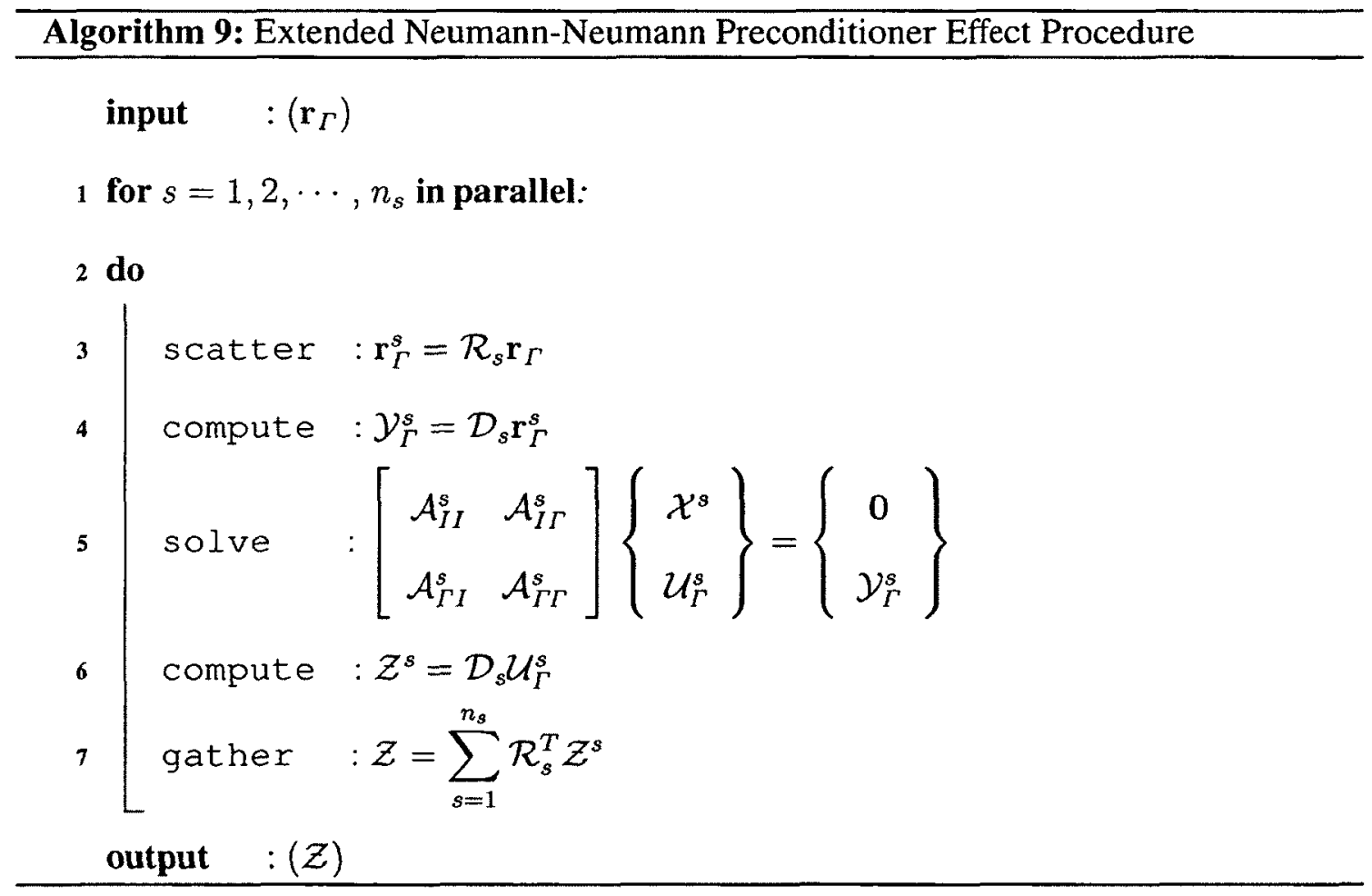


The regularization and Cholesky factorization of the subdomain level stiffness matrix is performed only once, and the solution of the local system proceeds by forward and backward substitutions.

\subsection{Summary}

One-level domain decomposition algorithms are described for the iterative solution of the coupled large-scale linear systems in the spectral stochastic finite element method. The extended Neumann-Neumann preconditioner proposed here is the essential ingredient to develop two-level domain decomposition algorithms for SPDEs. 


\section{Chapter 6}

\section{Two-Level Preconditioner for Stochastic}

\section{PDEs}

\subsection{Introduction}

This chapter is closely based on the references $[76,77]$. Recent advances in high performance computing systems and sensing technologies motivate computational simulations with extremely high resolution models with capabilities to quantify uncertainties for credible numerical predictions. To this end, a two-level domain decomposition method is reported in this chapter to tackle the large-scale linear system within the framework of the intrusive spectral stochastic finite element method (SSFEM). In particular, a two-level scalable preconditioner is introduced in order to iteratively solve the large-scale linear system in the intrusive SSFEM using an iterative substructuring based domain decomposition solver. The implementation of the algorithm involves solving a local problem on each subdomain 
that constructs the local part of the preconditioner and a coarse problem that propagates information globally among the subdomains. The two-level preconditioner may be construed to be a probabilistic extension of the Balancing Domain Decomposition by Constraints (BDDC) $[66,78]$ devised for deterministic PDEs.

\subsection{Solution of the Interface Problem using Two-Level Pre- conditioner}

In the iterative substructuring technique, the interface system $\mathcal{S U _ { \Gamma }}=\mathcal{G}_{\Gamma}$ is generally tackled by the preconditioned conjugate gradient method (PCGM). In the PCGM, a preconditioning matrix $\mathcal{M}^{-1}$ is employed to transform the original system into an equivalent system whereby the transformed system possesses a smaller condition number than that of the original system. Additionally the preconditioned system has eigenvalues clustered near one $[52,140,141]$. The preconditioned extended Schur complement system takes the following form

$$
\mathcal{M}^{-1} \mathcal{S U}_{\Gamma}=\mathcal{M}^{-1} \mathcal{G}_{\Gamma}
$$

In this chapter, we devise a two-level preconditioner to enhance the performance of iterative substructuring techniques to tackle Eq.(6.1). The preconditioner consists of a local part to smooth some components of the error and a global part to propagate the error globally over the subdomains. The general form of the two-level preconditioner is defined 


$$
\mathcal{M}^{-1}=\sum_{s=1}^{n_{s}} \mathcal{R}_{s}^{T} \mathcal{M}_{s}^{-1} \mathcal{R}_{s}+\mathcal{R}_{0}^{T} \mathcal{M}_{0}^{-1} \mathcal{R}_{0}
$$

Within the parallel PCGM algorithm, the two-level preconditioner can be implemented using Algorithm(10) [74, 79]. 


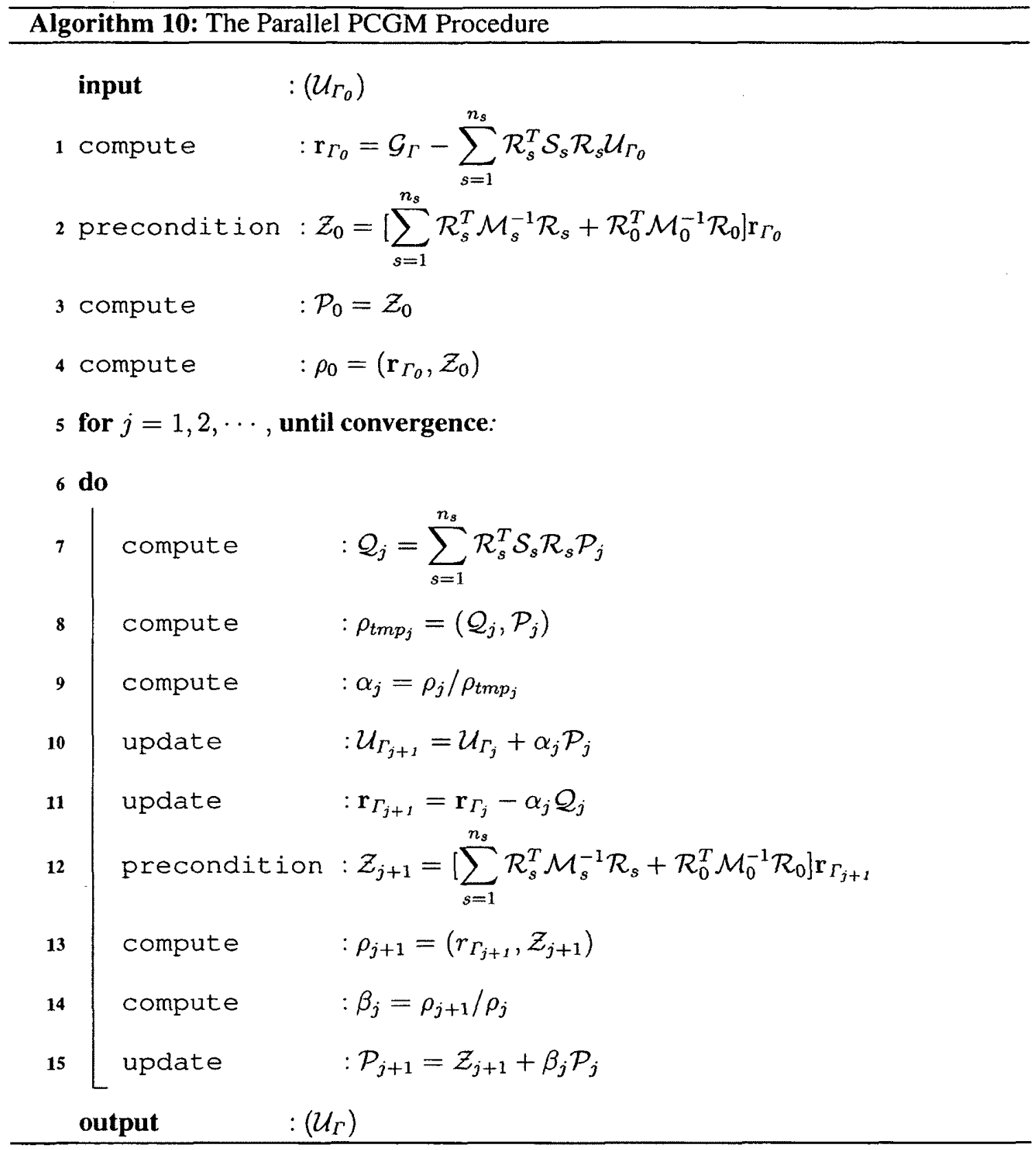

Neither the extended Schur complement matrix in step 7, nor the two-level preconditioner in step 12 need to be formed explicitly. The effect of the extended Schur complement matrix on a given vector is obtained by computing three subdomain level ma- 
trix vector products and solving local stochastic Dirichlet problems as outlined in Algorithm(11) $[74,79]$. Whereas the preconditioner effect on the residual is calculated by solving local stochastic Neumann problems and a global coarse problem.

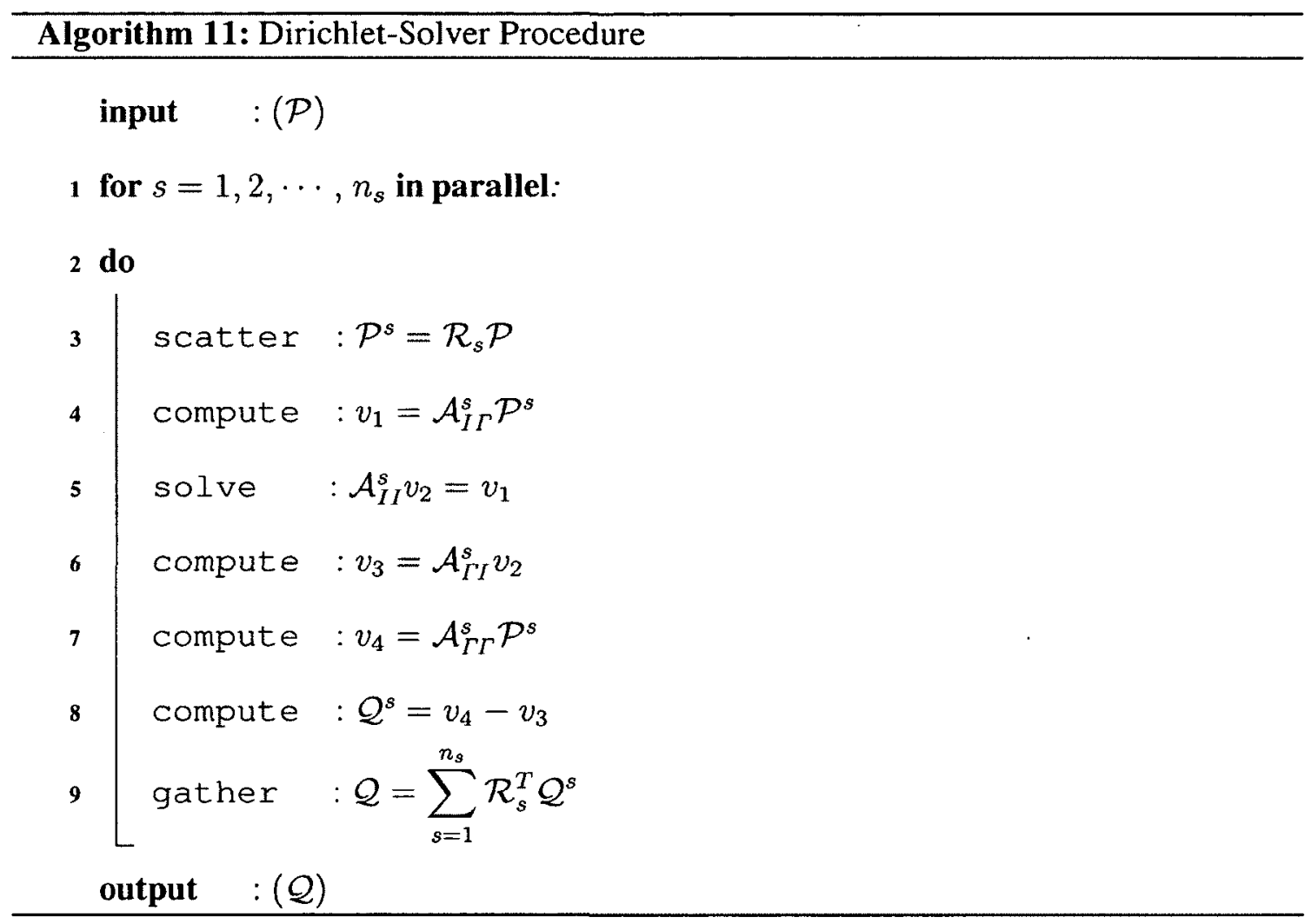

\section{Preconditioners for Stochastic PDEs}

For stochastic PDEs, the convergence rate of the PCGM equipped with efficient preconditioners should not be hampered by the level of uncertainty and order of the stochastic dimension. Furthermore, the PCG solvers must also demonstrate a scalable performance in relation to the physical problem size and number of subdomains. Such performance can be achieved by a two-level preconditioner suitably customized for the stochastic system as 
delineated next.

\section{Extended One-Level Preconditioner}

Previously, a one-level Neumann-Neumann preconditioner for stochastic PDEs is introduced in Chapter 5. The extended Neumann-Neumann preconditioner is defined as

$$
\mathcal{M}_{N N}^{-1}=\sum_{s=1}^{n_{s}} \mathcal{R}_{s}^{T} \mathcal{D}_{s}^{T} \mathcal{S}_{s}^{-1} \mathcal{D}_{s} \mathcal{R}_{s}
$$

where

$$
\mathcal{D}_{s}=\operatorname{blockdiag}\left(\mathbf{D}_{s}^{0}, \cdots, \mathbf{D}_{s}^{N}\right),
$$

and $\mathrm{D}_{s}^{k}$ is a diagonal scaling matrix defined by

$$
\sum_{s=1}^{n_{s}} \mathbf{R}_{s}^{T} \mathbf{D}_{s}^{k} \mathbf{R}_{s}=\mathbf{I}
$$

The diagonal entries of $D_{s}^{k}$ are the reciprocal of the number of subdomains that share the interface nodes [4-7].

The implementation of the extended Neumann-Neumann preconditioner entails solving a subdomain level stochastic Neumann problem. This step calculates the effect of the inverse of the local Schur complement matrix $\mathcal{S}_{s}^{-1}$ on the local residual vector $\mathbf{r}_{\Gamma}^{s}$ as

$$
\left[\begin{array}{cc}
\mathcal{A}_{I I}^{s} & \mathcal{A}_{I \Gamma}^{s} \\
\mathcal{A}_{\Gamma I}^{s} & \mathcal{A}_{\Gamma \Gamma}^{s}
\end{array}\right]\left\{\begin{array}{c}
\mathcal{X}^{s} \\
\mathcal{U}_{\Gamma}^{s}
\end{array}\right\}=\left\{\begin{array}{c}
0 \\
\mathrm{r}_{\Gamma}^{s}
\end{array}\right\},
$$

where $\mathbf{r}_{\Gamma}^{s}=\mathcal{R}_{s} \mathcal{D}_{s} \mathbf{r}_{\Gamma_{j}}$ and $\mathbf{r}_{\Gamma_{j}}$ denotes the global residual at the $j^{\text {th }}$ iteration of the PCGM. 
The PCGM equipped with a one-level Neumann-Neumann preconditioner exhibits a convergence rate that is almost insensitive to the level of uncertainty in the system parameters $[74,75]$. As no mechanism for global information exchange exists in the one-level Neumann-Neumann preconditioner, its performance deteriorates for relatively large number of subdomains. For scalability, the global communication is required to propagate information across the subdomains [4-7]. Another difficulty in the implementation of the one-level Neumann-Neumann preconditioner is that the local stochastic Neumann problem defined in Eq.(6.6) is singular for floating subdomains. In practice, the singularity of the local stiffness matrix is avoided by altering its diagonal entries with a small positive constant and the local Neumann problem is solved approximately $[6,7]$. However, such approximation may adversely affect the overall convergence rate of the algorithm [6].

\subsection{Extended Two-Level Preconditioner}

In the one-level Neumann-Neumann preconditioner, the information is only exchanged among the neighboring subdomains. To provide a mechanism for a global transfer of information, a coarse problem is required $[4,5,7]$. To this end, we describe a mathematical framework for a two-level preconditioner for the iterative substructuring to devise an iterative solver for the large-scale linear system in the intrusive SSFEM. This method bears similarity with a version of the Balancing Domain Decomposition by Constraints (BDDC) for deterministic PDEs [66, 78]. The two-level preconditioner is formulated by adding a coarse grid to the one-level Neumann-Neumann preconditioner as described next. 
The interface nodes of each subdomain $\left\{\Omega_{s}\right\}_{s=1}^{n_{s}}$ are partitioned into a set of remaining nodes (boundary nodes shared only between two adjacent subdomains) and corner nodes (boundary nodes shared among more than two subdomains plus the nodes at the ends of interface edges) $[64,66,67,78]$ as shown in Fig.(6.1).

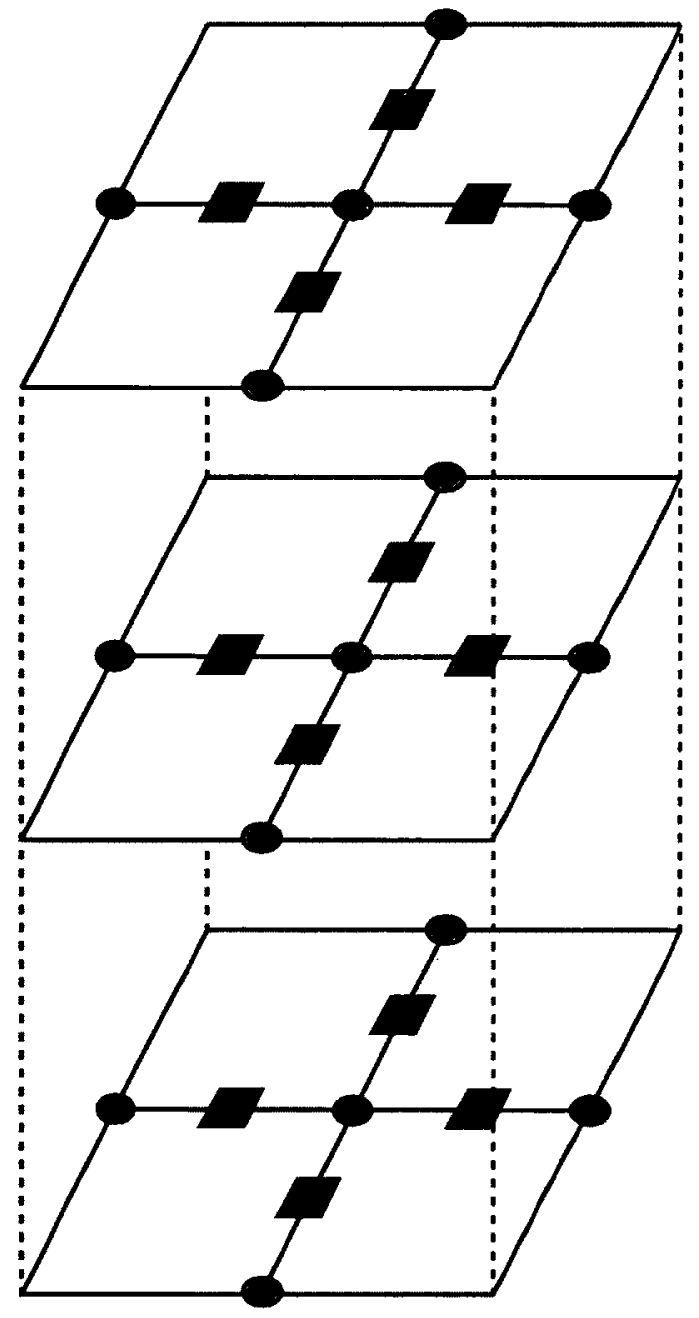

Figure 6.1: Partitioned interface nodes into remaining $(\square)$ and corner( $($ ) nodes.

Let $\mathcal{R}_{s}^{r}$ and $\mathcal{R}_{s}^{c}$ be the Boolean operators that split the local interface unknowns $\mathcal{U}_{\Gamma}^{s}$ into 
remaining $\mathcal{U}_{r}^{s}$ and corner $\mathcal{U}_{c}^{s}$ variables respectively as

$$
\left\{\begin{array}{l}
\mathcal{U}_{r}^{s} \\
\mathcal{U}_{c}^{s}
\end{array}\right\}=\left[\begin{array}{l}
\mathcal{R}_{s}^{r} \\
\mathcal{R}_{s}^{c}
\end{array}\right] \mathcal{U}_{\Gamma}^{s}
$$

where

$$
\begin{aligned}
& \mathcal{R}_{s}^{r}=\operatorname{blockdiag}\left(\mathbf{R}_{s}^{r, 0}, \ldots, \mathbf{R}_{s}^{r, N}\right) \\
& \mathcal{R}_{s}^{c}=\operatorname{blockdiag}\left(\mathbf{R}_{s}^{c, 0}, \ldots, \mathbf{R}_{s}^{c, N}\right)
\end{aligned}
$$

The deterministic restriction operators $\mathbf{R}_{s}^{r, k}$ and $\mathbf{R}_{s}^{c, k}$ for the $k^{\text {th }}$ polynomial chaos basis are defined as

$$
\begin{aligned}
& \mathbf{u}_{r, k}^{s}=\mathbf{R}_{s}^{r, k} \mathbf{u}_{\Gamma, k}^{s}, \\
& \mathbf{u}_{c, k}^{s}=\mathbf{R}_{s}^{c, k} \mathbf{u}_{\Gamma, k}^{s} .
\end{aligned}
$$

According to the partition defined in Eq.(6.7), the subdomain level Neumann problem given by Eq.(6.6), can be rewritten as

$$
\left[\begin{array}{ccc}
\mathcal{A}_{i i}^{s} & \mathcal{A}_{i r}^{s} & \mathcal{A}_{i c}^{s} \\
\mathcal{A}_{r i}^{s} & \mathcal{A}_{r r}^{s} & \mathcal{A}_{r c}^{s} \\
\mathcal{A}_{c i}^{s} & \mathcal{A}_{c r}^{s} & \mathcal{A}_{c c}^{s}
\end{array}\right]\left\{\begin{array}{c}
\mathcal{X}^{s} \\
\mathcal{U}_{r}^{s} \\
\mathcal{U}_{c}^{s}
\end{array}\right\}=\left\{\begin{array}{c}
0 \\
\mathcal{F}_{r}^{s} \\
\mathcal{F}_{c}^{s}
\end{array}\right\},
$$

where

$$
\begin{aligned}
& \mathcal{F}_{r}^{s}=\mathcal{R}_{s}^{r} \mathbf{r}_{\Gamma}^{s} \\
& \mathcal{F}_{c}^{s}=\mathcal{R}_{s}^{c} \mathbf{r}_{\Gamma}^{s}
\end{aligned}
$$


Performing a partial assembly to enforce the continuity condition at the corner nodes, we obtain the following linear system

$$
\left[\begin{array}{ccc}
\mathcal{A}_{i i}^{s} & \mathcal{A}_{i r}^{s} & \mathcal{A}_{i c}^{s} \mathcal{B}_{c}^{s} \\
\mathcal{A}_{r i}^{s} & \mathcal{A}_{r r}^{s} & \mathcal{A}_{r c}^{s} \mathcal{B}_{c}^{s} \\
\sum_{s=1}^{n_{s}} \mathcal{B}_{c}^{s T} \mathcal{A}_{c i}^{s} & \sum_{s=1}^{n_{s}} \mathcal{B}_{c}^{s T} \mathcal{A}_{c r}^{s} & \sum_{s=1}^{n_{s}} \mathcal{B}_{c}^{s T} \mathcal{A}_{c c}^{s} \mathcal{B}_{c}^{s}
\end{array}\right]\left\{\begin{array}{c}
\mathcal{X}^{s} \\
\mathcal{U}_{r}^{s} \\
\mathcal{U}_{c}
\end{array}\right\}=\left\{\begin{array}{c}
0 \\
\mathcal{F}_{r}^{s} \\
\sum_{s=1}^{n_{s}} \mathcal{B}_{c}^{s T} \mathcal{F}_{c}^{s}
\end{array}\right\},
$$

where $\mathcal{B}_{c}^{s}$ is a restriction operator that maps the global corner unknown $\mathcal{U}_{c}$ into its local components $\left\{\mathcal{U}_{c}^{s}\right\}_{s=1}^{n_{s}}$ as

$$
\mathcal{U}_{c}^{s}=\mathcal{B}_{c}^{s} \mathcal{U}_{c}
$$

where

$$
\mathcal{B}_{c}^{s}=\operatorname{blockdiag}\left(\mathbf{B}_{c, 0}^{s}, \ldots, \mathbf{B}_{c, N}^{s}\right)
$$

and $\mathbf{B}_{c, k}^{s}$ is defined by

$$
\mathbf{u}_{c, k}^{s}=\mathbf{B}_{c, k}^{s} \mathbf{u}_{c, k}
$$

Eliminating the local variables $\mathcal{X}^{s}$ and $\mathcal{U}_{r}^{s}$ in Eq.(6.9), we obtain the following symmetric positive definite coarse problem

$$
F_{c c} \mathcal{U}_{c}=d_{c}
$$

where

$$
\begin{aligned}
F_{c c} & =\sum_{s=1}^{n_{s}} \mathcal{B}_{c}^{s T}\left(\mathcal{S}_{c c}^{s}-\mathcal{S}_{c r}^{s}\left[\mathcal{S}_{r r}^{s}\right]^{-1} \mathcal{S}_{r c}^{s}\right) \mathcal{B}_{c}^{s} \\
d_{c} & =\sum_{s=1}^{n_{s}} \mathcal{B}_{c}^{s T}\left(\mathcal{F}_{c}^{s}-\mathcal{S}_{c r}^{s}\left[\mathcal{S}_{r r}^{s}\right]^{-1} \mathcal{F}_{r}^{s}\right)
\end{aligned}
$$


and $\mathcal{S}_{\alpha \beta}^{s}=\mathcal{A}_{\alpha \beta}^{s}-\mathcal{A}_{\alpha i}^{s}\left[\mathcal{A}_{i i}^{s}\right]^{-1} \mathcal{A}_{i \beta}^{s}$ where $\alpha$ and $\beta$ stand for the index $r$ and $c$.

The corner nodes artificially enforce Dirichlet boundary conditions to eliminate the singularity arising from the subdomain Schur complement matrix $\mathcal{S}_{r r}^{s}$ of the floating subdomains. The remaining interface variables $\mathcal{U}_{r}^{s}$ can be obtained next for each subdomain as

$$
\mathcal{S}_{r r}^{s} \mathcal{U}_{r}^{s}=\mathcal{F}_{r}^{s}-\mathcal{S}_{r c}^{s} \mathcal{U}_{c}^{s}
$$

Once the local interface variables $\mathcal{U}_{r}^{s}$ and $\mathcal{U}_{c}^{s}$ are available, the global interface unknowns $\mathcal{U}_{\Gamma}$ can be aggregated as

$$
\mathcal{U}_{\Gamma}=\sum_{s=1}^{n_{s}} \mathcal{R}_{s}^{T} \mathcal{D}_{s}\left(\mathcal{R}_{s}^{r T} \mathcal{U}_{r}^{s}+\mathcal{R}_{s}^{c T} \mathcal{U}_{c}^{s}\right)
$$

Substituting Eq.(6.11) and Eq.(6.12) into Eq.(6.13) and performing some algebraic manipulations, the two-level preconditioner can be expressed as

$$
\mathcal{M}_{N N C}^{-1}=\sum_{s=1}^{n_{s}} \mathcal{R}_{s}^{T} \mathcal{D}_{s} \mathcal{R}_{s}^{r^{T}}\left[\mathcal{S}_{r r}^{s}\right]^{-1} \mathcal{R}_{s}^{r} \mathcal{D}_{s} \mathcal{R}_{s}+R_{0}^{T}\left[F_{c c}\right]^{-1} R_{0}
$$

where the restriction operator $R_{0}$ mapping from fine to coarse grid, is defined by

$$
R_{0}=\sum_{s=1}^{n_{s}} \mathcal{B}_{c}^{s T}\left(\mathcal{R}_{s}^{c}-\mathcal{S}_{c r}^{s}\left[\mathcal{S}_{r r}^{s}\right]^{-1} \mathcal{R}_{s}^{r}\right) \mathcal{D}_{s} \mathcal{R}_{s}
$$

Evidently, the two-level Neumann-Neumann preconditioner in Eq.(6.14) is equipped with a coarse operator $\left[F_{c c}\right]^{-1}$ to scatter information globally. 


\subsection{Parallel Implementation}

The precondition extended Schur complement system in Eq.(6.1) is solved iteratively using PCGM (see Algorithm(10)) equipped with the aforementioned two-level NeumannNeumann preconditioner defined in Eq.(6.14). The preconditioned residual vectors at steps 2 and 12 of Algorithm (10) are computed in parallel as outlined in Algorithm(12). 


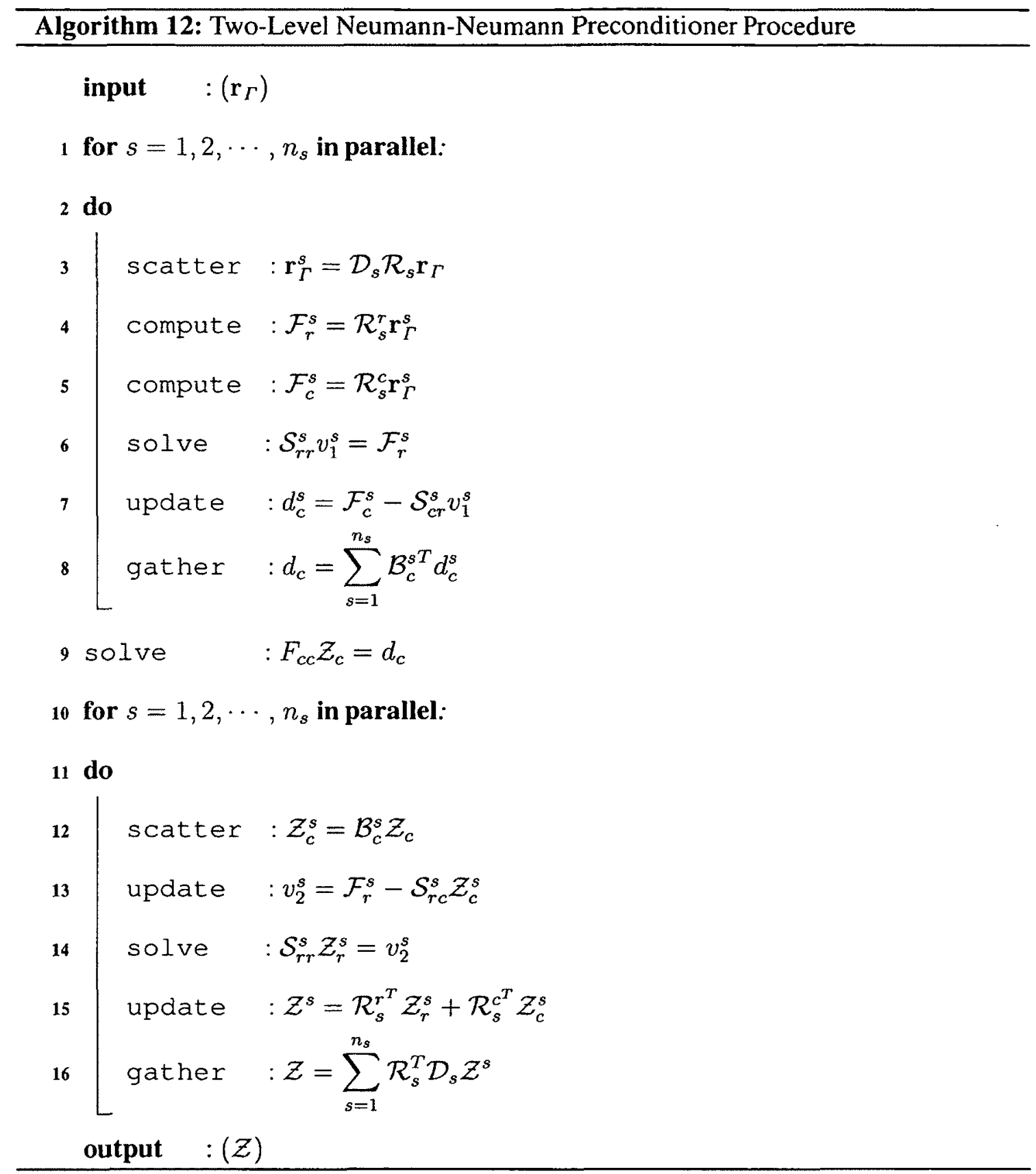

The implementation of Algorithm (12) requires solution of two local fine problems (steps 6 and 14) and a global coarse problem (step 9). The fine problems correspond to 
the local stochastic Neumann problems are solved using Algorithm (13).

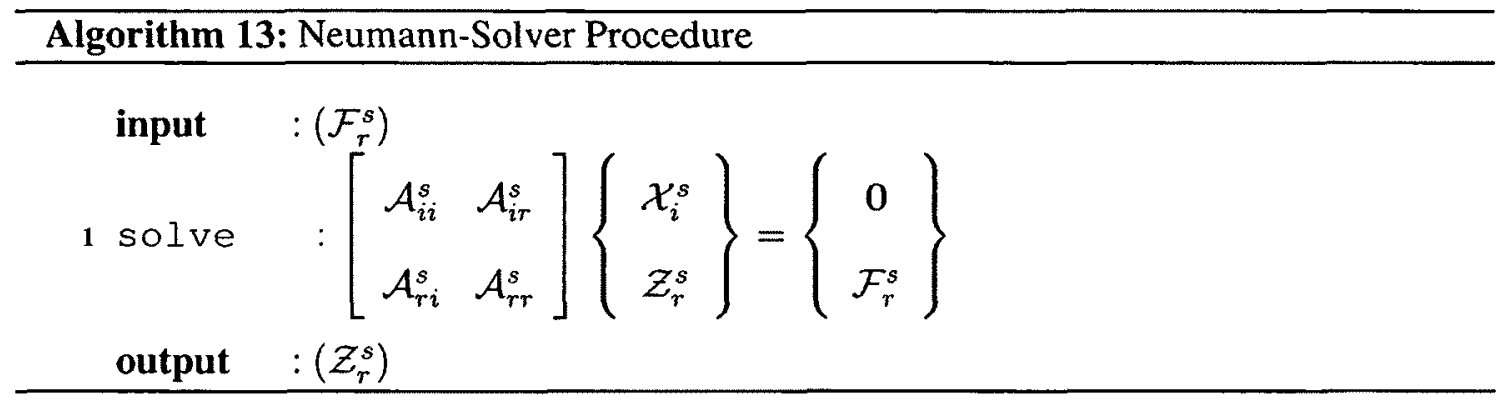

The size of the coarse problem in step 9 of Algorithm (12) is relatively small. However, an explicit construction of the coarse Schur complement matrix $F_{c c}$ is computationally intensive. In our implementation, the coarse problem is solved using a parallel PCGM with the Lumped preconditioner as

$$
\mathcal{M}_{c}^{-1} F_{c c} \mathcal{Z}_{c}=\mathcal{M}_{c}^{-1} d_{c}
$$

where

$$
\mathcal{M}_{c}^{-1}=\sum_{s=1}^{n_{s}} \mathcal{B}_{c}^{s T} \mathcal{A}_{c c}^{s} \mathcal{B}_{c}^{s} .
$$

A flow-chart of the two-level extended Neumann-Neumann algorithm is shown in Fig.(6.2) 


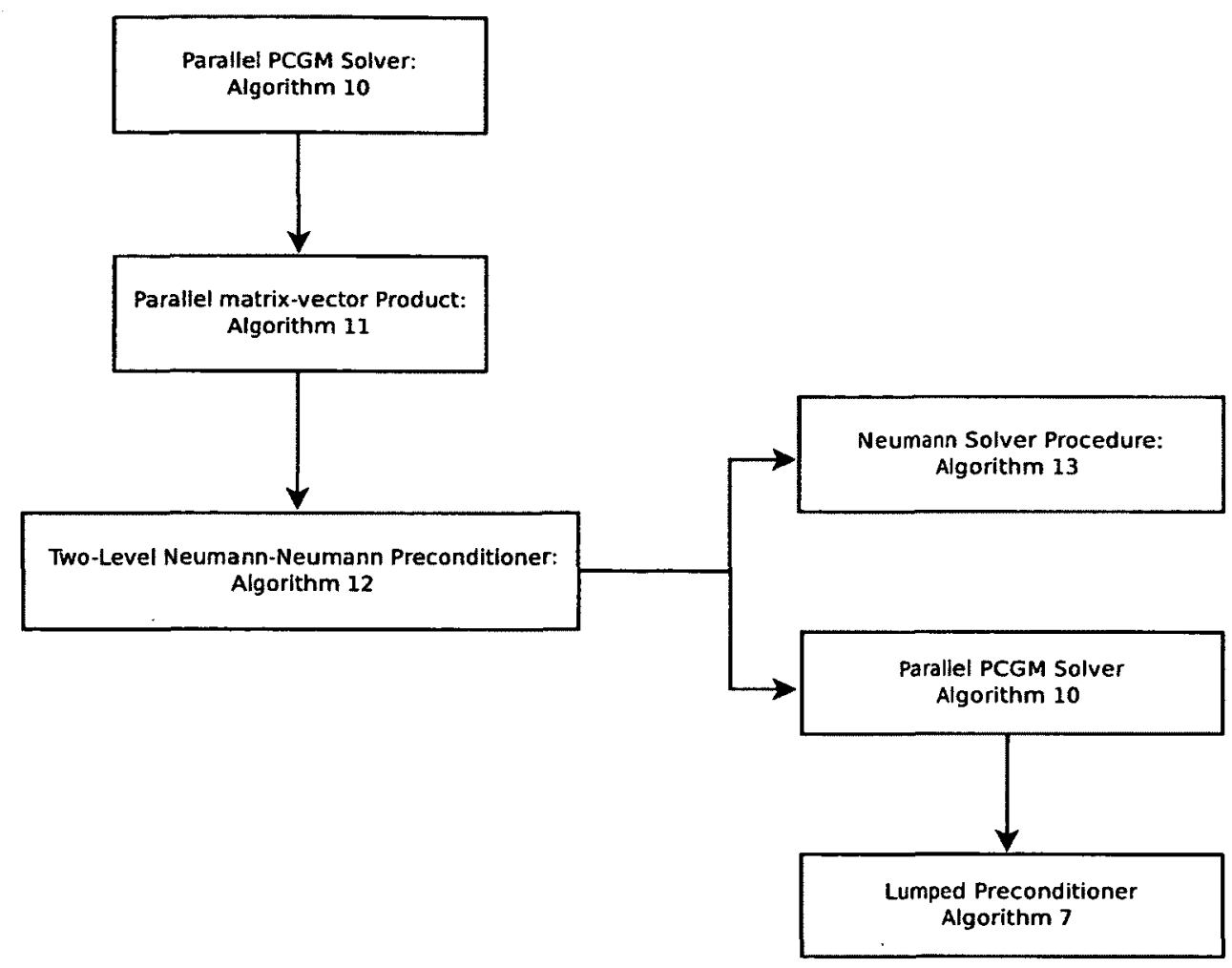

Figure 6.2: Flow chart of parallel implementation of the two-level extended Neumann-Neumann algorithm

\subsection{Summary}

We formulated a novel two-level domain decomposition preconditioner for the iterative substructuring of stochastic PDEs. Within the framework of the intrusive SSFEM, the twolevel preconditioner is constructed by adding a coarse problem to the one-level NeumannNeumann preconditioner. A collection of corner nodes at the subdomain interfaces constitute the coarse grid. Consequently, a coarse problem is solved at each iteration to spread information across all the subdomains. This information exchange achieved by the coarse 
grid makes the preconditioner scalable. The two-level preconditioner may be construed to be a probabilistic extension of the Balancing Domain Decomposition by Constraints (BDDC) devised for deterministic PDEs. 


\section{Chapter 7}

\section{Primal-Primal Domain Decomposition}

\section{Method for Stochastic PDEs}

\subsection{Introduction}

This chapter is closely based on the references $[79,80]^{1}$. In this chapter, we describe a novel primal version of the iterative substructuring technique for the solution of the largescale linear system arising from the stochastic finite element method. The algorithm offers a straightforward approach to formulate a two-level scalable preconditioner. In the primalprimal method, a reduced interface problem corresponding to the remaining interface nodes is solved iteratively using PCGM equipped with a two-level preconditioner.

In the preconditioning step of the primal-primal technique, the continuity condition is strictly enforced on the corner nodes, while weakly satisfied (in an average sense) on the

\footnotetext{
${ }^{I}$ The paper [79] received an award at the HPCS2010 conference for outstanding technical contribution.
} 
remaining interface nodes. The iterative method converges when the continuity of the solution field is satisfied across the entire interface. This approach naturally leads to a coarse problem connecting the subdomains globally via the corner nodes which makes the algorithm scalable.

\subsection{Primal-Primal Method for Stochastic PDEs}

Let the physical domain $\Omega$ be decomposed into $n_{s}$ non-overlapping subdomains $\left\{\Omega_{s}\right\}_{s=1}^{n_{s}}$, and the subdomain nodal vector $\mathbf{u}^{s}(\theta)$ be partitioned into a set of interior $\mathbf{u}_{i}^{s}(\theta)$, corner $\mathbf{u}_{c}^{s}(\theta)$ and remaining unknowns $\mathbf{u}_{r}^{s}(\theta)$, as schematically shown in Fig.(7.1).
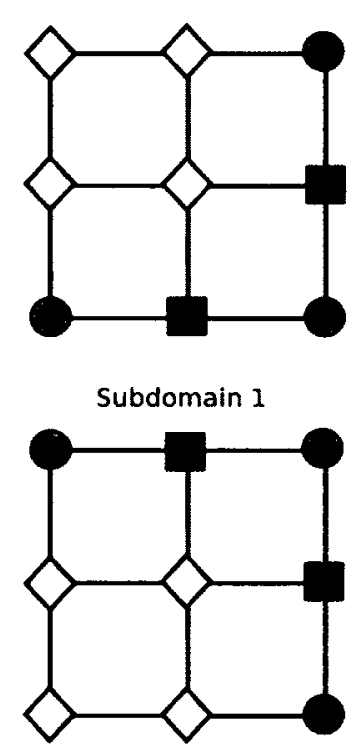

Subdomain 3
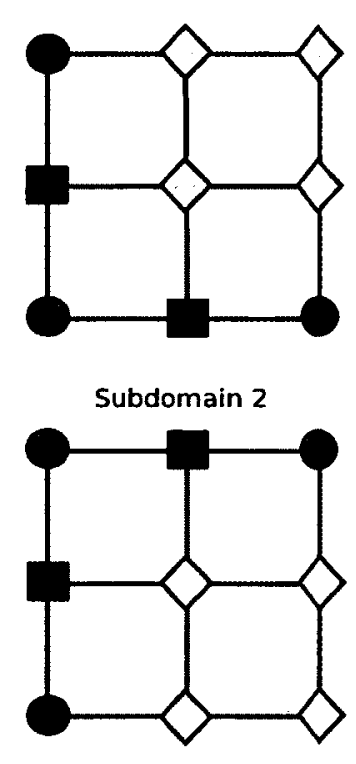

Subdomain 4

Figure 7.1: Partitioning the subdomain nodes into: interior $(\diamond)$, remaining

(ם) and corner( $(\mathbf{)}$ 
For a typical subdomain $\Omega_{s}$, the equilibrium equation can be expressed as

$$
\left[\begin{array}{lll}
\mathbf{A}_{i i}^{s}(\theta) & \mathbf{A}_{i r}^{s}(\theta) & \mathbf{A}_{i c}^{s}(\theta) \\
\mathbf{A}_{r i}^{s}(\theta) & \mathbf{A}_{r r}^{s}(\theta) & \mathbf{A}_{r c}^{s}(\theta) \\
\mathbf{A}_{c i}^{s}(\theta) & \mathbf{A}_{c r}^{s}(\theta) & \mathbf{A}_{c c}^{s}(\theta)
\end{array}\right]\left\{\begin{array}{c}
\mathbf{u}_{i}^{s}(\theta) \\
\mathbf{u}_{r}^{s}(\theta) \\
\mathbf{u}_{c}^{s}(\theta)
\end{array}\right\}=\left\{\begin{array}{c}
\mathbf{f}_{i}^{s} \\
\mathbf{f}_{r}^{s} \\
\mathbf{f}_{c}^{s}
\end{array}\right\}
$$

Expanding the stochastic system parameters and the solution process by PCE and then performing Galerkin projection, Eq.(7.1) can be expressed as

$$
\left[\begin{array}{ccc}
\mathcal{A}_{i i}^{s} & \mathcal{A}_{i r}^{s} & \mathcal{A}_{i c}^{s} \\
\mathcal{A}_{r i}^{s} & \mathcal{A}_{r r}^{s} & \mathcal{A}_{r c}^{s} \\
\mathcal{A}_{c i}^{s} & \mathcal{A}_{c r}^{s} & \mathcal{A}_{c c}^{s}
\end{array}\right]\left\{\begin{array}{l}
\mathcal{U}_{i}^{s} \\
\mathcal{U}_{r}^{s} \\
\mathcal{U}_{c}^{s}
\end{array}\right\}=\left\{\begin{array}{c}
\mathcal{F}_{i}^{s} \\
\mathcal{F}_{r}^{s} \\
\mathcal{F}_{c}^{s}
\end{array}\right\}
$$

where

$$
\left[\mathcal{A}_{\alpha \beta}^{s}\right]_{j k}=\sum_{l=0}^{L}\left\langle\Psi_{l} \Psi_{j} \Psi_{k}\right\rangle \mathbf{A}_{\alpha \beta, l}^{s}, \quad \mathcal{F}_{\alpha, k}^{s}=\left\langle\Psi_{k} \mathbf{f}_{\alpha}^{s}\right\rangle, \quad \mathcal{U}_{\alpha}^{s}=\left(\mathbf{u}_{\alpha, 0}^{s}, \cdots, \mathbf{u}_{\alpha, N}^{s}\right)^{T}
$$

The subscripts $\alpha$ and $\beta$ represent the index $i, r$ and $c$.

The continuity condition is enforced next by global assembly of the remaining and corner variables as

$$
\left[\begin{array}{ccc}
\mathcal{A}_{i i}^{s} & \mathcal{A}_{i r}^{s} \mathcal{B}_{r}^{s} & \mathcal{A}_{i c}^{s} \mathcal{B}_{c}^{s} \\
\sum_{s=1}^{n_{s}} \mathcal{B}_{r}^{s T} \mathcal{A}_{r i}^{s} & \sum_{s=1}^{n_{s}} \mathcal{B}_{r}^{s T} \mathcal{A}_{r r}^{s} \mathcal{B}_{r}^{s} & \sum_{s=1}^{n_{s}} \mathcal{B}_{r}^{s T} \mathcal{A}_{r c}^{s} \mathcal{B}_{c}^{s} \\
\sum_{s=1}^{n_{s}} \mathcal{B}_{c}^{s T} \mathcal{A}_{c i}^{s} & \sum_{s=1}^{n_{s}} \mathcal{B}_{c}^{s T} \mathcal{A}_{c r}^{s} \mathcal{B}_{r}^{s} & \sum_{s=1}^{n_{s}} \mathcal{B}_{c}^{s T} \mathcal{A}_{c c}^{s} \mathcal{B}_{c}^{s}
\end{array}\right]\left\{\begin{array}{l}
\mathcal{U}_{i}^{s} \\
\mathcal{U}_{r} \\
\mathcal{U}_{c}
\end{array}\right\}=\left\{\begin{array}{c}
\mathcal{F}_{i}^{s} \\
\sum_{s=1}^{n_{s}} \mathcal{B}_{r}^{s T} \mathcal{F}_{r}^{s} \\
\sum_{s=1}^{n_{s}} \mathcal{B}_{c}^{s T} \mathcal{F}_{c}^{s}
\end{array}\right\},
$$


where $\mathcal{B}_{r}^{s}$ is a Boolean rectangular matrix that maps the global remaining vector $\mathcal{U}_{r}$ to the local remaining vector $\mathcal{U}_{r}^{s}$ as

$$
\mathcal{U}_{r}^{s}=\mathcal{B}_{r}^{s} \mathcal{U}_{r}
$$

Similarly, the restriction operator $\mathcal{B}_{c}^{s}$ is a Boolean rectangular matrix that maps the global corner vector $\mathcal{U}_{c}$ to the local corner vector $\mathcal{U}_{c}^{s}$ as

$$
\mathcal{U}_{c}^{s}=\mathcal{B}_{c}^{s} \mathcal{U}_{c}
$$

The interior unknowns $\mathcal{U}_{i}^{s}$ can be obtained concurrently from Eq.(7.3) as

$$
\mathcal{U}_{i}^{s}=\left[\mathcal{A}_{i i}^{s}\right]^{-1}\left(\mathcal{F}_{i}^{s}-\mathcal{A}_{i r}^{s} \mathcal{B}_{r}^{s} \mathcal{U}_{r}-\mathcal{A}_{i c}^{s} \mathcal{B}_{c}^{s} \mathcal{U}_{c}\right)
$$

Substituting Eq.(7.6) into Eq.(7.3) leads to the following Schur compliment system corresponding to the remaining and corner nodes of the interface boundary, as shown schematically in Fig.(7.2).

$$
\left[\begin{array}{ll}
\sum_{s=1}^{n_{s}} \mathcal{B}_{r}^{s T} \mathcal{S}_{r r}^{s} \mathcal{B}_{r}^{s} & \sum_{s=1}^{n_{s}} \mathcal{B}_{r}^{s T} \mathcal{S}_{r c}^{s} \mathcal{B}_{c}^{s} \\
\sum_{s=1}^{n_{s}} \mathcal{B}_{c}^{s T} \mathcal{S}_{c r}^{s} \mathcal{B}_{r}^{s} & \sum_{s=1}^{n_{s}} \mathcal{B}_{c}^{s T} \mathcal{S}_{c c}^{s} \mathcal{B}_{c}^{s}
\end{array}\right]\left\{\begin{array}{l}
\mathcal{U}_{r} \\
\mathcal{U}_{c}
\end{array}\right\}=\left\{\begin{array}{l}
\sum_{s=1}^{n_{s}} \mathcal{B}_{r}^{s T} \mathcal{G}_{r}^{s} \\
\sum_{s=1}^{n_{s}} \mathcal{B}_{c}^{s T} \mathcal{G}_{c}^{s}
\end{array}\right\}
$$

where

$$
\begin{aligned}
\mathcal{S}_{\alpha \beta}^{s} & =\mathcal{A}_{\alpha \beta}^{s}-\mathcal{A}_{\alpha i}^{s}\left[\mathcal{A}_{i i}^{s}\right]^{-1} \mathcal{A}_{i \beta}^{s} \\
\mathcal{G}_{\alpha}^{s} & =\mathcal{F}_{\alpha}^{s}-\mathcal{A}_{\alpha i}^{s}\left[\mathcal{A}_{i i}^{s}\right]^{-1} \mathcal{F}_{i}^{s}
\end{aligned}
$$




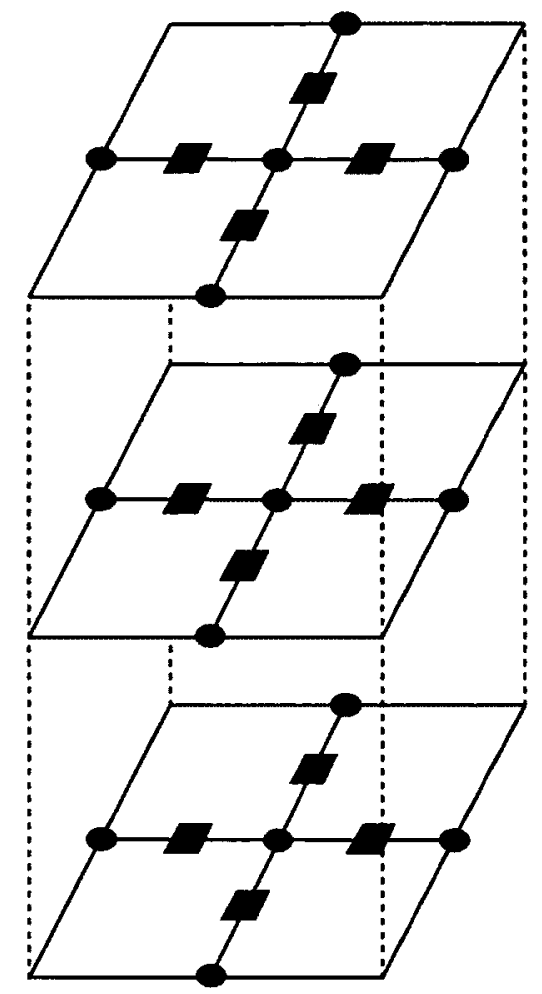

Figure 7.2: Fully assembled system: remaining $(\boldsymbol{\square})$ and corner $(\mathbf{)})$ nodes

Eliminating the corner unknowns $\mathcal{U}_{c}$ in Eq.(7.7) gives the following symmetric positive definite reduced interface problem

$$
\left(F_{r r}-F_{r c}\left[F_{c c}\right]^{-1} F_{c r}\right) \mathcal{U}_{r}=d_{r}-F_{r c}\left[F_{c c}\right]^{-1} d_{c}
$$

where

$$
\begin{aligned}
F_{\alpha \beta} & =\sum_{s=1}^{n_{s}} \mathcal{B}_{\alpha}^{s T} \mathcal{S}_{\alpha \beta}^{s} \mathcal{B}_{\beta}^{s} \\
d_{\alpha} & =\sum_{s=1}^{n_{s}} \mathcal{B}_{\alpha}^{s T} \mathcal{G}_{\alpha}^{s}
\end{aligned}
$$


The reduced interface problem defined in Eq.(7.10) can be solved iteratively using PCGM equipped with two-level preconditioner defined in the next section.

\subsection{Primal-Primal Preconditioner}

The two-level preconditioner for the reduced interface problem is constructed by strictly enforcing the continuity condition on the corner nodes, while for the remaining nodes the continuity is satisfied in an average sense. This fact is schematically illustrated in Fig.(7.3). As the iterations converge, the continuity condition at all interface nodes (both corner and remaining boundary nodes) is satisfied strictly.

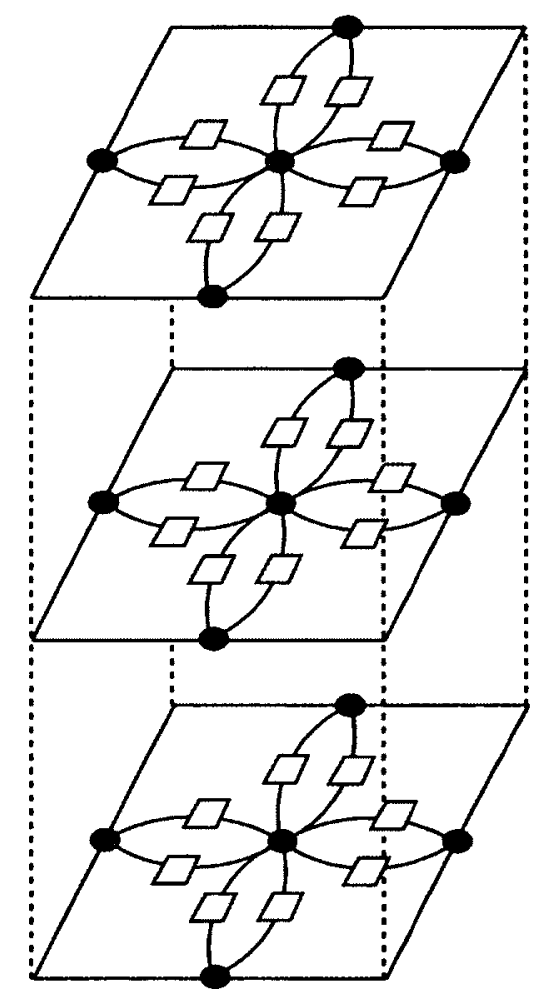

Figure 7.3: Partial assembly of the corner nodes $(\bullet)$ 
Performing a global assembly of the corner variables leads to the following system:

$$
\left[\begin{array}{ccc}
\mathcal{A}_{i i}^{s} & \mathcal{A}_{i r}^{s} & \mathcal{A}_{i c}^{s} \mathcal{B}_{c}^{s} \\
\mathcal{A}_{r i}^{s} & \mathcal{A}_{r r}^{s} & \mathcal{A}_{r c}^{s} \mathcal{B}_{c}^{s} \\
\sum_{s=1}^{n_{s}} \mathcal{B}_{c}^{s T} \mathcal{A}_{c i}^{s} & \sum_{s=1}^{n_{s}} \mathcal{B}_{c}^{s T} \mathcal{A}_{c r}^{s} \mathcal{B}_{r}^{s} & \sum_{s=1}^{n_{s}} \mathcal{B}_{c}^{s T} \mathcal{A}_{c c}^{s} \mathcal{B}_{c}^{s}
\end{array}\right]\left\{\begin{array}{c}
\mathcal{X}_{i}^{s} \\
\mathcal{U}_{r} \\
\mathcal{U}_{c}
\end{array}\right\}=\left\{\begin{array}{c}
0 \\
\mathcal{F}_{r}^{s} \\
0
\end{array}\right\},
$$

where

$$
\mathcal{F}_{r}^{s}=\mathcal{D}_{r}^{s} \mathcal{B}_{r}^{s} \mathbf{r}_{j}
$$

and $\mathbf{r}_{j}$ is the residual at the $j^{\text {th }}$ iteration of the iterative solver and $\mathcal{D}_{r}^{s}$ represents a block diagonal matrix defined as

$$
\mathcal{D}_{r}^{s}=\operatorname{blockdiag}\left(\mathbf{D}_{r, 0}^{s}, \cdots, \mathbf{D}_{r, N}^{s}\right)
$$

where $\mathrm{D}_{r, k}^{s}$ is a weighting matrix that satisfies the following property

$$
\sum_{s=1}^{n_{s}} \mathbf{B}_{r}^{s T} \mathbf{D}_{r}^{s} \mathbf{B}_{r}^{s}=\mathbf{I} .
$$

The diagonal entries of the scaling matrix $\mathbf{D}_{r}^{s}$ are the reciprocal of the number of subdomains that share the corresponding remaining interface boundary nodes.

Eliminating the local unknown vector $\mathcal{X}_{i}^{s}$ from Eq.(7.13) leads to the following partially assembled extended Schur complement system

$$
\left[\begin{array}{cc}
\mathcal{S}_{r r}^{s} & \mathcal{S}_{r c}^{s} \mathcal{B}_{c}^{s} \\
\sum_{s=1}^{n_{s}} \mathcal{B}_{c}^{s T} \mathcal{S}_{c r}^{s} \mathcal{B}_{r}^{s} & \sum_{s=1}^{n_{s}} \mathcal{B}_{c}^{s T} \mathcal{S}_{c c}^{s} \mathcal{B}_{c}^{s}
\end{array}\right]\left\{\begin{array}{c}
\mathcal{U}_{r}^{s} \\
\mathcal{U}_{c}
\end{array}\right\}=\left\{\begin{array}{c}
\mathcal{F}_{r}^{s} \\
0
\end{array}\right\} .
$$


Next, the local remaining unknown vector $\mathcal{U}_{r}^{s}$ can be eliminated concurrently from Eq.(7.17) leading to the following global coarse problem

$$
F_{c c} \mathcal{U}_{c}=d_{c}
$$

where

$$
\begin{aligned}
F_{c c} & =\sum_{s=1}^{n_{s}} \mathcal{B}_{c}^{s T}\left(\mathcal{S}_{c c}^{s}-\mathcal{S}_{c r}^{s}\left[\mathcal{S}_{r r}^{s}\right]^{-1} \mathcal{S}_{r c}^{s}\right) \mathcal{B}_{c}^{s} \\
d_{c} & =-\sum_{s=1}^{n_{s}} \mathcal{B}_{c}^{s T} \mathcal{S}_{c r}^{s}\left[\mathcal{S}_{r r}^{s}\right]^{-1} \mathcal{F}_{r}^{s}
\end{aligned}
$$

The continuity of the solution field over the remaining interface nodes is satisfied next by averaging the local results as

$$
\mathcal{U}_{r}=\sum_{s=1}^{n_{s}} \mathcal{B}_{r}^{s T} \mathcal{D}_{r}^{s} \mathcal{U}_{r}^{s}
$$

After some algebraic manipulations, the stochastic primal-primal preconditioner can be expressed as

$$
\mathcal{M}_{S P P M}^{-1}=\sum_{s=1}^{n_{s}} \mathcal{B}_{r}^{s T} \mathcal{D}_{r}^{s}\left[\mathcal{S}_{r r}^{s}\right]^{-1} \mathcal{D}_{r}^{s} \mathcal{B}_{r}^{s}+R_{0}^{T}\left[F_{c c}\right]^{-1} R_{0}
$$

where

$$
R_{0}=\sum_{s=1}^{n_{s}} \mathcal{B}_{c}^{s T} \mathcal{S}_{c r}\left[\mathcal{S}_{r r}^{s}\right]^{-1} \mathcal{D}_{r}^{s} \mathcal{B}_{r}^{s}
$$

Clearly, the primal-primal preconditioner consists of subdomain level problems $\left[\mathcal{S}_{r r}^{s}\right]^{-1}$ and a global coarse problem $\left[F_{c c}\right]^{-1}$. 


\subsection{Parallel Implementation}

The PCGM equipped with the primal-primal preconditioner is employed in parallel to tackle the reduced interface problem as

$$
\mathcal{M}_{S P P M}^{-1}\left(F_{r r}-F_{r c}\left[F_{c c}\right]^{-1} F_{c r}\right) \mathcal{U}_{r}=\mathcal{M}_{S P P M}^{-1}\left(d_{r}-F_{r c}\left[F_{c c}\right]^{-1} d_{c}\right)
$$

At the $j^{\text {th }}$ iteration of the parallel PCGM, the multiplication of the coefficient matrix by a vector is obtained using the following algorithm: 


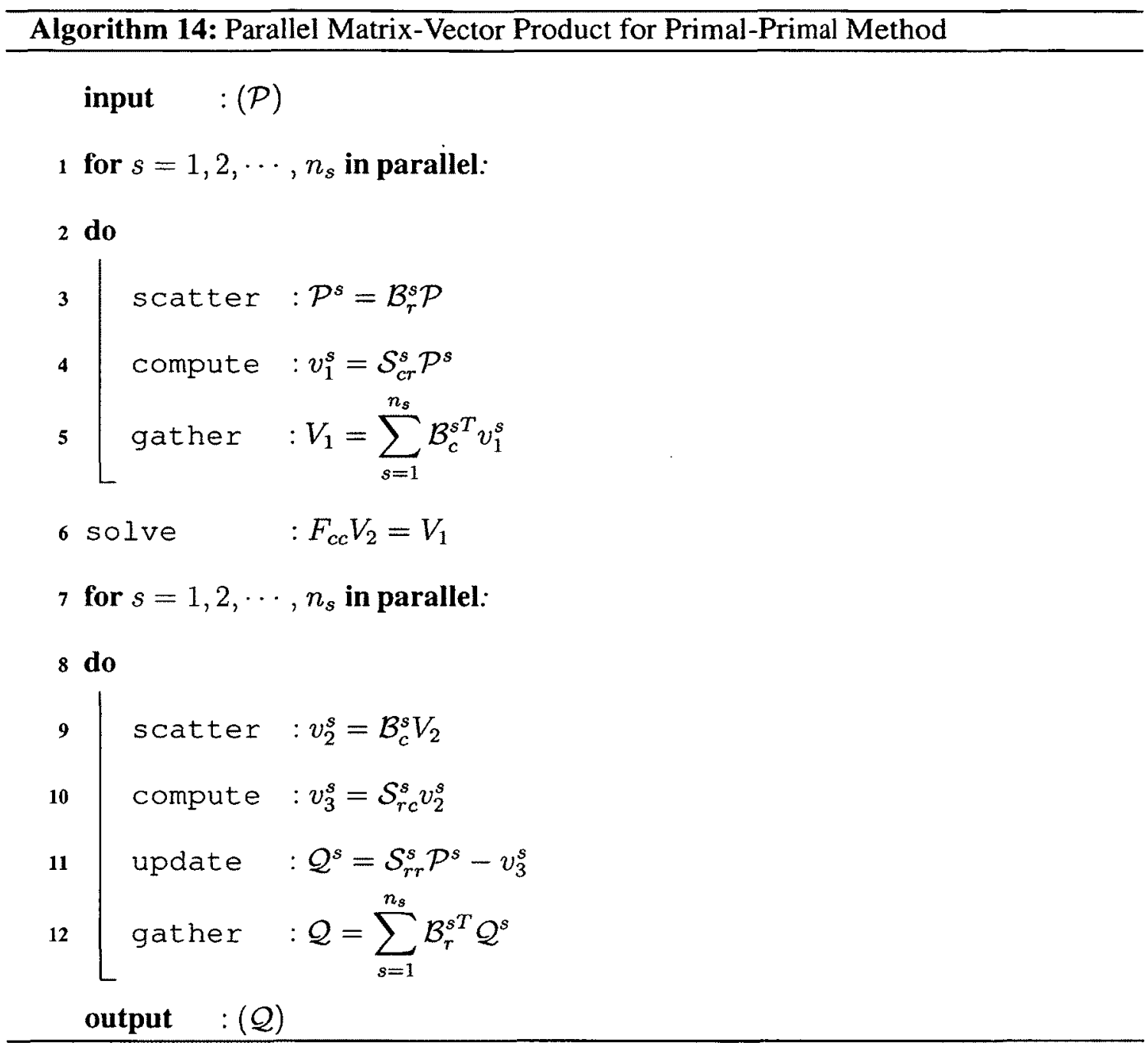

Multiplication of the extended Schur complement matrix by a vector in steps 4, 10 and 11 of Algorithm (14) is computed by solving a corresponding Dirichlet problem as

$$
\begin{aligned}
v_{\alpha}^{s} & =\mathcal{S}_{\alpha \beta}^{s} v_{\beta}^{s}, \\
v_{\alpha}^{s} & =\left(\mathcal{A}_{\alpha \beta}^{s}-\mathcal{A}_{\alpha i}^{s}\left[\mathcal{A}_{i i}^{s}\right]^{-1} \mathcal{A}_{i \beta}^{s}\right) v_{\beta}^{s} .
\end{aligned}
$$


This procedure is outlined in the following algorithm:

\begin{tabular}{l}
\hline Algorithm 15: Dirichlet-Solver Procedure \\
\hline input $\quad:\left(v_{\beta}^{s}, \mathcal{A}_{i \beta}^{s}, \mathcal{A}_{\alpha i}^{s}, \mathcal{A}_{\alpha \beta}^{s}, \mathcal{A}_{i i}^{s}\right)$ \\
1 compute $: v_{1}^{s}=\mathcal{A}_{i \beta}^{s} v_{\beta}^{s}$ \\
2 solve $\quad: \mathcal{A}_{i i}^{s} v_{2}^{s}=v_{1}^{s}$ \\
3 compute $: v_{3}^{s}=\mathcal{A}_{\alpha i}^{s} v_{2}^{s}$ \\
4 compute $: v_{4}^{s}=\mathcal{A}_{\alpha \beta}^{s} v_{\beta}^{s}$ \\
5 compute $: v_{\alpha}^{s}=v_{4}^{s}-v_{3}^{s}$ \\
output $\quad:\left(v_{\alpha}^{s}\right)$
\end{tabular}

The global problem in step 6 of Algorithm (14) is solved iteratively using PCGM equipped with lumped preconditioner as

$$
\mathcal{M}_{c c}^{-1} F_{c c} V_{2}=\mathcal{M}_{c c}^{-1} V_{1}
$$

where

$$
\mathcal{M}_{c c}^{-1}=\sum_{s=1}^{n_{s}} \mathcal{B}_{c}^{s T} \mathcal{A}_{c c}^{s} \mathcal{B}_{c}^{s}
$$

The effect of the primal-primal preconditioner is computed using the procedure outlined in the following algorithm: 


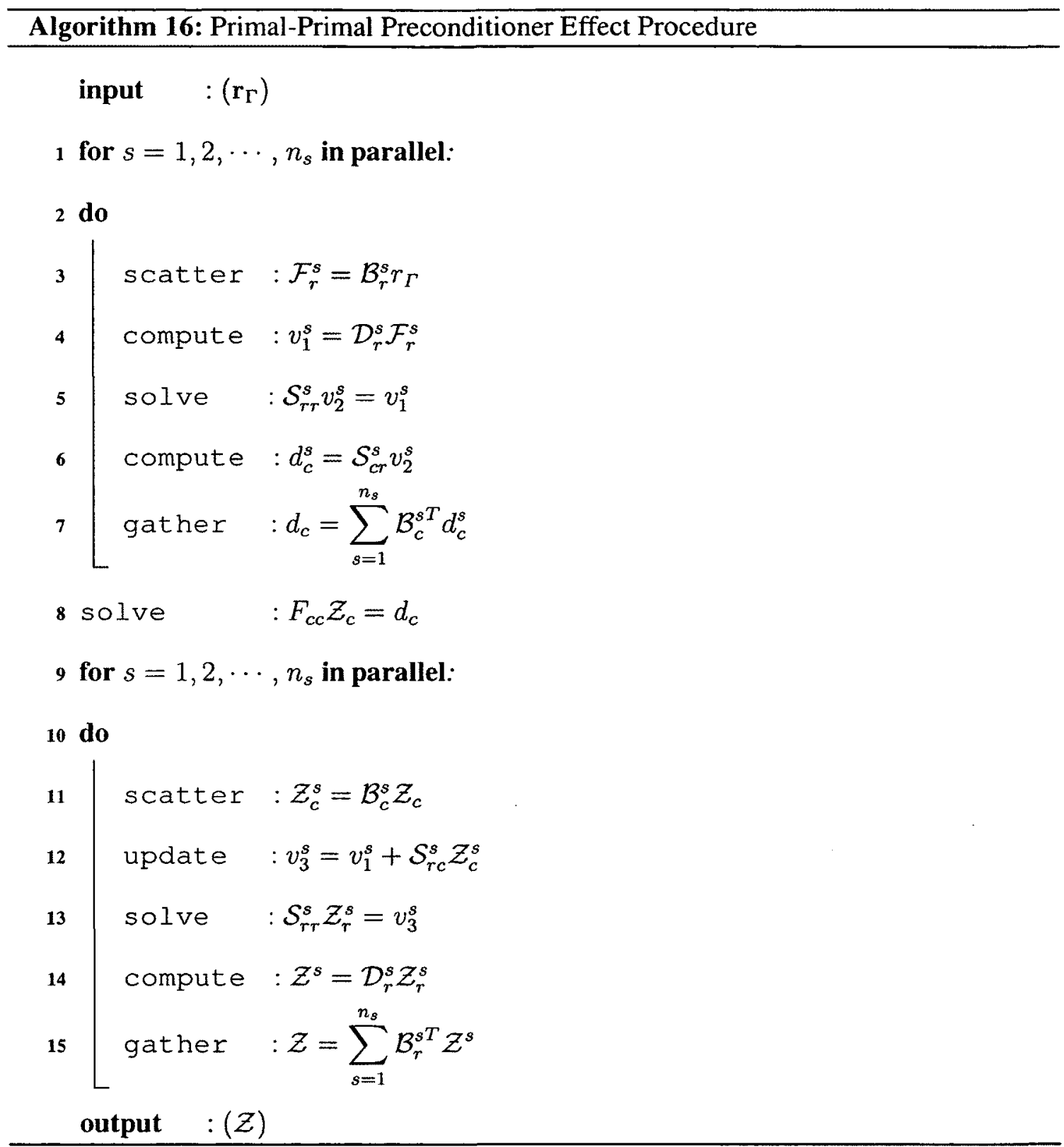

The local problems in steps 5 and 13 of Algorithm (16) constitute a subdomain level Neumann problem which can be solved using the following algorithm: 


\begin{tabular}{cc}
\hline Algorithm 17: Neumann-Solver Procedure \\
\hline input & $:\left(\mathcal{F}_{r}^{s}\right)$ \\
1 solve & $:\left[\begin{array}{cc}\mathcal{A}_{i i}^{s} & \mathcal{A}_{i r}^{s} \\
\mathcal{A}_{r i}^{s} & \mathcal{A}_{r r}^{s}\end{array}\right]\left\{\begin{array}{c}\mathcal{X}_{i}^{s} \\
\mathcal{U}_{r}^{s}\end{array}\right\}=\left\{\begin{array}{c}0 \\
\mathcal{F}_{r}^{s}\end{array}\right\}$ \\
\hline
\end{tabular}

The global coarse stochastic problem in step 8 of Algorithm (16) is solved iteratively using PCGM solver equipped with lumped preconditioner as

$$
\mathcal{M}_{c c}^{-1} F_{c c} \mathcal{Z}_{c}=\mathcal{M}_{c c}^{-1} d_{c}
$$

where

$$
\mathcal{M}_{c c}^{-1}=\sum_{s=1}^{n_{s}} \mathcal{B}_{c}^{s T} \mathcal{A}_{c c}^{s} \mathcal{B}_{c}^{s}
$$

Finally, we summarize the parallel implementation of the PCGM to solve the reduced interface problem using the primal-primal preconditioner in the following flow chart. 


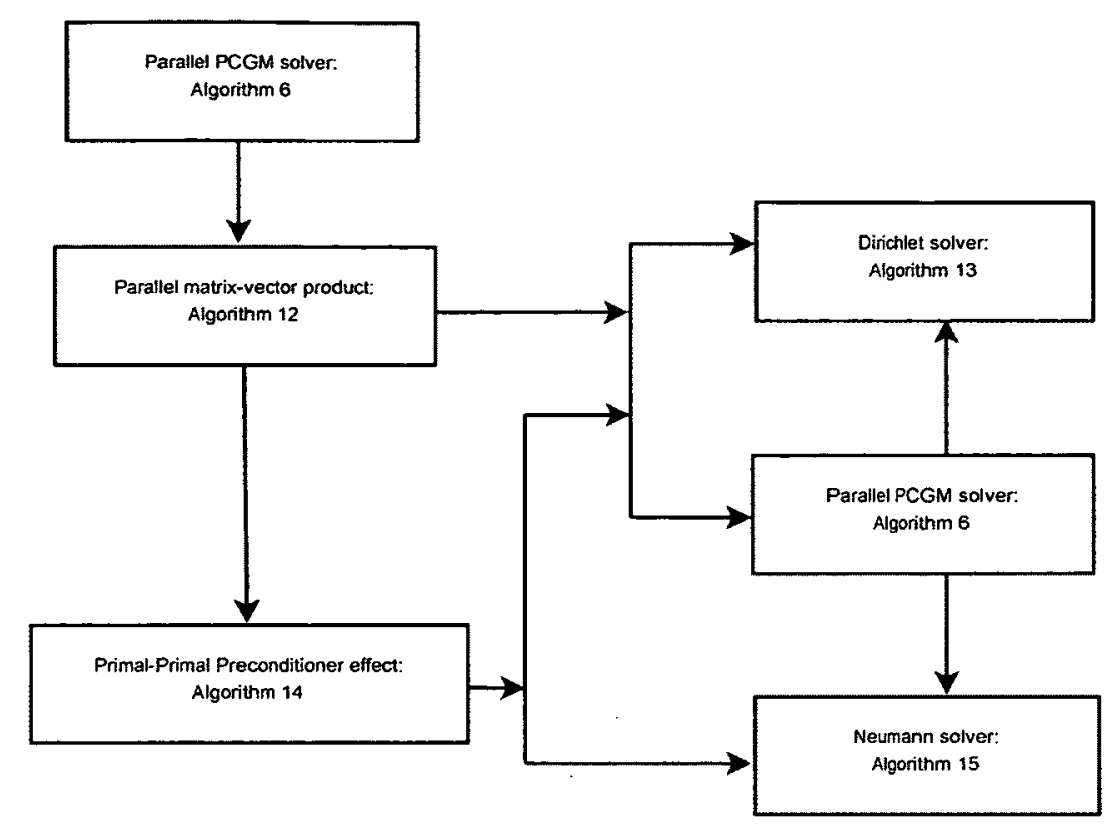

Figure 7.4: Flow chart of parallel implementation of the primal-primal method

\subsection{Summary}

A novel primal-primal iterative substructuring technique is introduced to solve the largescale linear system arising from the finite element discretization of stochastic PDEs. The algorithm offers a direct approach to formulate a two-level scalable preconditioner. Throughout the iteration, the primal-primal preconditioner strictly enforces the continuity condition on the corner nodes of the interface, while weakly satisfying the continuity condition over the remaining part of the interface. This approach naturally leads to a coarse grid connecting the subdomains globally and provides a mechanism to propagate information across the subdomains which makes the algorithm scalable. 


\section{Chapter 8}

\section{Dual-Primal Domain Decomposition Method for Stochastic PDEs}

\subsection{Introduction}

This chapter is closely based on the references $[79,80,86]$. In this chapter, we report a probabilistic version of the dual-primal domain decomposition method for the intrusive SSFEM in order to exploit high performance computing platforms for uncertainty quantification. In particular, we formulate a probabilistic version of the dual-primal finite element tearing and interconnect (FETI-DP) technique to solve the large-scale linear systems in the intrusive SSFEM. In the probabilistic setting, the operator of the dual interface system in the dual-primal approach contains a coarse problem. The introduction of the coarse problem leads to a scalable performance of the dual-primal iterative substructuring method for uncertainty quantification of large-scale computational models. 
For deterministic PDEs, BDDC $[78,107,108]$ and FETI-DP $[64,81]$ are perhaps the most popular non-overlapping domain decomposition techniques for the iterative solution of large-scale deterministic linear systems. BDDC provides a two-level preconditioner for the solution of the so-called primal interface problem (Schur complement system). On the contrary, FETI-DP iteratively solves a system of Lagrange multipliers arises in the so-called dual interface system. The coarse problem essential for scalability is built in the preconditioner of the BDDC, while it is embedded within the operator of FETIDP. For deterministic systems, it has already been demonstrated that the condition number and thus the parallel performance of BDDC and FETI-DP are quite similar $[65,67,78$, 83-85]. Indeed, the preconditioned operators in BDDC and its counterpart in FETI-DP have the same algebraic structure with identical eigenvalues except possibly for zero and one [67]. This fact indicates that the convergence properties of both BDDC and FETI-DP are identical when using the same primal constraints [83]. It is therefore natural to ask whether the similarity of BDDC and FETI-DP extends to stochastic systems.

\subsection{Dual-Primal Domain Decomposition Method for SPDEs}

Using a general framework of iterative substructuring techniques $[68,70,74-77,79,80]$, we formulate a probabilistic version of FETI-DP for the iterative solution of the deterministic linear system in the SSFEM $[79,80]$. In the dual-primal method for stochastic systems, the spatial domain is partitioned into a number of non-overlapping subdomains. The polynomial chaos expansions is employed to represent the solution process. For each 
subdomain, the solution vector is divided into the interior and interface unknowns [64, 81]. The interface unknowns are further split into sets of corner and remaining variables. In each iteration of PCGM for the probabilistic dual interface system, global assembly for the chaos coefficients of the solution process at the corner nodes is performed to exactly enforce the continuity conditions. On the other hand, Lagrange multipliers are utilized to weakly satisfy the continuity of the polynomial chaos coefficients on the remaining nodes of the interface. Consequently, a probabilistic coarse problem is embedded in the operator of the dual interface problem of FETI-DP. The introduction of the coarse problem in the probabilistic framework leads to a scalable performance of FETI-DP iterative substructuring based solver for large-scale stochastic systems. The methodology can be described as follows.

The physical domain $\Omega$ is partitioned into $n_{s}$ non-overlapping subdomains such that $\Omega=\bigcup_{s=1}^{n_{s}} \Omega_{s}$. For a typical subdomain $\Omega_{s}$, we divide the nodal solution vector as the interior unknown $\mathbf{u}_{i}^{s}(\theta)$, remaining $\mathbf{u}_{r}^{s}(\theta)$ and interface corner $\mathbf{u}_{c}^{s}(\theta)$ as shown in Fig.(8.1). 


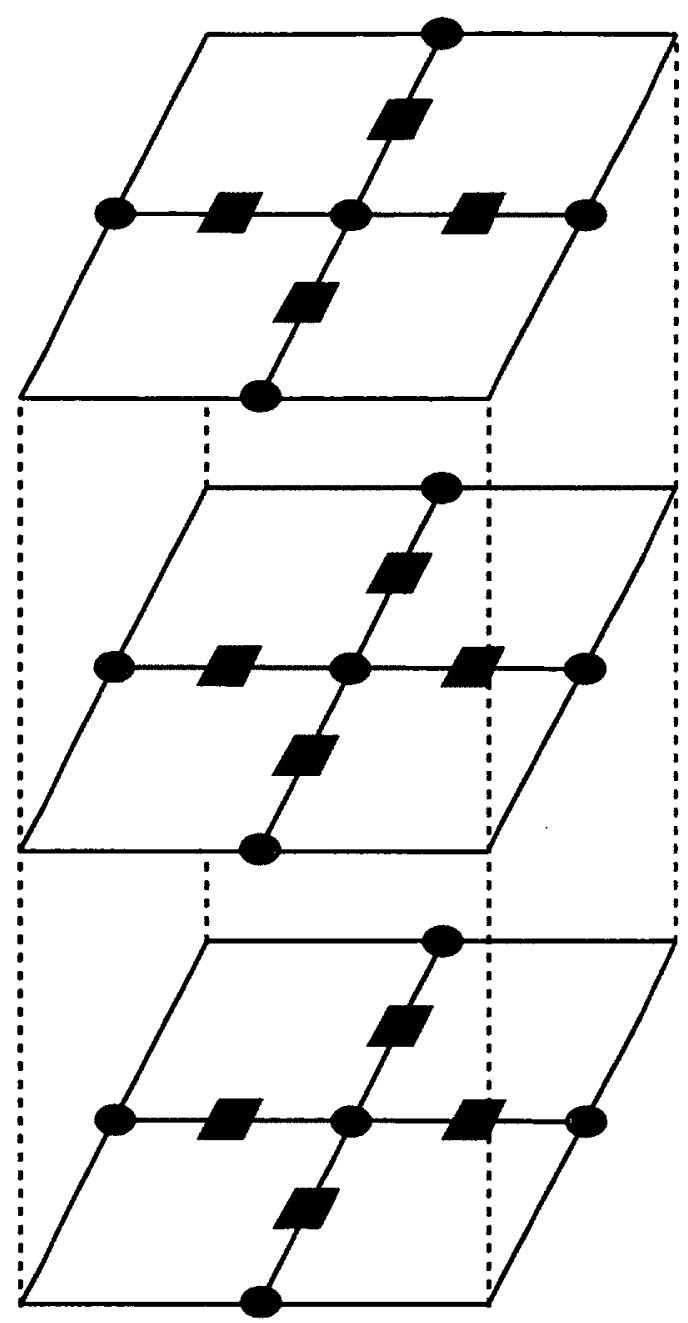

Figure 8.1: Partitioning the boundary nodes into: remaining ( $\boldsymbol{\square}$ ) and corner(@) nodes

Accordingly, the subdomain problem can be expressed as

$$
\left[\begin{array}{lll}
\mathbf{A}_{i i}^{s}(\theta) & \mathbf{A}_{i r}^{s}(\theta) & \mathbf{A}_{i c}^{s}(\theta) \\
\mathbf{A}_{r i}^{s}(\theta) & \mathbf{A}_{r r}^{s}(\theta) & \mathbf{A}_{r c}^{s}(\theta) \\
\mathbf{A}_{c i}^{s}(\theta) & \mathbf{A}_{c r}^{s}(\theta) & \mathbf{A}_{c c}^{s}(\theta)
\end{array}\right]\left\{\begin{array}{c}
\mathbf{u}_{i}^{s}(\theta) \\
\mathbf{u}_{r}^{s}(\theta) \\
\mathbf{u}_{c}^{s}(\theta)
\end{array}\right\}=\left\{\begin{array}{c}
\mathbf{f}_{i}^{s} \\
\mathbf{f}_{r}^{s} \\
\mathbf{f}_{c}^{s}
\end{array}\right\}
$$


Expanding the stochastic system parameters and the solution process by PCE and then performing Galerkin projection, Eq.(8.1) can be expressed as

where

$$
\left[\begin{array}{ccc}
\mathcal{A}_{i i}^{s} & \mathcal{A}_{i r}^{s} & \mathcal{A}_{i c}^{s} \\
\mathcal{A}_{r i}^{s} & \mathcal{A}_{r r}^{s} & \mathcal{A}_{r c}^{s} \\
\mathcal{A}_{c i}^{s} & \mathcal{A}_{c r}^{s} & \mathcal{A}_{c c}^{s}
\end{array}\right]\left\{\begin{array}{l}
\mathcal{U}_{i}^{s} \\
\mathcal{U}_{r}^{s} \\
\mathcal{U}_{c}^{s}
\end{array}\right\}=\left\{\begin{array}{c}
\mathcal{F}_{i}^{s} \\
\mathcal{F}_{r}^{s} \\
\mathcal{F}_{c}^{s}
\end{array}\right\}
$$

$$
\left[\mathcal{A}_{\alpha \beta}^{s}\right]_{j k}=\sum_{l=0}^{L}\left\langle\Psi_{l} \Psi_{j} \Psi_{k}\right\rangle \mathbf{A}_{\alpha \beta, l}^{s}, \quad \mathcal{F}_{\alpha, k}^{s}=\left\langle\Psi_{k} \mathbf{f}_{\alpha}^{s}\right\rangle, \quad \mathcal{U}_{\alpha}^{s}=\left(\mathbf{u}_{\alpha, 0}^{s}, \cdots, \mathbf{u}_{\alpha, N}^{s}\right)^{T},
$$

and the subscripts $\alpha$ and $\beta$ indicate $i, r$ and $c$.

Let $\mathcal{B}_{c}^{s}$ be a block-diagonal matrix used to scatter the global corner solution vector $\mathcal{U}_{c}$ to its local component as

$$
\mathcal{U}_{c}^{s}=\mathcal{B}_{c}^{s} \mathcal{U}_{c}
$$

where

$$
\mathcal{B}_{c}^{s}=\operatorname{blockdiag}\left(\mathbf{B}_{c, 0}^{s}, \ldots, \mathbf{B}_{c, N}^{s}\right)
$$

and

$$
\mathbf{u}_{c, j}^{s}=\mathbf{B}_{c, j}^{s} \mathbf{u}_{c, j}
$$

Next, introducing the Lagrange multipliers $\Lambda$ to satisfy the weak compatibility constraint on the remaining part of the interface and performing a global assembly of the polynomial chaos coefficients of the solution process at the corner nodes, we obtain the following system 


$$
\left[\begin{array}{cccc}
\mathcal{A}_{i i}^{s} & \mathcal{A}_{i r}^{s} & \mathcal{A}_{i c}^{s} \mathcal{B}_{c}^{s} & 0 \\
\mathcal{A}_{r i}^{s} & \mathcal{A}_{r r}^{s} & \mathcal{A}_{r c}^{s} \mathcal{B}_{c}^{s} & \mathcal{B}_{r}^{s T} \\
\sum_{s=1}^{n_{s}} \mathcal{B}_{c}^{s T} \mathcal{A}_{c i}^{s} & \sum_{s=1}^{n_{s}} \mathcal{B}_{c}^{s T} \mathcal{A}_{c r}^{s} & \sum_{s=1}^{n_{s}} \mathcal{B}_{c}^{s T} \mathcal{A}_{c c}^{s} \mathcal{B}_{c}^{s} & 0 \\
0 & \sum_{s=1}^{n_{s}} \mathcal{B}_{r}^{s} & 0 & 0
\end{array}\right]\left\{\begin{array}{c}
\mathcal{U}_{i}^{s} \\
\mathcal{U}_{r}^{s} \\
\mathcal{U}_{c} \\
\Lambda
\end{array}\right\}=\left\{\begin{array}{c}
\mathcal{F}_{i}^{s} \\
\mathcal{F}_{r}^{s} \\
\sum_{s=1}^{n_{s}} \mathcal{B}_{c}^{s T} \mathcal{F}_{c}^{s} \\
0
\end{array}\right\}
$$

where $\mathcal{B}_{r}^{s}$ is a block diagonal jump operator such that

$$
\sum_{s=1}^{n_{s}} \mathcal{B}_{r}^{s} \mathcal{U}_{r}^{s}=0
$$

and

$$
\Lambda=\left\{\lambda_{0}, \ldots, \lambda_{N}\right\}^{T} .
$$

The Lagrange multiplier system for the polynomial chaos coefficients of the solution process can be obtain from Eq.(8.6) as

$$
\left(\bar{F}_{r r}+\bar{F}_{r c}\left[\bar{F}_{c c}\right]^{-1} \bar{F}_{c r}\right) \Lambda=\bar{d}_{r}-\bar{F}_{r c}\left[\bar{F}_{c c}\right]^{-1} \bar{d}_{c}
$$

where

$$
\begin{array}{ll}
\bar{F}_{r r}=\sum_{s=1}^{n_{s}} \mathcal{B}_{r}^{s}\left[\mathcal{S}_{r r}^{s}\right]^{-1} \mathcal{B}_{r}^{s T}, & \bar{F}_{r c}=\sum_{s=1}^{n_{s}} \mathcal{B}_{r}^{s}\left[\mathcal{S}_{r r}^{s}\right]^{-1} \mathcal{S}_{r c}^{s} \mathcal{B}_{c}^{s} \\
\bar{F}_{c c}=\sum_{s=1}^{n_{s}} \mathcal{B}_{c}^{s T}\left(\mathcal{S}_{c c}^{s}-\mathcal{S}_{c r}^{s}\left[\mathcal{S}_{r r}^{s}\right]^{-1} \mathcal{S}_{r c}^{s}\right) \mathcal{B}_{c}^{s}, & \bar{F}_{c r}=\sum_{s=1}^{n_{s}} \mathcal{B}_{c}^{s T} \mathcal{S}_{c r}^{s}\left[\mathcal{S}_{r r}^{s}\right]^{-1} \mathcal{B}_{r}^{s T}, \\
\bar{d}_{c}=\sum_{s=1}^{n_{s}} \mathcal{B}_{c}^{s T}\left(\mathcal{G}_{c}^{s}-\mathcal{S}_{c r}^{s}\left[\mathcal{S}_{r r}^{s}\right]^{-1} \mathcal{G}_{r}^{s}\right), & \bar{d}_{r}=\sum_{s=1}^{n_{s}} \mathcal{B}_{r}^{s}\left[\mathcal{S}_{r r}^{s}\right]^{-1} \mathcal{G}_{r}^{s},
\end{array}
$$


and

$$
\begin{aligned}
\mathcal{S}_{\alpha \beta}^{s} & =\mathcal{A}_{\alpha \beta}^{s}-\mathcal{A}_{\alpha i}^{s}\left[\mathcal{A}_{i i}^{s}\right]^{-1} \mathcal{A}_{i \beta}^{s}, \\
\mathcal{G}_{\alpha}^{s} & =\mathcal{F}_{\alpha}^{s}-\mathcal{A}_{\alpha i}^{s}\left[\mathcal{A}_{i i}^{s}\right]^{-1} \mathcal{F}_{i}^{s} .
\end{aligned}
$$

The extended Lagrange multipliers system defined by Eq.(8.7) is symmetric and positive definite (e.g. $[11,52,64,81])$ and thus can be solved iteratively using PCGM. Similar to the deterministic version of FETI-DP $[64,81]$, the coarse problem $\bar{F}_{c c}^{-1}$ required for global communication is already embedded within the operator. In this case, the extended lumped $[70,75]$ or the extended Dirichlet preconditioner is sufficient for scalability. Physically, the extended Lagrange multiplier system relates to the nodal forces required to satisfy the compatibility condition of the polynomial chaos coefficients of the solution process at the remaining interface nodes as shown in Fig.(8.2). 


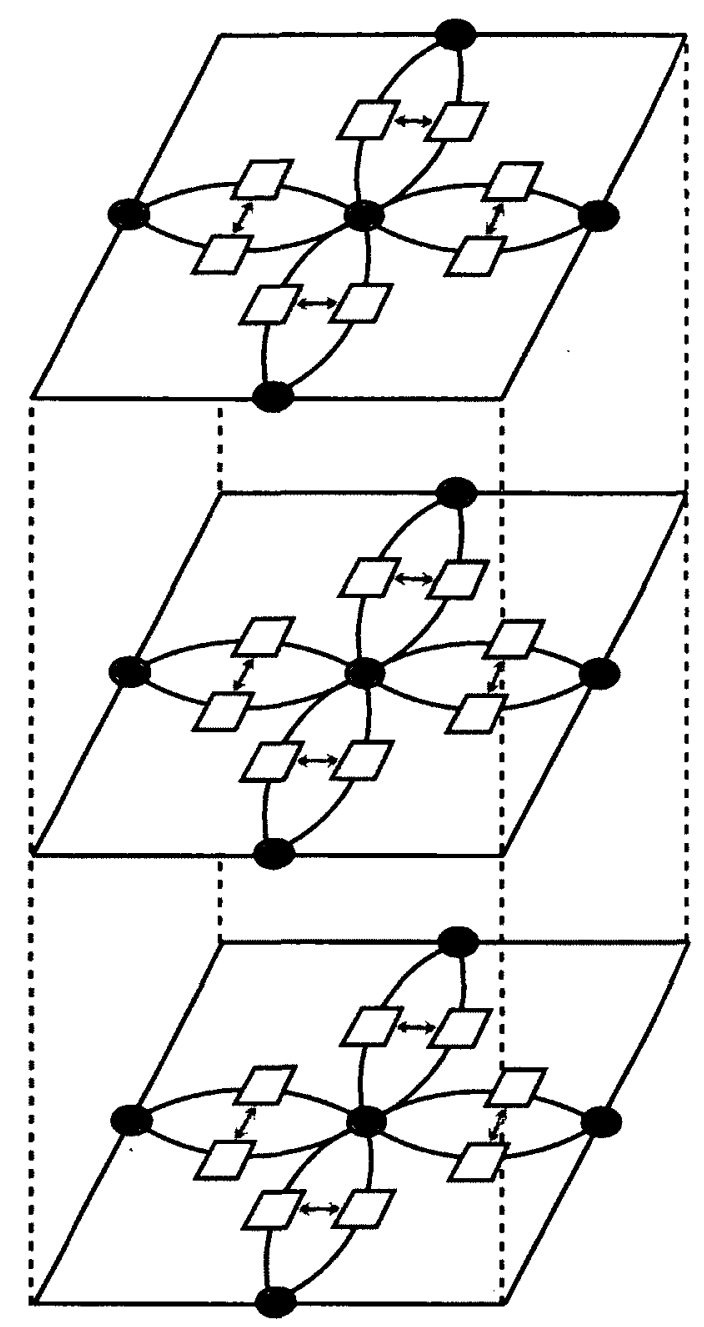

Figure 8.2: Lagrange multipliers: enforcing continuity of the polynomial chaos coefficients of the solution process

Once the Lagrange multipliers are computed, the polynomial chaos coefficients associated with the corner nodes can be recovered as

$$
\bar{F}_{c c} \mathcal{U}_{c}=\left(\bar{d}_{c}+\bar{F}_{c r} \Lambda\right)
$$

The subdomain level variables $\mathcal{U}_{i}^{s}$ and $\mathcal{U}_{r}^{s}$ can be obtained in parallel from the following 
local linear system

$$
\left[\begin{array}{cc}
\mathcal{A}_{i i}^{s} & \mathcal{A}_{i r}^{s} \\
\mathcal{A}_{r i}^{s} & \mathcal{A}_{r r}^{s}
\end{array}\right]\left\{\begin{array}{c}
\mathcal{U}_{i}^{s} \\
\mathcal{U}_{r}^{s}
\end{array}\right\}=\left\{\begin{array}{c}
\mathcal{F}_{i}^{s}-\mathcal{A}_{i c}^{s} \mathcal{B}_{c}^{s} \mathcal{U}_{c} \\
\mathcal{F}_{r}^{s}-\mathcal{A}_{r c}^{s} \mathcal{B}_{c}^{s} \mathcal{U}_{c}-\mathcal{B}_{r}^{s T} \Lambda
\end{array}\right\}
$$

\subsection{Preconditioners for the Probabilistic FETI-DP}

The preconditioned extended Lagrange multipliers system takes the following form

$$
\mathcal{M}\left(\bar{F}_{r r}+\bar{F}_{r c}\left[\bar{F}_{c c}\right]^{-1} \bar{F}_{c r}\right) \Lambda=\mathcal{M}\left(\bar{d}_{r}-\bar{F}_{r c}\left[\bar{F}_{c c}\right]^{-1} \bar{d}_{c}\right)
$$

where $\mathcal{M}$ is a one-level extended Dirichlet preconditioner defined as

$$
\mathcal{M}_{D}=\sum_{s=1}^{n_{s}} \mathcal{B}_{r}^{s} \mathcal{D}_{r}^{s} \mathcal{S}_{r r}^{s} \mathcal{D}_{r}^{s} \mathcal{B}_{r}^{s T}
$$

where

$$
\mathcal{D}_{r}^{s}=\operatorname{blockdiag}\left(\mathbf{D}_{r}^{s, 0}, \cdots, \mathbf{D}_{r}^{s, N}\right)
$$

and $\mathbf{D}_{r}^{s, k}$ is a diagonal averaging matrix defined as

$$
\sum_{s=1}^{n_{s}} \mathbf{R}_{r}^{s T} \mathbf{D}_{r}^{s, k} \mathbf{R}_{r}^{s}=\mathbf{I} .
$$

The implementation of the extended Dirichlet preconditioner within the PCGM involves solving a local stochastic Dirichlet problem as explained below.

We consider the following local system 


$$
\left[\begin{array}{cc}
\mathcal{A}_{i i}^{s} & \mathcal{A}_{i r}^{s} \\
\mathcal{A}_{r i}^{s} & \mathcal{A}_{r \tau}^{s}
\end{array}\right]\left\{\begin{array}{c}
\mathcal{X}_{i}^{s} \\
\mathcal{D}_{r}^{s} \mathcal{B}_{r}^{s T} \mathbf{r}_{r}
\end{array}\right\}=\left\{\begin{array}{c}
0 \\
\mathcal{Z}_{r}^{s}
\end{array}\right\},
$$

where $\mathbf{r}_{r}$ is the global residual vector at the $j^{\text {th }}$ iteration of PCGM.

A stochastic local Dirichlet problem is solved to compute $\mathcal{X}_{i}^{s}$ as

$$
\mathcal{X}_{i}^{s}=-\left[\mathcal{A}_{i i}^{s}\right]^{-1} \mathcal{A}_{i r}^{s} \mathcal{D}_{r}^{s} \mathcal{B}_{r}^{s T} \mathbf{r}_{r}
$$

Substituting Eq.(8.15) into the second row of Eq.(8.14), we obtain

$$
\mathcal{Z}_{r}^{s}=\left(\mathcal{A}_{r r}^{s}-\mathcal{A}_{r i}^{s}\left[\mathcal{A}_{i i}^{s}\right]^{-1} \mathcal{A}_{i r}^{s}\right) \mathcal{D}_{r}^{s} \mathcal{B}_{r}^{s T} \mathbf{r}_{r}
$$

Finally the global preconditioned residual is aggregated as

$$
\mathcal{Z}_{r}=\sum_{s=1}^{n_{s}} \mathcal{B}_{r}^{s} \mathcal{D}_{r}^{s} \mathcal{Z}_{r}^{s}
$$

\subsection{Parallel Implementation}

In this section, we detail the parallel implementation of the PCGM to solve the Lagrange multiplier system of the chaos coefficients. The distributed implementation of the matrixvector product $\mathcal{Q}_{j}=\left(\bar{F}_{r r}+\bar{F}_{r c}\left[\bar{F}_{c c}\right]^{-1} \bar{F}_{c r}\right) \mathcal{P}_{j}$ is listed in Algorithm (18). 


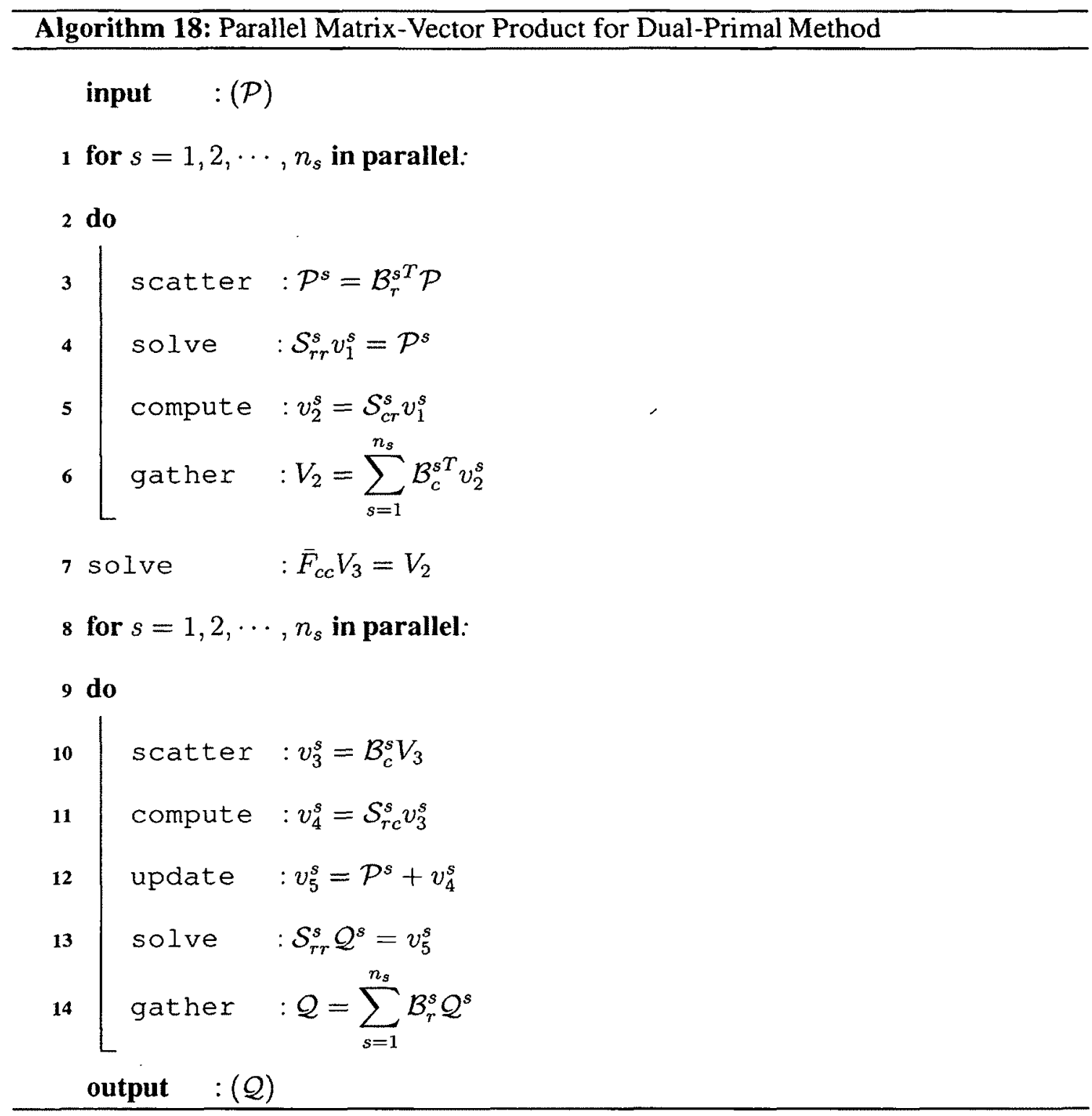

The steps 4 and 13 of Algorithm (18) are implemented by solving a local stochastic 
Neumann problem as outlined in Algorithm (19).

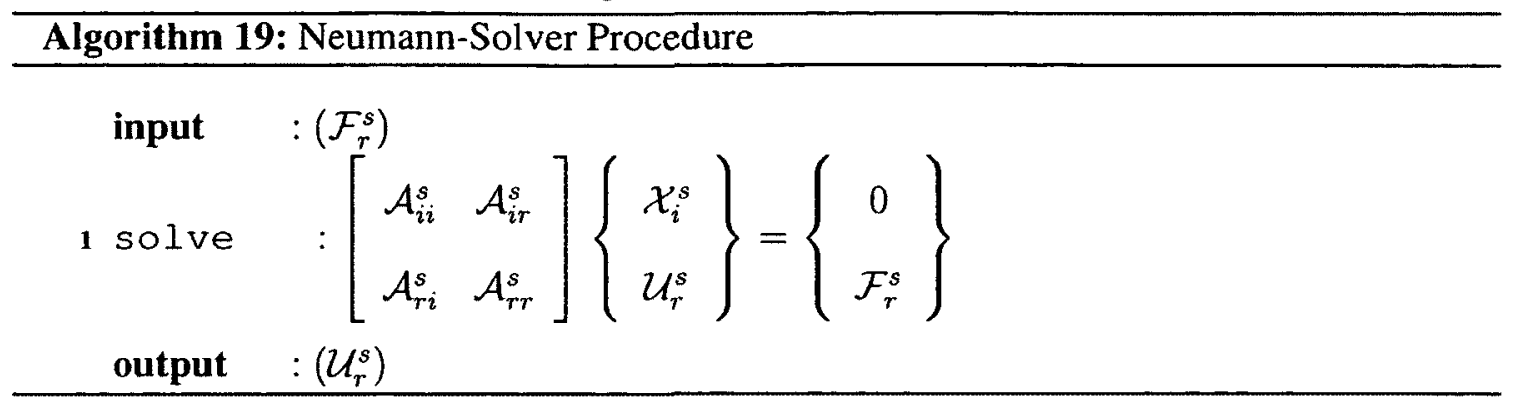

The multiplication of the extended Schur complement matrix by a vector in steps 5 and 11 of Algorithm (18) involves solving a stochastic local Dirichlet problem as outlined in Algorithm (20).

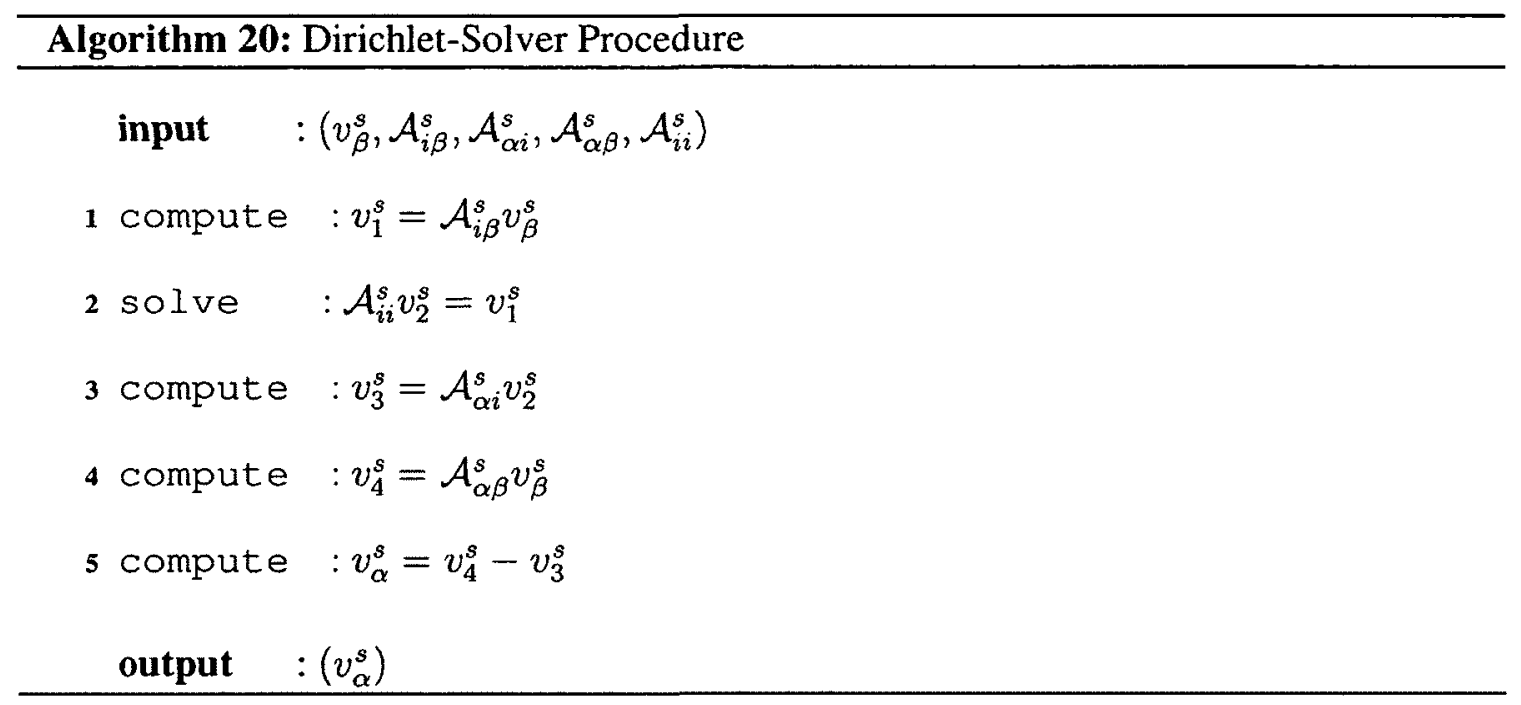

Note that steps 12,13 and 14 of Algorithm (18) can be explained as follows. Recall that the local vector $v_{4}^{s}$ in step 11 of Algorithm (18) can be written explicitly as

$$
v_{4}^{s}=\mathcal{S}_{r c}^{s} \mathcal{B}_{c}^{s}\left[\bar{F}_{c c}\right]^{-1} \sum_{s=1}^{n_{s}} \mathcal{B}_{c}^{s T} \mathcal{S}_{c r}^{s}\left[\mathcal{S}_{r r}^{s}\right]^{-1} \mathcal{B}_{r}^{s T} \mathcal{P}
$$


Accordingly, the local vector $v_{5}^{s}$ in step 12 of Algorithm (18) takes the following form

$$
\begin{aligned}
v_{5}^{s} & =\mathcal{P}^{s}+v_{4}^{s}, \\
& =\mathcal{B}_{r}^{s T} \mathcal{P}+\mathcal{S}_{r c}^{s} \mathcal{B}_{c}^{s}\left[\bar{F}_{c c}\right]^{-1} \sum_{s=1}^{n_{s}} \mathcal{B}_{c}^{s T} \mathcal{S}_{c r}^{s}\left[\mathcal{S}_{r r}^{s}\right]^{-1} \mathcal{B}_{r}^{s T} \mathcal{P}
\end{aligned}
$$

It follows that in step 13 of Algorithm (18), the local vector $\mathcal{Q}^{s}$ can be recast as

$$
\begin{aligned}
\mathcal{Q}^{s} & =\left[\mathcal{S}_{r r}^{s}\right]^{-1} v_{5}^{s}, \\
& =\left[\mathcal{S}_{r r}^{s}\right]^{-1} \mathcal{B}_{r}^{s T} \mathcal{P}+\left[\mathcal{S}_{r r}^{s}\right]^{-1} \mathcal{S}_{r c}^{s} \mathcal{B}_{c}^{s}\left[\bar{F}_{c c}\right]^{-1} \sum_{s=1}^{n_{s}} \mathcal{B}_{c}^{s T} \mathcal{S}_{c r}^{s}\left[\mathcal{S}_{r r}^{s}\right]^{-1} \mathcal{B}_{r}^{s T} \mathcal{P} .
\end{aligned}
$$

Finally, the global vector $\mathcal{Q}$ in step 14 of Algorithm (18) representing the the matrixvector product $\mathcal{Q}=\left(\bar{F}_{r r}+\bar{F}_{r c}\left[\bar{F}_{c c}\right]^{-1} \bar{F}_{c r}\right) \mathcal{P}$ is computed as

$$
\begin{aligned}
\mathcal{Q} & =\sum_{s=1}^{n_{s}} \mathcal{B}_{r}^{s} \mathcal{Q}^{s} \\
& =\left(\sum_{s=1}^{n_{s}} \mathcal{B}_{r}^{s}\left[\mathcal{S}_{r r}^{s}\right]^{-1} \mathcal{B}_{r}^{s T}+\sum_{s=1}^{n_{s}} \mathcal{B}_{r}^{s}\left[\mathcal{S}_{r r}^{s}\right]^{-1} \mathcal{S}_{r c}^{s} \mathcal{B}_{c}^{s}\left[\bar{F}_{c c}\right]^{-1} \sum_{s=1}^{n_{s}} \mathcal{B}_{c}^{s T} \mathcal{S}_{c r}^{s}\left[\mathcal{S}_{r r}^{s}\right]^{-1} \mathcal{B}_{r}^{s T}\right) \mathcal{P}
\end{aligned}
$$

The probabilistic coarse problem in step 7 of Algorithm (18) is solved in parallel using PCGM with the extended Lumped preconditioner as

$$
\mathcal{M}_{c c} \bar{F}_{c c} V_{3}=\mathcal{M}_{c c} V_{2}
$$

where

$$
\mathcal{M}_{c c}=\sum_{s=1}^{n_{s}} \mathcal{B}_{c}^{s T} \mathcal{A}_{c c}^{s} \mathcal{B}_{c}^{s}
$$


A flow-chart of the probabilistic FETI-DP algorithm is shown in Fig.(8.3)

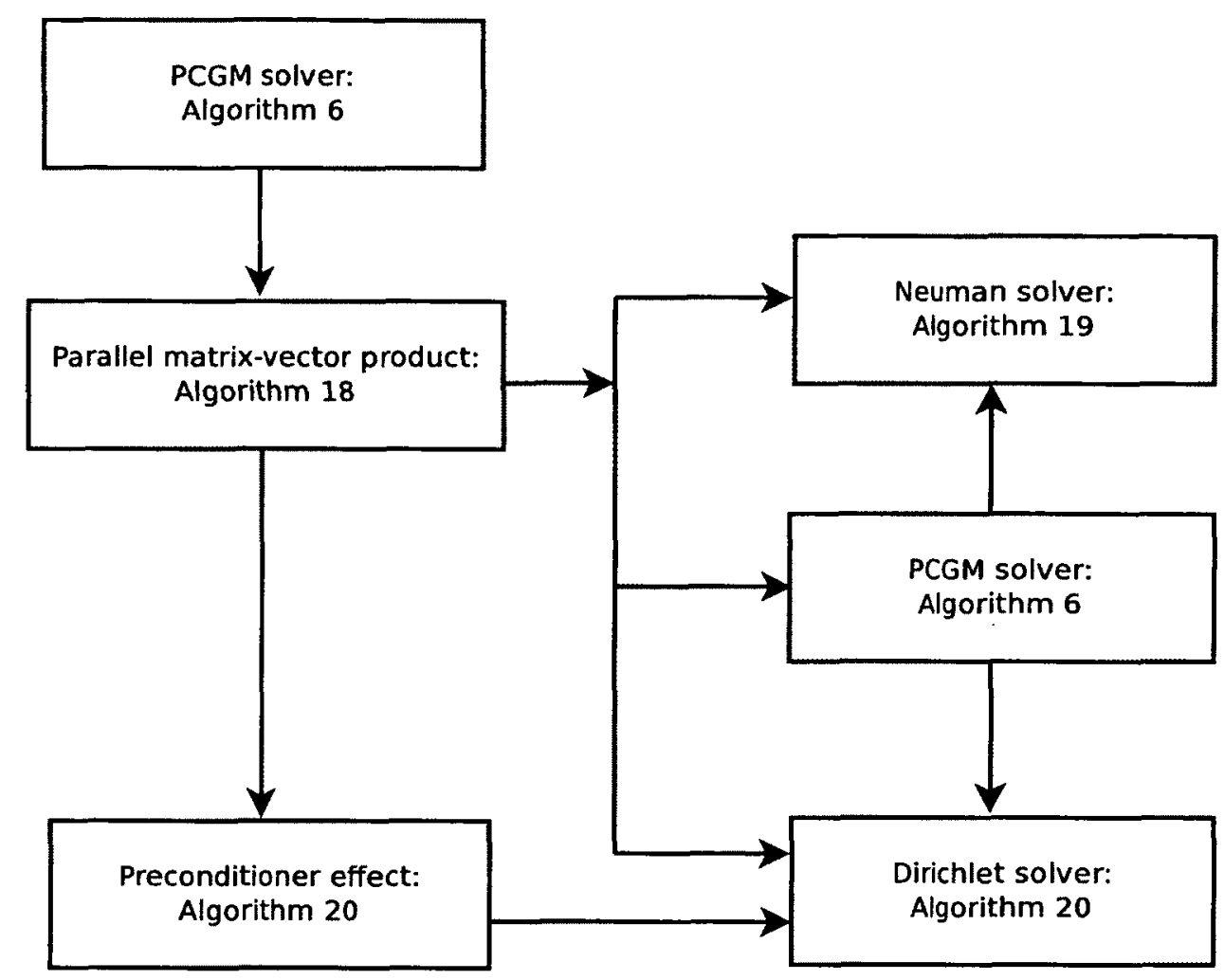

Figure 8.3: Flow chart of parallel implementation of FETI-DP algorithm

\subsection{Summary}

A novel dual-primal iterative substructuring technique is formulated to tackle the coupled deterministic linear systems in the intrusive spectral stochastic finite element discretization of large-scale SPDEs. In the dual-primal approach for SPDEs, the continuity of the polynomial chaos coefficients is strictly enforced at the interface corner nodes at each iteration of PCGM. For the remaining nodes on the interface, the Lagrange multipliers are used to weakly satisfy the continuity condition of the chaos coefficients during the PCGM 
iterations. The continuity of the polynomial chaos coefficients is satisfied along the entire interface as the PCGM converges. The proposed method may be viewed as the probabilistic version of FETI-DP. 


\section{Chapter 9}

\section{Numerical Results: Two-Dimensional}

\section{Problems}

\subsection{Introduction}

The numerical results presented in this chapter are from the references $[75,77,86]$. One of the most attractive features of domain decomposition method is its ability to offer parallel scalable algorithms $[7,72]$. The iterative algorithm is said to be scalable when its convergence rate does not deteriorate as the number of subdomains increases (or the size of subdomain $H$ decreases) $[4,5,7,64]$. On the other hand, the iterative algorithm is said to be optimal when its convergence rate is independent of the mesh size $h[4,5]$. However, the iterative algorithm is said to be quasi-optimal when its convergence rate only dependents on the ratio of the subdomain size and element size $(H / h)$ (i.e. the number of unknowns associated with one subdomain [4]). It worth emphasizing the fact that an optimal or scal- 
able preconditioner does not necessarily mean the computationally fastest preconditioner or a preconditioner with minimum computational complexity for a given problem [72].

To this end, we measure the numerical scalability of the algorithms: (a) firstly with respect to mesh size $h$ whereby we fix the number of subdomains used to solve the problem and increase the spatial mesh resolution; (b) secondly, with respect to subdomain size $H$ whereby we fix the spatial problem size and increase number of subdomains used to tackle the problem; (c) thirdly, with respect to the ratio of the number of elements to the number of subdomains $(H / h)$ whereby we fix problem size per subdomain and increase the overall problem size by increasing the number of subdomains. For all these scalability measurements, we report the results for different order of PCE to measure the sensitivity of the algorithms to the order of $\mathrm{PC}$ representation.

The absolute performance or the parallel scalability of the algorithms is measured in terms of the strong and weak scalability $[61,142]$. For the strong scalability test, we fix the global problem size and increase the number of CPUs used to solve the problem in order to reduce the total execution time. For the weak scalability test, we fix the problem size per subdomain and increase the global size of the problem by increasing the number of subdomains whereby the goal is to solve $n$-times larger problem using $n$-times larger computer (processors or cores) in nearly a constant CPU time.

For the numerical implementations, the PCGM solver is initialized with $\mathcal{U}_{\Gamma_{0}}=0$ and the iterations are terminated when the ratio of the $L_{2}$ norms of the current residual to the 
initial residual is less than $10^{-6}$ defined as

$$
\frac{\left\|\mathcal{G}_{\Gamma}-\mathcal{S} \mathcal{U}_{\Gamma}^{k}\right\|_{2}}{\left\|\mathcal{G}_{\Gamma}-\mathcal{S U}_{\Gamma_{0}}\right\|_{2}} \leqslant 10^{-6} .
$$

For efficient memory usage and minimum floating point operation, the numerical implementation of the algorithm exploits the multi-level sparsity structure of the coefficient matrix of the stochastic system, namely (a) the sparsity structure due to the finite element discretization and (b) the block sparsity structure due to the orthogonal representation and projection of the stochastic processes. PETSc [87] and MPI [88] parallel libraries are used for efficient parallel implementation of the proposed algorithm. The graph partitioning tool METIS [89] is used for optimal decomposition of the finite element mesh. The finite element meshes are generated using Gmsh software [143].

Firstly, we investigate the parallel performance of the one-level domain decomposition preconditioners, namely the extended Lumped (L), Weighted Lumped (WL) and NeumannNeumann (NN) domain decomposition preconditioners for flow through random media with spatially varying non-Gaussian random parameters. Secondly, we contrast the numerical and parallel scalabilities of the one-level preconditioner (NN) with the two-level preconditioner (NNC) for flow through random media and elasticity problems with spatially varying non-Gaussian material properties. Thirdly, we compare the convergence properties, numerical and parallel scalabilities of the two-level preconditioner (NNC) with probabilistic FETI-DP method using illustrations from flow through random media and linear elasticity with spatially varying non-Gaussian material properties. 
The numerical experiments are performed on a Linux cluster (SUSE Linux Enterprise Server $11 \mathrm{SP} 1$ ) consists of 22 nodes with an InfiniBand interconnect (2 Quad-Core $3.0 \mathrm{GHz}$ Intel Xeon processors and $32 \mathrm{~GB}$ of memory per node). PETSc version 2.3.3 and OpenMPI version 1.4.3 libraries are compiled with GNU compiler GCC version 4.3.4 without any optimization flags.

\subsection{One-Level Preconditioner}

In this section, we study the performance of the one-level extended preconditioners $(\mathrm{L}, \mathrm{WL}$ and NN) with respect to the level of uncertainty, the order of stochastic dimension, the spatial problem size, number of subdomains and fixed problem size per subdomain.

\subsubsection{Flow Through Random Media}

For the numerical illustrations, we consider a two-dimensional steady-state flow through random media with random hydraulic conductivity coefficient defined as

$$
\begin{aligned}
\nabla \cdot(\kappa(\mathbf{x}, \theta) \nabla u(\mathbf{x}, \theta)) & =f(\mathbf{x}, \theta), \quad \mathbf{x} \in \Omega \\
u(\mathbf{x}, \theta)) & =0, \quad \mathbf{x} \in \partial \Omega,
\end{aligned}
$$

where the coefficient $\kappa(\mathbf{x}, \theta)$ is modeled as a lognormal stochastic process obtained from underlying Gaussian process with an exponential covariance function and the forcing term is assumed to be a deterministic quantity defined as $f(\mathbf{x}, \theta)=1.0$. 


\section{Finite Element Discretization and Mesh Decomposition}

The maximum size of the finite element mesh considered consists of 512,154 elements and 256,081 nodes. The response is expanded using four-dimensional ( $L=3$ ) third order PCE $(N=34)$ leading to a linear system of order $8,962,835$. Typical finite element mesh is depicted in Fig.(9.1(a)). The computational domain partitioned into 160 subdomains is plotted in Fig.(9.1(b)).

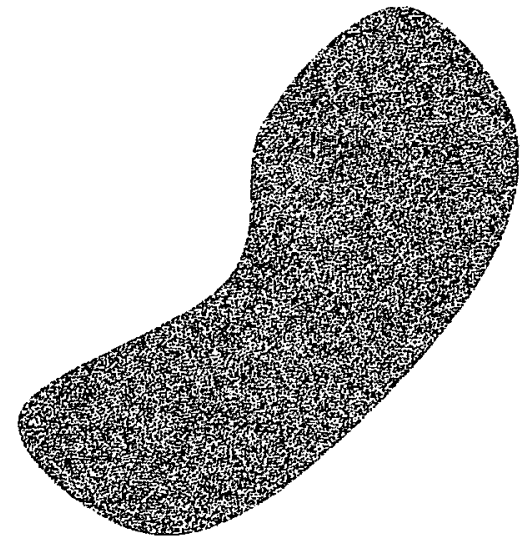

(a) FEM mesh

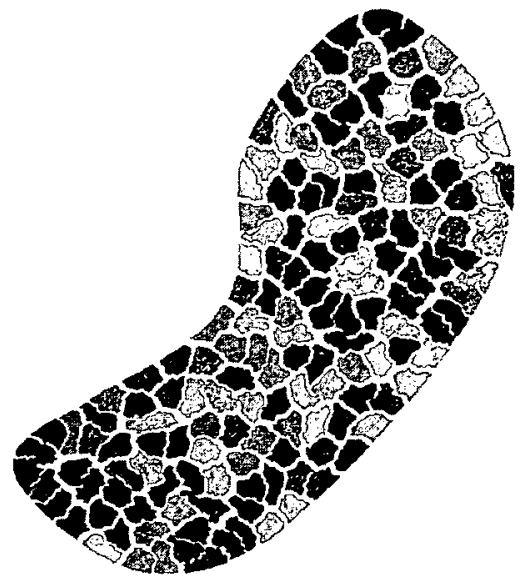

(b) Domain Decomposition

Figure 9.1: Finite element discretization and mesh decomposition

\section{Stochastic Field Representation}

The hydraulic conductivity coefficient $\kappa(\mathbf{x}, \theta)$ is modeled as a lognormal stochastic process obtained from the underlying Gaussian process with an exponential covariance function given by

$$
C_{\alpha \alpha}(\mathbf{x}, \mathbf{y})=\sigma^{2} \exp \left(\frac{-\left|x_{1}-y_{1}\right|}{b_{1}}+\frac{-\left|x_{2}-y_{2}\right|}{b_{2}}\right)
$$


where the numerical values used in the investigation are $\sigma=0.3$ and $b_{1}=b_{2}=1.0$.

The generalized eigenvalue problem associated with KLE decomposition is solved using FEM with a coarse mesh consists of 21,800 elements and 10,904 nodes. Fig.(9.2) shows the monotonic decay of the eigenvalues of the exponential covariance function. The first fourth eigenvalues correspond to about $90 \%$ energy of the random process. Fig.(9.3) shows the eigenfunctions associated with the first four eigenvalues. Fig.(9.4) shows some selected PC coefficients of the lognormal process.

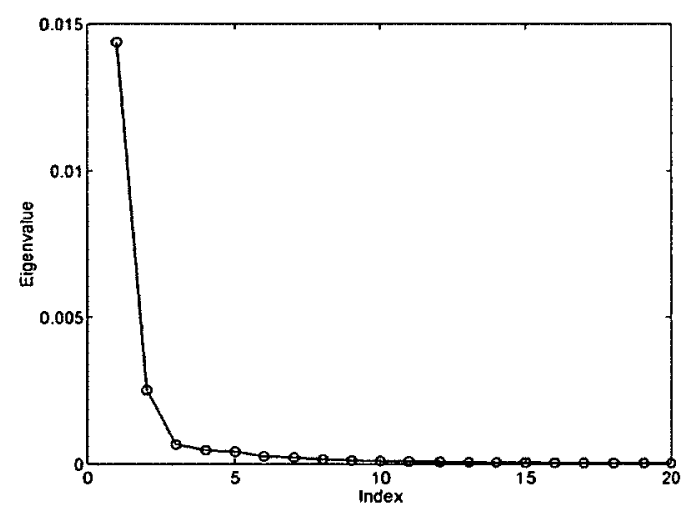

Figure 9.2: The decay of the eigenvalues of the exponential covariance function 

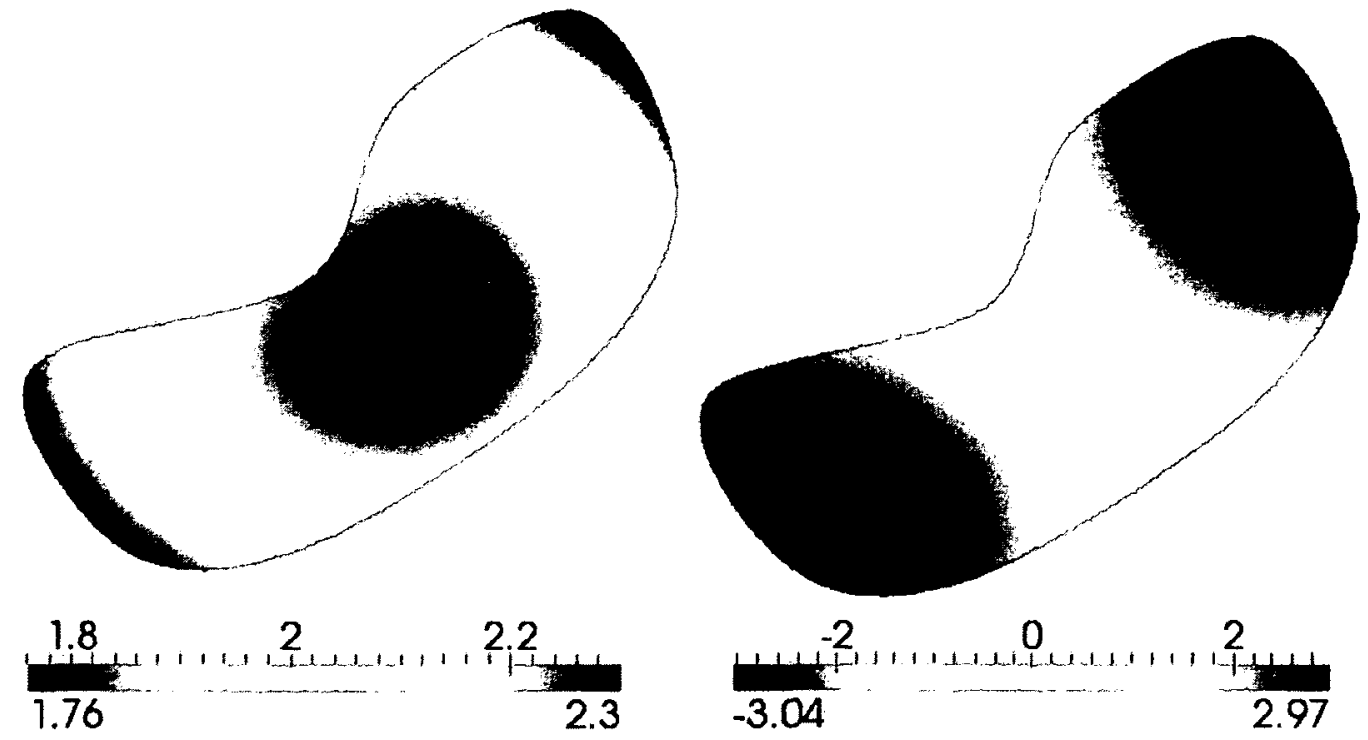

(a) $1 \mathrm{st}$

(b) 2nd

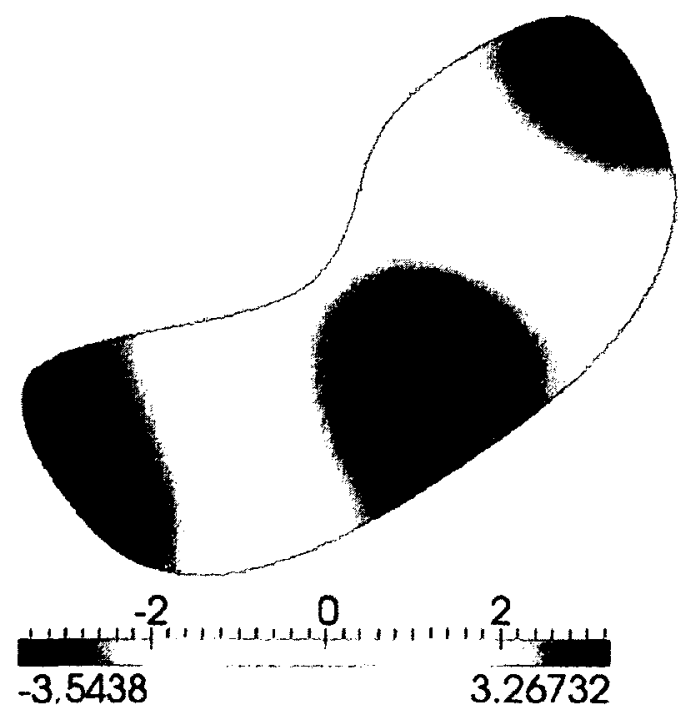

(c) 3rd

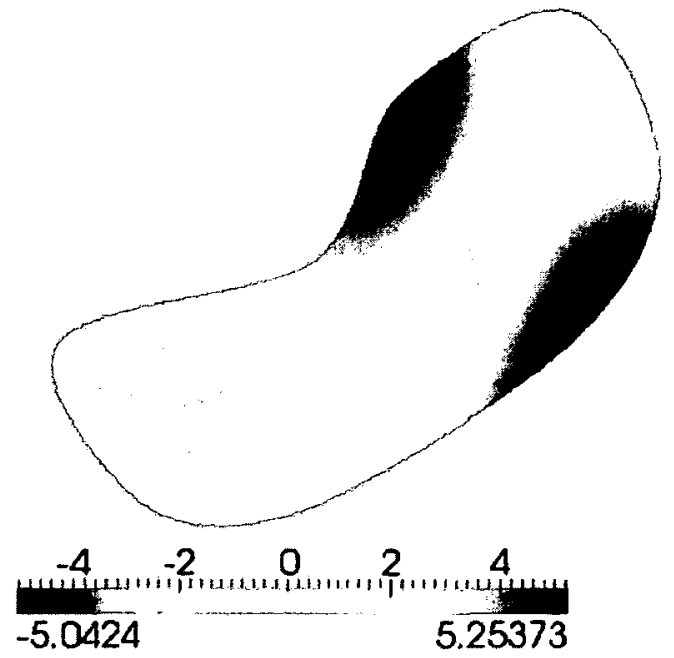

(d) 4 th

Figure 9.3: The first fourth eigenfunctions of the exponential covariance function 

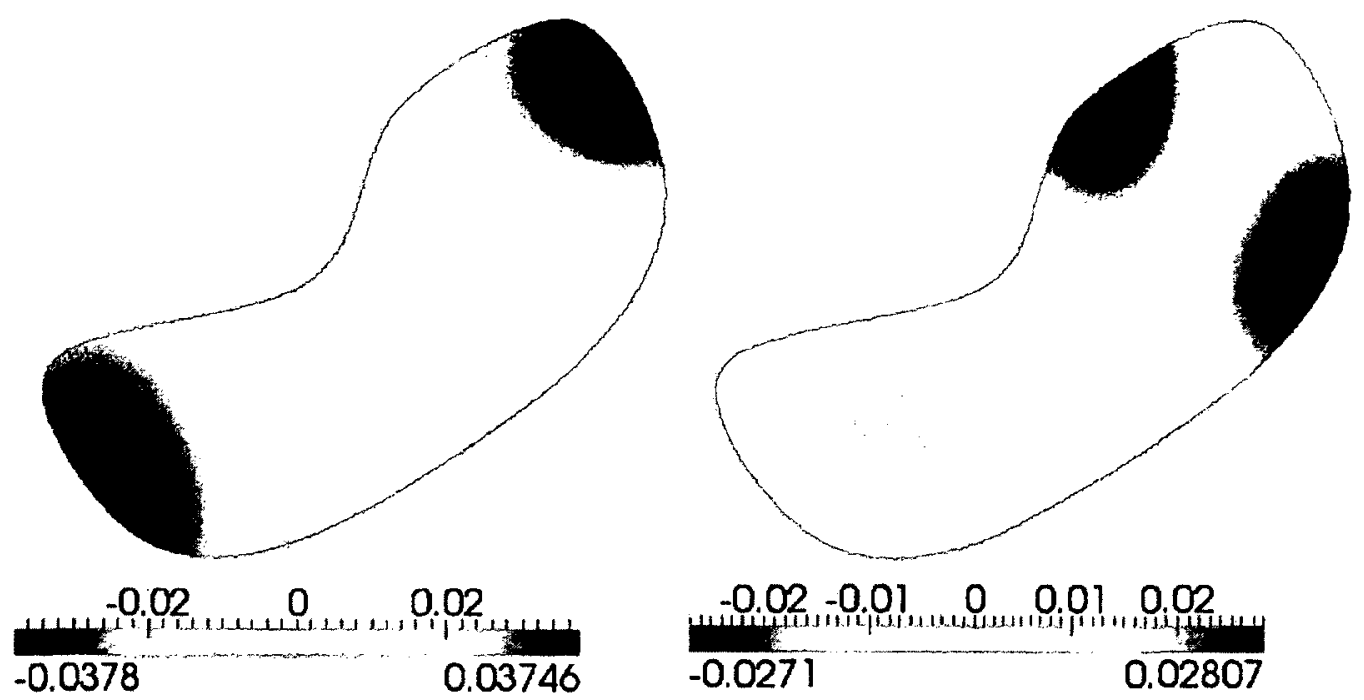

(a) Chaos coefficients: $l_{11}$

(b) Chaos coefficients: $l_{12}$
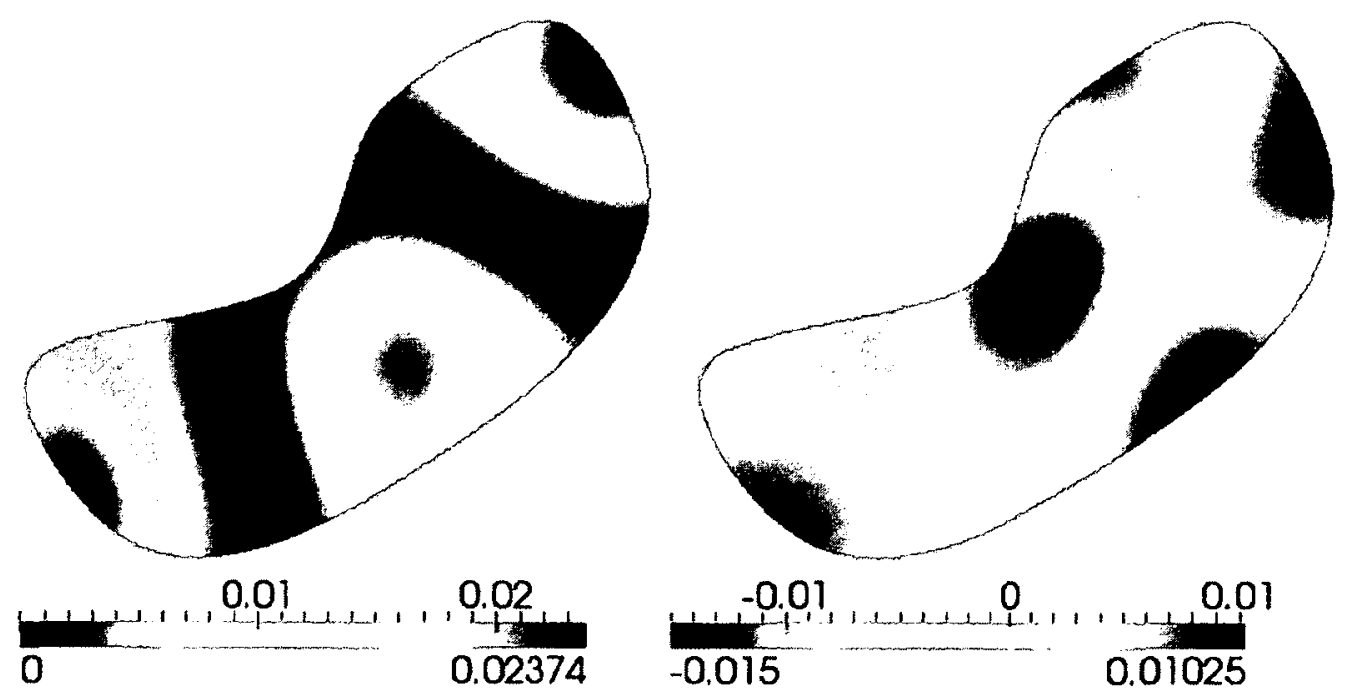

(c) Chaos coefficients: $l_{13}$

(d) Chaos coefficients: $l_{14}$

Figure 9.4: Selected PC coefficients of the hydraulic conductivity 


\section{Stochastic Characteristics of the Solution Process}

The mean and standard deviation of the hydraulic head are plotted in Fig.(9.5(a)) and Fig.(9.5(b)) respectively. The maximum coefficient of variation $(\mathrm{CoV})$ of the solution field is about $25 \%$ underscoring the effect of uncertainty. Some of the chaos coefficients of the solution process are shown in Fig.(9.6). Although the response is a positive quantity, some of these chaos coefficients have negative amplitudes.

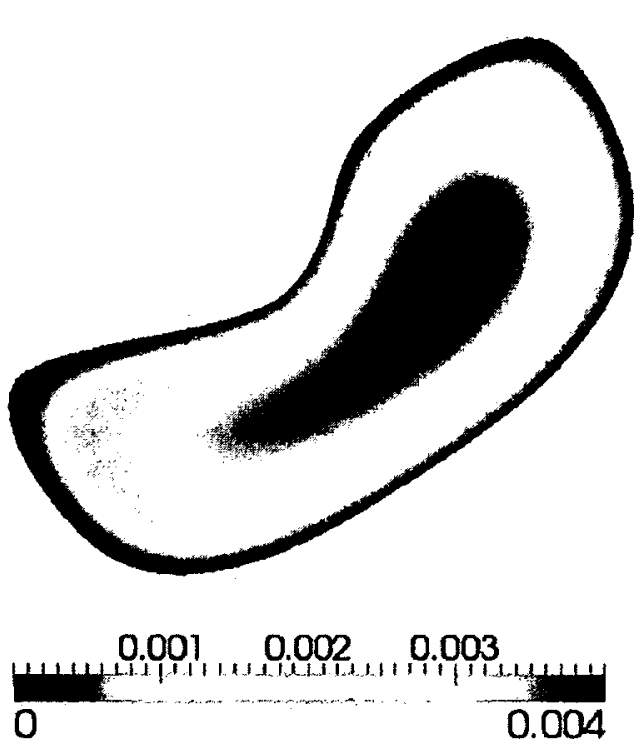

(a) Mean response
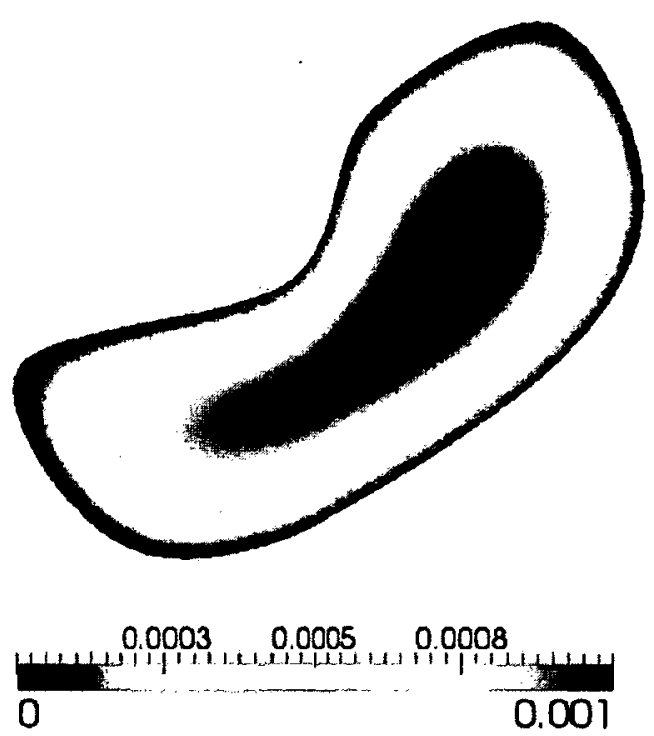

(b) Standard deviation

Figure 9.5: The mean and the standard deviation of the hydraulic head 


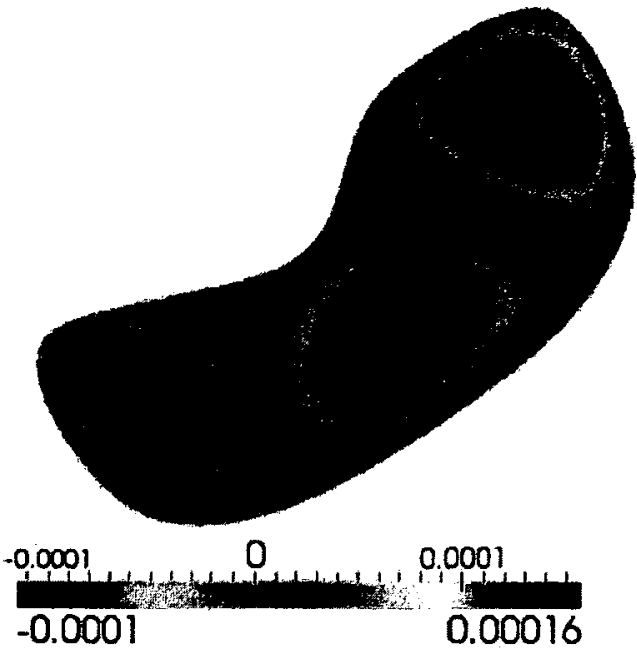

(a) Chaos coefficients: $\mathbf{u}_{4}$

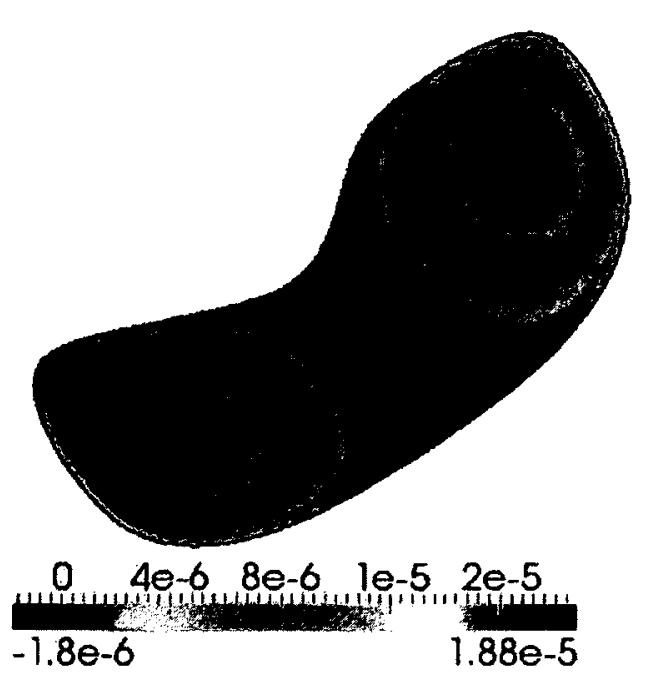

(c) Chaos coefficients: $\mathbf{u}_{10}$

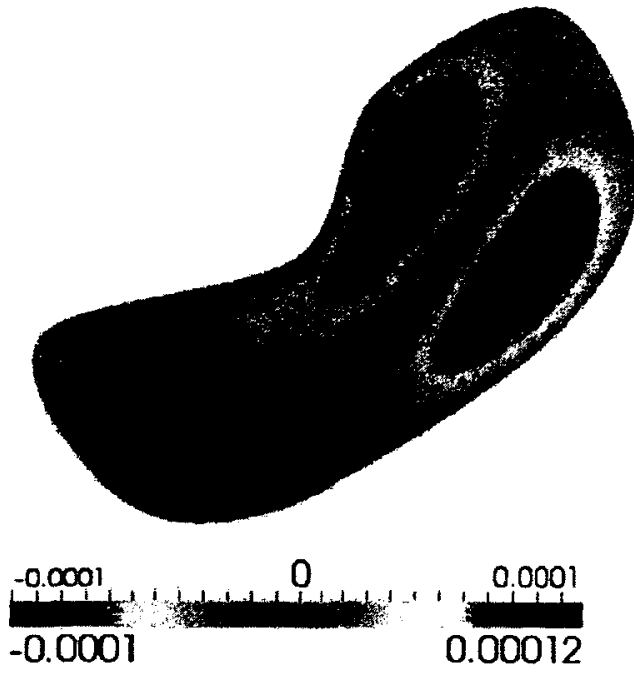

(b) Chaos coefficients: $\mathbf{u}_{5}$
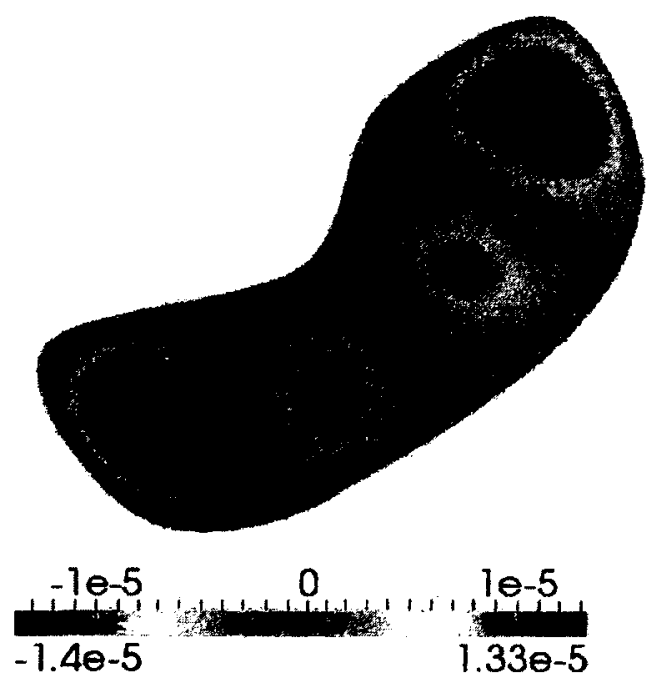

(d) Chaos coefficients: $\mathbf{u}_{\mathbf{1 1}}$

Figure 9.6: Selected PC coefficients of the hydraulic head 


\section{Solution Convergence}

Typical relative residual decay of PCGM equipped with Extended Lumped (L), Weighted Lumped (WL) and Neumann-Neumann (NN) preconditioners are plotted in Fig.(9.7). The WL preconditioner shows better convergence rate than the preliminary $\mathrm{L}$ preconditioner. This fact highlights the benefit of the weighting matrix in accelerating the convergence rate of the WL preconditioner. The NN preconditioner shows the fastest convergence rate with a smooth decay of the relative residual.

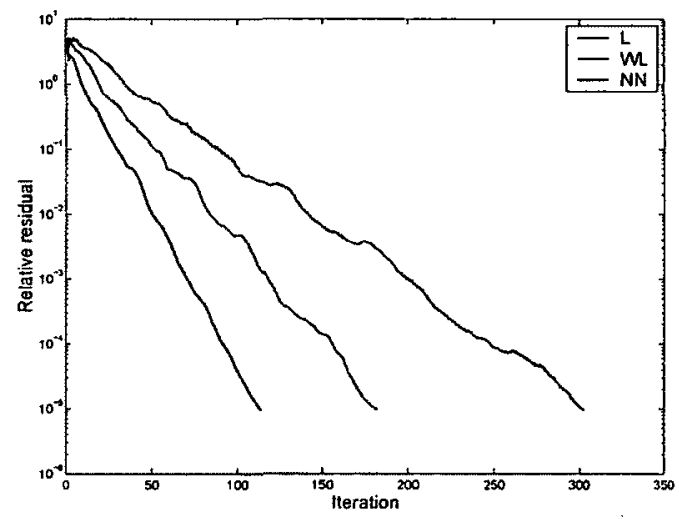

Figure 9.7: The history of the relative PCGM residual

\section{Effect of The Coefficient of Variation}

For a fixed problem size $n=16,808$, fixed number of subdomains $n_{s}=16$ and fixed order of PCE $N=15$, we investigate the performance of the one-level preconditioners with respect to the coefficient of variation $(\mathrm{CoV})$ of the diffusion coefficient. In Fig.(9.8), the PCGM iteration counts versus $\mathrm{CoVs}$ are plotted for various preconditioners. Increasing the $\mathrm{CoV}$ of the system parameter up to $60 \%$ does not degrade the performance of $\mathbf{L}$ and NN preconditioners as the number of PCGM iteration is nearly constant. For the WL 
preconditioner, we notice a slight growth in the iteration counts as the $C o V$ increases.

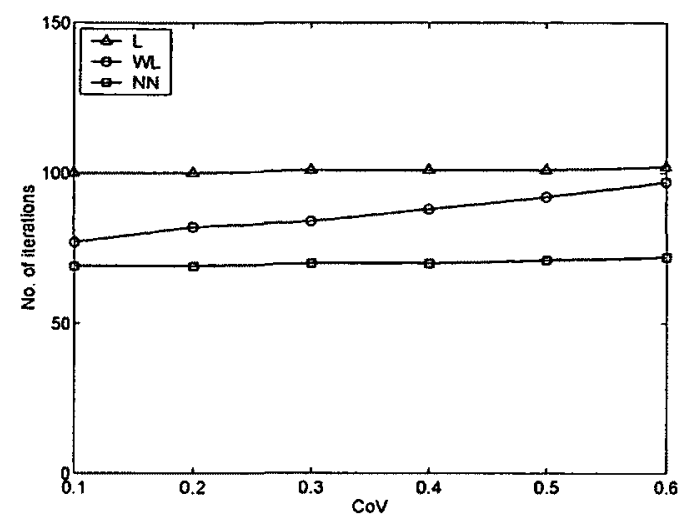

Figure 9.8: Iteration counts with respect to the $\mathrm{CoV}$

\section{Numerical scalability}

The scalability is measured (e.g. $[6,64,142]$ ) (a) firstly with respect to the size of the mesh $h$ whereby we fix the number of subdomains and increase the spatial mesh resolution; (b) secondly, with respect to subdomain size $H$ whereby we fix the spatial problem size and increase number of subdomains used to tackle the problem; (c) thirdly, with respect to the ratio of the number of elements to that of subdomains $(H / h)$ whereby we increase the total problem size by increasing the number of subdomains while maintaining a fixed problem size per subdomain. For these scalability tests, we report the results for different order of PCE to measure the sensitivity of the algorithms to the order of PC representation.

\section{Scalability with respect to mesh size $(h)$}

For this scalability study, the number of subdomains is fixed to 128 , the finite element mesh resolution is refined. Fig.(9.9) depicts the results for the one-level preconditioners for the 
first, second and third PCEs. Fig.(9.10), the same results are plotted again for L, WL and NN preconditioners separately against various PCEs. In general, increasing mesh resolution leads to nearly linear increase in iteration counts of PCGM solver. The NN preconditioner requires less number of iteration than those required by $\mathrm{L}$ and $\mathrm{WL}$ preconditioners. In contrast to $\mathrm{WL}$ preconditioner, the performances of $\mathrm{L}$ and $\mathrm{NN}$ preconditioners are nearly independent of the order of PCE as the numbers of iterations remain almost constant for a given mesh size. For a spatial problem size of order $n$, the first, second and third order PCE involve solving problem sizes of order $(5 \times n),(15 \times n)$ and $(35 \times n)$ respectively. 


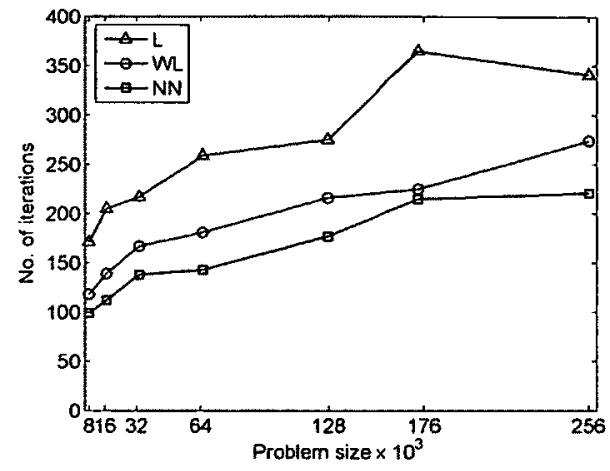

(a) 1st order PCE

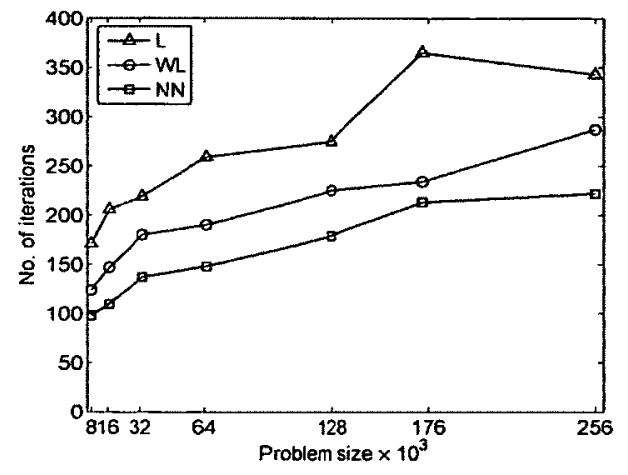

(b) 2nd order PCE

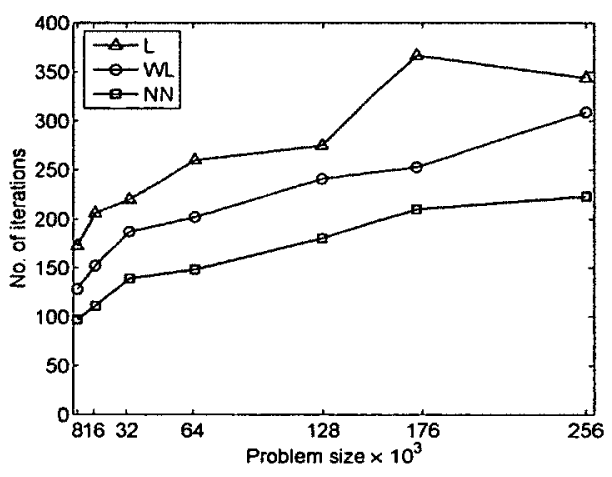

(c) 3rd order PCE

Figure 9.9: Iteration counts with respect to mesh size for fixed number of subdomains: Different order of PCE 


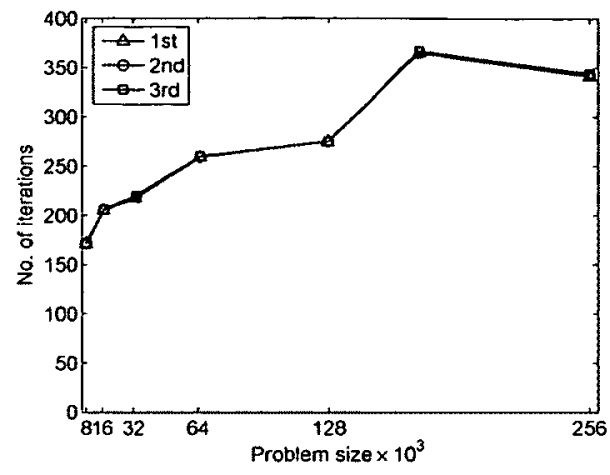

(a) $\mathrm{L}$

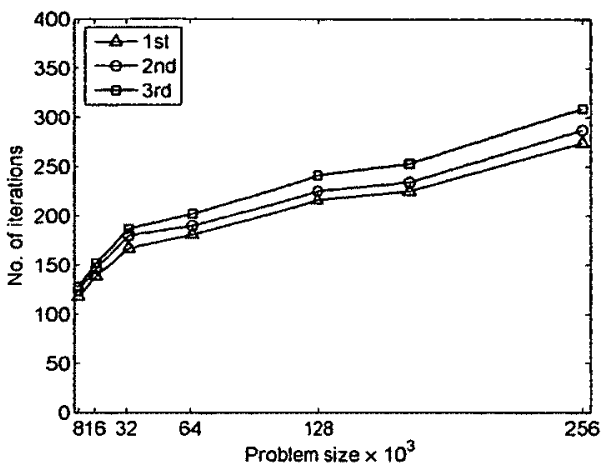

(b) WL

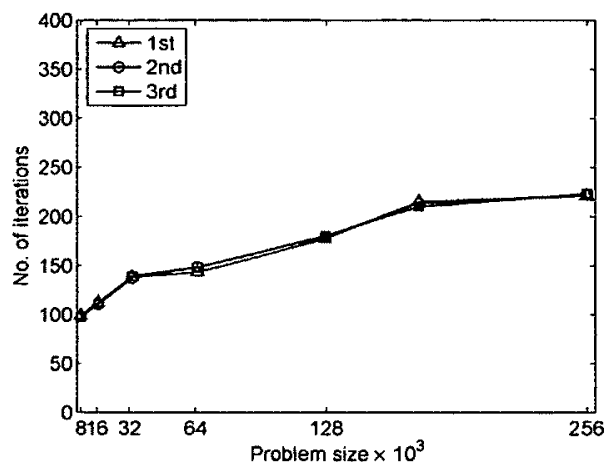

(c) NN

Figure 9.10: Iteration counts with respect to mesh size for fixed number of subdomains: Different preconditioners

\section{Scalability with respect to the number of subdomains $(H)$}

Next we maintain the problem size in the spatial dimension to $n=127,552$ while increasing the number of subdomains. In Fig.(9.11), we present the results for the first, second and third order PCEs for L, WL and NN preconditioners. In Fig.(9.12), the same results are plotted separately for various orders of PCE for $\mathrm{L}, \mathrm{WL}$ and $\mathrm{NN}$ preconditioners. As 
the number of subdomains is increased, the iteration counts grow linearly for the one-level preconditioners. The NN preconditioner demands less number of iterations compared to $\mathrm{L}$ and WL preconditioners.

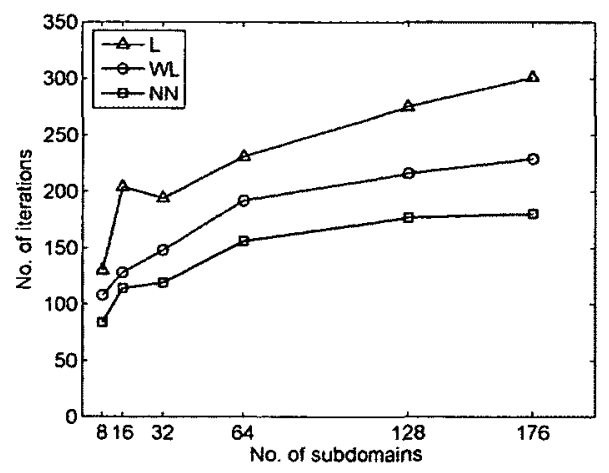

(a) 1st order PCE

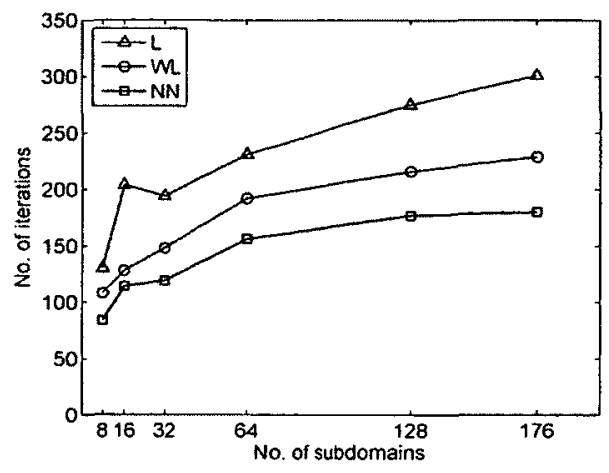

(b) 2nd order PCE

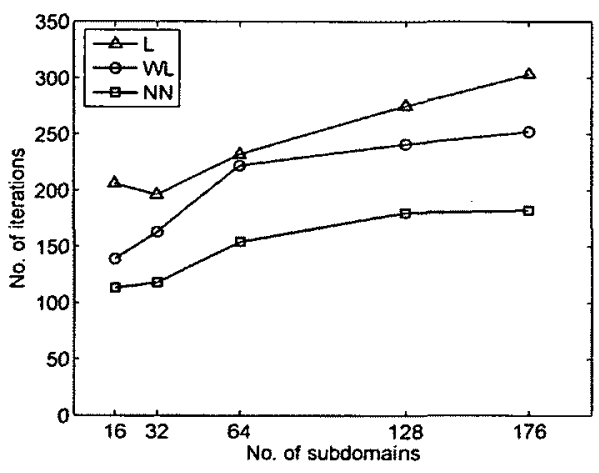

(c) 3rd order PCE

Figure 9.11: Iteration counts with respect to the number of subdomains for fixed problem size: Different order of PCE 


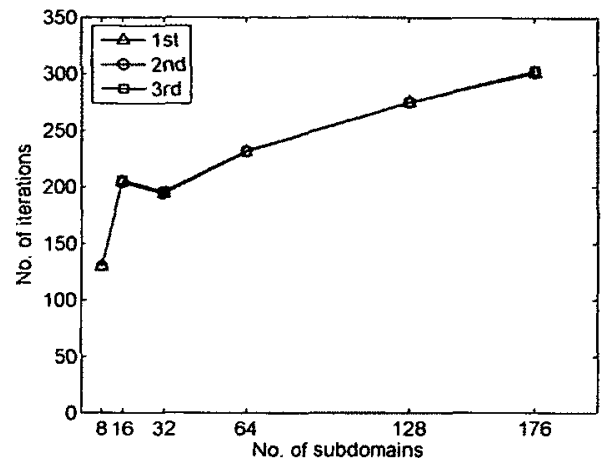

(a) $\mathrm{L}$

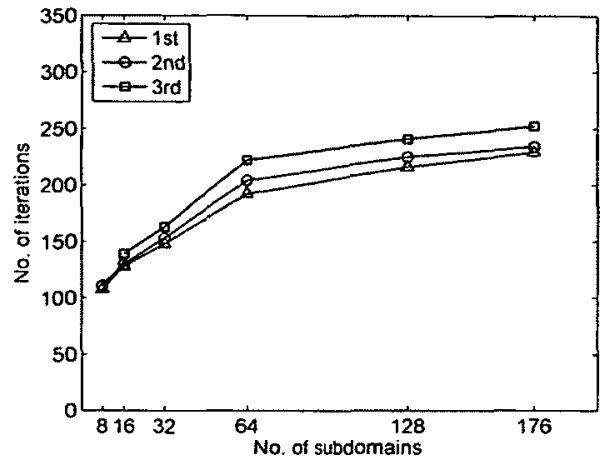

(b) WL

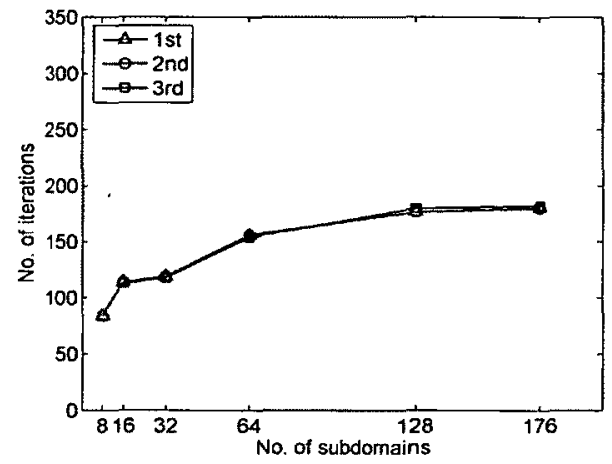

(c) $\mathrm{NN}$

Figure 9.12: Iteration counts with respect to the number of subdomains for fixed problem size: Different preconditioners

\section{Scalability with respect to fixed problem size per subdomain $(H / h)$}

Fig.(9.13) demonstrates the performance of the one-level preconditioners when the problem size per subdomain remains the same, but the total problem size increases due to the additional subdomains for various orders of PCE. The same results are plotted again for L, WL and NN preconditioners separately in Fig.(9.14). These performance results indi- 
cate that for fixed problem size per subdomain, the number of iterations of the one-level preconditioners increases almost linearly as the number of subdomains is increased.

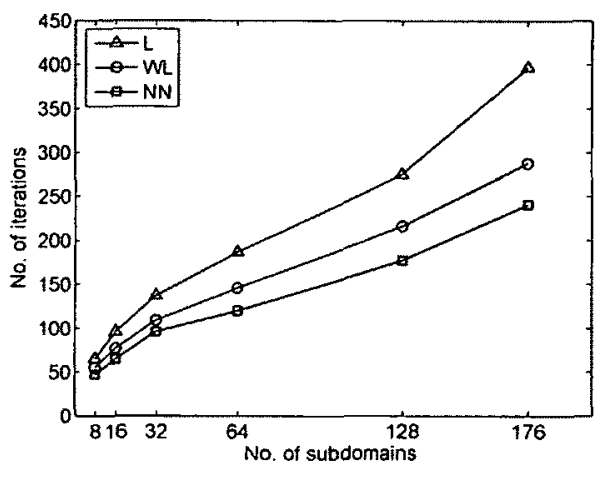

(a) 1st order PCE

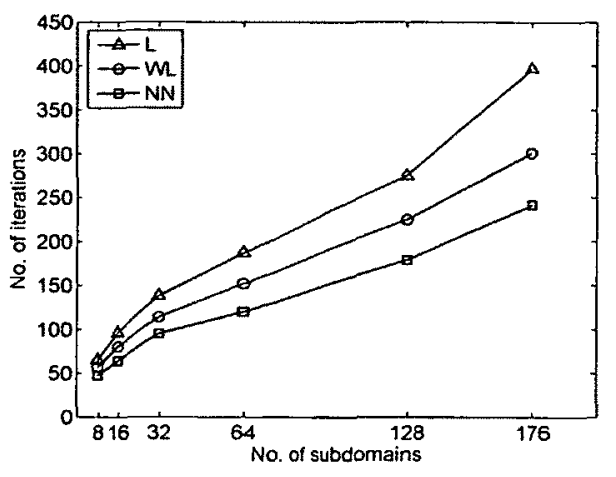

(b) 2nd order PCE

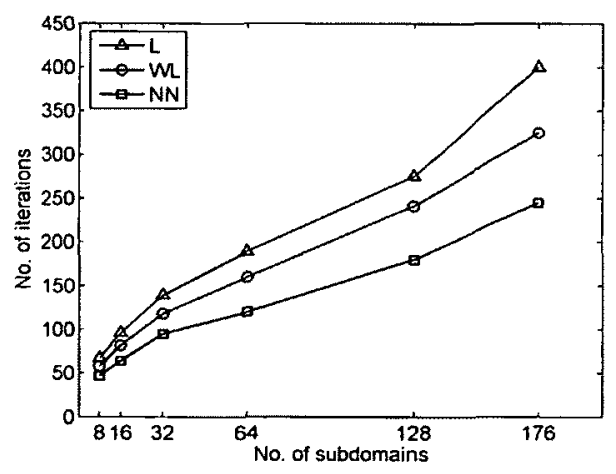

(c) 3rd order PCE

Figure 9.13: Iteration counts with respect to the number of subdomains for fixed problem size per subdomain: Different order of PCE 


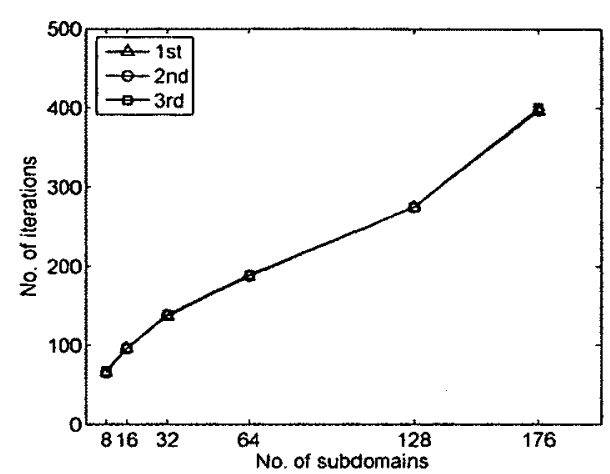

(a) $\mathrm{L}$

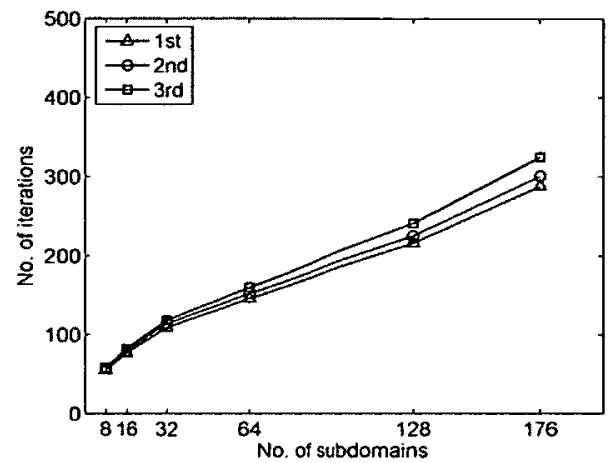

(b) WL

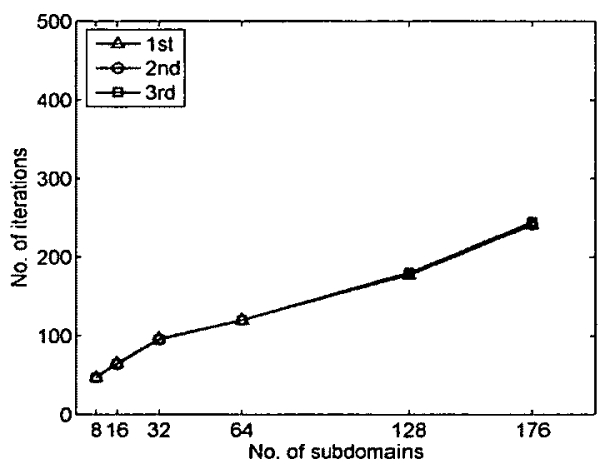

(c) $\mathrm{NN}$

Figure 9.14: Iteration counts with respect to the number of subdomains for fixed problem size per subdomain: Different preconditioners

\section{Parallel scalability}

In order to investigate the absolute performance of the algorithm, its parallel scalability is measured in terms of the strong and weak scalabilities [61, 142]. For the strong scalability test, we maintain a constant global problem size, but increase the number of processors/cores to tackle the problem in order to reduce the total execution time. For the 
weak scalability test, the problem size per subdomain is fixed, but the total problem size is increased by adding more subdomains. The goal of the weak scalability test is to tackle $n$-times larger system by exploiting $n$-times larger processors or cores while maintaining nearly a constant execution time (e.g. $[142,144]$.

\section{Strong scalability}

Fig.(9.15) shows the total execution times for the one-level preconditioners for various numbers of processors. The results are presented for the first, second and third order of PCE. Increasing number of processors greatly reduces the total execution time. For the specific problem size considered here, the optimal number of CPUs is 64 in the case of the first order PCE. In this case, if the number of CPUs is increased further, the communication overhead predominates over the cost of floating point operation. For the second and third order PCEs, the execution time steadily decreases up to 176 CPUs. This is because the computational cost consistently dominates the communication cost while tackling the system of increasingly larger size. 


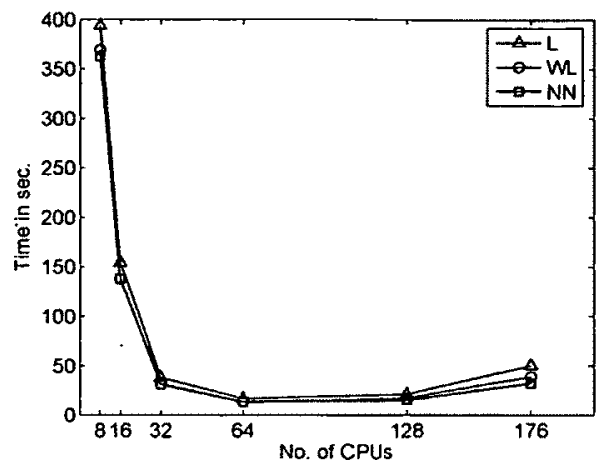

(a) 1st order PCE

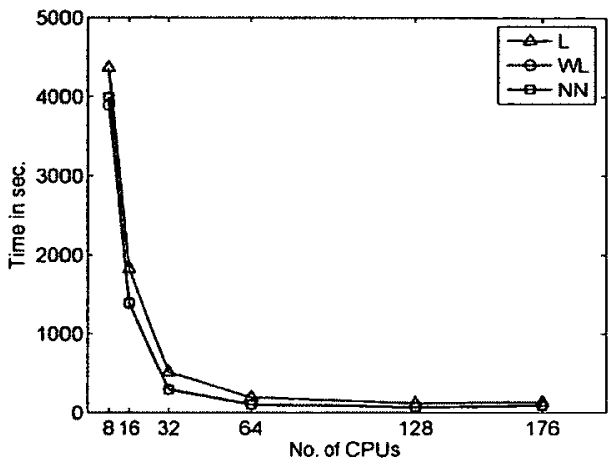

(b) 2nd order PCE

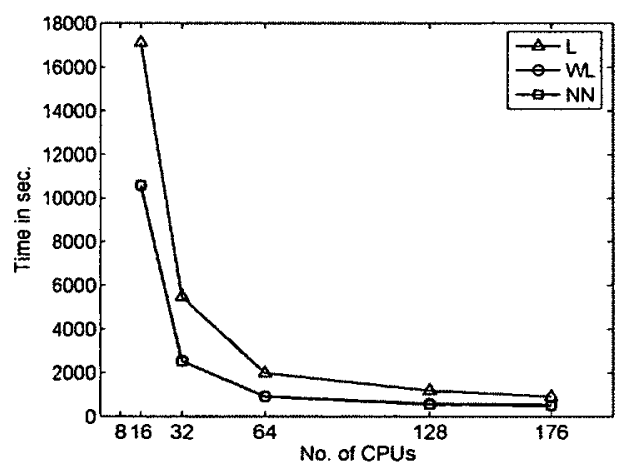

(c) 3rd order PCE

Figure 9.15: Strong scalability

\section{Weak scalability}

Fig.(9.16) shows the weak scalability of the one-level preconditioners for the first, second and third order PCEs. The results indicate that for the fixed problem size per processor, the total execution time grows almost linearly while using larger number of processors. The $\mathrm{NN}$ and WL preconditioners exhibit better performance than $\mathrm{L}$ preconditioner. 


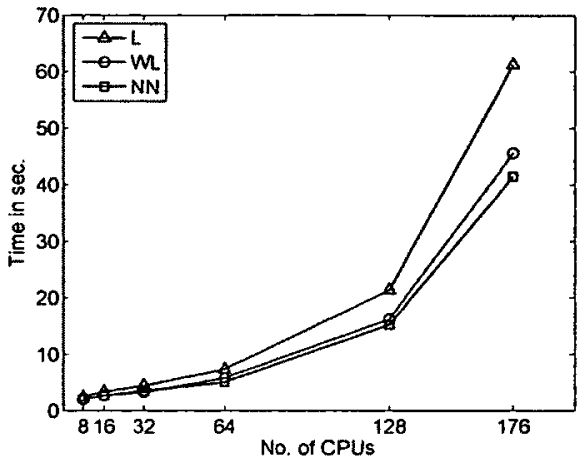

(a) 1st order PCE

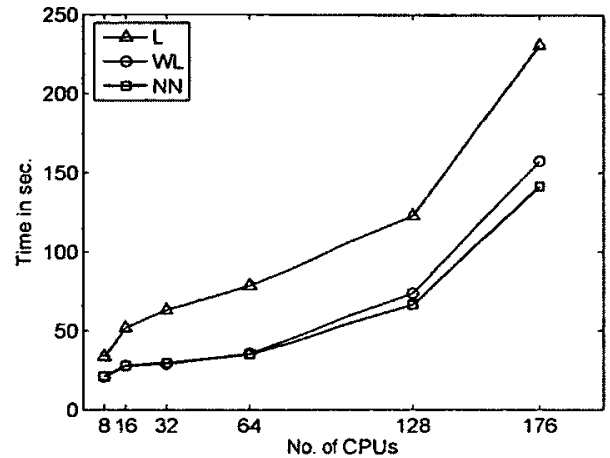

(b) 2nd order PCE

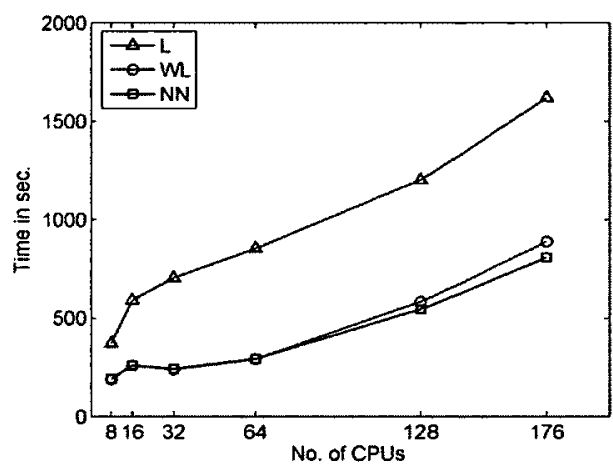

(c) 3rd order PCE

Figure 9.16: Weak scalability

\subsection{Two-Level Preconditioner}

In this section, we contrast the algorithmic performance of the one-level Neumann-Neumann preconditioner $(\mathrm{NN})$ and two-level Neumann-Neumann preconditioner (NNC) to highlight the beneficial effect of the coarse problem. For the numerical investigation, we consider flow through random media and linear elasticity problems with spatially varying nonGaussian material properties. 


\subsubsection{Flow Through Random Media}

\section{Convergence Study}

A typical relative residual decay of PCGM equipped with NN and NNC preconditioners are shown in Fig.(9.17). Evidently, the PCGM equipped with NNC preconditioner converges in fewer iterations compared to that of the NN preconditioner.

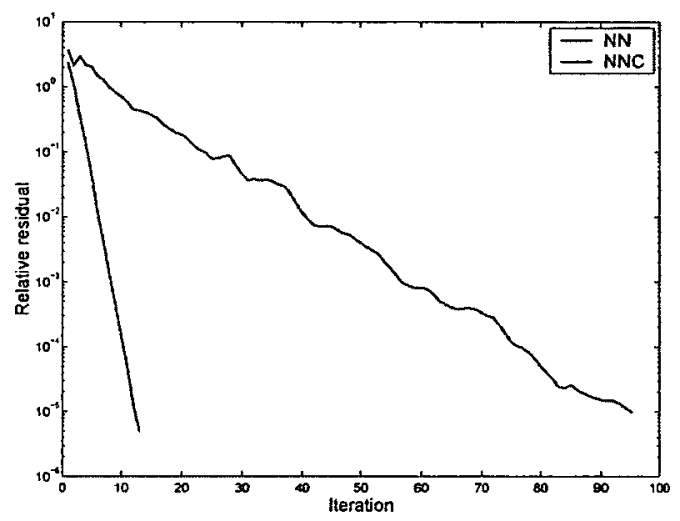

Figure 9.17: The history of the relative PCGM residual

\section{Numerical Scalability}

For the numerical scalability study, we report the iteration counts of the PCGM while (a) decreasing the finite element mesh size $(h)$ for fixed number of subdomain partitions; (b) increasing number of subdomains $(1 / H)$ for fixed spatial mesh resolution (c) increasing the total problem size by including more subdomains with fixed number of elements $(H / h)$. 


\section{Scalability with respect to mesh size $(h)$}

Fig.(9.18) plots the results for the NN and NNC preconditioners for 128 subdomains and third order PCE while increasing the finite element mesh resolution. Clearly, adding a coarse problem to NN preconditioner significantly reduces the number of iterations for fixed number of subdomains. Increasing problem size does not deteriorate the performance of the NNC preconditioner as the iteration counts remain constant. This fact indicates the numerical scalability of the NNC preconditioner with respect to the problem size.

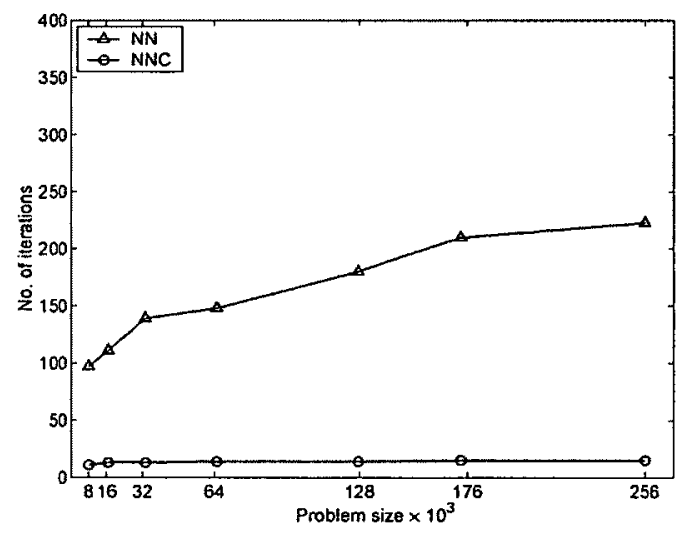

Figure 9.18: Iteration counts versus mesh size for fixed number of subdomains

\section{Scalability with respect to the number of subdomains $(H)$}

Fig.(9.19) plots the performance results of the NN and NNC preconditioners for the fixed spatial problem size and fixed order of PCE with increasing number of subdomains. The NNC preconditioner demands fewer iterations to converge compared to NN preconditioner. For the NNC preconditioner, the number of iterations does not grow with the number of subdomains. This fact indicates the numerical scalability of the NNC preconditioner in 
relation to the number of subdomains.

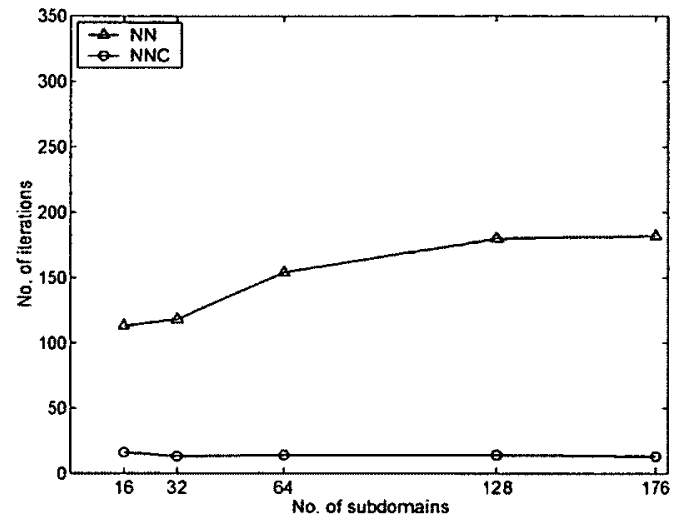

Figure 9.19: Iteration counts versus the number of subdomains for fixed problem size

\section{Scalability with respect to fixed problem size per subdomain $(H / h)$}

For fixed problem size per subdomain, the total problem size is increased next by adding more subdomains. The corresponding results are shown in Fig.(9.20). Increasing number of subdomains demonstrates a linear growth in the iteration counts for the NN preconditioner. But the iteration counts remain constant for the NNC preconditioner. This fact indicates the scalability of the NNC preconditioner in relation to the fixed problem size per subdomain. 


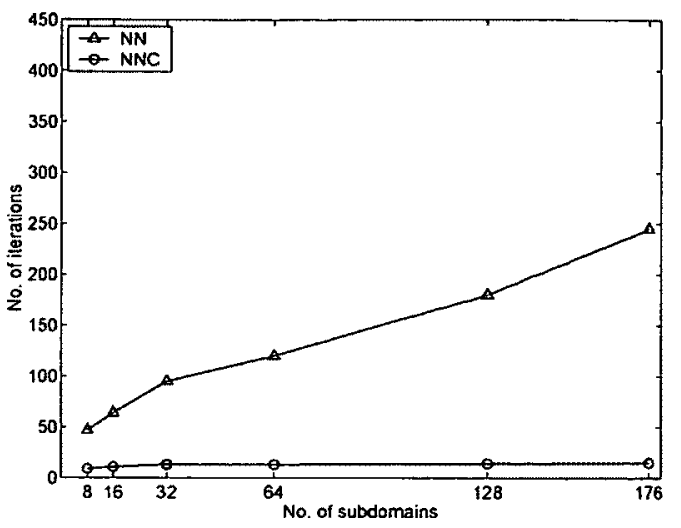

Figure 9.20: Iteration counts versus the number of subdomains for fixed problem size per subdomain

\section{Parallel Scalability}

\section{Strong scalability}

The strong scalability of the NN and NNC preconditioners is plotted in Fig.(9.21). For fixed problem size (strong scalability), the total execution time for both the NN and NNC preconditioners reduce steadily as expected.

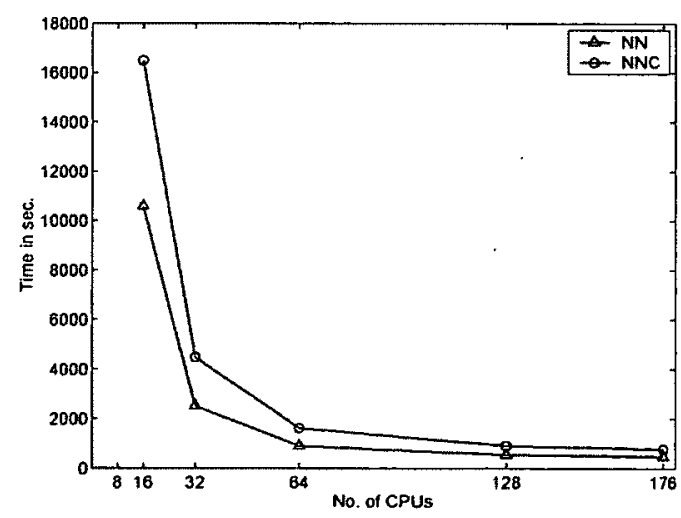

Figure 9.21: Strong scalability 


\section{Weak scalability}

The weak scalability of the NN and NNC preconditioners is presented Fig.(9.22). For fixed problem size per subdomain (weak scalability), the total execution time increases almost linearly in both the preconditioners as we add more processors.

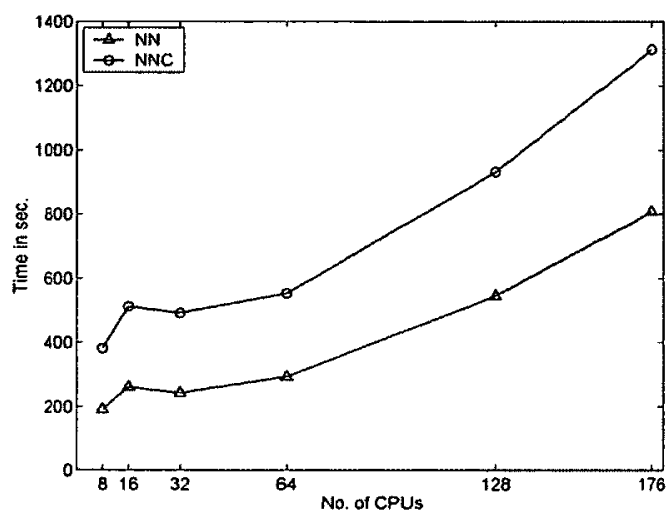

Figure 9.22: Weak scalability

It is worthwhile to point out that the NNC preconditioner takes longer to execute than the NN preconditioner. This is due to the extra computation incurred in solving the coarse problem at each iteration. Although the total execution time for the PCGM with the NN preconditioner is lower, the PCGM convergence criterion may not be satisfied with the NN preconditioner. The iteration count of the PCGM with the NN preconditioner increases rapidly with the number of subdomains. Furthermore, a scalable preconditioner does not necessarily mean the preconditioner with the minimum computational complexity [72], but it ensures convergence while tackling increasingly larger problems. More efficient algorithms that minimize the computational complexities of the coarse problem will reduce the total execution time of the PCGM equipped with the NNC preconditioner. This aspect 
will be the subject of future investigations.

\subsubsection{Two-Dimensional Elasticity}

A plane-stress problem is considered next to study the performance of both the $\mathrm{NN}$ and NNC preconditioners for two-dimensional elasticity problems. The geometry of the problem is shown in Fig.(9.23).

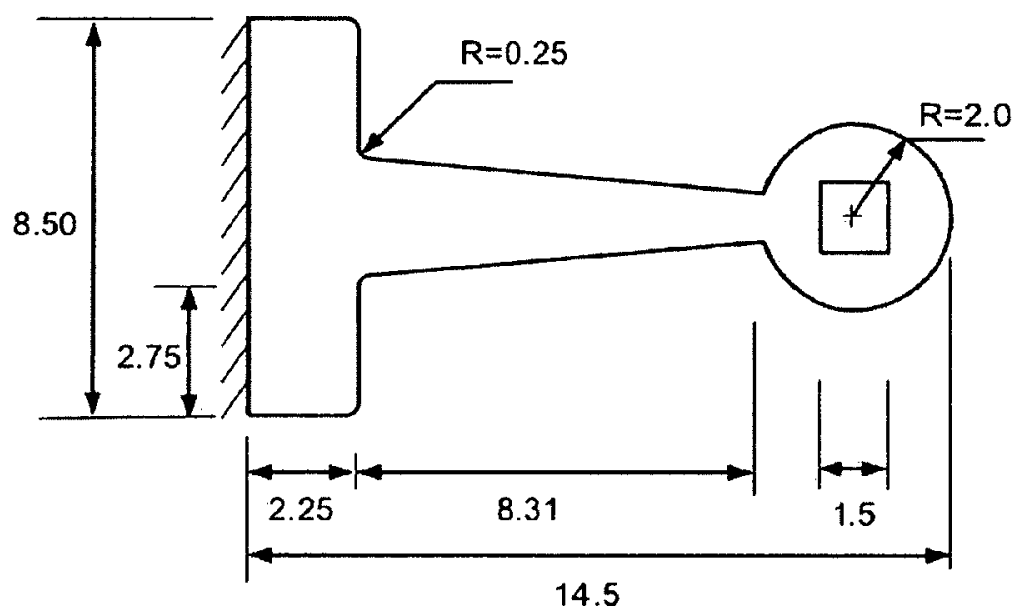

Figure 9.23: The geometry of the two-dimensional plane-stress problem (all dimensions in $\mathrm{cm}$ )

For plane-stress problem, the uncertain constitutive matrix can be represented as (e.g. [11])

$$
\mathbf{D}(\mathbf{x}, \theta)=\frac{E(\mathbf{x}, \theta)}{1-\nu^{2}(\mathbf{x}, \theta)}\left[\begin{array}{ccc}
1 & \nu(\mathbf{x}, \theta) & 0 \\
\nu(\mathbf{x}, \theta) & 1 & 0 \\
0 & 0 & \frac{1-\nu(\mathbf{x}, \theta)}{2}
\end{array}\right]
$$

where $E(\mathbf{x}, \theta)$ and $\nu(\mathbf{x}, \theta)$ are Young's modulus and Poisson's ratio respectively. For 
simplicity and clarity of presentation, we assume Poisson's ratio to be a spatially invariant deterministic quantity. The spatial uncertainty of the Young's modulus is modeled as a stochastic process. The methodology for simultaneous treatment of uncertainties in Young's modules and Poisson's ratio is described in details in [38]. However, the proposed algorithm is general and capable of handling arbitrary non-Gaussian models. We represent the Young's modules as a lognormal process defined as

$$
E(\mathbf{x}, \theta)=E_{0}(\mathbf{x}) \exp (g(\mathbf{x}, \theta))
$$

where $E_{0}(\mathbf{x})$ is the mean of Young's modulus assumed to be $20 \times 10^{9}$ and $g(\mathbf{x}, \theta)$ is the underlying Gaussian process with a squared exponential covariance function of the following form $[92,101]$

$$
C_{g g}(\mathbf{x}, \mathbf{y})=\sigma^{2} \exp \left(\frac{-\left(x_{2}-x_{1}\right)^{2}-\left(y_{2}-y_{1}\right)^{2}}{b^{2}}\right) .
$$

For the numerical investigation, two random variables are used to represent the underlying Gaussian process while the lognormal process is expanded using two-dimensional third order PCE (e.g [145]). The Gaussian process is assumed to have zero mean and $\sigma$ and $b$ are 0.35 and 12.65 respectively. Some of the chaos coefficients of the lognormal process are plotted in Fig.(9.24) 


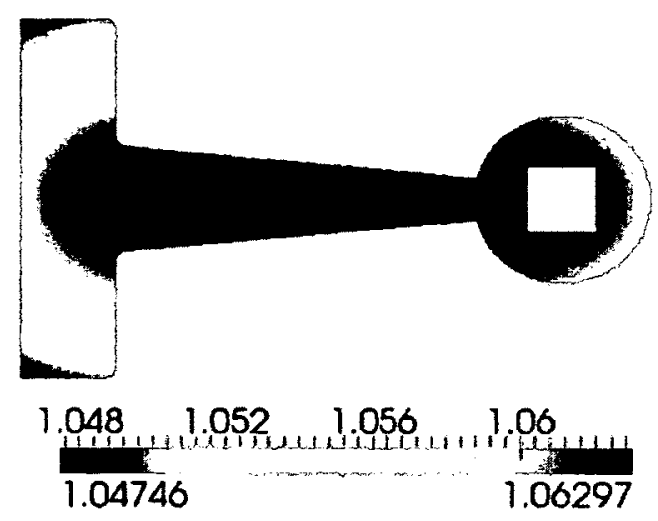

(a) Chaos coefficients: $l_{0}$

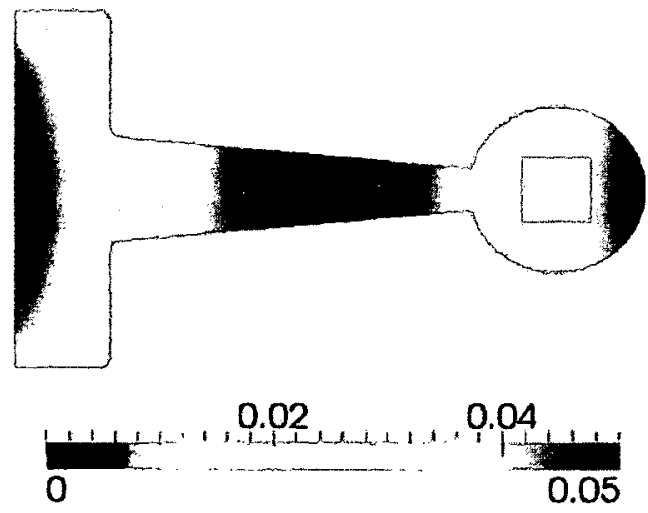

(c) Chaos coefficients: $l_{5}$
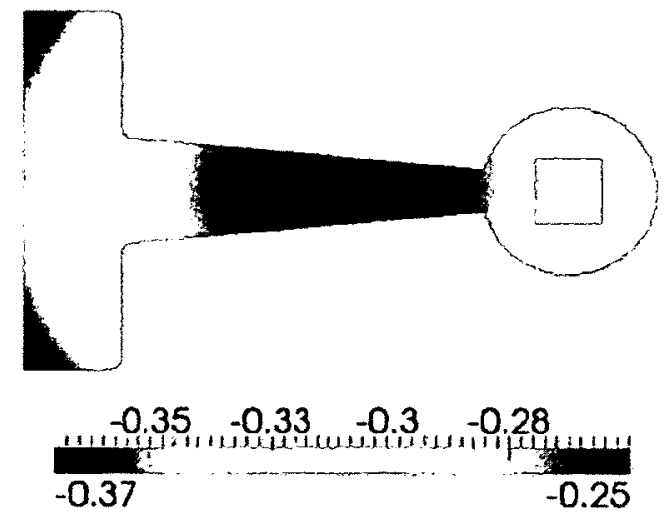

(b) Chaos coefficients: $l_{1}$
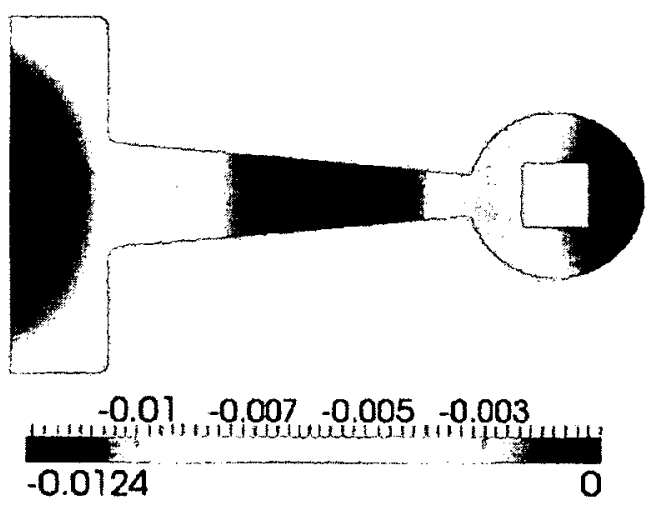

(d) Chaos coefficients: $l_{105}$

Figure 9.24: Selected PC coefficients of the Young's modules

\section{Stochastic Characteristics of the Solution Process}

The physical domain and its typical decomposition are shown in Fig.(9.25a) and Fig.(9.25b) respectively. Fig.(9.26a) and Fig.(9.26b) show the mean and standard deviation of the magnitude of the displacement field, respectively. At the free end, the coefficient of variation $(\mathrm{CoV})$ is about $30 \%$ underscoring the effect of uncertainty. We show a set of selected 
polynomial chaos coefficients of the magnitude of the displacement field in Fig.(9.27).

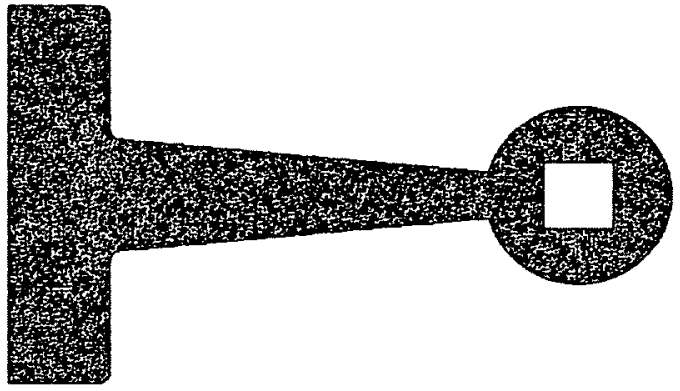

(a) FEM mesh

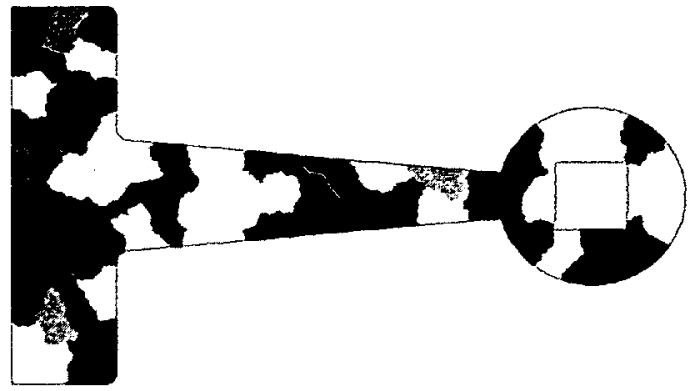

(b) Domain decomposition

Figure 9.25: Finite element discretization and domain decomposition

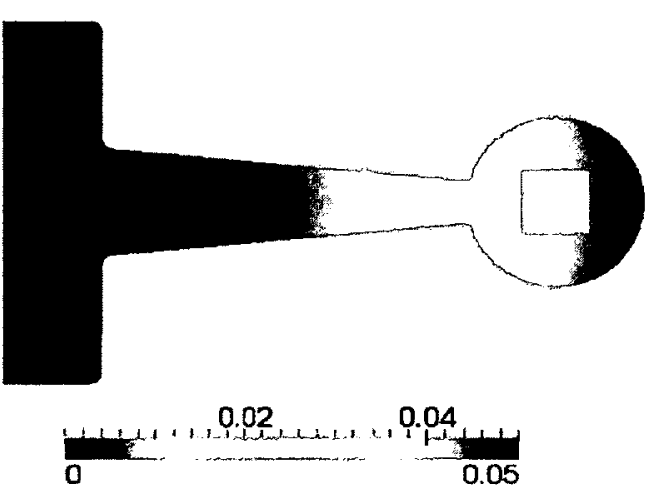

(a) Mean response

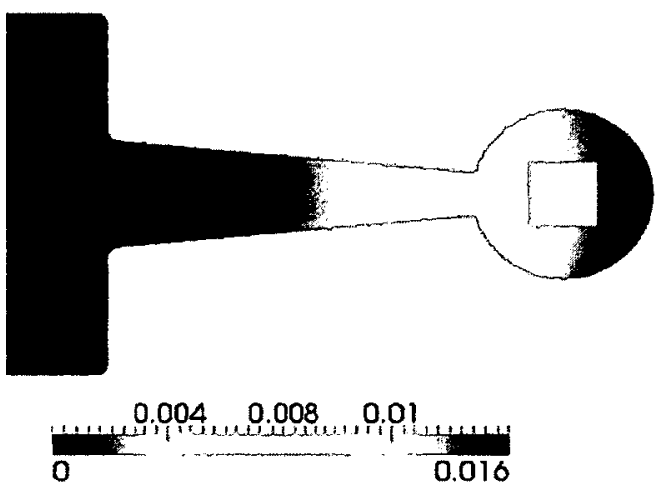

(b) Standard deviation

Figure 9.26: The mean and standard deviation of the magnitude of the displacement field 

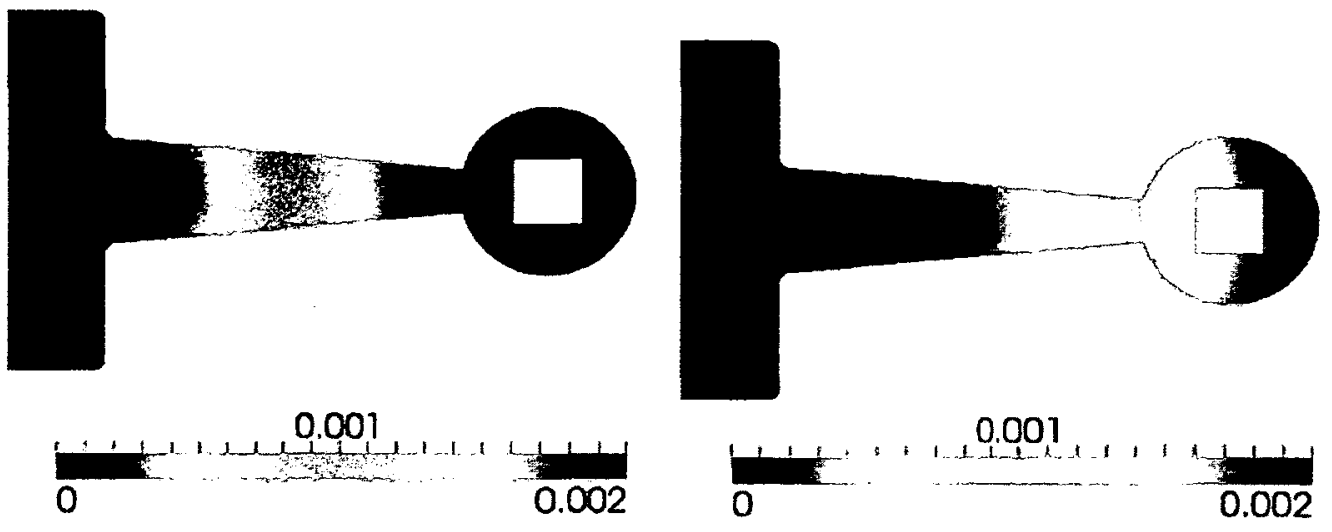

(a) Chaos coefficients: $\mathbf{u}_{2}$

(b) Chaos coefficients: $\mathbf{u}_{3}$
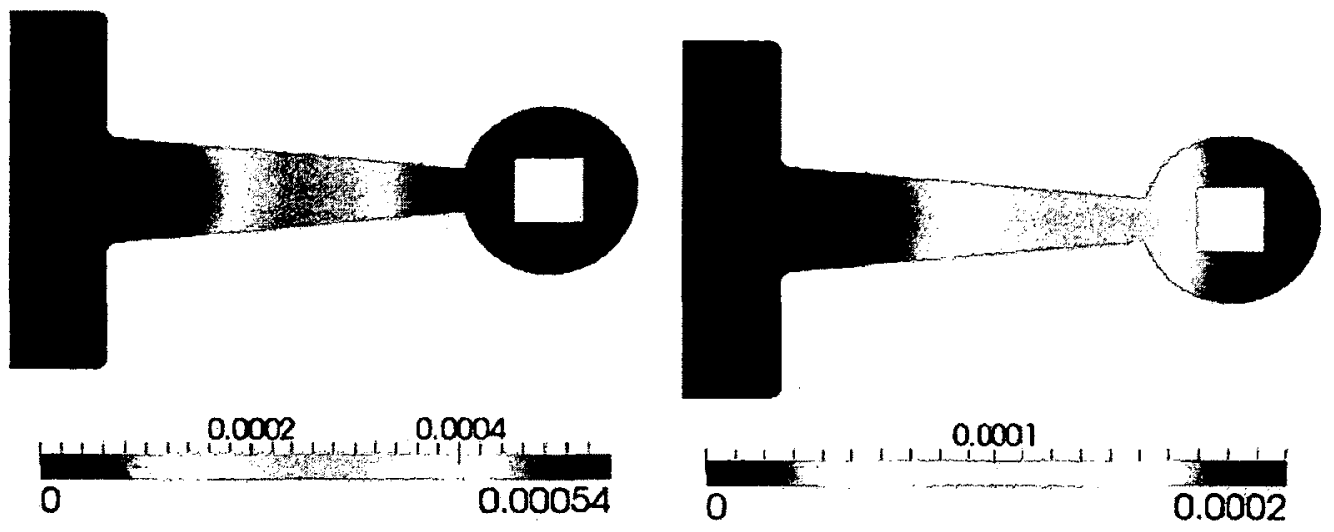

(c) Chaos coefficients: $u_{4}$

(d) Chaos coefficients: $\mathbf{u}_{5}$

Figure 9.27: Selected PC coefficients of the magnitude of the displacement field

\section{Numerical Scalability}

Fig.(9.28) demonstrates the performance of both the NN and NNC preconditioners by fixing the problem size and number of subdomains, but increasing the order of PCE of the solution process. Increasing the order of PCE exhibits a linear growth in the iteration 
counts for the NN preconditioner. However the iteration counts remain steady for the NNC preconditioner. This fact demonstrates that the NNC preconditioner is scalable to the order of PCE.

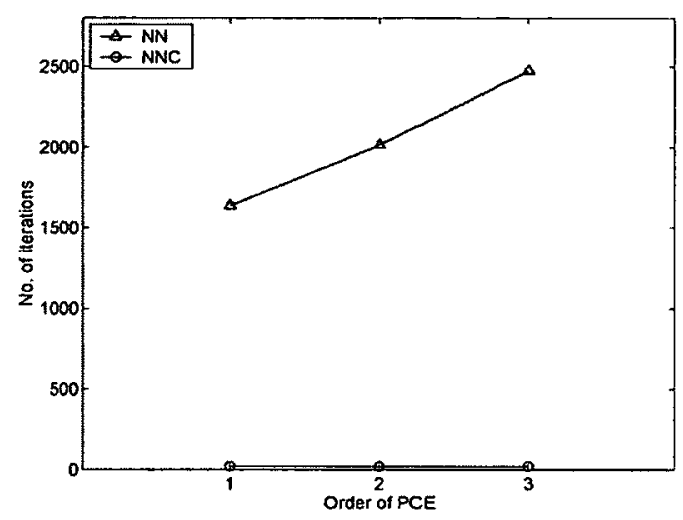

Figure 9.28: Iteration counts versus the order of PCE

In Fig.(9.29), the iteration counts for the PCGM with NN and NNC preconditioners, for fixed number of subdomains $\left(n_{s}=64\right)$ and the second order PCE, are plotted while refining the finite element mesh. Evidently, the problem size does not degrade the efficiency of the NNC preconditioner as the number of iterations required for convergence remains the same. The iteration counts increase almost linearly for the NN preconditioner as the problem size grows. The NNC preconditioner takes fewer iterations to converge compared to the NN preconditioner. 


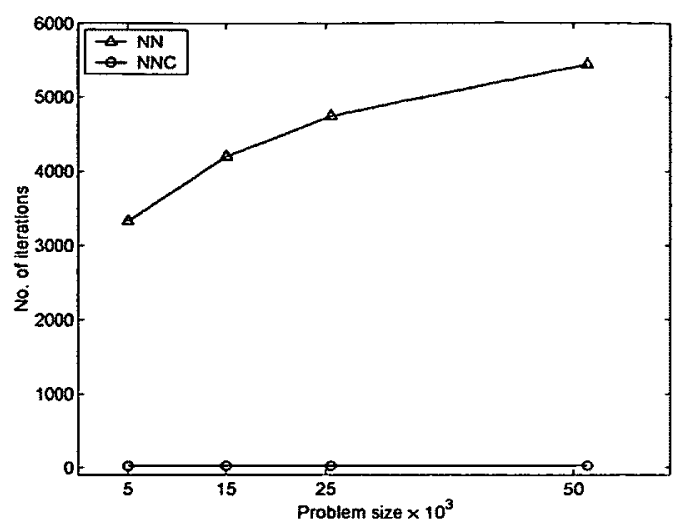

Figure 9.29: Iteration counts versus mesh size for fixed number of subdomains

In Fig.(9.30), we maintain the problem size in the spatial domain to 111,651 nodes with 223, 316 elements but add more subdomains for the second order PCE of the solution process. The results demonstrates that the $\mathrm{NN}$ preconditioner is scalable to the number of subdomains.

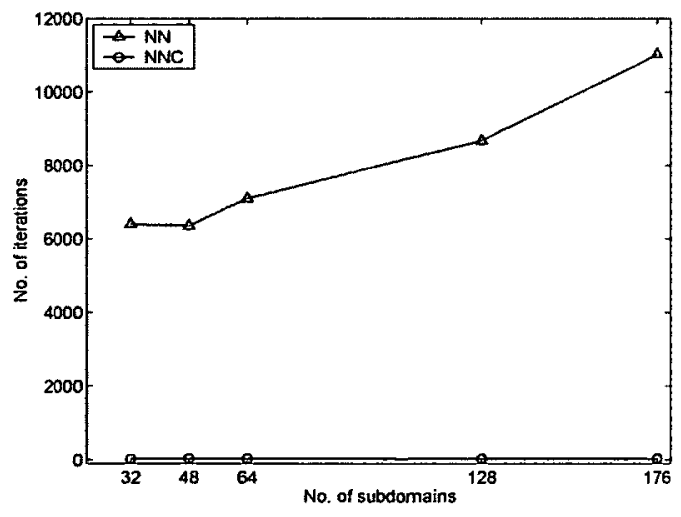

Figure 9.30: Iteration counts versus the number of subdomains for fixed problem size

The performance of the NN and NNC preconditioners is shown in Fig.(9.31) for fixed 
problem size per subdomain. Again these results highlight the fact that the NNC preconditioner is scalable to fixed problem size per subdomain. The performance of the NN preconditioner degrades rapidly as the number of subdomains grows.

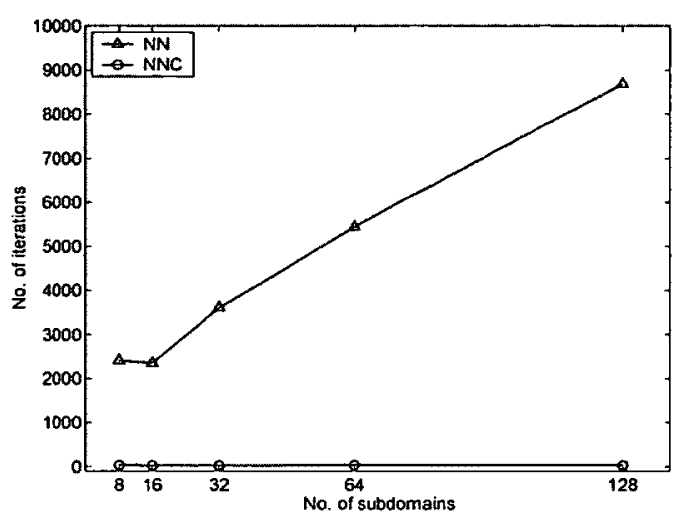

Figure 9.31: Iteration counts versus the number of subdomains for fixed

problem size per subdomain

\section{Parallel Scalability}

Fig.(9.32) and Fig.(9.33) demonstrate the strong and weak scalabilities for both NN and NNC preconditioners respectively. For a fixed mesh resolution, both the NN and NNC preconditioners steadily reduce the execution time when we exploit more processors to tackle the problem. For the weak scalability tests, the execution times for both the NN and NNC preconditioners increase linearly. As alluded to previously, the extra computation involved in solving the coarse problem leads to the higher execution time for the PCGM with the NNC preconditioner than that of the NN preconditioner. 


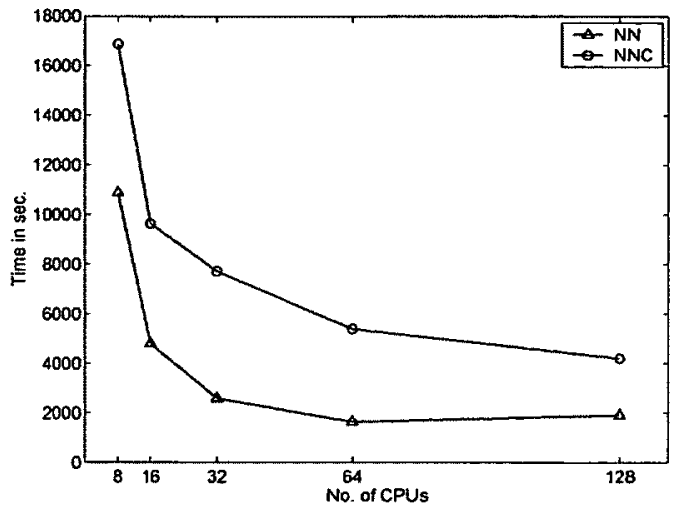

Figure 9.32: Strong scalability

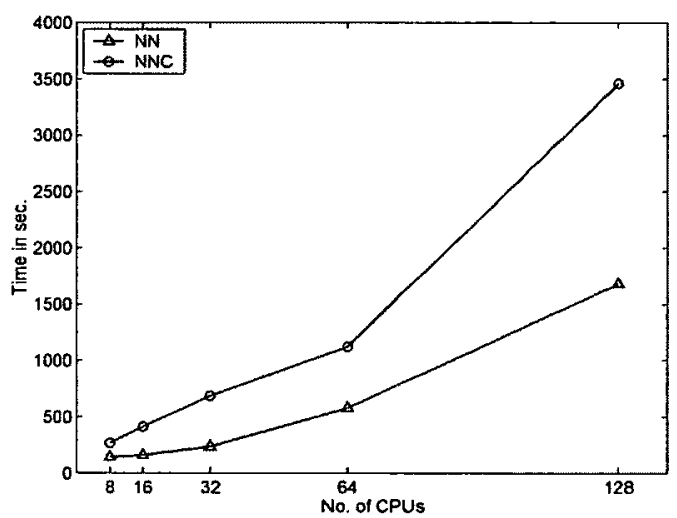

Figure 9.33: Weak scalability

\subsection{Dual-Primal Domain Decomposition Method}

In this section, we contrast the convergence properties, numerical and parallel scalabilities of both the probabilistic NNC and FETI-DP for flow through random media and linear elasticity problems with spatially varying system parameters modeled as non-Gaussian stochastic processes. 


\subsubsection{Flow Through Random Media and Elasticity Problems}

\section{Convergence Properties}

The convergence rate of the probabilistic NNC and FETI-DP methods for flow through random media and elasticity problems are show in Fig.(9.34). Clearly, the convergence rates of both the methods is similar. However, the probabilistic NNC method requires slightly higher iteration counts to converge compare to the probabilistic FETI-DP method. This discrepancy between the iteration counts of the two methods maybe attributed to the fact that the initial residual in the probabilistic NNC method is bigger than that of the probabilistic FETI-DP method.

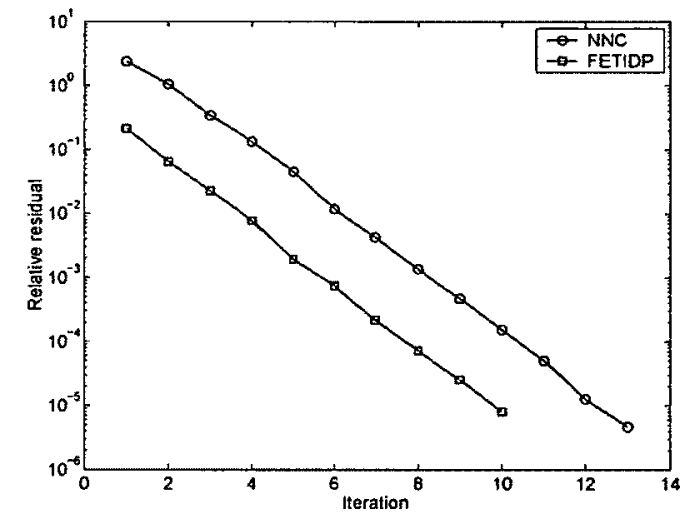

(a) Flow through random media

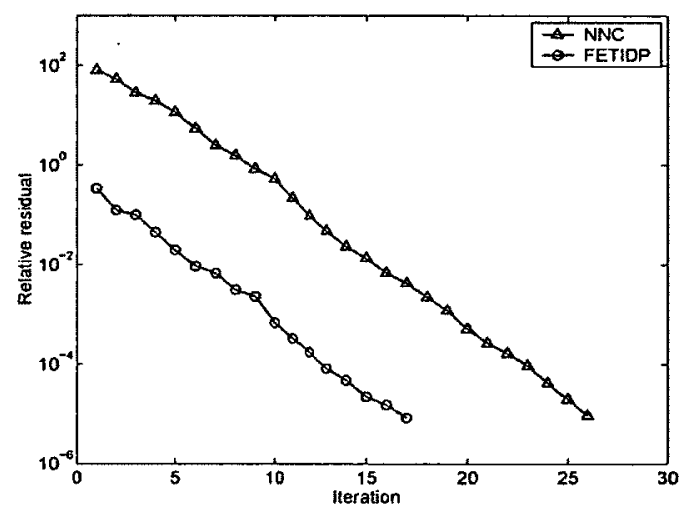

(b) Plane stress

Figure 9.34: The history of the relative PCGM residual

In Fig.(9.35), we plot the iteration counts for the probabilistic NNC and FETI-DP solvers versus the order of PCE used for the solution process. The iteration counts required for convergence is nearly independent of the order of the PCE for both the probabilistic NNC and FETI-DP methods. In general, the probabilistic NNC and FETI-DP solvers for elasticity 
require higher iteration counts to converge compare to Poisson's problem. This fact can be mainly attributed to the size of the coarse problem. In two-dimensional cases, there are two unknowns per each corner node for elasticity while one unknown per corner node for Poisson's problem.

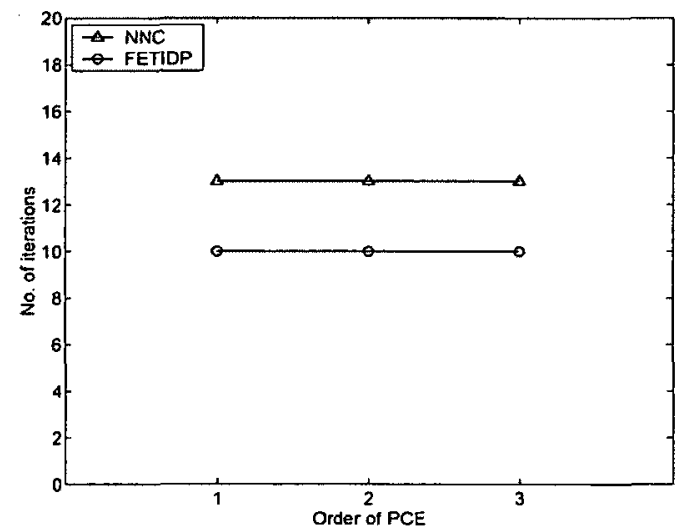

(a) Flow through random media

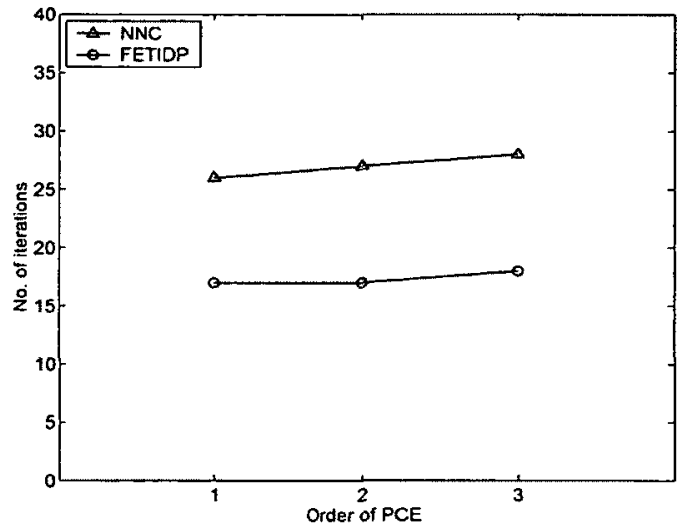

(b) Plane stress

Figure 9.35: Iteration counts with respect to the order of PCE

For fixed number of subdomains and order of PCE, we show the number of iterations required for the probabilistic NNC and FETI-DP methods in Fig.(9.36). The iteration counts for both the flow through random media and elasticity problems remain almost constant as we increase mesh resolution. This fact indicates that both the probabilistic NNC and FETI-DP methods are scalable with respect to the problem size. Once again the probabilistic NNC method requires more iteration than that of probabilistic FETI-DP method. The difference between the iteration counts of the probabilistic NNC and FETI-DP methods becomes larger in the elasticity problem. As alluded to previously, this behavior is attributed to the size of the coarse problem which is bigger in elasticity than that in Poisson's problem. 


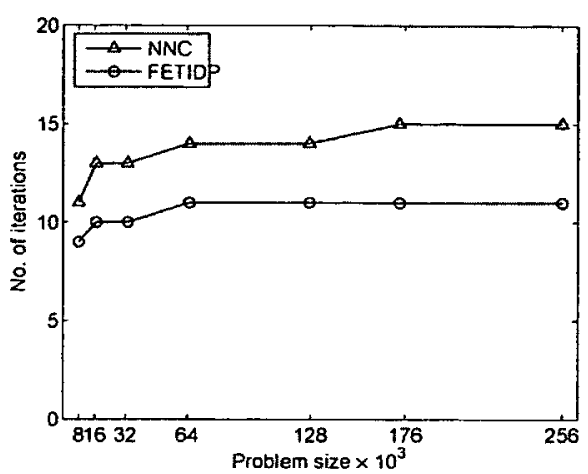

(a) Flow through random media

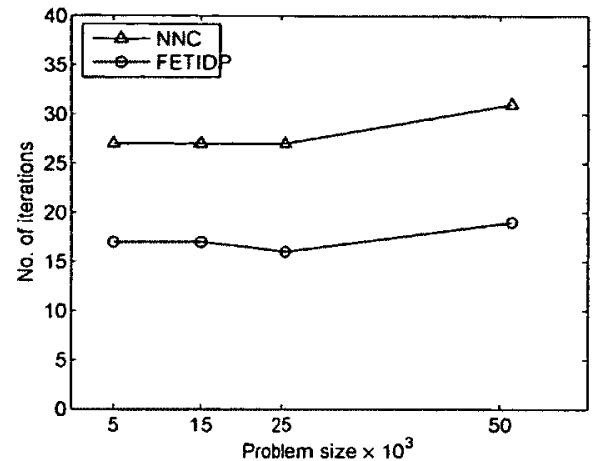

(b) Plane stress

Figure 9.36: Iteration counts with respect to mesh size for fixed number of subdomains

Next we fix the spatial problem size and order of PCE while increasing the number of subdomains/processors used to tackle the problem. The corresponding results are shown in Fig.(9.37). Note that the iteration counts for both the methods are not significantly influenced by the number of subdomains. 


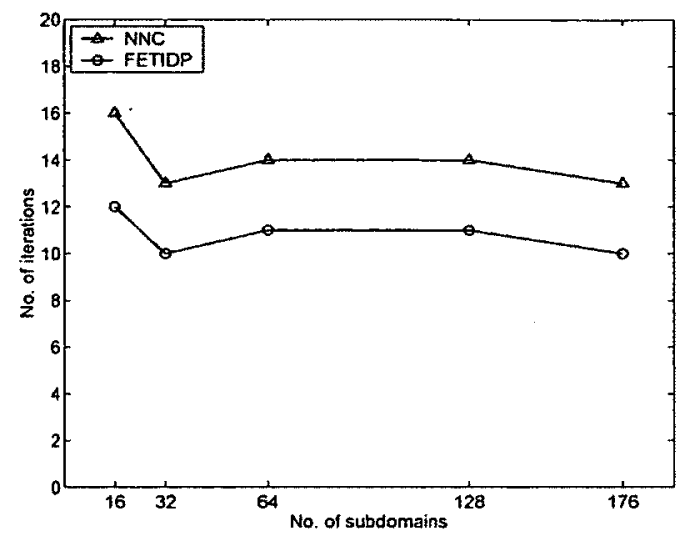

(a) Flow through random media

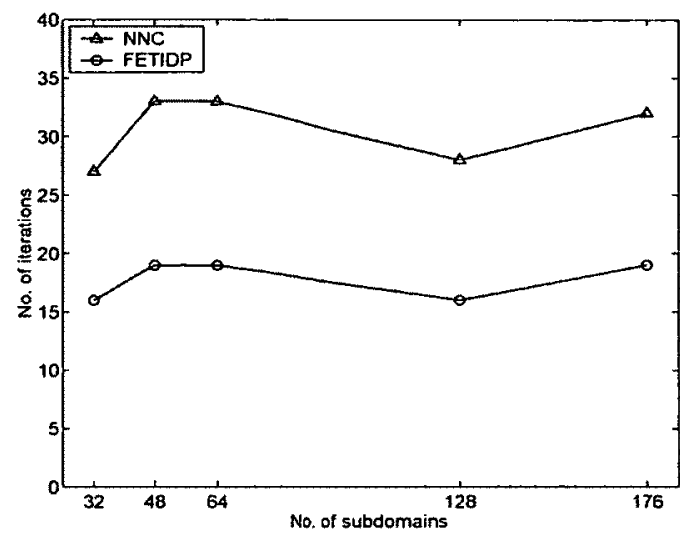

(b) Plane stress

Figure 9.37: Iteration counts with respect to number of subdomains for fixed mesh size

Next we fix the order of PCE and problem size per subdomain and increase the total problem size by adding more subdomains. Fig.(9.38) shows the iteration counts versus the number of subdomains. Clearly, the iteration counts for both probabilistic NNC and FETIDP methods do not change significantly with respect to the number of subdomains (or the global problem size). 


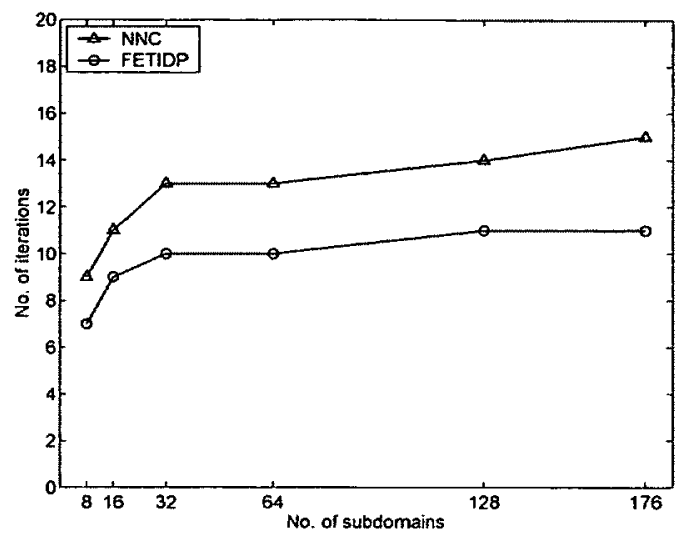

(a) Flow through random media

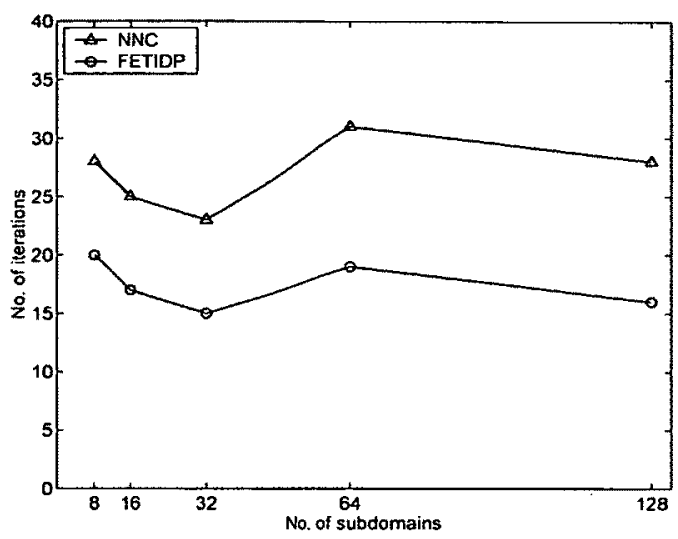

(b) Plane stress

Figure 9.38: Iteration counts with respect to the number of subdomains for fixed problem size per subdomain

Fig.(9.39) shows the reduction in the total execution time for both probabilistic NNC and FETI-DP methods when we increase the number of processors to tackle a fixed problem size. The spatial problem size considered for flow through random media and elasticity problems is 127,552 and 111,651 respectively. Both probabilistic NNC and FETI-DP methods exhibit similar behaviors for flow through random media and elasticity problems. 


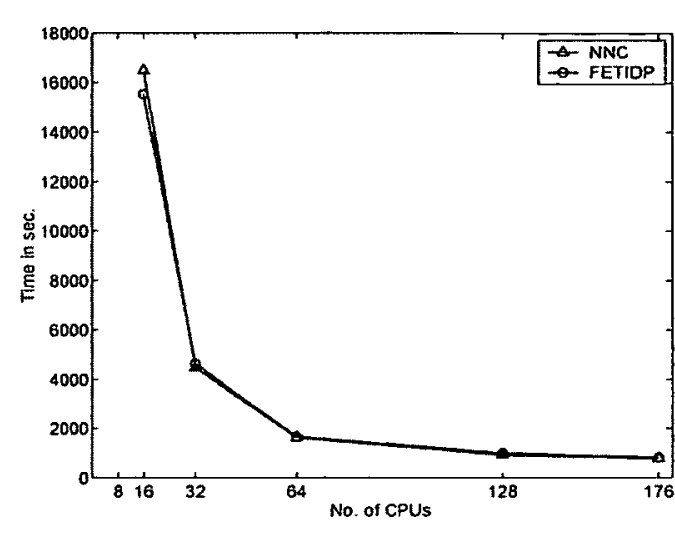

(a) Flow through random media

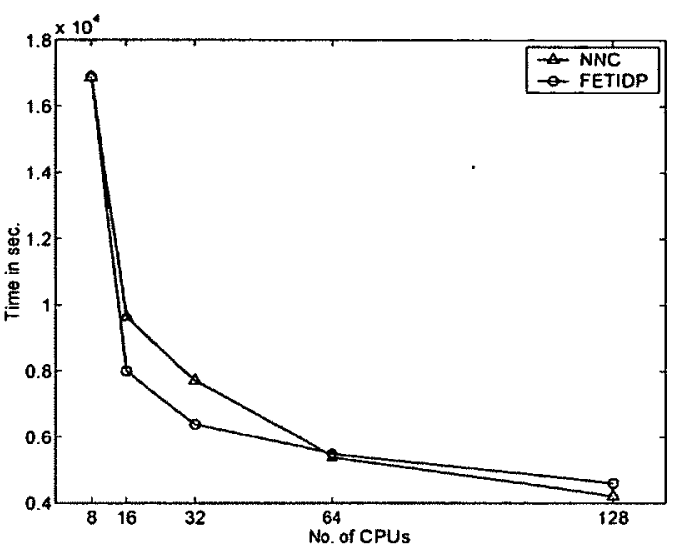

(b) Plane stress

Figure 9.39: Strong scalability

Next we compare the weak scalability of the probabilistic NNC and FETI-DP algorithms by increasing the overall problem size for fixed problem size per subdomain. Note the linear growth in the total execution time for both probabilistic NNC and FETI-DP algorithms for both flow through random media and elasticity problems as shown in Fig.(9.40). This fact indicates that the probabilistic NNC and FETI-DP algorithms are weakly scalable. 


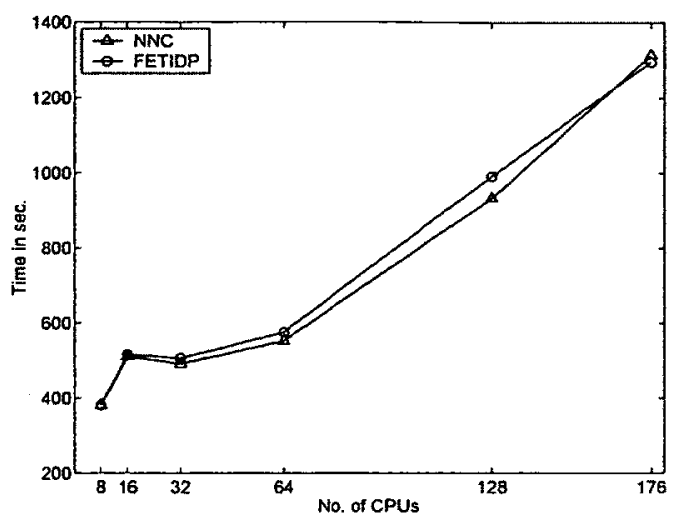

(a) Flow through random media

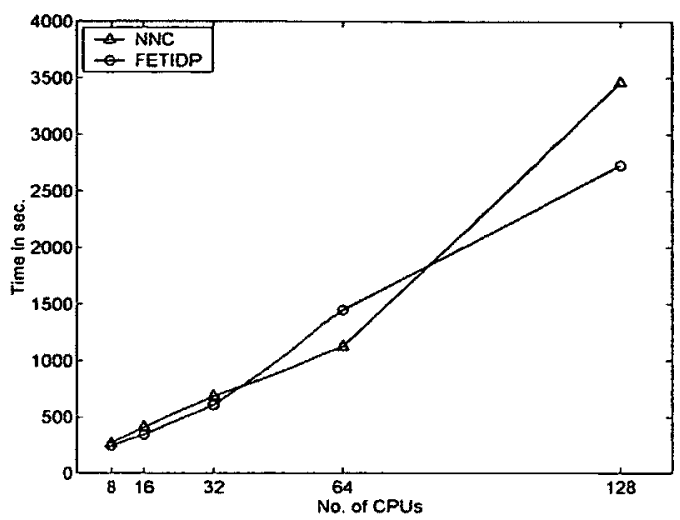

(b) Plane stress

Figure 9.40: Weak scalability

\subsection{Summary}

We investigate the convergence rate, numerical and parallel scalabilities of the one-level and two-level domain decomposition algorithms for two-dimensional flow through random media and elasticity problems with spatially varying non-Gaussian system parameters. The parallel performances of the algorithms are studied with respect to the level of uncertainty, order of stochastic dimension, spatial problem size, number of subdomains and fixed problem size per subdomain. The numerical results demonstrate that:

- The performance of the probabilistic algorithms are nearly independent of the level of uncertainty and the size of the stochastic dimension.

- The one-level domain decomposition algorithms demonstrate scalability for moderate range of processors for the problem size considered in the investigation. 
- The two-level preconditioner equipped with coarse problem exhibits a scalable performance with respect to the spatial problem size, number of subdomains and fixed problem size per subdomain.

- The probabilistic dual-primal approach shows similar convergence properties and parallel performance to the probabilistic BDDC type two-level domain decomposition preconditioner. 


\section{Chapter 10}

\section{Numerical Results: Three-Dimensional}

\section{Problems}

\subsection{Introduction}

The numerical results in this chapter are taken from the references [146, 147]. Constructing scalable domain decomposition algorithms for large-scale linear systems arising from three-dimensional problems is more challenging than that for two-dimensional problems. The difficulty arises due to the complicated coupling among the subdomains in three dimensions and the complex geometry of the interface boundary [7]. On the other hand, the resulting linear systems from finite element discretization of three-dimensional problems are often very ill-conditioned [4]. Therefore, simple domain decomposition preconditioners are inefficient especially for elasticity problems in three dimensions [4]. Uncertainty quantification by polynomial chaos approach adds additional complexities to the problem due to the 
block structure of the stochastic submatrices and the coupling among the polynomial chaos coefficients in three dimensions. Direct extension of the Schur complement based domain decomposition algorithms developed for two-dimensional to three-dimensional problems is feasible, but maintaining the same performance and convergence properties is not always guaranteed $[4,6]$. It has been demonstrated that iterative substructuring techniques can achieve a scalable performance only when equipped with a coarse problem that is required for global communication $[4-7,114]$. The coarse grid for three-dimensional problems is often more complicated to construct than that for the two-dimensional cases $[137,148]$.

In the following sections, we apply the probabilistic Neumann-Neumann with coarse problem (NNC), Primal-Primal Domain Decomposition Method (PP-DDM) and DualPrimal Domain Decomposition Method (DP-DDM) to quantify uncertainty in three- dimensional SPDEs. In our implementation, we select the primal constraints as the unknowns at the corner nodes of the subdomains (i.e. vertices only). This approach for constructing the coarse problem does not guarantee convergence for three-dimensional elasticity problems. In order to achieve better convergence properties for elasticity problems in three dimensions, the averages over faces or edges must be considered as additional constraints to the coarse problem. This aspect however, is not considered in the thesis and will be the subject of future investigations.

Firstly, we consider three-dimensional flow through random media and heat transfer problems with non-Gaussian system parameters. Next, we tackle three-dimensional elasticity problem having random constitutive properties. The parallel performance is investigated 
with respect to mesh size, number of subdomain and fixed problem size per subdomain.

The numerical experiments are performed using MPI and PETSc libraries on a Linux cluster (CentOS release 5.6) consists of 1200 compute nodes with 6 cores and 36 GBytes of memory per node with InfiniBand interconnect [149]. PETSc version 3.1 and MVAPICH2 version 1.6 libraries are compiled with Intel compiler version 12.0.4.

\subsection{Three-Dimensional Flow Through Random Media}

In this section, a three-dimensional steady-state flow through random media with a spatially varying non-Gaussian hydraulic conductivity coefficient is considered. The flow is modelled by three-dimensional stochastic Poisson equation as

$$
\begin{aligned}
\nabla \cdot(\kappa(\mathbf{x}, \theta) \nabla u(\mathbf{x}, \theta)) & =f(\mathbf{x}), \quad \mathbf{x} \in D \\
u(\mathbf{x}, \theta)) & =0, \quad \mathbf{x} \in \partial D
\end{aligned}
$$

The hydraulic conductivity coefficient is modeled as a lognormal stochastic process obtained from the underlying Gaussian field with zero mean and an exponential covariance function defined as $[11,145]$

$$
C_{g g}(\mathbf{x}, \mathbf{y})=\sigma^{2} \exp \left(\frac{-\left|x_{1}-x_{2}\right|}{b_{x}}+\frac{-\left|y_{1}-y_{2}\right|}{b_{y}}+\frac{-\left|z_{1}-z_{2}\right|}{b_{z}}\right)
$$

where $\sigma$ is the standard deviation of the process and $b_{x}, b_{y}$ and $b_{z}$ are the correlation lengths in $x, y$ and $z$ directions respectively. For numerical investigations, we use $\sigma=0.6$ and 
$f(\mathbf{x})=1.0$.

A typical finite element mesh of the computational domain is presented in Fig.(10.1(a)). A decomposition of the spatial domain into 128 subdomains is shown in Fig.(10.1(b)). The finite element mesh considered for the eigenvalue problem associated with KLE of the underlying Gaussian process consists of 26,614 linear tetrahedral elements and 5,654 nodes.

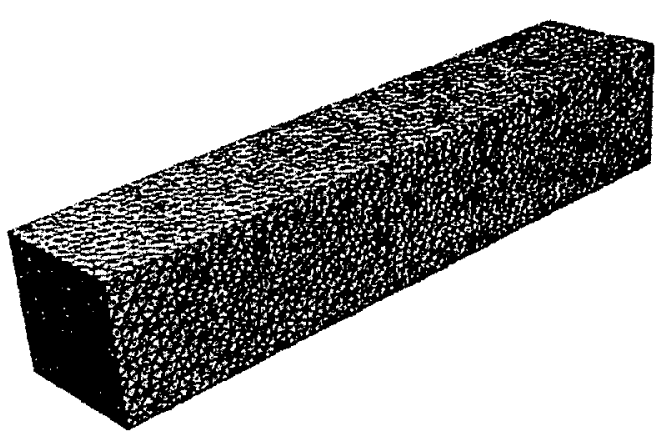

(a) FEM mesh

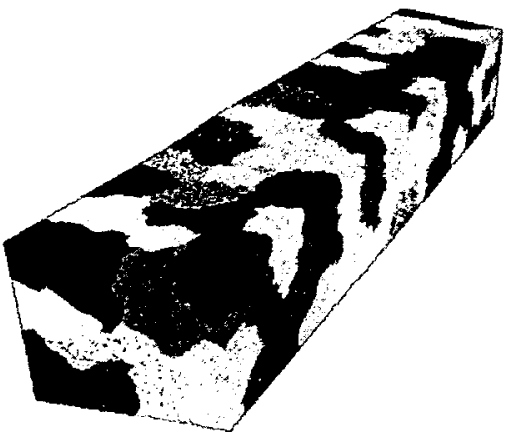

(b) Domain Decomposition

Figure 10.1: Finite element discretization and mesh decomposition

\section{Stochastic Field Representation}

Fig.(10.2) shows the first 20 eigenvalues of the exponential covariance function defined in Eq.(10.1), for different correlation lengths. Evidently, the eigenvalues exhibit larger decay rate as the correlation length increases. These results suggest that the decay rate of the eigenvalues is inversely proportional to the correlation length. For small correlation length (e.g. $b_{x}=b_{y}=b_{z}=b=0.1$ ), the decay rate of the eigenvalues is almost invisible. When the eigenvalues decay slowly, large number of terms in KLE is required to represent the 
stochastic process. Stated alternatively, the decay rate of the eigenvalues can be used as a guideline to determine the number of random variables required in KLE to sufficiently approximate a stochastic process.

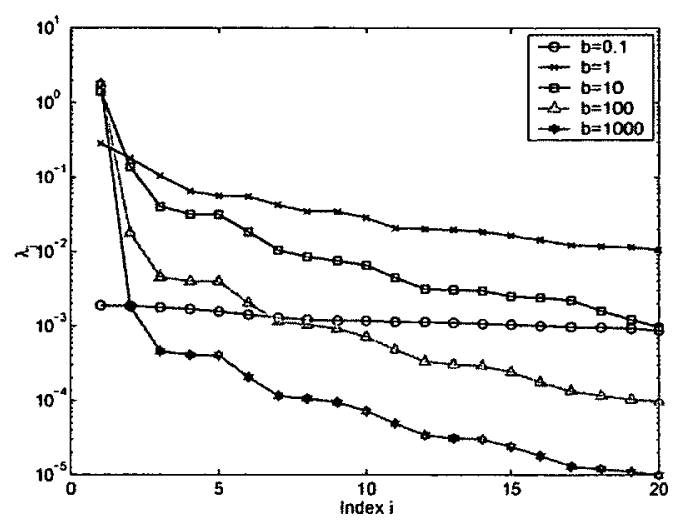

Figure 10.2: Eigenvalues $\lambda_{i}$ of the covariance function

For correlation lengths $\left(b_{x}=b_{y}=b_{z}=b=1\right)$ and $\left(b_{x}=b_{y}=b_{z}=b=10\right)$, selected eigenfunctions $\phi_{i}(\mathbf{x})$ of the exponential covariance kernel are shown in Fig.(10.3) and Fig.(10.4) respectively. As the index $i$ increases, the eigenfunctions $\phi_{i}(\mathbf{x})$ exhibit higher oscillations. These results emphasize the fact that the more random variables we use in KLE, the more scales of fluctuation we incorporate in the representation of the stochastic process. Clearly, a finer finite element mesh resolution is required to accurately capture high frequency oscillations as indicated in Fig.(10.3f) and Fig.(10.4f). 

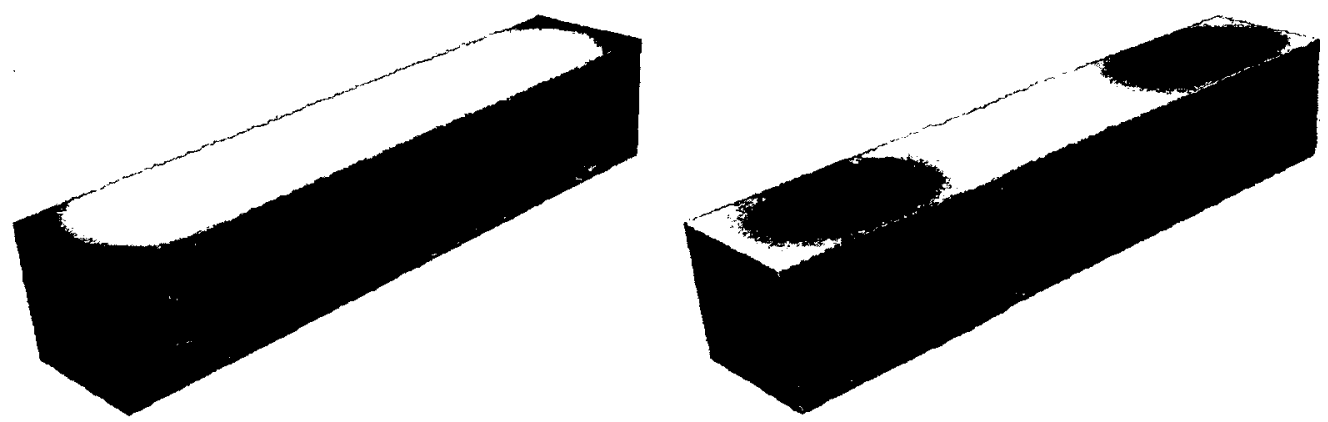

$\frac{0.2}{0.176}+0.3 \quad 0.4 \quad 0.5 \frac{0.6}{0.642}$

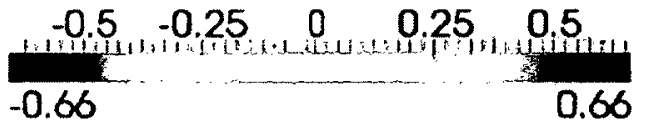

(a) $1 \mathrm{st}$

(b) 2nd
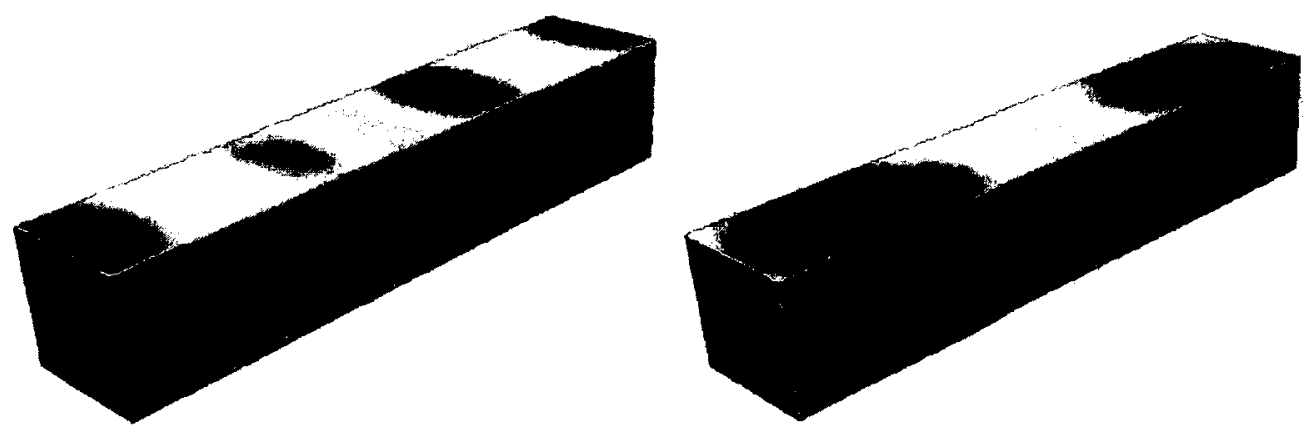

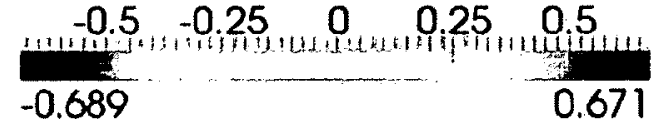

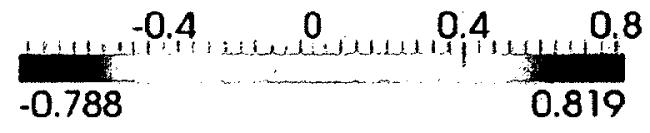

(c) $4 \mathrm{rd}$

(d) 9 th
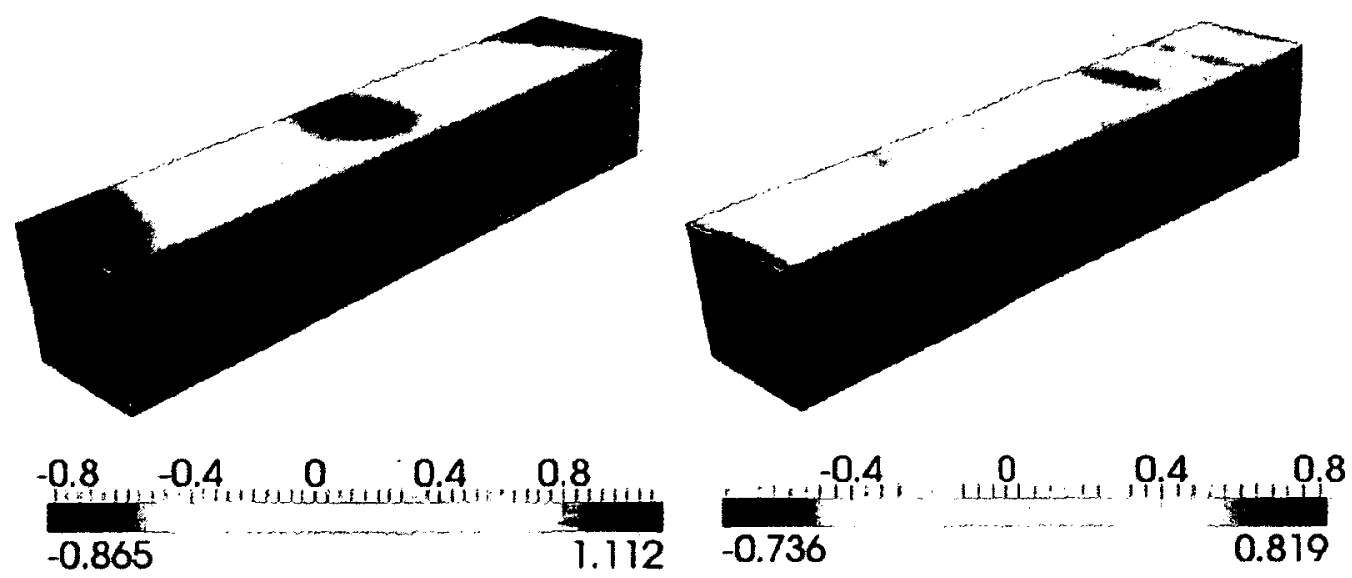

(e) 13 th

(f) 16 th

Figure 10.3: Selected eigenfunctions: $b_{x}=b_{y}=b_{z}=1$ 

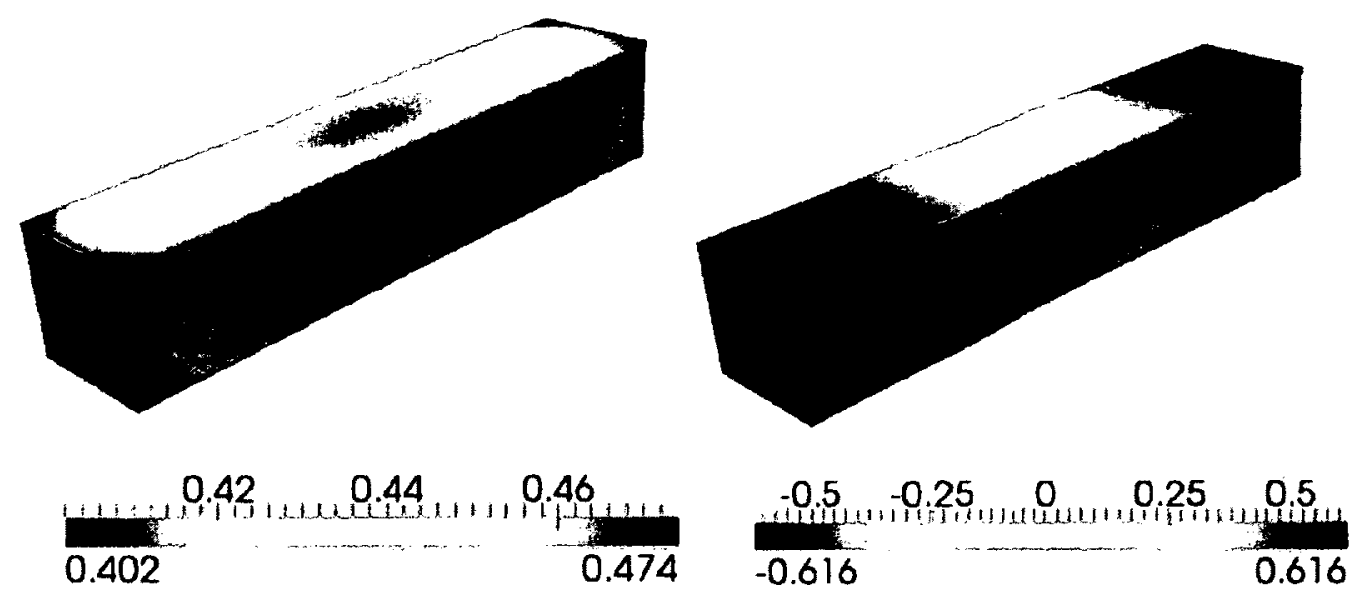

(a) $1 \mathrm{st}$

(b) 2nd
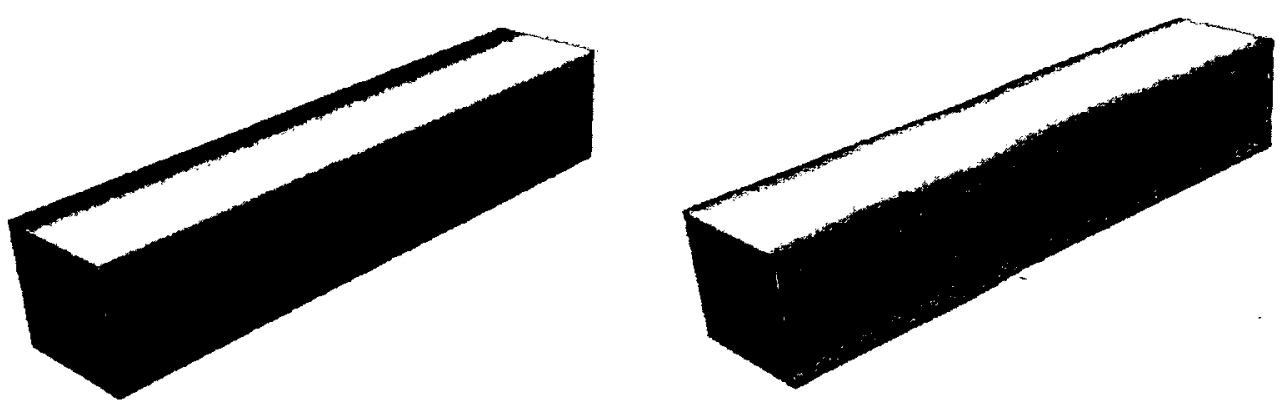

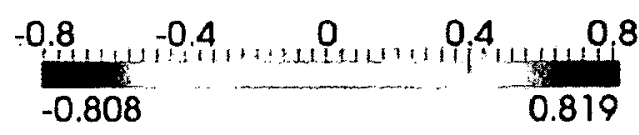

(c) $4 \mathrm{rd}$
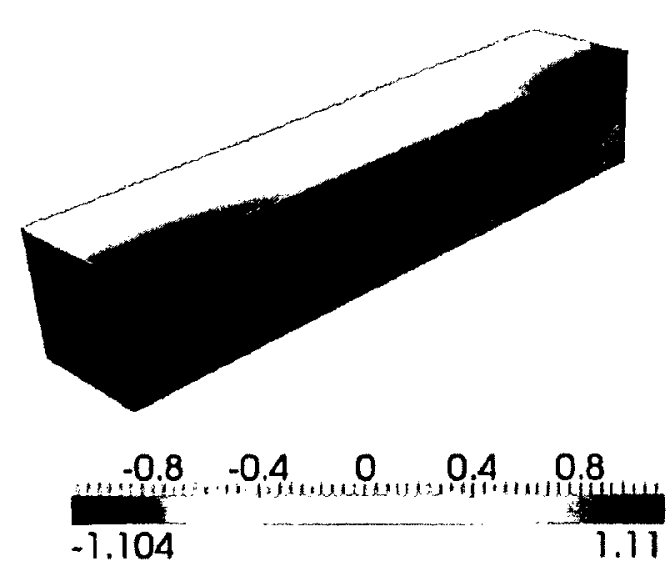

(e) 13 th

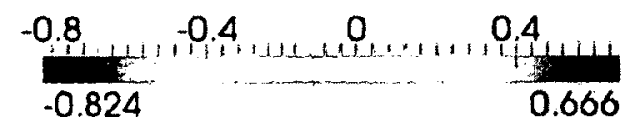

(d) 9 th
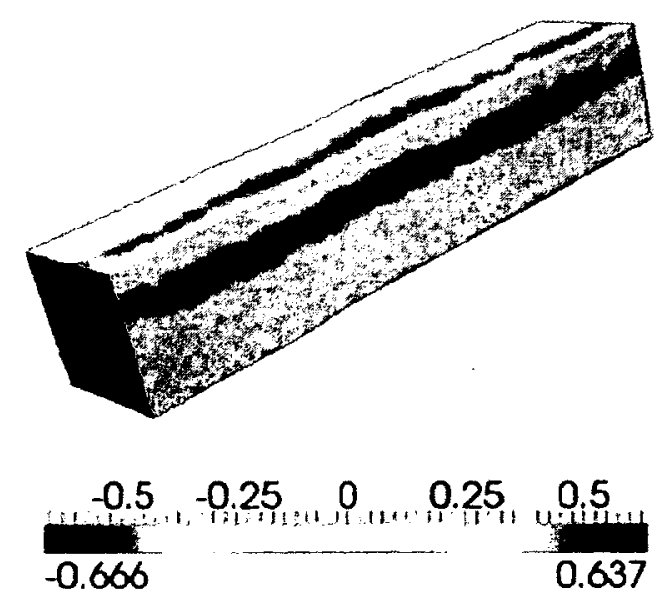

(f) 16th

Figure 10.4: Selected eigenfunctions: $b_{x}=b_{y}=b_{z}=10$ 
Some of the chaos coefficients of the lognormal process are plotted in Fig.(10.5) and Fig.(10.6) for correlation lengths $\left(b_{x}=b_{y}=b_{z}=1\right)$ and $\left(b_{x}=b_{y}=b_{z}=10\right)$ respectively. The number of terms in KLE is taken to be $L=4$. In Fig.(10.5a) and Fig.(10.6a), the first chaos coefficient $l_{0}$ representing the mean of the lognormal processes is defined as [145, 150]

$$
l_{0}(\mathbf{x})=\exp \left[g_{0}(\mathbf{x})+\frac{1}{2} \sum_{j=1}^{L} g_{j}^{2}(\mathbf{x})\right]
$$

where $g_{0}(\mathbf{x})$ represents the mean of the underlying Gaussian processes, $g_{j}(\mathbf{x})=\sqrt{\lambda_{i}} \phi_{i}(\mathbf{x})$, $\sqrt{\lambda_{i}}$ are the eigenvalues and $\phi_{i}(\mathbf{x})$ are eigenfunctions of the covariance kernel. Note that in the limit as $(L \rightarrow \infty)$, Eq.(10.2) converges to

$$
l_{0}=\exp \left[g_{0}(\mathbf{x})+\frac{1}{2} \sigma_{g}^{2}\right]
$$

For $g_{0}(\mathbf{x})=0$ and $\sigma_{g}=0.6$, the exact mean of the lognormal process defined in Eq.(10.3) become $l_{0}=1.1972$.

As shown in Fig.(10.5a) and Fig.(10.6a) the first chaos coefficient $l_{0}$ is almost constant. For $b=1$ the first PC coefficient $l_{0}$ underestimates the exact value of the mean. In contrast, the first PC coefficient $l_{0}$ sufficiently approximates the mean of the lognormal process for $b=10$. Fig.(10.2) demonstrated that for small correlation length such as $b=1$, the eigenvalues decay slowly and thus more terms in KLE are needed to adequately approximate the process. For this reason, higher dimensional PCE is required to sufficiently approximate the lognormal process using KLE of a Gaussian process with small correlation lengths. 
The PC coefficients $l_{j}$ where $j>0$ for small correlation length in Fig.(10.5) show finer structure compared to that for larger correlation length in Fig.(10.6). 

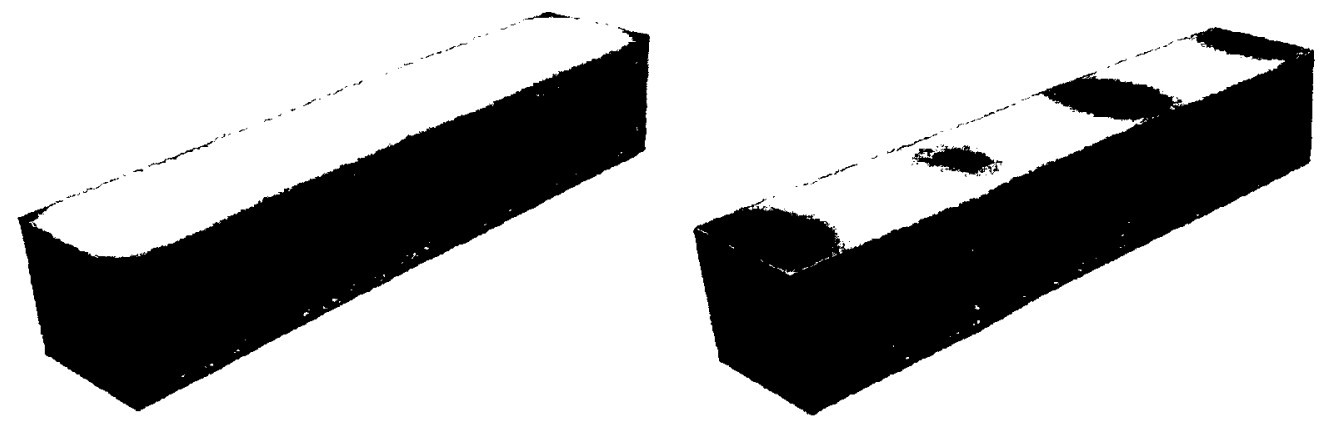

$\frac{1.041,1.06}{1.026}$

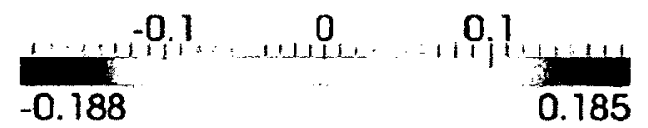

(a) $l_{0}$

(b) $l_{4}$
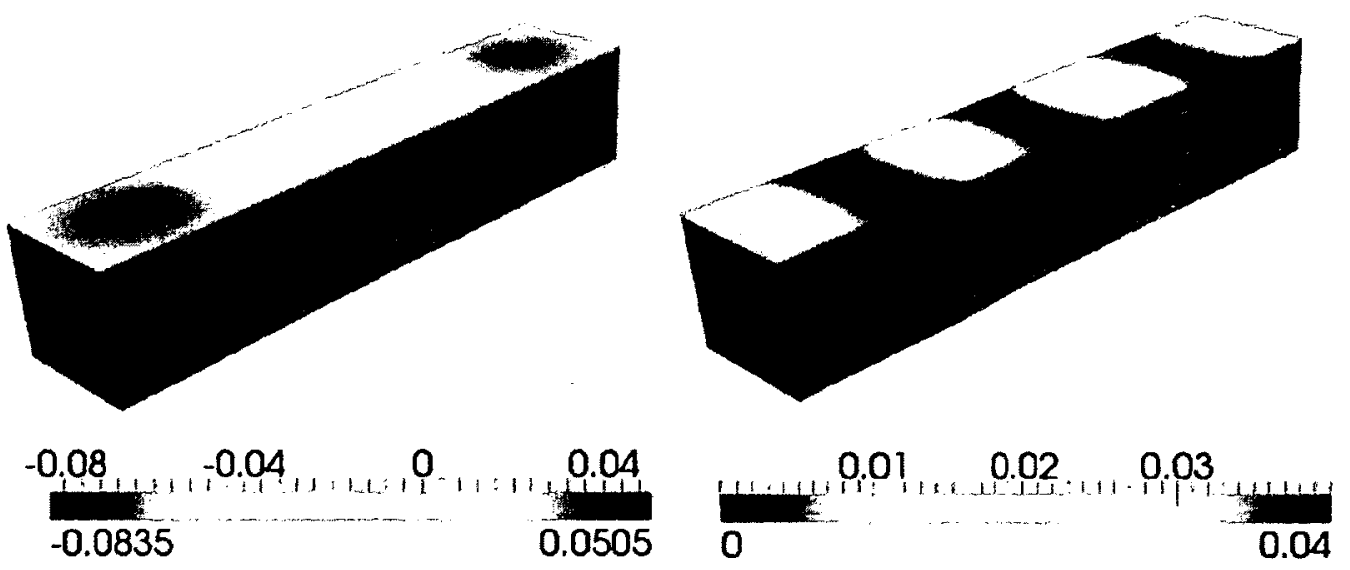

(c) $l_{7}$

(d) $l_{14}$
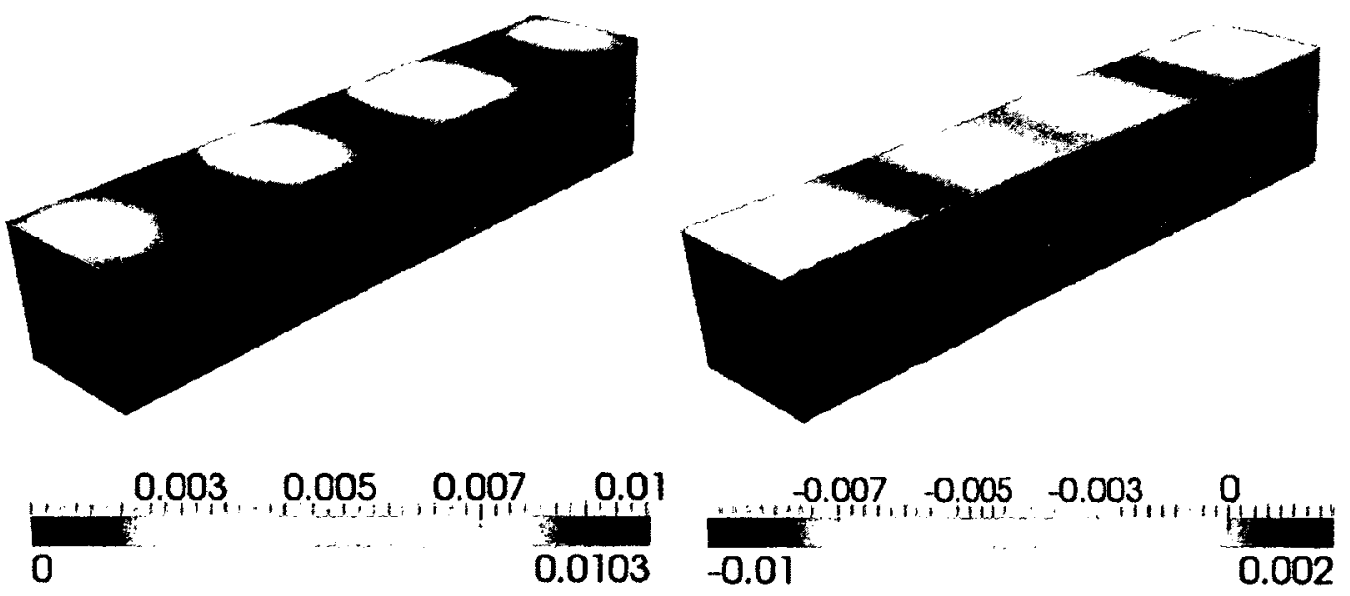

(e) $l_{24}$

(f) $l_{29}$

Figure 10.5: Selected PC coefficients of the hydraulic conductivity:

$$
b_{x}=b_{y}=b_{z}=1
$$



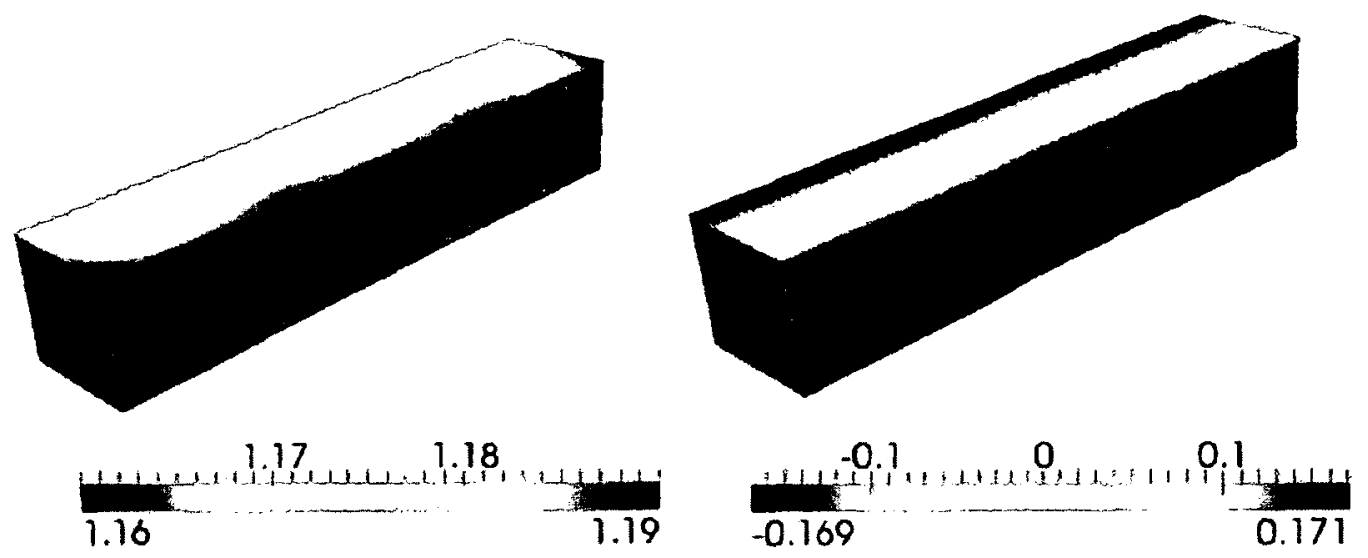

(a) $l_{0}$

(b) $l_{4}$
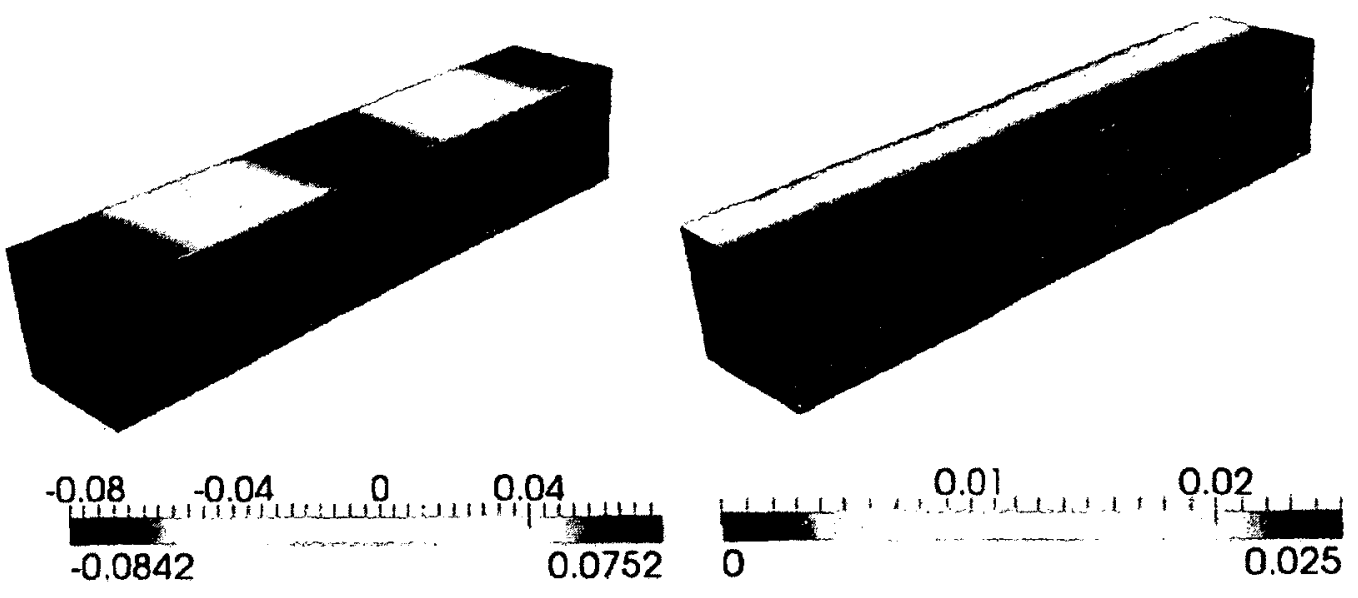

(c) $l_{7}$

(d) $l_{14}$
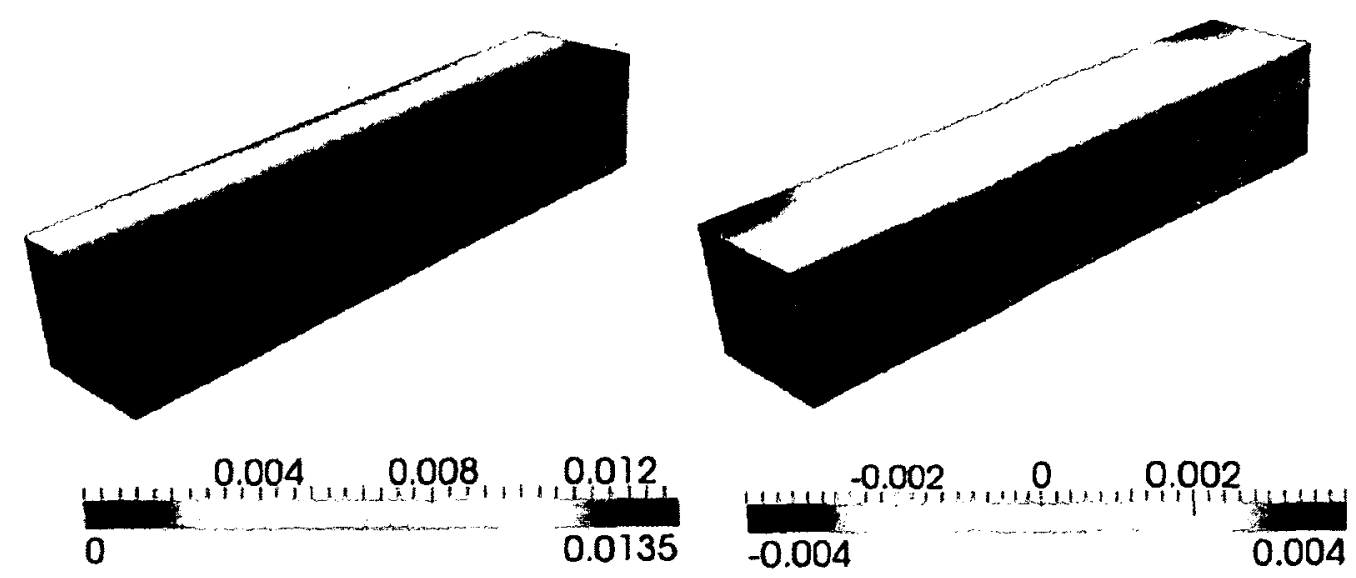

(e) $l_{24}$

(f) $l_{29}$

Figure 10.6: Selected PC coefficients of the hydraulic conductivity:

$$
b_{x}=b_{y}=b_{z}=10
$$


Realizations of the underlying Gaussian and lognormal processes are plotted in Fig.(10.7) and Fig.(10.8) for different correlation lengths. Evidently, the Gaussian process is not strictly positive. On the contrary, the lognormal process is strictly positive. This fact indicates that Gaussian process is inadequate to model uncertainty in a strictly positive quantity such as the hydraulic conductivity coefficient. Note that the fluctuations of the Gaussian and lognormal processes are around the corresponding mean values.

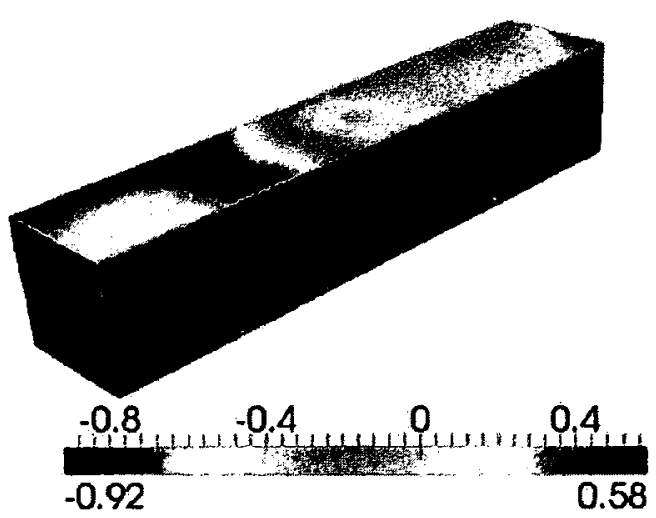

(a) Gaussian

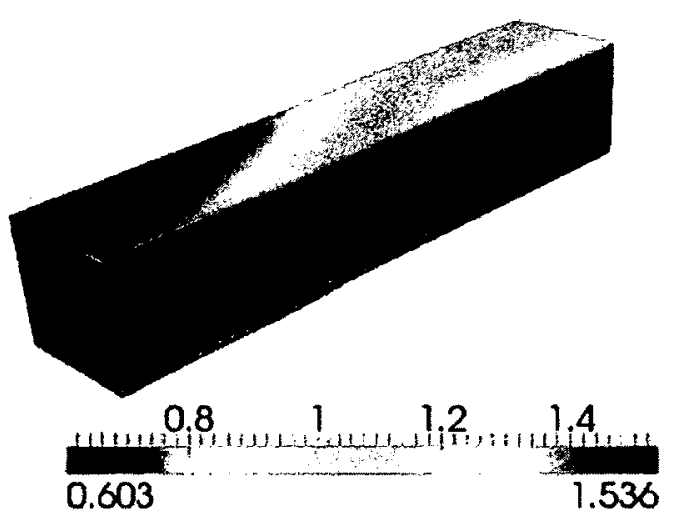

(b) Lognormal

Figure 10.7: Samples of the random hydraulic conductivity field:

$$
b_{x}=b_{y}=b_{z}=1
$$



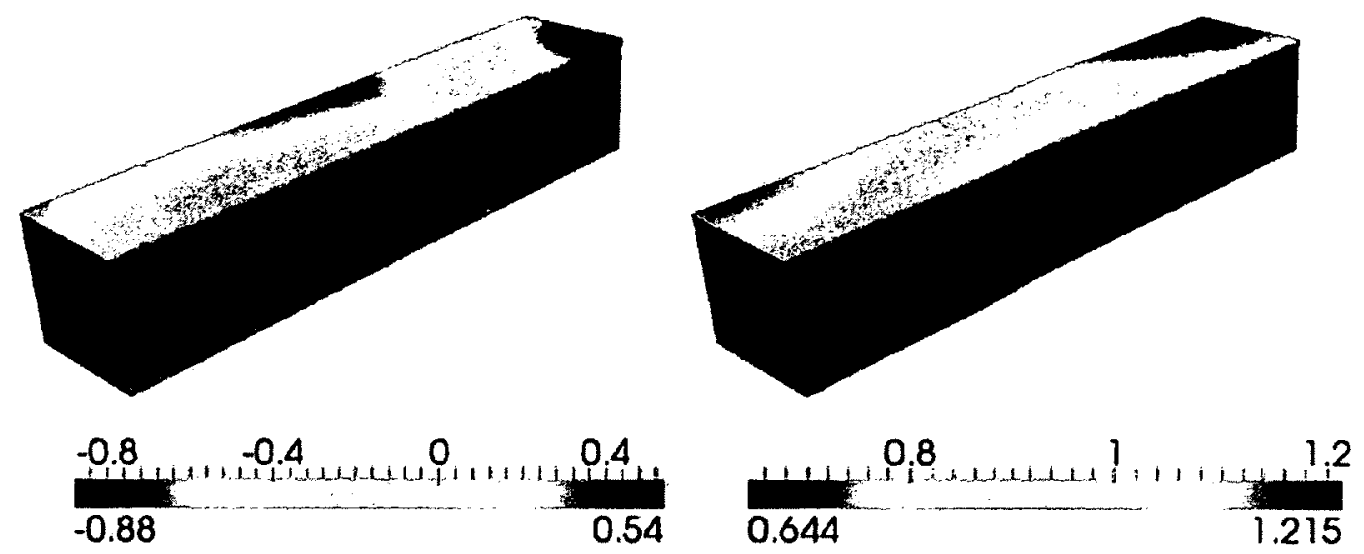

(a) Gaussian

(b) Lognormal

Figure 10.8: Samples of the random hydraulic conductivity field:

$$
b_{x}=b_{y}=b_{z}=10
$$

\subsubsection{Stochastic Features of the Solution Field}

Fig.(10.9) and Fig.(10.10) show the mean and standard deviation of the hydraulic head using different orders of PCE for two different correlation lengths. The first order PCE represents the Gaussian contribution to the solution process whereas the higher orders PC coefficients contain non-Gaussian effects. As the order of PCE increases the solution converges in terms of the mean and standard deviation. 

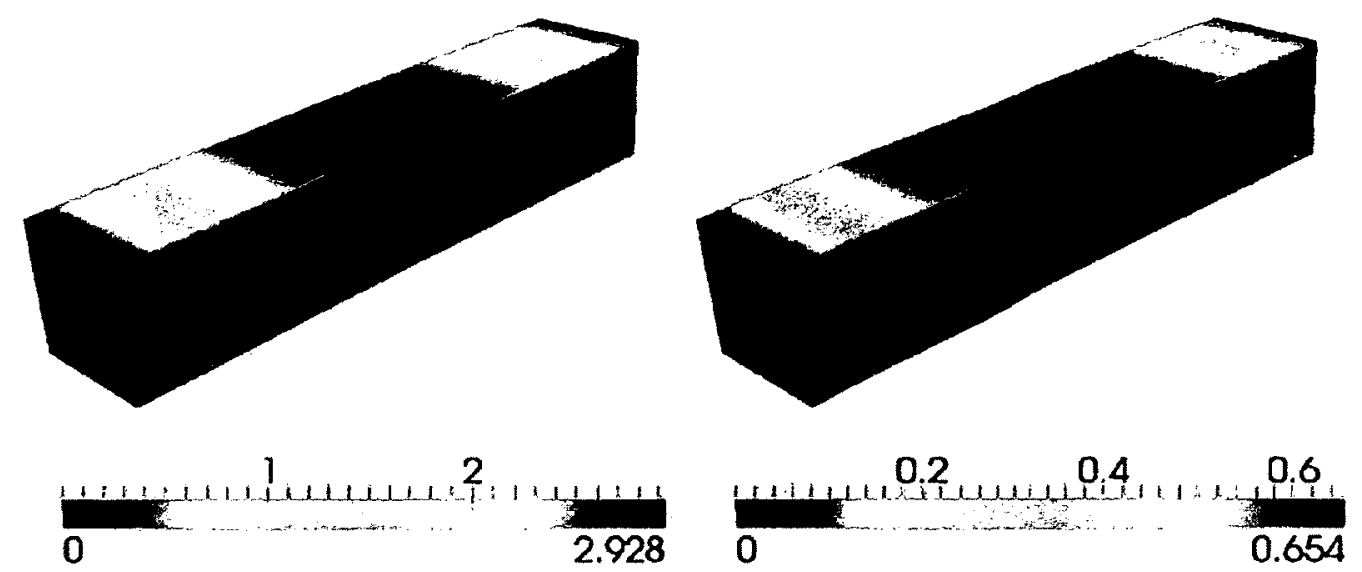

(a) mean: 1st order PCE

(b) standard deviation: 1st order PCE
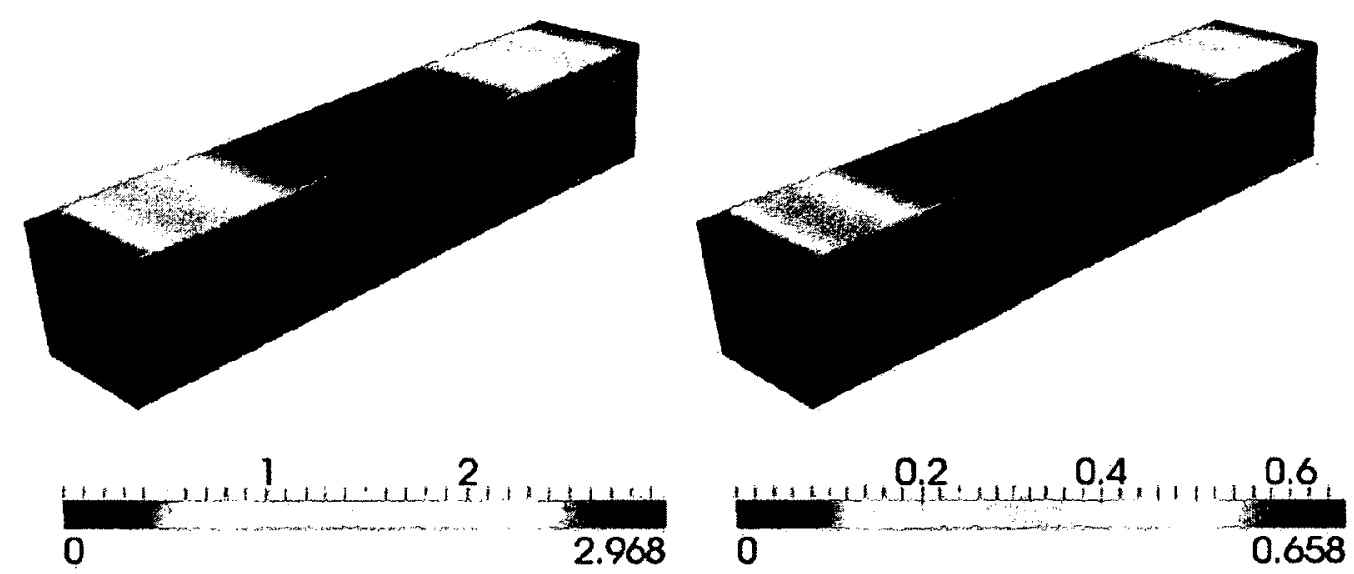

(c) mean: $2 n d$ order PCE

(d) standard deviation: 2 nd order PCE
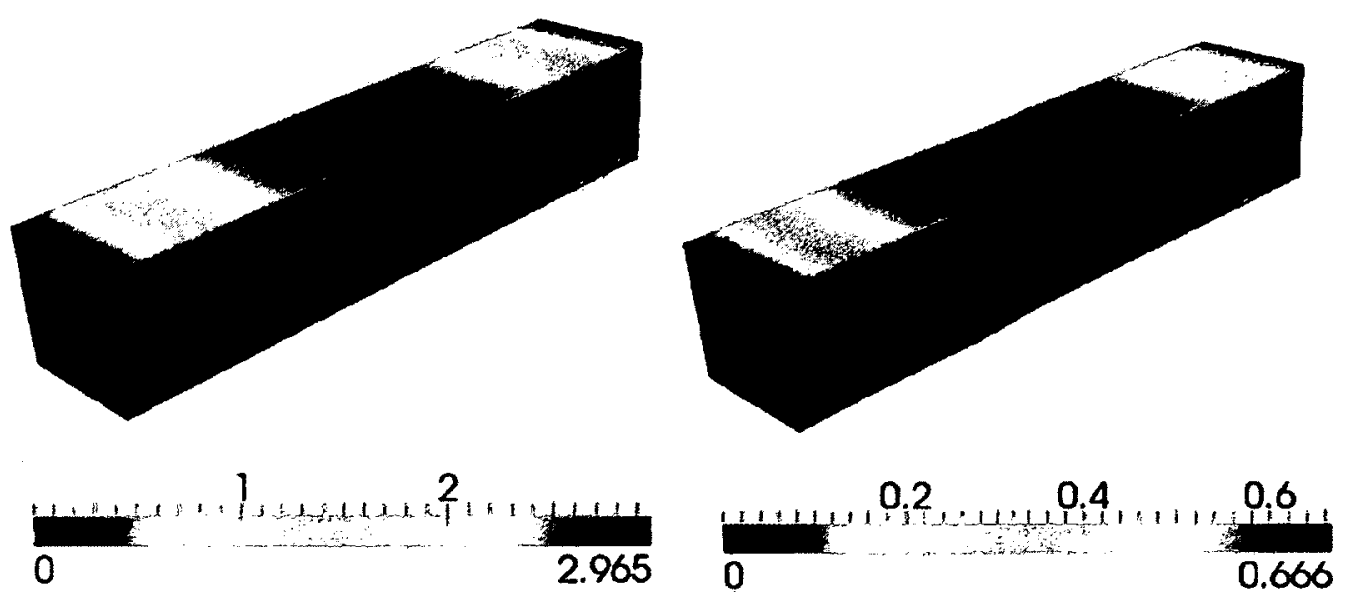

(e) mean: $3 r d$ order PCE

(f) standard deviation: $3 r d$ order PCE

Figure 10.9: The mean and standard deviation of the hydraulic head for different order of PCE: $b_{x}=b_{y}=b_{z}=1$ 

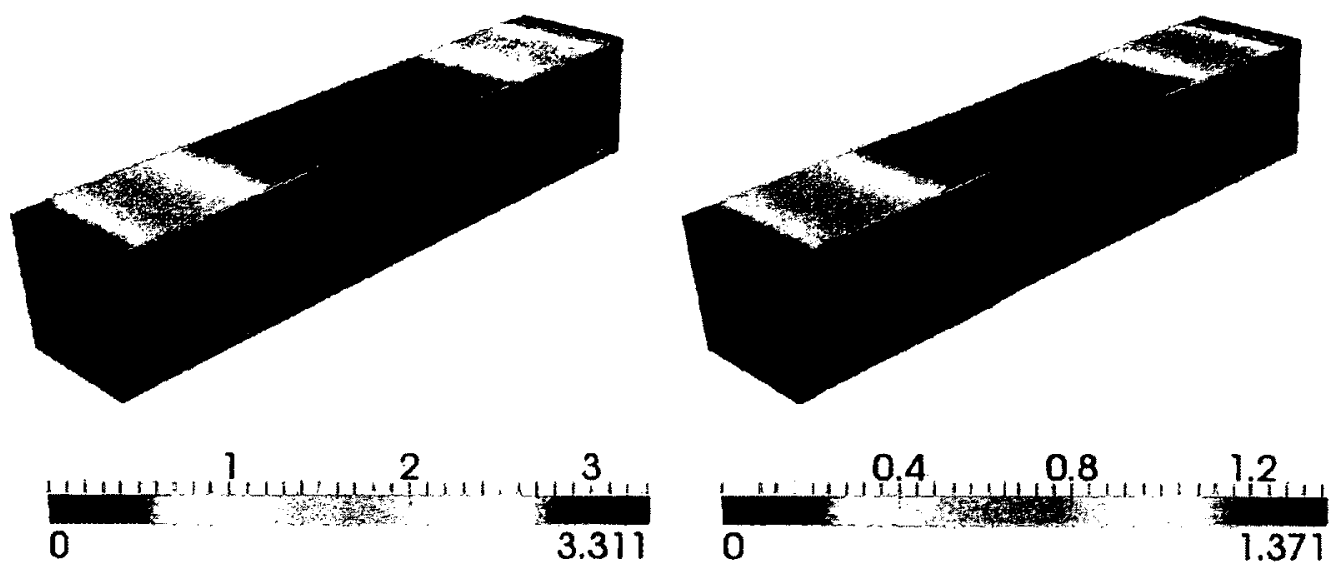

(a) mean: 1 st order PCE

(b) standard deviation: 1 st order PCE
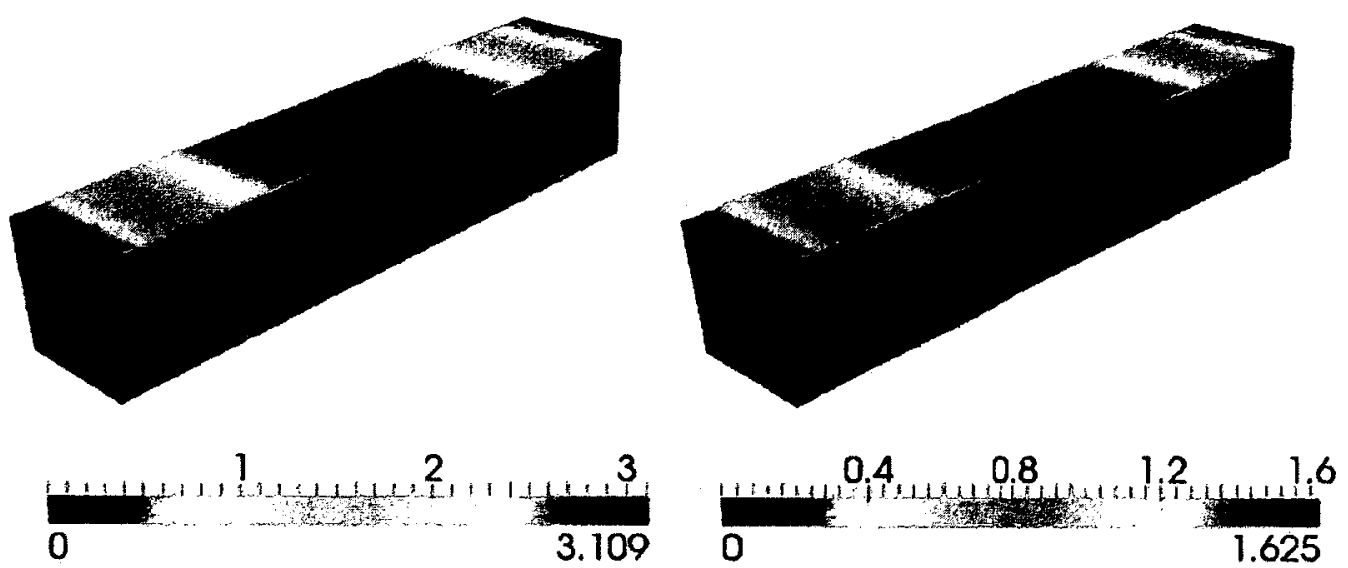

(c) mean: 2nd order PCE

(d) standard deviation: 2 nd order PCE
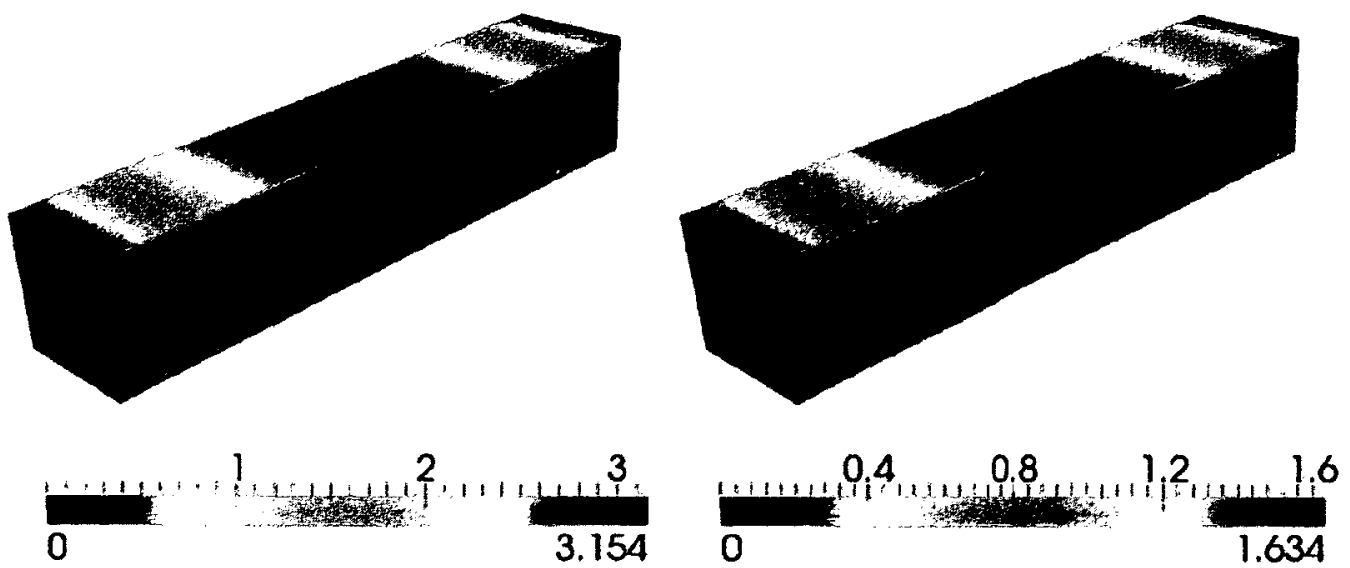

(e) mean: $3 r d$ order PCE

(f) standard deviation: $3 r d$ order PCE

Figure 10.10: The mean and standard deviation of the hydraulic head for different order of PCE: $b_{x}=b_{y}=b_{z}=10$ 
Some of the chaos coefficients of the hydraulic head are plotted in Fig.(10.11) and Fig.(10.12) for two correlation lengths. The chaos coefficients $\mathbf{u}_{j}$ exhibit oscillations whose fluctuations increase with increasing the index $j$. Finer structure can be observed for the case of $\left(b_{x}=b_{y}=b_{z}=1\right)$ compared to that for $\left(b_{x}=b_{y}=b_{z}=10\right)$. One can see that the higher the index $j$ the smaller the magnitude of the chaos coefficients. As mentioned before, higher order PC coefficients correspond to the non-Gaussian components of the response process. 

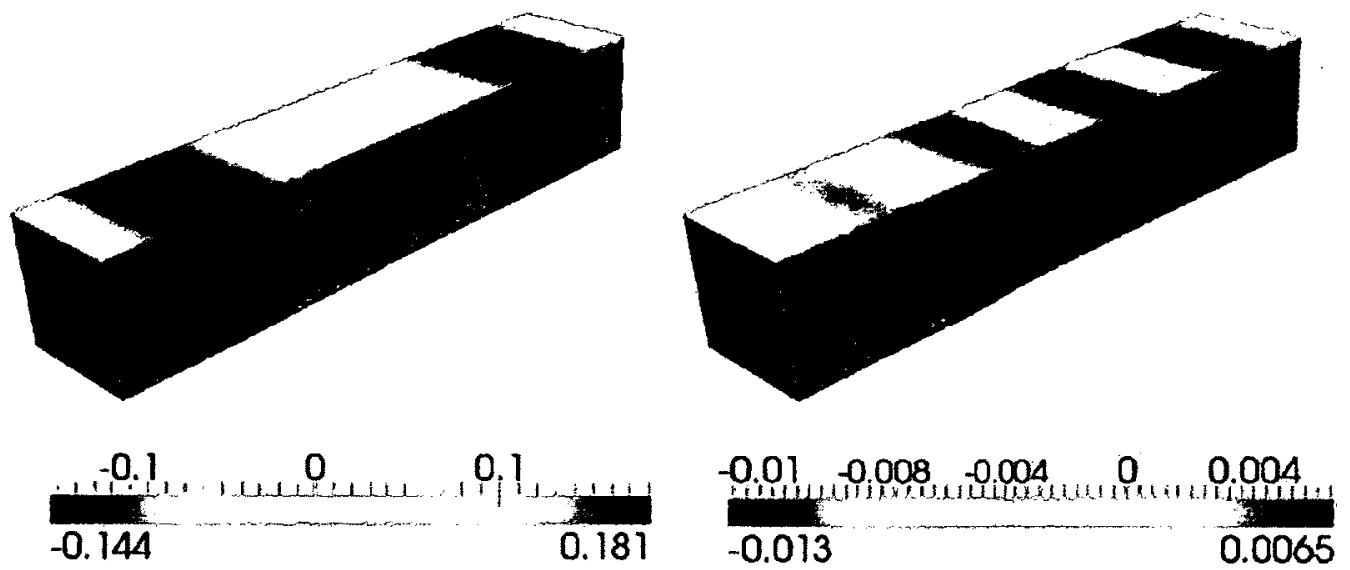

(a) Chaos coefficients: $\mathbf{u}_{\mathbf{4}}$

(b) Chaos coefficients: $\mathbf{u}_{10}$
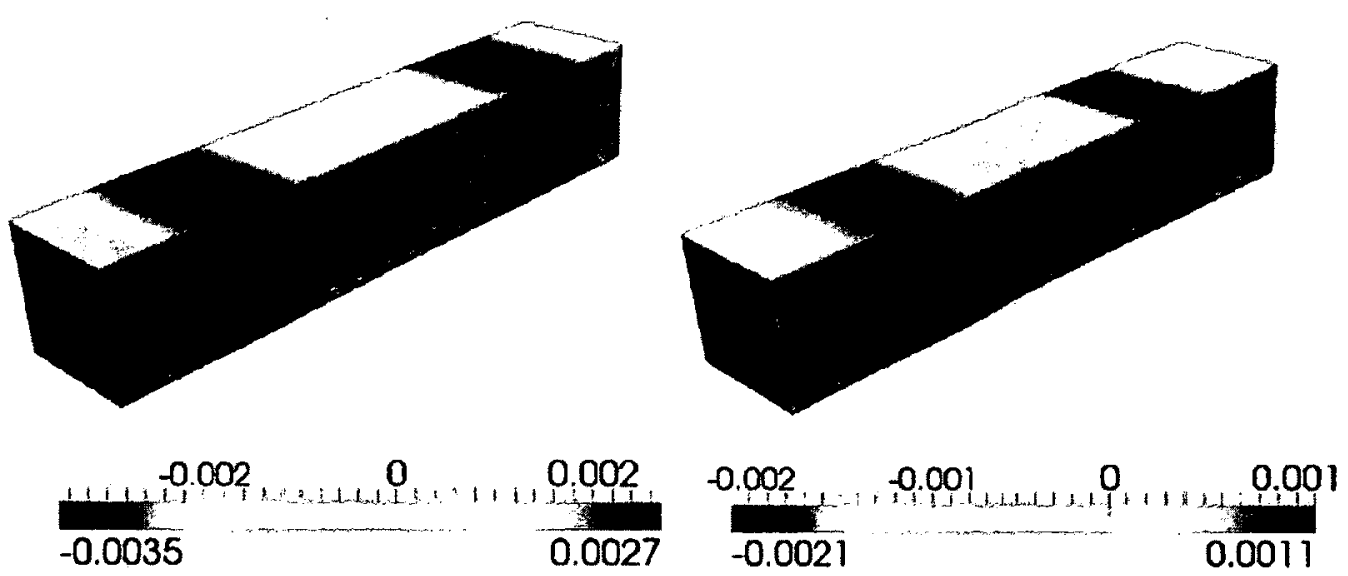

(c) Chaos coefficients: $\mathbf{u}_{18}$

(d) Chaos coefficients: $\mathbf{u}_{27}$
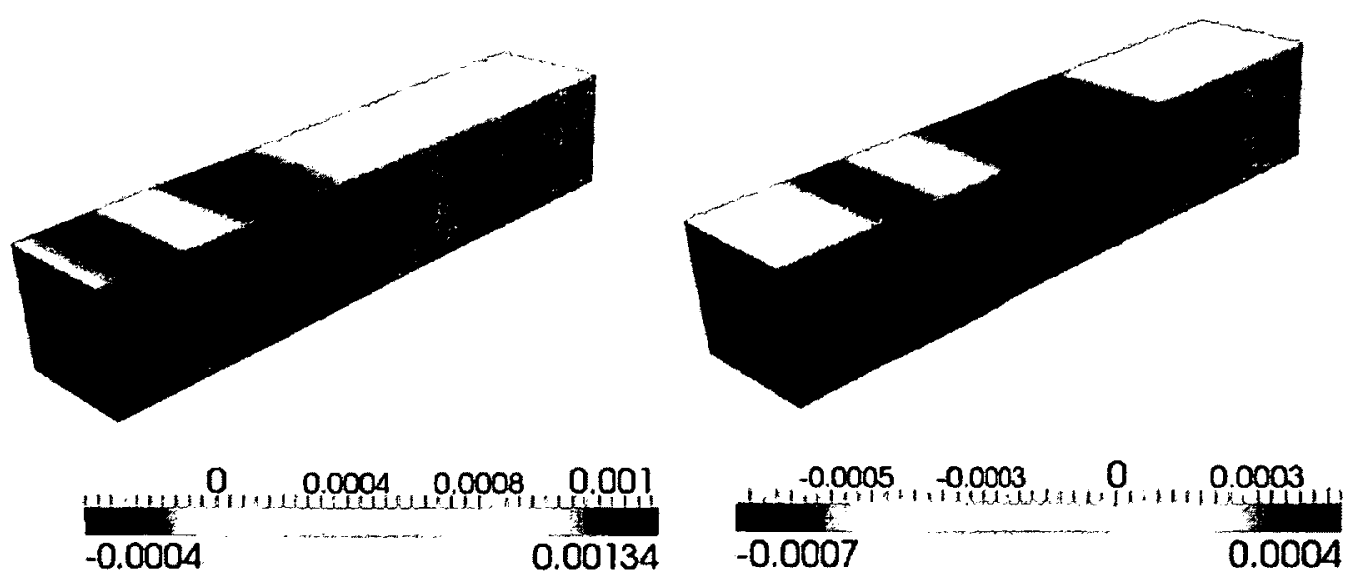

(e) Chaos coefficients: $u_{32}$

(f) Chaos coefficients: $\mathbf{u}_{33}$

Figure 10.11: Selected PC coefficients of the hydraulic head:

$$
b_{x}=b_{y}=b_{z}=1
$$



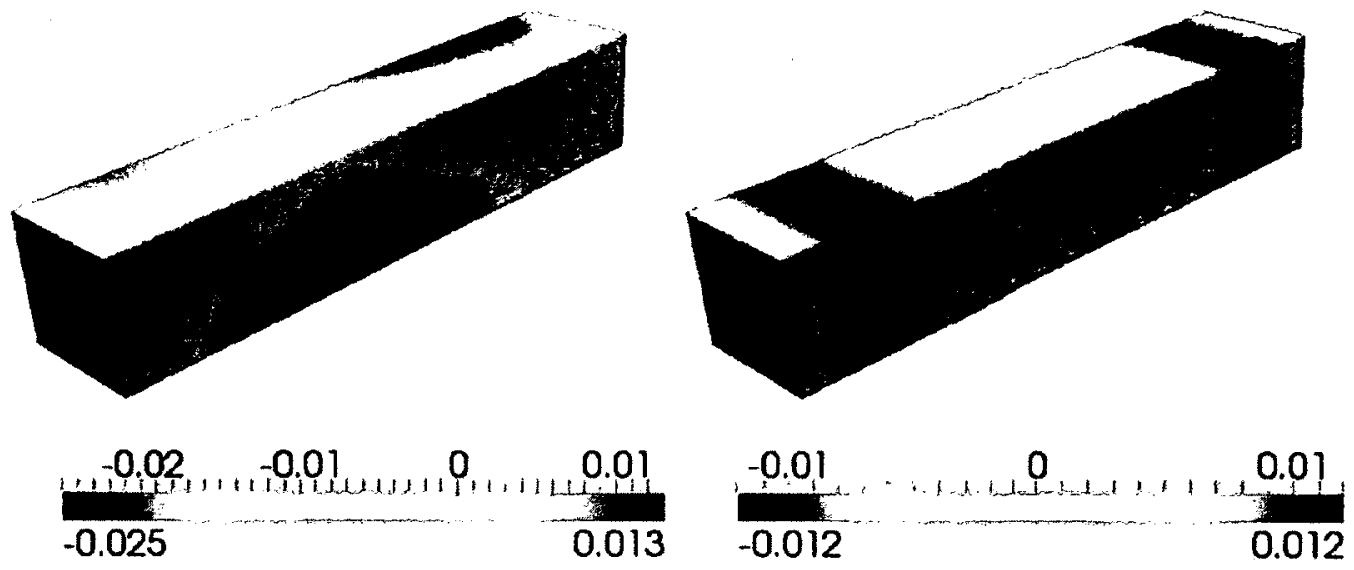

(a) Chaos coefficients: $u_{4}$

(b) Chaos coefficients: $\mathbf{u}_{10}$
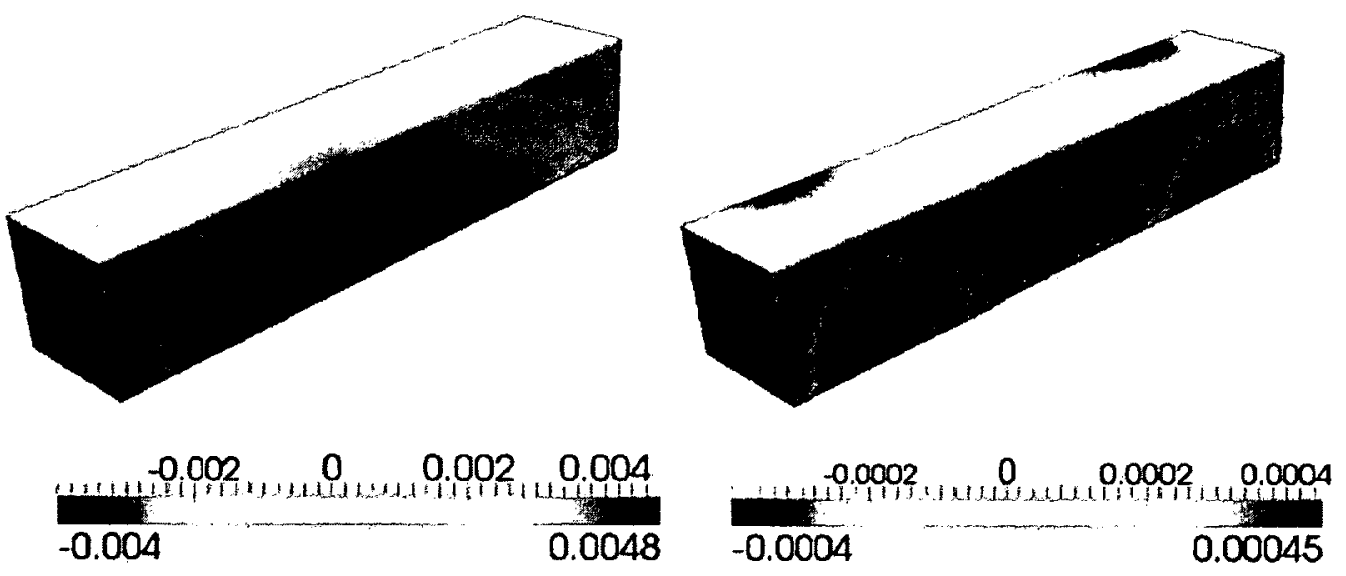

(c) Chaos coefficients: $\mathbf{u}_{18}$
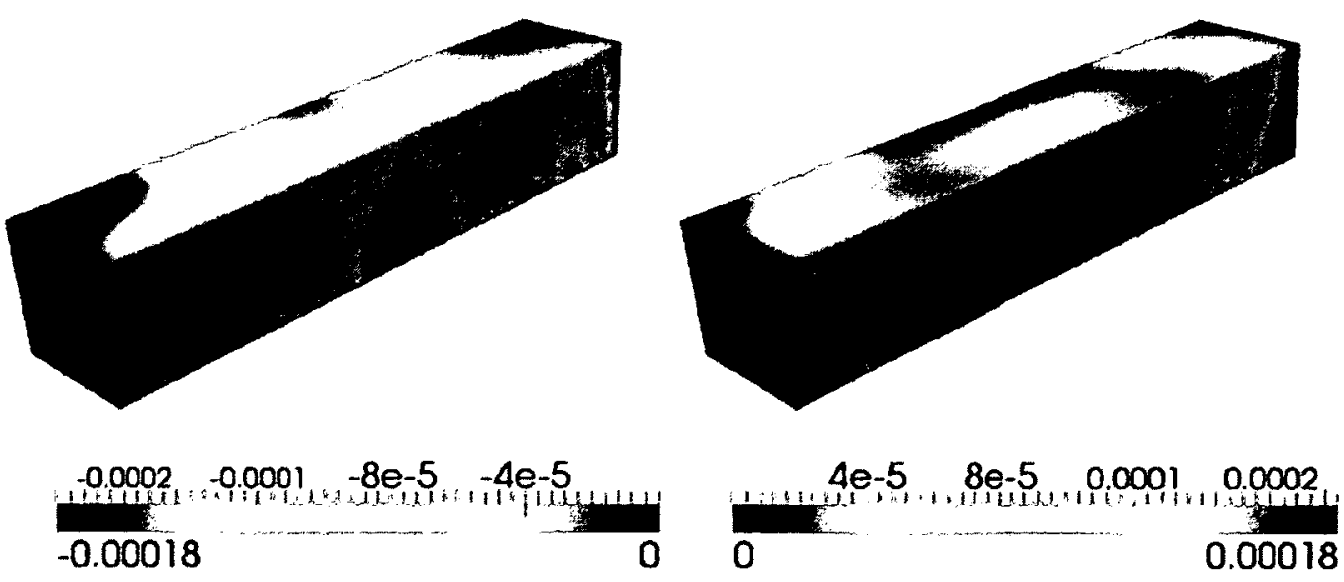

(e) Chaos coefficients: $\mathbf{u}_{32}$

(f) Chaos coefficients: $\mathbf{u}_{33}$

Figure 10.12: Selected PC coefficients of the hydraulic head:

$$
b_{x}=b_{y}=b_{z}=10
$$




\subsubsection{Numerical Scalability}

\section{Effect of PCE order}

For the fixed problem size $n=106,456$ and number of subdomains $n_{s}=128$, Fig.(10.13) shows the iteration counts for the probabilistic two-level methods NNC, PP-DDM and DPDDM versus the order of PCE used to represent the solution process. The probabilistic NNC and PP-DDM methods exhibit similar performances. Both the NNC and PP-DDM methods require more iteration to converge than that of DP-DDM. Nevertheless, the iteration counts required by the probabilistic two-level methods are nearly independent of the order of PCE. This fact demonstrates that the probabilistic two-level techniques are scalable to the order of PCE.

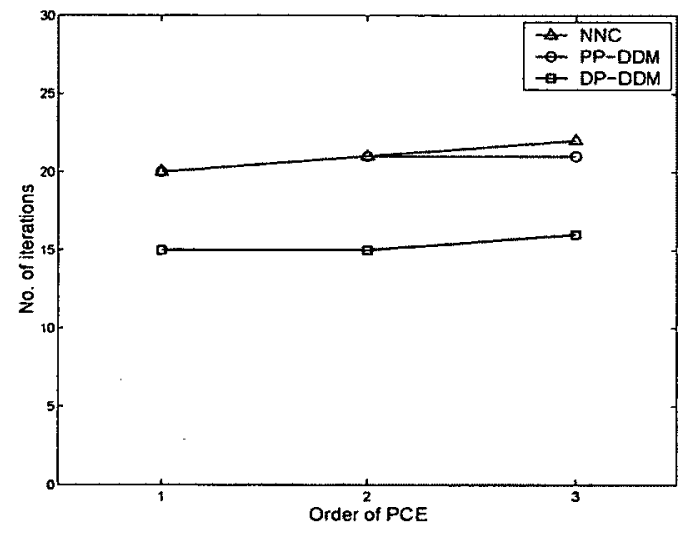

Figure 10.13: Iteration counts versus the order of PCE

In Fig.(10.14), we plot the total execution time for the probabilistic two-level techniques as the order of PCE increases. Clearly, all the two-level methods require almost the same time to run. Note that for a given spatial problem size $n$ the first, second and third order 
PCE involve solving problem size of order $(5 \times n),(15 \times n)$ and $(35 \times n)$ respectively. Undoubtedly, this rapid increase in the problem size demands longer running time.

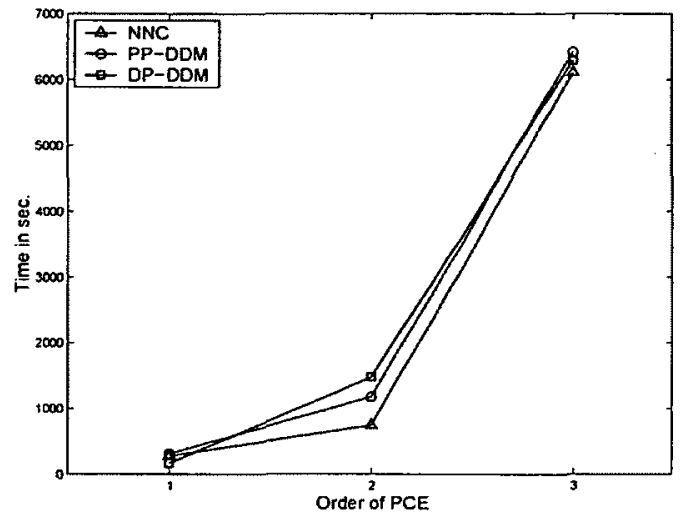

Figure 10.14: Running time versus the order of PCE

\section{Scalability with respect to mesh size $(h)$}

For the fixed number of subdomains $n_{s}=128$ and second order PCE, Fig.(10.15) plots the iteration counts of the probabilistic two-level methods against the finite element mesh resolution. Evidently, the size of the problem does not affect the performance of the two-level methods as the iteration counts required for convergence remain nearly constant. Again the NNC and PP-DDM methods show similar performances, and the DP-DDM requires less number of iterations to converge. 


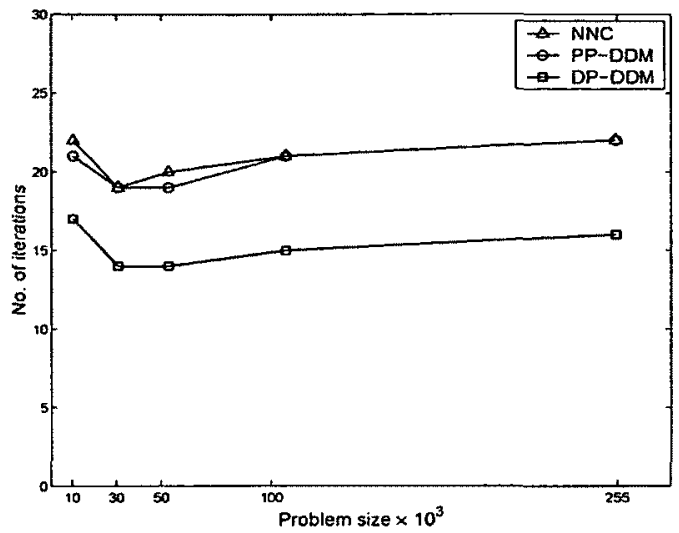

Figure 10.15: Iteration counts with respect to mesh size for fixed number of subdomains

\section{Scalability with respect to the number of subdomains $(H)$}

Fig.(10.16) shows the iteration counts versus number of subdomains for fixed problem size $n=106,456$ and second order PCE. Increasing number of subdomains does not degrade the performance of the probabilistic two-level methods as the number of iterations required for convergence remains almost constant. This fact suggests that the probabilistic two-level methods are scalable with respect to number of subdomains [129]. 


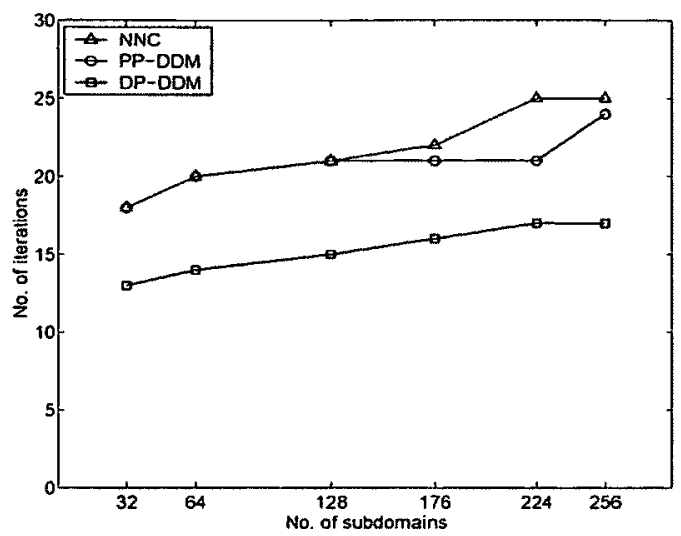

Figure 10.16: Iteration counts with respect to number of subdomains for fixed problem size

\section{Scalability with respect to fixed problem size per subdomain $(H / h)$}

In Fig.(10.17), we plot the iteration counts of the probabilistic two-level methods for fixed problem size per subdomain $n \approx 1,300$ and second order PCE while increasing the global problem size by adding more subdomains. The iteration counts remain bounded as we increase the total problem size by using more subdomains with fixed problem size per subdomain. 


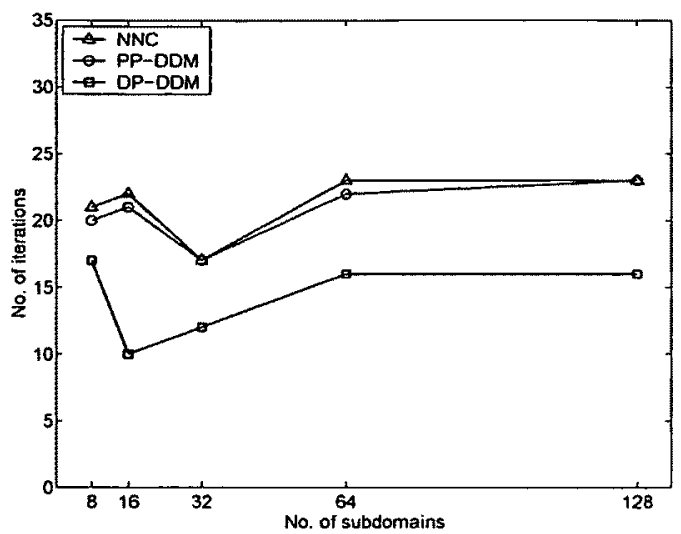

Figure 10.17: Iteration counts with respect to number of subdomains for fixed problem size per subdomain

\subsubsection{Parallel Scalability}

\section{Strong scalability}

The strong scalabilities of the probabilistic two-level domain decomposition methods are shown in Fig.(10.18) for problem size $n=106,456$ and second order PCE. As we use more processors to tackle the problem, the total execution time steadily decreases up to 256 processors. For this specific problem, as increasing the number of processors further, the communication overhead predominates over the cost of floating point operation and no reduction in the total execution time can be gained. 


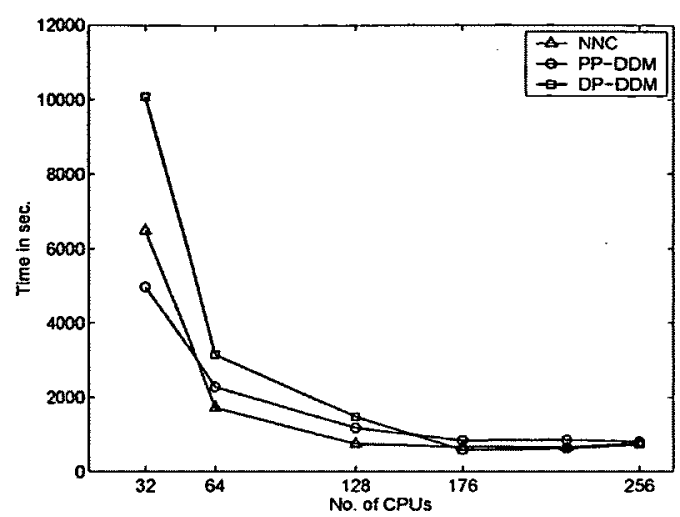

Figure 10.18: Strong scalability

\section{Weak scalability}

In Fig.(10.19), we show the weak scalabilities of the probabilistic two-level methods for fixed spatial problem size per subdomain $n \approx 1,300$ and second order PCE. All the methods exhibit almost the same performance in terms of total execution time. The run time increases almost linearly as we increase the total problem size by adding more processors/subdomains.

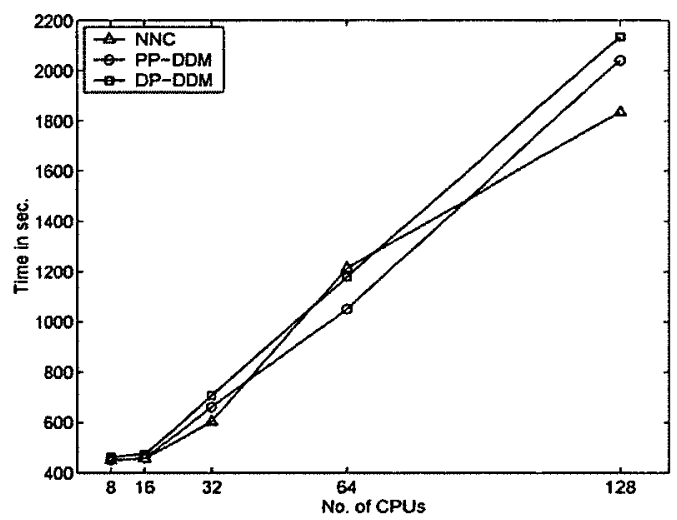

Figure 10.19: Weak scalability 


\subsection{Application to Large-Scale Heat Transfer Problem}

In this section, we apply the probabilistic two-level iterative substructuring techniques to quantify uncertainty in three-dimensional heat transfer problem in a crankshaft defined as

$$
\begin{aligned}
\nabla \cdot(\kappa(\mathbf{x}, \theta) \nabla u(\mathbf{x}, \theta)) & =f(\mathbf{x}, \theta), \quad \mathbf{x} \in \Omega \\
u(\mathbf{x}, \theta) & =0, \quad \mathbf{x} \in \partial \Omega_{D}
\end{aligned}
$$

where the thermal diffusivity coefficient $\kappa(\mathbf{x}, \theta)$ is modeled as a lognormal stochastic process obtained from the underlying Gaussian process with a given covariance function. The volumetric heat source is assumed to be spatially invariant deterministic quantity $f(\mathbf{x}, \theta)=1.0$. The geometry of the crankshaft is shown in Fig.(10.20).

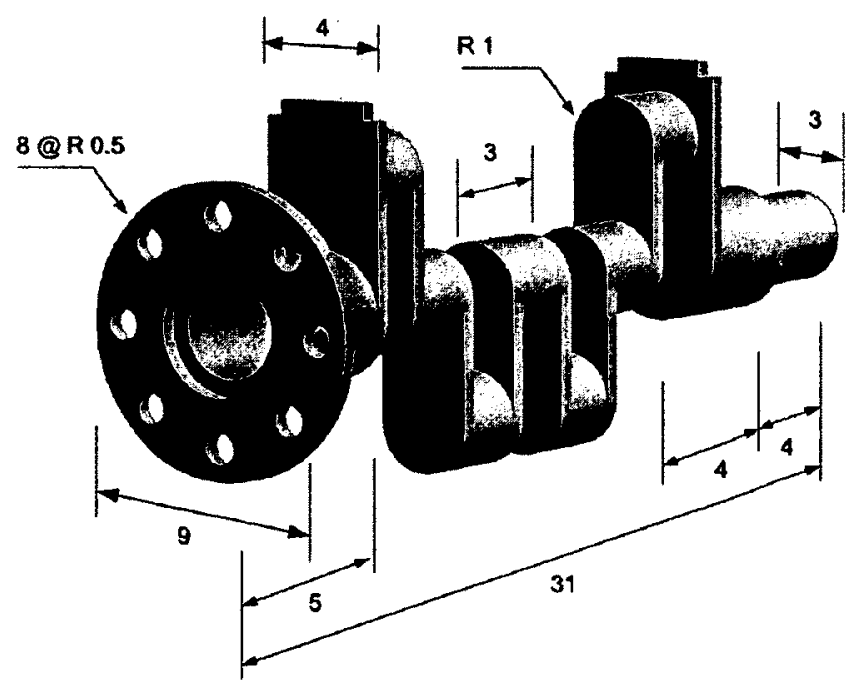

Figure 10.20: The geometry of the crankshaft (all dimensions in $\mathrm{cm}$ ) 


\subsubsection{Finite Element Discretization and Mesh Decomposition}

The crankshaft shown in Fig.(10.21(a)) is discretized using 92, 429 linear tetrahedral finite elements and 545,663 nodes. The response is expanded using four-dimensional $(L=4)$ third order PCE $(N=34)$ yielding a linear system of order $3,235,015$. The partitioned domain into 160 subdomains using METIS graph partitioning tool [89] is depicted in Fig.(10.21(b)) and the crankshaft is assumed to be insulated on the top surfaces.

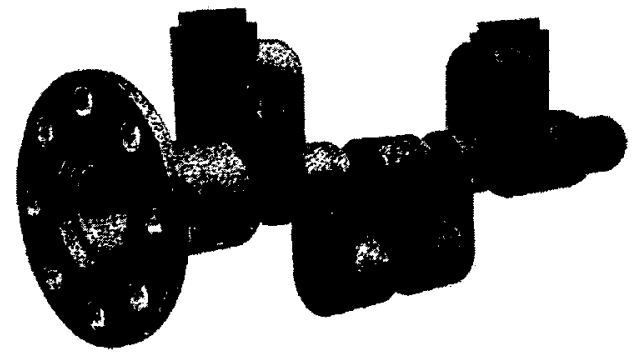

(a) FEM mesh

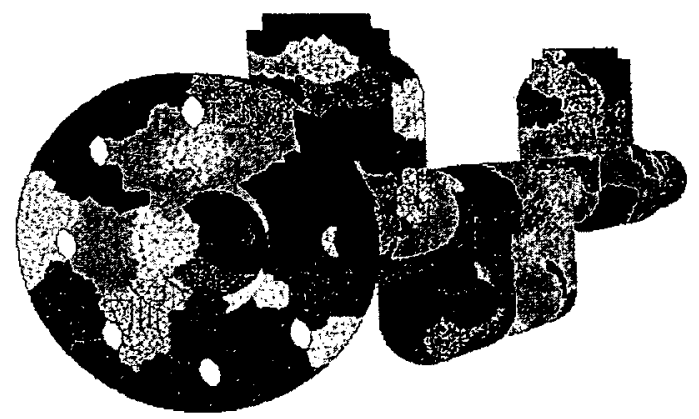

(b) Domain Decomposition

Figure 10.21: Finite element discretization and mesh decomposition

\subsubsection{Stochastic Field Representation}

The uncertain thermal diffusivity coefficient $\kappa(\mathbf{x}, \theta)$ is modeled as a lognormal stochastic process obtained from the underlying Gaussian process as

$$
\kappa(\mathbf{x}, \theta)=\exp (g(\mathbf{x}, \theta))
$$

The Gaussian process $g(\mathbf{x}, \theta)$ has a zero mean and an exponential covariance function defined as $[92,101]$ 


$$
C_{g g}(\mathbf{x}, \mathbf{y})=\sigma^{2} \exp \left(\frac{-\left|x_{1}-x_{2}\right|}{b_{x}}+\frac{-\left|y_{1}-y_{2}\right|}{b_{y}}+\frac{-\left|z_{1}-z_{2}\right|}{b_{z}}\right)
$$

The following numerical values used in the investigation are $\sigma=0.3$ and $b_{x}=b_{y}=$ $b_{z}=60$.

For computational efficiency, the eigenvalue analysis for KLE is performed on an extended domain which encapsulates the original geometry over which the problem is defined. The extended domain is discretized using 31,705 elements and 7,706 nodes. This mesh is coarse compared to the original finite element mesh of the problem. In addition, the PC coefficients of the lognormal process are computed on the same mesh. Then the finite element shape functions are used to extrapolate the PC coefficients from the coarse to the fine mesh used in the analysis. This approach avoids unnecessary complication for $\mathrm{KLE}$ involving the fine mesh.

In our implementation, we use LAPACK dense eigenvalue problem solver [151]. For the stochastic process with small correlation length, the contributions of higher order fluctuations become significant. Thus the eigenvalue analysis for KLE must be carried out on the fine mesh. In this context, parallel solvers for the eigenvalue problem can be useful [152].

In Fig.(10.22), we show the first four eigenfunctions of the covariance kernel. Fig.(10.23) and Fig.(10.24) show a set of selected chaos coefficients of the lognormal process (diffusivity coefficient) on the coarse and fine domains respectively. 


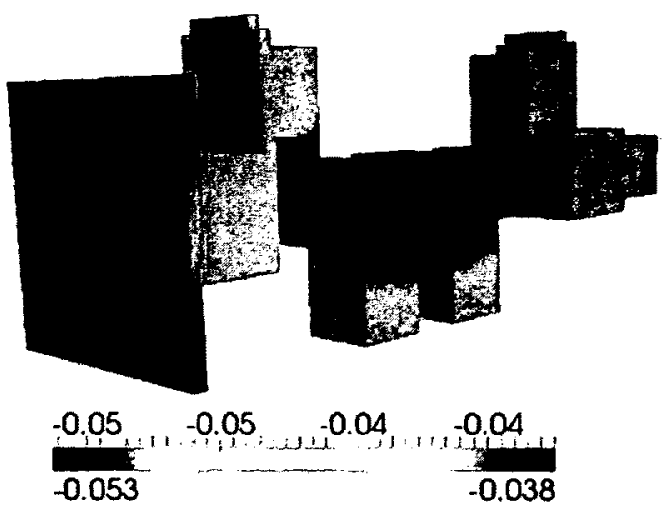

(a) $1 \mathrm{st}$

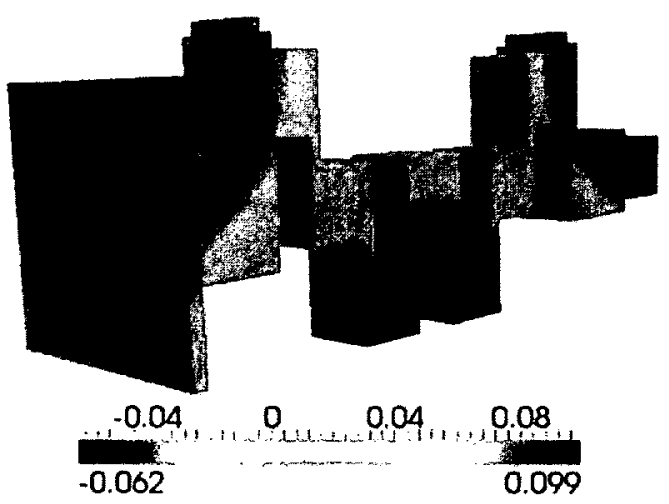

(c) 3rd

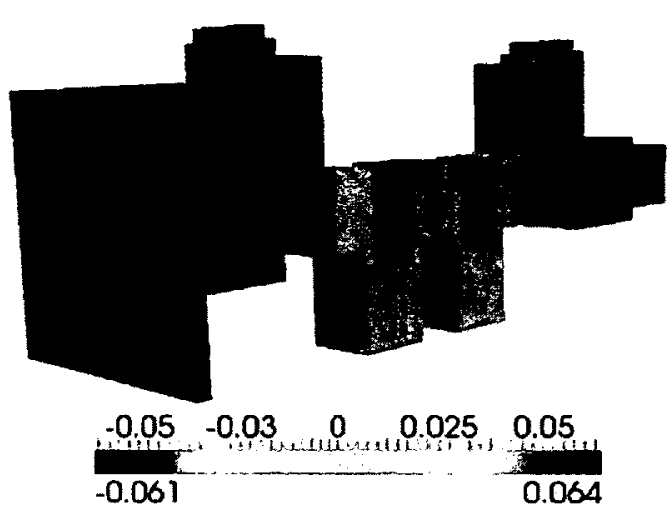

(b) 2nd

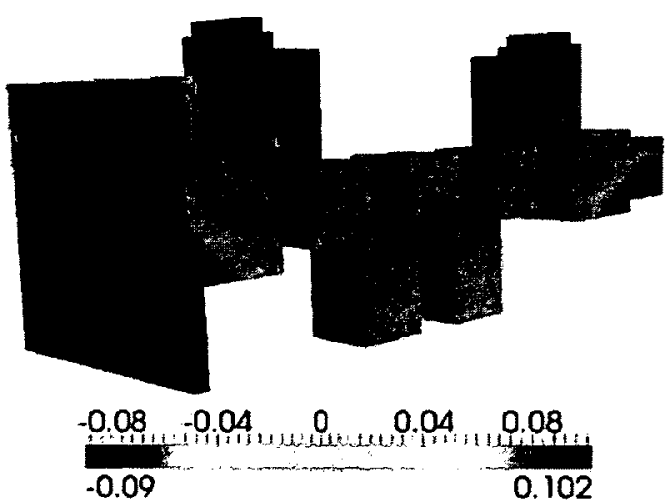

(d) 4 th

Figure 10.22: The first fourth eigenfunctions of the covariance function 


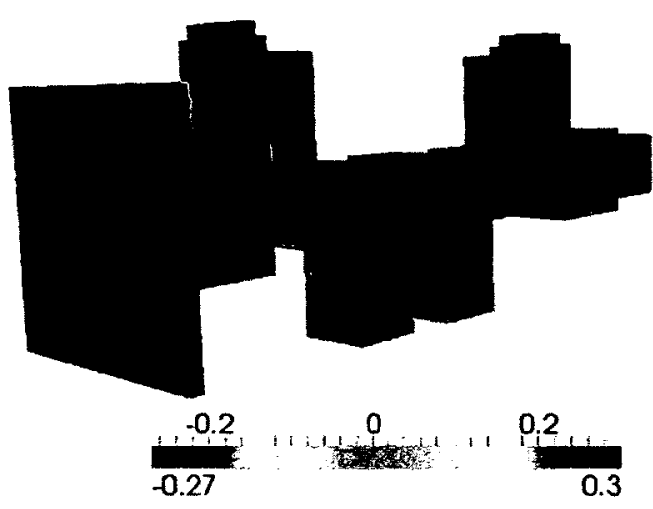

(a) Chaos coefficients: $l_{5}$

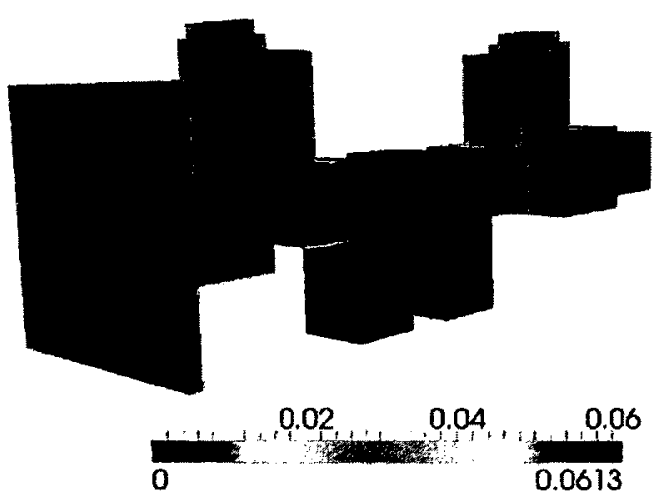

(c) Chaos coefficients: $l_{10}$

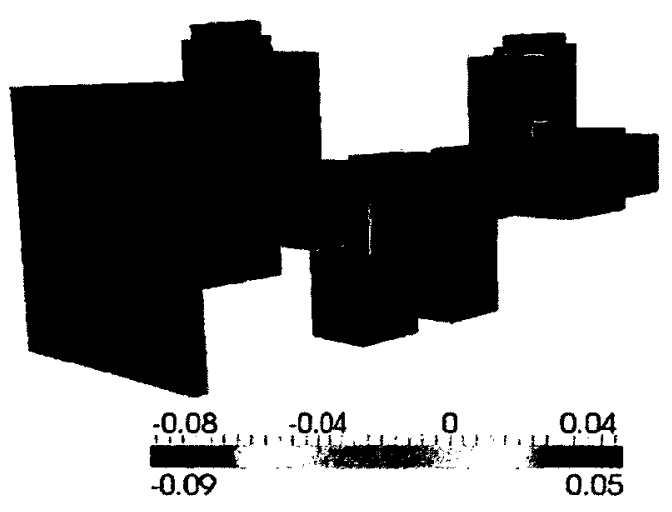

(b) Chaos coefficients: $l_{8}$

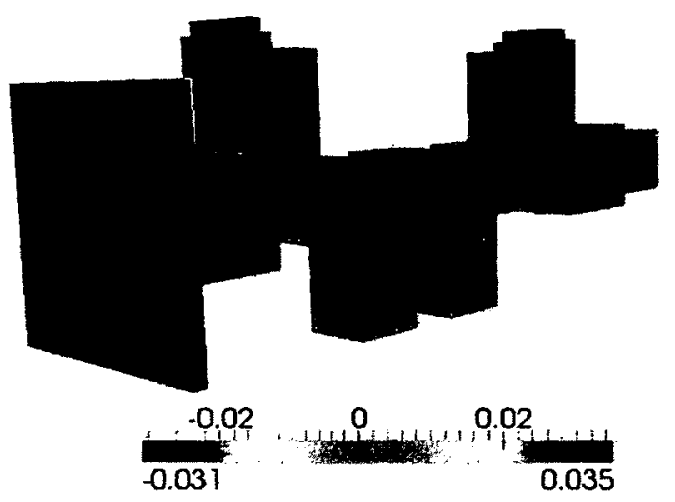

(d) Chaos coefficients: $l_{12}$

Figure 10.23: Selected PC coefficients of the diffusivity field on the extended domain 


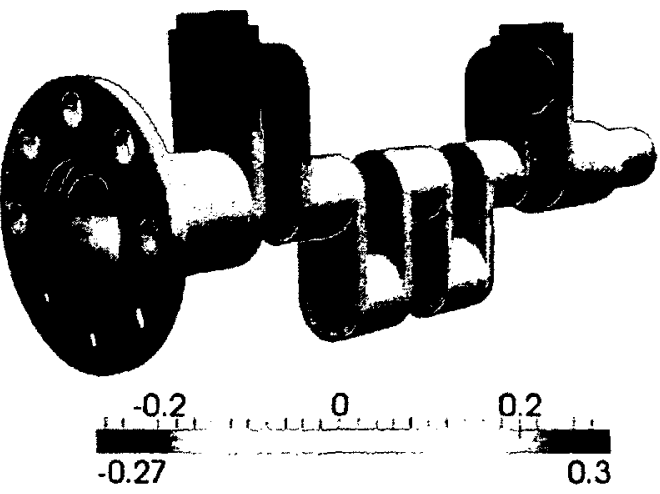

(a) Chaos coefficients: $l_{5}$

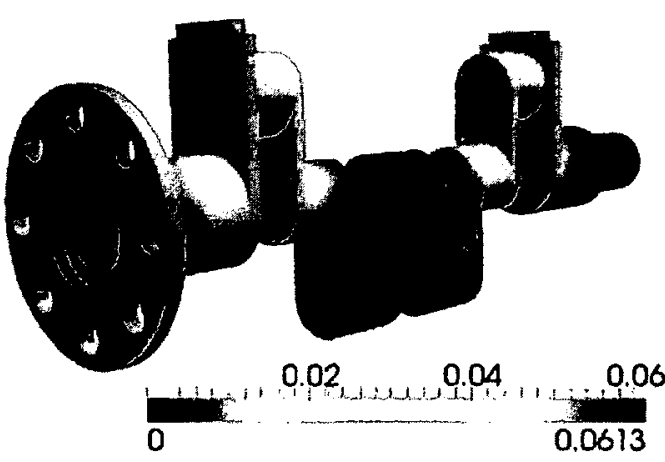

(c) Chaos coefficients: $l_{10}$

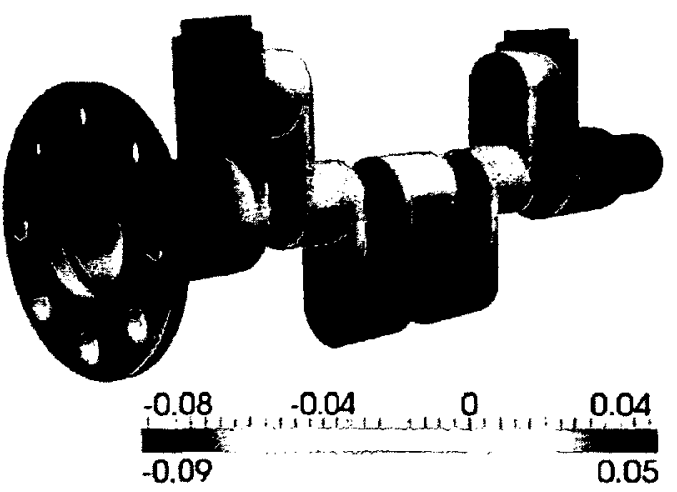

(b) Chaos coefficients: $l_{8}$

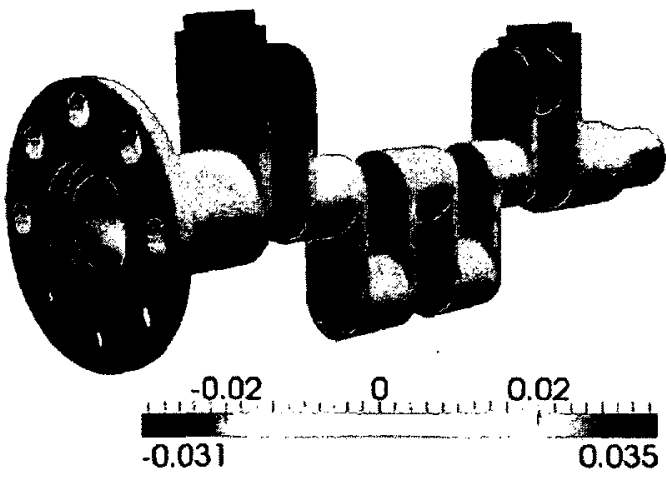

(d) Chaos coefficients: $l_{12}$

Figure 10.24: Selected PC coefficients of the diffusivity field on the original domain

\subsubsection{Stochastic Features of the Solution Field}

The mean and standard deviation of the temperature process are plotted in Fig.(10.25(a)) and Fig.(10.25(b)) respectively. The standard deviation has the same trend as the mean. The maximum coefficient of variation in the middle of the crankshaft is $21 \%$. Some of the chaos coefficients of the temperature process are shown in Fig.(10.26). The chaos coeffi- 
cients exhibit decay in magnitude as the index of the coefficients increases. In Fig.(10.27), we show Lagrange multipliers $\lambda_{j}$ corresponding to the first four PC coefficient of the temperature process. Clearly, the larger the index $j$, the smaller the magnitude of Lagrange multipliers $\lambda_{j}$.

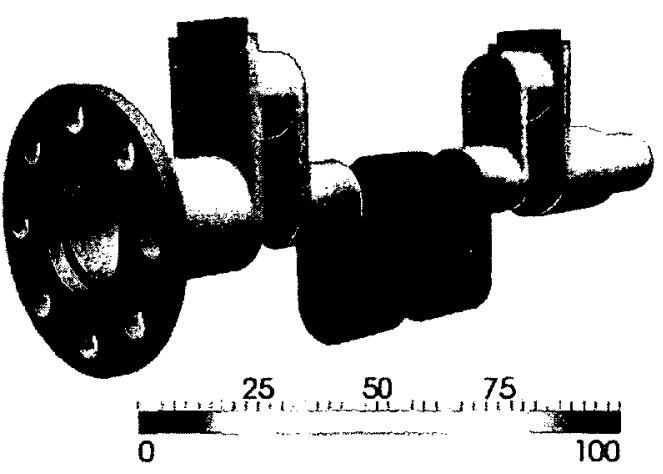

(a) Mean response

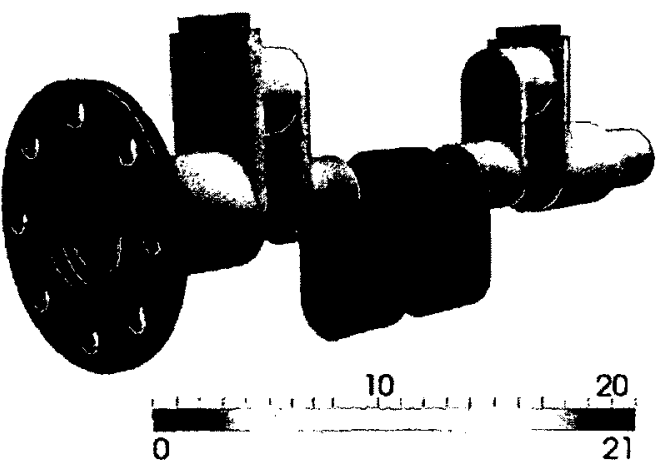

(b) Standard deviation

Figure 10.25: The mean and the standard deviation of the temperature field 

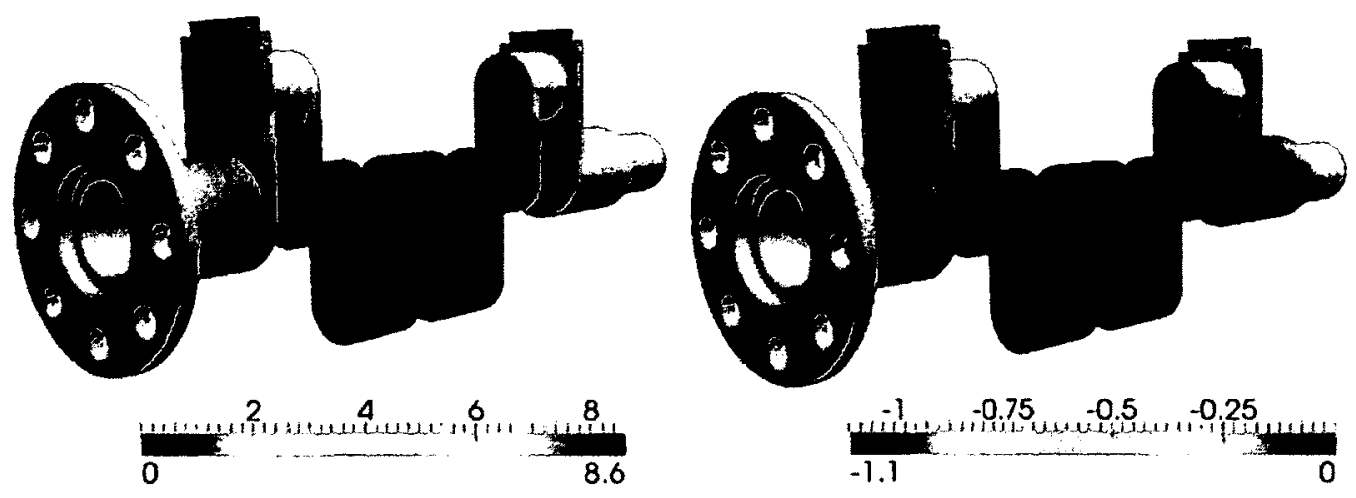

(a) Chaos coefficients: $\mathbf{u}_{4}$
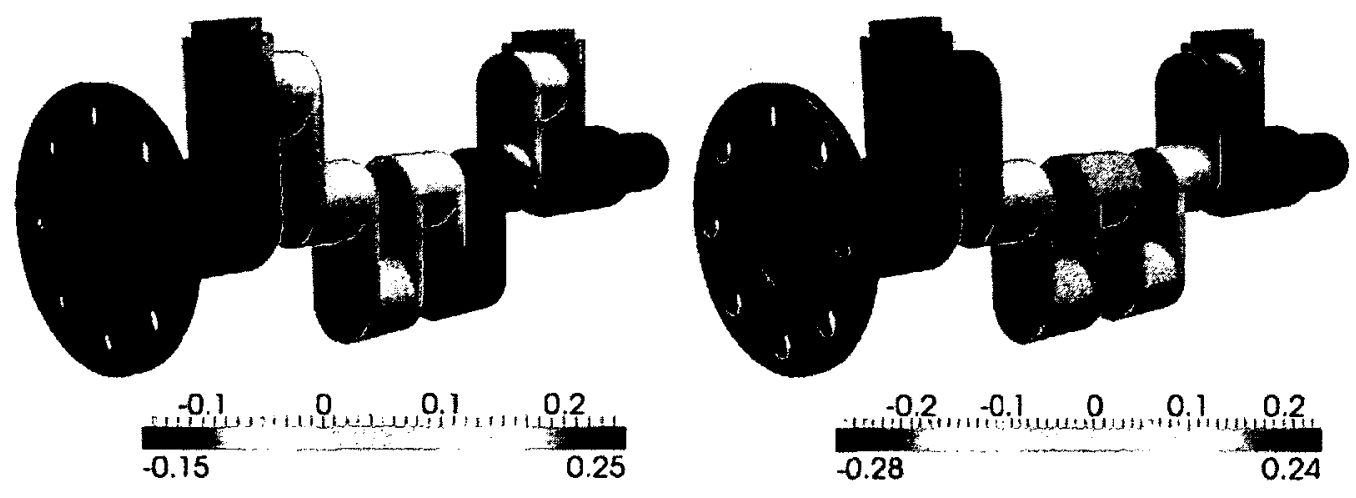

(c) Chaos coefficients: $\mathbf{u}_{10}$

(d) Chaos coefficients: $\mathbf{u}_{11}$

Figure 10.26: Selected PC coefficients of the temperature field 


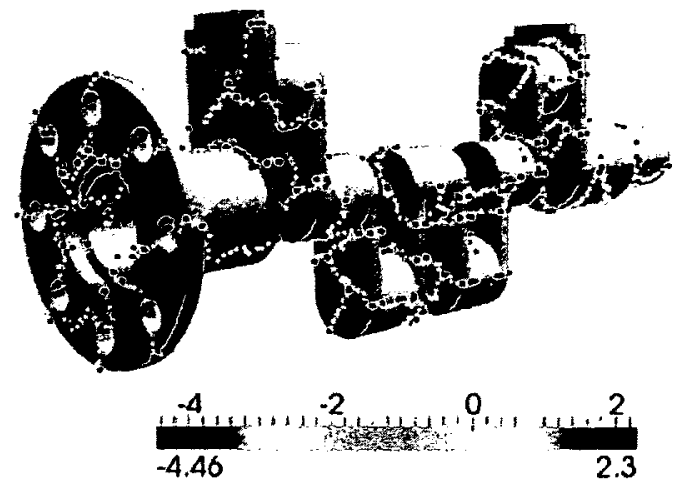

(a) Lagrange multipliers: $\lambda_{0}$

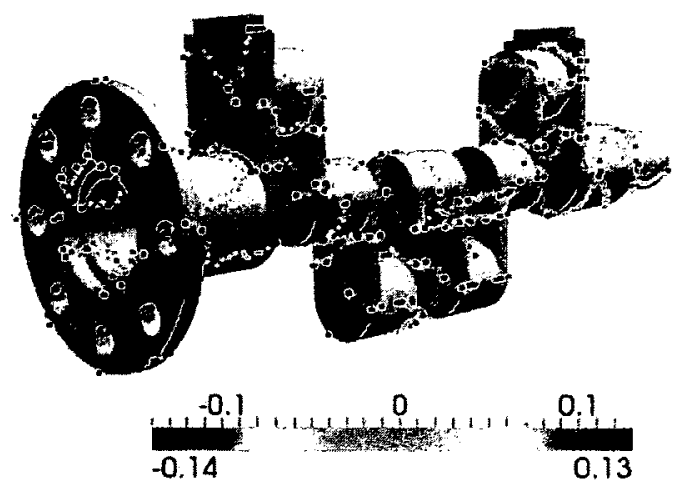

(c) Lagrange multipliers: $\boldsymbol{\lambda}_{2}$

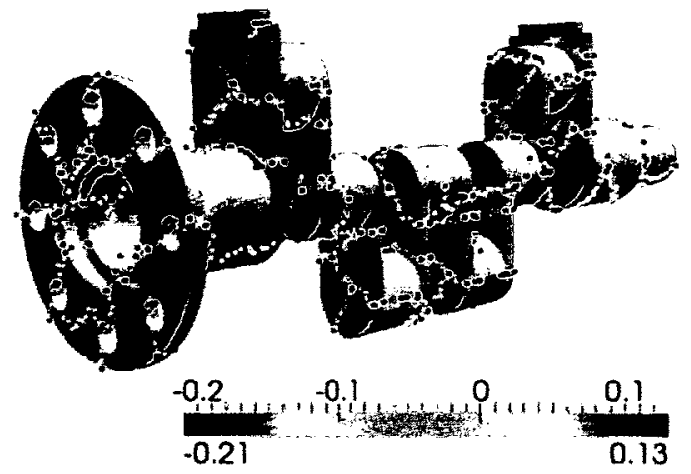

(b) Lagrange multipliers: $\lambda_{1}$

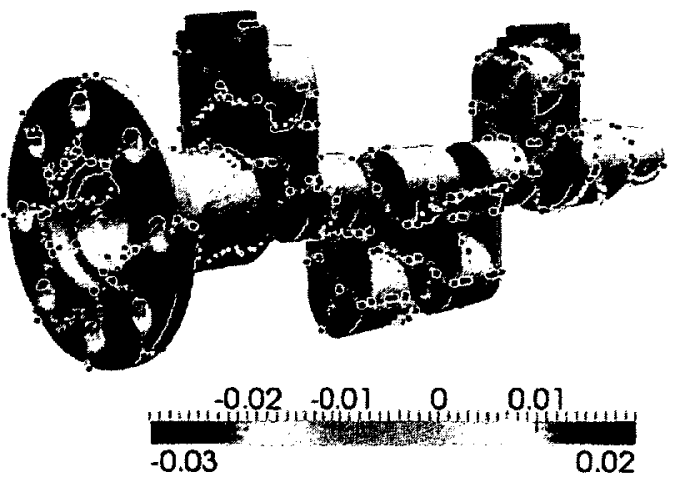

(d) Lagrange multipliers: $\boldsymbol{\lambda}_{\mathbf{3}}$

Figure 10.27: Lagrange multipliers corresponding to the first four PC coefficient of the temperature field 


\subsubsection{Numerical Scalability}

\section{Effect of the order of PCE}

In Fig.(10.28), we show the performances of the probabilistic two-level methods as we increase the order of PCE of the solution process. Increasing the order of PCE leads to a mild growth in the iteration counts of the probabilistic NNC and PP-DDM. However, the iteration counts remain constant for the probabilistic DP-DDM.

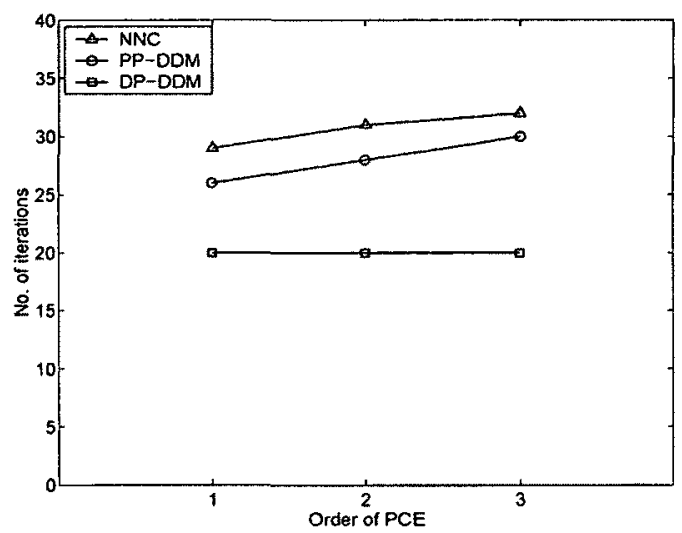

Figure 10.28: Iteration counts versus the order of PCE

\section{Scalability with respect to mesh size $(h)$}

In Fig.(10.29), we show the iteration counts of the probabilistic two-level methods when we fix the number of subdomains and order of PCE, but refine the finite element mesh. Evidently, the iteration counts required for convergence are nearly independent of the finite element mesh resolution. The probabilistic NNC and PP-DDM techniques exhibit similar performances. The probabilistic DP-DDM requires less number of iterations compared to the NNC and PP-DDM techniques. 


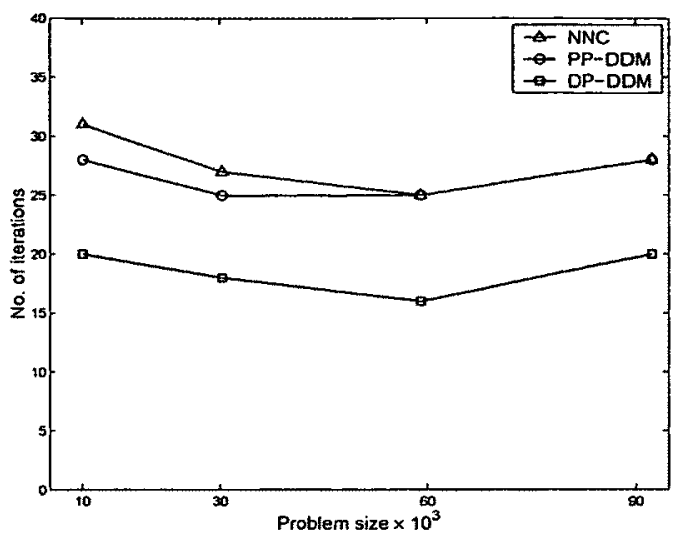

Figure 10.29: Iteration counts with respect to mesh size for fixed number of subdomains

\section{Scalability with respect to the number of subdomains $(H)$}

For fixed spatial mesh resolution and order of PCE, Fig.(10.30) shows the iteration counts of the probabilistic two-level methods for increasing number of subdomains. Once again, the probabilistic NNC and PP-DDM show similar performances while DP-DDM demands less number of iterations to converge. Nevertheless, the performances of all the probabilistic two-level methods are nearly independent of the number of subdomains. This fact indicates that the probabilistic two-level techniques show a scalable preformance with respect to the number of subdomains. 


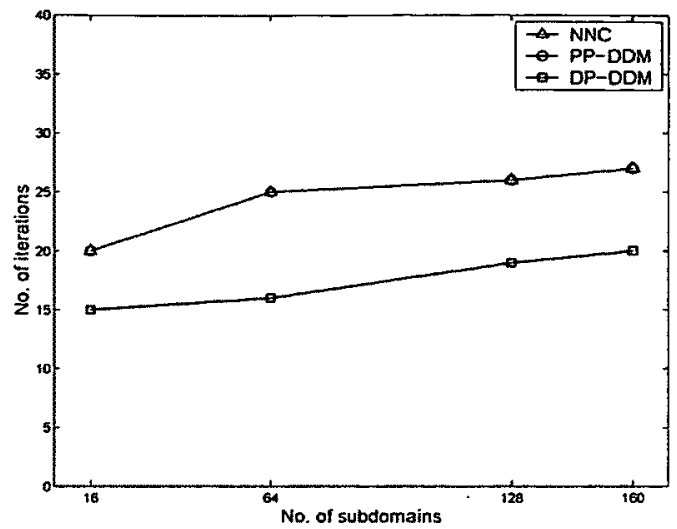

Figure 10.30: Iteration counts with respect to the number of subdomains for fixed problem size

\section{Scalability with respect to fixed problem size per subdomain $(H / h)$}

Fig.(10.31) demonstrates the performances of the probabilistic two-level domain decomposition techniques when the problem size per subdomain is kept constant, but the total problem size is increased by adding more subdomains $[64,153]$. The iteration counts increase almost linearly as we add more subdomains with fixed problem size. 


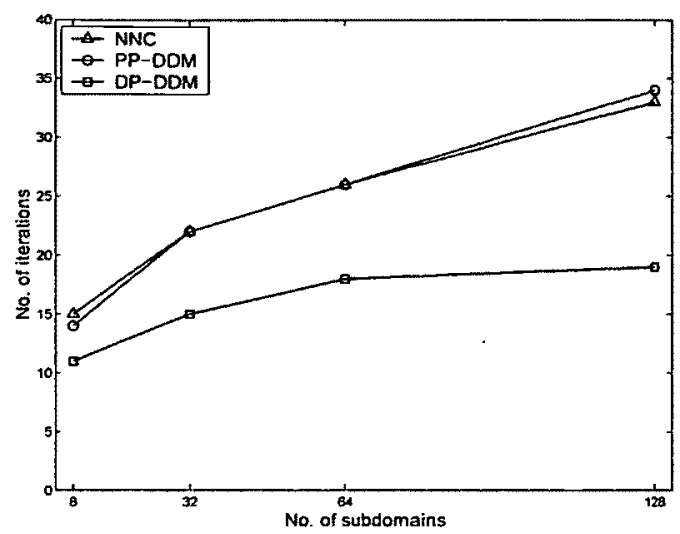

Figure 10.31: Iteration counts with respect to the number of subdomains for fixed problem size per subdomain

\subsubsection{Parallel Scalability}

\section{Strong scalability}

For fixed problem size, Fig.(10.32) shows the execution time for the probabilistic two-level methods when we increase the number of processors to tackle the problem. All the methods exhibit similar performances. Increasing the number of processors from 16 to 64 leads to about $80 \%$ reduction in the total execution time. However, increasing the number of processors further does not significantly reduce the total execution time as the computational cost dominates over the communication cost. 


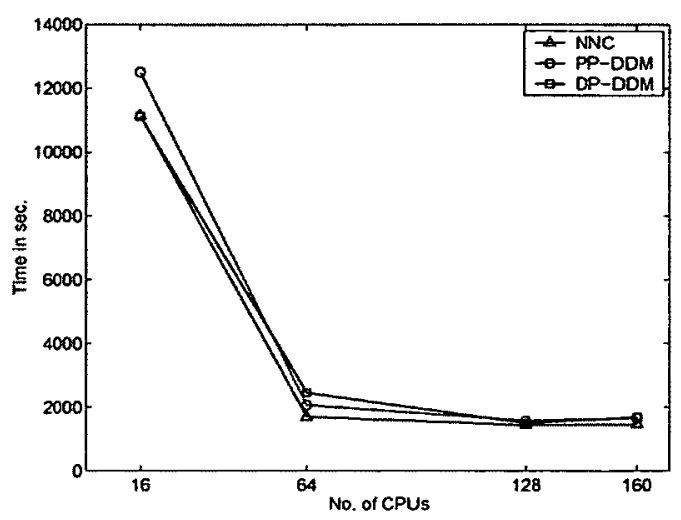

Figure 10.32: Strong scalability

\section{Weak scalability}

Fig.(10.33) shows the total execution time for the probabilistic two-level methods when we fix the problem size per subdomain and increase the global size of the problem by using more subdomains. The results indicate that for fixed problem size per processor, the total execution time grows almost linearly as we add more subdomains, thereby increasing the overall problem size.

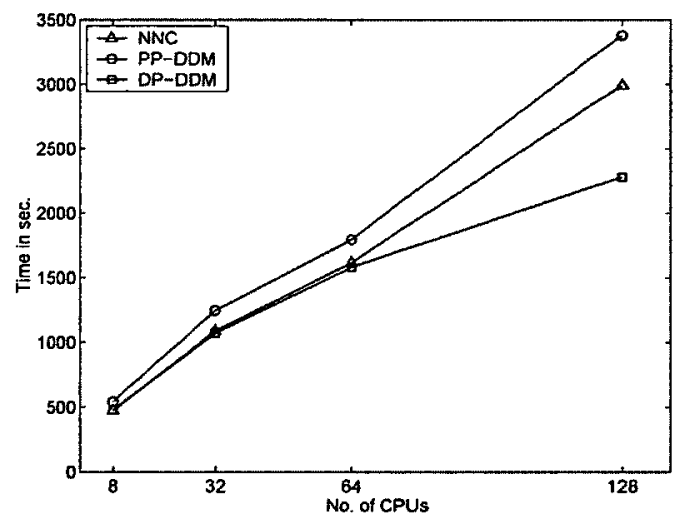

Figure 10.33: Weak scalability 


\subsubsection{Performance Study for Large-Scale Systems}

In this section, we demonstrate the ability of the probabilistic two-level methods to tackle a large-scale problem using increasingly large number of subdomains/processors. In particular, we refine the finite element mesh of the crankshaft to generate $2,074,627$ elements and 337,826 nodes. The response is expanded using four-dimensional $(L=4)$ second order PCE $(N=14)$ leading to a linear system of order $5,067,390$. We tackle the problem using the probabilistic dual-primal technique and the performance results are shown in Table.(10.1). These results are generated on a Linux cluster consists of 1200 compute nodes with 6 cores and 36 GBytes of memory per node with InfiniBand interconnect [149].

Table 10.1: Iteration counts and timing for the probabilistic DP-DDM

\begin{tabular}{|c|c|c|}
\hline No. of Processors & No. of Iterations & Time in sec. \\
\hline 128 & 16 & 5364.56 \\
\hline 256 & 19 & 3712.71 \\
\hline 512 & 25 & 2800.07 \\
\hline 640 & 20 & 1890.88 \\
\hline 768 & 22 & 1776.71 \\
\hline
\end{tabular}

\subsection{Three-Dimensional Elasticity Problem}

In this section a homogeneous, isotropic, linear elastic three-dimensional cantilever beam subjected to a self-weight loading is investigated. In Fig.(10.34), we show the dimensions and boundary conditions of the physical problem. 


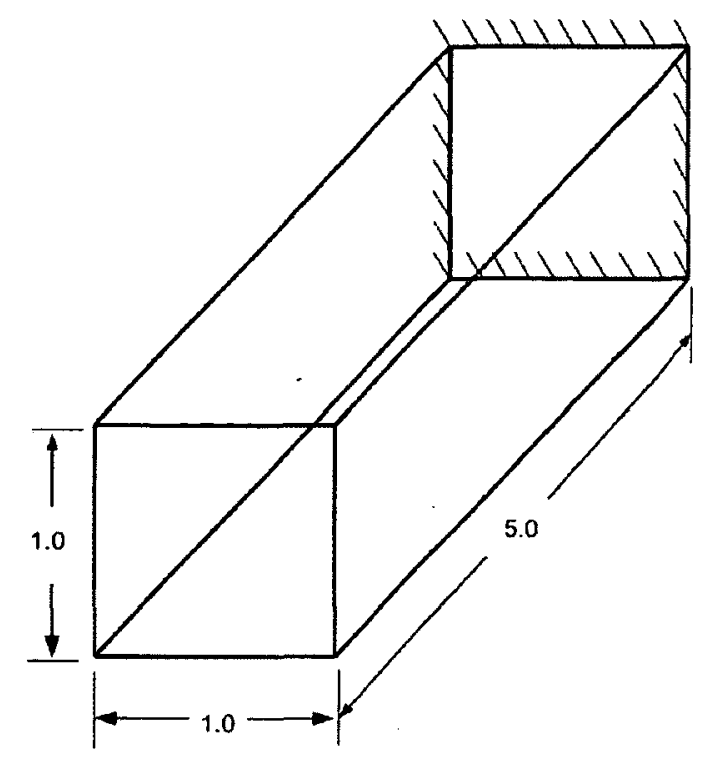

Figure 10.34: The geometry of three-dimensional cantilever beam (all dimensions in $\mathrm{m}$ )

For simplicity and clarity of presentation, we assume Poisson's ratio to be a spatially invariant deterministic quantity $\nu=0.35$. The spatial uncertainty of the Young's modulus is modeled as a stochastic process as

$$
E(\mathbf{x}, \theta)=E_{0}(\mathbf{x}) \exp (g(\mathbf{x}, \theta))
$$

where $E_{0}(\mathbf{x})$ is the mean of Young's modulus assumed to be $70 \mathrm{GPa}$ and $g(\mathbf{x}, \theta)$ is the underlying Gaussian process with covariance function defined in Eq.(10.1). Consequently, the uncertain constitutive matrix can be represented as (e.g. $[11,154])$ 


$$
\mathrm{D}(\mathrm{x}, \theta)=E(\mathbf{x}, \theta) \frac{(1-\nu)}{(1+\nu)(1-2 \nu)}\left[\begin{array}{ccccccc}
1 & \frac{\nu}{1-\nu} & \frac{\nu}{1-\nu} & 0 & 0 & 0 \\
\frac{\nu}{1-\nu} & 1 & \frac{\nu}{1-\nu} & 0 & 0 & 0 \\
\frac{\nu}{1-\nu} & \frac{\nu}{1-\nu} & 1 & 0 & 0 & 0 \\
0 & 0 & 0 & \frac{1-2 \nu}{2(1-\nu)} & 0 & 0 \\
0 & 0 & 0 & 0 & \frac{1-2 \nu}{2(1-\nu)} & 0 \\
0 & 0 & 0 & 0 & & \frac{1-2 \nu}{2(1-\nu)}
\end{array}\right] .
$$

The lognormal process and response are represented using four-dimensional $(L=4)$ second order PCE ( $N=14)$. We only consider a relatively small size problem. The finite element mesh used in the analysis consists of 10,376 nodes and 62,233 elements. Direct extension of the probabilistic dual-primal method to three-dimensional elasticity problem is implemented. The performance results for fixed problem size are shown in Table(10.2). Although the iteration counts remain nearly constant, the code takes about $3.5 \mathrm{H}$ to run (when using 64 processors) which is relatively long for the problem size considered. Increasing number of subdomains further does not improve the performance of iterative solver.

Table 10.2: Iteration counts and timing for 3D elasticity problem

\begin{tabular}{|c|c|c|}
\hline No. of Processors & No. of Iterations & Time in sec. \\
\hline 16 & 18 & 60210.33 \\
\hline 32 & 13 & 18565.41 \\
\hline 64 & 17 & 12536.05 \\
\hline 128 & 21 & 17997.63 \\
\hline
\end{tabular}


In Fig.(10.35), we show the magnitude, $x, y$ and $z$ components of the mean and standard deviation of displacement field. As predicted the maximum deflection occurs at the free end of the cantilever. The maximum coefficient of variation in the magnitude of the displacement field is about $45 \%$ which highlights the effect of uncertainty. The boundary conditions at the fixed end of the cantilever are satisfied with zero uncertainty. The components of the displacement vector exhibit more complex features than the magnitude.

Fig.(10.36) displays the magnitude and components of selected chaos coefficients of the displacement field. These PC coefficients represent the non-Gaussian contributions to the displacement field.

Selected Lagrange multipliers of the chaos coefficients of the displacement field are depicted in Fig.(10.37). As the index $j$ increase, Lagrange multipliers $\lambda_{j}$ exhibit decay in magnitude. Physically, the extended Lagrange multipliers relate to the nodal forces required to satisfy the compatibility condition of the polynomial chaos coefficients of the displacement field at the remaining interface nodes. 

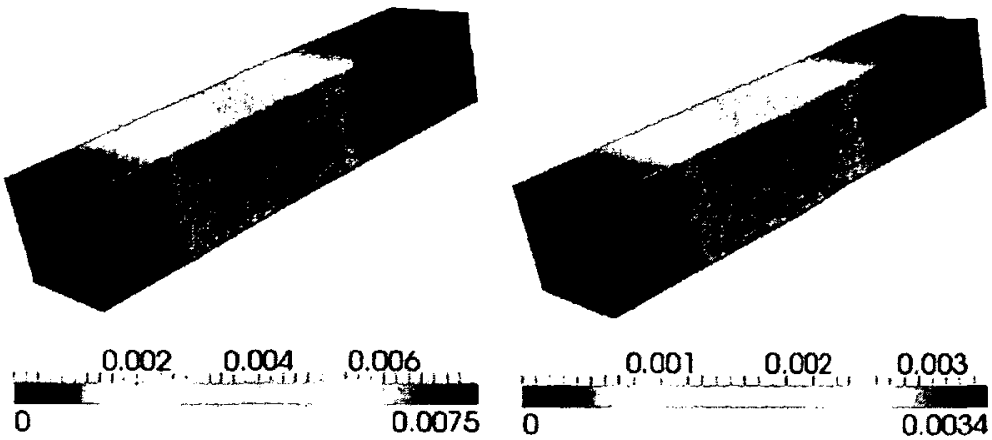

$\frac{1}{0} \frac{0.001,0.002}{0.003}$

(a) mean: magnitude

(b) standard deviation: magnitude
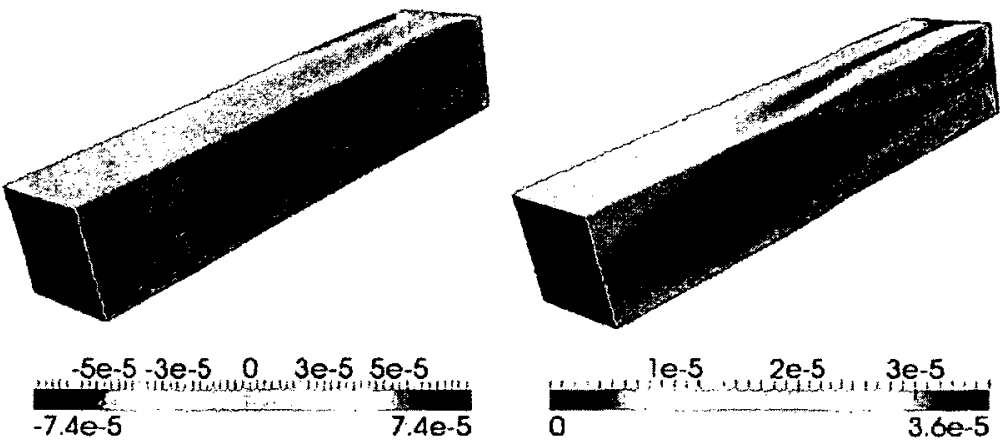

(c) mean: $x$-component

(d) standard deviation: $x$-component
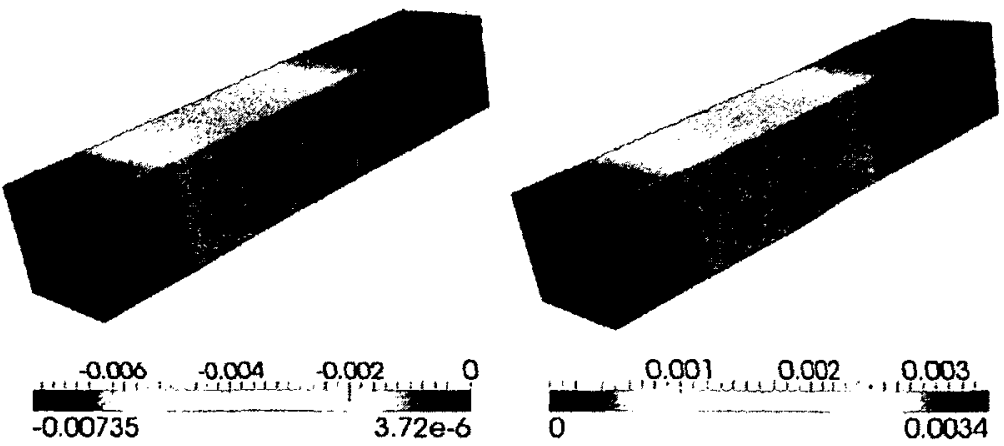

(e) mean: y-component

(f) standard deviation: $y$-component
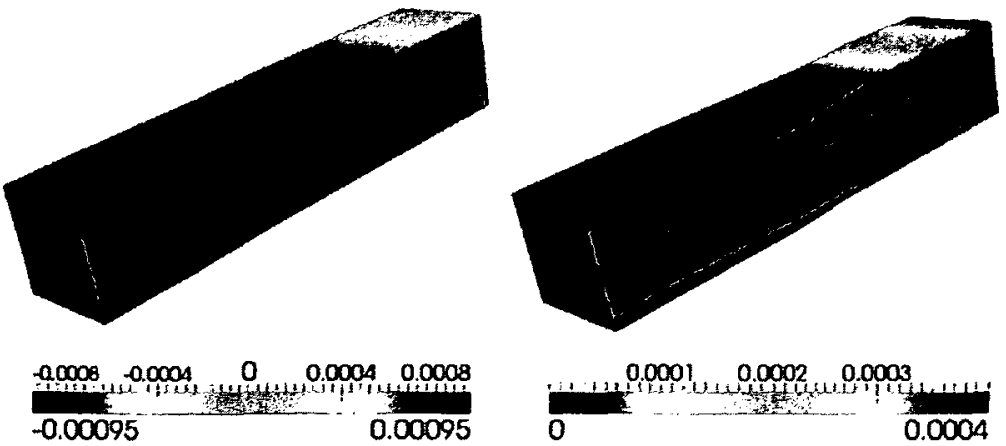

(g) mean: z-component

(h) standard deviation: z-component 

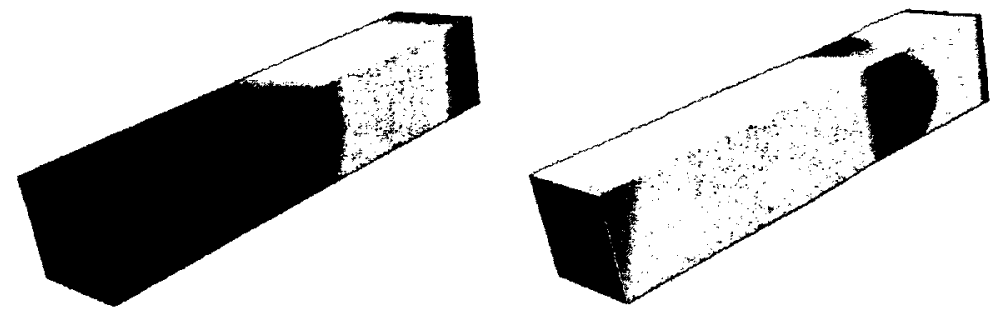

$\frac{1+20-6}{2}+5 e-6 \quad \frac{7 \theta-6}{1 \theta-5}$

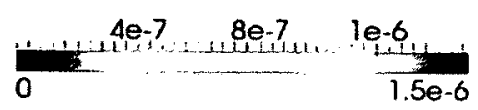

(a) $\mathbf{u}_{8}$ : magnitude

(b) $\mathbf{u}_{13}$ : magnitude
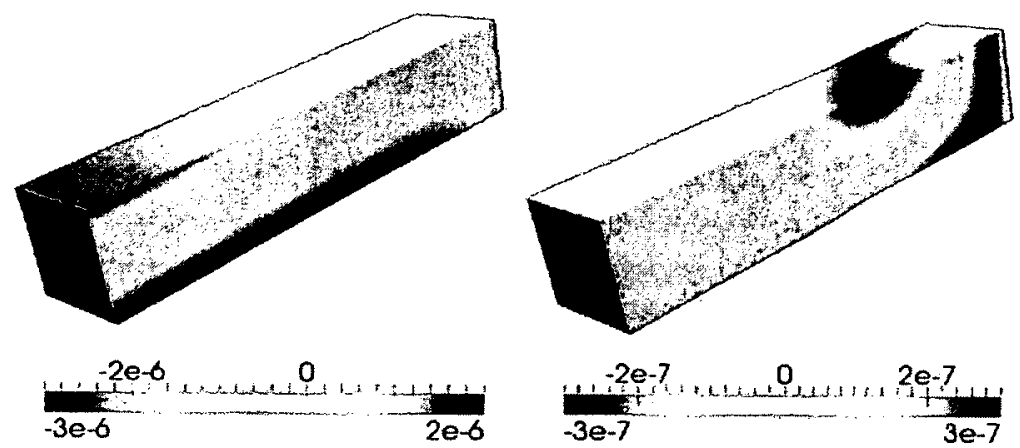

(c) $u_{8}: x$-component

(d) $u_{13}: x$-component
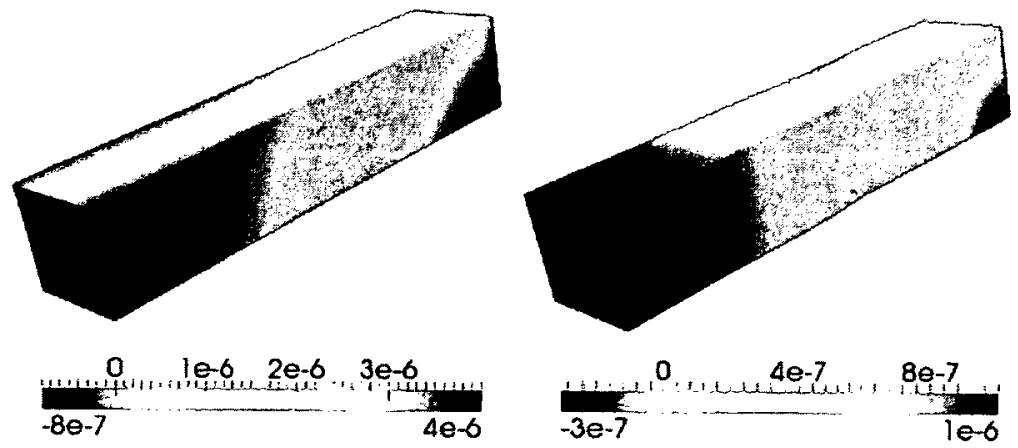

(e) $u_{8}: y$-component

(f) $u_{13}: y$-component
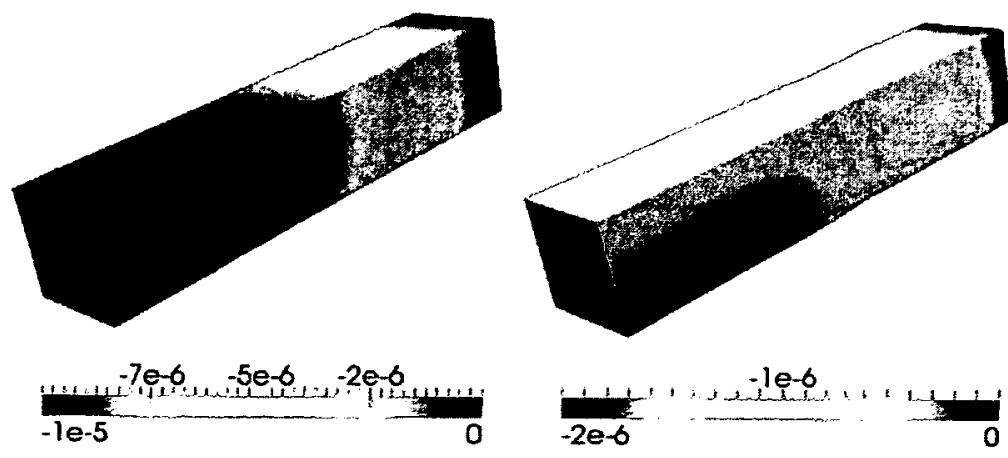

(g) $\mathbf{u}_{8}: z$-component

(h) $u_{13}: z$-component 


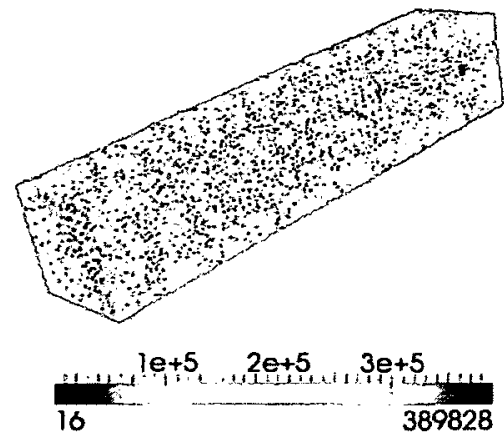

(a) $\lambda_{0}$ : magnitude

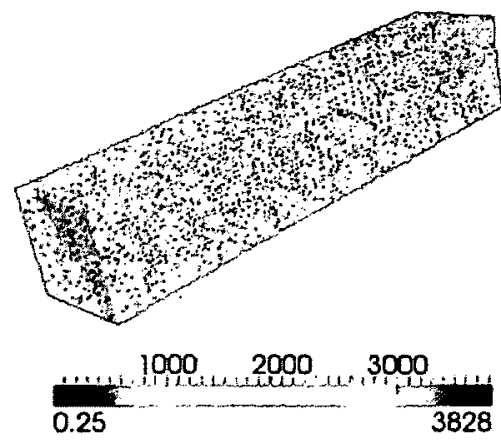

(c) $\lambda_{8}$ : magnitude

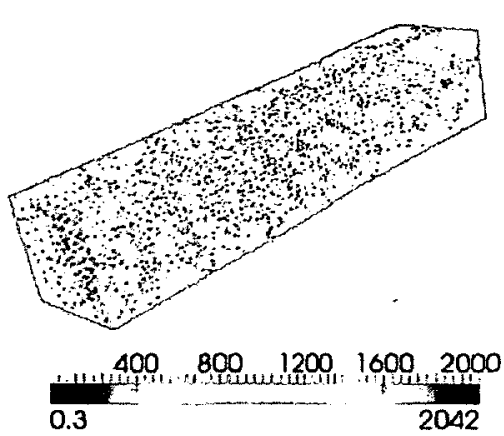

(b) $\lambda_{1}$ : magnitude

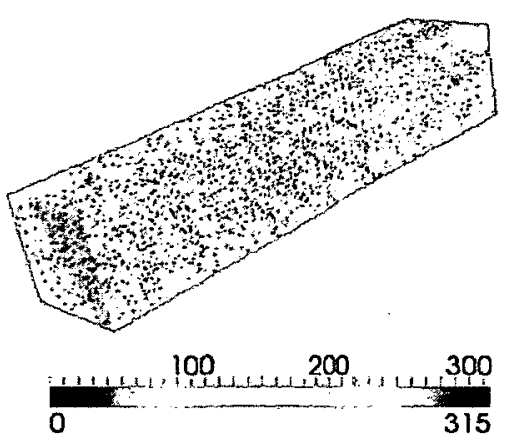

(d) $\lambda_{9}$ : magnitude

Figure 10.37: Selected Lagrange multipliers for the chaos coefficients of the displacement field 


\section{Discussion}

For three-dimensional elasticity problems, it is sometimes necessary to include edges and faces of the interface boundary into the coarse grid to achieve a rate of convergence that is independent on the number of subdomains (see Fig.(10.38)) [4, 6, 137, 148].

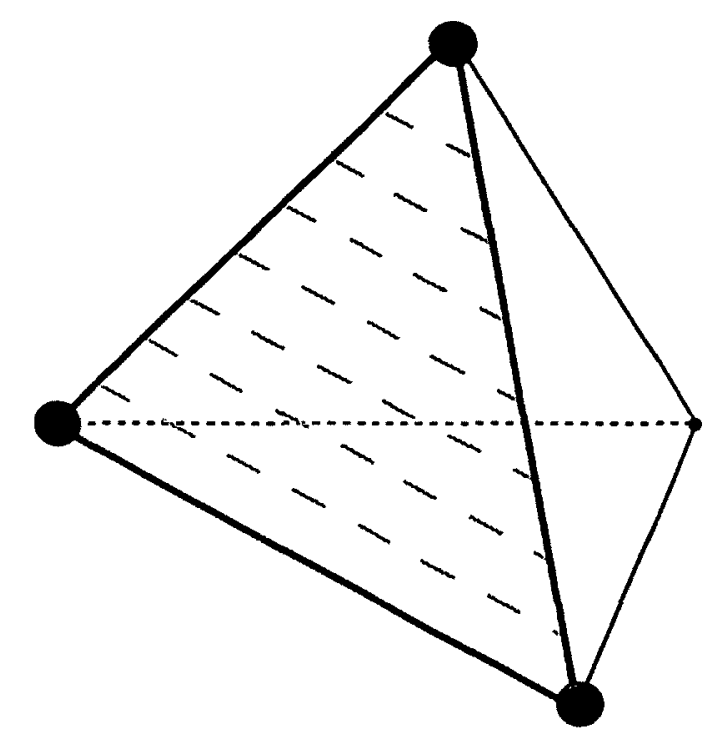

Figure 10.38: The interface boundary $\Gamma$ in three dimensions: Vertices $(\bullet)$,

Edges ( $\longrightarrow$ ) and Faces (三)

(adapted from Ref. [6])

In the domain decomposition methods for two-dimensional PDEs, the coarse grid is constructed based on a set of corner nodes. The corner nodes are selected such that the subdomain systems are non-singular. For three-dimensional elasticity problems however, the use of only the corner nodes to build the coarse problem may not be sufficient to ensure the solvability of the local problems and to achieve a scalable performance [135]. Therefore, some essential modifications in the construction of the coarse problem must 
be considered for elasticity problems in three dimensions. Such modifications may involve using additional constraints such as averages over faces and first-order moments over edges $[81,83,135,155,156]$. The implementation of the additional constraints can be performed by using optional Lagrange multipliers in the dual domain decomposition approach $[81,157]$ or transformation of basis in the primal domain decomposition approach $[155,156]$. This aspect however, is not considered in the thesis and will be the subject of future investigations.

\subsection{Summary}

We investigate the convergence rate, numerical and parallel scalabilities of the two-level domain decomposition algorithms for three-dimensional flow through porous media and elasticity problems with spatially varying non-Gaussian system parameters. The numerical results demonstrate that:

- The two-level methods exhibit a scalable performance with respect to the spatial problem size, number of subdomains and fixed problem size per subdomain for threedimensional flow through porous media.

- The probabilistic dual-primal approach shows almost similar convergence properties and parallel performance to the probabilistic BDDC type two-level preconditioners.

- For elasticity problems in three dimensions, additional constraints must be considered to achieve better convergence rate and scalability for large number of subdomains. 


\section{Chapter 11}

\section{Time-Domain Decomposition for}

\section{Stochastic ODEs}

\subsection{Introduction}

This chapter is closely based on the references $[158,159]$. For a fixed mesh resolution, spatial domain decomposition techniques cannot reduce the total solution time beyond a critical number of subdomains as the interprocessor communication overhead and memory bandwidth requirement predominate over floating point operations. In order to exploit the remaining idle processors in multi-processor computers, the recent research initiatives have been directed towards achieving time domain parallelism (e.g.,[90, 91]). Roughly speaking, the time domain parallelism involves the decomposition of the total simulation time into a number of time-slices, whereby a coarse integration is carried out serially. Next the system is integrated using a fine step size within each of these coarse time-slicès. The solu- 
tion from the coarse integration at the beginning of each time-slice are used as approximate initial conditions. A correction to these initial conditions is then made iteratively at the interface points. The solution converges when the error at each interface point reduces to a prescribed error tolerance.

Sufficient care must be taken to extend any algorithm for ODEs to the case of SDEs. The interpretation of the solutions of Ito SDEs is problematical in the classical sense as the solution is continuous everywhere but not differentiable anywhere in almost sure sense [160]. In contrast to the ODEs, the solution techniques of SDEs are more susceptible to numerical approximations. This is due to the fact that the Riemann sum approximating a deterministic integral in an ODE solver can be evaluated at any arbitrary point within the discretization interval. On the contrary, the integrals in an Ito SDE involving the Wiener process are evaluated at the left end point in the time interval [160].

In this chapter, we extend a parallel ODEs solver to tackle Ito SDEs describing noisy nonlinear oscillatory systems. In particular, a parallel version of Euler-Maruyama time integration scheme is described in the framework of parareal algorithm. More specifically, we study the ability of the stochastic parareal scheme to track sample paths of noisy nonlinear systems. For numerical illustration, we consider a Duffing oscillator driven by combined harmonic and random excitations. The stochastic parareal scheme is used to simulate the trajectories of two qualitatively different noisy limit cycle oscillations (LCOs) of the Duffing system. The efficiency and limitation of the stochastic parareal algorithm with respect to the strength of the random noise are brought out through an extensive numerical ex- 
perimentation. Furthermore, we demonstrate the scalability of the algorithm through its distributed implementation using Message Passing Interface (MPI) [88].

\subsection{Numerical Solution of Ito SDEs}

In the framework of Ito SDEs, nonlinear oscillators driven by broadband random noise can be represnted as

$$
d \mathbf{x}=\mathbf{f}(\mathbf{x}, t) d t+\mathbf{G}(\mathbf{x}, t) d \mathbf{w}
$$

where $\mathbf{x}$ and $\mathbf{f}(\mathbf{x}, t)$ denote $n$-dimensional state vector and random vector function respectively, $\mathbf{G}(\mathbf{x}, t)$ is a $n \times m$ - matrix valued random functions and $\mathbf{w}$ is $m$-dimensional vector Wiener process. It is well-known fact that the interpretation of the solutions of Eq.(11.1) is problematical in the classical sense as the solution $\mathbf{x}(t)$ is continuous everywhere but not differentiable anywhere with probability one.

One of the simplest time marching schemes of an Ito SDE is the Euler-Maruyama scheme [160]. This scheme leads to the following discrete representation of Eq.(11.1)

$$
\mathbf{x}_{i+1}=\mathbf{x}_{i}+\mathbf{f}\left(\mathbf{x}_{i}, t_{i}\right) \Delta t+\sqrt{\Delta t} \mathbf{G}\left(\mathbf{x}_{i}, t_{i}\right) \xi_{i}, i=0, \cdots, n
$$

where $\Delta t$ is the integration time step, $d \mathbf{w}$ defining Brownian path increment in Eq.(11.1) is replaced by $\mathbf{w}\left(t_{i+1}\right)-\mathbf{w}\left(t_{i}\right)=\sqrt{\Delta t} \xi_{i}$ and the elements of $\xi_{i} \sim \mathcal{N}(0,1)$ are independent random numbers sampled from a unit standard Gaussian random variable $\mathcal{N}(0,1)$. 
At this stage, we recall that the accuracy of a numerical integration of SDEs is measured using the notion of weak and strong convergence [160,161]. The following metric $\epsilon^{w e a k}(\Delta t)$ is generally used as an indicator of weak convergence:

$$
\epsilon^{w e a k}(\Delta t)=\sup _{0 \leq t_{n} \leq T}\left|E\left(\phi\left(\mathbf{x}_{n}\right)\right)-E\left(\phi\left(\mathbf{x}\left(t_{n}\right)\right)\right)\right| \leq C \Delta t^{\beta}
$$

where $E$ denotes expectation operator, $\phi$ is some polynomial, $C$ being some positive constant and $\beta$ is the order of convergence. Loosely speaking, the weak convergence implies that only certain statistical moments of the approximate and exact solution require to match with reasonable accuracy. On other hand, the strong convergence criterion is employed to estimate the closeness of the numerical approximation to the exact (strong) solution as given by $[160,161]$ :

$$
\epsilon^{\text {strong }}(\Delta t)=\sup _{0 \leq t_{n} \leq T} E\left(\left|\mathbf{x}_{n}-\mathbf{x}\left(t_{n}\right)\right|\right) \leq C \Delta t^{\beta}
$$

In general the strong convergence criterion is computationally expensive to satisfy than the weak convergence criterion $[160,161]$.

The current investigation relates to the strong convergence criterion as we approximate the sample paths (strong solutions) of noisy non-linear dynamical systems using a stochastic version of the parareal scheme. For convergence study, we conduct numerical experiments to evaluate the performance of the stochastic parareal scheme in relation its sequential counterpart. For a simple linear SDE, the theoretical convergence study is available in reference [162]. By contrast, a similar theoretical convergence analysis for the nonlinear SDE can be technically challenging. Therefore we compromise on analytical concern and 
undertake numerical experiment as adopted by others (e.g. [161]).

\subsection{Parareal Algorithm for SDEs}

In this section, we provide a concise description of a parareal algorithm adapted to Ito SDEs. The parareal algorithm was presented in reference [90] as a numerical method to solve ODEs in parallel computers. The mathematical analysis of this method was provided in reference [91]. However, the adaptation and applicability of the parareal algorithm in the context of SDEs are not widely reported in the open literature except the work in reference [162] which deals only with simple linear SDEs.

We present the adaptation of this parallel algorithm for ODEs to Ito SDEs in the framework of Euler-Murayama scheme. A parallel version of Euler-Murayama scheme involves splitting the time integration interval into $n_{c}$ number of time slices as shown in Figure (11.1). 


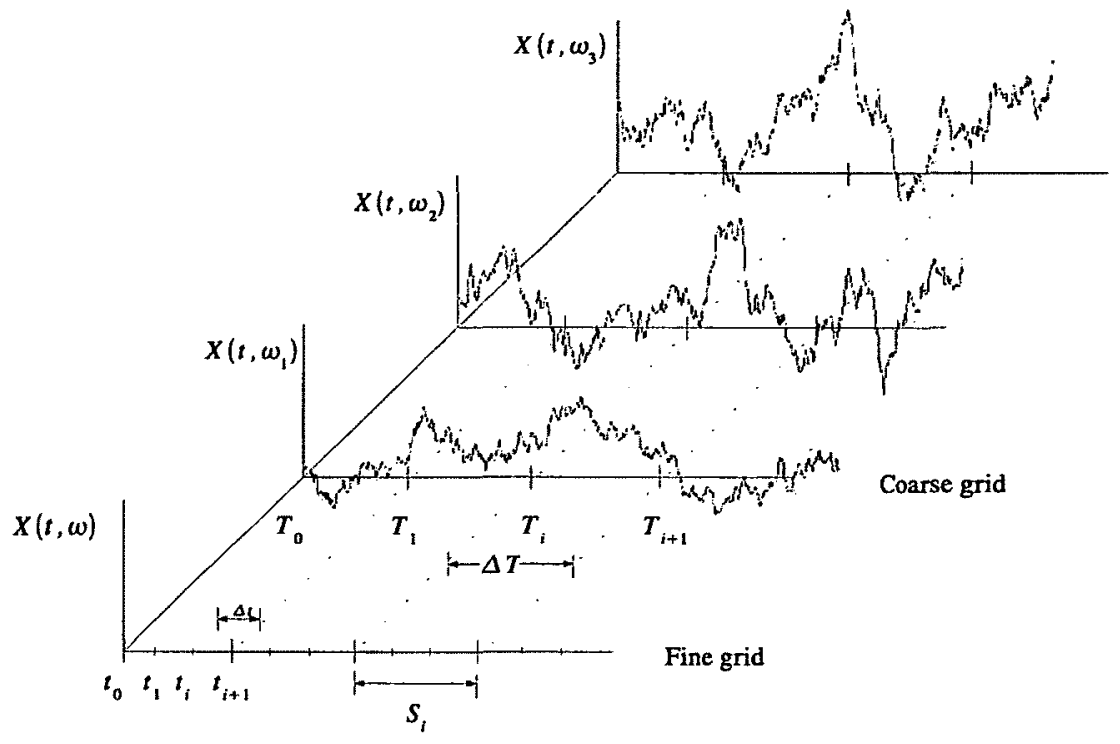

Figure 11.1: Schematic Diagram: $S_{i}$ is the time slice $i$ and $X\left(\omega_{j}, t\right)$ denotes $j$-th realization

This approach tackles $n_{c}$ SDEs over these time slices in parallel. Therefore Eq.(11.2) can be rewritten over each time slice as

$$
\mathbf{x}_{i+1, s}=\mathbf{x}_{i, s}+\mathbf{f}\left(\mathbf{x}_{i, s}, t_{i}\right) \Delta t+\sqrt{\Delta t} \mathbf{G}\left(\mathbf{x}_{i, s}, t_{i}\right) \xi_{i, s}^{f}, s=1, \cdots, n_{c}, i=0, \cdots, n_{f}
$$

where the superscript $f$ indicates the fine grid. Each of these initial value problems will be termed as the fine integral. 
To start the fine scale integrator over each time slice, $n_{c}$ initial conditions at the interface points must be specified. An approximation to these initial conditions is generated by solving the Ito SDE in Eq.(11.2) over a coarse scale time grid with step size $\Delta T$. The EulerMurayama numerical integration method for the coarse scale time grid can be expressed using explicit scheme as $[160,161]$

$$
\mathbf{X}_{i+1}=\mathbf{X}_{i}+\mathbf{f}\left(\mathbf{X}_{i}, T_{i}\right) \Delta T+\sqrt{\Delta T} \mathbf{G}\left(\mathbf{X}_{i}, T_{i}\right) \xi_{i}^{c}, i=0, \cdots, n_{c}
$$

or using semi-implicit scheme as $[160,161,163]$

$$
\mathbf{X}_{i+1}=\mathbf{X}_{i}+\mathbf{f}\left(\mathbf{X}_{i+1}, T_{i+1}\right) \Delta T+\sqrt{\Delta T} \mathbf{G}\left(\mathbf{X}_{i}, T_{i}\right) \xi_{i}^{c}, i=0, \cdots, n_{c}
$$

where the superscript $c$ relates to the coarse grid. In the semi-implicit method, the drift coefficient $\mathbf{f}(\mathbf{X}, T)$ is evaluated at the end of the integration step (i.e. implicitly) and the diffusion coefficient $\mathrm{G}(\mathrm{X}, T)$ is computed at the beginning of the integration step (i.e. explicitly) [161]. Note that the fully-implicit scheme where both the drift and diffusion coefficients are evaluated implicitly does not converge to the correct strong solution of the Ito SDEs [160, 161]. To achieve parallel efficiency, the coarse integral is performed using a relatively large time step $\Delta T$. For convergence and stability, the semi-implicit scheme is preferable than the explicit method for the coarse integral.

In the numerical implementation of this two-scale integrator, the fine and coarse integrator must remain on the same Brownian path. This condition enforces that $\xi_{i, s}^{f}$ is equal to $\xi_{i}^{c}$ at the interface points. The convergence is achieved when the errors at the interface points of the coarse and fine integrators reduce to a prescribed tolerance after a certain number of 
iterations. The adapted parareal scheme for Ito SDEs is summarized next.

\section{Fine Scale Integration}

The fine scale integration is carried out over each time slice concurrently as

$$
\mathbf{x}_{i+1, s}^{k}=\mathbf{x}_{i, s}^{k}+\mathbf{f}\left(\mathbf{x}_{i, s}^{k}, t_{i}\right) \Delta t+\sqrt{\Delta t} \mathbf{G}\left(\mathbf{x}_{i, s}^{k}, t_{i}\right) \xi_{i, s}^{f}, s=1, \cdots, n_{c}, i=0, \cdots, n_{f}
$$

where $k=1, . ., n_{\text {iter }}$ denotes the number of iterations. The initial conditions at the interface points is approximated by solving the coarse integral and given by

$$
\mathbf{x}_{0, s}^{k}=\mathrm{X}_{s-1}^{k}
$$

At the end of each fine integral, the error at each interface point is evaluated as

$$
\Delta_{s}^{k+1}=\mathbf{x}_{n_{f}, s}^{k}-\mathbf{X}_{s}^{k}+\Delta_{s}^{k}
$$

where

$$
\Delta_{s}^{0}=0
$$

The method converges as $\Delta^{k}=\sum_{k=1}^{n_{i t e r}} \Delta_{s}^{k}$ tends to zero (i.e. the difference in the coarse and fine integration results are indistinguishable in the graphical scale)

\section{Coarse Scale Integration}

At the interface points, the coarse integral is used to approximate the initial conditions for the fine integral. For the $k$-th iteration, the coarse integral as an explicit scheme is given by 


$$
\mathbf{X}_{i+1}^{k}=\mathbf{X}_{i}^{k}+\mathbf{f}\left(\mathbf{X}_{i}^{k}, T_{i}\right) \Delta T+\sqrt{\Delta T} \mathbf{G}\left(\mathbf{X}_{i}^{k}, T_{i}\right) \xi_{i}^{c}, i=0, \cdots, n_{c}
$$

When a semi-implicit scheme is used, the coarse integral takes the following form

$$
\mathbf{X}_{i+1}^{k}=\mathbf{X}_{i}^{k}+\mathbf{f}\left(\mathbf{X}_{i+1}^{k}, T_{i+1}\right) \Delta T+\sqrt{\Delta T} \mathbf{G}\left(\mathbf{X}_{i}^{k}, T_{i}\right) \xi_{i}^{c}, i=0, \cdots, n_{c}
$$

with the initial condition

$$
\mathbf{X}_{0}^{k}=\mathbf{X}\left(T=T_{0}\right)
$$

In contrast to the fine integral, the coarse integral is carried out serially. Furthermore, it is not an ordinary SDE solver as, after each step of coarse integration, a correction is performed as

$$
\mathbf{X}_{i+1}^{k}=\mathbf{X}_{i+1}^{k}+\Delta_{i+1}^{k}
$$

where $\Delta_{i+1}^{k}$ is substituted from Eq.(11.10).

\subsection{Parallel Implementation}

The adapted parareal algorithm for SDEs is implemented in parallel as listed in Algorithm (21). The parallel implementation of the algorithm requires two SDEs solvers: a coarse integrator $\mathcal{G}_{\Delta T}$ with a time step $\Delta T$ and a fine integrator $\mathcal{F}_{\Delta t}$ with a time step $\Delta t$. These numerical time integrators can be explicit or implicit and of any order such as RungeKutta [164] or Newmark methods [165]. As can be seen from Algorithm (21), the selection of the time integration method does not influence the parallel implementation, but only 
affects the stability and accuracy of the numerical scheme. For simplicity, we consider a semi-implicit Euler scheme for the coarse grid and an explicit Euler scheme for the fine grid. Message Passing Interface (MPI) libraries [88] are used to implement the algorithm on parallel computers. 


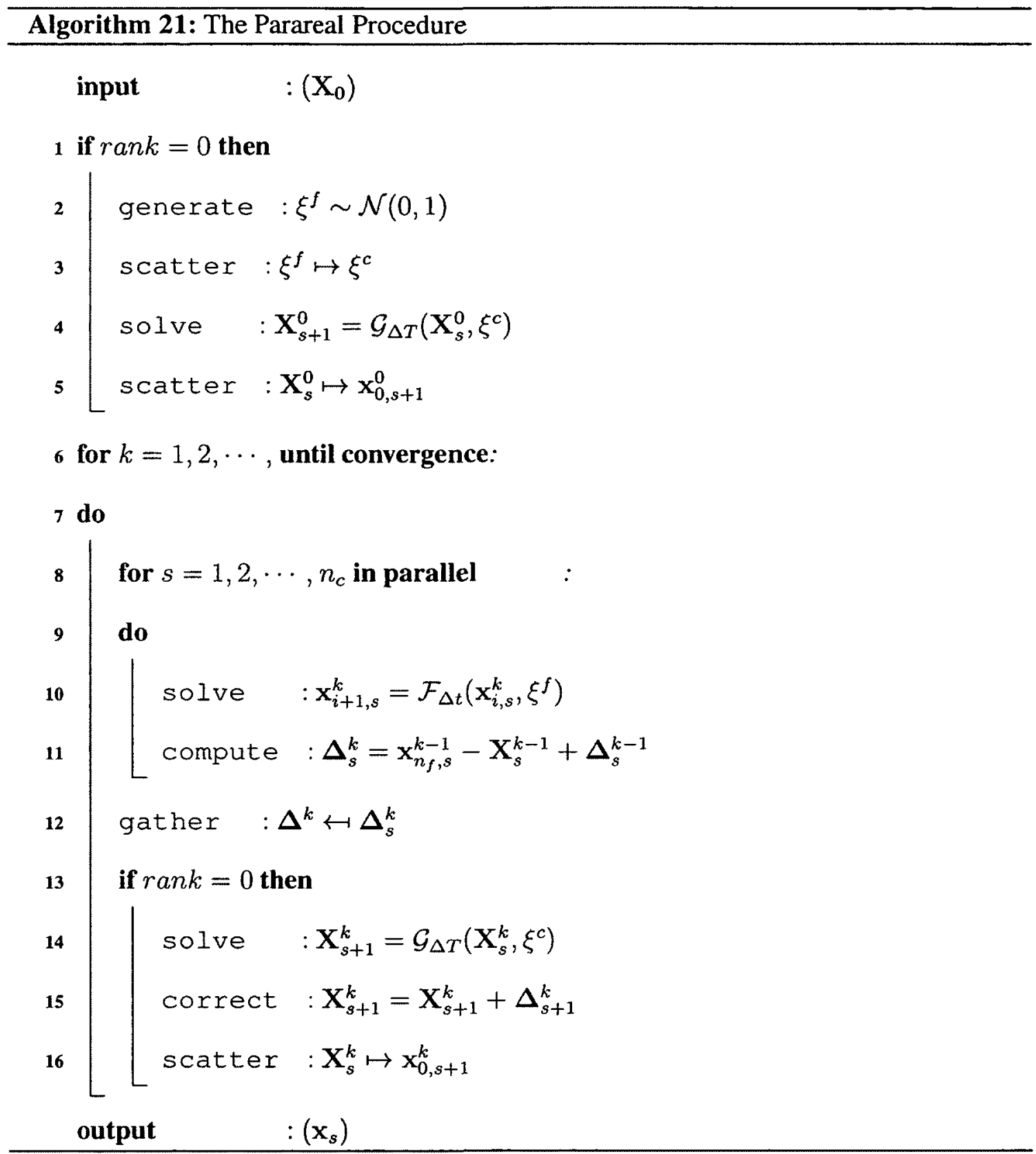




\subsection{Numerical Results}

For the numerical demonstration, we consider a Duffing oscillator (see [166-168]) driven by combined deterministic and random excitations as

$$
\ddot{u}+c \dot{u}+k_{1} u+k_{3} u^{3}=\Gamma \cos (\omega t)+\sigma \xi(t)
$$

where $u(t)$ is the displacement, $c$ denotes the damping coefficient, $k_{1}$ and $k_{3}$ represent the stiffness coefficients, $\Gamma$ is the amplitude and $\omega$ denotes the frequency of the input, $\xi(t)$ is a Gaussian white noise with unit strength and $\sigma$ denotes the strength of random noise. For a slight change in the model parameters, the Duffing oscillator displays a range of dynamical response [166-168]. Therefore it serves as a paradigm model to test the performance of the parareal algorithm without undue mathematical complexity.

Eq.(11.16) can be recast in the form of Eq.(11.1) as

$$
\left\{\begin{array}{c}
d x_{1} \\
d x_{2}
\end{array}\right\}=\left\{\begin{array}{c}
x_{2} \\
-\left[c x_{2}+k_{1} x_{1}+k_{3} x_{1}^{3}-\Gamma \cos (\omega t)\right]
\end{array}\right\} d t+\left[\begin{array}{cc}
0 & 0 \\
0 & \sigma
\end{array}\right]\left\{\begin{array}{c}
0 \\
d w
\end{array}\right\}
$$

where $x_{1}=u$ and $x_{2}=\dot{u}$.

Temporal explicit discretization with time step $\Delta t$ over the fine grid leads to the following discrete state evolution equations: 


$$
\begin{aligned}
\left\{\begin{array}{c}
x_{1} \\
x_{2}
\end{array}\right\}_{i+1}=\left\{\begin{array}{c}
x_{1} \\
x_{2}
\end{array}\right\}_{i}+\left[\begin{array}{cc}
0 & \Delta t \\
-\Delta t\left(k_{1}+k_{3} x_{1}^{2}\right) & -\Delta t c
\end{array}\right]_{i}\left\{\begin{array}{l}
x_{1} \\
x_{2}
\end{array}\right\}_{i}+ \\
\left\{\begin{array}{c}
0 \\
\Delta t \Gamma \cos (\omega t)
\end{array}\right\}_{i}+\left\{\begin{array}{c}
0 \\
\sigma d w
\end{array}\right\}
\end{aligned}
$$

where $d w=w\left(t_{i+1}\right)-w\left(t_{i}\right)$ is the Brownian path increment.

The semi-implicit discrete representation of the coarse grid integral with time step $\Delta T$ is defined as

$$
\begin{array}{r}
{\left[\begin{array}{cc}
1 & -\Delta T \\
\Delta T\left(k_{1}+k_{3} X_{1}^{2}\right) & 1+\Delta T c
\end{array}\right]_{i+1}\left\{\begin{array}{c}
X_{1} \\
X_{2}
\end{array}\right\}_{i+1}=\left\{\begin{array}{c}
X_{1} \\
X_{2}
\end{array}\right\}_{i}+} \\
\left\{\begin{array}{c}
0 \\
\Delta T \Gamma \cos (\omega T)
\end{array}\right\}_{i}+\left\{\begin{array}{c}
0 \\
\sigma d W
\end{array}\right\}
\end{array}
$$

where $d W=W\left(t_{i+1}\right)-W\left(t_{i}\right)$ is Brownian path increment.

The following system parameters are considered for the numerical investigation: $c=$ $0.3, k_{1}=-1, k_{3}=1, \omega=1.25$. Two values of $\Gamma$ are considered: $\Gamma=0.30$ and $\Gamma=0.37$. The underlying unforced (autonomous) system has three fixed points at $u=0, \pm 1[166-$ 168]. The fixed point at $u=0$ is unstable whereas the fixed points at $u= \pm 1$ are stable. In the absence of noise $(\sigma=0)$, the harmonically forced Duffing system exhibits a period-two (period $T=\frac{4 \pi}{\omega}$ ) and period-five (period $T=\frac{10 \pi}{\omega}$ ) subharmonic oscillations for $\Gamma=0.30$ 
and $\Gamma=0.37$ respectively $[168]$.

Next we investigate the performance of the stochastic parareal scheme to track the noisy limit cycle oscillations for $\Gamma=0.30$ and $\Gamma=0.37$ by varying the strength of random noise $\sigma$. We have taken the following integration parameters: $\Delta T=0.63, \Delta t=0.003$ for the integration interval $\left[0, \frac{40 \pi}{\omega}\right]$.

\section{Case I: Noisy period two subharmonic motion $\Gamma=0.30$}

Fig.(11.2) presents the system response corresponding to the noise free case $(\sigma=0.0)$. The figure shows the starting initial conditions $\mathbf{X}^{k=0}$ for the fine scale integral obtained using the coarse integrator given by Eq.(11.19) and the solution on the fine grid $\mathrm{x}^{k=n_{i t e r}}$ obtained from Eq.(11.18) where $n_{i t e r}$ represents the number of iterations required to converge as indicated in the figure $\left(n_{i t e r}=59\right)$. The method converges as $\sum_{k=1}^{k=n_{i t e r}} \Delta_{s}^{k}$ tends to zero (i.e. the difference in the coarse and fine integration results are indistinguishable in the graphical scale). Therefore the continuity of the coarse and fine scale integral is enforced at the interface points $\mathbf{X}_{s}=\mathbf{x}_{n_{f}, s}$. 


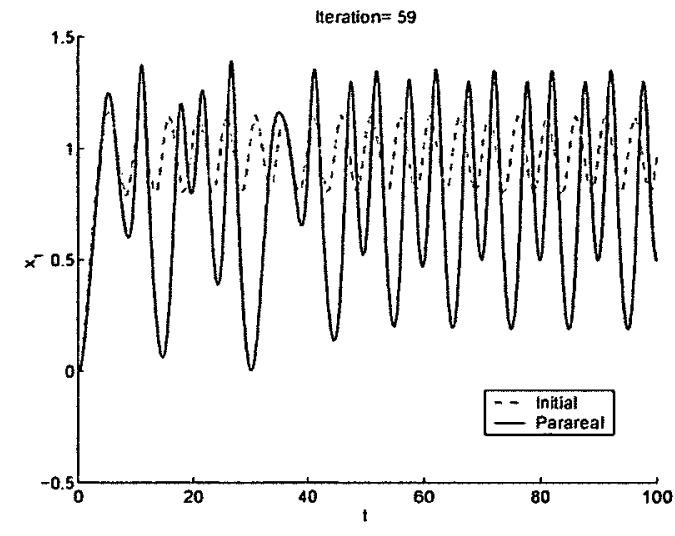

(a) Time history

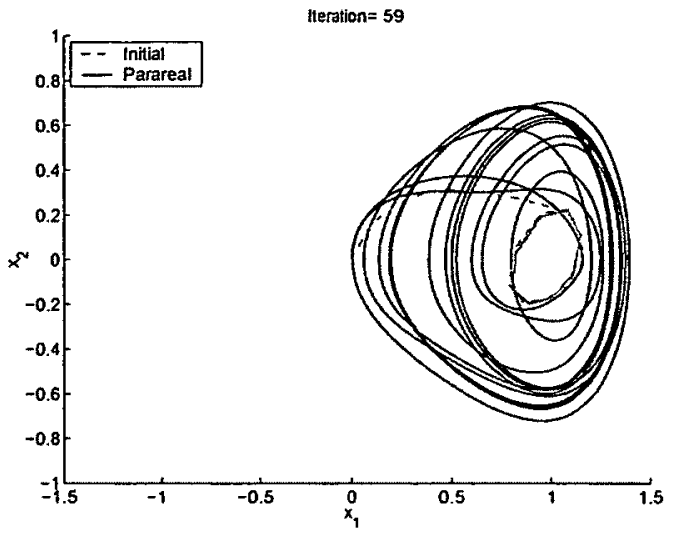

(b) Phase portrait

Figure 11.2: Performance of The Stochastic Parareal Integrator:

$$
\Gamma=0.3, \sigma=0
$$

The phase plane diagrams showing the convergence of the solution with respect to the iteration number $k$ are presented in Fig.(11.3). As expected, the convergence of the parareal algorithm improves as the iteration number increases. 

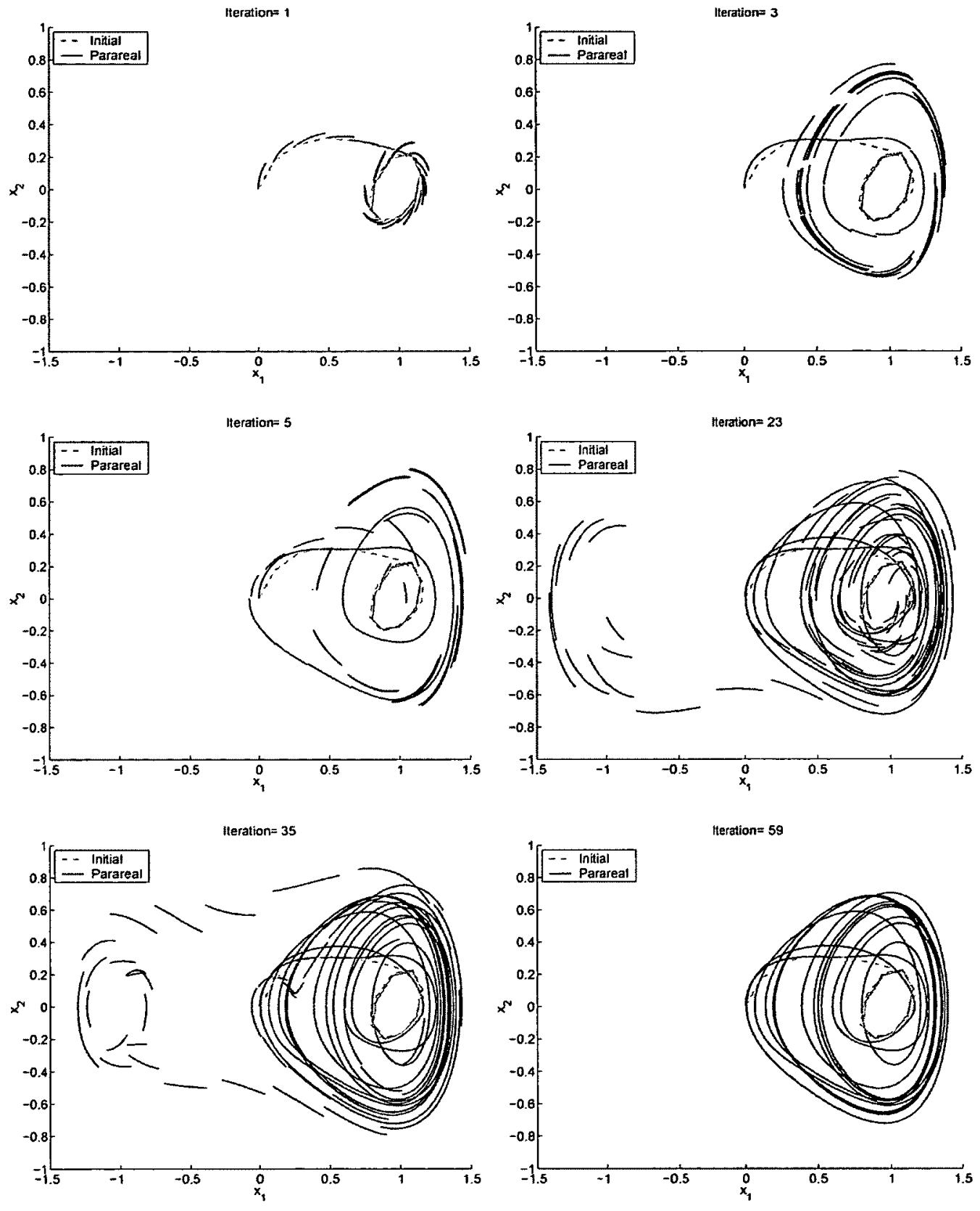

Figure 11.3: Convergence of the parareal algorithm $(k=1, k=3, k=5$,

$$
k=23, k=35 \text { and } k=59): \Gamma=0.3, \sigma=0
$$


Next we investigate the efficacy of the parareal integrator on capturing the trajectories (samples paths) of the Duffing system perturbed by combined harmonic and random inputs. Fig.(11.4) shows the results for mild noise strength corresponding to $\sigma=0.1 \Gamma$. The three panels in the plot present the response of the Duffing oscillator for three different random samples. In the figure, we show the starting initial conditions for the parallel fine scale integration scheme and the converged parareal solution after $n_{i t e r}$. Although the results presented for three different random samples, the starting initial conditions generated from the coarse integrator are almost the same. This fact can be attributed to the large time step $\Delta T$ used in the coarse integration method which affects the accuracy of the numerical scheme. Depending on the random sample, the transitions of the trajectories take place between two potential wells located at $u= \pm 1$ due to random perturbation. Interestingly the parareal method is able to capture the sample paths even when the starting initial conditions, denoted by the green lines, are grossly erroneous indicating the solution confined to the opposite potential wells. The results obtained from the parallel and sequential schemes (not shown) are almost identical and indistinguishable in the plot. 


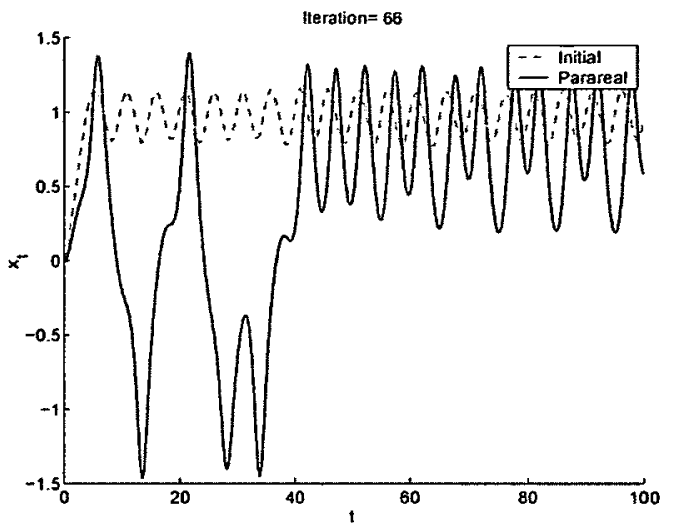

(a) Time history

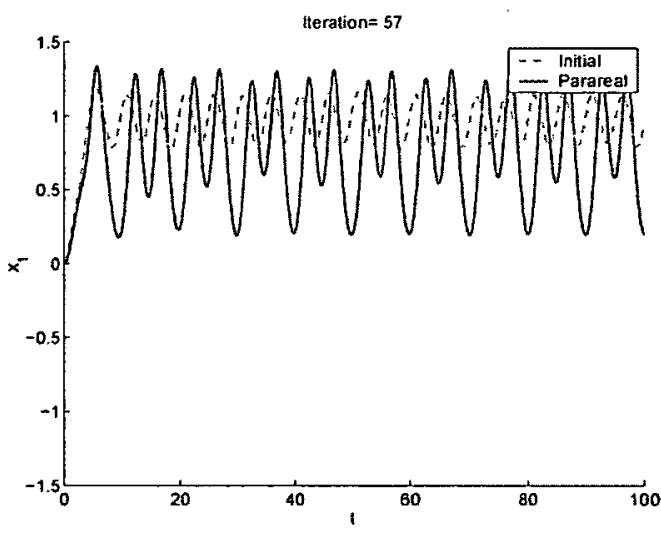

(c) Time history

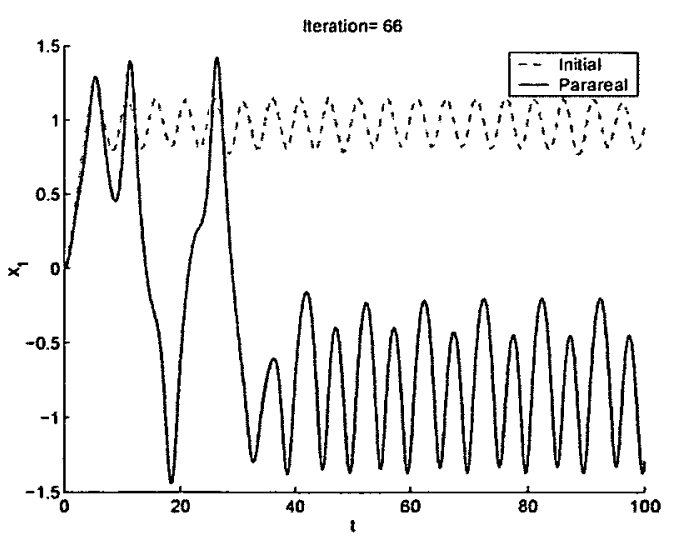

(e) Time history

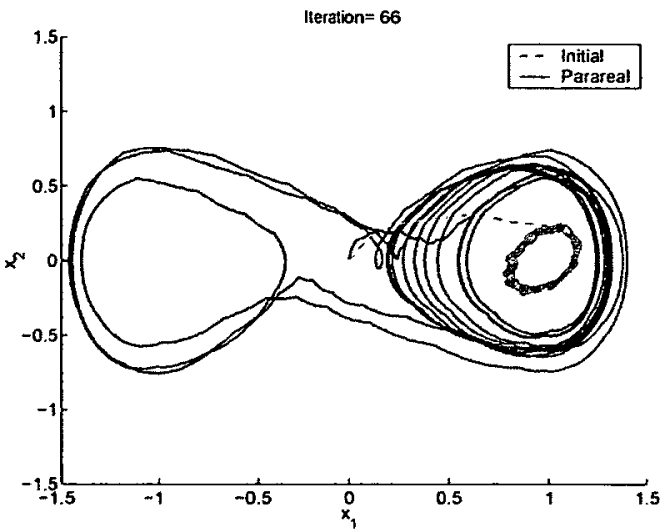

(b) Phase portrait

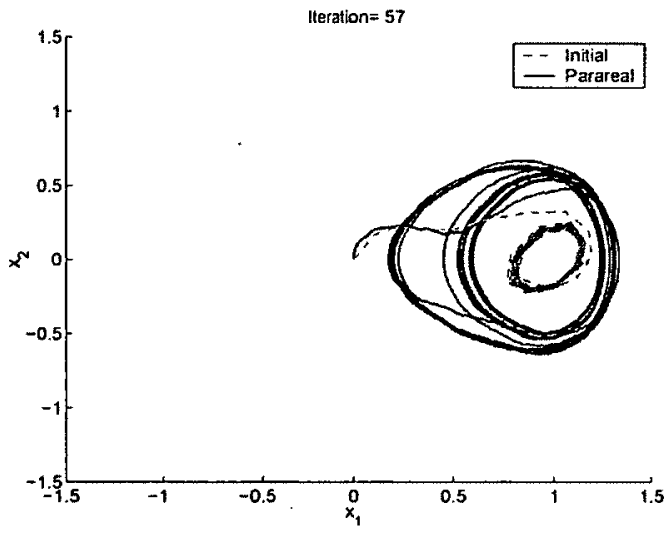

(d) Phase portrait

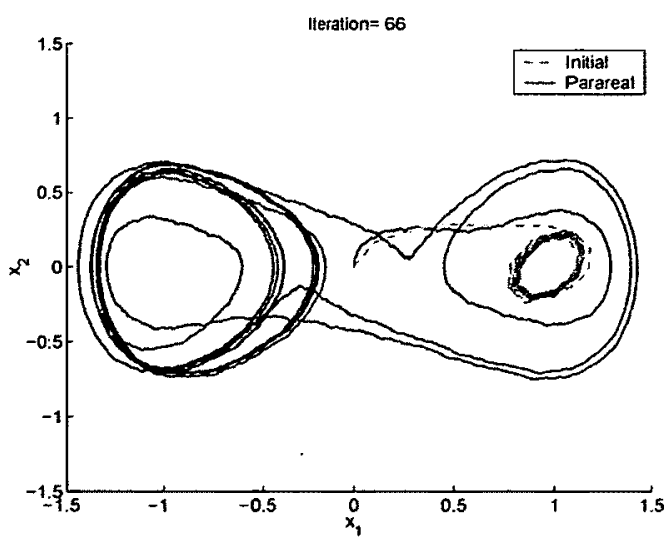

(f) Phase portrait

Figure 11.4: Samples of the response of the Duffing oscillator:

$$
\Gamma=0.3, \sigma=0.1 \Gamma
$$


Fig.(11.5) shows the response of the Duffing oscillator for the case of medium noise strength: $\sigma=0.5 \Gamma$. Despite starting with significantly erroneous initial conditions, the parareal method again effectively tracks the sample paths. Of course, the response is now significantly affected by the random noise as observed from the noisy phase portrait. Once again, the solutions obtained from the parallel and sequential scheme match identically in the scale of the graph. 


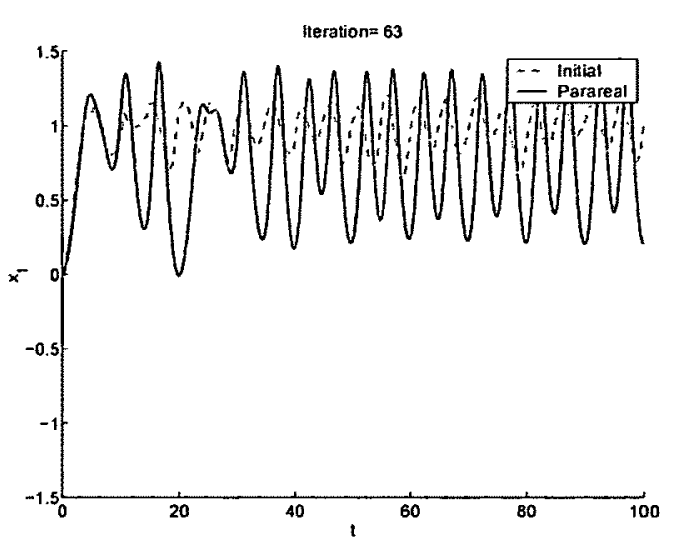

(a) Time history

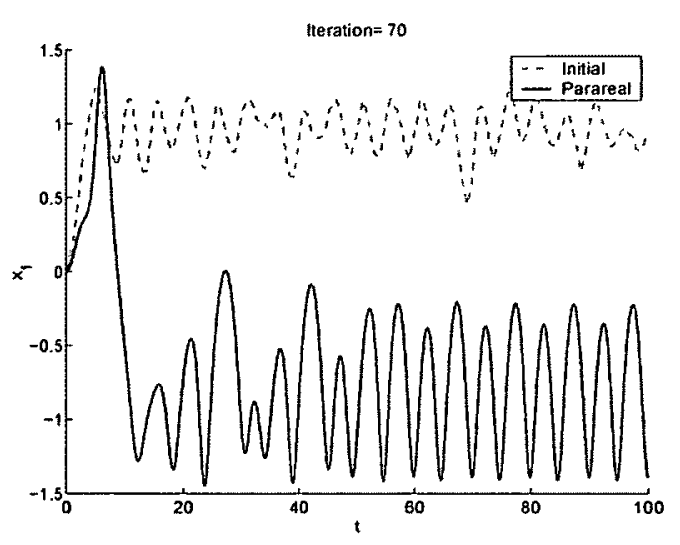

(c) Time history

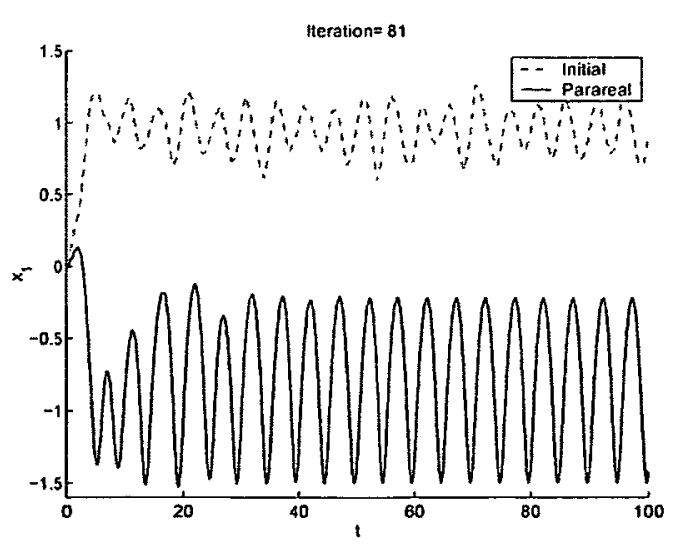

(e) Time history

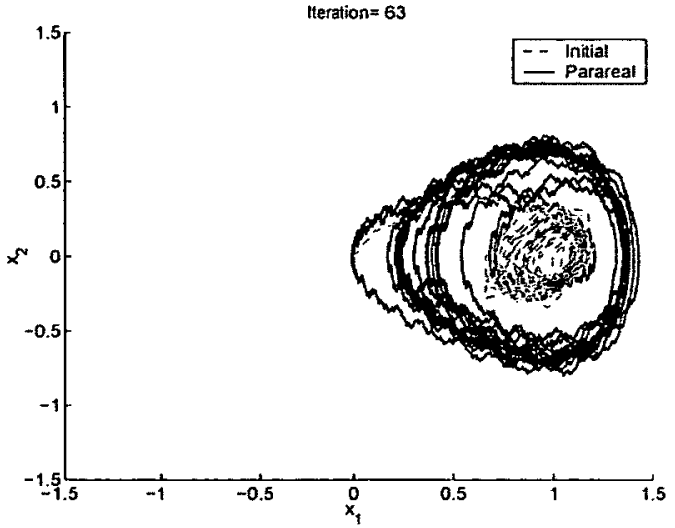

(b) Phase portrait

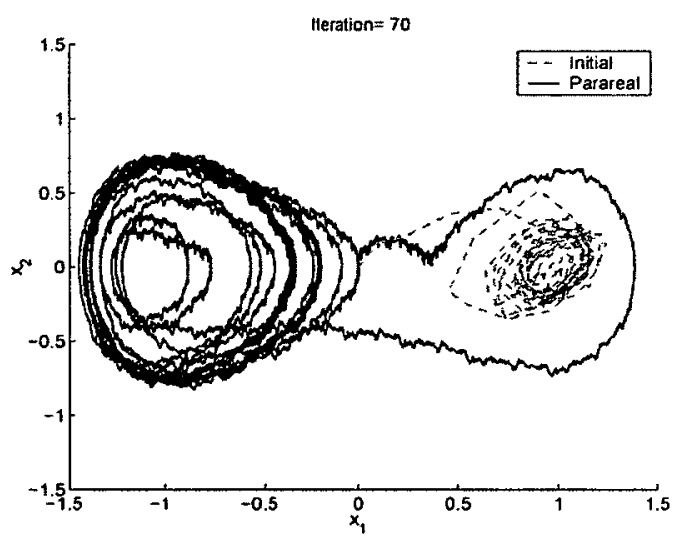

(d) Phase portrait

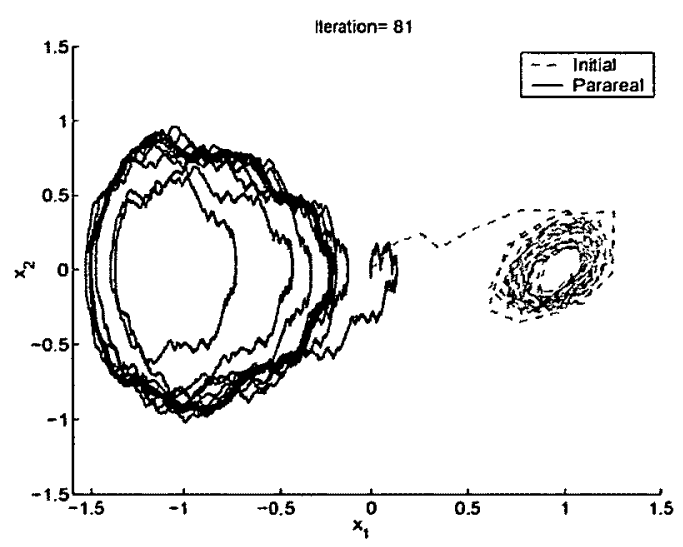

(f) Phase portrait

Figure 11.5: Samples of the response of the Duffing oscillator:

$$
\Gamma=0.3, \sigma=0.5 \Gamma
$$


Next we consider the input noise with high strength corresponding to $\sigma=0.9 \Gamma$. The related results are plotted in Fig.(11.6) for three random samples. Although the parareal scheme still works for two samples in the top and middle panels, it fails for the third sample shown in the bottom panel as maximum number of iterations (which is set to 100) is reached without convergence. We conjecture such failure is partly attributed to the numerical scheme adopted for the coarse integrator which demands much finer integration time step and/or more iterations to converge. 


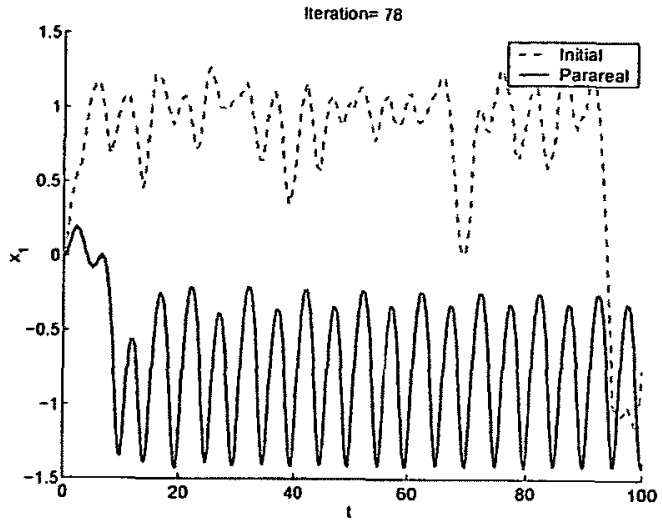

(a) Time history

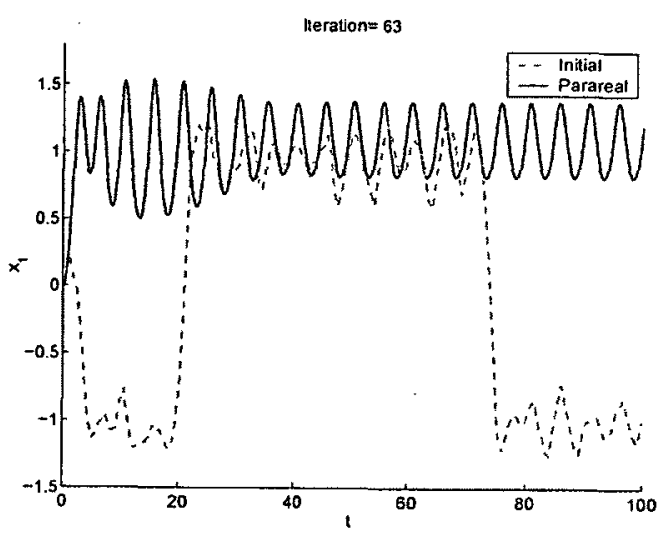

(c) Time history

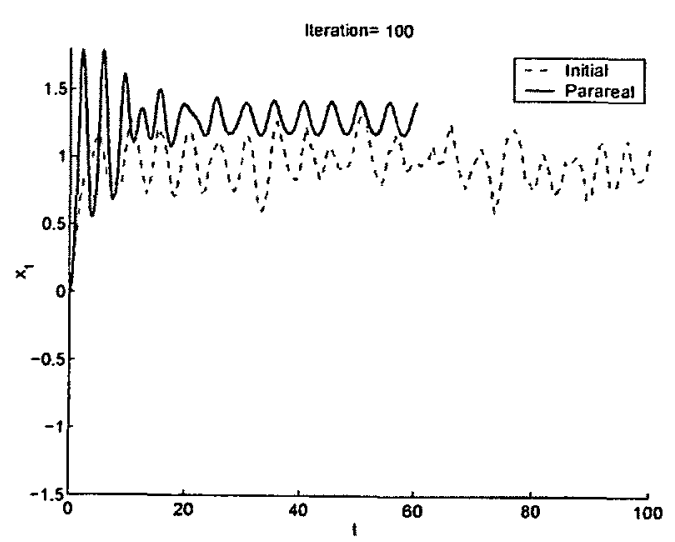

(e) Time history

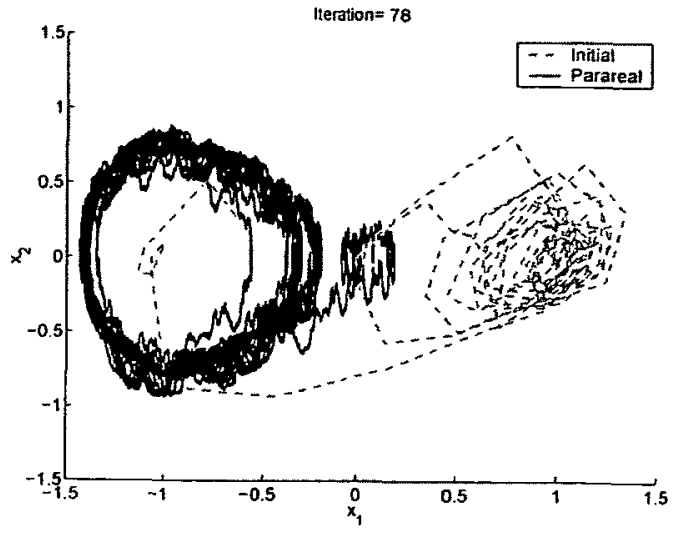

(b) Phase portrait

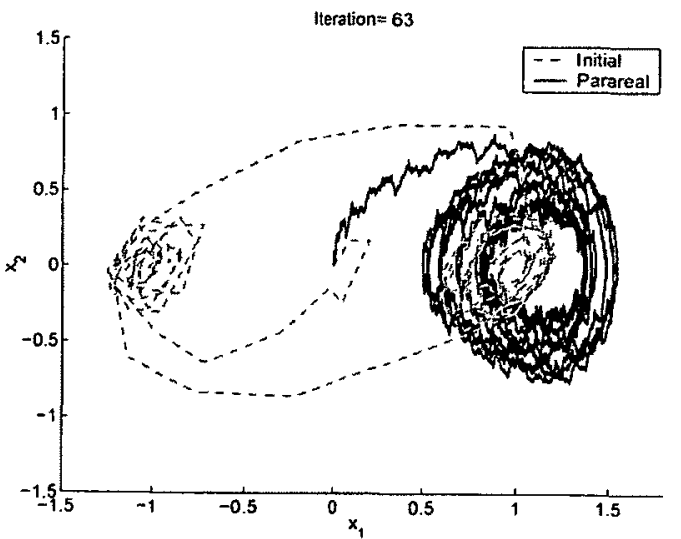

(d) Phase portrait

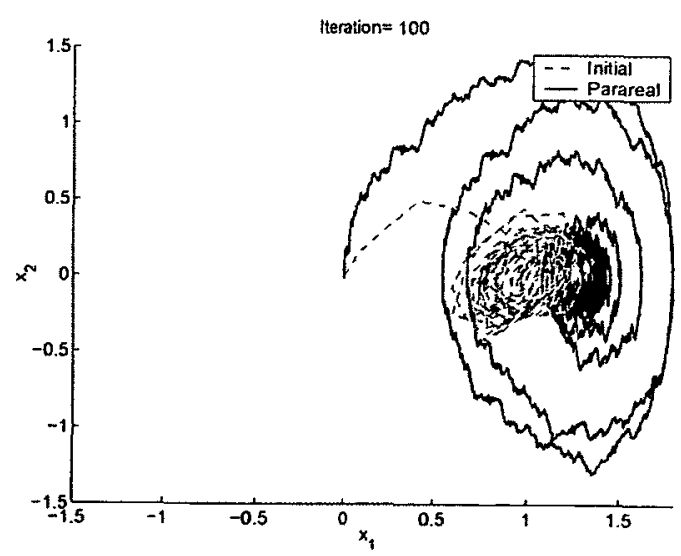

(f) Phase portrait

Figure 11.6: Samples of the response of the Duffing oscillator:

$$
\Gamma=0.3, \sigma=0.9 \Gamma
$$




\section{Case II: Noisy Period five subharmonic motion $\Gamma=0.37$}

In this section, we consider the case of $\Gamma=0.37$. In the absence of random perturbation, the system displays a period-five subharmonic oscillation (period $T=\frac{10 \pi}{\omega}$ ) leading to much slower dynamics compared to the case of $\Gamma=0.3$. In contrast to the previous case, the response now periodically spans both the potential wells located at $u= \pm 1$. The performance of the parallel integrator is studied next with respect the strength of random noise as explained in the subsequent paragraphs.

The displacement response and the phase portrait for the noise free case are plotted in Fig(11.7a) and Fig(11.7b) respectively. Notwithstanding starting with grossly inaccurate initial condition, the parareal scheme again follows true solution trajectory after 59 iterations.

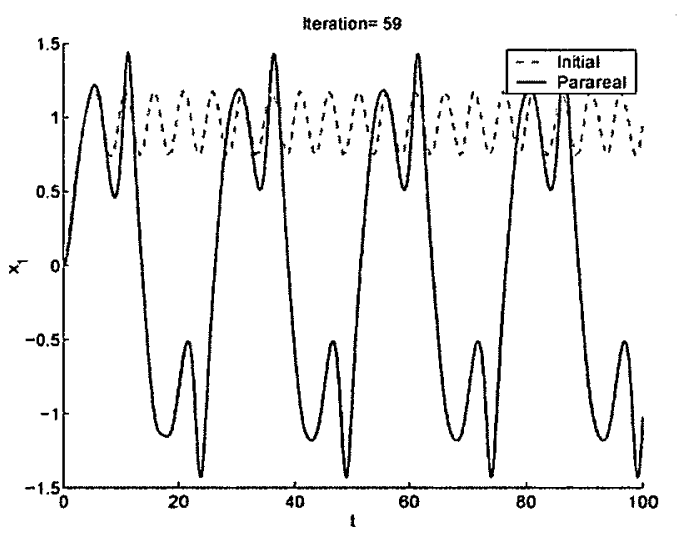

(a) Time history

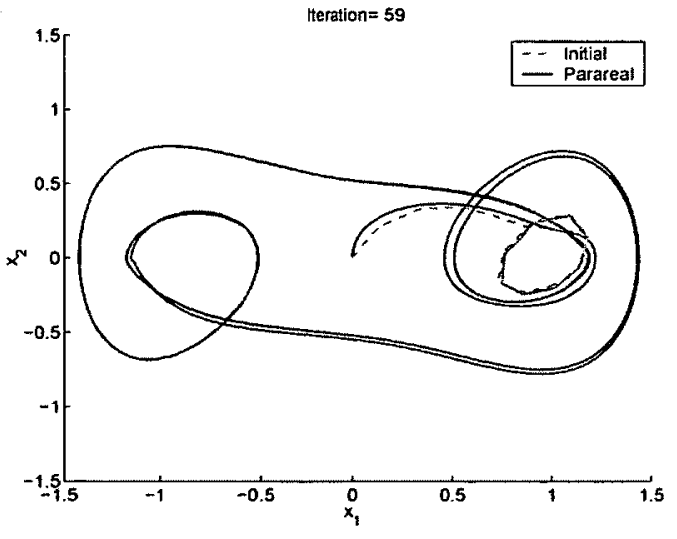

(b) Phase portrait

Figure 11.7: Performance of The Stochastic Parareal Integrator:

$$
\Gamma=0.37, \sigma=0
$$


The convergence of the parareal scheme with respect to the iteration number is shown in Fig.(11.8) for $k=1, k=3, k=5, k=6, k=35$ and $k=59$ iterations. 

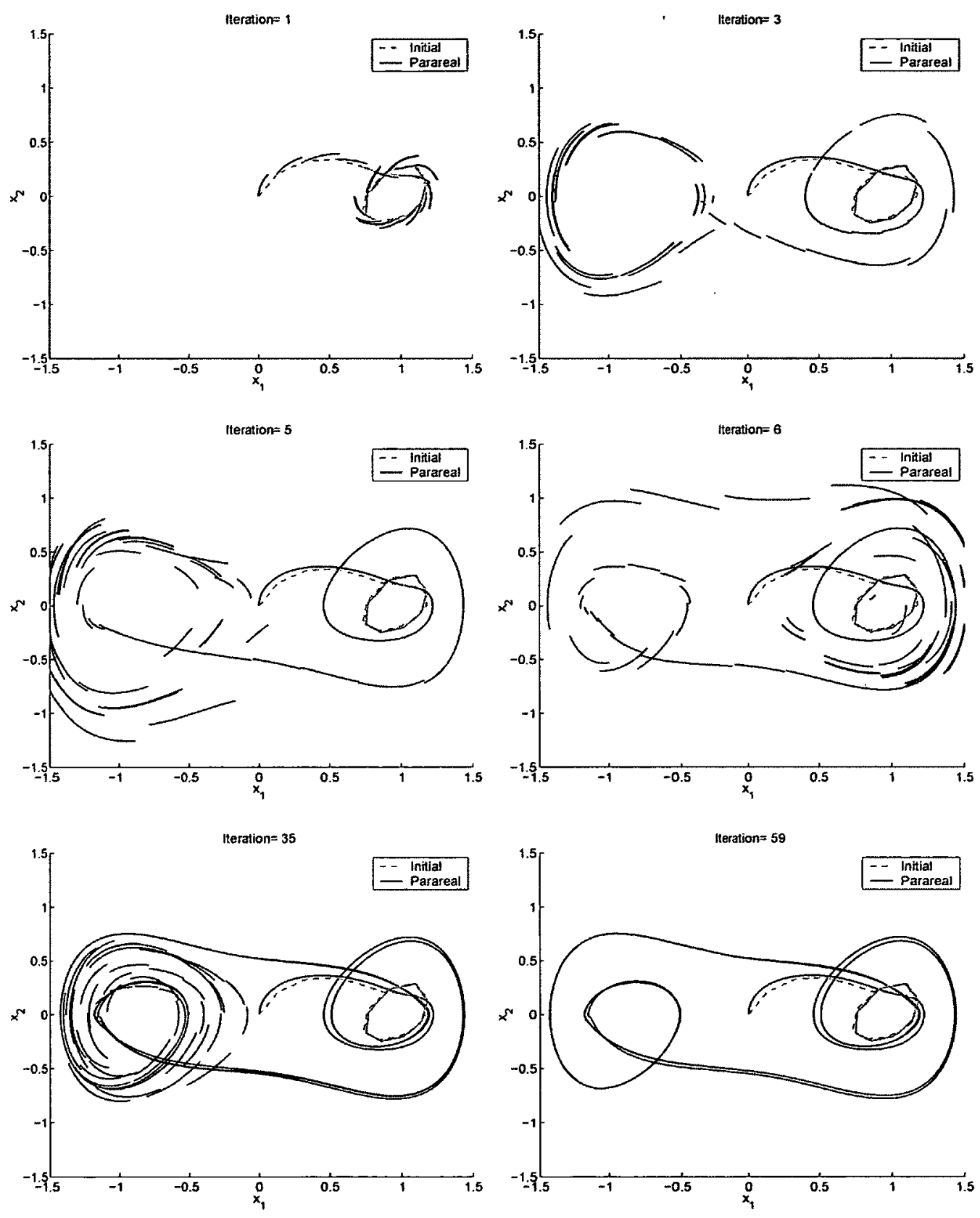

Figure 11.8: Convergence of the parareal algorithm $(k=1, k=3, k=5$,

$$
k=6, k=35 \text { and } k=59): \Gamma=0.37, \sigma=0
$$


For a noise strength $\sigma=0.1 \Gamma$, the samples of noisy limit cycle motion are plotted in Fig.(11.9). Interestingly the presence of random noise occasionally entrains the dynamics in the potential wells at $u= \pm 1$. Such occasional entrainment phenomenon is absent for the harmonic perturbation alone $(\sigma=0)$. Recall that such behavior induced by random noise is in contrary to the previous case related to $\Gamma=0.3$, whereby depending on the initial conditions, the dynamics is confined to a particular potential well located at either $u=1$ or $u=-1$ for $\sigma=0.1 \Gamma$. 


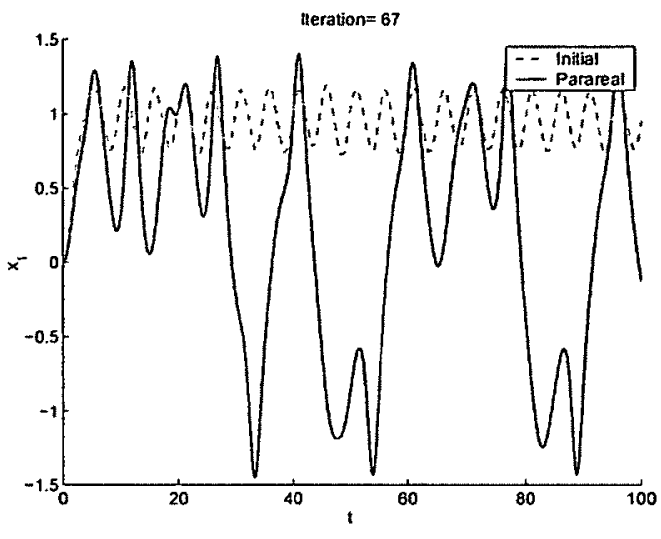

(a) Time history

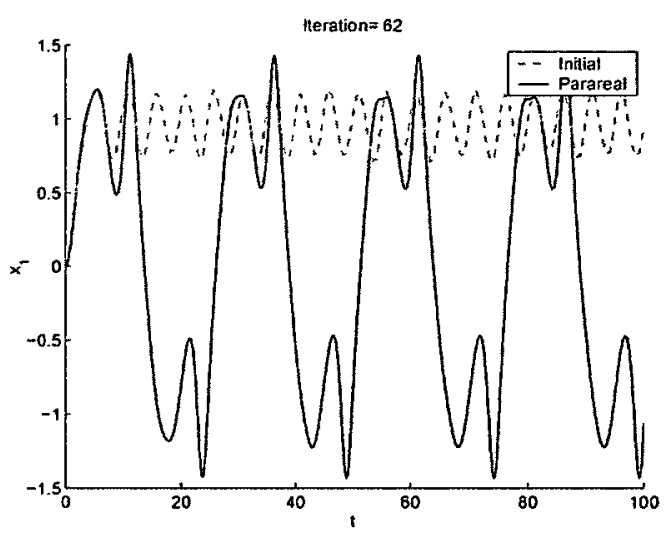

(c) Time history

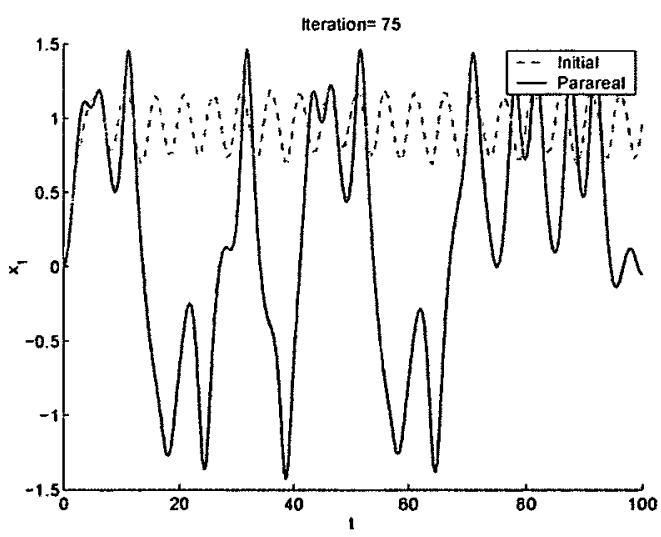

(e) Time history

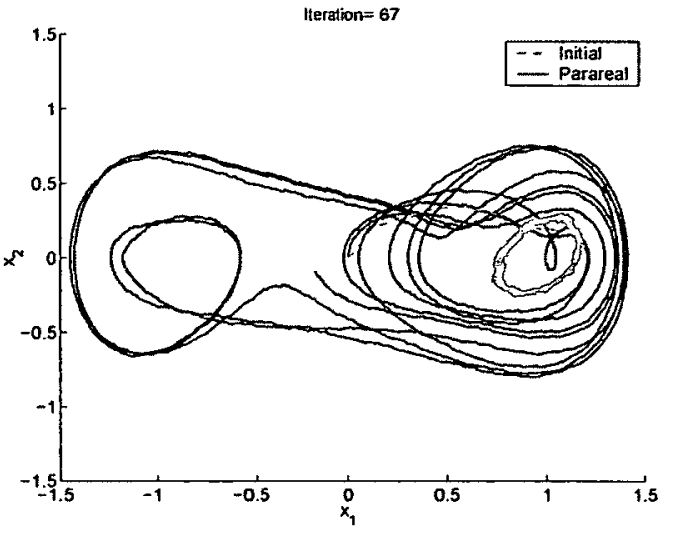

(b) Phase portrait

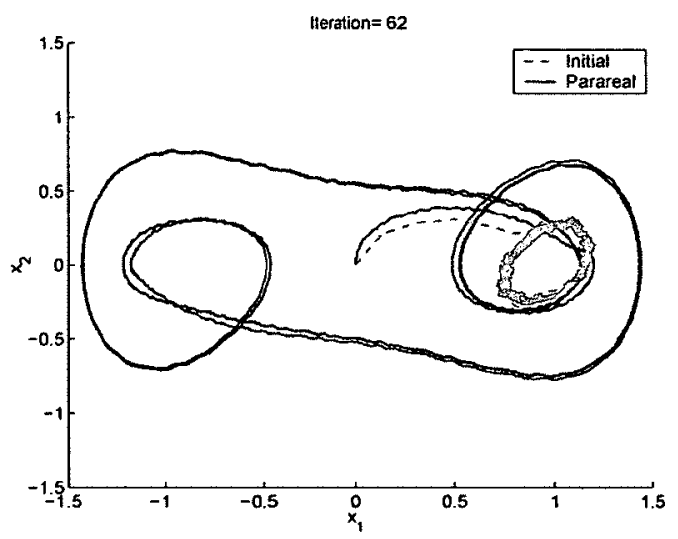

(d) Phase portrait

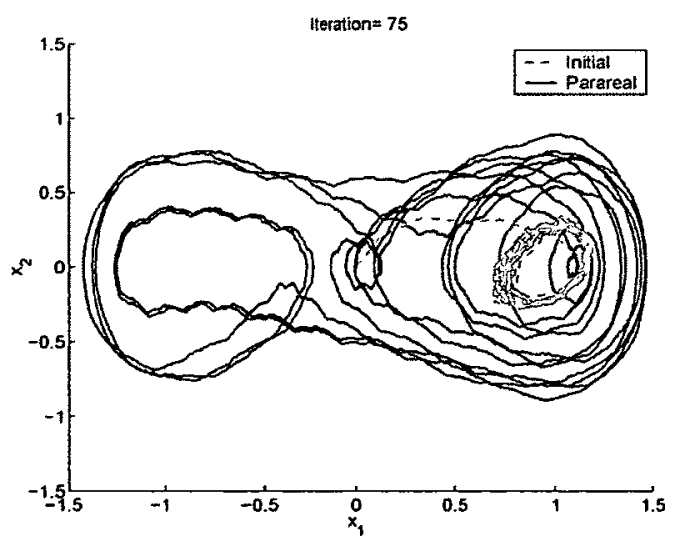

(f) Phase portrait

Figure 11.9: Samples of the response of the Duffing oscillator:

$$
\Gamma=0.37, \sigma=0.1 \Gamma
$$


The results for medium noise strength: $\sigma=0.5 \Gamma$ are presented in Fig.(11.10). As the noise strength increases, the presence of higher frequency oscillations become more apparent. This fact can be realized by comparing the phase portraits of those in Fig.(11.9) with Fig.(11.10). Evidently, the number of iterations required to converge depends on the random sample. 


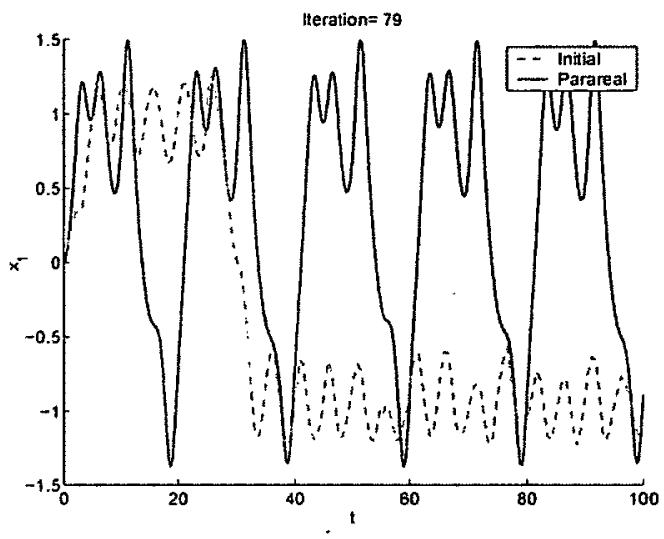

(a) Time history

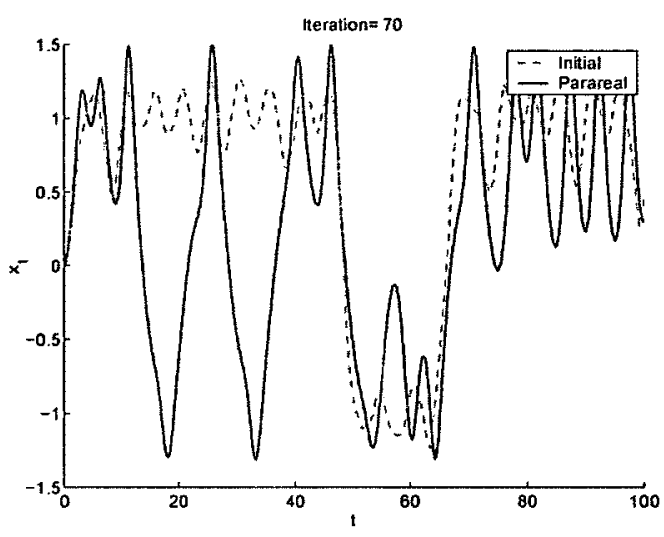

(c) Time history

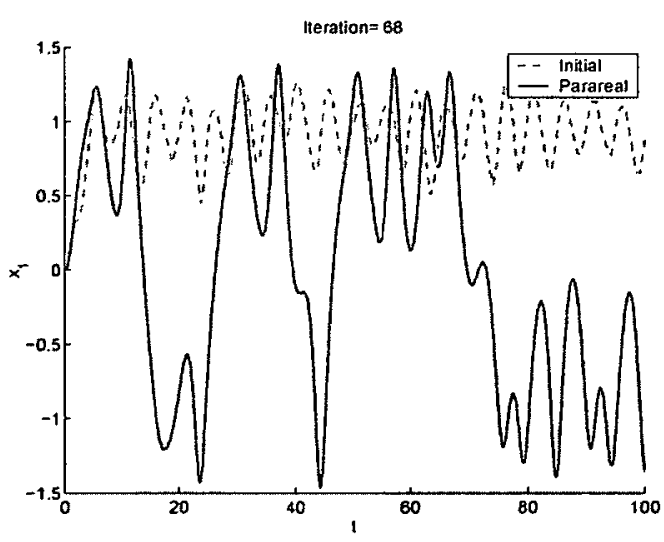

(e) Time history

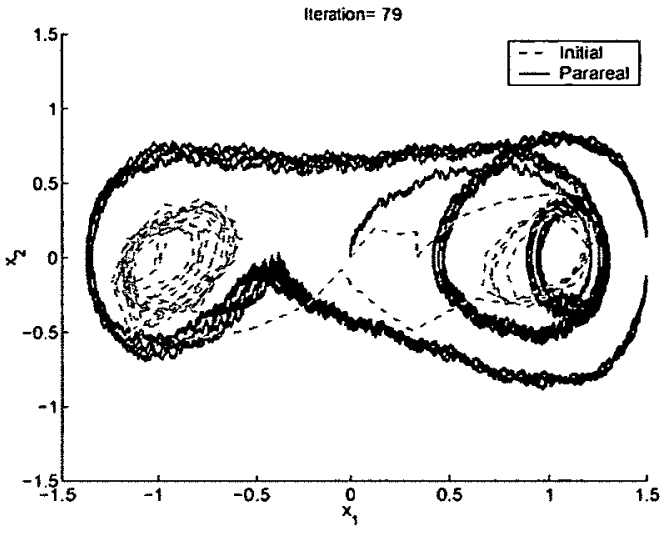

(b) Phase portrait

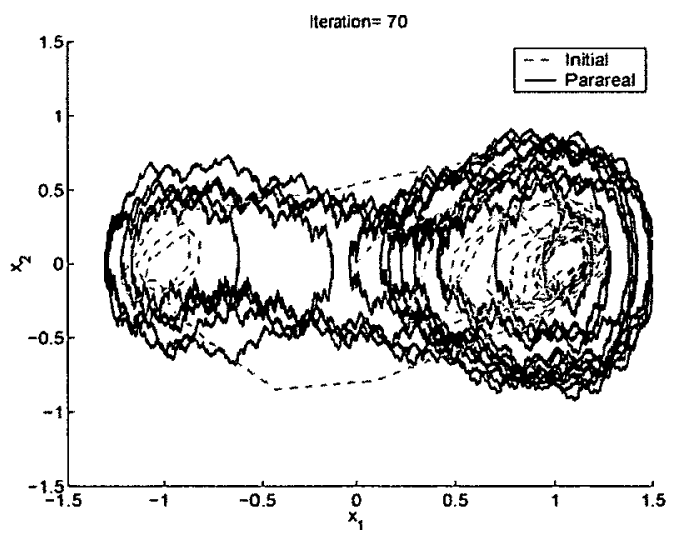

(d) Phase portrait

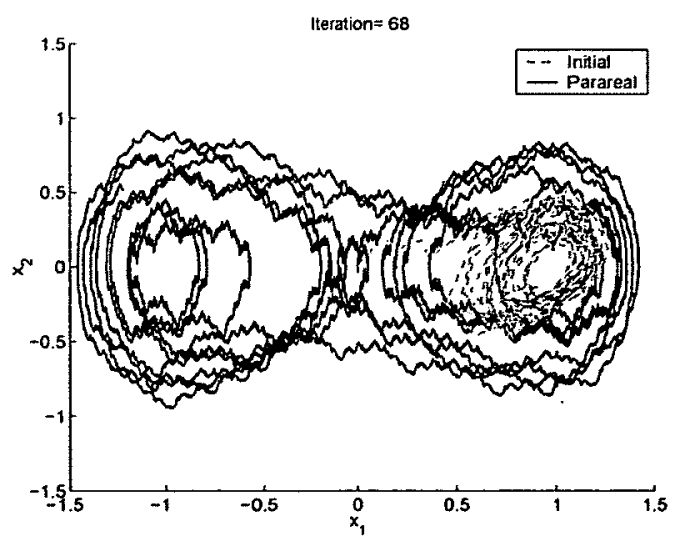

(f) Phase portrait

Figure 11.10: Samples of the response of the Duffing oscillator:

$$
\Gamma=0.37, \sigma=0.5 \Gamma
$$


Fig.(11.11) presents the results for sample trajectories for high strength of noise corresponding to $\sigma=0.9 \Gamma$, whereby the results obtained from parareal scheme become increasingly unwieldy. This phenomenon again can be attributed to the inadequacy of the coarse integrator as addressed in the previous case $(\Gamma=0.3)$. 


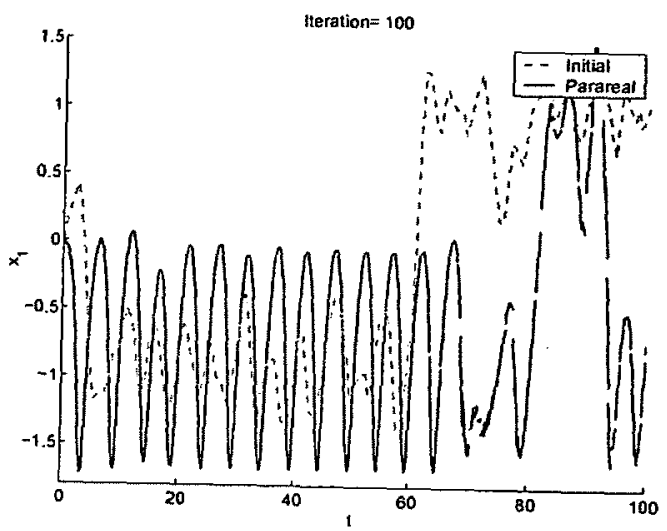

(a) Time history

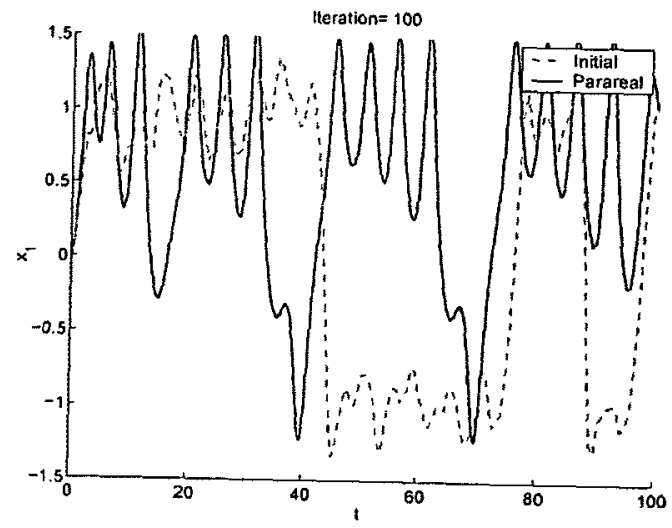

(c) Time history

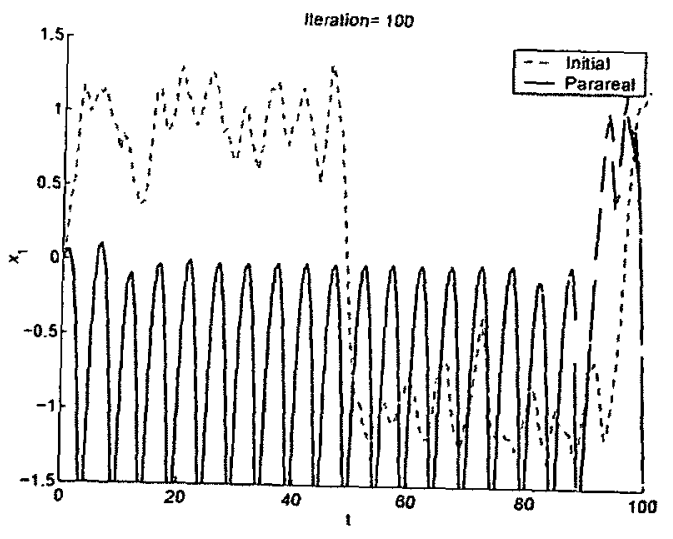

(e) Time history

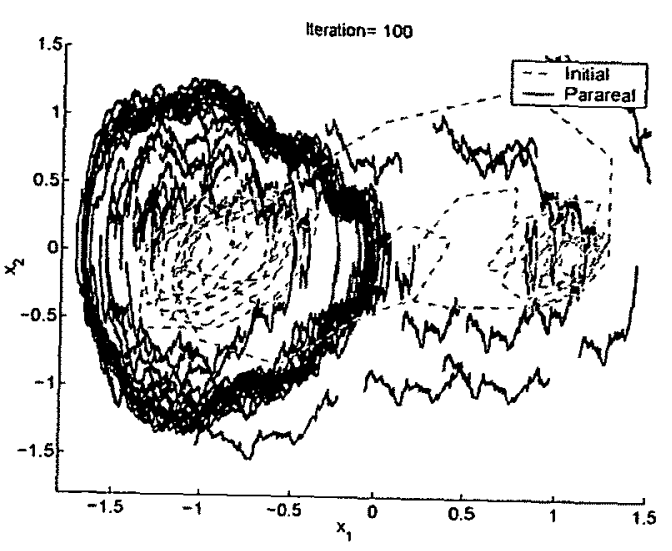

(b) Phase portrait

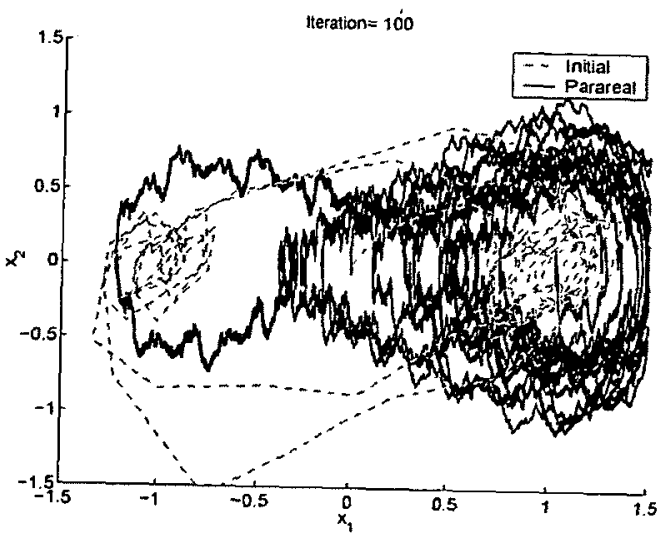

(d) Phase portrait

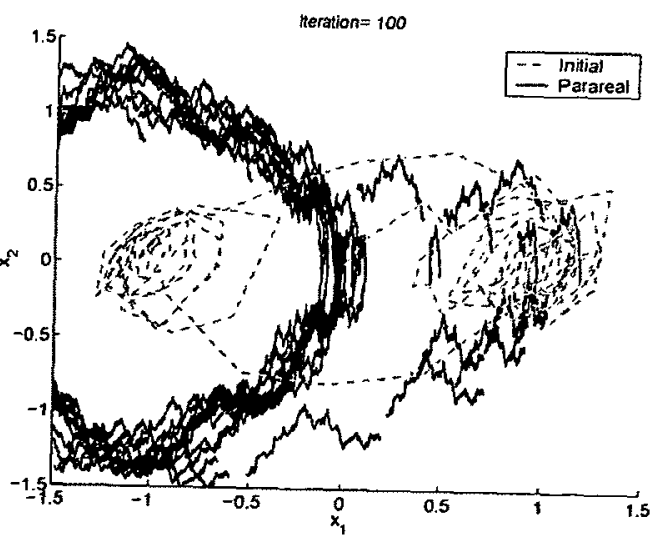

(f) Phase portrait

Figure 11.11: Samples of the response of the Duffing oscillator:

$$
\Gamma=0.37, \sigma=0.9 \Gamma
$$




\subsection{Parallel Performance}

We measure the parallel performance of the adapted parareal algorithm by the strong scalability and speedup. The aim of the strong scalability test is to show the ability of the parallel algorithm to reduce the total execution time as we increase the number of processors [7]. The speedup measures the amount of the execution speed gained by increasing the number of processors [7]. The total speedup $S_{t}(n p)$ of a parallel algorithm implemented on a parallel computer using $n p$ processors is defined as [7]

$$
S_{t}(n p)=\frac{T_{s}}{T(n p)},
$$

where $T_{s}$ refers to the total execution time of the sequential scheme using a single processor computer and $T(n p)$ is the total execution time of the parallel scheme executed on a parallel computer using $n p$ processors. When the execution time of the sequential algorithm $T_{s}$ is not available, the execution time of the parallel algorithm on a parallel computer using a single processor is used. In this case the speedup is called the relative speedup $S_{r}(n p)$ and it is defined as [7]

$$
S_{r}(n p)=\frac{T(1)}{T(n p)},
$$

where $T(1)$ and $T(n p)$ denote the total execution time of the parallel algorithm executed on a parallel computer using a single and $n p$ processors respectively [7].

At this stage, it is worth emphasizing the fact that the time domain parallelism is beneficial only for relatively long time simulations. For short time simulations, the interprocessor 
communication cost of the parallel algorithm cannot mask the computational cost. Indeed, when we use the previously used integration parameters $\left(T=\left[0, \frac{40 \pi}{\omega}\right], \Delta T=0.63\right.$ and $\Delta t=0.003$ ) the sequential algorithm takes no time (almost zero) to execute. The parareal algorithm, on the other hand, demands relatively longer time to execute. Although the total execution time is about fraction of a second, increasing the number of processors up to a certain value leads to a reduction in the total execution time as illustrated in Table(11.1).

Table 11.1: Execution time in second for the parareal algorithm

\begin{tabular}{|c|c|c|}
\hline No. of processors & Time: $\Gamma=0.3$ & Time: $\Gamma=0.37$ \\
\hline 1 & $8.489 \mathrm{E}-2$ & $8.646 \mathrm{E}-2$ \\
\hline 2 & $4.694 \mathrm{E}-2$ & $4.551 \mathrm{E}-2$ \\
\hline 4 & $2.217 \mathrm{E}-2$ & $2.101 \mathrm{E}-2$ \\
\hline 8 & $2.834 \mathrm{E}-2$ & $8.252 \mathrm{E}-2$ \\
\hline 16 & $12.178 \mathrm{E}-2$ & $22.941 \mathrm{E}-2$ \\
\hline
\end{tabular}

For such short simulation time, the stochastic parareal algorithm is not a preferable alternative to the sequential method. To demonstrate the usefulness of the parallel scheme for fast simulation, we increase the total simulation time to $T=500$. In addition, we take the coarse scale time step $\Delta T=0.03$ and the fine scale time step $\Delta t=1.5 \times 10^{-6}$. The parareal algorithm is implemented in a parallel environment using MPI libraries and executed on a Linux cluster consists of 1200 compute nodes with 6 cores and 36 GBytes of memory per node with InfiniBand interconnect [149]. A semi-implicit scheme is used for the serial algorithm and the results are generated using the above time integration grid (i.e. $T=500$ and $\Delta t=1.5 \times 10^{-6}$ ). 
Next we study the parallel performance of the algorithm, for different strength of the random noise. In Fig.(11.12) and Fig.(11.13) we show the strong scalability and the corresponding total and relative speedup of the stochastic parareal algorithm for $\Gamma=0.3$ and $\Gamma=0.37$ respectively. Clearly, increasing the number of processors leads to a great reduction in the total execution time as demonstrated in the left panels of Fig.(11.12) and Fig.(11.13). For this specific example, the stochastic parareal method is only efficient when we use roughly more than 24 processors. In these cases, the execution time of the parareal scheme is lower than that of the sequential method. Increasing the number of processors beyond certain thresholds ( 96 for the case of $\Gamma=0.3$ and 112 for the case of $\Gamma=0.37$ ) does not reduce the execution time further as the communication overhead predominates over the computational cost.

Depending on the random sample and the strength of the noise, the total speedup $S_{t}(n p)$ of the stochastic parareal scheme is between 2 and 3 as illustrated in the middle panels of Fig.(11.12) and Fig.(11.13). This means that the parallel method is faster than the sequential method by a factor between 2 to 3 . On the other hand, the stochastic parareal scheme can achieve a relative speedup $S_{r}(n p)$ up to 40 depending on the random sample and the strength of the noise as shown in the right panels of Fig.(11.12) and Fig.(11.13). 


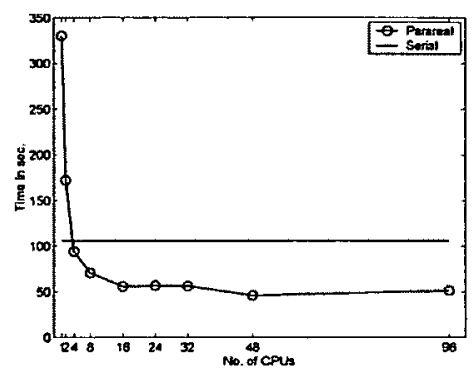

(a) Scalability: $\sigma=0$

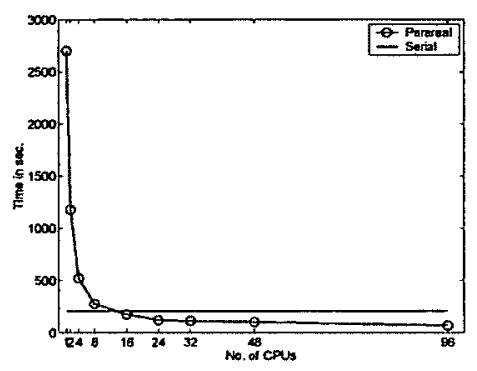

(d) Scalability: $\sigma=0.1 \Gamma$

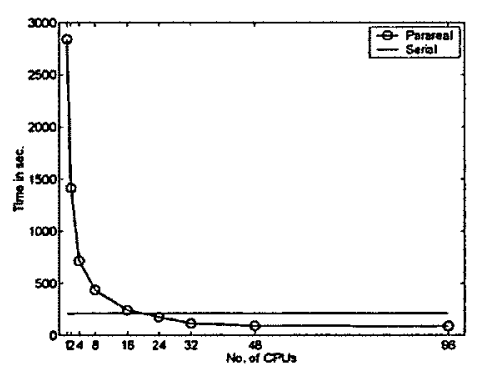

(g) Scalability: $\sigma=0.5 \Gamma$

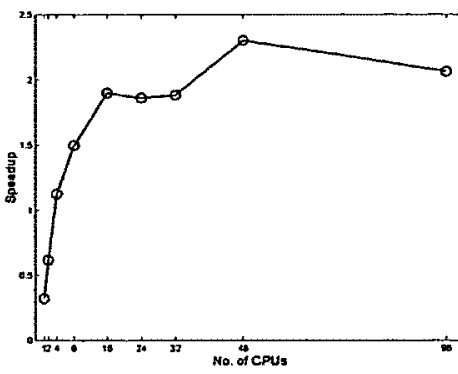

(b) Total speedup: $\sigma=0$

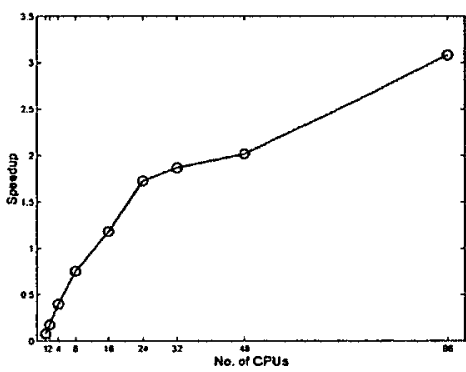

(e) Total speedup: $\sigma=0.1 \Gamma$

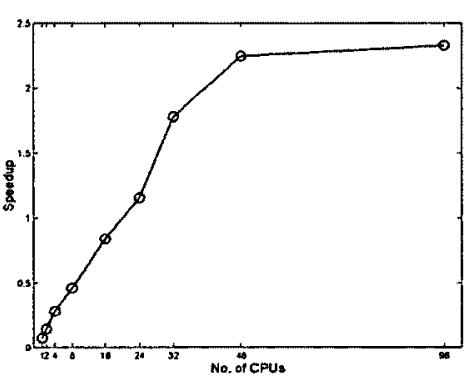

(h) Total speedup: $\sigma=0.5 \Gamma$

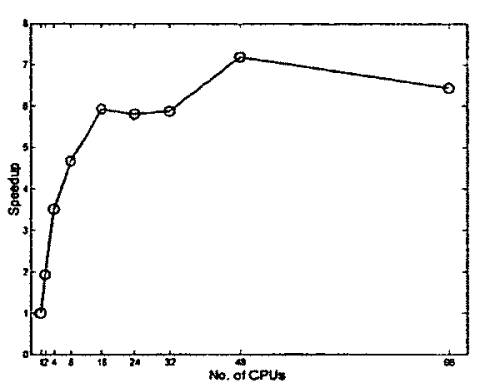

(c) Relative speedup: $\sigma=0$

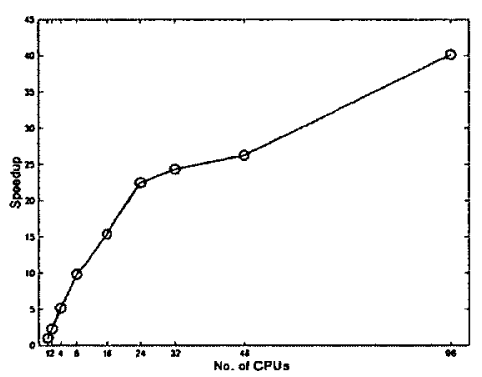

(f) Relative speedup: $\sigma=0.1 \Gamma$

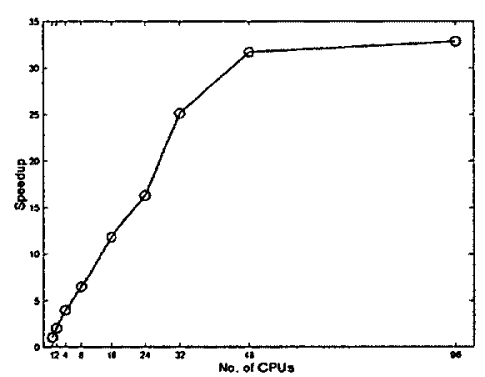

(i) Relative speedup: $\sigma=0.5 \Gamma$

Figure 11.12: Parallel scalability and speedup: $\Gamma=0.3$ 


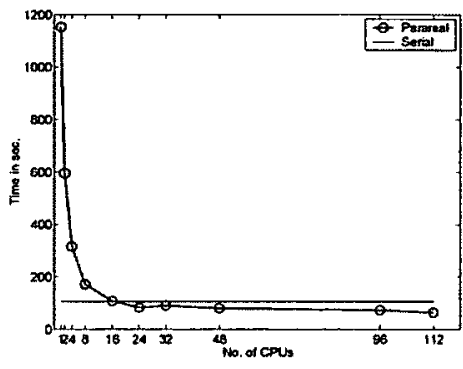

(a) Scalability: $\sigma=0$

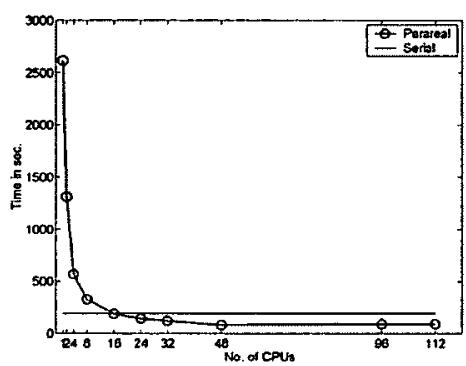

(d) Scalability: $\sigma=0.1 \Gamma$

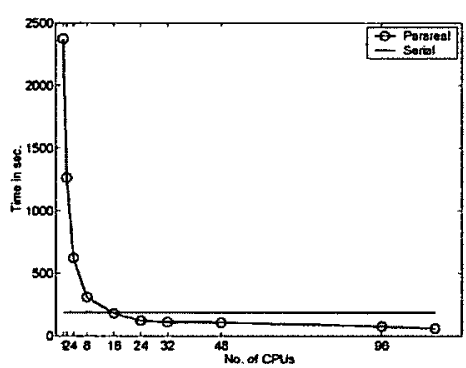

(g) Scalability: $\sigma=0.5 \Gamma$

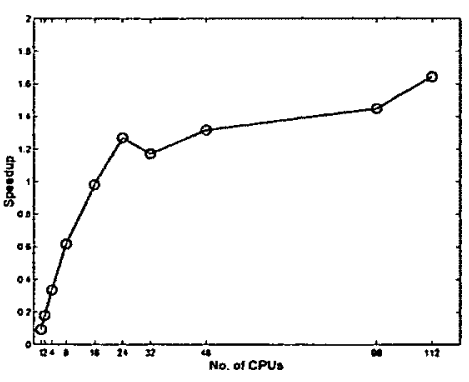

(b) Total speedup: $\sigma=0$

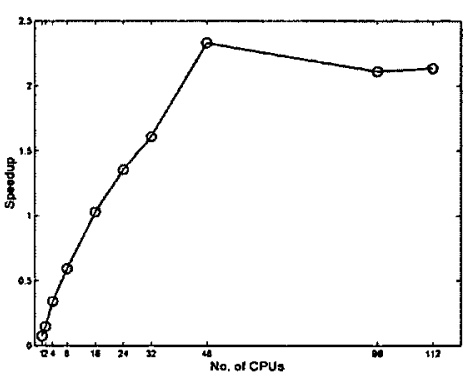

(e) Total speedup: $\sigma=0.1 \Gamma$

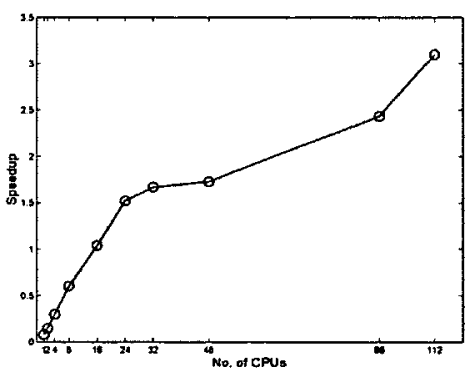

(h) Total speedup: $\sigma=0.5 \Gamma$

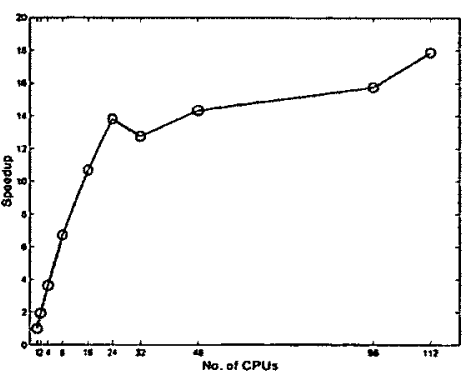

(c) Relative speedup: $\sigma=0$

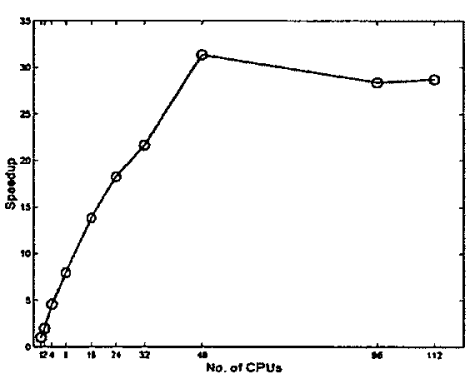

(f) Relative speedup: $\sigma=0.1 \Gamma$

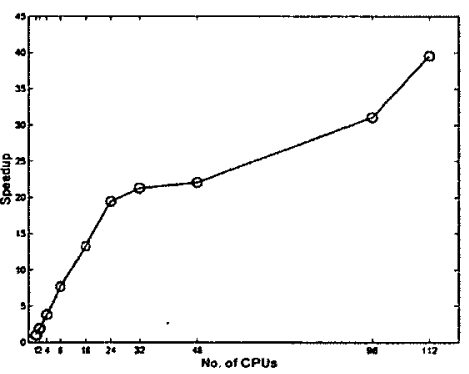

(i) Relative speedup: $\sigma=0.5 \Gamma$

Figure 11.13: Parallel scalability and speedup: $\Gamma=0.37$ 


\section{Discussion}

For the particular example considered here, the performance of the parareal scheme in terms of convergence rate and parallel scalability is dictated by the ratio $\frac{\Delta T}{\Delta t}$. For small $\Delta t$, relatively large amounts of computational work are performed independently by each processor. In other words, $\Delta t$ determines the computational cost of the parareal algorithm. On the other hand, taking large $\Delta T$ not only affects the stability of the coarse integral, but also increases the iteration counts of the parareal algorithm to converge. Each iteration of the parareal scheme involves two communication events as shown in (steps 12 and 16) of Algorithm(21). Consequently, $\Delta T$ quantifies in some sense the communication cost of the parallel algorithm (the larger $\Delta T$ leads to the higher communication cost). Furthermore, recall that the coarse integral and the correction to the initial conditions (steps 14 and 15 respectively) in Algorithm( refPararealAlgorithm) are performed serially on the master processor $($ rank $=0)$. For small $\Delta T$, the master processor may consume high CPU time, while the rest of the processors are idle. Therefore, the ratio $\frac{\Delta T}{\Delta t}$ can be used to qualitatively estimate the granularity of the parareal algorithm (i.e. computation/communication). For efficient parallel performance, one typically should maintain coarse granularity (e.g. rela-

tively large ratio of $\frac{\Delta T}{\Delta t}$ ) while satisfying the convergence criteria of both the fine and coarse integration methods. 


\subsection{Summary}

We made an initial attempt to study the efficiency and limitations of a stochastic parallel integration scheme for strongly nonlinear systems. At least for the Duffing oscillator considered here, the numerical results demonstrates the possible usefulness of the stochastic parareal scheme for efficient simulation of dynamical systems in parallel computers. For low to medium strength of noise, the parareal scheme tracks the response trajectories accurately. The failure of the scheme for high strength of input noise is also pointed out. The parallel implementation of the algorithm demonstrates that the parareal algorithm can accelerate the simulation time by a factor of 2 to 3 depending on the random sample and the strength of the noise.

In relation to some recent developments $[68,74-77,79,80]$, the parareal scheme may also be applied for efficient simulation of stochastic partial differential equation with random operator in conjunction with the spatial domain decomposition. On the other hand, the tracking sample path of randomly perturbed dynamical system subject to noisy observational data is most relevant to nonlinear filtering commonly used in data assimilation $[162,169-171]$. This fact may offer the possibility of near real-time forecast (prediction) of the dynamics of engineering and natural systems (e.g. [169-171]) although more research is needed in this direction. 


\section{Chapter 12}

\section{Conclusions and Future Directions}

\subsection{Summary of Findings}

Non-overlapping domain decomposition methods, namely iterative substructuring techniques are described for uncertainty quantification of large-scale computational models. The mathematical framework is based on non-overlapping domain decomposition in the geometric space and functional expansion along the stochastic dimension. For numerical illustrations, two-dimensional and three-dimensional SPDEs with spatially varying nonGaussian random coefficients are considered. The numerical scalability of the algorithms are investigated with respect to the mesh size, subdomain size, fixed problem size per subdomain, level of uncertainty and order of the stochastic dimension. For a fixed mesh resolution, the total solution time cannot be reduced beyond a critical number of subdomains as the interprocessor communication overhead and memory bandwidth requirement predominate over floating point operations. In order to exploit the remaining idle processors in multi-processor computers, the investigation is directed towards achieving time domain 
parallelism. To this end, a probabilistic version of a parallel time integration scheme is developed to simulate the trajectories of a noisy non-linear dynamical system described by SDEs. The primary contributions of this investigation are summarized as follows:

- Iterative substructuring techniques are developed to tackle the coupled large-scale linear systems arising in the context of the intrusive Spectral Stochastic Finite Element Method (SSFEM). The iterative algorithm circumvents the need of constructing both the local and global extended Schur complement matrices explicitly.

- A novel probabilistic one-level Neumann-Neumann domain decomposition preconditioner is introduced for the iterative solution of the coupled large-scale linear systems in the SSFEM. The performance of the algorithm is nearly independent of the level of uncertainty and the size of the stochastic dimension. The one-level domain decomposition algorithm demonstrates scalability for moderate range of processors/subdomains for the problem size considered in this investigation. The probabilistic Neumann-Neumann domain decomposition preconditioner is the essential ingredient to develop two-level domain decomposition algorithms for SPDEs.

- A novel two-level domain decomposition preconditioner is formulated for the iterative substructuring of SPDEs. Within the framework of the intrusive SSFEM, the two-level preconditioner is constructed by adding a probabilistic coarse problem to the one-level Neumann-Neumann preconditioner. The algorithm may be considered as a probabilistic version of the Balancing Domain Decomposition by Constraints (BDDC). The probabilistic two-level Neumann-Neumann preconditioner exhibits a scalable performance for problems in linear elasticity and flow through porous media 
with spatially varying non-Gaussian random parameters.

- A novel primal-primal iterative substructuring technique is introduced to solve the large-scale linear system arising from the finite element discretization of stochastic PDEs. The algorithm offers a direct approach to formulate a two-level scalable preconditioner. The algorithm can be viewed as another probabilistic version of BDDC. The algorithm shows a scalable performance with respect to the order of the stochastic expansion, the spatial problem size, number of subdomains and fixed problem size per subdomain.

- A novel dual-primal iterative substructuring technique is formulated to tackle the coupled deterministic linear systems in the intrusive SSFEM of large-scale SPDEs. The proposed method may be viewed as the probabilistic version of the Dual-Primal Finite Element Tearing and Interconnecting (FETI-DP). The numerical results illustrate that the proposed algorithm is scalable with respect to the order of the stochastic expansion, the spatial problem size, number of subdomains and fixed problem size per subdomain. The probabilistic FETI-DP method demonstrates convergence properties similar to the probabilistic BDDC type two-level domain decomposition preconditioner for SPDEs.

- A parallel time integration algorithm for ODEs is adapted to tackle SDEs describing noise-driven dynamical systems. For the Duffing oscillator considered in this investigation, the numerical results demonstrate the possible usefulness of the stochastic parareal scheme for efficient simulation of dynamical systems in parallel computers. For low to medium strength of noise, the parareal scheme tracks the response trajec- 
tories accurately. The parallel implementation of the algorithm demonstrates that the parareal algorithm can accelerate the simulation time by a factor of 2 to 3 depending on the random sample and the strength of the noise.

In the abstract form, the probabilistic two-level domain decomposition methods can be expressed as

$$
\mathcal{M}^{-1}=\sum_{s=1}^{n_{s}} \mathcal{H}_{f}^{s T}\left[\mathcal{S}_{f}^{s}\right]^{-1} \mathcal{H}_{f}^{s}+\mathcal{H}_{c}^{T}\left[\mathcal{S}_{c}\right]^{-1} \mathcal{H}_{c}
$$

where $\mathcal{M}^{-1}$ denotes the preconditioner in the primal methods (and operator in the dualprimal method), $\left[\mathcal{S}_{f}^{s}\right]^{-1}$ is the local (fine) problem, $\left[\mathcal{S}_{c}\right]^{-1}$ is the (global) coarse problem, and $\mathcal{H}_{f}^{s}$ and $\mathcal{H}_{c}$ are the restriction operators. A summary of the two-level domain decomposition methods for the stochastic problems is shown in Table (12.1). Clearly, the fine $\left[\mathcal{S}_{f}^{s}\right]^{-1}$ and coarse $\left[\mathcal{S}_{c}\right]^{-1}$ level problems are the same in all the methods. The structure of the fine $\mathcal{H}_{f}^{s}$ and coarse $\mathcal{H}_{c}$ level restriction operators are the same in both the probabilistic primal-primal and dual-primal methods. Note that the two-level Neumann-Neumann preconditioner is employed to precondition the extended Schur complement system, whereas the primal-primal preconditioner is used to precondition the reduced system by excluding the variables associated with the corner nodes of the interface boundary. The primal-primal preconditioner has the same size and structure of the dual-primal operator. 
Table 12.1: Connection among the two-level probabilistic Neumann-Neumann preconditioner, primal-primal preconditioner and dual-primal operator

\begin{tabular}{|l|l|l|l|l|}
\hline Method & $\mathcal{H}_{f}^{s}$ & {$\left[\mathcal{S}_{f}^{s}\right]^{-1}$} & $\mathcal{H}_{c}$ & {$\left[\mathcal{S}_{c}\right]^{-1}$} \\
\hline \hline$(a)$ & $\mathcal{R}_{s}^{r} \mathcal{D}_{s} \mathcal{R}_{s}$ & {$\left[\mathcal{S}_{r r}^{s}\right]^{-1}$} & $\sum_{s=1}^{n_{s}} \mathcal{B}_{c}^{s T}\left(\mathcal{R}_{s}^{c}-\mathcal{S}_{c r}^{s}\left[\mathcal{S}_{r r}^{s}\right]^{-1} \mathcal{R}_{s}^{r}\right) \mathcal{D}_{s} \mathcal{R}_{s}$ & $\sum_{s=1}^{n_{s}} \mathcal{B}_{c}^{s T}\left(\mathcal{S}_{c c}^{s}-\mathcal{S}_{c r}^{s}\left[\mathcal{S}_{r r}^{s}\right]^{-1} \mathcal{S}_{r c}^{s}\right) \mathcal{B}_{c}^{s}$ \\
\hline$(b)$ & $\mathcal{D}_{r}^{s} \mathcal{B}_{r}^{s}$ & {$\left[\mathcal{S}_{r r}^{s}\right]^{-1}$} & $\sum_{s=1}^{n_{s}} \mathcal{B}_{c}^{s T} \mathcal{S}_{c r}\left[\mathcal{S}_{r r}^{s}\right]^{-1} \mathcal{D}_{r}^{s} \mathcal{B}_{r}^{s}$ & $\sum_{s=1}^{n_{s}} \mathcal{B}_{c}^{s T}\left(\mathcal{S}_{c c}^{s}-\mathcal{S}_{c r}^{s}\left[\mathcal{S}_{r r}^{s}\right]^{-1} \mathcal{S}_{r c}^{s}\right) \mathcal{B}_{c}^{s}$ \\
\hline$(c)$ & $\mathcal{B}_{r}^{s T}$ & {$\left[\mathcal{S}_{r r}^{s}\right]^{-1}$} & $\sum_{s=1}^{n_{s}} \mathcal{B}_{c}^{s T} \mathcal{S}_{c r}^{s}\left[\mathcal{S}_{r r}^{s}\right]^{-1} \mathcal{B}_{r}^{s T}$ & $\sum_{s=1}^{n_{s}} \mathcal{B}_{c}^{s T}\left(\mathcal{S}_{c c}^{s}-\mathcal{S}_{c r}^{s}\left[\mathcal{S}_{r r}^{s}\right]^{-1} \mathcal{S}_{r c}^{s}\right) \mathcal{B}_{c}^{s}$ \\
\hline
\end{tabular}

(a) : Two-Level Neumann-Neumann Preconditioner.

(b) : Primal-Primal Preconditioner.

(c) : Dual-Primal Operator. 


\subsection{Directions for Future Research}

The computational framework of uncertainty analysis developed in this thesis demonstrates scalability for large range of processors. The rapid advances of high performance computing systems warrants further improvement of the aforementioned domain decomposition algorithms for SPDEs to exploit even larger (e.g. thousands) number of processors for uncertainty quantification of large-scale systems. To address this issue, the following future research directions are envisioned:

- The probabilistic coarse problem in the two-level domain decomposition methods for SPDEs plays the critical role to achieve a scalable performance. For large-scale three-dimensional problems, the size of the coarse problem increases rapidly with the number of subdomains. At each iteration of the two-level domain decomposition solver, the coarse problem have to be solved efficiently to spread information globally across the subdomains. The solution of the coarse problem becomes the performance bottleneck for large number of subdomains. For deterministic systems, this issue is addressed by the use of inexact solvers for the coarse problem [123, 131, 172]. For stochastic system, the Stochastic Reduced Basis Methods (SRBMs) [47-49] can be exploited to formulate a reduced-order probabilistic coarse problem which can be solved efficiently. This approach seems to be feasible as only a small number of basis vectors should be adequate to obtain a good approximate solution to the the coarse problem at much lower computational cost.

- To achieve better convergence properties for three-dimensional elasticity problems, additional primal constraints must be considered in constructing the probabilistic 
coarse problem. These constraints may include averages over faces or averages and first-order moments over edges $[81,83,135,155,156]$. Optional Lagrange multipliers $[81,157]$ and transformation of basis $[155,156]$ approaches can be utilized to implement the additional constraints in the probabilistic FETI-DP and BDDC type preconditioner respectively.

- For a given problem size, the spatial domain decomposition techniques cannot reduce the total solution time beyond a certain number of processors. This limitation is due to hardware related issues such as latency and bandwidth of the communication network. To take the full advantage of the massively parallel computers, the concurrency should be exploited in both temporal and spatial dimension simultaneously. The parareal scheme for parallel time integration performs reasonably at least for the one degree of freedom system considered in the thesis. The performance study of the parareal scheme in conjunction with the spatial domain decomposition for time dependent SPDEs using SSFEM merits future investigation.

- Hybrid CPU/GPU clusters are becoming cost-effective high performance computing systems for computationally intensive applications. To effectively utilize this hybrid computing environment, further adaptations to the probabilistic domain decomposition solvers need to be considered. Such adaptations may include exploiting two-level concurrency, namely data and task parallelisms. Each subdomain can be assigned to a pair of CPU and GPU in each compute node. The data parallelism achieved by domain decomposition constitutes the global level of parallelization. This data parallel approach can be implemented using MPI on the CPU. Within each 
compute node, the computational power of the GPU can be exploited to speedup the subdomain level computations. This local level of parallelization constitutes task parallelism to be delegated to the GPU.

- For deterministic PDEs, the scalability of a domain decomposition algorithm is estimated theoretically by the condition number bound of the preconditioned operator as $[4,5,7]$

$$
\kappa\left(\mathbf{M}^{-1} \mathbf{S}\right) \leq C\left(1+\log \frac{H}{h}\right)^{2}
$$

where $C$ is a positive constant (independent of the geometric parameters $h$ and $H$ ) and $h$ and $H$ are the finite element mesh size and subdomain size respectively. When the condition number is independent of the subdomain size $H$ (or number of subdomains $1 / H$ ), the method is said to be scalable $[4,5,7,64]$. For SPDEs, we conduct numerical experiments to demonstrate that the probabilistic two-level domain decomposition methods are scalable with respect to the number of subdomains [129]. A theoretical study to estimate the condition number bound for SPDEs is required. The condition number bound for SPDEs maybe cast in the following form

$$
\kappa\left(\mathcal{M}^{-1} \mathcal{S}\right) \leq f(h, H, L, N)
$$

where $L$ and $N$ denote the number of terms in KLE and PCE respectively. 


\section{Appendices}




\section{Appendix A}

\section{Uncertainty Representation by}

\section{Stochastic Processes}

The representation of uncertainty in the system parameters by PCE based on experimental data highly affects the accuracy of the response statistics $[24,173,174]$. Indeed, identification of the polynomial chaos coefficients from measurements is an active topic in the literature [24, 173-176]. Statistical estimation methods such as maximum likelihood estimation [175-177] and Bayesian inference $[24,174]$ are proposed to characterize the chaos coefficients from field observations. However, for cases when higher order of PCE is required to accurately represent random variables (e.g. multi-modal random variables), these approaches may by inefficient $[24,175]$ or become computationally intensive [173]. In order to reduce the computational complexity, an empirical projection technique is proposed in [173]. The methodology is based on representing a multi-modal random variable as a combination of uni-modal random variables [173]. Although the method may be compu- 
tationally efficient, it requires very large number of samples as the dimension of the PCE increases [173].

For an elementary exposition of PCE, we consider a stochastic Poisson's problem defined on a square domain. We assume that the sufficient statistical data is available to represent the the permeability coefficient as a random field. Consequently, we represent the permeability coefficient using PCE. Next, we propagate uncertainty in the model parameters through the system using both PCE and Monte Carlo simulation (MCS).

In Fig.(A.1), we show the discretized computational domain using 2093 nodes and 4028 elements. On this domain we consider Poisson's equation defined as

$$
\begin{aligned}
\nabla \cdot(\kappa(\mathbf{x}) \nabla u(\mathbf{x}, \theta)) & =f(\mathbf{x}), \quad \mathbf{x} \in D \\
u(\mathbf{x}) & =0, \quad \mathbf{x} \in \partial D
\end{aligned}
$$

where $f(\mathbf{x})=1.0$ and $\kappa(\mathbf{x}, \theta)$ is the permeability coefficient modelled as a stochastic process. 


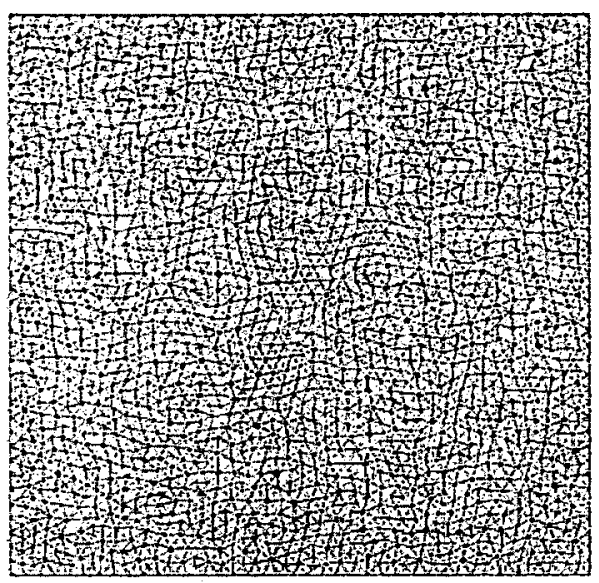

Figure A.1: The finite element mesh

Next we assume sufficient permeability tests are carried out which allow accurate representation of the permeability coefficient as a random field. As an ideal case, we assume the data is noise free and available at each point in the domain. In practice however, the available data is generally sparse and contains noise. For this case, maximum likelihood estimation [175-177] or Bayesian inference $[24,174]$ can be used to estimate the chaos coefficients of the permeability field. Samples of the permeability measurements are shown in Fig.(A.2). The samples are synthetically generated using a four dimensional third order PCE of a lognormal process (see Appendix B). 


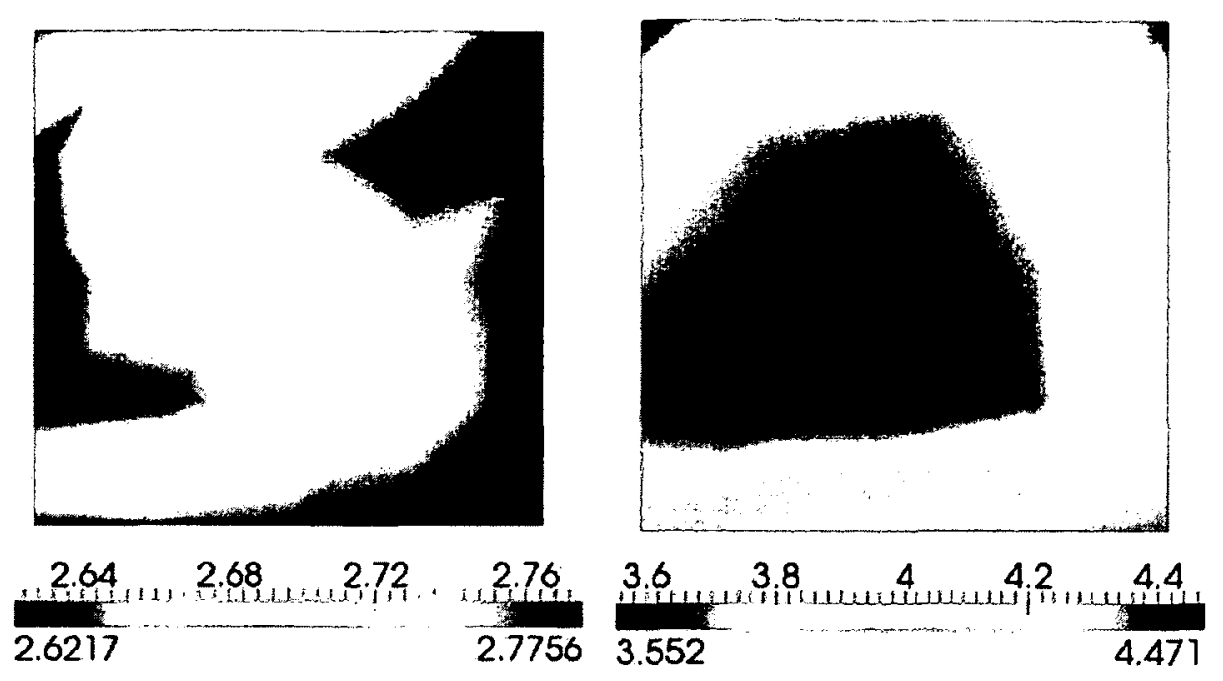

Figure A.2: Samples of the permeability measurements

The PCE of the permeability field $\kappa(\mathbf{x}, \theta)$ can be expressed as

$$
\kappa(\mathbf{x}, \theta)=\sum_{j=0}^{P} \kappa_{j}(\mathbf{x}) \Psi_{j}(\boldsymbol{\xi}) .
$$

For multi-modal random variables, very high-order PCE is needed to accurately represent the random field [173]. As an efficient alternative technique, multi-modal random variables can be represented as a mixture of uni-modal random variables [173]. The uni-modal random variables can be represented using low-order PCE and the expansion coefficients can be calculated using the empirical projection technique [173].

Assuming sufficient data is available and four dimensional third order PCE is sufficient to accurately represent the permeability field, the chaos coefficients $\kappa_{j}(\mathbf{x})$ can be obtained from the samples as 


$$
\widehat{\kappa}_{j}(\mathbf{x})=\frac{\left\langle\kappa(\mathbf{x}, \theta) \Psi_{i}(\boldsymbol{\xi})\right\rangle}{\left\langle\Psi_{i}^{2}(\boldsymbol{\xi})\right\rangle}
$$

The estimates of the selected chaos coefficients $\widehat{\kappa}_{j}(\mathbf{x})$ are shown in Fig.(A.3). Note that we use 1000 realizations to estimate the coefficients. The first coefficient $\widehat{\kappa}_{0}(\mathbf{x})$ denotes the mean of the stochastic process. The higher order coefficients represent the non-Gaussian effect. 


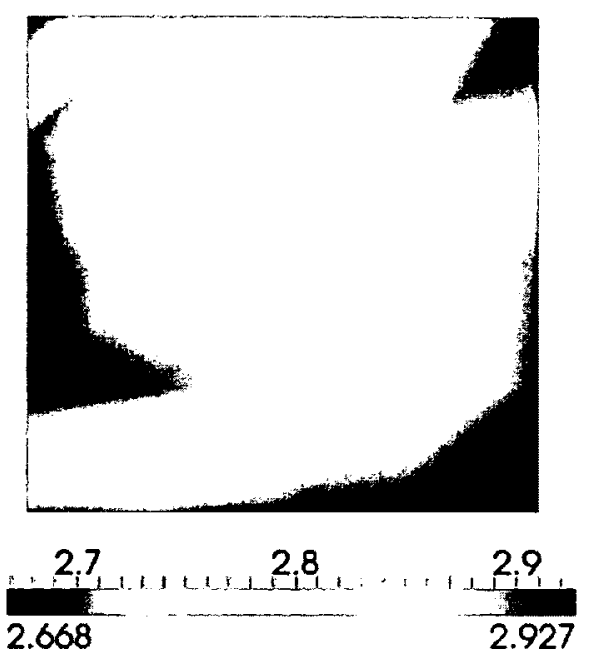

(a) $\widehat{\kappa}_{0}(\mathbf{x})$

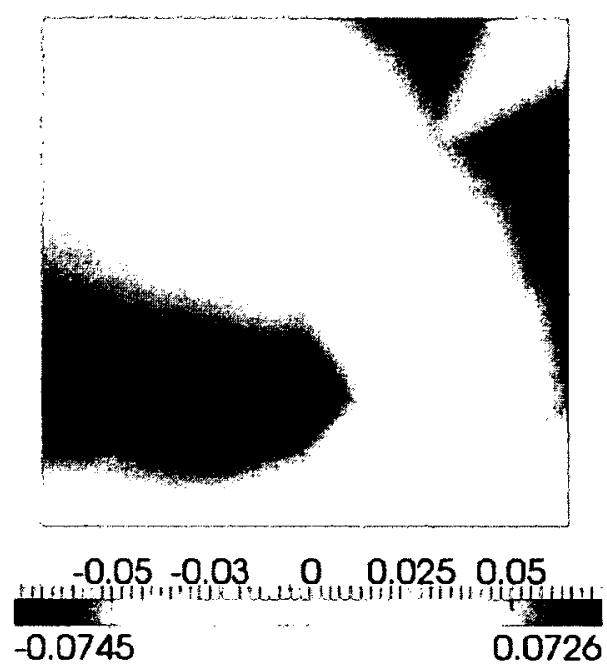

(c) $\widehat{\kappa}_{7}(\mathbf{x})$
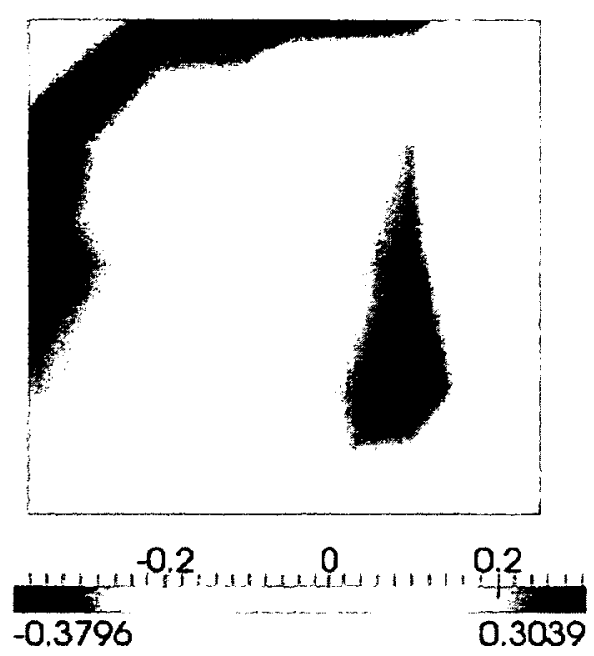

(b) $\widehat{\kappa}_{2}(\mathrm{x})$

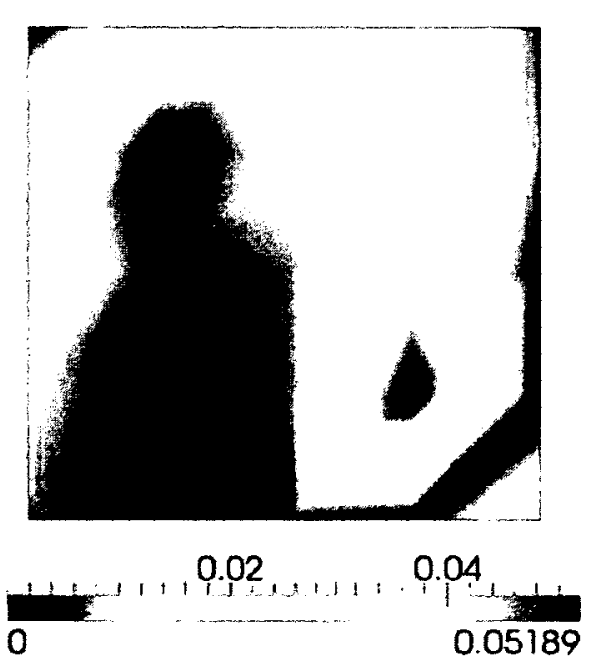

(d) $\widehat{\kappa}_{9}(\mathbf{x})$

Figure A.3: Selected PC coefficients of the permeability random field

Firstly, we deterministically represent the permeability coefficient using the mean of the stochastic process. The corresponding deterministic solution field is shown in Fig.(A.4). 
Secondly, we represent the permeability coefficient as a stochastic process using Eq.(A.3). The mean and standard deviation of the solution field obtained using PCE and MCS are plotted in Fig.(F.1). Acceptable agreement between PCE and MCS can be observed. The maximum $C o V$ in the middle of the domain is about $25 \%$. The mean and standard deviation have the same trend as the deterministic solution previously shown in Fig.(A.4).

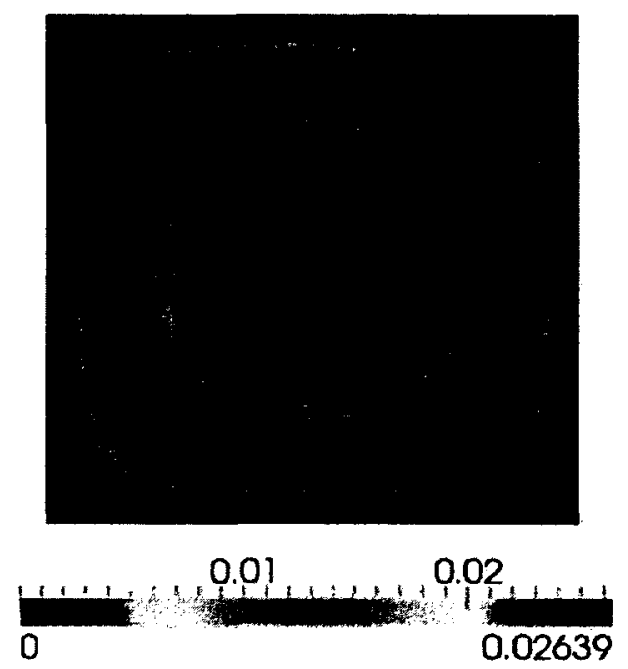

Figure A.4: The deterministic solution field 

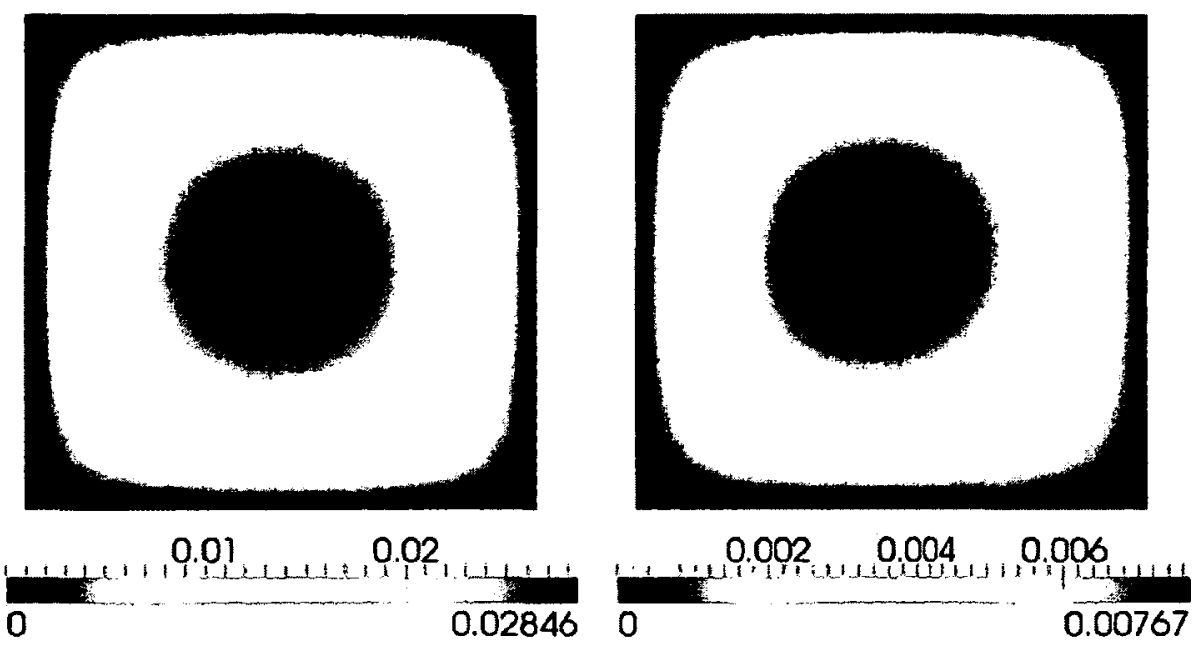

(a) mean: PCE

(b) standard deviation: PCE
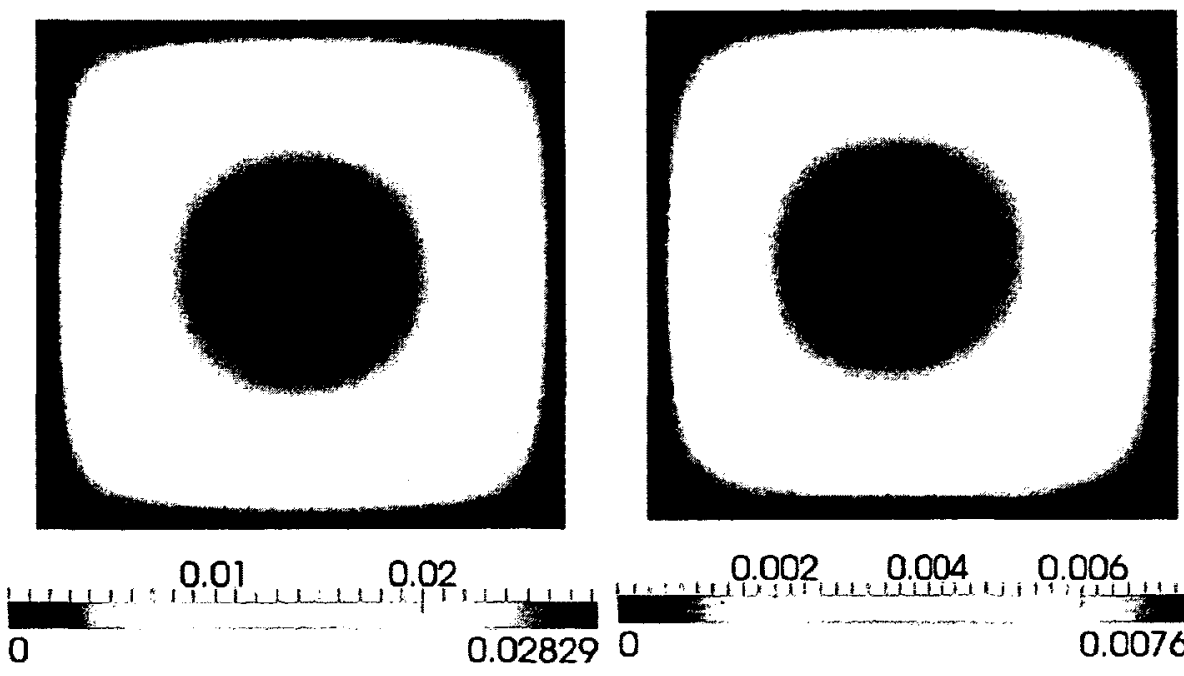

(c) mean: MCS

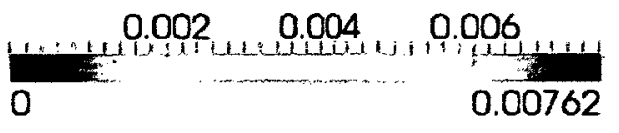

(d) standard deviation: MCS

Figure A.5: The mean and standard deviation of the solution process using PCE and MCS 


\section{Appendix B}

\section{Polynomial Chaos Expansion of}

\section{Lognormal Stochastic Process}

This section closely follows the references $[96,145]$. Let $g(\mathbf{x}, \theta)$ be a Gaussian stochastic process and $C_{g g}\left(\mathbf{x}_{1}, \mathbf{x}_{2}\right)$ its covariance function. The KLE of the Gaussian process $g(\mathbf{x}, \theta)$ can be obtained as

$$
g(\mathbf{x}, \theta)=g_{0}(\mathbf{x})+\sum_{i=1}^{L} \sqrt{\lambda_{i}} \phi_{i}(\mathbf{x}) \xi_{i}(\theta)
$$

Eq.(B.1) can be rewritten in compact form as

$$
g(\mathbf{x}, \theta)=\sum_{i=0}^{L} g_{i}(\mathbf{x}) \xi_{i}
$$

where

$$
\xi_{0}(\theta)=1 \quad \text { and } \quad g_{i}(\mathbf{x})=\sqrt{\lambda_{i}} \phi_{i}(\mathbf{x})
$$


Let $l(\mathbf{x}, \theta)$ be a lognormal stochastic process obtained from the Gaussian stochastic process $g(\mathbf{x}, \theta)$ as

$$
l(\mathbf{x}, \theta)=\exp [g(\mathbf{x}, \theta)]
$$

Substituting the KLE of the Gaussian process given by Eq.(B.2) into Eq.(B.4) leads to

$$
l(\mathbf{x}, \theta)=\exp \left[\sum_{i=0}^{L} g_{i}(\mathbf{x}) \xi_{i}\right]
$$

The PCE of the lognormal process $l(\mathbf{x}, \theta)$ can be defined as

$$
l(\mathbf{x}, \theta)=\sum_{i=0}^{P} l_{i}(\mathbf{x}) \Psi_{i}(\boldsymbol{\xi}),
$$

where

$$
\left\langle\Psi_{i}(\xi)\right\rangle=0, \quad\left\langle\Psi_{i}(\boldsymbol{\xi}) \Psi_{j}(\boldsymbol{\xi})\right\rangle=\left\langle\Psi_{i}^{2}(\boldsymbol{\xi})\right\rangle \delta_{i j}
$$

Galerkin projection of Eq.(B.6) on the PC basis leads to

$$
l_{i}(\mathbf{x})=\frac{\left\langle l(\mathbf{x}, \theta) \Psi_{i}(\boldsymbol{\xi})\right\rangle}{\left\langle\Psi_{i}^{2}(\boldsymbol{\xi})\right\rangle}
$$

The denominator in Eq.(B.8) can be evaluated analytically. The numerator is calculated as follows [145].

Using Eq.(B.5), the numerator in Eq.(B.8) can be expressed as [145] 


$$
\left\langle l(\mathbf{x}, \theta) \Psi_{i}(\boldsymbol{\xi})\right\rangle=\exp \left[g_{0}(\mathbf{x})\right] \int_{-\infty}^{+\infty} \exp \left[\sum_{j=1}^{L} g_{j}(\mathbf{x}) \xi_{j}\right] \Psi_{j}(\boldsymbol{\xi}) \exp \left[-\frac{1}{2} \sum_{j=1}^{L} \xi_{j} \xi_{j}\right] d \boldsymbol{\xi}
$$

The integration in Eq.(B.9) can be simplified to [145]

$$
\left\langle l(\mathbf{x}, \theta) \Psi_{i}(\boldsymbol{\xi})\right\rangle=\exp \left[g_{0}(\mathbf{x})+\frac{1}{2} \sum_{j=1}^{L} g_{j}^{2}(\mathbf{x})\right]\left\langle\Psi_{j}(\boldsymbol{\eta})\right\rangle
$$

where

$$
\eta_{j}=\xi_{j}-g_{j}(\mathbf{x})
$$

In a concise from, Eq.(B.10) can be expressed as

$$
\left\langle l(\mathbf{x}, \theta) \Psi_{i}(\boldsymbol{\xi})\right\rangle=l_{0}(\mathbf{x})\left\langle\Psi_{j}(\boldsymbol{\eta})\right\rangle
$$

where $l_{0}(\mathbf{x})$ is the mean of the lognormal stochastic process defined as

$$
l_{0}(\mathbf{x})=\exp \left[g_{0}(\mathbf{x})+\frac{1}{2} \sum_{j=1}^{L} g_{j}^{2}(\mathbf{x})\right]
$$

The quantity $\left\langle\Psi_{j}(\eta)\right\rangle$ in Eq.(B.12) is the expectation of the PC basis centered around the Gaussian coefficients $g_{i}(\mathbf{x})$. This expectation can be evaluated analytically as shown in Table.(B.1) [145]. 
Table B.1: The expectation and variance of the $\mathrm{PC}$ basis

\begin{tabular}{|l|c|l|}
\hline$\Psi_{i}(\boldsymbol{\xi})$ & $\left\langle\Psi_{i}^{2}(\boldsymbol{\xi})\right\rangle$ & $\left\langle\Psi_{i}(\boldsymbol{\eta})\right\rangle$ \\
\hline \hline$\xi_{1}$ & 1 & $g_{1}(\mathbf{x})$ \\
\hline$\xi_{2}$ & 1 & $g_{2}(\mathbf{x})$ \\
\hline$\xi_{1}^{2}-1$ & 2 & $g_{1}^{2}(\mathbf{x})$ \\
\hline$\xi_{1} \xi_{2}$ & 1 & $g_{1}(\mathbf{x}) g_{2}(\mathbf{x})$ \\
\hline$\xi_{2}^{2}-1$ & 2 & $g_{2}^{2}(\mathbf{x})$ \\
\hline$\xi_{1}^{3}-3 \xi_{1}$ & 6 & $g_{1}^{3}(\mathbf{x})$ \\
\hline$\xi_{1}^{2} \xi_{2}-\xi_{2}$ & 2 & $g_{1}^{2}(\mathbf{x}) g_{2}(\mathbf{x})$ \\
\hline$\xi_{1} \xi_{2}^{2}-\xi_{1}$ & 2 & $g_{1}(\mathbf{x}) g_{2}^{2}(\mathbf{x})$ \\
\hline$\xi_{2}^{3}-3 \xi_{2}$ & 6 & $g_{2}^{3}(\mathbf{x})$ \\
\hline
\end{tabular}

Using Eq.(B.12), the PC coefficients of the lognormal stochastic process in Eq.(B.8) can be rewritten as

$$
l_{i}(\mathbf{x})=l_{0}(\mathbf{x}) \frac{\left\langle\Psi_{i}(\boldsymbol{\eta})\right\rangle}{\left\langle\Psi_{i}^{2}(\boldsymbol{\xi})\right\rangle}
$$

Finally, the PCE of the lognormal stochastic process can be expressed as

$$
l(\mathbf{x}, \theta)=l_{0}(\mathbf{x}) \sum_{i=0}^{L} \frac{\left\langle\Psi_{i}(\boldsymbol{\eta})\right\rangle}{\left\langle\Psi_{i}^{2}(\boldsymbol{\xi})\right\rangle} \Psi_{i}(\boldsymbol{\xi}) .
$$

As a special case, let $g(\theta)$ be a Gaussian random variable defined as

$$
g(\theta)=\mu_{g}+\sigma_{g} \xi
$$


where $\mu_{g}$ is the mean and $\sigma_{g}$ denotes the standard deviation. According to Eq.(B.15) the lognormal random variable can be represented as

$$
\begin{aligned}
l(\theta) & =\mu_{l} \sum_{i=0}^{\infty} \frac{\sigma_{g}^{i}}{i !} \Psi_{i}(\xi) \\
& =\mu_{l}\left(1+\sigma_{g} \xi+\frac{\sigma_{g}^{2}}{2}\left(\xi^{2}-1\right)+\frac{\sigma_{g}^{3}}{6}\left(\xi^{3}-3 \xi\right)+\cdots\right),
\end{aligned}
$$

where the mean of the lognormal random variable is defined as

$$
\mu_{l}=\exp \left(\mu_{g}+\frac{1}{2} \sigma_{g}^{2}\right)
$$




\section{Appendix C}

\section{Spectral Stochastic Finite Element}

\section{Method}

This section closely follows the references $[3,11,17,37]$. Consider a linear elliptic stochastic PDE defined on a domain $\Omega$ with a given data on the boundary $\partial \Omega$ as

$$
\begin{aligned}
\nabla \cdot(\kappa(\mathbf{x}, \theta) \nabla u(\mathbf{x}, \theta)) & =f(\mathbf{x}) \quad \mathbf{x} \in \Omega, \\
u(\mathbf{x}, \theta) & =0 \quad \mathbf{x} \in \partial \Omega,
\end{aligned}
$$

where $\theta$ denotes the stochastic dimension, $\kappa(\mathbf{x}, \theta)$ is the random permeability coefficient, $u(\mathbf{x}, \theta)$ is the stochastic response and $f(\mathbf{x})$ represents the forcing term. The finite element discretization in the spatial domain leads to the following stochastic linear system $[3,11$, $17,37]$

$$
\mathbf{A}(\theta) \mathbf{u}(\theta)=\mathbf{f},
$$


where $A(\theta)$ represents the global stiffness matrix with random coefficients. The global stiffness matrix can be expressed as a summation of the element stiffness matrices as

$$
\mathbf{A}(\theta)=\sum_{e=1}^{n_{e}} \mathbf{A}^{e}(\theta),
$$

where $n_{e}$ denotes the total number of elements associated with the spatial discretization and $\mathrm{A}^{e}(\theta)$ is the element stiffness matrix defined as $[3,11,17,37]$

$$
\mathbf{A}^{e}(\theta)=\int_{\Omega^{e}} \mathbf{B}^{T} \mathrm{D}(\mathbf{x}, \theta) \mathbf{B} \mathrm{d} \Omega^{e}
$$

where $\mathbf{B}$ is strain matrix and $\mathbf{D}(x, \theta)$ represents the random constitutive matrix. Without loss of generality, the random properties is assumed to be isotropic field. Thus, $\mathbf{D}(\mathbf{x}, \theta)$ can be expressed as $[3,11,17,37]$

$$
\mathbf{D}(\mathbf{x}, \theta)=\kappa(\mathbf{x}, \theta) \mathbf{I}
$$

where $I$ is the identity matrix. For general non-Gaussian material properties, the PCE can be used to represent the random permeability coefficient $\kappa(\mathbf{x}, \theta)$ as $[3,11,17,37]$

$$
\kappa(\mathbf{x}, \theta)=\sum_{i=0}^{L} \kappa_{i}(\mathbf{x}) \Psi_{i}
$$

where $\kappa_{i}(\mathbf{x})$ are the PCE coefficients and $\Psi_{i}$ are a set of Hermite polynomials with the following properties

$$
\left\langle\Psi_{0}\right\rangle=1, \quad\left\langle\Psi_{i}\right\rangle=0 \text { for } i \neq 0 \quad \text { and } \quad\left\langle\Psi_{i} \Psi_{j}\right\rangle=\delta_{i j}\left\langle\Psi_{i}^{2}\right\rangle
$$

Substituting Eq.(C.6) and Eq.(C.7) into Eq.(C.5), we obtain 


$$
\mathrm{A}^{e}(\theta)=\sum_{i=0}^{L} \mathrm{~A}_{i}^{e} \Psi_{i}(\theta)
$$

where

$$
\mathbf{A}_{i}^{e}=\int_{\Omega^{e}} \mathbf{B}^{T}\left[\kappa_{i}(\mathbf{x}) \mathbf{I}\right] \mathbf{B} \mathrm{d} \Omega^{e}
$$

Substituting Eq.(C.9) into Eq.(C.4), we obtain

$$
\mathbf{A}(\theta)=\sum_{i=0}^{L} \sum_{e=1}^{n_{e}} \mathbf{A}_{i}^{e} \Psi_{i} .
$$

Eq.(C.11) can be rearranged as

$$
\mathbf{A}(\theta)=\sum_{i=0}^{L} \mathbf{A}_{i} \Psi_{i}
$$

where

$$
\mathbf{A}_{i}=\sum_{e=1}^{n_{e}} \mathbf{A}_{i}^{e}
$$

The solution process can be expanded using the same PC basis as

$$
\mathbf{u}(\theta)=\sum_{j=0}^{N} \mathbf{u}_{j} \Psi_{j}
$$

Substituting Eq.(C.12) and Eq.(C.14) into Eq.(C.3), we obtain

$$
\sum_{i=0}^{L} \sum_{j=0}^{N} \Psi_{i} \Psi_{j} \mathbf{A}_{i} \mathbf{u}_{j}=\mathbf{f} .
$$


Performing Galerkin projection over the space spanned by the polynomial chaoses $\Psi_{k}$, the following coupled deterministic system for the PC coefficients of the solution process is obtained $[3,11,17,37]$

$$
\sum_{j=0}^{N} \sum_{i=0}^{L}\left\langle\Psi_{i} \Psi_{j} \Psi_{k}\right\rangle \mathbf{A}_{i} \mathbf{u}_{j}=\left\langle\Psi_{k} \mathbf{f}\right\rangle, \quad k=0,1, \ldots, N
$$

Equivalently, Eq.(C.16) can be reexpressed in a compact form as

$$
\mathcal{A U}=\mathcal{F}
$$

where

$$
\mathcal{A}_{j, k}=\sum_{i=0}^{L}\left\langle\Psi_{i} \Psi_{j} \Psi_{k}\right\rangle \mathbf{A}_{i}, \quad \mathcal{U}=\left(\mathbf{u}_{0}, \ldots, \mathbf{u}_{N}\right)^{T} \quad \text { and } \quad \mathcal{F}_{k}=\left\langle\Psi_{k} \mathbf{f}\right\rangle
$$

Eq.(C.17) can be expanded as

$$
\left[\begin{array}{cccc}
\mathcal{A}_{0,0} & \mathcal{A}_{0,1} & \ldots & \mathcal{A}_{0, N} \\
\mathcal{A}_{1,0} & \mathcal{A}_{1,1} & \ldots & \mathcal{A}_{1, N} \\
\vdots & \vdots & \ddots & \vdots \\
\mathcal{A}_{N, 0} & \mathcal{A}_{N, 1} & \ldots & \mathcal{A}_{N, N}
\end{array}\right]\left\{\begin{array}{c}
\mathbf{u}_{0} \\
\mathbf{u}_{1} \\
\vdots \\
\mathbf{u}_{N}
\end{array}\right\}=\left\{\begin{array}{c}
\mathcal{F}_{0} \\
\mathcal{F}_{1} \\
\vdots \\
\mathcal{F}_{N}
\end{array}\right\} .
$$

The solution of the coupled linear system defined in Eq.(C.19) provides the chaos coefficients of the solution process $\left(\mathbf{u}_{0}, \ldots, \mathbf{u}_{N}\right)^{T}$. The mean and variance of the solution process can be obtained from Eq.(C.14) respectively as

$$
\boldsymbol{\mu}_{u}=\sum_{j=0}^{N}\left\langle\Psi_{j}\right\rangle \mathbf{u}_{j}=\mathbf{u}_{0}
$$

and

$$
\sigma_{u}^{2}=\left\langle\left(\mathbf{u}(\theta)-\mu_{u}\right)^{2}\right\rangle=\sum_{j=1}^{N}\left\langle\Psi_{j}^{2}\right\rangle \mathbf{u}_{j}^{2}
$$




\section{Appendix D}

\section{Domain Decomposition Methods for}

\section{SPDEs}

This section closely follows the references $[68,74]$. Consider a linear elliptic stochastic PDE defined on a domain $\Omega$ with a given data on the boundary $\partial \Omega$. The finite element approximation of the stochastic PDE leads to the following discrete linear system

$$
\mathbf{A}(\theta) \mathbf{u}(\theta)=\mathbf{f}
$$

where $\mathbf{A}(\theta)$ is the stiffness matrix with random coefficients, $\mathbf{u}(\theta)$ is the stochastic process representing the response vector and $\mathbf{f}$ denotes the applied force. Let the spatial domain $\Omega$ be partitioned into two non-overlapping subdomains such that $\Omega=\Omega_{1} \cup \Omega_{2}, \Omega_{1} \cap \Omega_{2}=0$ and $\Gamma=\partial \Omega_{1} \cap \partial \Omega_{2}$ where $\Gamma$ defines the interface of $\Omega_{1}$ and $\Omega_{2}$ [178]. Accordingly, the equilibrium system for a typical subdomain can be expressed as 


$$
\left[\begin{array}{cc}
\mathbf{A}_{I I}^{s}(\theta) & \mathbf{A}_{I \Gamma}^{s}(\theta) \\
\mathbf{A}_{\Gamma I}^{s}(\theta) & \mathbf{A}_{\Gamma \Gamma}^{s}(\theta)
\end{array}\right]\left\{\begin{array}{l}
\mathbf{u}_{I}^{s}(\theta) \\
\mathbf{u}_{\Gamma}^{s}(\theta)
\end{array}\right\}=\left\{\begin{array}{c}
\mathbf{f}_{I}^{s} \\
\mathbf{f}_{\Gamma}^{s}
\end{array}\right\}
$$

where the partitions $I$ and $\Gamma$ relate to the interior and interface degrees of freedom, respectively. The uncertain system parameters can be expanded using PCE as

$$
\sum_{i=0}^{L} \Psi_{i}\left[\begin{array}{cc}
\mathbf{A}_{I I, i}^{s} & \mathbf{A}_{I \Gamma, i}^{s} \\
\mathbf{A}_{\Gamma I, i}^{s} & \mathbf{A}_{\Gamma \Gamma, i}^{s}
\end{array}\right]\left\{\begin{array}{c}
\mathbf{u}_{I}^{s}(\theta) \\
\mathbf{u}_{\Gamma}^{s}(\theta)
\end{array}\right\}=\left\{\begin{array}{c}
\mathbf{f}_{I}^{s} \\
\mathrm{f}_{\Gamma}^{s}
\end{array}\right\} .
$$

Enforcing the transmission conditions along the interface boundary, the equilibrium equation of the stochastic system defined by two subdomains can be represented as

$$
\sum_{i=0}^{L} \Psi_{i}\left[\begin{array}{ccc}
\mathbf{A}_{I I, i}^{1} & 0 & \mathbf{A}_{I \Gamma, i}^{1} \\
0 & \mathbf{A}_{I I, i}^{2} & \mathbf{A}_{I \Gamma, i}^{2} \\
\mathbf{A}_{\Gamma I, i}^{1} & \mathbf{A}_{\Gamma I, i}^{2} & \mathbf{A}_{\Gamma \Gamma, i}^{1}+\mathbf{A}_{\Gamma \Gamma, i}^{2}
\end{array}\right]\left\{\begin{array}{c}
\mathbf{u}_{I}^{1}(\theta) \\
\mathbf{u}_{I}^{2}(\theta) \\
\mathbf{u}_{\Gamma}(\theta)
\end{array}\right\}=\left\{\begin{array}{c}
\mathbf{f}_{I}^{1} \\
\mathbf{f}_{I}^{2} \\
\mathbf{f}_{\Gamma}^{1}+\mathbf{f}_{\Gamma}^{2}
\end{array}\right\}
$$

The solution process can be expanding using the same PCE basis as

$$
\left\{\begin{array}{c}
\mathbf{u}_{I}^{1}(\theta) \\
\mathbf{u}_{I}^{2}(\theta) \\
\mathbf{u}_{\Gamma}(\theta)
\end{array}\right\}=\sum_{j=0}^{N} \Psi_{j}\left\{\begin{array}{c}
\mathbf{u}_{I, j}^{1} \\
\mathbf{u}_{I, j}^{2} \\
\mathbf{u}_{\Gamma, j}
\end{array}\right\}
$$

Substituting Eq.(D.5) in Eq.(D.4) leads to

$$
\sum_{i=0}^{L} \sum_{j=0}^{N} \Psi_{i} \Psi_{j}\left[\begin{array}{ccc}
\mathbf{A}_{I I, i}^{1} & 0 & \mathbf{A}_{I \Gamma, i}^{1} \\
0 & \mathbf{A}_{I I, i}^{2} & \mathbf{A}_{I \Gamma, i}^{2} \\
\mathbf{A}_{\Gamma I, i}^{1} & \mathbf{A}_{\Gamma I, i}^{2} & \mathbf{A}_{\Gamma \Gamma, i}^{1}+\mathbf{A}_{\Gamma \Gamma, i}^{2}
\end{array}\right]\left\{\begin{array}{c}
\mathbf{u}_{I, j}^{1} \\
\mathbf{u}_{I, j}^{2} \\
\mathbf{u}_{\Gamma, j}
\end{array}\right\}=\left\{\begin{array}{c}
\mathbf{f}_{I}^{1} \\
\mathbf{f}_{I}^{2} \\
\mathbf{f}_{\Gamma}^{1}+\mathbf{f}_{\Gamma}^{2}
\end{array}\right\}
$$


Performing Galerkin projection on the space of the polynomial chaos $\left\{\Psi_{i}\right\}_{i=1}^{N}$ leads to

where

$$
\left[\begin{array}{ccc}
\mathcal{A}_{I I}^{1} & 0 & \mathcal{A}_{I \Gamma}^{1} \\
0 & \mathcal{A}_{I I}^{2} & \mathcal{A}_{I \Gamma}^{2} \\
\mathcal{A}_{\Gamma I}^{1} & \mathcal{A}_{\Gamma I}^{2} & \mathcal{A}_{\Gamma \Gamma}^{1}+\mathcal{A}_{\Gamma \Gamma}^{2}
\end{array}\right]\left\{\begin{array}{c}
\mathcal{U}_{I}^{1} \\
\mathcal{U}_{I}^{2} \\
\mathcal{U}_{\Gamma}
\end{array}\right\}=\left\{\begin{array}{c}
\mathcal{F}_{I}^{1} \\
\mathcal{F}_{I}^{2} \\
\mathcal{F}_{\Gamma}^{1}+\mathcal{F}_{\Gamma}^{2}
\end{array}\right\}
$$

$$
\begin{array}{r}
{\left[\mathcal{A}_{\alpha \beta}^{s}\right]_{j k}=\sum_{i=0}^{L}\left\langle\Psi_{i} \Psi_{j} \Psi_{k}\right\rangle \mathbf{A}_{\alpha \beta, i}^{s} ; \quad \mathcal{F}_{\alpha, k}^{s}=\left\langle\Psi_{k} \mathbf{f}_{\alpha}^{s}\right\rangle,} \\
\mathcal{U}_{I}^{m}=\left(\mathbf{u}_{I, 0}^{m}, \ldots, \mathbf{u}_{I, N}^{m}\right)^{T} \quad ; \quad \mathcal{U}_{\Gamma}=\left(\mathbf{u}_{\Gamma, 0}, \ldots, \mathbf{u}_{\Gamma, N}\right)^{T} .
\end{array}
$$

The subscripts $\alpha$ and $\beta$ can assume the index $I$ and $\Gamma$. Block Gaussian elimination reduces Eq.(D.7) into the following system

where

$$
\left[\begin{array}{ccc}
\mathcal{A}_{I I}^{1} & 0 & \mathcal{A}_{I \Gamma}^{1} \\
0 & \mathcal{A}_{I I}^{2} & \mathcal{A}_{I \Gamma}^{2} \\
0 & 0 & \mathcal{S}^{1}+\mathcal{S}^{2}
\end{array}\right]\left\{\begin{array}{c}
\mathcal{U}_{I}^{1} \\
\mathcal{U}_{I}^{2} \\
\mathcal{U}_{\Gamma}
\end{array}\right\}=\left\{\begin{array}{c}
\mathcal{F}_{I}^{1} \\
\mathcal{F}_{I}^{2} \\
\mathcal{G}_{\Gamma}^{1}+\mathcal{G}_{\Gamma}^{2}
\end{array}\right\}
$$

$$
\begin{aligned}
& \mathcal{S}^{s}=\mathcal{A}_{\Gamma \Gamma}^{s}-\mathcal{A}_{\Gamma I}^{s}\left(\mathcal{A}_{I I}^{s}\right)^{-1} \mathcal{A}_{I \Gamma}^{s}, \quad s=1,2 \\
& \mathcal{G}_{\Gamma}^{s}=\mathcal{F}_{\Gamma}^{s}-\mathcal{A}_{\Gamma I}^{s}\left(\mathcal{A}_{I I}^{s}\right)^{-1} \mathcal{F}_{I}^{s}, \quad s=1,2
\end{aligned}
$$

Eq.(D.9) can be rearranged as

$$
\begin{aligned}
\sum_{s=1}^{2} \mathcal{S}^{s} \mathcal{U}_{\Gamma} & =\sum_{s=1}^{2} \mathcal{G}_{\Gamma}^{s} \\
\mathcal{A}_{I I}^{1} \mathcal{U}_{I}^{1} & =\mathcal{F}_{I}^{1}-\mathcal{A}_{I \Gamma}^{1} \mathcal{U}_{\Gamma} \\
\mathcal{A}_{I I}^{2} \mathcal{U}_{I}^{2} & =\mathcal{F}_{I}^{2}-\mathcal{A}_{I \Gamma}^{2} \mathcal{U}_{\Gamma}
\end{aligned}
$$


Once the interface variables $\mathcal{U}_{\Gamma}$ obtained from Eq.(D.12), the interior variables $\mathcal{U}_{I}^{1}$ and $\mathcal{U}_{I}^{2}$ can be obtained in parallel from Eq.(D.13) and Eq.(D.14) respectively.

For the case of many subdomains, we define $\mathbf{R}_{s}$ as a restriction operator that maps the global interface unknown $\mathbf{u}_{\Gamma}$ to the local interface vector $\mathbf{u}_{\Gamma}^{s}$ as

$$
\mathbf{u}_{\Gamma}^{s}=\mathbf{R}_{s} \mathbf{u}_{\Gamma}
$$

In parallel implementation $\mathbf{R}_{s}$ acts as a scatter operator while $\mathrm{R}_{s}^{T}$ acts as a gather operator and is not constructed explicitly. Enforcing the transmission conditions (compatibility and equilibrium) along the interfaces, the global equilibrium equation of the stochastic system can be expressed in the following block linear systems of equations

$$
\sum_{i=0}^{L} \Psi_{i}\left[\begin{array}{cccc}
\mathbf{A}_{I I, i}^{1} & \ldots & 0 & \mathbf{A}_{I \Gamma, i}^{1} \mathbf{R}_{1} \\
\vdots & \ddots & \vdots & \vdots \\
0 & \ldots & \mathbf{A}_{I I, i}^{n_{s}} & \mathbf{A}_{I \Gamma, i}^{n_{s}} \mathbf{R}_{n_{s}} \\
\mathbf{R}_{1}^{T} \mathbf{A}_{\Gamma I, i}^{1} & \ldots & \mathbf{R}_{n_{s}}^{T} \mathbf{A}_{\Gamma I, i}^{n_{s}} & \sum_{s=1}^{n_{s}} \mathbf{R}_{s}^{T} \mathbf{A}_{\Gamma \Gamma, i}^{s} \mathbf{R}_{s}
\end{array}\right]\left\{\begin{array}{c}
\mathbf{u}_{I}^{1}(\theta) \\
\vdots \\
\mathbf{u}_{I}^{n_{s}}(\theta) \\
\mathbf{u}_{\Gamma}(\theta)
\end{array}\right\}=\left\{\begin{array}{c}
\mathbf{f}_{I}^{1} \\
\vdots \\
\mathbf{f}_{I}^{n_{s}} \\
\sum_{s=1}^{n_{s}} \mathbf{R}_{s}^{T} \mathbf{f}_{\Gamma}^{s}
\end{array}\right\} .
$$

The solution process can be expanded using the same polynomial chaos basis as

$$
\left\{\begin{array}{c}
\mathbf{u}_{I}^{1}(\theta) \\
\vdots \\
\mathbf{u}_{I}^{n_{s}}(\theta) \\
\mathbf{u}_{\Gamma}(\theta)
\end{array}\right\}=\sum_{j=0}^{N} \Psi_{j}\left\{\begin{array}{c}
\mathbf{u}_{I, j}^{1} \\
\vdots \\
\mathbf{u}_{I, j}^{n_{s}} \\
\mathbf{u}_{\Gamma, j}
\end{array}\right\}
$$


Substituting Eq.(D.17) into Eq.(D.16) and performing the Galerkin projection to minimize the error over the space spanned by the chaos basis, the following coupled deterministic systems of equations is obtained

$$
\left[\begin{array}{cccc}
\mathcal{A}_{I I}^{1} & \ldots & 0 & \mathcal{A}_{I \Gamma}^{1} \mathcal{R}_{1} \\
\vdots & \ddots & \vdots & \vdots \\
0 & \ldots & \mathcal{A}_{I I}^{n_{s}} & \mathcal{A}_{I \Gamma}^{n_{s}} \mathcal{R}_{n_{s}} \\
\mathcal{R}_{1}^{T} \mathcal{A}_{\Gamma I}^{1} & \ldots & \mathcal{R}_{n_{s}}^{T} \mathcal{A}_{\Gamma I}^{n_{s}} & \sum_{s=1}^{n_{s}} \mathcal{R}_{s}^{T} \mathcal{A}_{\Gamma \Gamma}^{s} \mathcal{R}_{s}
\end{array}\right]\left\{\begin{array}{c}
\mathcal{U}_{I}^{1} \\
\vdots \\
\mathcal{U}_{I}^{n_{s}} \\
\mathcal{U}_{\Gamma}
\end{array}\right\}=\left\{\begin{array}{c}
\mathcal{F}_{I}^{1} \\
\vdots \\
\mathcal{F}_{I}^{n_{s}} \\
\sum_{s=1}^{n_{s}} \mathcal{R}_{s}^{T} \mathcal{F}_{\Gamma}^{s}
\end{array}\right\}
$$

The coefficient matrix in Eq.(D.18) is of order $n(N+1) \times n(N+1)$ where $n$ and $(N+1)$ denote the number of the physical degrees of freedom for a typical subdomain and chaos coefficients, respectively. The stochastic counterpart of the restriction operator in Eq.(D.18) takes the following form

$$
\mathcal{R}_{s}=\operatorname{blockdiag}\left(\mathbf{R}_{s}^{0}, \ldots, \mathbf{R}_{s}^{N}\right)
$$

where $\left(\mathbf{R}_{s}^{0}, \ldots, \mathbf{R}_{s}^{N}\right)$ are the deterministic restriction operators given by Eq.(D.15). Block Gaussian elimination reduces the system in Eq.(D.18) to the following Schur complement system for the interface variable $\mathcal{U}_{\Gamma}$ as

$$
\mathcal{S} \mathcal{U}_{\Gamma}=\mathcal{G}_{\Gamma}
$$

where the global Schur complement matrix $\mathcal{S}$ is given by

$$
\mathcal{S}=\sum_{s=1}^{n_{s}} \mathcal{R}_{s}^{T}\left[\mathcal{A}_{\Gamma \Gamma}^{s}-\mathcal{A}_{\Gamma I}^{s}\left(\mathcal{A}_{I I}^{s}\right)^{-1} \mathcal{A}_{I \Gamma}^{s}\right] \mathcal{R}_{s}
$$


and the corresponding right hand side vector $\mathcal{G}_{\Gamma}$ is

$$
\mathcal{G}_{\Gamma}=\sum_{s=1}^{n_{s}} \mathcal{R}_{s}^{T}\left[\mathcal{F}_{\Gamma}^{s}-\mathcal{A}_{\Gamma I}^{s}\left(\mathcal{A}_{I I}^{s}\right)^{-1} \mathcal{F}_{I}^{s}\right]
$$

Once the interface unknowns $\mathcal{U}_{\Gamma}$ is available, the interior unknowns can be obtained concurrently by solving the interior problem on each subdomain as

$$
\mathcal{A}_{I I}^{s} \mathcal{U}_{I}^{s}=\mathcal{F}_{I}^{s}-\mathcal{A}_{\Gamma I}^{s} \mathcal{R}_{s} \mathcal{U}_{\Gamma}
$$




\section{Appendix E}

\section{Numerical Validation}

We present numerical validations of the parallel code developed in this study in this section. Firstly, we validate the results of the KLE and domain decomposition method for SPDEs using those obtained by serial codes in Ref. [179]. Secondly, we contrast the performance of the domain decomposition method of SPDEs and MC simulation using the domain decomposition solver. Two- and three-dimensional elliptic SPDEs are considered.

\section{Comparison of the Parallel and Serial Results}

Using the parallel code of domain decomposition for SPDEs developed in this study, we reproduced the results reported in Ref. [179]. In particular, we consider a linear elliptic SPDE defined on L-shaped domain $\Omega$ with a given data on the boundary $\partial \Omega$ as 


$$
\begin{array}{r}
\nabla \cdot(\kappa(\mathbf{x}, \theta) \nabla u(\mathbf{x}, \theta))=f(\mathbf{x}) \quad \mathbf{x} \in \Omega \\
u(\mathbf{x}, \theta)=0 \quad \mathbf{x} \in \partial \Omega
\end{array}
$$

where the coefficient $\kappa(\mathbf{x}, \theta)$ is modeled as a Gaussian process with an exponential covariance function defined as [179]

$$
C_{\kappa \kappa}(\mathbf{x}, \mathbf{y})=\exp \left(\frac{-4\left|x_{1}-y_{1}\right|^{2}}{b}+\frac{-4\left|x_{2}-y_{2}\right|^{2}}{b}\right)
$$

For the numerical implementation, the correlation length is assumed to be $b=0.5$.

Fig.(E.1) shows the monotonic decay of the eigenvalues of the exponential covariance function. Selected eigenfunctions of the covariance function are plotted in Fig.(E.2). The mean and standard deviation of the solution process are shown in Fig.(E.3). In this graphical scale, these results identically match with the corresponding results reported in Ref. [179].

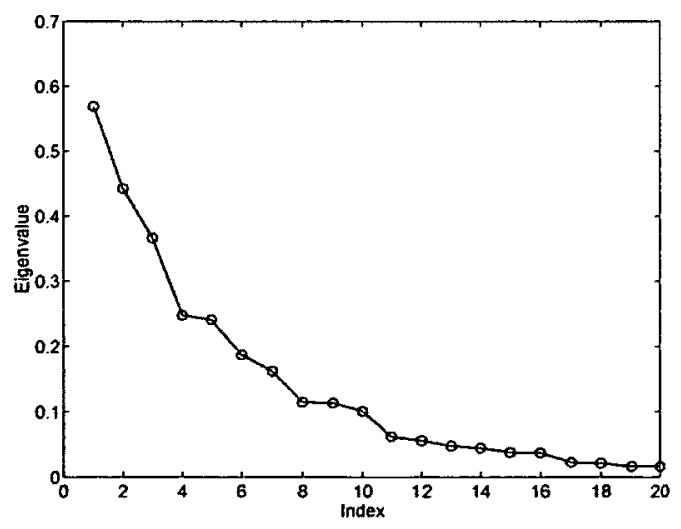

Figure E.1: The decay of the eigenvalues of the covariance function 


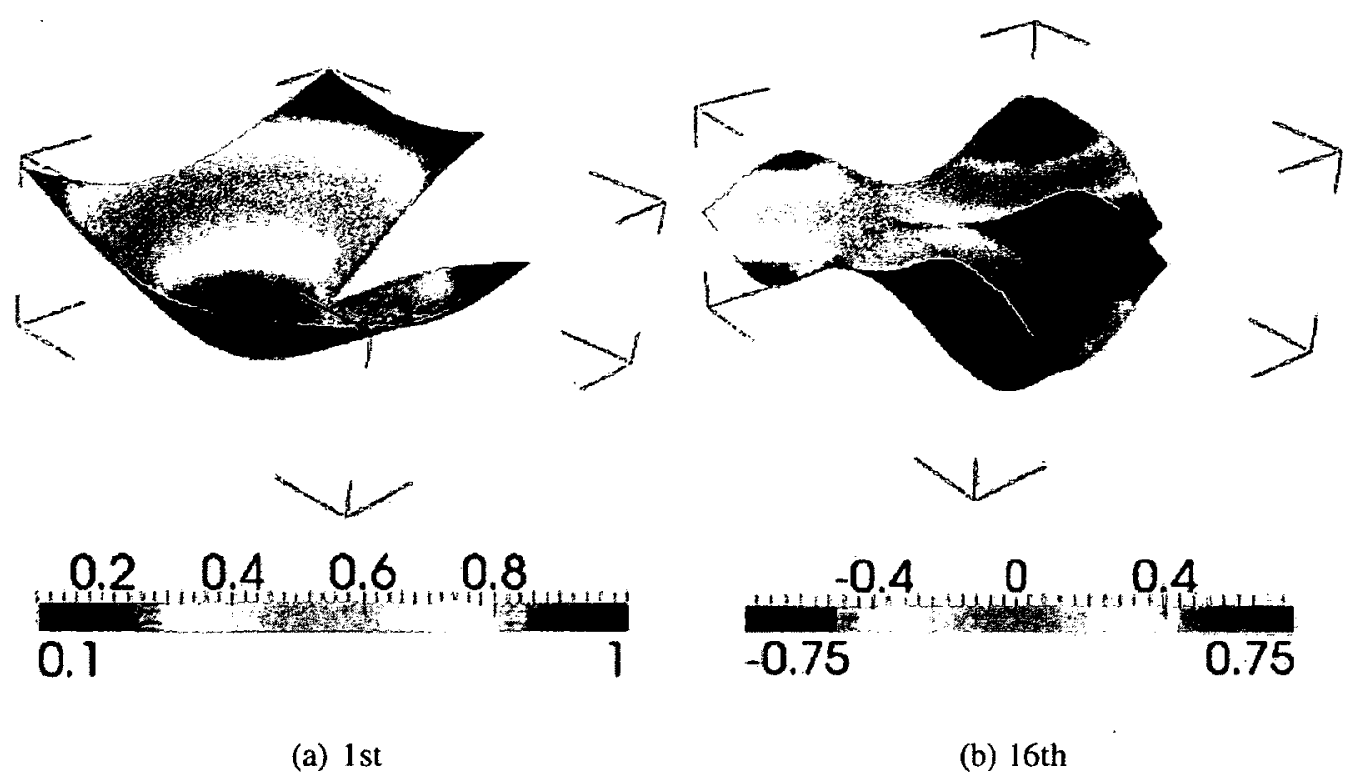

Figure E.2: Selected eigenfunctions of the covariance function

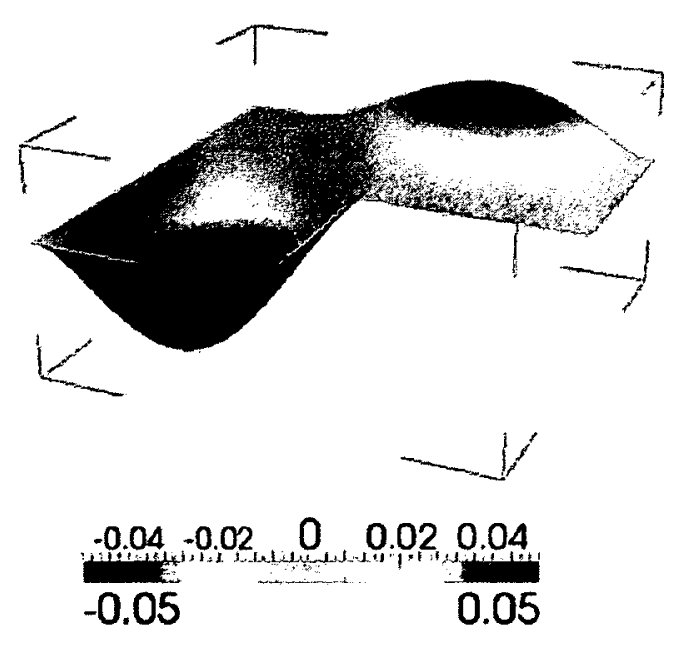

(a) Mean response

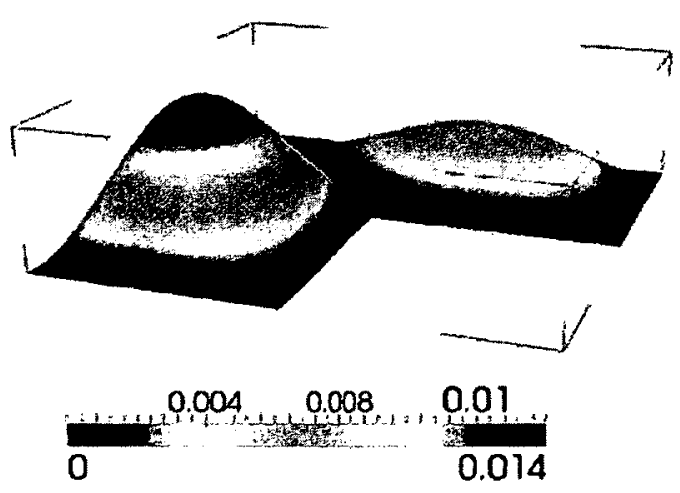

(b) Standard deviation

Figure E.3: The mean and standard deviation of the solution process 


\section{Comparison of PCE and MCS}

\section{Two-dimensional case:}

In this section, we contrast the results obtained from the domain decomposition method of SPDEs and MC simulation using domain decomposition solver. Firstly, we consider the elliptic SPDE defined in Eq.(E.1) where the permeability coefficient $\kappa(\mathbf{x}, \theta)$ is modeled as a lognormal random variable. The Gaussian image of the random variable has a mean $\mu=1.0$ and standard deviation $\sigma=0.25$. Spatial discretization with linear triangular elements leads to 606,089 nodes. The decomposed computational domain is shown in Fig.(E.4).

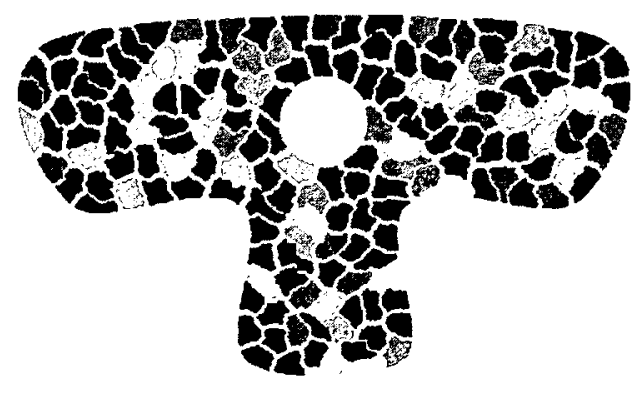

Figure E.4: The decomposed domain

The mean response and standard deviation are plotted in Fig.(E.5). The results obtained from the domain decomposition method of SPDEs and MC simulation are identical in the scale of the graph. Latin Hypercube Sampling (LHS) [20] is used for efficient random sampling. 


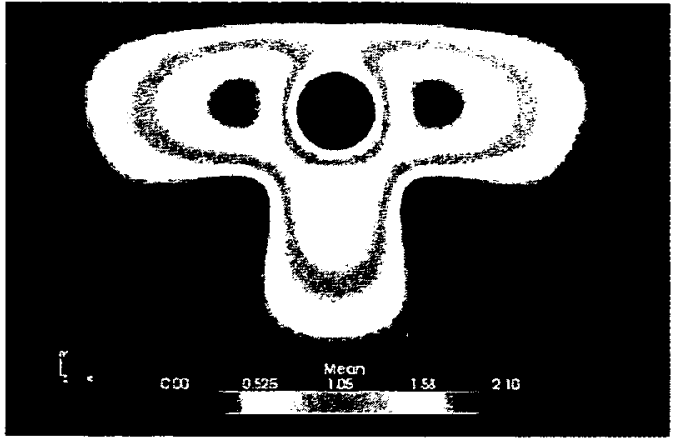

(a) Mean response

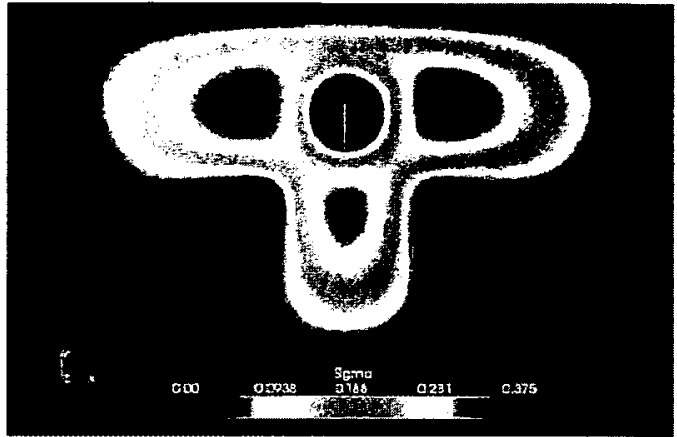

(b) Standard deviation

Figure E.5: The mean and standard deviation of the solution process

The convergence behavior of MC simulation with deterministic domain decomposition method and domain decomposition of SPDEs are shown in Fig.(E.6). The $L_{2}$ norm of the standard deviation vector is considered as the metric for convergence. The difference between the norms of the third order and fourth order PC expansions is indistinguishable in the plot meaning that PC expansion converges at the third order. The total execution time of MCS and PCE are shown in Fig.(E.7). As the order of PC expansion increases, the execution time increases almost linearly. 


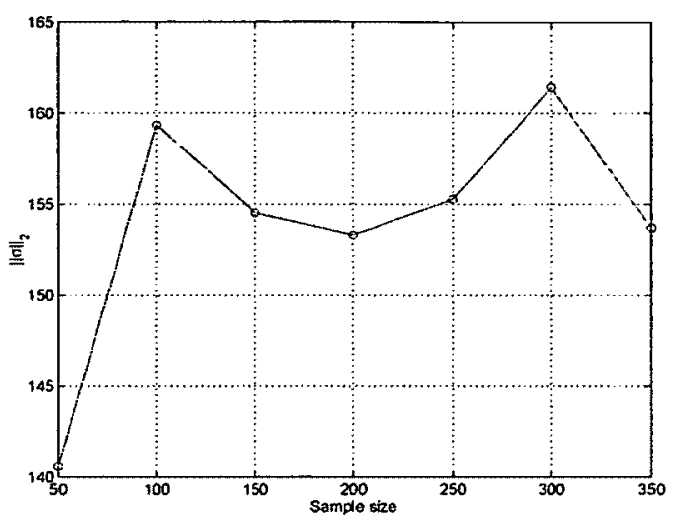

(a) MCS

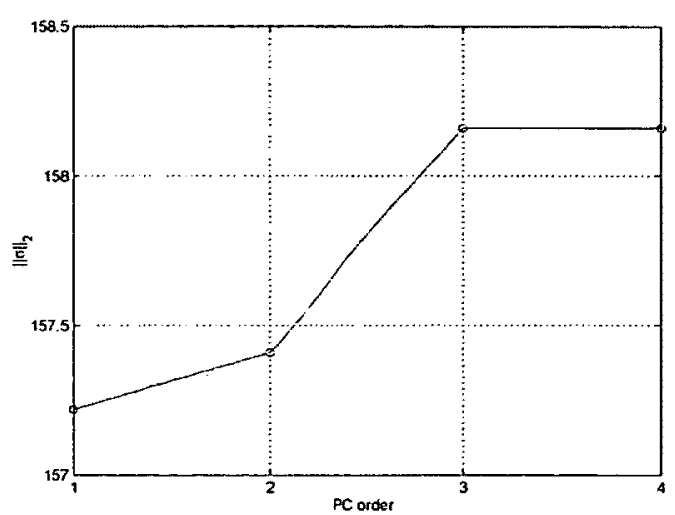

(b) PCE

Figure E.6: The convergence of MCS and PCE

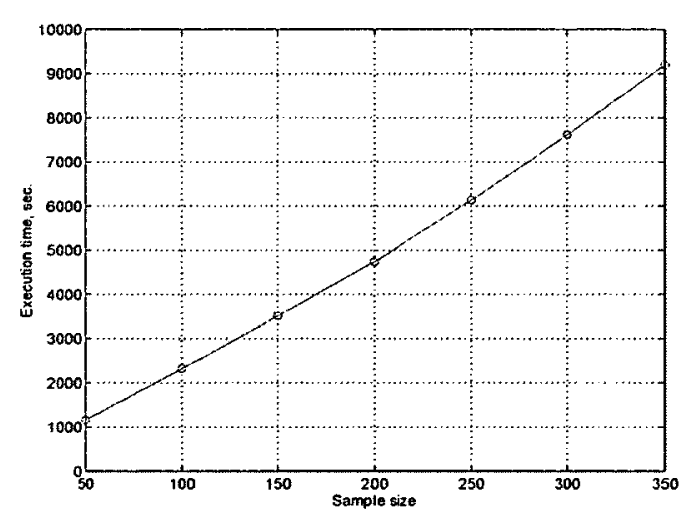

(a) MCS

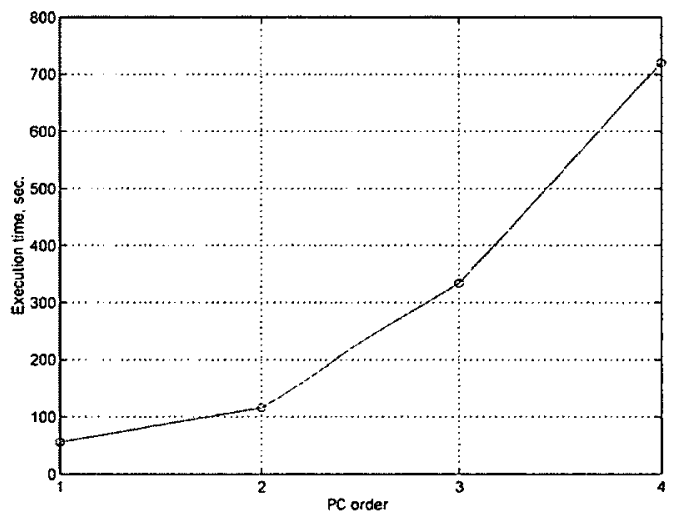

(b) PCE

Figure E.7: The total execution time of MCS and PCE

\section{Three-dimensional case:}

Next, we consider a three-dimensional steady-state heat transfer problem where the thermal diffusivity coefficient $\kappa(\mathbf{x}, \theta)$ is modeled as a lognormal stochastic process obtained from 
the underlying Gaussian process with an exponential covariance function. The volumetric heat source is assumed to be spatially invariant deterministic quantity $f(\mathbf{x}, \theta)=1.0$. The mean and standard deviation of the solution process are plotted in Fig.(E.8). Once again, the MCS and PCE results match identically in the scale of the graph. Fig.(E.9) shows the convergence of the MCS and PCE. The associated total execution times are plotted in Fig.(E.10). Evidently, the PCE approach is about 10 times faster than MCS.

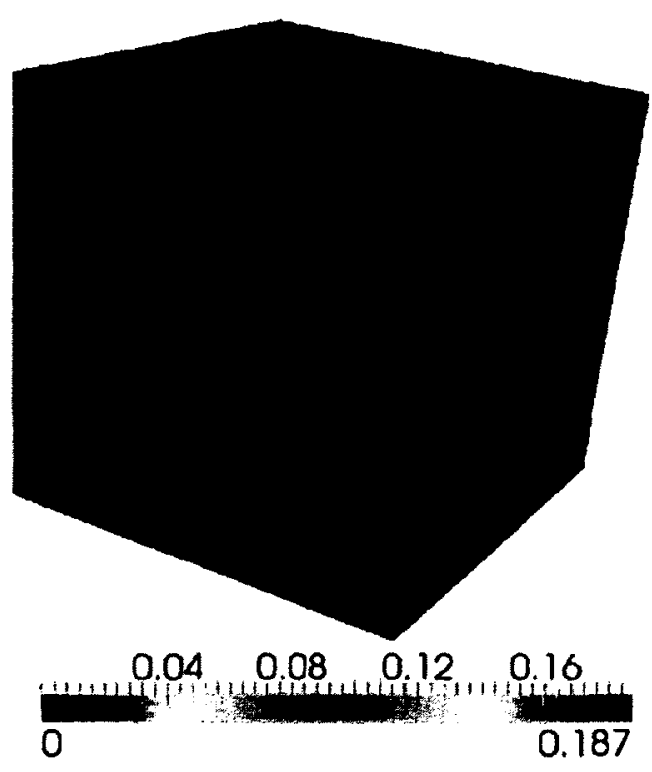

(a) Mean

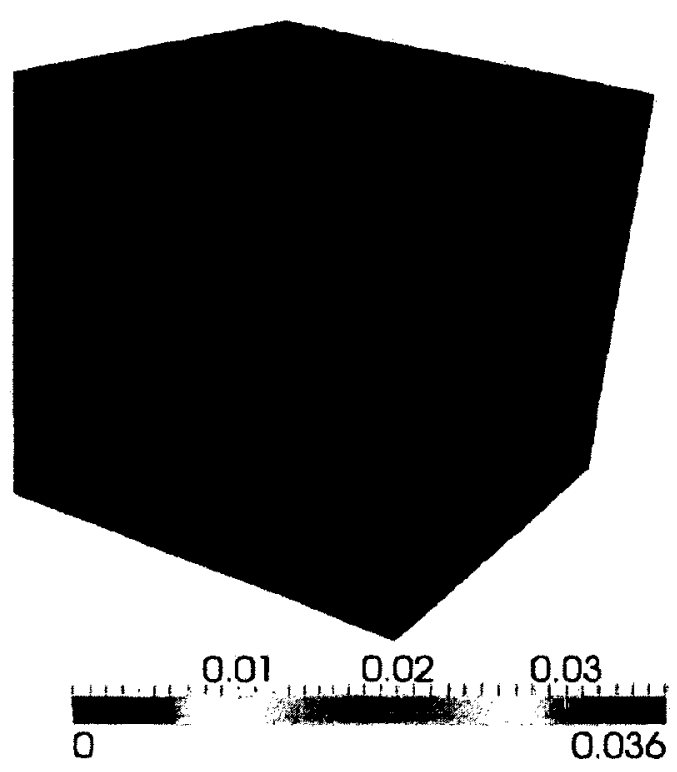

(b) Standard deviation

Figure E.8: The mean and standard deviation of the solution process 


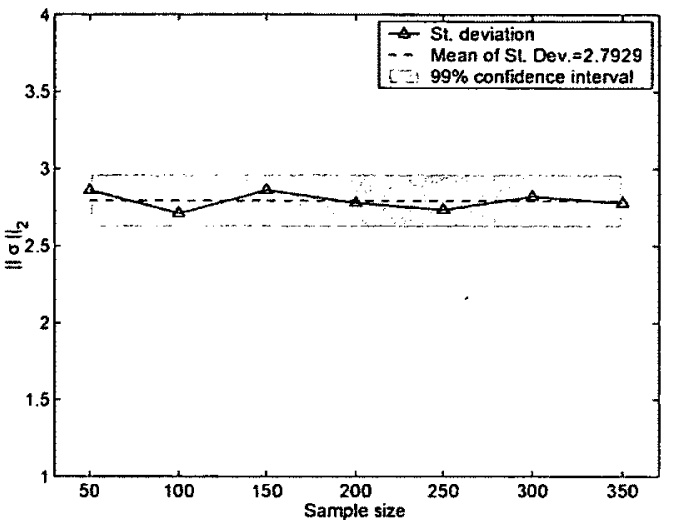

(a) MCS

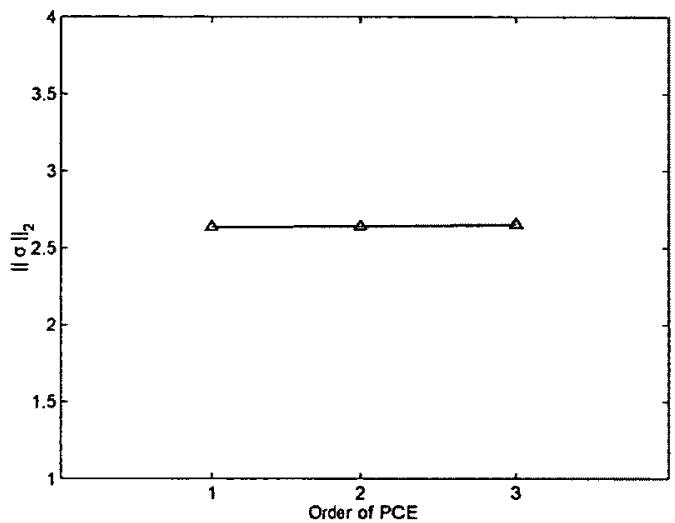

(b) PCE

Figure E.9: The convergence of MCS and PCE

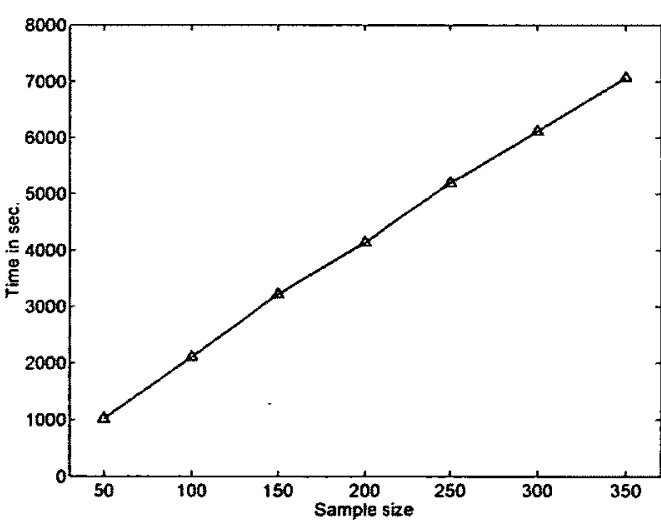

(a) MCS

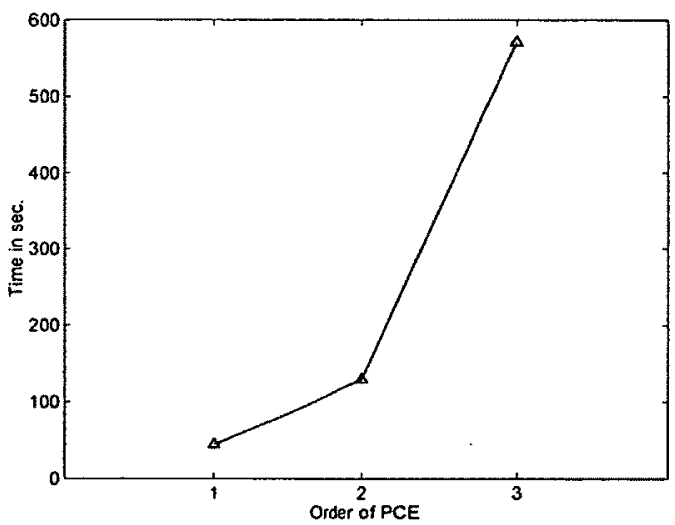

(b) PCE

Figure E.10: The total execution time of MCS and PCE 


\section{Appendix F}

\section{Comparison among Jacobi, Block-Jacobi}

\section{and Lumped Preconditioners}

In this section, we compare the performance of the PCGM equipped with Jacobi, BlockJacobi and Lumped preconditioners to tackle the extended Schur complement system. In particular, we tackle the extended Schur complement system defined as

$$
\mathcal{M}^{-1} \mathcal{S} \mathcal{U}_{\Gamma}=\mathcal{M}^{-1} \mathcal{G}_{\Gamma}
$$

where $\mathcal{M}^{-1}$ is the preconditioning matrix. For Jacobi, Block-Jacobi and Lumped preconditioners $\mathcal{M}^{-1}$ is defined respectively as 


$$
\begin{aligned}
& \mathcal{M}_{J}^{-1}=\operatorname{diag}\left(\mathcal{S}^{-1}\right) \\
& \mathcal{M}_{B J}^{-1}=\operatorname{blockdiag}\left(\mathbf{S}_{0}^{-1}, \ldots, \mathbf{S}_{0}^{-1}\right) \\
& \mathcal{M}_{L}^{-1}=\sum_{s=1}^{n_{s}} \mathcal{R}_{s}^{T}\left[\mathcal{A}_{\Gamma \Gamma}^{s}\right]^{-1} \mathcal{R}_{s}
\end{aligned}
$$

where $\mathrm{S}_{0}$ is the deterministic Schur complement matrix corresponding to the mean material properties.

For the numerical experiment, we again consider a two-dimensional steady-state flow through random media (with stochastic permeability coefficient) defined as

$$
\begin{aligned}
\nabla \cdot(\boldsymbol{\kappa}(\mathbf{x}, \theta) \nabla u(\mathbf{x}, \theta)) & =f(\mathbf{x}, \theta), \quad \mathbf{x} \in \Omega \\
u(\mathbf{x}, \theta) & =0, \quad \mathbf{x} \in \partial \Omega
\end{aligned}
$$

For simplicity, we represent the coefficient $\kappa(\mathbf{x}, \theta)$ as a longnormal random variable. Its polynomial chaos representation is given by $[96,145]$

$$
\kappa(\theta)=\mu_{\kappa} \sum_{i=0}^{L} \frac{\sigma_{g}^{i}}{i !} \Psi_{i}
$$

where

$$
\mu_{\kappa}=\exp \left(\mu_{g}+\frac{1}{2} \sigma_{g}^{2}\right)
$$

where $\mu_{g}$ and $\sigma_{g}$ are the mean and standard deviation of the underlying Gaussian random variable, respectively (see Appendix B).

The mean and standard deviation of the solution process for $\mu_{g}=0.0$ and $\sigma_{g}=0.25$ are shown in Fig.(F.1). 


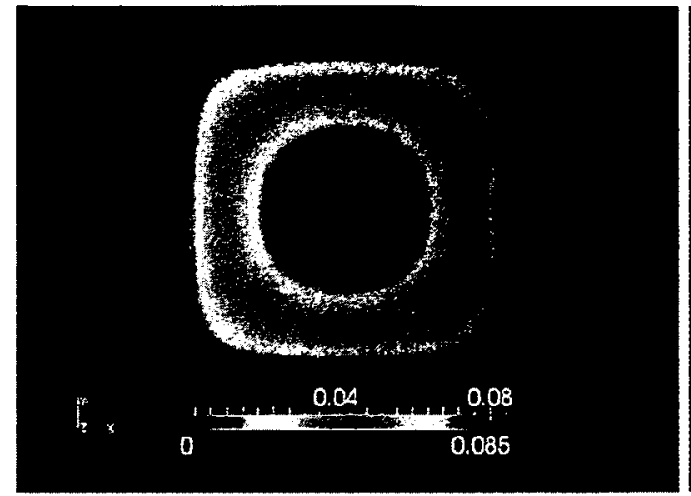

(a) Mean

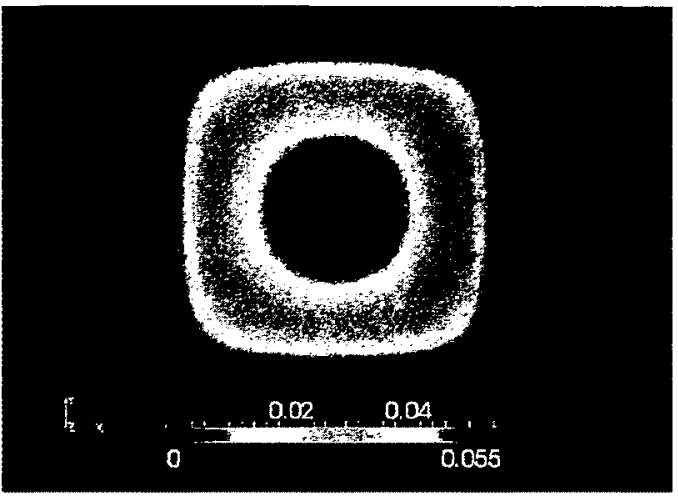

(b) Standard deviation

Figure F.1: The mean and standard deviation of the solution process

In Fig.(F.2), we show the performance of the Jacobi, Block-Jacobi and Lumped preconditioners for a fixed value of the $\mathrm{CoV}$ while increasing the order of PCE. For two subdomains, the iteration counts for both the Jacobi and Block-Jacobi preconditioners grow linearly as the order of PCE increases. For the lumped preconditioner, the iteration counts remain constant as we increase the order of PCE.

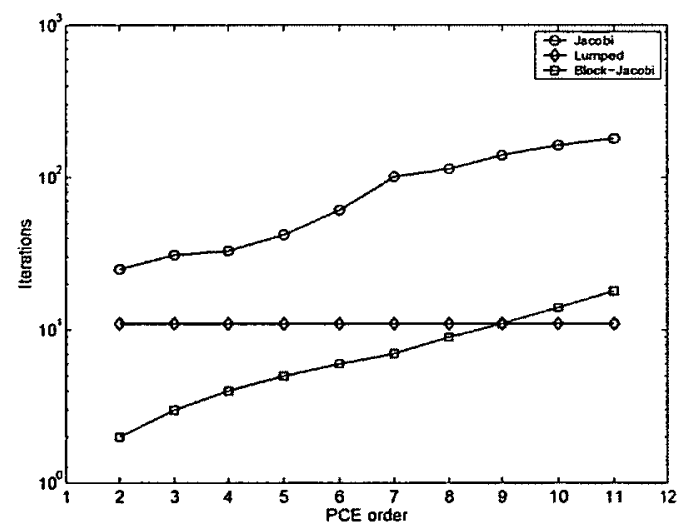

Figure F.2: Iteration counts for fixed $\mathrm{CoV}$ while increasing the order of PCE 
The performances of the Jacobi, Block-Jacobi and Lumped preconditioners for a fixed order of PCE while increasing the value of the $\mathrm{CoV}$ of the permeability coefficient are shown in Fig.(F.3). Clearly, increasing the level of uncertainty of the input parameter does not affect the performance of the Block-Jacobi and Lumped preconditioners. Although Block-Jacobi requires less number of iterations than the Lumped preconditioner, the explicit construction of Block-Jacobi preconditioner is computationally intensive.

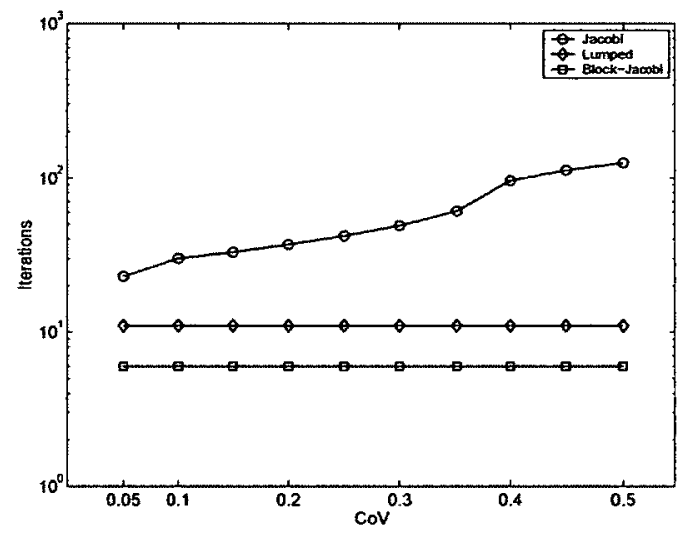

Figure F.3: Iteration counts for fixed order of PCE while increasing the value of the $C o V$ 


\section{Bibliography}

[1] I. Babuska, R. Tempone, and G. Zouraris. Solving elliptic boundary value problems with uncertain coefficients by the finite element method: the stochastic formulation. Computer Methods in Applied Mechanics and Engineering, 194:1251-1294, 2005.

[2] I. Babuska, R. Tempone, and G. Zouraris. Galerkin finite element approximations of stochastic elliptic partial differential equations. SIAM Journal on Numerical Analysis, 42:800-825, 2004.

[3] O. Maitre and O. Knio. Spectral Methods for Uncertainty Quantification With Applications to Computational Fluid Dynamics. Springer, New York, 2010.

[4] A. Toselli and O. Widlund. Domain Decomposition Methods - Algorithms and Theory, volume 34 of Springer Series in Computational Mathematics. Springer, Berlin, 2005.

[5] A. Quarteroni and A. Valli. Domain Decomposition Methods for Partial Differential Equations. Numerical Mathematics and Scientific Computation. Oxford University Press, USA, 1999.

[6] B. Smith, P. Bjorstad, and W. Gropp. Domain Decomposition: Parallel Multilevel Methods for Elliptic Partial Differential Equations. Cambridge University Press, New York, 1996.

[7] T. Mathew. Domain Decomposition Methods for the Numerical Solution of Partial Differential Equations. Number 61 in Lecture Notes in Computational Science and Engineering. Springer, Berlin, 2008.

[8] I. Babuska, F. Nobile, and R. Tempone. A stochastic collocation method for elliptic partial differential equations with random input data. SIAM Journal on Numerical Analysis, 45:1005-1034, 2007.

[9] A. Nouy. Recent developments in spectral stochastic methods for the numerical solution of stochastic partial differential equations. Archives of Computational Methods in Engineering, 16(3):251-285, 2009. 
[10] J. Faragher. Probabilistic methods for the quantification of uncertainty and error in computational fluid dynamics simulations, 2004. Australian Government, Department of Defence, Defence Science and Technology Organisation.

[11] R. Ghanem and P. Spanos. Stochastic Finite Element: A Spectral Approach. Springer-Verlag, New York, 1991.

[12] R. Ghanem. Uncertainty quantification in computational and prediction science. International Journal for Numerical Methods in Engineering, 80:671-672, 2009.

[13] M. Eldred and J. Burkardt. Comparison of non-intrusive polynomial chaos and stochastic collocation methods for uncertainty quantification. 47th AlAA Aerospace Sciences Meeting Including the New Horizons Forum and Aerospace Exposition, 2009.

[14] M. Eiermann and O. Ernst. Computational aspect of the stochastic finite element method. Computing and Visualization in Science. Sci., 10(1):3-15, 2007.

[15] M. Reagan, H. Najm, R. Ghanem, and O. Knio. Uncertainty quantification in reacting-flow simulations through non-intrusive spectral projection. Combustion and Flame, 132:545-555, 2009.

[16] O. Kino and O. Maitre. Uncertainty propagation in CFD using polynomial chaos decomposition. Fluid Dynamics Research, 38:616-640, 2006.

[17] D. Xiu. Numerical Methods for Stochastic Computations: A Spectral Method Approach. Princeton University Press, New Jersey, 2010.

[18] D. Xiu and G. Karniadakis. Modeling uncertainty in steady state diffusion problems via generalized polynomial chaos. Computer Methods in Applied Mechanics and Engineering, 191:4927-4948, 2002.

[19] O. Maitre, O. Knio, H. Najm, and R. Ghanem. A stochastic projection method for fluid flow: I. basic formulation. Journal of Computational Physics, 173:481-511, 2001 .

[20] X. Zhu. Uncertainty simulation using domain decomposition and stratified sampling. Master's thesis, Civil and Environmental Engineering Department, Carleton University, Ottawa, ON,Canada, 2006.

[21] C. Powell. Robust preconditioning for second-order elliptic PDEs with random field coefficients. Technical report, Manchester Institute for Mathematical Sciences, School of Mathematics, University of Manchester, 2006. Technical Report MIMS EPrint 2006.419. 
[22] I. Babuska and P. Chatzipantelidis. On solving elliptic stochastic partial differential equations. Computer Methods in Applied Mechanics and Engineering, 191:40934122, 2002.

[23] A. Keese. A review of recent developments in the numerical solution of stochastic partial differential equations (stochastic finite elements). Technical report, Institute of Scientific Computing Technical University Braunschweig, Brunswick, Germany, 2003. Technical Research Report: Informatikbericht Nr.: 2003-06.

[24] R. Ghanem and A. Doostan. On the construction and analysis of stochastic models: characterization and propagation of the errors associated with limited data. Journal of Computational Physics, 217:63-81, 2006.

[25] R. Ghanem. Ingredients for a general purpose stochastic finite elements implementation. Computer Methods in Applied Mechanics and Engineering, 168:19-34, 1999.

[26] M. Schevenels, G. Lombaert, and G. Degrande. Application of the stochastic finite element method for gaussian and non-gaussian systems. Proceeding of ISMA2004, pages 3299-3314, 2004.

[27] D. Ghosh, Ph. Avery, and C. Farhat. A FETI-preconditioned conjugate gradient method for large-scale stochastic finite element problems. International Journal for Numerical Methods in Engineering, 80:914-931, 2009.

[28] G. Stefanou. The stochastic finite element method: Past, present and future. Computer Methods in Applied Mechanics and Engineering, 198:1031-1051, 2009.

[29] P. Dosterta, Y. Efendieva, and T.Y. Houb. Multiscale finite element methods for stochastic porous media flow equations and application to uncertainty quantification. Computer Methods in Applied Mechanics and Engineering, 197:3445-3455, 2008.

[30] H. Najm. Uncertainty quantification and polynomial chaos techniques in computational fluid dynamics. Annual Review of Fluid Mechanics, 41:35-52, 2009.

[31] R. Ghanem and S. Dham. Stochastic finite element analysis for multiphase flow in heterogeneous porous media. Porous Media, 32:239-262, 1998.

[32] D. Xiu, D. Lucor, C. Su, and G. Karniadakis. Stochastic modeling of flow-structure interactions using generalized polynomial chaos. Journal of Fluids Engineering, 124(1):51-59, 2002.

[33] M. Reagan, H. Najm, B. Debusschere, O. Le Maitre, O. Knio, and R. Ghanem. Spectral stochastic uncertainty quantification in chemical systems. Proceedings of the Physical Society, 8:607-632, 2004. 
[34] H.Najm, B. Debusschere, Y. Marzouk, S. Widmer, and O. Le Maitre. Uncertainty quantification in chemical systems. International Journal for Numerical Methods in Engineering, 80(6-7):789-814, 2009.

[35] S. Geneser, R. Kirby, and R. MacLeod. Application of stochastic finite element methods to study the sensitivity of ecg forward modeling to organ conductivity. IEEE Transactions on Biomedical Engineering, 55(1):31-40, 2008.

[36] H. Elman, O. Ernst, D. O'Leary, and M. Stewart. Efficient iterative algorithms for the stochastic finite element method with application to acoustic scattering. Computer Methods in Applied Mechanics and Engineering, 194:1037-1055, 2005.

[37] R. Ghanem and R. Kruger. Numerical solution of spectral stochastic finite element systems. Computer Methods in Applied Mechanics and Engineering, 129(3):289303, 1996.

[38] R. Ghanem, G. Saad, and A. Doostan. Efficient solution of stochastic systems: Application to the embankment dam problem. Structural Safety, 29(3):238 - 251, 2007. A Benchmark Study on Reliability in High Dimensions.

[39] R. Ghanem and P Spanos. Polynomial chaos in stochastic finite elements. Journal of Applied Mechanics, 57(1):197-202, 1990.

[40] M. Deb, I. Babuska, and J. Oden. Solution of stochastic partial differential equations using galerkin finite element techniques. Computer Methods in Applied Mechanics and Engineering, 190:6359-6372, 2001.

[41] Nitin Agarwal and N.R. Aluru. Stochastic modeling of coupled electromechanical interaction for uncertainty quantification in electrostatically actuated MEMS. Computer Methods in Applied Mechanics and Engineering, 197(43-44):3456 - 3471, 2008.

[42] D. Xiu. Efficient collocational approach for parametric uncertainty analysis. Communications in Computational Physics, 2:293-309, 2007.

[43] D. Xiu and J.S. Hesthaven. High-order collocation methods for differential equations with random inputs. SIAM Journal on Scientific Computing, 27:1118-1139, 2005.

[44] H. Elman, Ch. Miller, E. Phipps, and R. Tuminaro. Assessment of collocation and galerkin approaches to linear diffusion equations with random data. International Journal for Uncertainty Quantification, 1:19-33, 2011.

[45] D. Xiu. Fast numerical methods for stochastic computations: A review. Communications in Computational Physics, 5:242-272, 2009. 
[46] J. Back, F. Nobile L. Tamellini, and R. Tempone. Stochastic spectral galerkin and collocation methods for pdes with random coefficients: A numerical comparison. In Spectral and High Order Methods for Partial Differential Equations, volume 76 of Lecture Notes in Computational Science and Engineering, pages 43-62. Springer Berlin Heidelberg, 2011.

[47] P. Nair and A. Keane. Stochastic reduced basis methods. AlAA Journal, 40(8):1653$1664,2002$.

[48] S. Sachdeva, P. Nair, and A. Keane. Comparative study of projection schemes for stochastic finite element analysis. Computer Methods in Applied Mechanics and Engineering, 195(19-22):2371-2392, 2006.

[49] S. Sachdeva, P. Nair, and A. Keane. Hybridization of stochastic reduced basis methods with polynomial chaos expansions. Probabilistic Engineering Mechanics, 21(2):182-192, 2006.

[50] M. Pellissetti and R. Ghanem. Iterative solution of systems of linear equations arising in the context of stochastic finite elements. Advances in Engineering Software, 31(8-9):607-616, 2000.

[51] O. Maitre, O. Knio, B. Debusschere, H. Najm, and R. Ghanem. A multigrid solver for two-dimensional stochastic diffusion equations. Computer Methods in Applied Mechanics and Engineering, 192(41-42):4723-4744, 2003.

[52] Y. Saad. Iterative methods for sparse linear systems. SIAM, Philadelphia, 2 edition, 2003.

[53] D. Chung, M. Gutirrez, L. Graham-Brady, and F. Lingen. Efficient numerical strategies for spectral stochastic finite element models. International Journal for Numerical Nethods in Engineering, 64:1334-1349, 2005.

[54] A. Keese and H.Matthies. Parallel computation of stochastic groundwater flow. In NIC Symposium, volume 20, pages 399-408, John von Neumann Institute for Computing, 2004.

[55] H. Elman and D. Furnival. Solving the stochastic steady-state diffusion problem using multigrid. IMA Journal of Numerical Analysis, 27:675-688, 2007.

[56] E. Rosseel, T. Boonen, and S. Vandewalle. Algebraic multigrid for stationary and time-dependent partial differential equations with stochastic coefficients. Numerical Linear Algebra With Applications, 15(2-3):141-163, 2008.

[57] C. Powell and H. Elman. Block-diagonal preconditioning for spectral stochastic finite-element systems. IMA Journal of Numerical Analysis, 29:350-375, 2009. 
[58] S. Geneser, R. Kirby, and R. MacLeod. Application of stochastic finite element methods to study the sensitivity of ecg forward modeling to organ conductivity. IEEE Transactions on Biomedical Engineering, 55(1):31-40, 2008.

[59] W. Gropp. Parallel computing and domain decomposition. In Fifth International Symposium on Domain Decomposition Methods for Partial Differential Equations, pages 394-361. Society for Industrial and Applied Mathematics, 1992.

[60] 1. Navon and Y. Cai. Domain decomposition and parallel processing of a finite element model of the shallow water equations. Computer Methods in Applied Mechanics and Engineering, 106:179-212, 1993.

[61] A. Klawonn and O. Rheinbach. Highly scalable parallel domain decomposition methods with an application to biomechanics. ZAMM - Journal of Applied Mathematics and Mechanics / Zeitschrift fr Angewandte Mathematik und Mechanik, 90:5$32,2010$.

[62] K. Pierson, G. Reese, and P. Raghavan. Experiences with FETI-DP in a production level finite element application. In Fourteenth International Conference on Domain Decomposition Methods, pages 233-240. Springer, 2003.

[63] V. Korneev and U. Langer. Domain decomposition methods and preconditioning. In Encyclopedia of Computational Mechanics, pages 617-647. John Wiley and Sons Ltd, 2004.

[64] C. Farhat, M. Lesoinne, and K. Pierson. A scalable dual-primal domain decomposition method. Numerical Linear Algebra with Applications, 7:687-714, 2000.

[65] Y. Fragakis and M. Papadrakakis. The mosaic of high performance domain decomposition methods for structural mechanics: Formulation, interrelation and numerical efficiency of primal and dual methods. Computer Methods in Applied Mechanics and Engineering, 192:3799-3830, 2003.

[66] J. Cros. A preconditioer for the schur complement domain decomposition method. -In Fourteenth International Conference on Domain Decomposition Methods, pages 373-380. National Autonomous University of Mexico (UNAM), 2003.

[67] J. Mandel, C. Dohrmann, and R. Tezaur. An algebraic theory for primal and dual substructuring methods by constraints. Applied Numerical Mathematics, 54:167$193,2005$.

[68] A. Sarkar, N. Benabbou, and R. Ghanem. Domain decomposition of stochastic PDEs: Theoretical formulations. International Journal for Numerical Methods in Engineering, 77:689-701, 2009. 
[69] A. Sarkar, N. Benabbou, and R. Ghanem. Domain decomposition of stochastic PDEs: Performance study. International Journal of High Performance Computing Applications, 2010. Accepted.

[70] W. Subber, H. Monajemi, M. Khalil, and A. Sarkar. A scalable parallel uncertainty analysis and data assimilation framework applied to some hydraulic problems. In International Symposium on Uncertainties in Hydrologic and Hydraulic, Montreal, Canada, 2008.

[71] De. Roeck and P. Tallec. Analysis and test of a local domain decomposition preconditioner. In Fourth International Symposium on Domain Decomposition Methods for Partial Differential Equations, pages 112-128, Philadelphia, 1991. SIAM.

[72] T. Chan and T. Mathew. Domain decomposition algorithms. Acta Numerica, 3:61$143,1994$.

[73] O. Axelsson, R. Radim, and M. Neytcheva. Preconditioning of boundary value problems using elementwise schur complements. SIAM J. Matrix Anal. Appl., 31(2):767$789,2009$.

[74] W. Subber and A. Sarkar. Domain decomposition of stochastic PDEs: A novel preconditioner and its parallel performance. In High Performance Computing Symposium, volume 5976 of Lecture Notes in Computer Science, pages 251-268. Springer, 2010.

[75] W. Subber and A. Sarkar. Domain decomposition method of stochastic PDEs: An iterative solution technique using one-level parallel preconditioner. Journal of Probabilistic Engineering Mechanics, 2012. Submitted.

[76] W. Subber and A. Sarkar. Domain decomposition methods of stochastic PDEs: A two-level scalable preconditioner. Journal of Physics: Conference Series, 341(1), 2012.

[77] W. Subber and A. Sarkar. A domain decomposition method of stochastic PDEs: An iterative solution techniques using a two-level scalable preconditioner. Journal of Computational Physics, 2012. Submitted.

[78] B. Sousedik and J. Mandel. On the equivalence of primal and dual substructuring preconditioners. Electronic Transactions in Numerical Analysis, 31:384-402, 2008.

[79] W. Subber and A. Sarkar. Primal and dual-primal iterative substructuring methods of stochastic PDEs. Journal of Physics: Conference Series, 256(1), 2010. 
[80] W. Subber and A. Sarkar. Domain decomposition methods of stochastic PDEs. In The Twentieth International Conference on Domain Decomposition Methods (DD20), California, USA, 2011. Accepted.

[81] C. Farhat, M. Lesoinne, P. LeTallec, K. Pierson, and D. Rixen. FETI-DP: a dual-primal unified FETI method-part I: A faster alternative to the two-level FETI method. Numerical Methods in Engineering, 50:1523-1544, 2001.

[82] Xuemin Tu. Three-level BDDC in three dimensions. SIAM J. Sci. Comput., 29(4):1759-1780, June 2007.

[83] J. Li and O. Widlund. FETI-DP, BDDC, and block Cholesky methods. International Journal for Numerical Methods in Engineering, 66:250-271, 2006.

[84] S. Brenner and L. Sung. BDDC and FETI-DP without matrices or vectors. Computer Methods in Applied Mechanics and Engineering, 196:1429-1435, 2007.

[85] J. Mandel and B. Sousedik. BDDC and FETI-DP under minimalist assumptions. Computing, 81:269-280, 2007.

[86] W. Subber and A. Sarkar. Dual-primal domain decomposition method for uncertainty quantification. Journal of Computer Methods in Applied Mechanics and Engineering, 2012. To be Submitted.

[87] S. Balay, K. Buschelman, W. Gropp, D. Kaushik, M. Knepley, L. McInnes, B. Smith, and H. Zhang. PETSc Web page, 2009. http://www.mcs.anl.gov/petsc.

[88] Message passing interface forum, 2009. http://www.mpi-forum.org.

[89] G. Karypis and V. Kumar. METIS unstructured graph partitioning and sparse matrix ordering system, 1995.

[90] J. L. Lions, Y. Maday, and G. Turinici. Re solution d'edp par un sche ma en teps parare el. C.R. Acad. Sci. Paris Ser. I Math, 332:661-668, 2001.

[91] C. Farhat and M. Chandesris. Time-decomposed parallel time-integrators: theory and feasibility studies for fluid, structure, and fluid-structure application. International Journal for Numerical Methods in Engineering, 58:1397-1434, 2003.

[92] D. Griffiths and G. Fenton, editors. Probabilistic Methods in Geotechnical Engineering. Number 491 in CISM International Centre for Mechanical Sciences. Springer, 2007.

[93] R. H. Cameron and W. T. Martin. The orthogonal development of nonlinear functionals in series of Fourier-Hermite functionals. Ann. Math, 48:385 - 392, 1947. 
[94] R. Ghanem and M. Pellissetti. Adaptive data refinement in the spectral stochastic finite element method. Communications in Numerical Methods in Engineering, 18(2):141-151, 2002.

[95] R. Ghanem. Probabilistic characterization of transport in heterogeneous media. Computer Methods in Applied Mechanics and Engineering, 158:199-220, 1998.

[96] R. Ghanem. Stochastic finite elements with multiple random non-gaussian properties. Journal of Engineering Mechanics, 125:26-41, 1998.

[97] G. Schueller. A state-of-the-art report on computational stochastic mechanics. Probabilistic Engineering Mechanics, 12(4):197 - 321, 1997.

[98] C. Blanze and $\mathrm{Ph}$. Rouch. Analysis of structures with stochastic interfaces in the medium-frequency range. Journal of Computational Acoustics, 13(04):711-729, 2005.

[99] S. Bitzarakis, M. Papadrakakis, and A. Kotsopulos. Parallel solution techniques in computational structural mechanics. Computer Methods in Applied Mechanics and Engineering, 148(1-2):75 - 104, 1997.

[100] Ch. Jin, X. Cai, and C. Li. Parallel domain decomposition methods for stochastic elliptic equations. SIAM Journal on Scientific Computing, 29:2096-2114, 2007.

[101] E. Ullmann. A kronecker product preconditioner for stochastic galerkin finite element discretizations. SIAM Journal for Scientific Computation, 32:923-946, 2010.

[102] L. Formaggia, M. Sala, and F. Saleri. Domain decomposition techniques. In Numerical Solution of Partial Differential Equations on Parallel Computers, volume 51 of Lecture Notes in Computational Science and Engineering, pages 135-164. Springer, 2005.

[103] Greenbaum Anne. Iterative Methods for Solving Linear Systems. Society for Industrial and Applied Mathematics, 1997.

[104] H. Vereecken, O. Neuendorf, G. Lindenmayr, and A. Basermann. A schwarz domain decomposition method for solution of transient unsaturated water flow on parallel computers. Ecological Modelling, 93:275-289, 1996.

[105] Y. Zhuang and X. Sun. Stable, globally non-iterative, non-overlapping domain decomposition parallel solvers for parabolic problems. In Proceedings of the 2001 ACM/IEEE conference on Supercomputing (CDROM), Supercomputing '01, pages 19-19, New York, NY, USA, 2001. ACM. 
[106] G. Kahou, E. Kamgnia, and B. Philippe. An explicit formulation of the multiplicative schwarz preconditioner. Appl. Numer. Math., 57(11-12):1197-1213, November 2007.

[107] C. Dohrmann. A preconditioner for substructuring based on constrained energy minimization. SIAM Journal on Scientific Computing, 25:246-258, 2003.

[108] J. Mandel and C. Dohrmann. Convergence of a balancing domain decomposition by constraints and energy minimization. Numerical Linear Algebra with Applications, 10:639-659, 2003.

[109] M. Papadrakakis, G. Stavroulakis, and A. Karatarakis. A new era in scientific computing: Domain decomposition methods in hybrid CPU-GPU architectures. Computer Methods in Applied Mechanics and Engineering, 200(13 - 16):1490 - 1508, 2011.

[110] P. Gosselet and Chr. Rey. Non-overlapping domain decomposition methods in structural mechanics. Archives of Computational Methods in Engineering, 13:515-572, 2007.

[111] T. Chan. Domain decomposition algorithms and computational fluid dynamics. International Journal of High Performance Computing Applications, 2:72-83, 1988.

[112] W. Gropp and D. Keyes. Domain decomposition methods in computational fluid dynamics. International Journal for Numerical Methods in Fluids, 14:147-165, 1992.

[113] C. Farhat, Ph. Avery, R. Tezaur, and J. Li. FETI-DPH: A dual-primal domain decomposition method for acoustic scattering. Journal of Computational Acoustics, 13:499-524, 2005.

[114] F. Magoules. Mesh Partitioning Techniques and Domain Decomposition Methods. SAXE-COBURG Prublications, Scotland, 2007.

[115] T. Barth, T. Chan, and W. Tang. Parallel domain-decomposition preconditioning for computational fluid dynamics. In Vector and Parallel Processing VECPAR'98, volume 1573 of Lecture Notes in Computer Science, pages 176-202. Springer Berlin Heidelberg, 1999.

[116] J. Mandel. Balancing domain decomposition. Communication in Numerical Methods in Engineering, 9:233-241, 1993.

[117] J. Mandel and M. Brezina. Balancing domain decomposition for problems with large jumps in coefficients. Mathematics of Computation, 65:1387-1401, 1996. 
[118] Z. Zheng, B. Simeon, and L. Petzold. A stabilized explicit Lagrange multiplier based domain decomposition method for parabolic problems. Journal of Computational Physics, 227(10):5272 - 5285, 2008.

[119] C. Farhat and F. Roux. A method of finite element tearing and interconnecting and its parallel solution algorithm. International Journal for Numerical Methods in Engineering, 32:1205-1227, 1991.

[120] C. Farhat, J. Mandel, and F. Roux. Optimal convergence properties of the FETI domain decomposition method. Computer Methods in Applied Mechanics and Engineering, 115:365-385, 1994.

[121] H. Schwarz. Gesammelte mathematische abhandlungen, 1890. First published in Vierteljahrsschrift der Naturforschenden Gesellschaft in Zurich, volume 15, 1870, pp. 272-286.

[122] M. Storti, L. Dalcin, R. Paz, A. Yommi, V. Sonzogni, and N. Nigro. A preconditioner for the schur complement matrix. Advances in Engineering Software, 37:754-762, 2006.

[123] C. Dohrmann. An approximate BDDC preconditioner. Numerical Linear Algebra with Applications, 14:149-168, 2007.

[124] J. Sisteka, J. Novotny, J. Mandel, M. Certikovad, and P. Burdad. BDDC by a frontal solver and the stress computation in a hip joint replacementn. Mathematics and Computers in Simulation, 80:1310-1323, 2010.

[125] C. Brenner. A functional analytic framework for BDDC and FETI-DP. In Domain decomposition methods in science and engineering XVII, pages 239-246. Springer, 2008.

[126] J. Mandel and R. Tezaur. On the convergence of a substructuring method with lagrange multipliers. Numerische Mathematik, 73:473-487, 1996.

[127] C. Farhat, A. Macedo, M. Lesoinne, F. Roux, F. Magoules, and A. Bourdonnaie. Two-level domain decomposition methods with lagrange multipliers for the fast iterative solution of acoustic scattering problems. Computer Methods in Applied Mechanics and Engineering, 184(24):213 - 239, 2000.

[128] C. Farhat and J. Mandel. The two-level FETI method for static and dynamic plate problems part. I: An optimal iterative solver for biharmonic systems. Computer Methods in Applied Mechanics and Engineering, 155(12):129 - 151, 1998. 
[129] C. Farhat, K. Pierson, and M. Lesoinne. The second generation FETI methods and their application to the parallel solution of large-scale linear and geometrically nonlinear structural analysis problems. Computer Methods in Applied Mechanics and Engineering, 184(2-4):333 - 374, 2000.

[130] W. Yao, M. Jin, and T. Krein. A highly efficient domain decomposition method applied to 3-D finite-element analysis of electromechanical and electric machine problems. IEEE Transactions on Energy Conversion, 27:1078 - 1086, 2012.

[131] A. Klawonn and O. Rheinbach. Inexact FETI-DP methods. International Journal for Numerical Methods in Engineering, 69:284-307, 2007.

[132] J. Mandel, R. Tezaur, and C. Farhat. A scalable substructuring method by lagrange multipliers for plate bending problems. SIAM J. Numer. Anal., 36(5):1370-1391, July 1999.

[133] A. Klawonn and O. Widlund. Selecting constraints in dual-primal feti methods for elasticity in three dimensions. In Domain Decomposition Methods in Science and Engineering, volume 40 of Lecture Notes in Computational Science and Engineering, pages 67-81. Springer Berlin Heidelberg, 2005.

[134] C. Farhat, J. Li, and Ph. Avery. A FETI-DP method for the parallel iterative solution of indefinite and complex-valued solid and shell vibration problems. International Journal for Numerical Methods in Engineering, 63(3):398-427, 2005.

[135] O. Rheinbach. Parallel iterative substructuring in structural mechanics. Archives of Computational Methods in Engineering, 16:425-463, 2009.

[136] J. Mandel and R. Tezaur. On the convergence of a dual-primal substructuring method. Numerische Mathematik, 88:543-558, 2001.

[137] A. Klawonn, O. Widlund, and M. Dryja. Dual-primal FETI methods for threedimensional elliptic problems with heterogeneous coefficients. SIAM Journal on Numerical Analysis, 40:159-179, 2002.

[138] C. Farhat and J. Li. An iterative domain decomposition method for the solution of a class of indefinite problems in computational structural dynamics. Applied Numerical Mathematics, 54(2):150-166, 2005.

[139] J. Shewchuk. An introduction to the conjugate gradient method without the agonizing pain. Technical report, School of Computer Science, Carnegie Mellon University, 1994. http://www.cs.cmu.edu/ quake-papers/painless-conjugate-gradient.pdf.

[140] C. Kelley. terative Methods for Linear and Nonlinear Equations. SIAM, Philadelphia, 1995. 
[141] M. Benzi. Preconditioning techniques for large linear systems: A survey. Journal of Computational Physics, 182:418-477, 2002.

[142] D. Keyes. How scalable is domain decomposition in practice? In 11th International Conference on Domain Decomposition Methods, pages 286-297, 1998.

[143] J. Remacle. C. Geuzaine. Gmsh: a three-dimensional finite element mesh generator with built-in pre- and post-processing facilities. Journal for Numerical Methods in Engineering, 79:1309-1331, 2009.

[144] M. Bhardwaj, D. Day, C. Farhat, M. Lesoinne, K. Pierson, and D. Rixen. Application of the FETI method to ASCI problems scalability results on 1000 processors and discussion of highly heterogeneous problems. International Journal for Numerical Methods in Engineering, 47(1-3):513-535, 2000.

[145] R. Ghanem. The nonlinear gaussian spectrum of log-normal stochastic processes and variables. Journal of Applied Mechanics, Transactions ASME, 66:964-973, 1999.

[146] W. Subber and A. Sarkar. Two-level domain decomposition methods for uncertainty quantification. In 54th AIAA/ASME/ASCE/AHS/ASC Structures, Structural Dynamics, and Materials Conference. Submitted, 2013.

[147] W. Subber and A. Sarkar. Scalable two-level domain decomposition algorithms for stochastic systems. In 11th International Conference on Structural Safety and Reliability. Submitted, 2013.

[148] O. Widlund. Exotic coarse spaces for schwarz methods for lower order and spectral finite elements. Contemporary Mathematics, 180:131-136, 1994.

[149] Clumeq, 2012. http://www.clumeq.ca/index.php/en/about/computers/guillimin.

[150] R. Ghanem. Ingredients for a general purpose stochastic finite elements implementation. Computer Methods in Applied Mechanics and Engineering, 168:19-34, 1999.

[151] E. Anderson, Z. Bai, C. Bischof, S. Blackford, J. Demmel, J. Dongarra, J. Du Croz, A. Greenbaum, S. Hammarling, A. McKenney, and D. Sorensen. LAPACK Users' Guide. Society for Industrial and Applied Mathematics, Philadelphia, PA, third edition, 1999.

[152] V. Hernandez, J. Roman, and V. Vidal. SLEPc: A scalable and flexible toolkit for the solution of eigenvalue problems. ACM Transactions on Mathematical Software, 31(3):351-362, 2005. 
[153] Ph. Avery and C. Farhat. The FETI family of domain decomposition methods for inequality-constrained quadratic programming: Application to contact problems with conforming and nonconforming interfaces. Computer Methods in Applied Mechanics and Engineering, 198(21-26):1673 - 1683, 2009.

[154] D. Griffiths I. Smith. Programming The Finite Element Method. John Wiley and Sons, West Sussex, England, 4 edition, 2004.

[155] A. Klawonn and O. Rheinbach. A parallel implementation of dual-primal FETI methods for three-dimensional linear elasticity using transformation of basis. SIAM Journal on Scientific Computing, 28:1886-1906, 2006.

[156] A. Klawonn and O. Widlund. Dual-primal FETI methods for linear elasticity. Communications on Pure and Applied Mathematics, 59:1523-1572, 2006.

[157] C. Farhat, P. Chen, F. Risler, and F. Roux. A unified framework for accelerating the convergence of iterative substructuring methods with lagrange multipliers. Numerical Methods in Engineering, 42:257-288, 1998.

[158] W. Subber and A. Sarkar. Performance of a parallel time integrator for noisy nonlinear system. In Non-Deterministic Approaches Forum, 49th AIAA/ASME/ASCE/AHS/ASC Structures, Structural Dynamics, and Materials Conference. Paper no: AIAA-2008-1924, 2008.

[159] W. Subber and A. Sarkar. A parallel time integrator for noisy nonlinear system. Journal of Probabilistic Engineering Mechanics, 2008. Accepted pending revision.

[160] P. E. Kloeden and E. Platen. Numerical Solution of Stochastic Differential Equations. Springer-Verlag, 1992.

[161] K. Burrage and T. Tian. Numerical methods for strong solutions of stochastic differential equations: An overview. Royal Society of London Proceedings Series A, 460:373-402, 2004.

[162] G. Bal. Parallelization in time of (stochastic) ordinary differential equations, 2003. www.columbia.edu/gb2030/PAPERS/paralleltime.pdf.

[163] D. Higham and P. Kloeden. Convergence and stability of implicit methods for jump-diffusion systems. International Journal of Numerical Analysis and Modeling, 3(2):125-140, 2006.

[164] K. Burrage P. Burrage. Order conditions of stochastic Runge-Kutta methods by BSeries. SIAM Journal of Numerical Analysis, 38:1626-1646, 2000. 
[165] D. Roy. A family of weak stochastic newmark methods for simplified and efficient monte carlo simulations of oscillators. International Journal for Numerical Methods in Engineering, 67:364-399, 2006.

[166] J. Guckenheimer and Ph. Holmes. Nonlinear Oscillations, Dynamical Systems, and Bifurcations of Vector Fields, volume 42. Springer, 1983.

[167] S. Strogatz. Nonlinear Dynamics And Chaos. Westview Press, 2008.

[168] S. Lynch. Dynamical Systems with Applications using MATLAB. Springer, 2004.

[169] G. Evensen. Data Assimilation: The Ensemble Kalman Filter. Springer, 2006.

[170] M. Khalil, A. Sarkar, and S. Adhikari. Tracking noisy limit cycle oscillations with nonlinear filters. Journal of Sound and Vibration, 329:150-170, 2010.

[171] M. Khalil, A. Sarkar, and S. Adhikari. Nonlinear filters for chaotic oscillatory systems. Journal of Nonlinear Dynamics, 55:113-137, 2009.

[172] J. Li and O. Widlund. On the use of inexact subdomain solvers for BDDC algorithms. Computer Methods in Applied Mechanics and Engineering, 196:1415-1428, 207.

[173] A. Nouy. Identification of multi-modal random variables through mixtures of polynomial chaos expansions. Comptes Rendus Mecanique, 338:698-703, 2010.

[174] M. Arnst, R. Ghanem, and C. Soize. Identification of bayesian posteriors for coefficients of chaos expansions. Journal of Computational Physics, 229(9):3134-3154, May 2010.

[175] R. Ghanem, A. Doostan, and J. Red-Horse. A probabilistic construction of model validation. Computer Methods in Applied Mechanics and Engineering, 197:25852595, 2008.

[176] C. Desceliers, R. Ghanem, and C. Soize. Maximum likelihood estimation of stochastic chaos representations from experimental data. International Journal of Numerical Method in Engineering, 66:978-1001, 2006.

[177] G. Stefanou, A. Nouy, and A. Clement. Identification of random shapes from images through polynomial chaos expansion of random level set functions. International Journal of Numerical Method in Engineering, 79:127-155, 2009.

[178] A. Sarkar, N. Benabbou, and R. Ghanem. Domain decompostion of stochastic PDEs and its parallel. In Proceedings of The 20th International Symposium on HighPerformance Computing in an Advanced Collaborative Environment. IEEE Conference Publications, 2006. 
[179] Ph. Frauenfelder, Ch. Schwab, and R. Todor. Finite elements for elliptic problems with stochastic coefficients. Computer Methods in Applied Mechanics and Engineering, 194:205-228, 2005. 